\title{
Rhodacyclopentanones as Linchpins for the Atom Economical Assembly of Diverse Polyheterocycles
}

\author{
Gang-Wei Wang, ${ }^{\dagger}$ Olivia Boyd,${ }^{\dagger}$ Tom A. Young, ${ }^{\dagger}$ Sophie M. Bertrand ${ }^{\dagger}$ and John F. Bower ${ }^{*},{ }^{\dagger}$ \\ ${ }^{\dagger}$ School of Chemistry, University of Bristol, Bristol, BS8 1TS, United Kingdom \\ ¥ GlaxoSmithKline R\&D, Medicines Research Centre, Gunnels Wood Road, Stevenage, Hertfordshire SG1 \\ 2NY, United Kingdom
}

\section{Supporting Information}

\section{$\underline{\text { Table of Contents }}$}

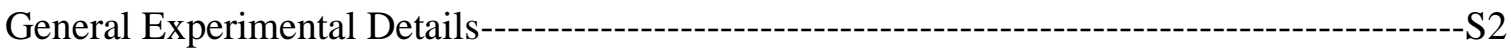

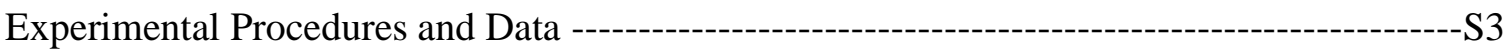

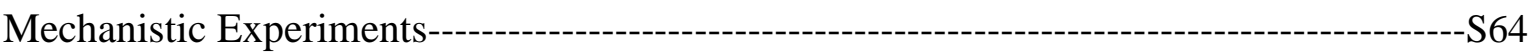

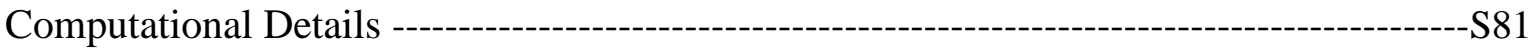

Copies of ${ }^{1} \mathrm{H}$ and ${ }^{13} \mathrm{C}$ NMR for Novel Compounds----------------------------------------------S118

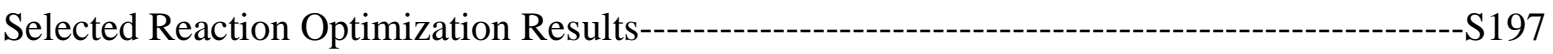

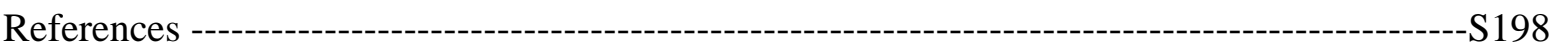

Experiments and data specifically referred to in the main paper as being in the SI are highlighted in blue. 
General Experimental Details. Starting materials sourced from commercial suppliers were used as received unless otherwise stated. Dry solvents, where necessary, were obtained by distillation using standard procedures or by passage through a column of anhydrous alumina using equipment from Anhydrous Engineering based on the Grubb's design. ${ }^{1}$ Petrol refers to the fraction of petroleum ether boiling in the range of $40-60{ }^{\circ} \mathrm{C}$. The removal of solvents in vacuo was achieved using both a Büchi rotary evaporator (bath temperatures up to $45^{\circ} \mathrm{C}$ ) at a pressure of either $15 \mathrm{mmHg}$ (diaphragm pump) or $0.1 \mathrm{mmHg}$ (oil pump), as appropriate and a high vacuum line at room temperature. Reactions requiring anhydrous conditions were run under an atmosphere of dry nitrogen or argon; glassware, syringes and needles were either flame dried immediately prior to use or placed in an oven $\left(200{ }^{\circ} \mathrm{C}\right)$ for at least $2 \mathrm{~h}$ and allowed to cool either in a desiccator or under an atmosphere of nitrogen or argon; liquid reagents, solutions or solvents were added via syringe through rubber septa; solid reagents were added via Schlenk type adapters. Commercially available Merck Kieselgel $60 \mathrm{~F}_{254}$ aluminium backed plates were used for TLC analysis. Visualisation was achieved by either UV fluorescence, basic $\mathrm{KMnO}_{4}$ solution and heat. Flash column chromatography (FCC) was performed using silica gel (Aldrich 40-63 $\mu \mathrm{m}, 230-400 \mathrm{mesh}$ ). The crude material was applied to the column as a solution in $\mathrm{CH}_{2} \mathrm{Cl}_{2}$ or by pre-adsorption onto silica, as appropriate. Melting points were determined using a Reichert melting point table and temperature controller and are uncorrected. Infra-red spectra were recorded in the range $4000-600 \mathrm{~cm}^{-1}$ on a Perkin Elmer Spectrum either as neat films or solids compressed onto a diamond window. Abbreviations used are: w (weak), m (medium) or s (strong). NMR spectra were recorded using either a Varian 400-MR, Varian VNMR S500, Bruker Nano 400, Jeol ECS 300 or Bruker Avance III HD 500 Cryo. Chemical shifts $(\delta)$ are quoted in parts per million (ppm), coupling constants $(J)$ are given in $\mathrm{Hz}$ to the nearest $0.5 \mathrm{~Hz}$. Other abbreviations used are $\mathrm{s}$ (singlet), d (doublet), $\mathrm{t}$ (triplet), $\mathrm{m}$ (multiplet) and br. (broad). ${ }^{1} \mathrm{H}$ and ${ }^{13} \mathrm{C}$ NMR spectra were referenced to the appropriate residual solvent peak. ${ }^{19} \mathrm{~F}$ spectra were referenced to $\mathrm{CCl}_{3} \mathrm{~F}$ as an external standard. Assignments of ${ }^{1} \mathrm{H}$ NMR and ${ }^{13} \mathrm{C}$ NMR signals were made, where possible, using COSY, HMQC, HMBC and NOE experiments. Numbering systems for NMR signal assignments are specified on the structure and are not related to those used for the compound names. Mass spectra were determined by the University of Bristol mass spectrometry service by electron impact $\left(\mathrm{ESI}^{+}\right)$using a Bruker Daltonics FT-ICR-MS Apex 4e 7.0T FT-MS. Chiral SFC was performed using the racemate as a standard on an Agilent 
1290 Infinity system equipped with a quaternary pump, diode array detector and column thermostat under the conditions specified in each case.

\section{$\underline{\text { Experimental Procedures and Data }}$}

General procedure A for protection of indole or pyrrole with carbamoyl chloride derivatives

A flame-dried round-bottomed flask was charged with $\mathrm{NaH}$ (60\% dispersion in mineral oil, $200 \mathrm{~mol} \%)$ and this was suspended in anhydrous THF (1.00 M) under nitrogen. The suspension was cooled to $0{ }^{\circ} \mathrm{C}$ and a solution of substrate $(100 \mathrm{~mol} \%)$ in THF $(0.50 \mathrm{M})$ was added dropwise over 10 minutes. The solution was warmed to room temperature and stirred for 1 hour, followed by dropwise addition of carbamoyl chloride derivatives (110 - 250 mol\%) in THF $(0.50 \mathrm{M})$ over 10 minutes. The solution was then stirred for 4 hours at room temperature. The reaction was quenched by the addition of saturated aqueous $\mathrm{NH}_{4} \mathrm{Cl}$ (3 $\mathrm{mL} / \mathrm{mmol})$ and extracted with EtOAc $(3 \times 3 \mathrm{~mL} / \mathrm{mmol})$. The organic extracts were combined, washed with brine $(5 \mathrm{~mL} / \mathrm{mmol})$, dried over $\mathrm{Na}_{2} \mathrm{SO}_{4}$, filtered and concentrated in vacuo. The residue was purified by column chromatography under the conditions noted to yield the desired product.

General procedure B for amide couplings of amines with $1 \mathrm{H}$-indole-1-carbonyl chloride or $1 H$-pyrrole-1-carbonyl chloride

To a solution of amine $(100 \mathrm{~mol} \%)$ and $\mathrm{K}_{2} \mathrm{CO}_{3}(200 \mathrm{~mol} \%)$ in acetone $(0.40 \mathrm{M})$ and water $(1.70 \mathrm{M})$ at $0{ }^{\circ} \mathrm{C}, 1 \mathrm{H}$-indole-1-carbonyl chloride $(200 \mathrm{~mol} \%)$ or $1 \mathrm{H}$-pyrrole-1-carbonyl chloride $(200 \mathrm{~mol} \%)$ in acetone $(2.00 \mathrm{M})$ was added dropwise over 5 minutes. The reaction mixture was warmed to room temperature and stirred for 16 hours. The solution was concentrated in vacuo to remove acetone, then water $(5 \mathrm{~mL} / \mathrm{mmol})$ was added. The solution was extracted with $\mathrm{CH}_{2} \mathrm{Cl}_{2}(3 \times 5 \mathrm{~mL} / \mathrm{mmol})$ and the organic extracts were combined, washed with brine $(5 \mathrm{~mL} / \mathrm{mmol})$, dried over $\mathrm{Na}_{2} \mathrm{SO}_{4}$ and concentrated in vacuo. The product was purified by flash column chromatography, under the conditions noted, to afford the title compound.

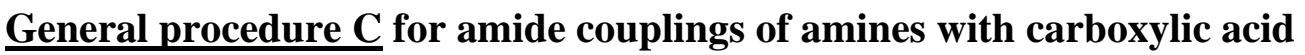

To a suspension of the specified carboxylic acid $(110 \mathrm{~mol} \%)$ in $\mathrm{CH}_{2} \mathrm{Cl}_{2}(0.30 \mathrm{M})$ at room temperature was added EDCI (110 mol\%), DMAP (10 mol\%) and corresponding amine (100 
mol\%). The resulting solution was stirred at room temperature for 18 hours. $1 \mathrm{M}$ aqueous $\mathrm{HCl}(5 \mathrm{~mL} / \mathrm{mmol})$ was added and the solution was extracted with $\mathrm{CH}_{2} \mathrm{Cl}_{2}(2 \times 3 \mathrm{~mL} / \mathrm{mmol})$. The organic extracts were combined, washed with water $(5 \mathrm{~mL} / \mathrm{mmol})$, brine $(5 \mathrm{~mL} / \mathrm{mmol})$, dried over $\mathrm{Na}_{2} \mathrm{SO}_{4}$ and concentrated in vacuo. The residue was purified by column chromatography under the conditions noted to yield the desired product.

General procedure D: An oven dried reaction tube, fitted with a magnetic stirrer, was charged with $\left[\mathrm{Rh}(\operatorname{cod})_{2}\right]$ OTf $(7.5-10 \mathrm{~mol} \%)$, 4-(dimethylamino)benzoic acid (30 mol\%), $\mathrm{Na}_{2} \mathrm{SO}_{4}(100 \mathrm{~mol} \%)$ and substrate $(100 \mathrm{~mol} \%)$. The tube was fitted with a rubber septum and purged with argon. Anhydrous 1,2-DCB $(0.10-0.20 \mathrm{M})$ was added by syringe. The reaction vessel was purged with $\mathrm{CO}$ for 10 minutes and the solution was subsequently sparged with $\mathrm{CO}$ for approximately 20 seconds. The mixture was then heated at the specified temperature, under a $\mathrm{CO}$ atmosphere (with $\mathrm{CO}$ balloon attached) (1 atm), for the specified reaction time. The mixture was cooled to room temperature, concentrated in vacuo and the residue was purified by flash column chromatography, under the conditions noted, to afford the desired product.

General procedure E: An oven dried reaction tube, fitted with a magnetic stirrer, was charged with $\left[\mathrm{Rh}(\operatorname{cod})_{2}\right]$ OTf $(7.5 \mathrm{~mol} \%)$, 4-(dimethylamino)benzoic acid (15 mol\%), tris(3,5dimethylphenyl)phosphine (15 mol\%), $\mathrm{Na}_{2} \mathrm{SO}_{4}(100 \mathrm{~mol} \%)$ and substrate (100 mol\%). The tube was fitted with a rubber septum and purged with argon. Anhydrous 1,2-DCB (0.10 M) was added by syringe. The reaction vessel was purged with $\mathrm{CO}$ for 10 minutes and the solution was subsequently sparged with $\mathrm{CO}$ for approximately 20 seconds. The mixture was then heated at the specified temperature, under a $\mathrm{CO}$ atmosphere (with $\mathrm{CO}$ balloon attached) (1 atm), for the specified reaction time. The mixture was cooled to room temperature, concentrated in vacuo and the residue was purified by flash column chromatography, under the conditions noted, to afford the desired product.

General procedure F: An oven dried reaction tube, fitted with a magnetic stirrer, was charged with $\left[\mathrm{Rh}(\mathrm{cod})_{2}\right] \mathrm{OTf}(7.5 \mathrm{~mol} \%), 2$-nitrobenzoic acid (100 mol\%), tris(4fluorophenyl)phosphine $(15 \mathrm{~mol} \%)$ and substrate $(100 \mathrm{~mol} \%)$. The tube was fitted with a rubber septum and purged with argon. Specified amount of benzonitrile was added by syringe. The reaction vessel was purged with $\mathrm{CO}$ for 10 minutes and the solution was subsequently sparged with CO for approximately 20 seconds. The mixture was then heated at the specified temperature, under a $\mathrm{CO}$ atmosphere (with $\mathrm{CO}$ balloon attached) (1 atm), for the specified 
reaction time. The mixture was cooled to room temperature, concentrated in vacuo and the residue was purified by flash column chromatography under the conditions noted to afford the desired product.

\section{$N$-Benzyl- $N$-cyclopropyl-1H-indole-1-carboxamide 3a}

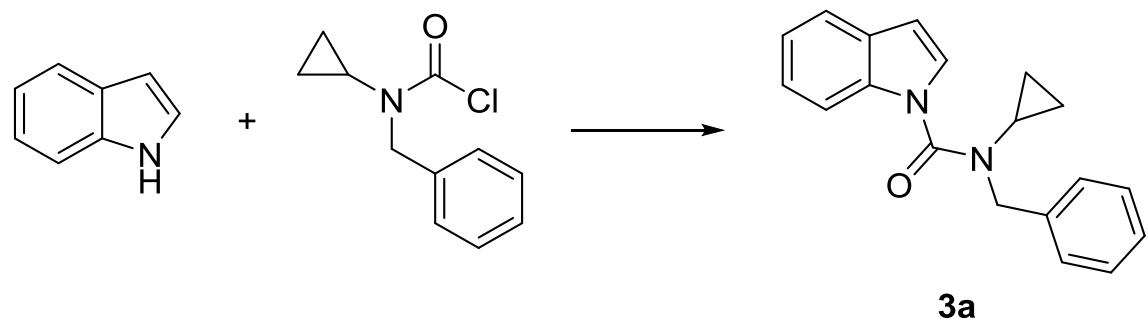

General procedure A: Indole (400 $\mathrm{mg}, 3.40 \mathrm{mmol}$ ) and benzyl(cyclopropyl)carbamic chloride (prepared according to the literature procedure) ${ }^{1}(781 \mathrm{mg}, 3.74 \mathrm{mmol})$ were employed. The crude mixture was purified by flash column chromatography (40\% toluene/hexane then $100 \%$ EtOAc) to afford the title compound 3a $(897 \mathrm{mg}, 91 \%)$ as a colourless solid; m.p. 50-52 ${ }^{\circ} \mathrm{C}\left(\mathrm{CH}_{2} \mathrm{Cl}_{2}\right) ; v_{\max } / \mathrm{cm}^{-1}$ : 1673 (s), 1453 (s), 1405 (m), 1319 (s), 1295 (s), 1210 (s); ${ }^{1} \mathrm{H}$ NMR (400 MHz, $\left.\mathrm{CDCl}_{3}\right): \delta 7.80$ (dd, $\left.J=8.4,1.0 \mathrm{~Hz}, 1 \mathrm{H}\right), 7.62$ (ddd, $J$ $=7.8,1.0,1.0 \mathrm{~Hz}, 1 \mathrm{H}), 7.47(\mathrm{~d}, J=3.6 \mathrm{~Hz}, 1 \mathrm{H}), 7.42-7.28(\mathrm{~m}, 6 \mathrm{H}), 7.23(\mathrm{ddd}, J=8.0,7.2$, $1.0 \mathrm{~Hz}, 1 \mathrm{H}), 6.62(\mathrm{dd}, J=3.6,1.0 \mathrm{~Hz}, 1 \mathrm{H}), 4.73(\mathrm{~s}, 2 \mathrm{H}), 2.66$ (tt, $J=6.8,4.0 \mathrm{~Hz}, 1 \mathrm{H}), 0.71-$ $0.68(\mathrm{~m}, 2 \mathrm{H}), 0.64-0.62(\mathrm{~m}, 2 \mathrm{H}) ;{ }^{13} \mathrm{C} \mathrm{NMR}\left(101 \mathrm{MHz}, \mathrm{CDCl}_{3}\right): \delta 155.1,136.9,135.6$, 129.5, 128.7, 128.3 125.9, 123.6, 121.9, 120.8, 113.8, 105.7, 77.2 , 77.0, 76.7, 53.3, 31.3, 9.1; $m / z\left(\mathrm{ESI}^{+}\right)$HRMS: Calculated for $\mathrm{C}_{19} \mathrm{H}_{19} \mathrm{~N}_{2} \mathrm{O}: 291.1493$. Found $[\mathrm{M}+\mathrm{H}]^{+}: 291.1488$.

$\left(3 \mathrm{a} S^{*}, 11 \mathrm{a} S^{*}\right)$-4-Benzyl-2,3,3a,4-tetrahydro-11H-cyclopenta[4,5]imidazo[1,5-a]indole1,5-dione 4a

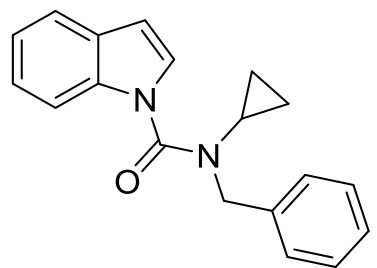

$3 a$

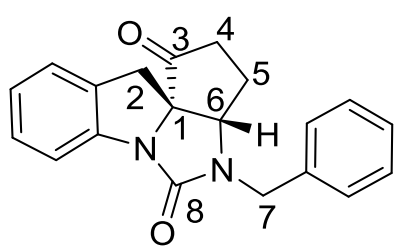

$4 a$

General procedure D: Compound 3a $(43.5 \mathrm{mg}, 0.15 \mathrm{mmol}),\left[\mathrm{Rh}(\operatorname{cod})_{2}\right] \mathrm{OTf}(5.28 \mathrm{mg}, 7.5$ mol\%) and anhydrous 1,2-DCB (1.50 mL) were employed and the reaction was stirred for 72 hours at $130{ }^{\circ} \mathrm{C}$. The crude mixture was purified by flash column chromatography (5\% EtOAc/toluene) to yield the title compound $\mathbf{4 a}(39.1 \mathrm{mg}, 82 \%)$ as a colourless solid; m.p. 
126-127 ${ }^{\circ} \mathrm{C}\left(\mathrm{CDCl}_{3}\right) ; v_{\max } / \mathrm{cm}^{-1}: 2919$ (m), 1748 (s), 1710 (s), 1480 (s), 1409 (s), 1290 (s), 1158 (s); ${ }^{1} \mathrm{H}$ NMR (400 MHz, $\left.\mathrm{CDCl}_{3}\right) \delta 7.51$ (d, J=7.9 Hz, 1H, $\left.1 \times \mathrm{ArC} \underline{\mathrm{H}}\right), 7.34-7.17$ (m, $7 \mathrm{H}, 7 \times \mathrm{ArC} \underline{\mathrm{H}}), 7.06(\mathrm{dd}, J=7.5,7.5 \mathrm{~Hz}, 1 \mathrm{H}, 1 \times \mathrm{ArC} \underline{\mathrm{H}}), 4.66(\mathrm{~d}, J=15.2 \mathrm{~Hz}, 1 \mathrm{H}, 1 \times \mathrm{C} 7-$ $\left.\underline{\mathrm{H}}_{2}\right), 4.36\left(\mathrm{~d}, J=15.2 \mathrm{~Hz}, 1 \mathrm{H}, 1 \times \mathrm{C} 7-\underline{\mathrm{H}}_{2}\right), 4.09(\mathrm{~d}, J=5.3 \mathrm{~Hz}, 1 \mathrm{H}, 1 \times \mathrm{C6}-\underline{\mathrm{H}}), 3.34(\mathrm{~d}, J=$ $\left.16.1 \mathrm{~Hz}, 1 \mathrm{H}, 1 \times \mathrm{C} 2-\underline{\mathrm{H}}_{2}\right), 3.08\left(\mathrm{~d}, J=16.1 \mathrm{~Hz}, 1 \mathrm{H}, 1 \times \mathrm{C} 2-\underline{\mathrm{H}}_{2}\right), 2.61$ (ddd, $J=17.7,13.6,9.1$ $\left.\mathrm{Hz}, 1 \mathrm{H}, 1 \times \mathrm{C} 4-\underline{\mathrm{H}}_{2}\right), 2.35-2.20$ (m, 2H, $\left.1 \times \mathrm{C} 4-\underline{\mathrm{H}}_{2}, 1 \times \mathrm{C} 5-\underline{\mathrm{H}}_{2}\right), 1.91-1.81$ (m, $1 \mathrm{H}, 1 \times \mathrm{C} 5-$ $\left.\underline{\mathrm{H}}_{2}\right) .{ }^{13} \mathrm{C}$ NMR (101 MHz, $\mathrm{CDCl}_{3}$ ): $\delta 212.3$ (C3), 159.1 (C8), 142.1 (CAr), 136.1 (CAr), 130.1 (CAr), 128.8 (CAr), 128.1 (CAr), 128.0 (CAr), 127.8 (CAr), 124.6 (CAr), 124.2 (CAr), 115.9 (CAr), 70.0 (C1), 63.8 (C6), 45.8 (C7), 35.6 (C2), 33.1 (C4), 23.2 (C5); m/z (ESI $\left.{ }^{+}\right)$ HRMS: Calculated for $\mathrm{C}_{20} \mathrm{H}_{19} \mathrm{~N}_{2} \mathrm{O}_{2:}$ 319.1441; Found $[\mathrm{M}+\mathrm{H}]^{+}: 319.1433$.

\section{$N$-Butyl- $N$-cyclopropyl-1H-indole-1-carboxamide $3 b$}
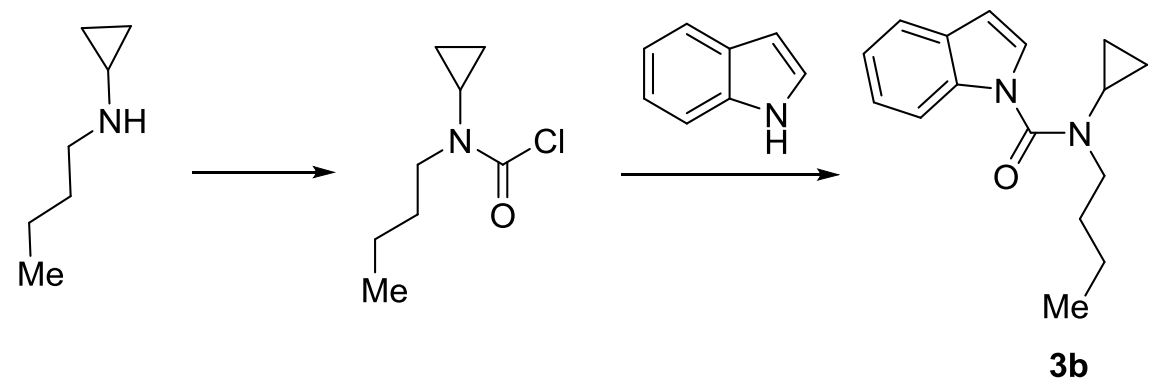

Pyridine $(1.91 \mathrm{~mL}, 24.0 \mathrm{mmol})$ was added dropwise to a solution of triphosgene $(2.24 \mathrm{~g}, 7.66$ mmol) in $\mathrm{CH}_{2} \mathrm{Cl}_{2}(50 \mathrm{~mL})$ over 10 minutes at $0{ }^{\circ} \mathrm{C}$. To the resulting suspension was added a solution of $\mathrm{N}$-butylcyclopropanamine (prepared according to the literature procedure $)^{2}(2.26$ $\mathrm{g}, 21.0 \mathrm{mmol})$ in $\mathrm{CH}_{2} \mathrm{Cl}_{2}(3.00 \mathrm{~mL})$, the resulting solution was warmed to room temperature and stirred for 2 hours. The reaction was quenched by the addition of saturated aqueous $\mathrm{NaHCO}_{3}(30 \mathrm{~mL})$ and extracted with $\mathrm{Et}_{2} \mathrm{O}(2 \times 40 \mathrm{~mL})$. The organic layers were combined and washed with $0.2 \mathrm{M}$ aqueous $\mathrm{HCl}(30 \mathrm{~mL}), \mathrm{H}_{2} \mathrm{O}(30 \mathrm{~mL})$, brine $(30 \mathrm{~mL})$, dried over $\mathrm{Na}_{2} \mathrm{SO}_{4}$ and concentrated in vacuo to give butyl(cyclopropyl)carbamic chloride (1.98 g, 67\%) as a yellow oil which was used without further purification. A flame-dried round-bottomed flask was charged with $\mathrm{NaH}(240 \mathrm{mg}, 6.00 \mathrm{mmol})$ and this was suspended in anhydrous THF $(10 \mathrm{~mL})$ under nitrogen. The suspension was cooled to $0{ }^{\circ} \mathrm{C}$ and indole $(351 \mathrm{mg}, 3.00 \mathrm{mmol})$ was added portionwise over 5 minutes. The solution was warmed to room temperature and stirred for 1 hour, followed by dropwise addition of butyl(cyclopropyl)carbamic chloride $(578 \mathrm{mg}, 3.30 \mathrm{mmol})$ in THF $(7.00 \mathrm{~mL})$ over 10 minutes. The solution was then stirred for 4 hours at room temperature. The reaction was quenched by the addition of saturated aqueous 
$\mathrm{NH}_{4} \mathrm{Cl}(10 \mathrm{~mL})$ and extracted with EtOAc $(3 \times 10 \mathrm{~mL})$. The organic extracts were combined, washed with brine $(20.0 \mathrm{~mL})$, dried over $\mathrm{Na}_{2} \mathrm{SO}_{4}$, filtered and concentrated in vacuo. The crude mixture was purified by flash column chromatography (10\% toluene/hexane then $100 \%$ EtOAc) to afford the title compound $\mathbf{3 b}(699 \mathrm{mg}, 91 \%)$ as a colourless oil; $v_{\max } / \mathrm{cm}^{-1}: 2958$ (m), 1671 (s), 1524 (m), 1452 (s), 1407 (s), 1210 (s); ${ }^{1} \mathrm{H}$ NMR (400 MHz, $\mathrm{CDCl}_{3}$ ): $\delta 7.72$ $(1 \mathrm{H}, \mathrm{dd}, J=8.2,1.0 \mathrm{~Hz}), 7.59(1 \mathrm{H}, \mathrm{ddd}, J=7.8,1.0,1.0 \mathrm{~Hz}), 7.41(1 \mathrm{H}, \mathrm{d}, J=3.6 \mathrm{~Hz}), 7.29-$ $7.25(1 \mathrm{H}, \mathrm{m}), 7.19(1 \mathrm{H}, \mathrm{ddd}, J=8.2,7.2,1.0 \mathrm{~Hz}), 6.57(1 \mathrm{H}, \mathrm{dd}, J=3.6,1.0 \mathrm{~Hz}), 3.52(2 \mathrm{H}, \mathrm{t}$, $J=7.2 \mathrm{~Hz}), 2.84(1 \mathrm{H}, \mathrm{tt}, J=6.9,3.9 \mathrm{~Hz}), 1.76-1.68(2 \mathrm{H}, \mathrm{m}), 1.43-1.34(2 \mathrm{H}, \mathrm{m}), 0.94(3 \mathrm{H}$, $\mathrm{t}, J=7.4 \mathrm{~Hz}), 0.75-0.70(2 \mathrm{H}, \mathrm{m}), 0.57-0.53(2 \mathrm{H}, \mathrm{m}) ;{ }^{13} \mathrm{C} \mathrm{NMR}\left(101 \mathrm{MHz}, \mathrm{CDCl}_{3}\right): \delta$ 155.3, 135.5, 129.4, 126.0, 123.4, 121.7, 120.8, 113.7, 105.4, 49.2, 30.9, 30, 20.0, 13.8, 8.9; $\mathrm{m} / \mathrm{z}\left(\mathrm{ESI}^{+}\right) \mathrm{HRMS}$ : Calculated for $\mathrm{C}_{16} \mathrm{H}_{20} \mathrm{~N}_{2} \mathrm{NaO}: 279.1468$. Found $[\mathrm{M}+\mathrm{Na}]^{+}:$279.1483.

$\left(3 \mathrm{a} S^{*}, 11 \mathrm{a} S^{*}\right)-4-B u t y l-2,3,3 \mathrm{a}, 4-t e t r a h y d r o-11 H$-cyclopenta[4,5]imidazo[1,5-a]indole-1,5dione $4 b$

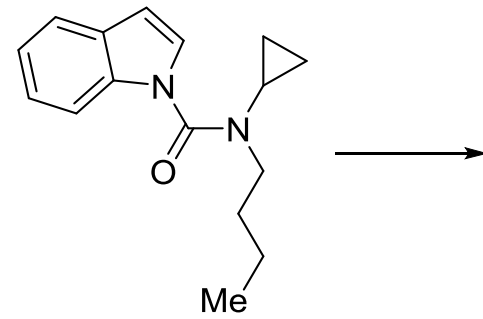

3b

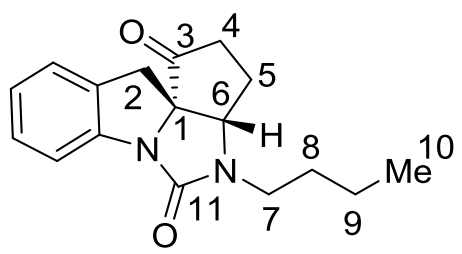

4b

General procedure D: Compound $3 \mathbf{b}(38.4 \mathrm{mg}, 0.15 \mathrm{mmol})$, [Rh(cod) $\left.)_{2}\right] \mathrm{OTf}(5.28 \mathrm{mg}, 7.5$ mol\%) and anhydrous 1,2-DCB $(1.50 \mathrm{~mL})$ were employed and the reaction was stirred for 72 hours at $130{ }^{\circ} \mathrm{C}$. The crude mixture was purified by flash column chromatography $(15 \%$ EtOAc/toluene) to yield the title compound $\mathbf{4 b}(22.6 \mathrm{mg}, 53 \%)$ as a yellow oil; $v_{\max } / \mathrm{cm}^{-1}$ : 2930 (m), 1750 (s), 1708 (s), 1480 (s), 1409 (s), 1292 (s), 1160 (s); ${ }^{1} \mathrm{H}$ NMR (400 MHz, $\left.\mathrm{CDCl}_{3}\right) \delta 7.45(1 \mathrm{H}, \mathrm{d}, J=7.5 \mathrm{~Hz}, 1 \times \mathrm{ArC} \underline{\mathrm{H}}), 7.23-7.18(2 \mathrm{H}, \mathrm{m}, 2 \times \mathrm{ArC} \underline{\mathrm{H}}), 7.04(1 \mathrm{H}, \mathrm{ddd}$, $J=7.5,7.5,1.1 \mathrm{~Hz}, 1 \times \mathrm{ArC} \underline{\mathrm{H}}), 4.24(1 \mathrm{H}, \mathrm{d}, J=5.3 \mathrm{~Hz}, 1 \times \mathrm{C6}-\underline{\mathrm{H}}), 3.46-3.38(2 \mathrm{H}, \mathrm{m}, 1 \times$ $\left.\mathrm{C} 2-\underline{\mathrm{H}}_{2}, 1 \times \mathrm{C} 7-\underline{\mathrm{H}}_{2}\right), 3.19-3.12\left(2 \mathrm{H}, \mathrm{m}, 1 \times \mathrm{C} 2-\underline{\mathrm{H}}_{2}, 1 \times \mathrm{C} 7-\underline{\mathrm{H}}_{2}\right), 2.67$ (1H, ddd, $J=17.6,13.7$, $\left.9.1 \mathrm{~Hz}, 1 \times \mathrm{C} 4-\underline{\mathrm{H}}_{2}\right), 2.41-2.29\left(2 \mathrm{H}, \mathrm{m}, 1 \times \mathrm{C} 4-\underline{\mathrm{H}}_{2}, 1 \times \mathrm{C} 5-\underline{\mathrm{H}}_{2}\right), 2.05-1.93(1 \mathrm{H}, \mathrm{m}, 1 \times \mathrm{C} 5-$ $\left.\underline{\mathrm{H}}_{2}\right), 1.64-1.42\left(2 \mathrm{H}, \mathrm{m}, 2 \times \mathrm{C} 8-\underline{\mathrm{H}}_{2}\right), 1.38-1.28\left(2 \mathrm{H}, \mathrm{m}, 2 \times \mathrm{C} 9-\underline{\mathrm{H}}_{2}\right), 0.93(3 \mathrm{H}, \mathrm{t}, J=7.3 \mathrm{~Hz}$, $\left.3 \times \mathrm{C10}-\underline{\mathrm{H}}_{3}\right) .{ }^{13} \mathrm{C} \mathrm{NMR}\left(101 \mathrm{MHz}, \mathrm{CDCl}_{3}\right): \delta 212.3$ (C3), 159.0 (C11), 142.5 (CAr), 129.9 (CAr), 127.9 (CAr), 124.6 (CAr), 124.1 (CAr), 115.8 (CAr), 70.0 (C1), 63.9 (C6), 41.5 
(C7), 35.7 (C2), 33.1 (C4), 29.4 (C8), 23.6 (C5), 20.1 (C9), 13.7(C10); m/z (ESI ${ }^{+}$) HRMS:

Calculated for $\mathrm{C}_{17} \mathrm{H}_{21} \mathrm{~N}_{2} \mathrm{O}_{2:}$ 285.1598; Found $[\mathrm{M}+\mathrm{H}]^{+}: 285.1601$.

$N$-Benzyl- $N$-cyclopropyl-4-fluoro-1 $H$-indole-1-carboxamide $3 \mathrm{c}$
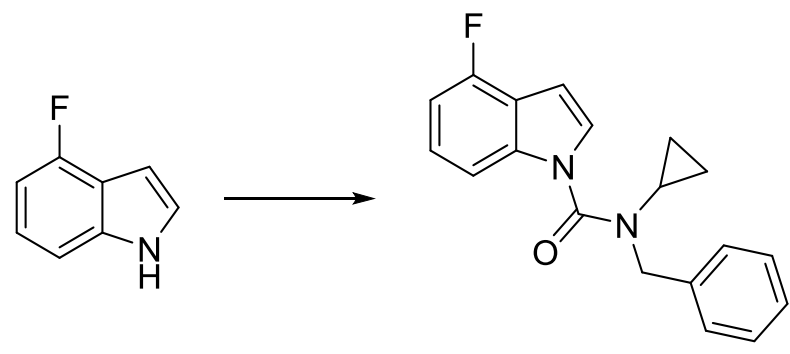

3c

General procedure A: 4-Fluoro- $1 H$-indole $\quad(405 \quad \mathrm{mg}, \quad 3.00 \mathrm{mmol})$ and benzyl(cyclopropyl)carbamic chloride $(752 \mathrm{mg}, 3.60 \mathrm{mmol})$ were employed. The crude mixture was purified by flash column chromatography ( $6 \%$ toluene/hexane) to afford the title compound 3c (825 mg, 89\%) as a colourless solid; m.p. 86-88 ${ }^{\circ} \mathrm{C}\left(\mathrm{CH}_{2} \mathrm{Cl}_{2}\right) ; v_{\max } / \mathrm{cm}^{-1}: 1679$ (s), 1406 (s), 1372 (s), 1269 (s), 1219 (s), 747 (s); ${ }^{1} \mathrm{H}$ NMR (400 MHz, CDCl 3 ): $\delta 7.54$ (1H, d, $J=8.3 \mathrm{~Hz}), 7.41-7.30(6 \mathrm{H}, \mathrm{m}), 7.20(1 \mathrm{H}, \mathrm{ddd}, J=8.1,5.3,5.3 \mathrm{~Hz}), 6.88(1 \mathrm{H}, \mathrm{ddd}, J=$ 9.9, 8.0, 0.7 Hz), $6.68(1 \mathrm{H}, \mathrm{dd}, J=3.6,0.7 \mathrm{~Hz}), 4.71(2 \mathrm{H}, \mathrm{s}), 2.64(1 \mathrm{H}, \mathrm{tt}, J=6.8,4.0 \mathrm{~Hz})$, $0.70-0.64(2 \mathrm{H}, \mathrm{m}), 0.63-0.56(2 \mathrm{H}, \mathrm{m}) ;{ }^{13} \mathrm{C} \mathrm{NMR}\left(101 \mathrm{MHz}, \mathrm{CDCl}_{3}\right): \delta 155.9$ (d, $J=247.4$ $\mathrm{Hz}), 154.8,138.0(\mathrm{~d}, J=10.1 \mathrm{~Hz}), 136.8,128.8,128.3,127.8,125.8,124.3$ (d, $J=7.5 \mathrm{~Hz})$, 118.4(d, $J=22.4 \mathrm{~Hz}), 110.0(\mathrm{~d}, J=3.7 \mathrm{~Hz}), 107.0(\mathrm{~d}, J=17.7 \mathrm{~Hz}), 101.4,53.3,31.4,9.2 ;{ }^{19} \mathrm{~F}$ NMR (377 MHz, $\left.\mathrm{CDCl}_{3}\right): \delta-122.1(\mathrm{dd}, J=9.9,5.3 \mathrm{~Hz}) ; \mathrm{m} / z\left(\mathrm{ESI}^{+}\right) \mathrm{HRMS}$ : Calculated for $\mathrm{C}_{19} \mathrm{H}_{18} \mathrm{~N}_{2} \mathrm{FO}: 309.1398$. Found $[\mathrm{M}+\mathrm{H}]^{+}: 309.1392$.

$\left(3 \mathrm{a} S^{*}, 11 \mathrm{a} S^{*}\right)$-4-Benzyl-10-fluoro-2,3,3a,4-tetrahydro-11 $H$-cyclopenta[4,5]imidazo[1,5a]indole-1,5-dione 4c

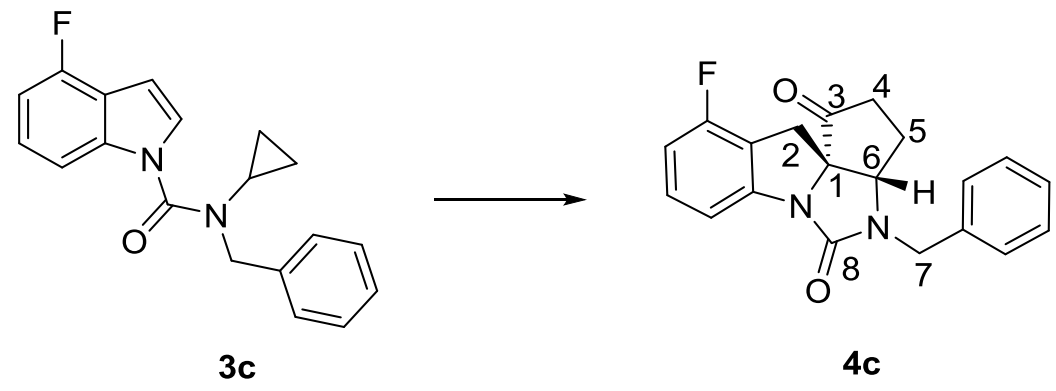

General procedure D: Compound $3 \mathbf{c}(42.6 \mathrm{mg}, 0.15 \mathrm{mmol}),\left[\mathrm{Rh}(\operatorname{cod})_{2}\right] \mathrm{OTf}(5.28 \mathrm{mg}, 7.5$ mol\%) and anhydrous 1,2-DCB $(1.50 \mathrm{~mL})$ were employed and the reaction was stirred for 72 hours at $130{ }^{\circ} \mathrm{C}$. The crude mixture was purified by column chromatography $(5 \%$ 
EtOAc/toluene) to yield the title compound 4c (34.7 mg, 69\%) as a yellow solid; m.p. 146$148{ }^{\circ} \mathrm{C}\left(\mathrm{CDCl}_{3}\right) ; v_{\max } / \mathrm{cm}^{-1}: 2923$ (m), 1752 (s), 1710 (s), 1468 (s), 1407 (s), 1241 (s); ${ }^{1} \mathrm{H}$ NMR (400 MHz, $\left.\mathrm{CDCl}_{3}\right) \delta 7.28-7.11(7 \mathrm{H}, \mathrm{m}, 7 \times \mathrm{ArC} \underline{\mathrm{H}}), 6.71-6.67(1 \mathrm{H}, \mathrm{m}, 1 \times \mathrm{ArC} \underline{\mathrm{H}})$, $4.58\left(1 \mathrm{H}, \mathrm{d}, J=15.2 \mathrm{~Hz}, 1 \times \mathrm{C} 7-\underline{\mathrm{H}}_{2}\right), 4.29\left(1 \mathrm{H}, \mathrm{d}, J=15.2 \mathrm{~Hz}, 1 \times \mathrm{C} 7-\underline{\mathrm{H}}_{2}\right), 4.04(1 \mathrm{H}, \mathrm{d}, J=$ $5.3 \mathrm{~Hz}, 1 \times \mathrm{C} 6-\underline{\mathrm{H}}), 3.31\left(1 \mathrm{H}, \mathrm{d}, J=16.3 \mathrm{~Hz}, 1 \times \mathrm{C} 2-\underline{\mathrm{H}}_{2}\right), 2.99(1 \mathrm{H}, \mathrm{d}, J=16.3 \mathrm{~Hz}, 1 \times \mathrm{C} 2-$ $\left.\underline{\mathrm{H}}_{2}\right), 2.52\left(1 \mathrm{H}, \mathrm{ddd}, J=17.8,13.7,9.1 \mathrm{~Hz}, 1 \times \mathrm{C} 4-\underline{\mathrm{H}}_{2}\right), 2.28-2.13\left(2 \mathrm{H}, \mathrm{m}, 1 \times \mathrm{C} 4-\underline{\mathrm{H}}_{2}, 1 \times\right.$ C5- $\left.\underline{\mathrm{H}}_{2}\right), 1.82-1.78\left(1 \mathrm{H}, \mathrm{m}, 1 \times \mathrm{C} 5-\underline{\mathrm{H}}_{2}\right) ;{ }^{13} \mathrm{C} \mathrm{NMR}\left(101 \mathrm{MHz}, \mathrm{CDCl}_{3}\right): \delta 211.80(\mathrm{C3}), 158.8$ $(\mathrm{d}, J=247.4 \mathrm{~Hz})(\mathrm{CAr}), 158.7$ (C8), 144.6 (d, $J=7.6 \mathrm{~Hz})(\mathrm{CAr}), 135.92$ (CAr), 129.9 (d, $J=$ 8.0 Hz) (CAr), 128.90 (CAr), 128.1 (CAr), 128.0 (CAr), 116.5 (d, $J=20.8$ Hz) (CAr), 111.6 $(\mathrm{d}, J=3.4 \mathrm{~Hz})(\mathrm{CAr}), 111.1(\mathrm{~d}, J=20.1 \mathrm{~Hz})(\mathrm{CAr}), 70.5$ (C1), 63.7 (C6), 45.9 (C7), 33.0 (C4), 32.3 (C2), $23.2(\mathrm{C5}) ;{ }^{19} \mathrm{~F}$ NMR (377 MHz, $\left.\mathrm{CDCl}_{3}\right): \delta-116.90(\mathrm{dd}, J=8.7,5.3 \mathrm{~Hz}) ; \mathrm{m} / \mathrm{z}$ $\left(\mathrm{ESI}^{+}\right)$HRMS: Calculated for $\mathrm{C}_{20} \mathrm{H}_{18} \mathrm{~N}_{2} \mathrm{FO}_{2}$ : 337.1347. Found $[\mathrm{M}+\mathrm{H}]^{+}: 337.1340$.

\section{$N$-Benzyl- $N$-cyclopropyl-4-methyl-1H-indole-1-carboxamide 3d}
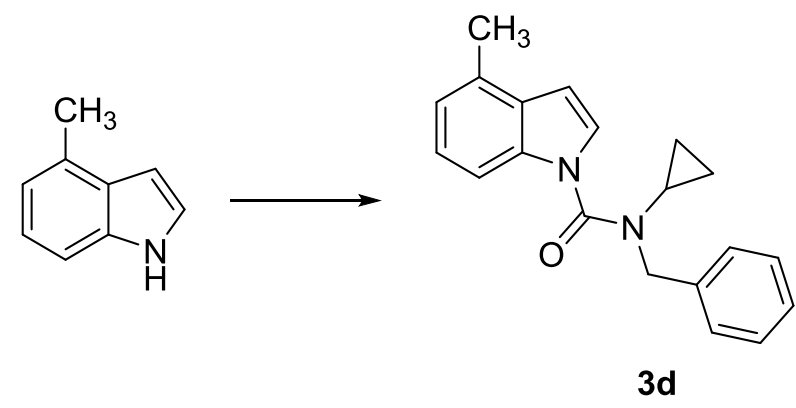

General procedure A: 4-Methylindole (131 $\mathrm{mg}, \quad 1.00 \quad \mathrm{mmol})$ and benzyl(cyclopropyl)carbamic chloride $(250 \mathrm{mg}, 1.20 \mathrm{mmol})$ were employed. The crude mixture was purified by flash column chromatography (20\% EtOAc/hexane) to afford the title compound 3d (258 mg, 85\%) as a colourless oil; $v_{\max } / \mathrm{cm}^{-1}: 1672(\mathrm{~s}), 1406(\mathrm{~s}), 1315$ (s), 1273 (s), 1158 (s) 755 (s); ${ }^{1} \mathrm{H}$ NMR (400 MHz, $\left.\mathrm{CDCl}_{3}\right): \delta 7.62(1 \mathrm{H}, \mathrm{dd}, J=8.3,1.0 \mathrm{~Hz}), 7.45$ $(1 \mathrm{H}, \mathrm{d}, J=3.6 \mathrm{~Hz}), 7.41-7.31(5 \mathrm{H}, \mathrm{m}), 7.21(1 \mathrm{H}, \mathrm{dd}, J=8.3,7.3 \mathrm{~Hz}), 7.03(1 \mathrm{H}, \mathrm{dd}, J=7.3$, $1.0 \mathrm{~Hz}), 6.64(1 \mathrm{H}, \mathrm{d}, J=3.6 \mathrm{~Hz}), 4.72(2 \mathrm{H}, \mathrm{s}), 2.65(1 \mathrm{H}, \mathrm{tt}, J=6.8,4.0 \mathrm{~Hz}), 2.57(3 \mathrm{H}, \mathrm{s})$, $0.71-0.59$ (4H, m); $\left.{ }^{13} \mathrm{C} \mathrm{NMR} \mathrm{(101} \mathrm{MHz}, \mathrm{CDCl}_{3}\right): \delta 155.2,137.0,135.3,130.2,129.2,128.7$, 128.3, 127.7, 125.3, 123.6, 122.2, 111.3, 104.1, 53.3, 31.2, 18.6, 18.5, 9.1; $\mathrm{m} / \mathrm{z}\left(\mathrm{ESI}^{+}\right) \mathrm{HRMS}$ : Calculated for $\mathrm{C}_{20} \mathrm{H}_{21} \mathrm{~N}_{2} \mathrm{O}$ : 305.1648. Found $[\mathrm{M}+\mathrm{H}]^{+}: 305.1649$. 


\section{a]indole-1,5-dione $4 \mathrm{~d}$}

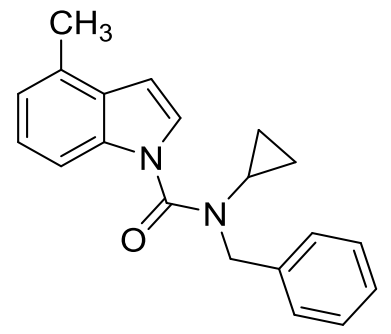

3d

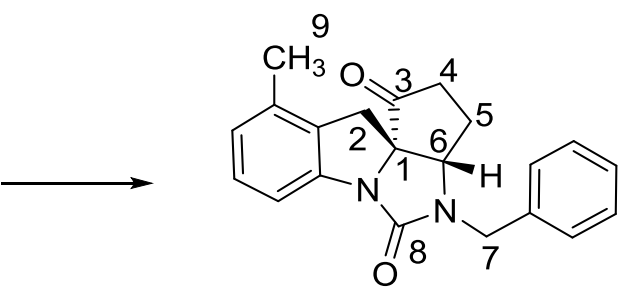

4d

General procedure D: Compound 3d $(45.6 \mathrm{mg}, 0.15 \mathrm{mmol}),\left[\mathrm{Rh}(\operatorname{cod})_{2}\right] \mathrm{OTf}(5.28 \mathrm{mg}, 7.5$ mol\%) and anhydrous 1,2-DCB $(1.50 \mathrm{~mL})$ were employed and the reaction was stirred for 72 hours at $140{ }^{\circ} \mathrm{C}$. The crude mixture was purified by flash column chromatography $(15 \%$ EtOAc/toluene) to yield the title compound $4 \mathbf{d}(29.4 \mathrm{mg}, 59 \%)$ as a yellow oil; $v_{\max } / \mathrm{cm}^{-1}$ : 2914 (m), 1750 (s), 1704 (s), 1409 (s), 1288 (s), 1158 (s); ${ }^{1} \mathrm{H}$ NMR (400 MHz, CDCl 3 ) $\delta 7.28$ $-7.19(6 \mathrm{H}, \mathrm{m}, 6 \times \mathrm{ArCH}), 7.07(1 \mathrm{H}, \mathrm{d}, J=7.7 \mathrm{~Hz}, 1 \times \mathrm{ArCH}), 6.80(1 \mathrm{H}, \mathrm{d}, J=7.5 \mathrm{~Hz}, 1 \times$ $\operatorname{ArC} \underline{\mathrm{H}}), 4.59\left(1 \mathrm{H}, \mathrm{d}, J=15.2 \mathrm{~Hz}, 1 \times \mathrm{C} 7-\underline{\mathrm{H}}_{2}\right), 4.28\left(1 \mathrm{H}, \mathrm{d}, J=15.2 \mathrm{~Hz}, 1 \times \mathrm{C} 7-\underline{\mathrm{H}}_{2}\right), 4.01(1 \mathrm{H}$, $\mathrm{d}, J=5.2 \mathrm{~Hz}, 1 \times \mathrm{C} 6-\underline{\mathrm{H}}), 3.18\left(1 \mathrm{H}, \mathrm{d}, J=16.1 \mathrm{~Hz}, 1 \times \mathrm{C} 2-\underline{\mathrm{H}}_{2}\right), 2.89(1 \mathrm{H}, \mathrm{d}, J=16.1 \mathrm{~Hz}, 1 \times$ C2- $\left.\underline{\mathrm{H}}_{2}\right), 2.52\left(1 \mathrm{H}, \mathrm{ddd}, J=17.7,13.7,9.1 \mathrm{~Hz}, 1 \times \mathrm{C} 4-\underline{\mathrm{H}}_{2}\right), 2.27-2.14\left(5 \mathrm{H}, \mathrm{m}, 1 \times \mathrm{C} 4-\underline{\mathrm{H}}_{2}, 1\right.$ $\left.\times \mathrm{C} 5-\underline{\mathrm{H}}_{2}, 3 \times \mathrm{C} 9-\underline{\mathrm{H}}_{3}\right), 1.84-1.76\left(1 \mathrm{H}, \mathrm{m}, 1 \times \mathrm{C} 5-\underline{\mathrm{H}}_{2}\right) ;{ }^{13} \mathrm{C} \mathrm{NMR}\left(101 \mathrm{MHz}, \mathrm{CDCl}_{3}\right): \delta 212.6$ (C3), 159.2 (C8), 142.0 (CAr), 136.2 (CAr), 134.2 (CAr), 128.9 (CAr), 128.8 (CAr), 128.2 (CAr), 128.1 (CAr), 127.8 (CAr), 125.2 (CAr), 113.2 (CAr), 70.0 (C1), 64.0 (C6), 45.8 (C7), 34.7 (C2), 33.1 (C4), 23.2 (C5), 18.8 (C9); m/z (ESI ${ }^{+}$) HRMS: Calculated for $\mathrm{C}_{21} \mathrm{H}_{21} \mathrm{~N}_{2} \mathrm{O}_{2:}$ 333.1598; Found [M+H] $]^{+}: 333.1603$.

\section{Methyl 1-(benzyl(cyclopropyl)carbamoyl)-1H-indole-4-carboxylate 3e}
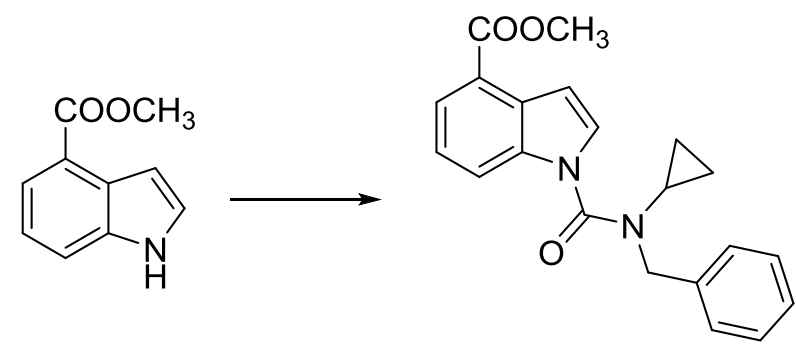

$3 e$

General procedure A: Methyl $1 H$-indole-4-carboxylate $(525 \mathrm{mg}, 3.00 \mathrm{mmol})$ and benzyl(cyclopropyl)carbamic chloride $(752 \mathrm{mg}, 3.60 \mathrm{mmol})$ were employed. The crude mixture was purified by flash column chromatography (10\% toluene/hexane) to afford the 
title compound 3e (667 mg, 64\%) as a yellow oil; $v_{\max } / \mathrm{cm}^{-1}: 1713(\mathrm{~s}), 1679$ (s), 1428 (s), 1406 (s), 1267 (s), 1174 (s); ${ }^{1} \mathrm{H}$ NMR (400 MHz, CDCl $)$ ) $\delta 8.03-7.96(2 \mathrm{H}, \mathrm{m}), 7.55$ (1H, d, $J=3.6 \mathrm{~Hz}), 7.40-7.27(7 \mathrm{H}, \mathrm{m}), 4.72(2 \mathrm{H}, \mathrm{s}), 3.98(3 \mathrm{H}, \mathrm{s}), 2.65(1 \mathrm{H}, \mathrm{tt}, J=6.8,4.0 \mathrm{~Hz}), 0.70$ - $0.61(2 \mathrm{H}, \mathrm{m}), 0.60-0.54(2 \mathrm{H}, \mathrm{m}) ;{ }^{13} \mathrm{C} \mathrm{NMR}\left(101 \mathrm{MHz}, \mathrm{CDCl}_{3}\right): \delta 167.5,154.7,136.8$, 136.4, 129.3, 128.8, 128.3, 127.8, 127.7, 125.0, 123.0, 121.9, 118.6, 106.4, 53.2, 51.8, 31.6, 9.3; $m / z\left(\mathrm{ESI}^{+}\right) \mathrm{HRMS}$ : Calculated for $\mathrm{C}_{21} \mathrm{H}_{21} \mathrm{~N}_{2} \mathrm{O}_{3}$ : 349.1547. Found $[\mathrm{M}+\mathrm{H}]^{+}: 349.1543$.

Methyl

$\left(3 \mathrm{a} S^{*}, 11 \mathrm{a} S^{*}\right)-4-b e n z y l-1,5-d i o x o-1,2,3,3 \mathrm{a}, 4,5-h e x a h y d r o-11 H-$ cyclopenta[4,5]imidazo[1,5-a]indole-10-carboxylate $4 \mathrm{e}$

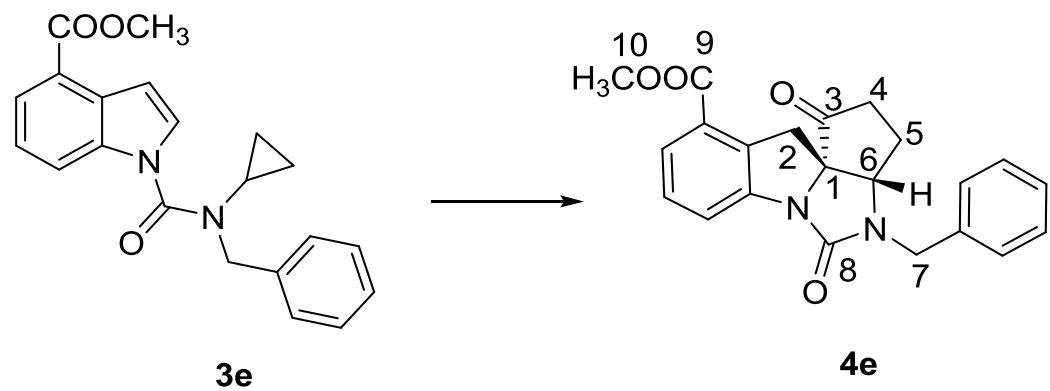

General procedure D: Compound $3 \mathbf{e}(52.2 \mathrm{mg}, 0.15 \mathrm{mmol}),\left[\mathrm{Rh}(\operatorname{cod})_{2}\right] \mathrm{OTf}(5.28 \mathrm{mg}, 7.5$ mol\%) and anhydrous 1,2-DCB $(1.50 \mathrm{~mL})$ were employed and the reaction was stirred for 72 hours at $130{ }^{\circ} \mathrm{C}$. The crude mixture was purified by column chromatography $(15 \%$ EtOAc/toluene) to yield the title compound $4 \mathrm{e}(31.7 \mathrm{mg}, 56 \%)$ as an off-white solid; m.p. 168-170 ${ }^{\circ} \mathrm{C}\left(\mathrm{CDCl}_{3}\right) ; v_{\max } / \mathrm{cm}^{-1}: 1751$ (s), 1711 (s), 1455 (s), 1407 (s), 1288 (s), 1274 (s); ${ }^{1} \mathrm{H}$ NMR (400 MHz, $\left.\mathrm{CDCl}_{3}\right): \delta 7.67-7.61(2 \mathrm{H}, \mathrm{m}, 2 \times \mathrm{ArC} \underline{\mathrm{H}}), 7.27-7.17(6 \mathrm{H}, \mathrm{m}, 6 \times \mathrm{ArC} \underline{\mathrm{H}})$, $4.59\left(1 \mathrm{H}, \mathrm{d}, J=15.2 \mathrm{~Hz}, 1 \times \mathrm{C} 7-\underline{\mathrm{H}}_{2}\right), 4.30\left(1 \mathrm{H}, \mathrm{d}, J=15.2 \mathrm{~Hz}, 1 \times \mathrm{C} 7-\underline{\mathrm{H}}_{2}\right), 4.05(1 \mathrm{H}, \mathrm{d}, J=$ $5.2 \mathrm{~Hz}, 1 \times \mathrm{C} 6-\underline{\mathrm{H}}), 3.79\left(3 \mathrm{H}, \mathrm{s}, 3 \times \mathrm{C10}-\underline{\mathrm{H}}_{3}\right), 3.67\left(1 \mathrm{H}, \mathrm{d}, J=18.0 \mathrm{~Hz}, 1 \times \mathrm{C} 2-\underline{\mathrm{H}}_{2}\right), 3.30(1 \mathrm{H}$, $\left.\mathrm{d}, J=18.0 \mathrm{~Hz}, 1 \times \mathrm{C} 2-\underline{\mathrm{H}}_{2}\right), 2.51\left(1 \mathrm{H}, \mathrm{ddd}, J=17.9,13.5,9.1 \mathrm{~Hz}, 1 \times \mathrm{C} 4-\underline{\mathrm{H}}_{2}\right), 2.29-2.14$ $\left(2 \mathrm{H}, \mathrm{m}, 1 \times \mathrm{C} 4-\underline{\mathrm{H}}_{2}, 1 \times \mathrm{C} 5-\underline{\mathrm{H}}_{2}\right), 1.88-1.78\left(1 \mathrm{H}, \mathrm{m}, 1 \times \mathrm{C} 5-\underline{\mathrm{H}}_{2}\right) ;{ }^{13} \mathrm{C} \mathrm{NMR}(101 \mathrm{MHz}$, $\mathrm{CDCl}_{3}$ ): $\delta 212.5$ (C3), 166.4 (C9), 159.1 (C8), 143.4 (CAr), 135.9 (CAr), 132.9 (CAr), 128.9 (CAr), 128.2 (CAr), 128.0 (CAr), 127.9 (CAr), 126.6 (CAr), 125.6 (CAr), 119.9 (CAr), 70.2 (C1), 64.0 (C6), 51.9 (C10), 45.9 (C7), 37.2 (C2), 33.1 (C4), 23.2 (C5); m/z (ESI ${ }^{+}$) HRMS: Calculated for $\mathrm{C}_{22} \mathrm{H}_{21} \mathrm{~N}_{2} \mathrm{O}_{4}: 377.1496$; Found $[\mathrm{M}+\mathrm{H}]^{+}: 377.1493$. 

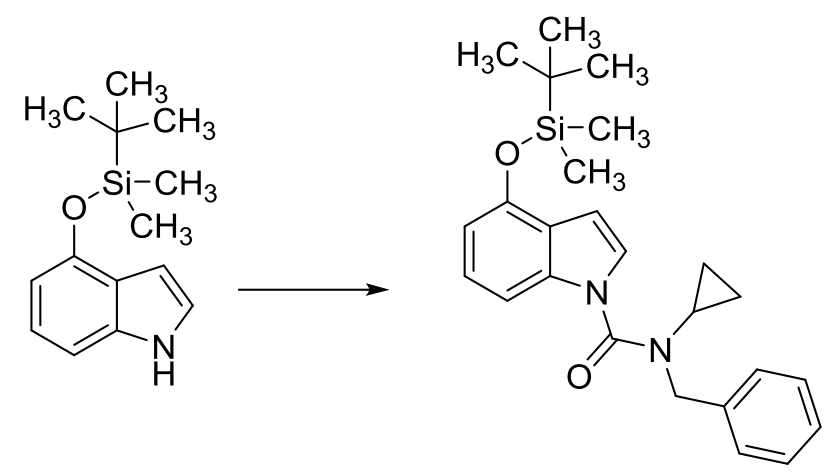

$3 \mathbf{f}$

General procedure A: 4-((tert-Butyldimethylsilyl)oxy)-1H-indole (988 mg, $4.07 \mathrm{mmol})$ and benzyl(cyclopropyl)carbamic chloride $(1.19 \mathrm{~g}, 5.70 \mathrm{mmol})$ were employed. The crude mixture was purified by flash column chromatography ( $20 \%$ toluene/hexane) to afford the title compound $3 f(1.22 \mathrm{~g}, 71 \%)$ as a yellow oil; $v_{\max } / \mathrm{cm}^{-1}: 2929(\mathrm{~m}), 1679$ (s), 1482 (s), 1407 (s), 1262 (s), 837 (s); ${ }^{1} \mathrm{H}$ NMR (400 MHz, $\left.\mathrm{CDCl}_{3}\right): \delta 7.38-7.31(7 \mathrm{H}, \mathrm{m}), 7.11(1 \mathrm{H}, \mathrm{dd}$, $J=8.0,8.0 \mathrm{~Hz}), 6.63-6.61(2 \mathrm{H}, \mathrm{m}), 4.68(2 \mathrm{H}, \mathrm{s}), 2.62(1 \mathrm{H}, \mathrm{tt}, J=6.8,4.0 \mathrm{~Hz}), 1.05(9 \mathrm{H}, \mathrm{s})$, $0.23(6 \mathrm{H}, \mathrm{s}) ;{ }^{13} \mathrm{C} \mathrm{NMR}\left(101 \mathrm{MHz}, \mathrm{CDCl}_{3}\right): \delta 155.3,148.8,137.4,137.0,128.8,128.3,127.7$, 124.5, 124.3, 123.0, 111.2, 107.4, 103.3, 53.4, 31.3, 25.8, 18.3, 9.1, -4.3; $\mathrm{m} / z\left(\mathrm{ESI}^{+}\right) \mathrm{HRMS}$ : Calculated for $\mathrm{C}_{25} \mathrm{H}_{33} \mathrm{~N}_{2} \mathrm{O}_{2} \mathrm{Si}: 421.2306$. Found $[\mathrm{M}+\mathrm{H}]^{+}: 421.2299$.

$\left(3 \mathrm{a} S^{*}, 11 \mathrm{a} S^{*}\right)-4-B e n z y l-10-(($ tert-butyldimethylsilyl)oxy)-2,3,3a,4-tetrahydro-11Hcyclopenta[4,5]imidazo[1,5-a]indole-1,5-dione $4 \mathrm{f}$

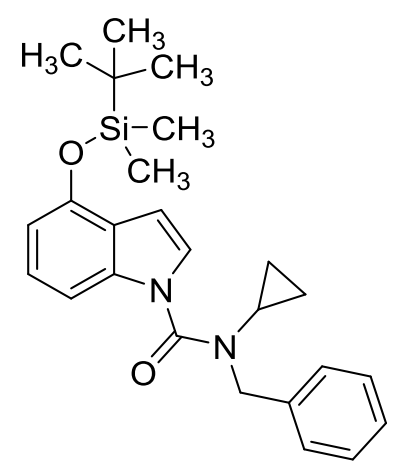

$3 \mathbf{f}$

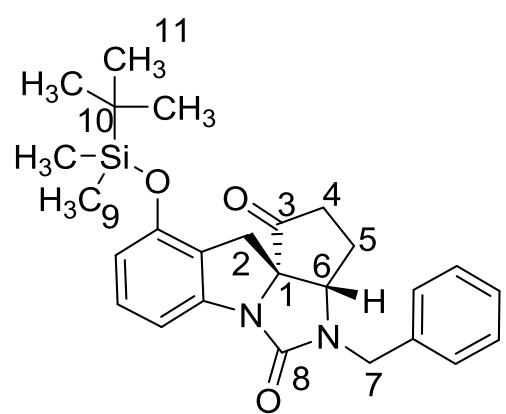

4f

General procedure D: Compound $3 f(63.0 \mathrm{mg}, 0.15 \mathrm{mmol}),\left[\mathrm{Rh}(\mathrm{cod})_{2}\right] \mathrm{OTf}(5.28 \mathrm{mg}, 7.5$ mol\%) and anhydrous 1,2-DCB $(1.50 \mathrm{~mL})$ were employed and the reaction was stirred for 72 hours at $130{ }^{\circ} \mathrm{C}$. The crude mixture was purified by column chromatography $(10 \%$ EtOAc/toluene) to yield the title compound $\mathbf{4 f}(30.3 \mathrm{mg}, 45 \%)$ as a yellow solid; m.p. 159- 
$160{ }^{\circ} \mathrm{C}\left(\mathrm{CDCl}_{3}\right) ; v_{\max } / \mathrm{cm}^{-1}: 1752$ (s), 1712 (s), 1598 (s), 1460 (s), 1407 (s), 1253 (s); ${ }^{1} \mathrm{H}$ NMR (400 MHz, $\left.\mathrm{CDCl}_{3}\right): \delta 7.14-7.05(5 \mathrm{H}, \mathrm{m}, 5 \times \mathrm{ArC} \underline{\mathrm{H}}), 6.94-6.88(2 \mathrm{H}, \mathrm{m}, 2 \times \mathrm{ArC} \underline{\mathrm{H}})$, $6.32(1 \mathrm{H}, \mathrm{dd}, J=7.1,1.9 \mathrm{~Hz}, 1 \times \mathrm{ArC} \underline{\mathrm{H}}), 4.44\left(1 \mathrm{H}, \mathrm{d}, J=15.2 \mathrm{~Hz}, 1 \times \mathrm{C} 7-\underline{\mathrm{H}}_{2}\right), 4.16(1 \mathrm{H}, \mathrm{d}, J$ $\left.=15.2 \mathrm{~Hz}, 1 \times \mathrm{C} 7-\underline{\mathrm{H}}_{2}\right), 3.88(1 \mathrm{H}, \mathrm{d}, J=5.1 \mathrm{~Hz}, 1 \times \mathrm{C} 6-\underline{\mathrm{H}}), 3.06(1 \mathrm{H}, \mathrm{d}, J=16.2 \mathrm{~Hz}, 1 \times \mathrm{C} 2-$ $\left.\underline{\mathrm{H}}_{2}\right), 2.75\left(1 \mathrm{H}, \mathrm{d}, J=16.2 \mathrm{~Hz}, 1 \times \mathrm{C} 2-\underline{\mathrm{H}}_{2}\right), 2.38\left(1 \mathrm{H}, \mathrm{ddd}, J=17.8,13.6,9.0 \mathrm{~Hz}, 1 \times \mathrm{C} 4-\underline{\mathrm{H}}_{2}\right)$, $2.15-1.96\left(2 \mathrm{H}, \mathrm{m}, 1 \times \mathrm{C} 4-\underline{\mathrm{H}}_{2}, 1 \times \mathrm{C} 5-\underline{\mathrm{H}}_{2}\right), 1.71-1.61\left(1 \mathrm{H}, \mathrm{m}, 1 \times \mathrm{C} 5-\underline{\mathrm{H}}_{2}\right), 0.76(9 \mathrm{H}, \mathrm{s}, 3 \times$ $\left.3 \times \mathrm{C} 11-\underline{\mathrm{H}}_{3}\right), 0.02\left(3 \mathrm{H}, \mathrm{s}, 3 \times \mathrm{C} 9-\underline{\mathrm{H}}_{3}\right), 0.00\left(3 \mathrm{H}, \mathrm{s}, 3 \times \mathrm{C} 9-\underline{\mathrm{H}}_{3}\right) ;{ }^{13} \mathrm{C} \mathrm{NMR}\left(101 \mathrm{MHz}, \mathrm{CDCl}_{3}\right)$ : $\delta 212.4$ (C3), 159.2 (C8), 151.9 (CAr), 143.8 (CAr), 136.2 (CAr), 129.2 (CAr), 128.8 (CAr), 128.1 (CAr), 127.8 (CAr), 120.3 (CAr), 114.8 (CAr), 109.0 (CAr), 70.3 (C1), 64.0 (C6), 45.9 (C7), 33.5 (C2), 33.1 (C4), 25.6 (C11), 23.1 (C5), 18.0 (C10), -4.3 (C9); m/z $\left(\mathrm{ESI}^{+}\right)$HRMS: Calculated for $\mathrm{C}_{26} \mathrm{H}_{33} \mathrm{~N}_{2} \mathrm{O}_{3} \mathrm{Si}$ : 449.2255; Found $[\mathrm{M}+\mathrm{H}]^{+}$: 449.2250 .

$N$-Benzyl- $N$-cyclopropyl-5-fluoro- $1 H$-indole-1-carboxamide $3 g$

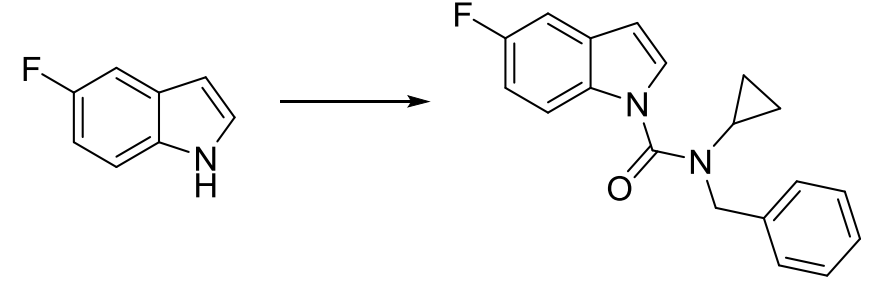

$3 \mathbf{g}$

General procedure A: 5-Fluoroindole (142 $\mathrm{mg}, \quad 1.05 \quad \mathrm{mmol})$ and benzyl(cyclopropyl)carbamic chloride $(209 \mathrm{mg}, 1.10 \mathrm{mmol})$ were employed. The crude mixture was purified by flash column chromatography (40\% toluene/hexane then $100 \%$ EtOAc) to afford the title compound $3 \mathbf{g}(280 \mathrm{mg}, 91 \%)$ as a yellow oil; $v_{\max } / \mathrm{cm}^{-1}: 2931(\mathrm{~m})$, 1675 (s), 1468 (s), 1405 (s), 1268 (s), 1199 (s); ${ }^{1} \mathrm{H}$ NMR (400 MHz, CDCl $): \delta 7.75$ - 7.72 $(1 \mathrm{H}, \mathrm{m}), 7.48-7.47(1 \mathrm{H}, \mathrm{m}), 7.40-7.28(5 \mathrm{H}, \mathrm{m}), 7.23(1 \mathrm{H}, \mathrm{dd}, J=9.0,2.6 \mathrm{~Hz}), 7.02(1 \mathrm{H}$, ddd, $J=9.0,2.6,2.6 \mathrm{~Hz}), 6.54(1 \mathrm{H}, \mathrm{dd}, J=3.5,0.7 \mathrm{~Hz}), 4.70(2 \mathrm{H}, \mathrm{s}), 2.63(1 \mathrm{H}, \mathrm{tt}, J=6.8$, $3.9 \mathrm{~Hz}), 0.1-0.56(4 \mathrm{H}, \mathrm{m}) ;{ }^{13} \mathrm{C} \mathrm{NMR}\left(101 \mathrm{MHz}, \mathrm{CDCl}_{3}\right): \delta 158.9(\mathrm{~d}, J=237.9 \mathrm{~Hz}), 154.9$, 136.9, 132.2, 130.1 (d, $J=10.2 \mathrm{~Hz}), 128.8,128.3,127.8,127.4,114.8$ (d, $J=9.4 \mathrm{~Hz}), 111.7$ $(\mathrm{d}, J=25.6 \mathrm{~Hz}), 106.1(\mathrm{~d}, J=24.0 \mathrm{~Hz}), 105.5$ (d, $J=3.9 \mathrm{~Hz}), 53.3,31.5,9.3 ;{ }^{19} \mathrm{~F} \mathrm{NMR}(377$ $\left.\mathrm{MHz}, \mathrm{CDCl}_{3}\right): \delta-121.9(\mathrm{ddd}, J=9.1,9.0,4.6 \mathrm{~Hz}) ; \mathrm{m} / z\left(\mathrm{ESI}^{+}\right) \mathrm{HRMS}: \mathrm{C}_{19} \mathrm{H}_{17} \mathrm{FN}_{2} \mathrm{NaO}$ : 331.1217. Found $[\mathrm{M}+\mathrm{Na}]^{+}: 331.1228$. 

a]indole-1,5-dione $4 \mathrm{~g}$

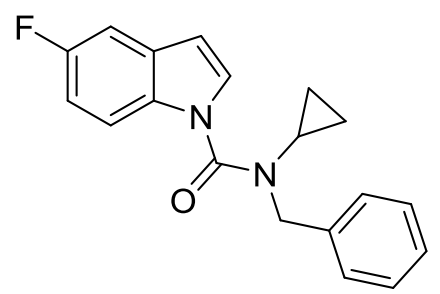

$3 \mathbf{g}$

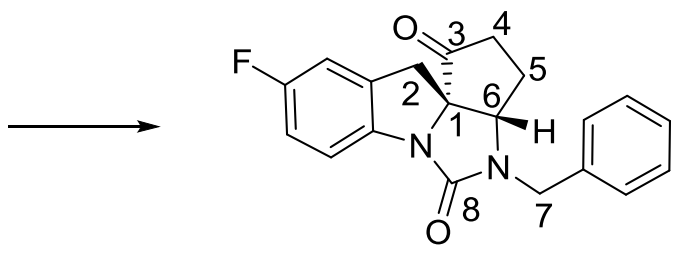

$4 \mathrm{~g}$

General procedure D: Compound $3 \mathrm{~g}(46.2 \mathrm{mg}, 0.15 \mathrm{mmol}),\left[\mathrm{Rh}(\operatorname{cod})_{2}\right] \mathrm{OTf}(5.28 \mathrm{mg}, 7.5$ mol\%) and anhydrous 1,2-DCB $(1.50 \mathrm{~mL})$ were employed and the reaction was stirred for 72 hours at $130{ }^{\circ} \mathrm{C}$. The crude mixture was purified by flash column chromatography $(10 \%$ EtOAc/toluene) to yield the title compound $4 \mathrm{~g}(27.0 \mathrm{mg}, 54 \%)$ as a colourless oil; $v_{\max } / \mathrm{cm}^{-1}$ : 2922 (m), 1751 (s), 1710 (s), 1485 (s), 1408 (s), 1308 (s), 1261 (s), 1160 (s); ${ }^{1} \mathrm{H}$ NMR (400 $\left.\mathrm{MHz}, \mathrm{CDCl}_{3}\right) \delta 7.42(1 \mathrm{H}, \mathrm{dd}, J=8.6,4.6 \mathrm{~Hz}, 1 \times \mathrm{ArC} \underline{\mathrm{H}}), 7.36-7.25(5 \mathrm{H}, \mathrm{m}, 5 \times \mathrm{ArC} \underline{\mathrm{H}})$, $6.96-6.88(2 \mathrm{H}, \mathrm{m}, 2 \times \mathrm{ArC} \underline{\mathrm{H}}), 4.68\left(1 \mathrm{H}, \mathrm{d}, J=15.2 \mathrm{~Hz}, 1 \times \mathrm{C} 7 \underline{-}_{2}\right), 4.37(1 \mathrm{H}, \mathrm{d}, J=15.2$ $\left.\mathrm{Hz}, 1 \times \mathrm{C} 7-\underline{\mathrm{H}}_{2}\right), 4.09(1 \mathrm{H}, \mathrm{d}, J=5.4 \mathrm{~Hz}, 1 \times \mathrm{C} 6-\underline{\mathrm{H}}), 3.34\left(1 \mathrm{H}, \mathrm{d}, J=16.4 \mathrm{~Hz}, 1 \times \mathrm{C} 2-\underline{\mathrm{H}}_{2}\right)$, $3.07\left(1 \mathrm{H}, \mathrm{dd}, J=16.4,1.2 \mathrm{~Hz}, 1 \times \mathrm{C} 2-\underline{\mathrm{H}}_{2}\right), 2.64\left(1 \mathrm{H}, \mathrm{ddd}, J=17.7,13.6,9.2 \mathrm{~Hz}, 1 \times \mathrm{C} 4-\underline{\mathrm{H}}_{2}\right)$, $2.35-2.19\left(2 \mathrm{H}, \mathrm{m}, 1 \times \mathrm{C} 4-\underline{\mathrm{H}}_{2}, 1 \times \mathrm{C} 5-\underline{\mathrm{H}}_{2}\right), 1.92-1.81\left(1 \mathrm{H}, \mathrm{m}, 1 \times \mathrm{C} 5-\underline{\mathrm{H}}_{2}\right) ;{ }^{13} \mathrm{C} \mathrm{NMR}(101$ $\left.\mathrm{MHz}, \mathrm{CDCl}_{3}\right): \delta 211.7(\mathrm{C3}), 160.2(\mathrm{~d}, J=242.4 \mathrm{~Hz})(\mathrm{CAr}), 159.2(\mathrm{C8}), 138.2(\mathrm{~d}, J=2.0 \mathrm{~Hz})$ (CAr), 136.0 (CAr), 132.0 (d, $J=8.7 \mathrm{~Hz}$ ) (CAr), 128.9 (CAr), 128.1 (CAr), 128.0 (CAr), $116.7(\mathrm{~d}, J=8.8 \mathrm{~Hz})(\mathrm{CAr}), 114.5(\mathrm{~d}, J=23.6 \mathrm{~Hz})(\mathrm{CAr}), 112.0(\mathrm{~d}, J=24.7 \mathrm{~Hz})(\mathrm{CAr}), 63.4$ (C1), 46.0 (C6), 35.6 (C7), 35.6 (C2), 33.1 (C4), 23.4 (C5); ${ }^{19} \mathrm{~F}$ NMR (377 MHz, $\mathrm{CDCl}_{3}$ ): $\delta$ 119.1 (ddd, $J=8.6,8.6,4.6 \mathrm{~Hz}$ ); HRMS: $\left(\mathrm{ESI}^{+}\right.$) Calculated for $\mathrm{C}_{20} \mathrm{H}_{17} \mathrm{FN}_{2} \mathrm{NaO}_{2}: 359.1184$. Found $[\mathrm{M}+\mathrm{Na}]^{+}: 359.1184$.

\section{$N$-Benzyl-5-bromo- $N$-cyclopropyl-1H-indole-1-carboxamide $3 \mathrm{~h}$}

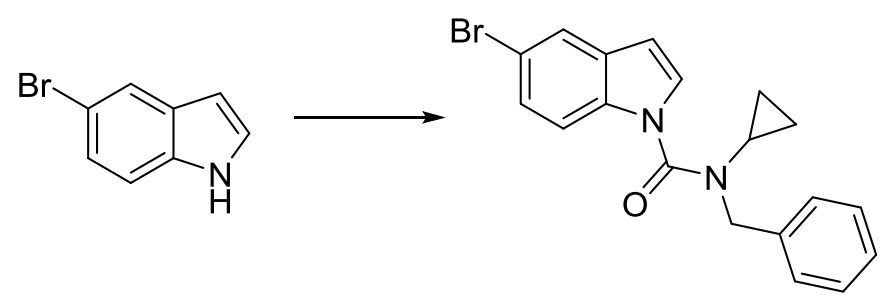

$3 \mathbf{h}$

General procedure A: 5-Bromo- $1 H$-indole $\quad(588 \quad \mathrm{mg}, \quad 3.00 \mathrm{mmol})$ and benzyl(cyclopropyl)carbamic chloride $(752 \mathrm{mg}, 3.60 \mathrm{mmol})$ were employed. The crude $\mathrm{S} 14$ 
mixture was purified by flash column chromatography ( $6 \%$ toluene/hexane) to afford the title compound 3h (842 mg, 76\%) as an off-white solid; m.p. $110-112{ }^{\circ} \mathrm{C}\left(\mathrm{CH}_{2} \mathrm{Cl}_{2}\right) ; v_{\max } / \mathrm{cm}^{-1}$ : 1673 (s), 1446 (s), 1403 (s), 1317 (s), 1200 (s), 700 (s); ${ }^{1} \mathrm{H}$ NMR (400 MHz, $\mathrm{CDCl}_{3}$ ): $\delta 7.72$ $(1 \mathrm{H}, \mathrm{d}, J=1.9 \mathrm{~Hz}), 7.66(1 \mathrm{H}, \mathrm{d}, J=8.8 \mathrm{~Hz}), 7.44-7.26(7 \mathrm{H}, \mathrm{m}), 6.52(1 \mathrm{H}, \mathrm{d}, J=3.6 \mathrm{~Hz})$, $4.70(2 \mathrm{H}, \mathrm{s}), 2.62(1 \mathrm{H}, \mathrm{tt}, J=7.0,4.0 \mathrm{~Hz}), 0.70-0.61(2 \mathrm{H}, \mathrm{m}), 0.59-0.55(2 \mathrm{H}, \mathrm{m}) ;{ }^{13} \mathrm{C}$ NMR (101 MHz, $\left.\mathrm{CDCl}_{3}\right): \delta 154.6,136.8,134.4,131.1,128.8,128.3,127.8,127.0,126.5$, 123.4, 115.3, 115.2, 105.0, 53.2, 31.5, 9.2; m/z (ESI $\left.{ }^{+}\right)$HRMS: $\mathrm{C}_{19} \mathrm{H}_{18}{ }^{79} \mathrm{BrN}_{2} \mathrm{O}: 369.0597$. Found $[\mathrm{M}+\mathrm{H}]^{+}: 369.0593$.

$\left(3 \mathrm{a} S^{*}, 11 \mathrm{a} S^{*}\right)$-4-Benzyl-9-bromo-2,3,3a,4-tetrahydro-11H-cyclopenta[4,5]imidazo[1,5a]indole-1,5-dione $4 \mathrm{~h}$

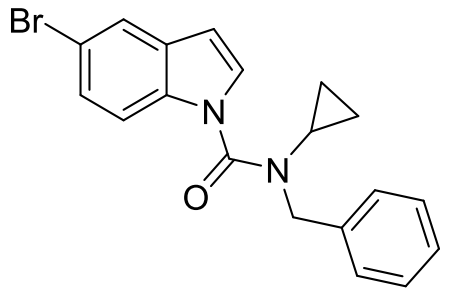

$3 \mathrm{~h}$

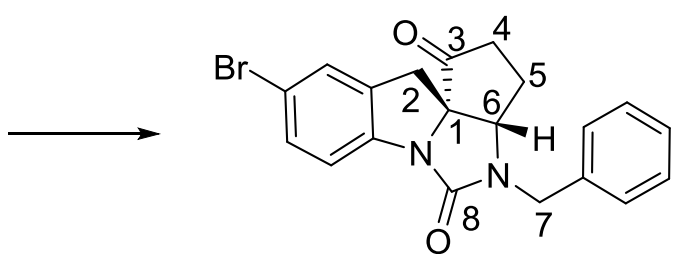

$4 h$

General procedure D: Compound $3 \mathbf{h}(55.2 \mathrm{mg}, 0.15 \mathrm{mmol}),\left[\mathrm{Rh}(\operatorname{cod})_{2}\right] \mathrm{OTf}(5.28 \mathrm{mg}, 7.5$ mol\%) and anhydrous 1,2-DCB $(1.50 \mathrm{~mL})$ were employed and the reaction was stirred for 72 hours at $130{ }^{\circ} \mathrm{C}$. The crude mixture was purified by column chromatography (5\% EtOAc/toluene) to yield the title compound $\mathbf{4 h}(33.7 \mathrm{mg}, 57 \%)$ as a colourless solid; m.p. 178-180 ${ }^{\circ} \mathrm{C}\left(\mathrm{CDCl}_{3}\right) ; v_{\max } / \mathrm{cm}^{-1}: 1751$ (s), 1709 (s), 1475 (s), 1404 (s), 1273 (s), 1158 (s); ${ }^{1} \mathrm{H}$ $\operatorname{NMR}\left(400 \mathrm{MHz}, \mathrm{CDCl}_{3}\right) \delta 7.30-7.16(8 \mathrm{H}, \mathrm{m}, 8 \times \mathrm{ArCH}), 4.60(1 \mathrm{H}, \mathrm{d}, J=15.2 \mathrm{~Hz}, 1 \times \mathrm{C} 7-$ $\left.\underline{\mathrm{H}}_{2}\right), 4.27\left(1 \mathrm{H}, \mathrm{d}, J=15.2 \mathrm{~Hz}, 1 \times \mathrm{C} 7-\underline{\mathrm{H}}_{2}\right), 4.01(1 \mathrm{H}, \mathrm{d}, J=5.4 \mathrm{~Hz}, 1 \times \mathrm{C} 6-\underline{\mathrm{H}}), 3.25(1 \mathrm{H}, \mathrm{d}, J$ $\left.=16.4 \mathrm{~Hz}, 1 \times \mathrm{C} 2-\underline{\mathrm{H}}_{2}\right), 2.99\left(1 \mathrm{H}, \mathrm{d}, J=16.4 \mathrm{~Hz}, 1 \times \mathrm{C} 2-\underline{\mathrm{H}}_{2}\right), 2.52(1 \mathrm{H}, \mathrm{ddd}, J=17.8,13.7$, $\left.9.1 \mathrm{~Hz}, 1 \times \mathrm{C} 4-\underline{\mathrm{H}}_{2}\right), 2.27-2.12\left(2 \mathrm{H}, \mathrm{m}, 1 \times \mathrm{C} 4-\underline{\mathrm{H}}_{2}, 1 \times \mathrm{C} 5-\underline{\mathrm{H}}_{2}\right), 1.83-1.73(1 \mathrm{H}, \mathrm{m}, 1 \times \mathrm{C} 5-$ $\left.\underline{\mathrm{H}}_{2}\right) ;{ }^{13} \mathrm{C} \mathrm{NMR}\left(101 \mathrm{MHz}, \mathrm{CDCl}_{3}\right): \delta 211.8$ (C3), 158.7 (C8), 141.4 (CAr), 135.9 (CAr), 132.4 (CAr), 130.9 (CAr), 128.9 (CAr), 128.0 (CAr), 128.0 (CAr), 127.8 (CAr), 117.2 (CAr), 116.9 (CAr), 70.2 (C1), 63.5 (C6), 45.9 (C7), 35.4 (C2), 33.0 (C4), 23.2 (C5); m/z $\left(\mathrm{ESI}^{+}\right)$HRMS: $\mathrm{C}_{19} \mathrm{H}_{18}{ }^{79} \mathrm{BrN}_{2} \mathrm{O}: 397.0546$. Found $[\mathrm{M}+\mathrm{H}]^{+}: 397.0539$. 


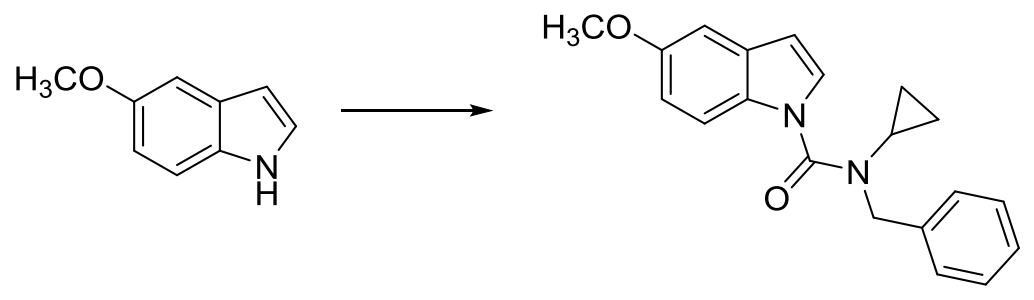

3i

General procedure A: 5-Methoxyindole (147 $\mathrm{mg}, \quad 1.00 \quad \mathrm{mmol})$ and benzyl(cyclopropyl)carbamic chloride $(220 \mathrm{mg}, 1.10 \mathrm{mmol})$ were employed. The crude mixture was purified by flash column chromatography (90\% toluene/hexane then $100 \%$ EtOAc) to afford the title compound $3 \mathbf{i}(280 \mathrm{mg}, 91 \%)$ as a light brown oil; $v_{\max } / \mathrm{cm}^{-1}: 2940$ (m), 1673 (s), 1473 (s), 1406 (s), 1266 (s), 1149 (s); ${ }^{1} \mathrm{H}$ NMR (400 MHz, $\left.\mathrm{CDCl}_{3}\right): \delta 7.69$ (1H, d, $J=9.0 \mathrm{~Hz}), 7.43(1 \mathrm{H}, \mathrm{d}, J=3.5 \mathrm{~Hz}), 7.39-7.28(5 \mathrm{H}, \mathrm{m}), 7.05(1 \mathrm{H}, \mathrm{d}, J=2.5 \mathrm{~Hz}), 6.92$ $(1 \mathrm{H}, \mathrm{dd}, J=9.0,2.5 \mathrm{~Hz}), 6.51(1 \mathrm{H}, \mathrm{dd}, J=3.5,0.7 \mathrm{~Hz}), 4.69(2 \mathrm{H}, \mathrm{s}), 3.86(3 \mathrm{H}, \mathrm{s}), 2.63(1 \mathrm{H}$, $\mathrm{tt}, J=6.7,4.0 \mathrm{~Hz}), 0.71-0.57(4 \mathrm{H}, \mathrm{m}) ;{ }^{13} \mathrm{C} \mathrm{NMR}\left(101 \mathrm{MHz}, \mathrm{CDCl}_{3}\right): \delta 155.5,155.1,137.0$, 130.6, 130.2, 128.7, 128.2, 127.7, 126.5, 114.7, 112.9, 105.7, 105.6, 103.0, 55.7, 53.2, 31.4, 9.2; $\mathrm{m} / z\left(\mathrm{ESI}^{+}\right)$HRMS: $\mathrm{C}_{20} \mathrm{H}_{20} \mathrm{~N}_{2} \mathrm{NaO}: 343.1417$. Found $[\mathrm{M}+\mathrm{Na}]^{+}: 343.1417$.

$\left(3 \mathrm{a} S^{*}, 11 \mathrm{a} S^{*}\right)$-4-Benzyl-9-methoxy-2,3,3a,4-tetrahydro-11H-cyclopenta[4,5]imidazo[1,5a]indole-1,5-dione $4 \mathrm{i}$

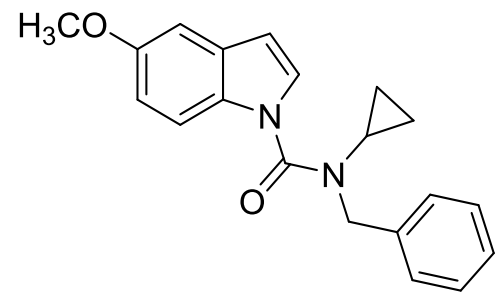

3i

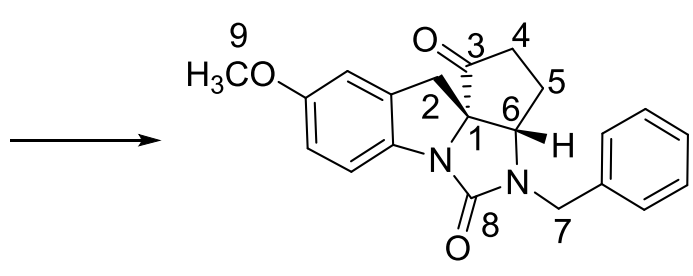

4i

General procedure D: Compound 3i $(48.0 \mathrm{mg}, 0.15 \mathrm{mmol}),\left[\mathrm{Rh}(\mathrm{cod})_{2}\right] \mathrm{OTf}(5.28 \mathrm{mg}, 7.5$ mol\%) and anhydrous 1,2-DCB $(1.50 \mathrm{~mL})$ were employed and the reaction was stirred for 72 hours at $130{ }^{\circ} \mathrm{C}$. The crude mixture was purified by flash column chromatography (15\% EtOAc/toluene) to yield the title compound $4 \mathbf{i}(34.3 \mathrm{mg}, 66 \%)$ as a colourless oil; $v_{\max } / \mathrm{cm}^{-1}$ : 2921 (m), 1750 (s), 1709 (s), 1496 (s), 1406 (s), 1155 (s), 1027 (s); ${ }^{1} \mathrm{H}$ NMR (400 MHz, $\left.\mathrm{CDCl}_{3}\right): \delta 7.39(1 \mathrm{H}, \mathrm{dd}, J=8.5,1.4 \mathrm{~Hz}, 1 \times \mathrm{ArC} \underline{\mathrm{H}}), 7.36-7.26(5 \mathrm{H}, \mathrm{m}, 5 \times \mathrm{ArC} \underline{\mathrm{H}}), 6.79-$ $6.75(2 \mathrm{H}, \mathrm{m}, 2 \times \mathrm{ArC} \underline{\mathrm{H}}), 4.68\left(1 \mathrm{H}, \mathrm{d}, J=15.4 \mathrm{~Hz}, 1 \times \mathrm{C} 7-\underline{\mathrm{H}}_{2}\right), 4.35(1 \mathrm{H}, \mathrm{dd}, J=15.4,1.4 \mathrm{~Hz}$, $\left.1 \times \mathrm{C} 7-\underline{\mathrm{H}}_{2}\right), 4.07(1 \mathrm{H}, \mathrm{d}, J=5.5 \mathrm{~Hz}, 1 \times \mathrm{C} 6-\underline{\mathrm{H}}), 3.76\left(3 \mathrm{H}, \mathrm{s}, 3 \times \mathrm{C} 9-\underline{\mathrm{H}}_{3}\right), 3.31(1 \mathrm{H}, \mathrm{d}, J=16.2$ 
$\left.\mathrm{Hz}, 1 \times \mathrm{C} 2-\underline{\mathrm{H}}_{2}\right), 3.05\left(1 \mathrm{H}, \mathrm{d}, J=16.2 \mathrm{~Hz}, 1 \times \mathrm{C} 2-\underline{\mathrm{H}}_{2}\right), 2.70-2.60\left(1 \mathrm{H}, \mathrm{m}, 1 \times \mathrm{C} 4-\underline{\mathrm{H}}_{2}\right), 2.33-$ $2.18\left(2 \mathrm{H}, \mathrm{m}, 1 \times \mathrm{C} 4-\underline{\mathrm{H}}_{2}, 1 \times \mathrm{C} 5-\underline{\mathrm{H}}_{2}\right), 1.91-1.80\left(1 \mathrm{H}, \mathrm{m}, 1 \times \mathrm{C} 5-\underline{\mathrm{H}}_{2}\right) ;{ }^{13} \mathrm{C} \mathrm{NMR}(101 \mathrm{MHz}$, $\left.\mathrm{CDCl}_{3}\right): \delta 211.9(\mathrm{C3}), 159.6$ (C8), 157.2 (CAr), 136.2 (CAr), 135.7 (CAr), 131.6 (CAr), 128.8 (CAr), 128.1 (CAr), 127.8 (CAr), 116.5 (CAr), 113.0 (CAr), 110.9 (CAr), 70.5 (C1), 63.3 (C6), 55.7 (C9), 45.9 (C7), 35.8 (C2), 33.1 (C4), 23.5 (C5); m/z HRMS: (ESI $\left.{ }^{+}\right)$ Calculated for $\mathrm{C}_{21} \mathrm{H}_{21} \mathrm{~N}_{2} \mathrm{O}_{3}: 349.1547$. Found $[\mathrm{M}+\mathrm{H}]^{+}: 349.1551$.

\section{$N$-Benzyl- $N$-cyclopropyl-6-isopropyl- $1 H$-indole-1-carboxamide $3 \mathrm{j}$}

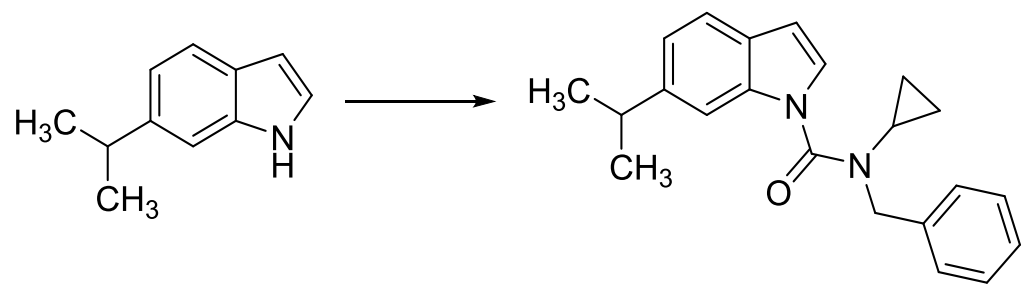

3j

General procedure A: 6-Isopropylindole $\quad(318 \mathrm{mg}, 2.00 \mathrm{mmol})$ and benzyl(cyclopropyl)carbamic chloride $(502 \mathrm{mg}, 2.40 \mathrm{mmol})$ were employed. The crude mixture was purified by flash column chromatography (90\% toluene/hexane) to afford the title compound 3j (610 mg, 92\%) as a brown oil; $v_{\max } / \mathrm{cm}^{-1}: 2958(\mathrm{~m}), 1671$ (s), 1437 (s), 1404 (s), 1318 (s), 1210 (s); ${ }^{1} \mathrm{H}$ NMR (400 MHz, $\mathrm{CDCl}_{3}$ ): $\delta 7.65$ (1H, dd, $J=1.6,0.8 \mathrm{~Hz}$ ), $7.50(1 \mathrm{H}, \mathrm{dd}, J=8.1,0.8 \mathrm{~Hz}), 7.40-7.31(6 \mathrm{H}, \mathrm{m}), 7.10(1 \mathrm{H}, \mathrm{dd}, J=8.1,1.6 \mathrm{~Hz}), 6.53(1 \mathrm{H}$, $\mathrm{dd}, J=3.6,0.8 \mathrm{~Hz}), 4.72(2 \mathrm{H}, \mathrm{s}), 3.01(1 \mathrm{H}, \mathrm{d}, J=6.9 \mathrm{~Hz}), 2.63(1 \mathrm{H}, \mathrm{tt}, J=6.7,4.0 \mathrm{~Hz}), 1.29$ $(6 \mathrm{H}, \mathrm{d}, J=6.9 \mathrm{~Hz}), 0.70-0.59(4 \mathrm{H}, \mathrm{m}) ;{ }^{13} \mathrm{C} \mathrm{NMR}\left(101 \mathrm{MHz}, \mathrm{CDCl}_{3}\right): \delta 155.3,144.9,137.0$, 136.1, 128.8, 128.4, 127.7, 127.6, 125.4, 121.2, 120.4, 111.4, 105.6, 53.2, 34.5, 31.4, 24.4, 9.2; $m / z$ (ESI $\left.{ }^{+}\right) \mathrm{HRMS}: \mathrm{C}_{22} \mathrm{H}_{24} \mathrm{~N}_{2} \mathrm{NaO}: 355.1781$. Found $[\mathrm{M}+\mathrm{Na}]^{+}: 355.1791$.

$\left(3 \mathrm{a} S^{*}, 11 \mathrm{a} S^{*}\right)$-4-Benzyl-8-isopropyl-2,3,3a,4-tetrahydro-11H-cyclopenta[4,5]imidazo[1,5a]indole-1,5-dione $4 \mathbf{j}$

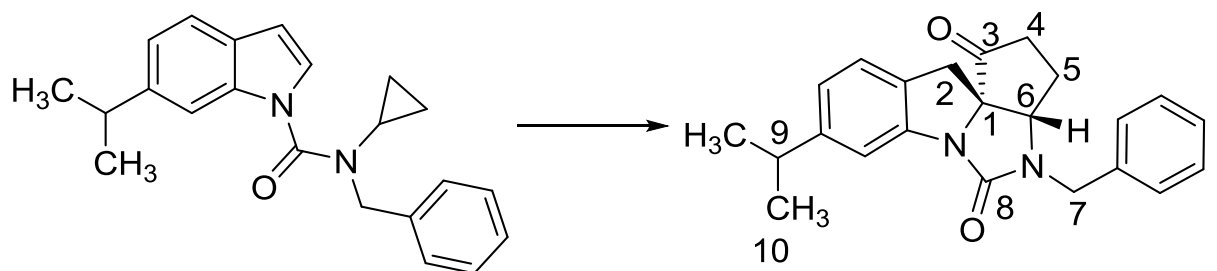

3j

$4 \mathbf{j}$ 
General procedure D: Compound $3 \mathbf{j}(50 \mathrm{mg}, 0.15 \mathrm{mmol}),\left[\mathrm{Rh}(\operatorname{cod})_{2}\right] \mathrm{OTf}(5.28 \mathrm{mg}, 7.5$ mol\%) and anhydrous 1,2-DCB $(1.50 \mathrm{~mL})$ were employed and the reaction was stirred for 72 hours at $130{ }^{\circ} \mathrm{C}$. The crude mixture was purified by flash column chromatography $(15 \%$ EtOAc/toluene) to yield the title compound $\mathbf{4 j}(45.0 \mathrm{mg}, 83 \%)$ as a colourless solid; m.p. 186-189 ${ }^{\circ} \mathrm{C}$ (EtOAc/Petroleum ether); $v_{\max } / \mathrm{cm}^{-1}: 2956$ (m), 1751 (s), 1698 (s), 1493 (s), 1410 (s), 1286 (s), 1153 (s); ${ }^{1} \mathrm{H}$ NMR (400 MHz, $\left.\mathrm{CDCl}_{3}\right) \delta 7.40-7.26(6 \mathrm{H}, \mathrm{m}, 6 \times \mathrm{ArC} \underline{\mathrm{H}}), 7.09$ $(1 \mathrm{H}, \mathrm{d}, J=7.7 \mathrm{~Hz}, 1 \times \mathrm{ArC} \underline{\mathrm{H}}), 6.94(1 \mathrm{H}, \mathrm{dd}, J=7.7,1.7 \mathrm{~Hz}, 1 \times \operatorname{ArC} \underline{\mathrm{H}}), 4.63(1 \mathrm{H}, \mathrm{d}, J=$ $\left.15.2 \mathrm{~Hz}, 1 \times \mathrm{C} 7-\underline{\mathrm{H}}_{2}\right), 4.40\left(1 \mathrm{H}, \mathrm{d}, J=15.2 \mathrm{~Hz}, 1 \times \mathrm{C} 7-\underline{\mathrm{H}}_{2}\right), 4.09(1 \mathrm{H}, \mathrm{d}, J=5.1 \mathrm{~Hz}, 1 \times \mathrm{C} 6-$ $\underline{\mathrm{H}}), 3.30\left(1 \mathrm{H}, \mathrm{dd}, J=15.8,1.0 \mathrm{~Hz}, 1 \times \mathrm{C} 2-\underline{\mathrm{H}}_{2}\right), 3.05\left(1 \mathrm{H}, \mathrm{dd}, J=15.8,1.0 \mathrm{~Hz}, 1 \times \mathrm{C} 2-\underline{\mathrm{H}}_{2}\right)$, $2.93(1 \mathrm{H}, \mathrm{d}, J=6.9 \mathrm{~Hz}, 1 \times \mathrm{C} 9-\underline{\mathrm{H}}), 2.59\left(1 \mathrm{H}, \mathrm{ddd}, J=17.7,13.7,9.1 \mathrm{~Hz}, 1 \times \mathrm{C} 4-\underline{\mathrm{H}}_{2}\right), 2.34-$ $2.19\left(2 \mathrm{H}, \mathrm{m}, 1 \times \mathrm{C} 4-\underline{\mathrm{H}}_{2}, 1 \times \mathrm{C} 5-\underline{\mathrm{H}}_{2}\right), 1.91-1.80\left(1 \mathrm{H}, \mathrm{m}, 1 \times \mathrm{C} 5-\underline{\mathrm{H}}_{2}\right), 1.26(6 \mathrm{H}, \mathrm{d}, J=6.9 \mathrm{~Hz}$, $\left.2 \times 3 \times \mathrm{C10}-\underline{\mathrm{H}}_{3}\right) ;{ }^{13} \mathrm{C} \mathrm{NMR}\left(101 \mathrm{MHz}, \mathrm{CDCl}_{3}\right): \delta 212.7$ (C3), 159.3 (C8), 149.5 (CAr), 142.5 (CAr), 136.4 (CAr), 129.0 (CAr), 128.3 (CAr), 128.0 (CAr), 127.5 (CAr), 124.5 (CAr), 122.5 (CAr), 114.2 (CAr), 70.6 (C1), 64.1 (C6), 46.1 (C7), 35.6 (C2), 34.3 (C9), 33.3 (C4), 24.4 (C10), 24.0 (C10), 23.4 (C5); HRMS: (ESI ${ }^{+}$) Calculated for $\mathrm{C}_{23} \mathrm{H}_{25} \mathrm{~N}_{2} \mathrm{O}_{2}$ : 361.1911. Found $[\mathrm{M}+\mathrm{H}]^{+}:$361.1921. The structure of this compound was determined unambiguously by $X$-ray crystallography.

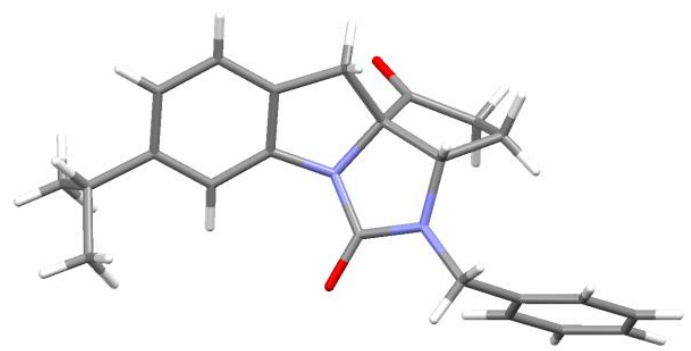

\section{$N$-Benzyl- $N$-cyclopropyl-7-methyl-1H-indole-1-carboxamide 3k}
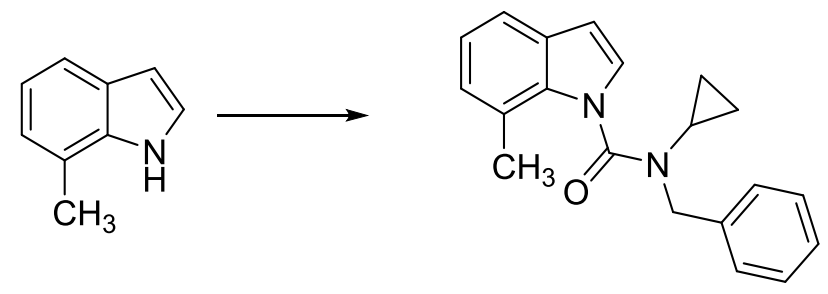

$3 \mathbf{k}$

General procedure A: 7-Methyl- $1 H$-indole $\quad(196 \quad \mathrm{mg}, \quad 1.50 \mathrm{mmol})$ and benzyl(cyclopropyl)carbamic chloride $(407 \mathrm{mg}, 1.95 \mathrm{mmol}$ ) were employed. The crude 
mixture was purified by flash column chromatography (100\% toluene) to afford the title compound 3k (454 mg, 98\%) as a yellow oil; $v_{\max } / \mathrm{cm}^{-1}: 1681$ (s), 1456 (s), 1402 (s), 1309 (s), 1240 (s), 701 (s); ${ }^{1} \mathrm{H}$ NMR (400 MHz, $\left.\mathrm{CDCl}_{3}\right): \delta 7.46(1 \mathrm{H}, \mathrm{ddd}, J=7.5,1.0,1.0 \mathrm{~Hz})$, $7.39-7.30(5 \mathrm{H}, \mathrm{m}), 7.20(1 \mathrm{H}, \mathrm{d}, J=3.5 \mathrm{~Hz}), 7.13(1 \mathrm{H}, \mathrm{dd}, J=7.5,7.5 \mathrm{~Hz}), 7.07$ (1H, ddd, $J$ $=7.5,1.0,1.0 \mathrm{~Hz}), 6.59(1 \mathrm{H}, \mathrm{d}, J=3.5 \mathrm{~Hz}), 4.70(2 \mathrm{H}, \mathrm{s}, b r), 2.66-2.60(1 \mathrm{H}, \mathrm{m}), 2.42(3 \mathrm{H}$, s), $0.66(4 \mathrm{H}, \mathrm{s}, b r) ;{ }^{13} \mathrm{C} \mathrm{NMR}\left(101 \mathrm{MHz}, \mathrm{CDCl}_{3}\right): \delta 155.2,137.0,134.9,130.2,128.7,128.3$, $127.8,126.3,125.9,123.2,122.0,118.7,105.7,53.0,31.0,19.9,8.7 ; \mathrm{m} / \mathrm{z}\left(\mathrm{ESI}^{+}\right) \mathrm{HRMS}:$ $\mathrm{C}_{20} \mathrm{H}_{21} \mathrm{~N}_{2} \mathrm{O}: 305.1648$. Found $[\mathrm{M}+\mathrm{H}]^{+}:$305.1645.

$\left(3 a S^{*}, 11 a S^{*}\right)-4-B e n z y l-7-m e t h y l-2,3,3 a, 4-t e t r a h y d r o-11 H$-cyclopenta[4,5]imidazo[1,5a]indole-1,5-dione $4 \mathrm{k}$

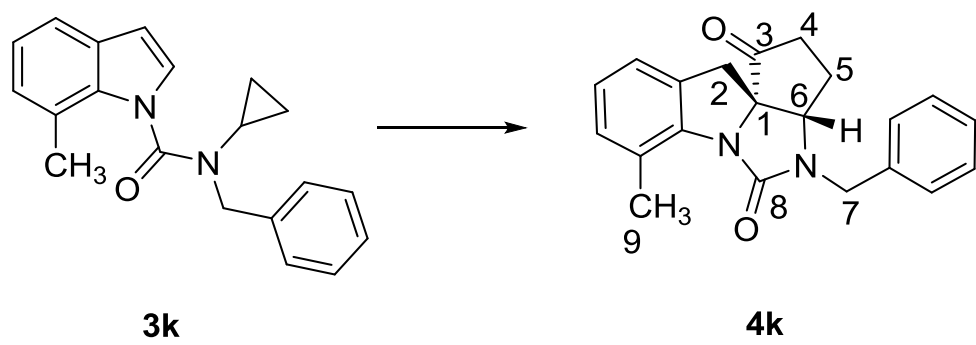

General procedure D: Compound 3k $(45.6 \mathrm{mg}, 0.15 \mathrm{mmol}),\left[\mathrm{Rh}(\operatorname{cod})_{2}\right] \mathrm{OTf}(5.28 \mathrm{mg}, 7.5$ mol\%) and anhydrous 1,2-DCB $(1.50 \mathrm{~mL})$ were employed and the reaction was stirred for 72 hours at $130{ }^{\circ} \mathrm{C}$. The crude mixture was purified by column chromatography $(10 \%$ toluene/hexane) to yield the title compound $4 \mathbf{k}(10.6 \mathrm{mg}, 21 \%)$ as a yellow oil; $v_{\max } / \mathrm{cm}^{-1}$ : 2919 (m), 1751 (s), 1710 (s), 1409 (s), 1267 (s), 1160 (s); ${ }^{1} \mathrm{H}$ NMR (400 MHz, CDCl 3 ) $\delta 7.37$ $-7.25(5 \mathrm{H}, \mathrm{m}, 5 \times \mathrm{ArCH}), 7.09-6.98(3 \mathrm{H}, \mathrm{m}, 3 \times \mathrm{ArCH}), 4.79(1 \mathrm{H}, \mathrm{d}, J=15.1 \mathrm{~Hz}, 1 \times \mathrm{C} 7-$ $\left.\underline{\mathrm{H}}_{2}\right), 4.27\left(1 \mathrm{H}, \mathrm{d}, J=15.1 \mathrm{~Hz}, 1 \times \mathrm{C} 7-\underline{\mathrm{H}}_{2}\right), 4.00(1 \mathrm{H}, \mathrm{d}, J=6.4 \mathrm{~Hz}, 1 \times \mathrm{C} 6-\underline{\mathrm{H}}), 3.23(1 \mathrm{H}, \mathrm{d}, J$ $\left.=15.8 \mathrm{~Hz}, 1 \times \mathrm{C} 2-\underline{\mathrm{H}}_{2}\right), 3.07\left(1 \mathrm{H}, \mathrm{d}, J=15.8 \mathrm{~Hz}, 1 \times \mathrm{C} 2-\underline{\mathrm{H}}_{2}\right), 2.82(1 \mathrm{H}, \mathrm{ddd}, J=17.3,12.4$, $\left.9.6 \mathrm{~Hz}, 1 \times \mathrm{C} 4-\underline{\mathrm{H}}_{2}\right), 2.56\left(3 \mathrm{H}, \mathrm{s}, 3 \times \mathrm{C} 9-\underline{\mathrm{H}}_{3}\right), 2.37-2.26\left(1 \mathrm{H}, \mathrm{m}, 1 \times \mathrm{C} 4-\underline{\mathrm{H}}_{2}\right), 2.23-2.11(1 \mathrm{H}$, m, $\left.1 \times \mathrm{C} 5-\underline{\mathrm{H}}_{2}\right), 1.93\left(1 \mathrm{H}\right.$, dddd, $\left.J=14.3,12.4,8.0,6.4 \mathrm{~Hz}, 1 \times \mathrm{C} 5-\underline{\mathrm{H}}_{2}\right) ;{ }^{13} \mathrm{C} \mathrm{NMR}(101 \mathrm{MHz}$, $\mathrm{CDCl}_{3}$ ): $\delta 210.8$ (C3), 159.8 (C8), 140.8 (CAr), 136.2 (CAr), 131.8 (CAr), 130.3 (CAr), 129.0 (CAr), 128.9 (CAr), 128.1 (CAr), 127.9 (CAr), 125.4 (CAr), 121.7 (CAr), 71.0 (C1), 61.0 (C6), 46.0 (C7), 35.4 (C2), 33.5 (C4), 24.3 (C5), 19.4 (C9); HRMS: (ESI ${ }^{+}$) Calculated for $\mathrm{C}_{21} \mathrm{H}_{21} \mathrm{~N}_{2} \mathrm{O}_{2}: 333.1598$. Found $[\mathrm{M}+\mathrm{H}]^{+}: 333.1595$. 


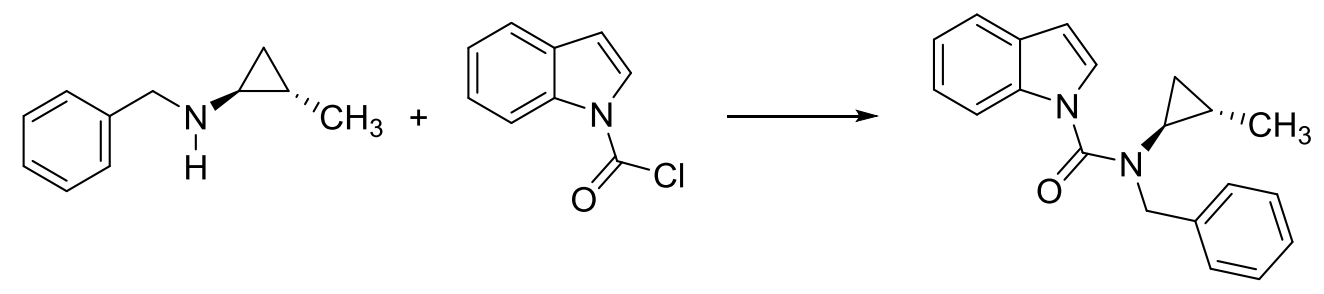

$3 \mathbf{3}$

General procedure B: $\left(1 S^{*}, 2 S^{*}\right)-N$-Benzyl-2-methylcyclopropan-1-amine (306 mg, 1.90 mmol) (prepared according to the literature procedure) ${ }^{3}$ and $1 H$-indole-1-carbonyl chloride (684 $\mathrm{mg}, 3.80 \mathrm{mmol}$ ) (prepared according to the literature procedure) ${ }^{4}$ were employed. The crude mixture was purified by flash column chromatography (75\% toluene/hexane) to afford the title compound 31 (472 mg, 82\%) as a yellow oil; $v_{\max } / \mathrm{cm}^{-1}: 1672(\mathrm{~s}), 1452$ (s), 1406 (s), 1311 (s), 1294 (s), 1210 (s); ${ }^{1} \mathrm{H}$ NMR (400 MHz, $\mathrm{CDCl}_{3}$ ): $\delta 7.66$ (1H, ddd, $J=8.3,1.0,1.0$ $\mathrm{Hz}), 7.51(1 \mathrm{H}, \mathrm{ddd}, J=7.5,1.0,1.0 \mathrm{~Hz}), 7.34-7.17(7 \mathrm{H}, \mathrm{m}), 7.11(1 \mathrm{H}, \mathrm{ddd}, J=7.5,7.5,1.0$ Hz), $6.51(1 \mathrm{H}, \mathrm{dd}, J=3.6,0.8 \mathrm{~Hz}), 4.76(1 \mathrm{H}, \mathrm{d}, J=14.4 \mathrm{~Hz}), 4.46(1 \mathrm{H}, \mathrm{d}, J=14.4 \mathrm{~Hz}), 2.21$ $(1 \mathrm{H}, \mathrm{ddd}, J=7.0,3.6,3.6 \mathrm{~Hz}), 0.81-0.73(1 \mathrm{H}, \mathrm{m}), 0.67-0.62(4 \mathrm{H}, \mathrm{m}), 0.33(1 \mathrm{H}, \mathrm{ddd}, J=$ 7.0, 5.9, $5.9 \mathrm{~Hz}) ;{ }^{13} \mathrm{C} \mathrm{NMR}\left(101 \mathrm{MHz}, \mathrm{CDCl}_{3}\right): \delta 155.0,137.0,135.6,129.5,128.7,128.4$, $127.7,125.9,123.5,121.9,120.8,113.8,105.7,52.9,38.7,17.4,16.8,16.6 ; \mathrm{m} / \mathrm{z}\left(\mathrm{ESI}^{+}\right)$ HRMS: $\mathrm{C}_{20} \mathrm{H}_{20} \mathrm{~N}_{2} \mathrm{NaO}$ : 327.1468. Found [M+Na] $]^{+}$327.1477.

$\left(3 S^{*}, 3 \mathrm{a} R^{*}, 11 \mathrm{a} R^{*}\right)-4-B e n z y l-3-m e t h y l-2,3,3 \mathrm{a}, 4-$ tetrahydro-11H-

\section{cyclopenta[4,5]imidazo[1,5-a]indole-1,5-dione 41}

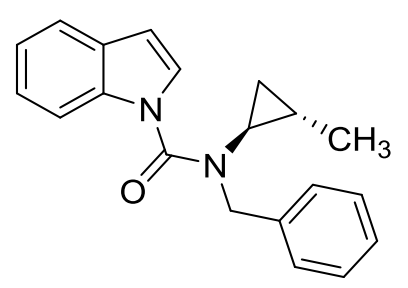

31

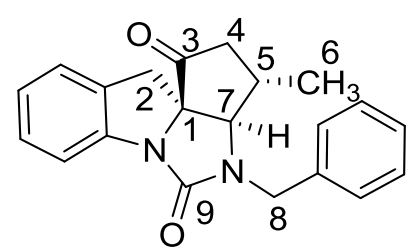

4!

General procedure D: Compound 31 (45.6 mg, $0.15 \mathrm{mmol})$, [Rh(cod) $\left.)_{2}\right] \mathrm{OTf}(5.28 \mathrm{mg}, 7.5$ mol\%) and anhydrous 1,2-DCB $(1.50 \mathrm{~mL})$ were employed and the reaction was stirred for 96 hours at $130{ }^{\circ} \mathrm{C}$. The crude mixture was purified by column chromatography $(10 \%$ EtOAc/toluene) to yield the title compound $\mathbf{4 l}(33.8 \mathrm{mg}, 68 \%)$ as a colourless solid; m.p. 135-137 ${ }^{\circ} \mathrm{C}\left(\mathrm{CDCl}_{3}\right) ; v_{\max } / \mathrm{cm}^{-1}: 1751$ (s), 1708 (s), 1481 (s), 1406 (s), 1291 (s), 1160 (s); ${ }^{1} \mathrm{H}$ NMR $\left(400 \mathrm{MHz}, \mathrm{CDCl}_{3}\right) \delta 7.43(1 \mathrm{H}, \mathrm{d}, J=7.9 \mathrm{~Hz}, 1 \times \mathrm{ArC} \underline{\mathrm{H}}), 7.28-7.09(6 \mathrm{H}, \mathrm{m}, 6 \times$ 
$\operatorname{ArC} \underline{\mathrm{H}}), 7.10(1 \mathrm{H}, \mathrm{d}, J=7.5 \mathrm{~Hz}, 1 \times \mathrm{ArC} \underline{\mathrm{H}}), 6.98(1 \mathrm{H}, \mathrm{dd}, J=7.5,7.5 \mathrm{~Hz}, 1 \times \operatorname{ArC} \underline{\mathrm{H}}), 4.60$ $\left(1 \mathrm{H}, \mathrm{d}, J=15.2 \mathrm{~Hz}, 1 \times \mathrm{C} 8-\underline{\mathrm{H}}_{2}\right), 4.30\left(1 \mathrm{H}, \mathrm{d}, J=15.2 \mathrm{~Hz}, 1 \times \mathrm{C} 8-\underline{\mathrm{H}}_{2}\right), 3.61(1 \mathrm{H}, \mathrm{s}, 1 \times \mathrm{C} 7-$ $\underline{\mathrm{H}}), 3.31\left(1 \mathrm{H}, \mathrm{d}, J=16.1 \mathrm{~Hz}, 1 \times \mathrm{C} 2-\underline{\mathrm{H}}_{2}\right), 3.02\left(1 \mathrm{H}, \mathrm{d}, J=16.1 \mathrm{~Hz}, 1 \times \mathrm{C} 2-\underline{\mathrm{H}}_{2}\right), 2.75(1 \mathrm{H}, \mathrm{dd}$, $\left.J=17.2,7.7 \mathrm{~Hz}, 1 \times \mathrm{C} 4-\underline{\mathrm{H}}_{2}\right), 2.48-2.40(1 \mathrm{H}, \mathrm{m}, 1 \times \mathrm{C} 5-\underline{\mathrm{H}}), 1.98(1 \mathrm{H}, \mathrm{d}, J=17.2 \mathrm{~Hz}, 1 \times$ C4- $\left.\underline{\mathrm{H}}_{2}\right), 0.84\left(3 \mathrm{H}, \mathrm{d}, J=7.4 \mathrm{~Hz}, 3 \times \mathrm{C6}-\underline{\mathrm{H}}_{3}\right) ;{ }^{13} \mathrm{C} \mathrm{NMR}\left(101 \mathrm{MHz}, \mathrm{CDCl}_{3}\right): \delta 211.8(\mathrm{C} 3)$, 158.9 (C9), 142.0 (CAr), 136.1 (CAr), 130.3 (CAr), 128.9 (CAr), 128.2 (CAr), 128.0 (CAr), 127.9 (CAr), 124.6 (CAr), 124.3 (CAr), 115.9 (CAr), 70.4 (C7), 69.4 (C1), 46.2 (C8), 41.1 (C4), 36.7 (C2), 29.6 (C5), 18.9 (C6); HRMS: (ESI ${ }^{+}$) Calculated for $\mathrm{C}_{21} \mathrm{H}_{20} \mathrm{~N}_{2} \mathrm{NaO}_{2}$ : 355.1420. Found $[\mathrm{M}+\mathrm{Na}]^{+}:$355.1421. The structure of this compound was determined unambiguously by X-ray crystallography.

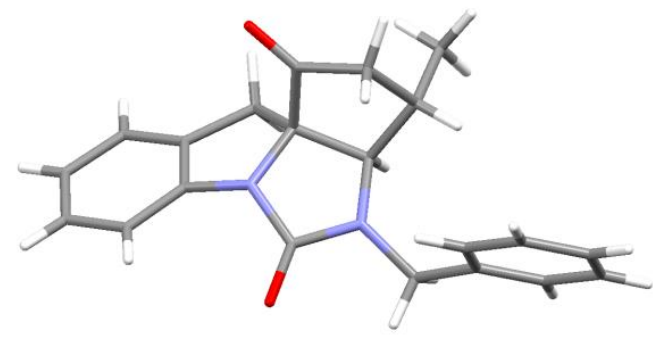

$N$-Benzyl- $N$-((1S*, 2S*)-2-benzylcyclopropyl)-1H-indole-1-carboxamide 3m<smiles>C/C=C\C(=C/C)C[C@@H]1C[C@H]1NCc1ccccc1</smiles><smiles>O=C(N(Cc1ccccc1)[C@H]1C[C@H]1Cc1ccccc1)n1ccc2ccccc21</smiles>

$3 m$

General procedure B: $\left(1 S^{*}, 2 S^{*}\right)-N, 2$-Dibenzylcyclopropan-1-amine $(1.28 \mathrm{~g}, 5.40 \mathrm{mmol})$ (prepared according to the literature procedure $)^{3}$ and $1 H$-indole-1-carbonyl chloride (1.94 g, $10.8 \mathrm{mmol}$ ) were employed. The crude mixture was purified by flash column chromatography (80\% toluene/hexane) to afford the title compound $\mathbf{3 m}(1.56 \mathrm{~g}, 76 \%)$ as a yellow oil; $v_{\max } / \mathrm{cm}^{-1}$ : 1674 (s), 1453 (s), 1408 (s), 1319 (s), 1210 (s), 746 (s); ${ }^{1} \mathrm{H}$ NMR (400 $\left.\mathrm{MHz}, \mathrm{CDCl}_{3}\right): \delta 7.62(1 \mathrm{H}, \mathrm{d}, J=8.3 \mathrm{~Hz}), 7.52(1 \mathrm{H}, \mathrm{d}, J=7.7 \mathrm{~Hz}), 7.34(1 \mathrm{H}, \mathrm{d}, J=3.5 \mathrm{~Hz})$, $7.29-7.18(4 \mathrm{H}, \mathrm{m}), 7.17-7.08(6 \mathrm{H}, \mathrm{m}), 6.88-6.86(2 \mathrm{H}, \mathrm{m}), 6.53(1 \mathrm{H}, \mathrm{d}, J=3.5 \mathrm{~Hz}), 4.58$ 
$(1 \mathrm{H}, \mathrm{d}, J=14.6 \mathrm{~Hz}), 4.50(1 \mathrm{H}, \mathrm{d}, J=14.6 \mathrm{~Hz}), 2.43-2.32(2 \mathrm{H}, \mathrm{m}), 2.01(1 \mathrm{H}, \mathrm{dd}, J=14.4$, $8.2 \mathrm{~Hz}), 1.15-1.07(1 \mathrm{H}, \mathrm{m}), 0.69(1 \mathrm{H}, \mathrm{ddd}, J=9.7,5.9,4.0 \mathrm{~Hz}), 0.54-0.49(1 \mathrm{H}, \mathrm{m}) ;{ }^{13} \mathrm{C}$ NMR $\left(101 \mathrm{MHz}, \mathrm{CDCl}_{3}\right): \delta 155.1,139.8,136.9,135.4,129.6,128.7,128.5,128.4,128.3$, 127.7, 126.2, 125.9, 123.6, 121.9, 120.9, 113.6, 105.8, 53.1, 37.4, 37.4, 23.8, 15.7; m/z (ESI $\left.{ }^{+}\right)$ HRMS: $\mathrm{C}_{26} \mathrm{H}_{24} \mathrm{~N}_{2} \mathrm{NaO}: 403.1780$. Found $[\mathrm{M}+\mathrm{Na}]^{+}: 403.1788$.

$\left(3 S^{*}, 3 \mathrm{a} R^{*}, 11 \mathrm{a} R^{*}\right)-3,4-D i b e n z y l-2,3,3 \mathrm{a}, 4-t e t r a h y d r o-11 H$-cyclopenta[4,5]imidazo[1,5a]indole-1,5-dione $4 \mathrm{~m}$

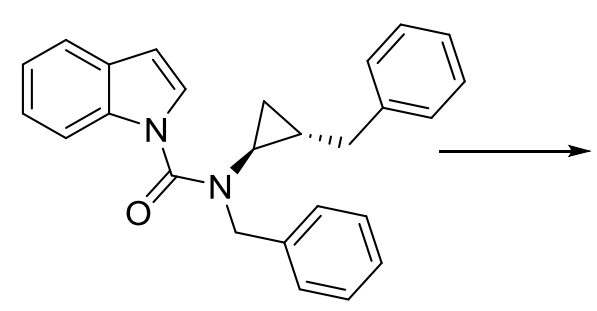

$3 m$

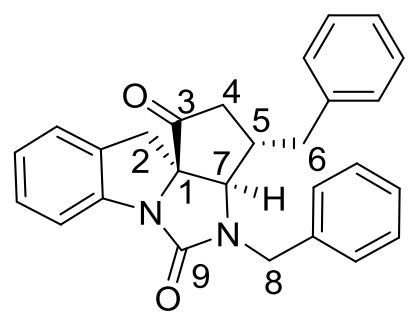

$4 \mathrm{~m}$

General procedure D: Compound $3 \mathrm{~m}(38.0 \mathrm{mg}, 0.10 \mathrm{mmol}),\left[\mathrm{Rh}(\mathrm{cod})_{2}\right] \mathrm{OTf}(3.52 \mathrm{mg}, 7.5$ mol\%) and anhydrous 1,2-DCB $(1.00 \mathrm{~mL})$ were employed and the reaction was stirred for 96 hours at $130{ }^{\circ} \mathrm{C}$. The crude mixture was purified by column chromatography $(10 \%$ EtOAc/toluene) to yield the title compound $\mathbf{4 m}(21.2 \mathrm{mg}, 52 \%)$ as a colourless oil; $v_{\max } / \mathrm{cm}^{-}$ 1: 1752 (s), 1708 (s), 1480 (s), 1407 (s), 1292 (s), 1162 (s); ${ }^{1} \mathrm{H}$ NMR (500 MHz, CDCl $) \delta$ $7.49(1 \mathrm{H}, \mathrm{d}, J=7.9 \mathrm{~Hz}, 1 \times \mathrm{ArC} \underline{\mathrm{H}}), 7.34-7.29(3 \mathrm{H}, \mathrm{m}, 3 \times \mathrm{ArC} \underline{\mathrm{H}}), 7.26-7.18(5 \mathrm{H}, \mathrm{m}, 5 \times$ $\operatorname{ArC} \underline{\mathrm{H}}), 7.08-7.02(5 \mathrm{H}, \mathrm{m}, 5 \times \operatorname{ArC} \underline{\mathrm{H}}), 4.54\left(1 \mathrm{H}, \mathrm{d}, J=15.0 \mathrm{~Hz}, 1 \times \mathrm{C8}-\underline{\mathrm{H}}_{2}\right), 4.22(1 \mathrm{H}, \mathrm{d}, J$ $\left.=15.0 \mathrm{~Hz}, 1 \times \mathrm{C} 8-\underline{\mathrm{H}}_{2}\right), 3.85(1 \mathrm{H}, \mathrm{s}, 1 \times \mathrm{C} 7-\underline{\mathrm{H}}), 3.41\left(1 \mathrm{H}, \mathrm{d}, J=16.0 \mathrm{~Hz}, 1 \times \mathrm{C} 2-\underline{\mathrm{H}}_{2}\right), 3.13$ $\left(1 \mathrm{H}, \mathrm{d}, J=16.0 \mathrm{~Hz}, 1 \times \mathrm{C} 2-\underline{\mathrm{H}}_{2}\right), 2.89\left(1 \mathrm{H}, \mathrm{dd}, J=17.3,8.0 \mathrm{~Hz}, 1 \times \mathrm{C} 6-\underline{\mathrm{H}}_{2}\right), 2.71-2.66(1 \mathrm{H}$, m, $1 \times \mathrm{C} 5-\underline{H}), 2.58\left(1 \mathrm{H}, \mathrm{dd}, J=14.0,7.1 \mathrm{~Hz}, 1 \times \mathrm{C} 4-\underline{\mathrm{H}}_{2}\right), 2.36(1 \mathrm{H}, \mathrm{dd}, J=14.0,9.6 \mathrm{~Hz}, 1 \times$ C4- $\left.\underline{\mathrm{H}}_{2}\right), 2.22\left(1 \mathrm{H}, \mathrm{d}, J=17.3 \mathrm{~Hz}, 1 \times \mathrm{C} 6-\underline{\mathrm{H}}_{2}\right) ;{ }^{13} \mathrm{C} \mathrm{NMR}\left(126 \mathrm{MHz}, \mathrm{CDCl}_{3}\right): \delta 211.4(\mathrm{C} 3)$, 158.9 (C9), 141.9 (CAr), 137.6 (CAr), 135.9 (CAr), 130.3 (CAr), 129.0 (CAr), 128.9 (CAr), 128.8 (CAr), 128.3 (CAr), 128.0 (CAr), 127.8 (CAr), 127.0 (CAr), 124.6 (CAr), 124.4 (CAr), 116.0 (CAr), 69.4 (C1), 67.2 (C7), 46.1 (C8), 39.6 (C6), 39.2 (C4), 37.0 (C5), 36.3 (C2); HRMS: $\left(\mathrm{ESI}^{+}\right)$Calculated for $\mathrm{C}_{27} \mathrm{H}_{24} \mathrm{~N}_{2} \mathrm{NaO}_{2}$ : 431.1730. Found [M+Na] $]^{+}: 431.1721$. 


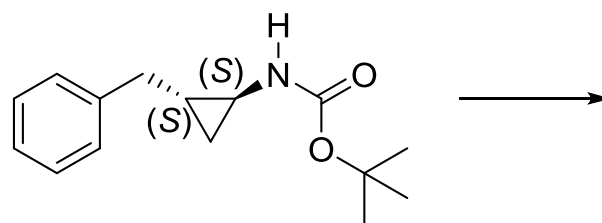

98.5:1.5 e.r.

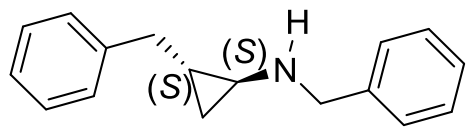

98.5:1.5 e.r.

To a stirred solution of tert-butyl ((1S,2S)-2-benzylcyclopropyl)carbamate (1.93 g, 7.51 mmol) (98.5:1.5 e.r.) (prepared according to the literature procedure from $(R)-(2,3-$ epoxypropyl)benzene (98.5:1.5 e.r.) $)^{3}$ in $\mathrm{CH}_{2} \mathrm{Cl}_{2}(25 \mathrm{~mL})$ was added trifluoroacetic acid (6.00 $\mathrm{mL}, 75.1 \mathrm{mmol}$ ) dropwise over 5 minutes. The reaction mixture was stirred at room temperature for 4 hours before concentrating in vacuo. Water $(40 \mathrm{~mL})$ was added to the crude material and the solution was extracted with $\mathrm{CH}_{2} \mathrm{Cl}_{2}(40 \mathrm{~mL})$. The aqueous portion was adjusted to $\mathrm{pH} 12$ by addition of $2 \mathrm{M}$ aqueous $\mathrm{NaOH}$ and then extracted with $\mathrm{CH}_{2} \mathrm{Cl}_{2}(3 \times 20$ $\mathrm{mL}$ ). The organic extracts were combined, dried over $\mathrm{Na}_{2} \mathrm{SO}_{4}$ and concentrated in vacuo. The residue was dissolved in $\mathrm{MeOH}(40 \mathrm{~mL}) \cdot \mathrm{NaHCO}_{3}(2.52 \mathrm{~g}, 30 \mathrm{mmol})$ and benzaldehyde $(0.72 \mathrm{~mL}, 7.13 \mathrm{mmol})$ were added and the solution was heated at reflux for $24 \mathrm{~h}$. The reaction mixture was cooled to $0{ }^{\circ} \mathrm{C}$ and $\mathrm{NaBH}_{4}(379 \mathrm{mg}, 9.76 \mathrm{mmol})$ was added portionwise over 5 minutes. The reaction was warmed to room temperature and stirred for 18 hours, then concentrated in vacuo. Saturated aqueous $\mathrm{NaHCO}_{3}(60 \mathrm{~mL})$ and $\mathrm{CH}_{2} \mathrm{Cl}_{2}(50 \mathrm{~mL})$ were added, the layers were separated and the aqueous portion was further extracted with $\mathrm{CH}_{2} \mathrm{Cl}_{2}(2 \times 30$ $\mathrm{mL})$. The organic extracts were combined, washed with brine $(50 \mathrm{~mL})$, dried over $\mathrm{Na}_{2} \mathrm{SO}_{4}$ and concentrated in vacuo. The crude mixture was purified by flash column chromatography (20\% EtOAc/hexane) to afford the title compound $(1.00 \mathrm{~g}, 55 \%)$ as a colourless oil. The enantiopurity of this compound was confirmed after the subsequent step.

$[\boldsymbol{\alpha}]_{\mathbf{D}}{ }^{19}+2.17\left(\mathrm{c}=0.64, \mathrm{CHCl}_{3}\right)$. All other analytical data were identical to reported literature. $^{3}$

(R)-(2,3-Epoxypropyl)benzene (98.5:1.5 e.r.) was prepared according to the literature procedure. ${ }^{5}$ The enantiopurity of this compound was determined by chiral SFC (Chiralpak IC, isocratic $\mathrm{CO}_{2}$-IPA 99:1, $3.0 \mathrm{~mL} / \mathrm{min}, 40{ }^{\circ} \mathrm{C}$ ) against a racemic standard; ( $\mathrm{t}_{\mathrm{R}}$ major $-6.3 \mathrm{~min}$ and $t_{R}$ (minor) $\left.-7.4 \mathrm{~min}\right)$. 


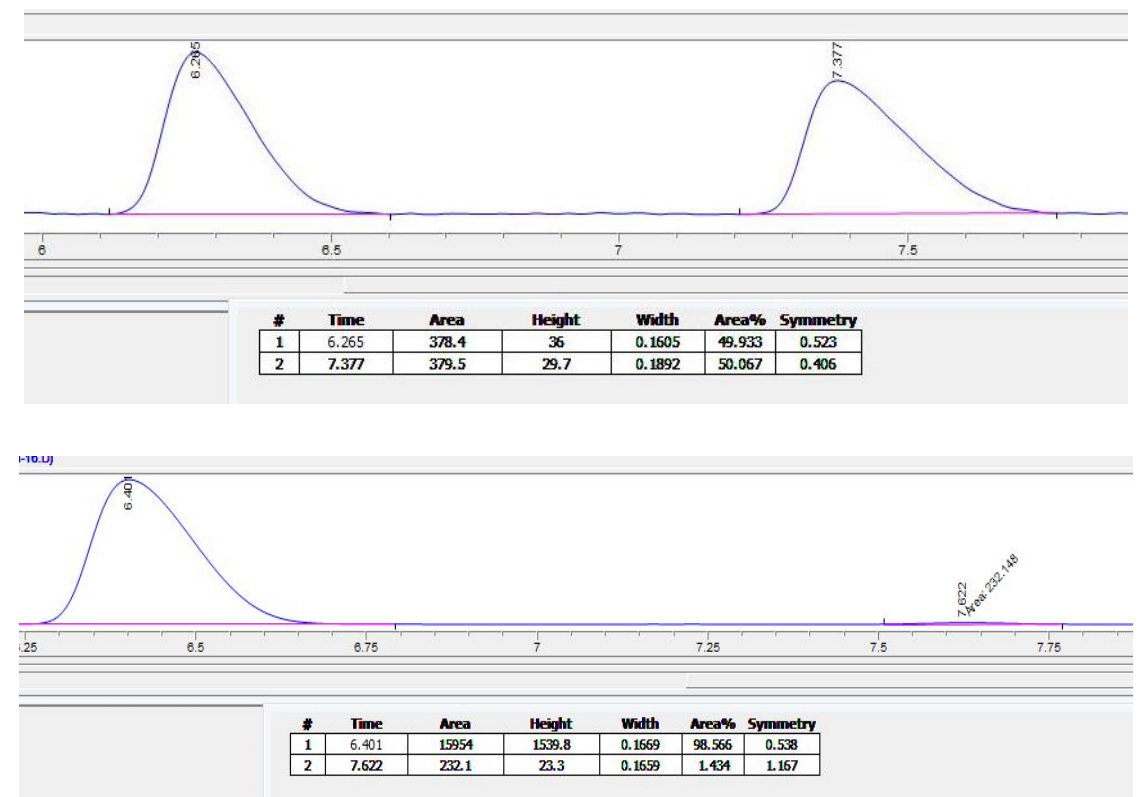

$N$-Benzyl- $N$-((1S,2S)-2-benzylcyclopropyl)-1H-indole-1-carboxamide

$3 \mathbf{m}$ (enantioenriched)<smiles>c1ccc(CNC2(Cc3ccccc3)CC2)cc1</smiles>

98.5:1.5 e.r

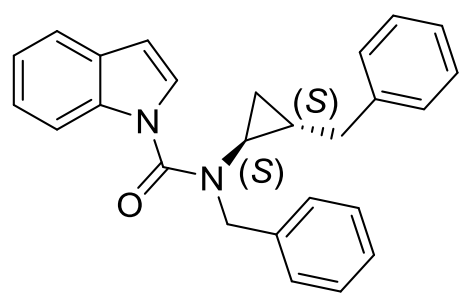

$3 m, 98.5: 1.5$ e.r.

General procedure B: $(1 S, 2 S)-N, 2$-Dibenzylcyclopropan-1-amine (98.5:1.5 e.r., $350 \mathrm{mg}$, $1.48 \mathrm{mmol}$ ) and $1 \mathrm{H}$-indole-1-carbonyl chloride (532 $\mathrm{mg}, 3.00 \mathrm{mmol})$ were employed. The crude mixture was purified by flash column chromatography ( $80 \%$ toluene/hexane) to afford the title compound 3m (enantioenriched) as a yellow oil (384 mg, 75\%). The enantiopurity of this compound was confirmed after the subsequent step.

$[\boldsymbol{\alpha}]_{\mathbf{D}}{ }^{23}+12.11\left(\mathrm{c}=0.99, \mathrm{CHCl}_{3}\right)$. All other analytical data were identical to those reported earlier. 


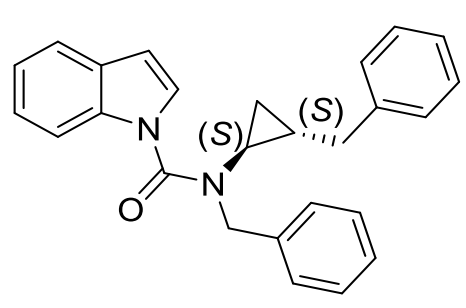

$3 m$

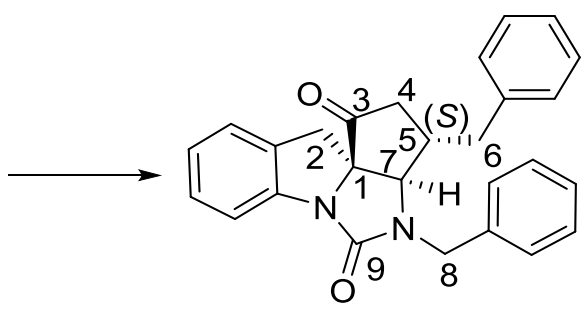

$4 \mathrm{~m}, 98.5: 1.5$ e.r.

General procedure D: Compound 3m (enantioenriched) $(38.0 \mathrm{mg}, 0.10 \mathrm{mmol}$ ), $\left[\mathrm{Rh}(\mathrm{cod})_{2}\right] \mathrm{OTf}(3.52 \mathrm{mg}, 7.5 \mathrm{~mol} \%)$ and anhydrous 1,2-DCB $(1.00 \mathrm{~mL})$ were employed and the reaction was stirred for 96 hours at $130{ }^{\circ} \mathrm{C}$. The crude mixture was purified by column chromatography (10\% EtOAc/toluene) to yield the title compound $\mathbf{4 m}$ (enantioenriched) $(21.0 \mathrm{mg}, 52 \%)$ as a colourless oil.

$[\boldsymbol{\alpha}]_{\mathbf{D}}{ }^{23}+42.16\left(\mathrm{c}=0.90, \mathrm{CHCl}_{3}\right)$. All other analytical data were identical to those reported earlier.

The enantiopurity of this compound was determined by chiral SFC (Chiralpak IB, isocratic $\mathrm{CO}_{2}-\mathrm{MeOH} 80: 20,2.0 \mathrm{~mL} / \mathrm{min}, 25{ }^{\circ} \mathrm{C}$ ) against a racemic standard; ( $\mathrm{t}_{\mathrm{R}}$ major $-10.4 \mathrm{~min}$ and $\left.\mathrm{t}_{\mathrm{R}}(\operatorname{minor})-8.2 \mathrm{~min}\right)$.

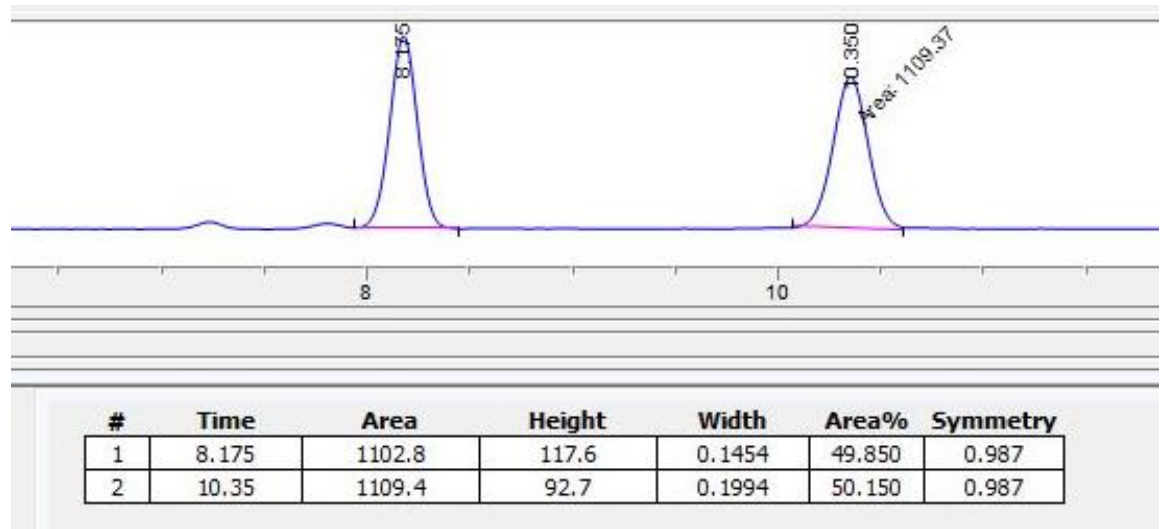




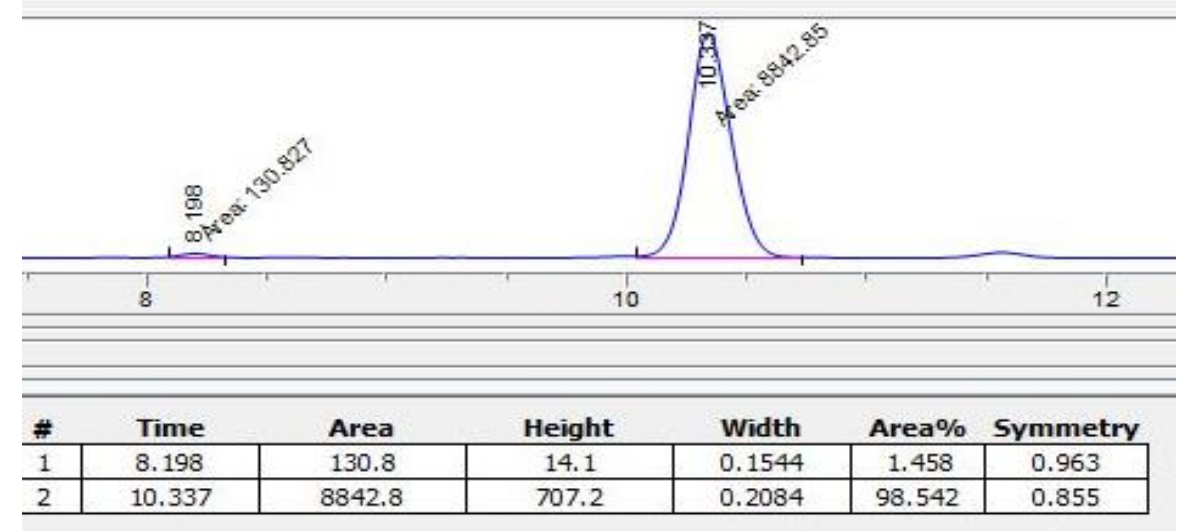

$N$-Benzyl- $N$-((1S*,2S*)-2-butylcyclopropyl)-1H-indole-1-carboxamide 3n
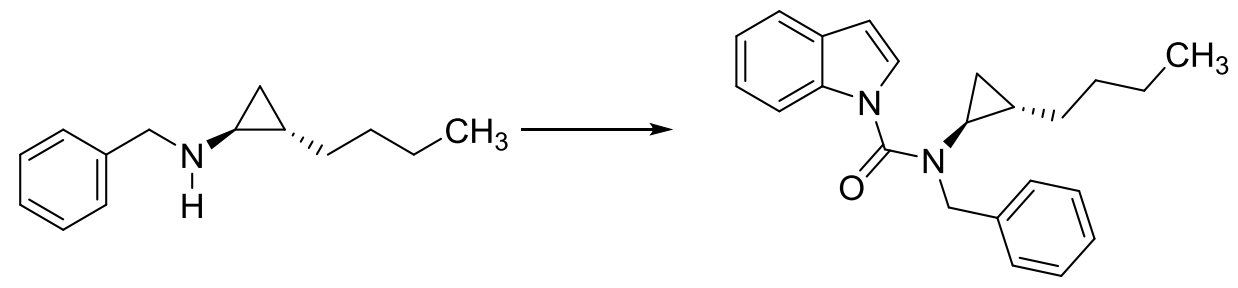

$3 n$

General procedure B: $\left(1 S^{*}, 2 S^{*}\right)-N$-Benzyl-2-butylcyclopropan-1-amine (1.01 mg, 5.00 mmol) (prepared according to the literature procedure) ${ }^{3}$ and $1 H$-indole-1-carbonyl chloride (1.80 g, $10.0 \mathrm{mmol})$ were employed. The crude mixture was purified by flash column chromatography (80\% toluene/hexane) to afford the title compound $\mathbf{3 n}(1.41 \mathrm{~g}, 82 \%)$ as a yellow oil; $v_{\max } / \mathrm{cm}^{-1}: 2925$ (s), 1675 (s), 1452 (s), 1408 (s), 1294 (s), 1210 (s); ${ }^{1} \mathrm{H}$ NMR $\left(400 \mathrm{MHz} \mathrm{CDCl}_{3}\right): \delta 7.64(1 \mathrm{H}, \mathrm{ddd}, J=8.3,1.0,1.0 \mathrm{~Hz}), 7.51(1 \mathrm{H}, \mathrm{ddd}, J=7.7,1.0,1.0 \mathrm{~Hz})$, $7.34(1 \mathrm{H}, \mathrm{d}, J=3.6 \mathrm{~Hz}), 7.31-7.29(1 \mathrm{H}, \mathrm{m}), 7.28-7.21(4 \mathrm{H}, \mathrm{m}), 7.19-7.17(1 \mathrm{H}, \mathrm{m}), 7.13$ - $7.09(1 \mathrm{H}, \mathrm{m}), 6.51(1 \mathrm{H}, \mathrm{dd}, J=3.6,0.8 \mathrm{~Hz}), 4.61(2 \mathrm{H}, \mathrm{s}), 2.29(1 \mathrm{H}, \mathrm{ddd}, J=7.1,3.6,3.6$ $\mathrm{Hz}), 1.10-1.02(5 \mathrm{H}, \mathrm{m}), 0.86-0.79(1 \mathrm{H}, \mathrm{m}), 0.74-0.68(4 \mathrm{H}, \mathrm{m}), 0.61(1 \mathrm{H}, \mathrm{ddd}, J=9.5$, 5.8, $3.8 \mathrm{~Hz}), 0.36(1 \mathrm{H}, \mathrm{ddd}, J=7.0,5.8,5.8 \mathrm{~Hz}) ;{ }^{13} \mathrm{C} \mathrm{NMR}\left(101 \mathrm{MHz}, \mathrm{CDCl}_{3}\right): \delta 155.1$, 137.0, 135.5, 129.6, 128.7, 128.2, 127.7, 126.0, 123.5, 121.9, 120.8, 113.7, 105.7, 53.1, 37.8, 31.6, 30.5, 22.9, 22.2, 15.6, 13.8; m/z (ESI $\left.{ }^{+}\right)$HRMS: $\mathrm{C}_{23} \mathrm{H}_{26} \mathrm{~N}_{2} \mathrm{O}: 347.2118$. Found $[\mathrm{M}+\mathrm{H}]^{+}$: 347.2125. 


\section{[1,5-a]indole-1,5-dione 4n}

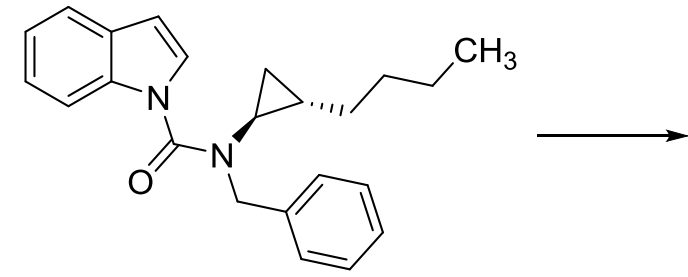

$3 n$

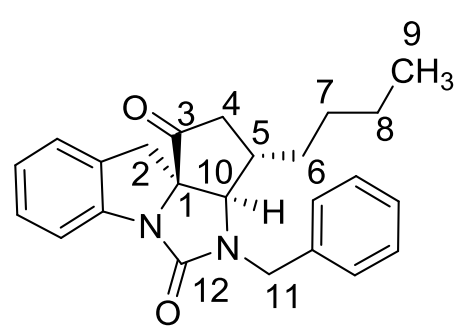

$4 n$

General procedure D: Compound $3 \mathbf{n}(51.9 \mathrm{mg}, 0.15 \mathrm{mmol}),\left[\mathrm{Rh}(\operatorname{cod})_{2}\right] \mathrm{OTf}(5.28 \mathrm{mg}, 7.5$ mol\%) and anhydrous 1,2-DCB $(1.50 \mathrm{~mL})$ were employed and the reaction was stirred for 96 hours at $130{ }^{\circ} \mathrm{C}$. The crude mixture was purified by column chromatography $(10 \%$ EtOAc/Hex) to yield the title compound $4 \mathbf{n}(31.4 \mathrm{mg}, 56 \%)$ as a yellow oil; $v_{\max } / \mathrm{cm}^{-1}: 2727$ (m), 1752 (s), 1710 (s), 1481 (s), 1408 (s), 1291 (s); ${ }^{1} \mathrm{H}$ NMR (400 MHz, CDCl 3 ) $\delta 7.42$ (1H, d, $J=7.8 \mathrm{~Hz}, 1 \times \operatorname{ArC} \underline{\mathrm{H}}), 7.27-7.14(6 \mathrm{H}, \mathrm{m}, 6 \times \mathrm{ArC} \underline{\mathrm{H}}), 7.10(1 \mathrm{H}, \mathrm{d}, J=7.8 \mathrm{~Hz}, 1 \times$ $\operatorname{ArC} \underline{H}), 6.98(1 \mathrm{H}, \mathrm{ddd}, J=7.5,7.5,1.1 \mathrm{~Hz}, 1 \times \mathrm{ArC} \underline{\mathrm{H}}), 4.64(1 \mathrm{H}, \mathrm{d}, J=15.0 \mathrm{~Hz}, 1 \times \mathrm{C} 11-$ $\left.\underline{\mathrm{H}}_{2}\right), 4.26\left(1 \mathrm{H}, \mathrm{d}, J=15.0 \mathrm{~Hz}, 1 \times \mathrm{C} 11-\underline{\mathrm{H}}_{2}\right), 3.69(1 \mathrm{H}, \mathrm{s}, 1 \times \mathrm{C10}-\underline{\mathrm{H}}), 3.29(1 \mathrm{H}, \mathrm{d}, J=16.0$ $\left.\mathrm{Hz}, 1 \times \mathrm{C} 2-\underline{\mathrm{H}}_{2}\right), 3.00\left(1 \mathrm{H}, \mathrm{d}, J=16.0 \mathrm{~Hz}, 1 \times \mathrm{C} 2-\underline{\mathrm{H}}_{2}\right), 2.74(1 \mathrm{H}, \mathrm{dd}, J=17.3,8.0 \mathrm{~Hz}, 1 \times \mathrm{C} 4-$ $\left.\underline{\mathrm{H}}_{2}\right), 2.28-2.22(1 \mathrm{H}, \mathrm{m}, 1 \times \mathrm{C} 5-\underline{\mathrm{H}}), 2.07\left(1 \mathrm{H}, \mathrm{d}, J=17.3 \mathrm{~Hz}, 1 \times \mathrm{C} 4-\underline{\mathrm{H}}_{2}\right), 1.23-1.00(6 \mathrm{H}, \mathrm{m}$, $\left.2 \times \mathrm{C} 6-\underline{\mathrm{H}}_{2}, 2 \times \mathrm{C} 7-\underline{\mathrm{H}}_{2}, 2 \times \mathrm{C} 8-\underline{\mathrm{H}}_{2}\right), 0.79\left(3 \mathrm{H}, \mathrm{t}, J=6.9 \mathrm{~Hz}, 3 \times \mathrm{C} 9-\underline{\mathrm{H}}_{3}\right) ;{ }^{13} \mathrm{C} \mathrm{NMR}(101 \mathrm{MHz}$, $\mathrm{CDCl}_{3}$ ): $\delta 211.8(\mathrm{C3}), 159.0$ (C12), 142.0 (CAr), 136.1 (CAr), 130.3 (CAr), 128.8 (CAr), 128.2 (CAr), 128.0 (CAr), 127.9 (CAr), 124.6 (CAr), 124.3 (CAr), 116.0 (CAr), 69.3 (C1), 68.9 (C10), 46.1 (C11), 39.6 (C4), 36.5 (C2), 34.9 (C5), 32.8 (C6), 29.0 (C7), 22.4 (C8), 13.8 (C9); HRMS: $\left(\mathrm{ESI}^{+} \text {) Calculated for } \mathrm{C}_{24} \mathrm{H}_{26} \mathrm{~N}_{2} \mathrm{NaO}_{2} \text { : 397.1886. Found [M+Na] }\right]^{+}: 397.1895$.

\section{$N$-Benzyl- $N$-((1R* $\left.{ }^{*}, 5 S^{*}, 6 r^{*}\right)$-bicyclo[3.1.0]hexan-6-yl)-1H-indole-1-carboxamide 3o}
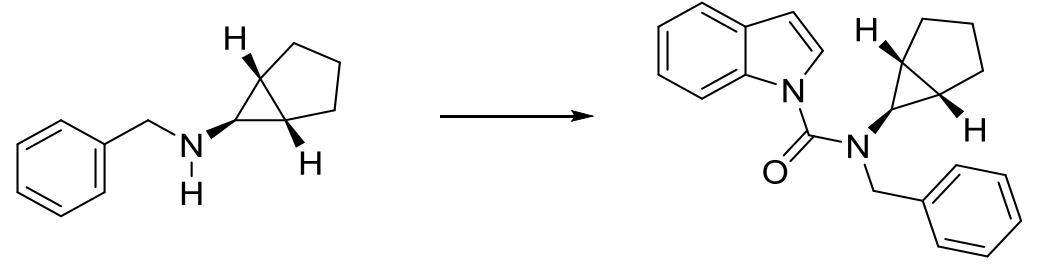

30

General procedure B: $\left(1 R^{*}, 5 S^{*}, 6 r^{*}\right)-N$-Benzylbicyclo[3.1.0]hexan-6-amine (840 mg, 4.50 mmol) (prepared according to the literature procedure $)^{3}$ and $1 H$-indole-1-carbonyl chloride 
(1.62 g, $9.00 \mathrm{mmol}$ ) were employed. The crude mixture was purified by flash column chromatography (5\% EtOAc/hexane) to afford the title compound 30 (457 $\mathrm{mg}, 31 \%$ ) as a yellow oil; $v_{\max } / \mathrm{cm}^{-1}: 1671$ (s), 1452 (s), 1395 (s), 1315 (s), 1296 (s), 1211 (s); ${ }^{1} \mathrm{H}$ NMR (400 MHz, $\left.\mathrm{CDCl}_{3}\right): \delta 7.69(1 \mathrm{H}, \mathrm{d}, J=8.2 \mathrm{~Hz}), 7.52(1 \mathrm{H}, \mathrm{d}, J=7.8 \mathrm{~Hz}), 7.32-7.10(8 \mathrm{H}, \mathrm{m})$, $6.51(1 \mathrm{H}, \mathrm{d}, J=3.6 \mathrm{~Hz}), 4.62(2 \mathrm{H}, \mathrm{s}), 2.14(1 \mathrm{H}, \mathrm{d}, J=2.3 \mathrm{~Hz}), 1.49-1.30(7 \mathrm{H}, \mathrm{m}), 0.83-$ $0.70(1 \mathrm{H}, \mathrm{m}) ;{ }^{13} \mathrm{C} \mathrm{NMR}\left(101 \mathrm{MHz}, \mathrm{CDCl}_{3}\right): \delta 154.8,136.9,135.6,129.5,128.7,128.6,127.7$, 125.8, 123.5, 121.9, 120.8, 114.0, 105.6, 52.8, 38.7, 28.6, 27.1, 21.3; m/z (ESI $\left.{ }^{+}\right)$HRMS: $\mathrm{C}_{22} \mathrm{H}_{23} \mathrm{~N}_{2} \mathrm{O}: 331.1805$. Found $[\mathrm{M}+\mathrm{H}]^{+}: 331.1818$.

$\left(3 \mathrm{a} R^{*}, 3 \mathrm{~b} S^{*}, 11 \mathrm{a} S^{*}, 12 \mathrm{a} S^{*}\right)-4-B e n z y l-2,3,3 \mathrm{a}, 3 \mathrm{~b}, 4,12 \mathrm{a}-h$ exahydro-1H,11Hpentaleno[1',2':4,5] imidazo[1,5-a] indole-5,12-dione 4o

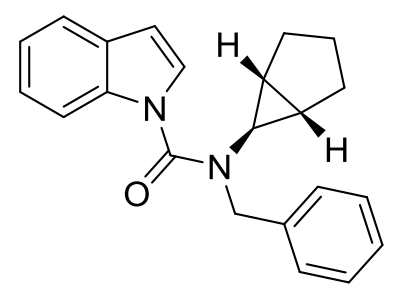

30

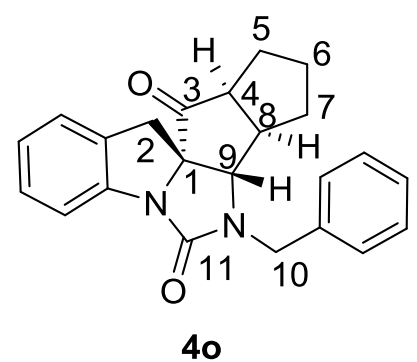

40

General procedure D: Compound $30(66.2 \mathrm{mg}, 0.20 \mathrm{mmol}),\left[\mathrm{Rh}(\operatorname{cod})_{2}\right] \mathrm{OTf}(9.39 \mathrm{mg}, 10$ mol\%) and anhydrous 1,2-DCB $(1.00 \mathrm{~mL})$ were employed and the reaction was stirred for 96 hours at $130{ }^{\circ} \mathrm{C}$. The crude mixture was purified by column chromatography $(5 \%$ EtOAc/toluene) to yield the title compound 40 (39.6 mg, 55\%) as a yellow solid; m.p. 168$168{ }^{\circ} \mathrm{C}\left(\mathrm{CDCl}_{3}\right) ; v_{\max } / \mathrm{cm}^{-1}: 1746$ (s), 1707 (s), 1481 (s), 1407 (s), 1288 (s), 1153 (s); ${ }^{1} \mathrm{H}$ NMR (400 MHz, $\left.\mathrm{CDCl}_{3}\right) \delta 7.42(1 \mathrm{H}, \mathrm{d}, J=7.9 \mathrm{~Hz}, 1 \times \mathrm{ArC} \underline{\mathrm{H}}), 7.30-7.08(6 \mathrm{H}, \mathrm{m}, 6 \times$ $\operatorname{ArC} \underline{H}), 7.09(1 \mathrm{H}, \mathrm{d}, J=7.4 \mathrm{~Hz}, 1 \times \operatorname{ArC} \underline{\mathrm{H}}), 6.98(1 \mathrm{H}, \mathrm{dd}, J=7.4,7.4 \mathrm{~Hz}, 1 \times \operatorname{ArC} \underline{\mathrm{H}}), 4.72$ $\left(1 \mathrm{H}, \mathrm{d}, J=15.1 \mathrm{~Hz}, 1 \times \mathrm{C10}-\underline{\mathrm{H}}_{2}\right), 4.24\left(1 \mathrm{H}, \mathrm{d}, J=15.1 \mathrm{~Hz}, 1 \times \mathrm{C10}-\underline{\mathrm{H}}_{2}\right), 3.70(1 \mathrm{H}, \mathrm{s}, 1 \times \mathrm{C} 9-$ $\underline{\mathrm{H}}), 3.22\left(1 \mathrm{H}, \mathrm{d}, J=16.0 \mathrm{~Hz}, 1 \times \mathrm{C} 2-\underline{\mathrm{H}}_{2}\right), 3.03-2.93\left(2 \mathrm{H}, \mathrm{m}, 1 \times \mathrm{C} 2-\underline{\mathrm{H}}_{2},, 1 \times \mathrm{C} 4-\underline{\mathrm{H}}\right), 2.72-$ $2.65(1 \mathrm{H}, \mathrm{m}, 1 \times \mathrm{C} 8-\underline{\mathrm{H}}), 1.98-1.77\left(3 \mathrm{H}, \mathrm{m}, 2 \times \mathrm{C} 5-\underline{\mathrm{H}}_{2}, 1 \times \mathrm{C} 7-\underline{\mathrm{H}}_{2}\right), 1.59-1.48(2 \mathrm{H}, \mathrm{m}, 2 \times$ C6- $\left.\underline{\mathrm{H}}_{2}\right), 0.91-0.81\left(1 \mathrm{H}, \mathrm{m}, 1 \times \mathrm{C}^{-}-\underline{\mathrm{H}}_{2}\right) ;{ }^{13} \mathrm{C} \mathrm{NMR}\left(101 \mathrm{MHz}, \mathrm{CDCl}_{3}\right): \delta 215.8(\mathrm{C} 3), 158.8$ (C11), 142.3 (CAr), 136.1 (CAr), 130.5 (CAr), 128.9 (CAr), 128.2 (CAr), 128.0 (CAr), 127.9 (CAr), 124.6 (CAr), 124.4 (CAr), 116.3 (CAr), 71.6 (C1), 66.0 (C9), 49.4 (C4), 45.9 (C10), 43.1 (C8), 37.4 (C2), 31.0 (C7), 27.8 (C5), 25.3 (C6); HRMS: (ESI ${ }^{+}$) Calculated for $\mathrm{C}_{23} \mathrm{H}_{22} \mathrm{~N}_{2} \mathrm{O}_{2}$ : 381.1573. Found $[\mathrm{M}+\mathrm{H}]^{+}:$381.1578. The structure of this compound was determined unambiguously by X-ray crystallography. 


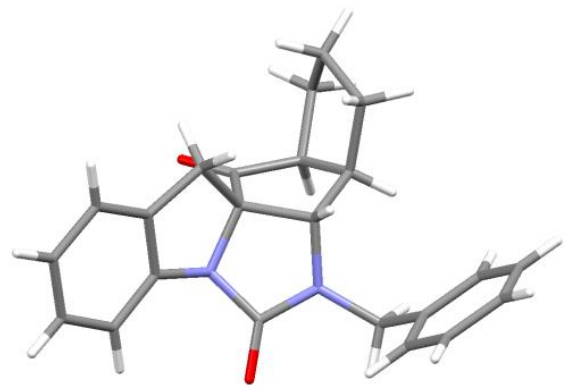

$N$-Benzyl- $N$-((1 $\left.R^{*}, 6 S^{*}, 7 r^{*}\right)$-bicyclo[4.1.0]heptan-7-yl)-1H-indole-1-carboxamide 3p

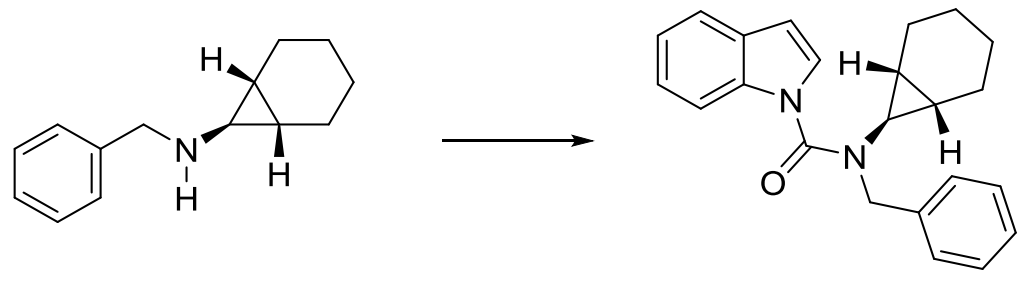

$3 p$

General procedure B: $\left(1 R^{*}, 6 S^{*}, 7 r^{*}\right)-N$-Benzylbicyclo[4.1.0]heptan-7-amine $(1.00 \mathrm{~g}, 5.00$ mmol) (prepared according to the literature procedure) ${ }^{2}$ and $1 H$-indole-1-carbonyl chloride (1.80 g, $10.0 \mathrm{mmol}$ ) were employed. The crude mixture was purified by flash column chromatography (80\% toluene/hexane) to afford the title compound $\mathbf{3 p}(1.52 \mathrm{~g}, 86 \%)$ as a yellow oil; m.p. 78-80 ${ }^{\circ} \mathrm{C}\left(\mathrm{CDCl}_{3}\right) ; v_{\max } / \mathrm{cm}^{-1}: 1672$ (s), 1451 (s), 1397 (s), 1294 (s), 1211 (s), $746(\mathrm{~s}) ;{ }^{1} \mathrm{H}$ NMR (400 MHz, $\left.\mathrm{CDCl}_{3}\right): \delta 7.63(1 \mathrm{H}, \mathrm{dd}, J=8.2,1.0 \mathrm{~Hz}), 7.50(1 \mathrm{H}, \mathrm{ddd}, J=$ 7.7, 1.0, $1.0 \mathrm{~Hz}), 7.34-7.23(6 \mathrm{H}, \mathrm{m}), 7.20-7.16(1 \mathrm{H}, \mathrm{m}), 7.10(1 \mathrm{H}, \mathrm{ddd}, J=7.7,7.7,1.0$ $\mathrm{Hz}), 6.51(1 \mathrm{H}, \mathrm{dd}, J=3.6,0.8 \mathrm{~Hz}), 4.62(2 \mathrm{H}, \mathrm{s}), 2.15(1 \mathrm{H}, \mathrm{dd}, J=3.6,3.6 \mathrm{~Hz}), 1.58-1.43$ $(2 \mathrm{H}, \mathrm{m}), 1.11-0.89(6 \mathrm{H}, \mathrm{m}), 0.84-0.75(2 \mathrm{H}, \mathrm{m}) ;{ }^{13} \mathrm{C} \mathrm{NMR}\left(101 \mathrm{MHz}, \mathrm{CDCl}_{3}\right): \delta 154.9$, 137.0, 135.5, 129.6, 128.8, 128.7, 127.7, 125.9, 123.5, 121.8, 120.8, 113.9, 105.6, 52.6, 42.7, 21.9, 21.1, 21.0; $\mathrm{m} / z\left(\mathrm{ESI}^{+}\right) \mathrm{HRMS}: \mathrm{C}_{23} \mathrm{H}_{25} \mathrm{~N}_{2} \mathrm{O}: 345.1961$. Found $[\mathrm{M}+\mathrm{H}]^{+}: 345.1972$. 


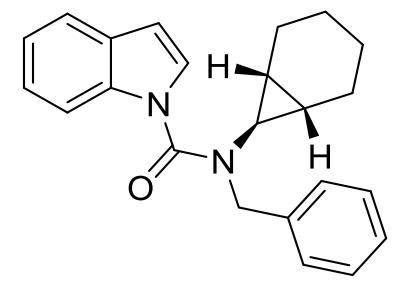

$3 p$

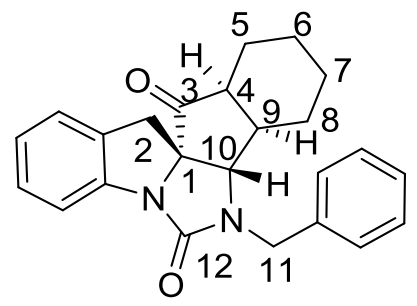

$4 p$

General procedure D: Compound 3p $(34.6 \mathrm{mg}, 0.10 \mathrm{mmol}),\left[\mathrm{Rh}(\operatorname{cod})_{2}\right] \mathrm{OTf}(4.69 \mathrm{mg}, 10$ mol\%) and anhydrous 1,2-DCB $(1.00 \mathrm{~mL})$ were employed and the reaction was stirred for 96 hours at $150{ }^{\circ} \mathrm{C}$. The crude mixture was purified by column chromatography $(5 \%$ EtOAc/toluene) to yield the title compound $\mathbf{4 p}(15.0 \mathrm{mg}, 40 \%)$ as a colourless oil; $v_{\max } / \mathrm{cm}^{-1}$ : 2931 (m), 1750 (s), 1712 (s), 1481 (s), 1410 (s), 1288 (s); ${ }^{1} \mathrm{H}$ NMR (400 MHz, $\mathrm{CDCl}_{3}$ ) $\delta 7.42$ - $7.40(1 \mathrm{H}, \mathrm{m}, 1 \times \mathrm{ArC} \underline{\mathrm{H}}), 7.28-7.09(7 \mathrm{H}, \mathrm{m}, 7 \times \mathrm{ArC} \underline{\mathrm{H}}), 6.98(1 \mathrm{H}, \mathrm{ddd}, J=7.5,7.5,1.1$ $\mathrm{Hz}, 1 \times \mathrm{ArC} \underline{\mathrm{H}}), 4.69\left(1 \mathrm{H}, \mathrm{d}, J=15.0 \mathrm{~Hz}, 1 \times \mathrm{C} 11-\underline{\mathrm{H}}_{2}\right), 4.24(1 \mathrm{H}, \mathrm{d}, J=15.0 \mathrm{~Hz}, 1 \times \mathrm{C} 11-$ $\left.\underline{\mathrm{H}}_{2}\right), 3.50(1 \mathrm{H}, \mathrm{s}, 1 \times \mathrm{C} 10-\underline{\mathrm{H}}), 3.36\left(1 \mathrm{H}, \mathrm{d}, J=16.1 \mathrm{~Hz}, 1 \times \mathrm{C} 2-\underline{\mathrm{H}}_{2}\right), 2.98(1 \mathrm{H}, \mathrm{d}, J=16.1 \mathrm{~Hz}$, $\left.1 \times \mathrm{C} 2-\underline{\mathrm{H}}_{2}\right), 2.87-2.83(1 \mathrm{H}, \mathrm{m}, 1 \times \mathrm{C} 4-\underline{\mathrm{H}}), 2.28(1 \mathrm{H}, \mathrm{ddd}, J=13.4,7.0,4.7 \mathrm{~Hz}, 1 \times \mathrm{C} 9-\underline{\mathrm{H}})$, $2.11\left(1 \mathrm{H}, \mathrm{ddd}, J=13.8,4.0,2.0 \mathrm{~Hz}, 1 \times \mathrm{C} 5-\underline{\mathrm{H}}_{2}\right), 1.63-1.56\left(2 \mathrm{H}, \mathrm{m}, 1 \times \mathrm{C} 8-\underline{\mathrm{H}}_{2}, 1 \times \mathrm{C} 7-\underline{\mathrm{H}}_{2}\right)$, $1.50-1.43\left(1 \mathrm{H}, \mathrm{m}, 1 \times \mathrm{C} 6-\underline{\mathrm{H}}_{2}\right), 1.38-1.28\left(1 \mathrm{H}, \mathrm{m}, 1 \times \mathrm{C} 5-\underline{\mathrm{H}}_{2}\right), 1.15-1.04(1 \mathrm{H}, \mathrm{m}, 1 \times \mathrm{C} 7-$ $\left.\underline{\mathrm{H}}_{2}\right), 1.02-0.92\left(1 \mathrm{H}, \mathrm{m}, 1 \times \mathrm{C} 6-\underline{\mathrm{H}}_{2}\right), 0.52-0.41\left(1 \mathrm{H}, \mathrm{m}, 1 \times \mathrm{C} 8-\underline{\mathrm{H}}_{2}\right) ;{ }^{13} \mathrm{C} \mathrm{NMR}(101 \mathrm{MHz}$, $\mathrm{CDCl}_{3}$ ): $\delta 211.2(\mathrm{C3}), 159.0$ (C12), 142.2 (CAr), 136.1 (CAr), 130.6 (CAr), 128.9 (CAr), 128.3 (CAr), 127.9 (CAr), 127.9 (CAr), 124.6 (CAr), 124.3 (CAr), 116.0 (CAr), 69.5 (C1), 67.5 (C10), 46.0 (C11), 43.4 (C4), 36.8 (C2), 36.2 (C9), 28.3 (C8), 24.5 (C7), 21.8 (C5), 21.4 (C6); HRMS: $\left(\mathrm{ESI}^{+}\right)$Calculated for $\mathrm{C}_{24} \mathrm{H}_{24} \mathrm{~N}_{2} \mathrm{O}_{2}$ : 395.1730. Found $[\mathrm{M}+\mathrm{H}]^{+}: 395.1741$.

\section{$\left(1 S^{*}, 1 \mathrm{a} R^{*}, 6 \mathrm{a} R^{*}\right)-N$-Benzyl-1,1a,6,6a-tetrahydrocyclopropa[a]inden-1-amine}

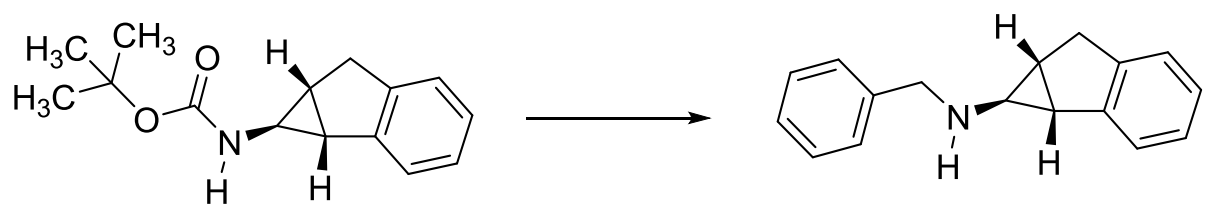

To a solution of tert-butyl $\left(\left(1 S^{*}, 1 \mathrm{a} R^{*}, 6 \mathrm{a} R^{*}\right)-1,1 a, 6,6 a\right.$-tetrahydrocyclopropa[ $\left.a\right]$ inden-1yl)carbamate (1.84 g, $7.51 \mathrm{mmol}$ ) (prepared according to the literature procedure) ${ }^{6}$ in $\mathrm{CH}_{2} \mathrm{Cl}_{2}$ $(24.0 \mathrm{~mL})$ was added trifluoroacetic acid $(5.80 \mathrm{~mL}, 75.1 \mathrm{mmol})$, the reaction was stirred at 
room temperature for 18 hours. The reaction mixture was concentrated in vacuo. Water (25 $\mathrm{mL})$ was added and the solution was extracted with $\mathrm{CH}_{2} \mathrm{Cl}_{2}(2 \times 25 \mathrm{~mL})$. The aqueous portion was adjusted to $\mathrm{pH} 12$ by addition of $2 \mathrm{M}$ aqueous $\mathrm{NaOH}$ and then extracted with $\mathrm{CH}_{2} \mathrm{Cl}_{2}(3 \times 15 \mathrm{~mL})$. The organic extracts were combined, dried over $\mathrm{Na}_{2} \mathrm{SO}_{4}$ and concentrated in vacuo. The residue was dissolved in $\mathrm{MeOH}(40 \mathrm{~mL}) . \mathrm{NaHCO}_{3}(2.52 \mathrm{~g}, 18.3$ mmol $)$ and benzaldehyde $(0.72 \mathrm{~mL}, 7.13 \mathrm{mmol})$ were added and the solution was heated at reflux for $24 \mathrm{~h}$. The reaction mixture was cooled to $0{ }^{\circ} \mathrm{C}$ and $\mathrm{NaBH}_{4}(379 \mathrm{mg}, 9.76 \mathrm{mmol})$ was added portionwise over 5 minutes. The reaction was warmed to room temperature, stirred for 18 hours and then concentrated in vacuo. Saturated aqueous $\mathrm{NaHCO}_{3}(50 \mathrm{~mL})$ and $\mathrm{CH}_{2} \mathrm{Cl}_{2}(35.0 \mathrm{~mL})$ were added, the layers were separated and the aqueous portion was further extracted with $\mathrm{CH}_{2} \mathrm{Cl}_{2}(2 \times 20 \mathrm{~mL})$. The organic extracts were combined, washed with brine (35 mL), dried over $\mathrm{Na}_{2} \mathrm{SO}_{4}$ and concentrated in vacuo. The crude mixture was purified by flash column chromatography (20\% EtOAc/hexane) to afford the title compound (1.67 $\mathrm{g}$, 90\%) as a colourless oil; $v_{\max } / \mathrm{cm}^{-1}: 3316(\mathrm{~m}), 2901(\mathrm{~m}), 1660$ (s), 1454 (s), 1076 (s), 731(s); ${ }^{1} \mathrm{H}$ NMR $\left(400 \mathrm{MHz}, \mathrm{CDCl}_{3}\right): \delta 7.39-7.24(6 \mathrm{H}, \mathrm{m}), 7.13-7.05(3 \mathrm{H}, \mathrm{m}), 3.90(1 \mathrm{H}, \mathrm{d}, J=$ $13.1 \mathrm{~Hz}), 3.86(1 \mathrm{H}, \mathrm{d}, J=13.1 \mathrm{~Hz}), 3.17(1 \mathrm{H}, \mathrm{dd}, J=17.1,7.0 \mathrm{~Hz}), 2.94(1 \mathrm{H}, \mathrm{d}, J=17.1 \mathrm{~Hz})$, $2.42(1 \mathrm{H}, \mathrm{ddd}, J=6.8,1.6,1.6 \mathrm{~Hz}), 1.90-1.85(2 \mathrm{H}, \mathrm{m}) ;{ }^{13} \mathrm{C} \mathrm{NMR}\left(101 \mathrm{MHz}, \mathrm{CDCl}_{3}\right): \delta$ 144.2 , 142.9, 140.2, 128.5, 128.4, 127.0, 126.0, 125.5, 124.9, 123.4, 53.5, 46.9, 34.8, 33.7, 24.7; m/z $\left(\mathrm{ESI}^{+}\right) \mathrm{HRMS}: \mathrm{C}_{17} \mathrm{H}_{18} \mathrm{~N}: 236.1434$. Found $[\mathrm{M}+\mathrm{H}]^{+}: 236.1428$.

\section{$N$-Benzyl- $N$-((1S*,1a $\left.R^{*}, 6 \mathrm{a} R^{*}\right)-1,1 \mathrm{a}, 6,6 \mathrm{a}-$ tetrahydrocyclopropa[a]inden-1-yl)-1H-indole-}

\section{1-carboxamide 3q}
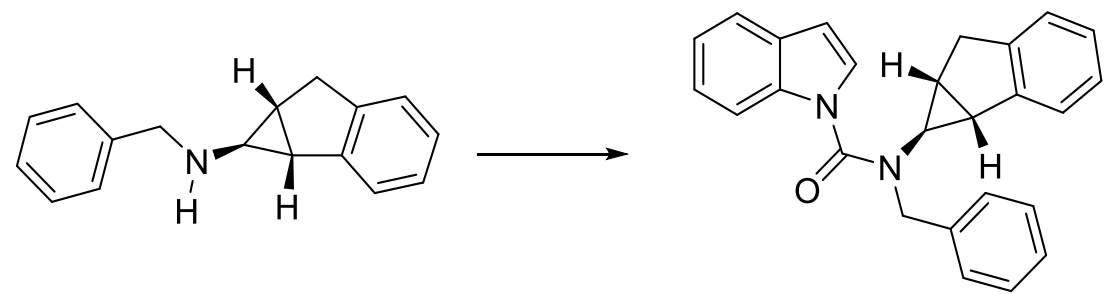

$3 q$

General procedure B: $\left(1 S^{*}, 1 \mathrm{a} R^{*}, 6 \mathrm{a} R^{*}\right)-N$-Benzyl-1,1a,6,6a-tetrahydrocyclopropa $[a]$ inden1-amine (705 mg, $3.00 \mathrm{mmol})$ and $1 H$-indole-1-carbonyl chloride (1.08 g, $6.00 \mathrm{mmol})$ were employed. The crude mixture was purified by flash column chromatography (80\% toluene/hexane then $8 \%$ EtOAc/hexane) to afford the title compound 3q (594 mg, 53\%) as a colourless solid; m.p. $134-136{ }^{\circ} \mathrm{C}\left(\mathrm{CDCl}_{3}\right) ; v_{\max } / \mathrm{cm}^{-1}: 1674$ (s), 1452 (s), 1409 (s), 1387 (s), 
1320 (s), 1210 (s); ${ }^{1} \mathrm{H}$ NMR (400 MHz, $\left.\mathrm{CDCl}_{3}\right): \delta 7.80(1 \mathrm{H}, \mathrm{d}, J=8.1 \mathrm{~Hz}), 7.62(1 \mathrm{H}, \mathrm{d}, J=$ $7.6 \mathrm{~Hz}), 7.38-7.19(8 \mathrm{H}, \mathrm{m}), 7.01-6.99(3 \mathrm{H}, \mathrm{m}), 6.89-6.87(1 \mathrm{H}, \mathrm{m}), 6.62(1 \mathrm{H}, \mathrm{d}, J=3.5$ $\mathrm{Hz}), 4.88(1 \mathrm{H}, \mathrm{d}, J=14.3 \mathrm{~Hz}), 4.69(1 \mathrm{H}, \mathrm{d}, J=14.3 \mathrm{~Hz}), 2.97(1 \mathrm{H}, \mathrm{dd}, J=17.4,6.9 \mathrm{~Hz})$, $2.61(1 \mathrm{H}, \mathrm{d}, J=17.4 \mathrm{~Hz}), 2.42(1 \mathrm{H}, \mathrm{d}, J=7.1 \mathrm{~Hz}), 1.99(1 \mathrm{H}, \mathrm{s}, b r), 1.90-1.86(1 \mathrm{H}, \mathrm{m}) ;{ }^{13} \mathrm{C}$ NMR (101 MHz, $\left.\mathrm{CDCl}_{3}\right): \delta 154.4,142.1,136.6,135.7,129.5,128.8,128.6,127.9,126.4$, 126.3, 125.8, 125.7, 124.9, 124.1, 123.8, 122.1, 120.9, 114.1, 106.0, 52.7, 46.3, 35.0, 34.8, 26.9; m/z $\left(\mathrm{ESI}^{+}\right)$HRMS: $\mathrm{C}_{26} \mathrm{H}_{22} \mathrm{~N}_{2} \mathrm{NaO}: 401.1624$. Found $[\mathrm{M}+\mathrm{Na}]^{+}: 401.1631$.

$\left(7 \mathrm{a} S^{*}, 7 \mathrm{~b} R^{*}, 12 \mathrm{~b} R^{*}, 13 \mathrm{a} S^{*}\right)-7-B e n z y l-7 \mathrm{a}, 7 \mathrm{~b}, 8,12 \mathrm{~b}-$ tetrahydro-6H,14Hbenzo[4',5']pentaleno[1',2':4,5]imidazo[1,5-a]indole-6,13(7H)-dione $4 \mathrm{q}$

And

$\left(7 \mathrm{a} S^{*}, 7 \mathrm{~b} S^{*}, 12 \mathrm{a} S^{*}, 13 \mathrm{a} S^{*}\right)-7-B e n z y l-7 \mathrm{a}, 7 \mathrm{~b}, 12,12 \mathrm{a}-t e t r a h y d r o-6 H, 14 H-$ benzo[5',6']pentaleno[1',2':4,5]imidazo[1,5-a]indole-6,13(7H)-dione 4q',

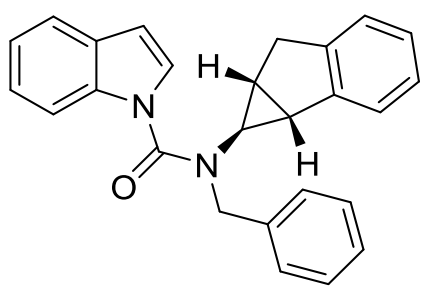

$3 \mathbf{q}$

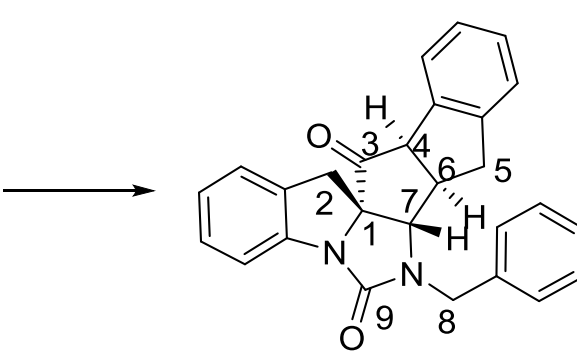

$4 q$

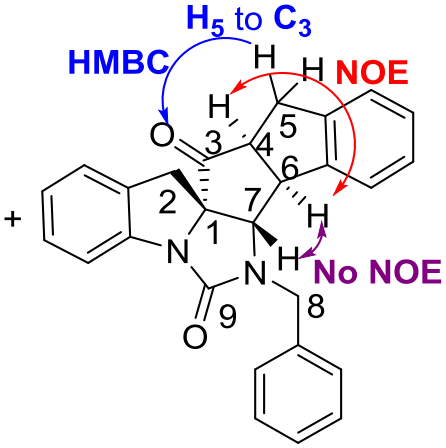

$4 q^{\prime}$

General procedure D: Compound 3q $(37.8 \mathrm{mg}, 0.10 \mathrm{mmol}),\left[\mathrm{Rh}(\operatorname{cod})_{2}\right] \mathrm{OTf}(4.69 \mathrm{mg}, 10$ mol\%) and anhydrous 1,2-DCB $(1.00 \mathrm{~mL})$ were employed and the reaction was stirred for 96 hours at $125{ }^{\circ} \mathrm{C}$. The crude mixture was purified by column chromatography (5\% EtOAc/toluene) to yield the title compounds $\mathbf{4 q}$ and $\mathbf{4 q}$ ' (22.4 $\mathrm{mg}, 52 \%)$ in a 10:1 ratio and as a colourless solid. An analytical sample of $\mathbf{4 q}$ was subsequently obtained by repeated column chromatography. The minor product $\mathbf{4} \mathbf{q}$ ' could not be isolated in pure form.

The structure of major product $\mathbf{4 q}$ was determined unambiguously by X-ray crystallography; The regiochemistry of minor product $\mathbf{4 q}$ ' was confirmed by HMBC analysis (as indicated on the compound structure). The relative stereochemistry of minor product $4 q^{\prime}$ was corroborated by nOe experiments (as indicated on the compound structure 4q'). nOe correlations were observed between $\mathrm{C4}-\mathrm{H}$ and $\mathrm{C6}-\mathrm{H}$ and no nOe correlations were observed 
between C6-H and C7-H. At the same time, the ${ }^{1} H$ NMR signal for $C 7-\underline{H}$ is $4.38(1 H, s)$. These results indicate that there is a cis relationship between $\mathrm{C4}-\mathrm{H}$ and $\mathrm{C6}-\mathrm{H}$ and a trans relationship between $\mathrm{C}$ - $\mathrm{H}$ and $\mathrm{C6}-\mathrm{H}$.

4q: m.p. 210-212 ${ }^{\circ} \mathrm{C}\left(\mathrm{CDCl}_{3}\right) ; v_{\max } / \mathrm{cm}^{-1}: 1748$ (s), 1710 (s), 1481 (s), 1407 (s), 1292 (s), 730 (s); ${ }^{1} \mathrm{H}$ NMR $\left(400 \mathrm{MHz}, \mathrm{CDCl}_{3}\right) \delta 7.52(1 \mathrm{H}, \mathrm{d}, J=7.9 \mathrm{~Hz}, 1 \times \mathrm{ArC} \underline{\mathrm{H}}), 7.39-7.27(6 \mathrm{H}, \mathrm{m}, 6$ $\times \operatorname{ArC} \underline{\mathrm{H}}), 7.25-7.17(4 \mathrm{H}, \mathrm{m}, 4 \times \operatorname{ArC} \underline{\mathrm{H}}), 7.05-7.00(2 \mathrm{H}, \mathrm{m}, 2 \times \operatorname{ArC} \underline{\mathrm{H}}), 4.82(1 \mathrm{H}, \mathrm{d}, J=$ $\left.15.1 \mathrm{~Hz}, 1 \times \mathrm{C} 8-\underline{\mathrm{H}}_{2}\right), 4.36\left(1 \mathrm{H}, \mathrm{d}, J=15.1 \mathrm{~Hz}, 1 \times \mathrm{C} 8-\underline{\mathrm{H}}_{2}\right), 4.06(1 \mathrm{H}, \mathrm{d}, J=8.0 \mathrm{~Hz}, 1 \times \mathrm{C} 4-$ $\underline{\mathrm{H}}), 3.79(1 \mathrm{H}, \mathrm{d}, J=1.3 \mathrm{~Hz}, 1 \times \mathrm{C} 7-\underline{\mathrm{H}}), 3.35-3.27\left(2 \mathrm{H}, \mathrm{m}, 1 \times \mathrm{C} 5-\underline{H}_{2}, 1 \times \mathrm{C6}-\underline{\mathrm{H}}\right), 2.98(1 \mathrm{H}$, d, $\left.J=16.2 \mathrm{~Hz}, 1 \times \mathrm{C} 2-\underline{\mathrm{H}}_{2}\right), 2.90\left(1 \mathrm{H}, \mathrm{d}, J=16.2 \mathrm{~Hz}, 1 \times \mathrm{C} 2-\underline{\mathrm{H}}_{2}\right), 2.80-2.67(1 \mathrm{H}, \mathrm{m}, 1 \times \mathrm{C} 5-$ $\left.\underline{\mathrm{H}}_{2}\right) ;{ }^{13} \mathrm{C} \mathrm{NMR}\left(101 \mathrm{MHz}, \mathrm{CDCl}_{3}\right): \delta 212.2$ (C3), 158.5 (C9), 142.5 (CAr), 141.6 (CAr), 138.0 (CAr), 135.8 (CAr), 130 (CAr), 128.9 (CAr), 128.3 (CAr), 128.1 (CAr), 128.0 (CAr), 127.7 (CAr), 125.1 (CAr), 124.8 (CAr), 124.5 (CAr), 124.3 (CAr), 116.2 (CAr), 72.5 (C1), 68.9 (C7), 56.9 (C4), 46.0 (C8), 42.6 (C6), 38.5 (C2), 37.8 (C5); One aromatic carbon signal is overlapped; HRMS: $\left(\mathrm{ESI}^{+}\right)$Calculated for $\mathrm{C}_{27} \mathrm{H}_{22} \mathrm{~N}_{2} \mathrm{NaO}_{2}$ : 429.1573. Found $[\mathrm{M}+\mathrm{Na}]^{+}$: 429.1574.

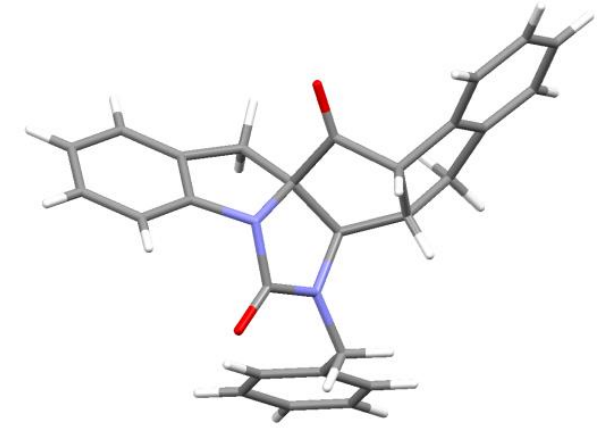

4q': Characteristic signals only: ${ }^{1} \mathrm{H} \mathrm{NMR}\left(500 \mathrm{MHz}, \mathrm{CD}_{3} \mathrm{CN}\right) \delta 4.81(1 \mathrm{H}, \mathrm{d}, J=15.4 \mathrm{~Hz}, 1$ $\left.\times \mathrm{C8}-\underline{\mathrm{H}}_{2}\right), 4.56\left(1 \mathrm{H}, \mathrm{d}, J=15.4 \mathrm{~Hz}, 1 \times \mathrm{C} 8-\underline{\mathrm{H}}_{2}\right), 4.38(1 \mathrm{H}, \mathrm{s}, 1 \times \mathrm{C} 7-\underline{\mathrm{H}}), 4.13(1 \mathrm{H}$, partly overlapped, $1 \times \mathrm{C6}-\underline{\mathrm{H}}), 4.13(1 \mathrm{H}$, partly overlapped, $1 \times \mathrm{C} 4-\underline{\mathrm{H}}), 3.17(2 \mathrm{H}$, partly overlapped, $\left.2 \times \mathrm{C} 5-\underline{\mathrm{H}}_{2}\right), 2.66\left(1 \mathrm{H}, \mathrm{d}, J=16.5 \mathrm{~Hz}, 1 \times \mathrm{C} 2-\underline{\mathrm{H}}_{2}\right) ;{ }^{13} \mathrm{C} \mathrm{NMR}\left(126 \mathrm{MHz}, \mathrm{CD}_{3} \mathrm{CN}\right): \delta 218.7$ (C3), 71.3 (C1), 67.5 (C7), 48.4 (C4), 47.8 (C6), 45.8 (C8), 36.3 (C5), 34.6 (C2). 


\section{$N$-(2-(1,3-Dioxolan-2-yl)ethyl)- $N$-cyclopropyl-1H-indole-1-carboxamide}

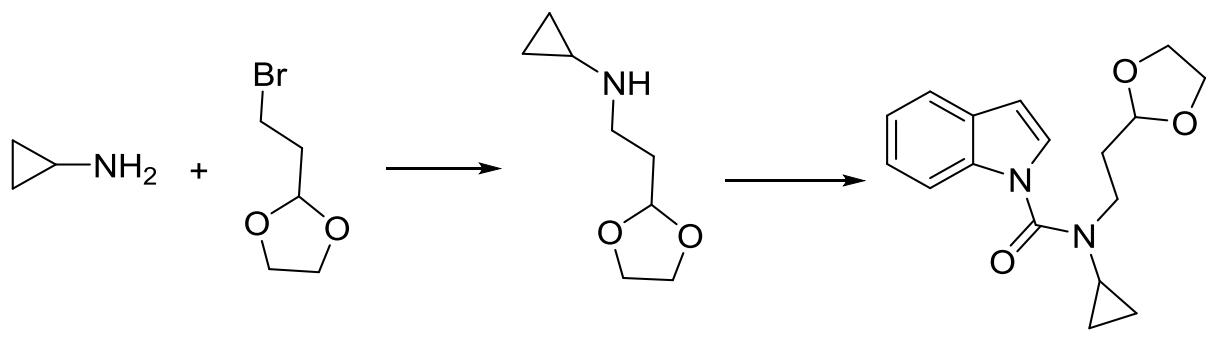

To a solution of cyclopropanamine $(2.76 \mathrm{~mL}, 40 \mathrm{mmol})$ and 2-(2-bromoethyl)-1,3-dioxolane $(2.34 \mathrm{~mL}, 20.0 \mathrm{mmol})$ in DMF $(40 \mathrm{~mL})$ was added $\mathrm{Et}_{3} \mathrm{~N}(5.55 \mathrm{~mL}, 40 \mathrm{mmol})$ at $0{ }^{\circ} \mathrm{C}$. The reaction mixture was warmed to room temperature and stirred for 16 hours. The reaction was quenched by the addition of saturated aqueous $\mathrm{NaHCO}_{3}(100 \mathrm{~mL})$ and extracted with EtOAc $(3 \times 50 \mathrm{~mL})$. The organic extracts were combined, washed with water $(100 \mathrm{~mL})$, brine $(50$ $\mathrm{mL}$ ), dried over $\mathrm{Na}_{2} \mathrm{SO}_{4}$ and concentrated in vacuo to yield $\mathrm{N}$-(2-(1,3-dioxolan-2yl)ethyl)cyclopropanamine and $N, N$-bis(2-(1,3-dioxolan-2-yl)ethyl)cyclopropanamine (1.52g, $48 \%, 6: 1)$. The mixture was dissolved in acetone $(20.0 \mathrm{~mL})$ and water $(4.40 \mathrm{~mL})$ and cooled to $0{ }^{\circ} \mathrm{C} . \mathrm{K}_{2} \mathrm{CO}_{3}(1.93 \mathrm{~g}, 14.0 \mathrm{mmol})$ and $1 \mathrm{H}$-indole-1-carbonyl chloride $(1.88 \mathrm{~g}, 10.5 \mathrm{mmol})$ were added. The reaction mixture was warmed to room temperature and stirred for 16 hours and then the reaction mixture was concentrated in vacuo to remove acetone. Water $(25 \mathrm{~mL})$ was added and the solution was extracted with $\mathrm{CH}_{2} \mathrm{Cl}_{2}(3 \times 20 \mathrm{~mL})$. The organic extracts were combined, washed with brine $(50 \mathrm{~mL})$, dried over $\mathrm{Na}_{2} \mathrm{SO}_{4}$ and concentrated in vacuo. The crude mixture was purified by flash column chromatography (15\% EtOAc/hexane to $20 \%$ EtOAc/hexane) to afford $N$-(2-(1,3-dioxolan-2-yl)ethyl)- $N$-cyclopropyl-1 $H$-indole-1carboxamide (1.65 g, 79\%) as a yellow oil; $v_{\max } / \mathrm{cm}^{-1}: 2884(\mathrm{~m}), 1670(\mathrm{~s}), 1452(\mathrm{~m}), 1407$ (m), 1029 (s), 747 (s); ${ }^{1} \mathrm{H}$ NMR (400 MHz, $\left.\mathrm{CDCl}_{3}\right): \delta 7.70(1 \mathrm{H}, \mathrm{d}, J=8.2 \mathrm{~Hz}), 7.50(1 \mathrm{H}, \mathrm{d}, J$ $=7.7 \mathrm{~Hz}), 7.38(1 \mathrm{H}, \mathrm{d}, J=3.6 \mathrm{~Hz}), 7.20-7.16(1 \mathrm{H}, \mathrm{m}), 7.12-7.08(1 \mathrm{H}, \mathrm{m}), 6.48(1 \mathrm{H}, \mathrm{d}, J=$ $3.6 \mathrm{~Hz}), 4.89(1 \mathrm{H}, \mathrm{t}, J=4.4 \mathrm{~Hz}), 3.89-3.83(2 \mathrm{H}, \mathrm{m}), 3.79-3.73(2 \mathrm{H}, \mathrm{m}), 3.63(2 \mathrm{H}, \mathrm{t}, J=$ $6.7 \mathrm{~Hz}), 2.05(2 \mathrm{H}, \mathrm{td}, J=6.7,4.4 \mathrm{~Hz}), 0.63-0.58(2 \mathrm{H}, \mathrm{m}), 0.46-0.42(2 \mathrm{H}, \mathrm{m}) ;{ }^{13} \mathrm{C} \mathrm{NMR}$ $\left(101 \mathrm{MHz}, \mathrm{CDCl}_{3}\right): \delta 155.0,135.6,129.4,126.0,123.4,121.7,120.6,113.8,105.2,102.9$, 64.9, 44.4, 31.6, 31.2, 9.0; m/z (ESI $\left.{ }^{+}\right)$HRMS: $\mathrm{C}_{17} \mathrm{H}_{21} \mathrm{~N}_{2} \mathrm{O}_{3}$ : 301.1547. Found $[\mathrm{M}+\mathrm{H}]^{+}$: 301.1551 . 


\section{$N$-Cyclopropyl- $N$-(3-oxopropyl)-1H-indole-1-carboxamide}<smiles>Cc1cccc2c1ccn2C(=O)N(CCC=O)C1CC1</smiles>

$N$-(2-(1,3-Dioxolan-2-yl)ethyl)- $N$-cyclopropyl-1 $H$-indole-1-carboxamide (1.82g, $4.27 \mathrm{mmol})$ was dissolved in water $(10 \mathrm{~mL})$ and THF $(10 \mathrm{~mL})$. HOAc $(10 \mathrm{~mL})$ and $6 \mathrm{M}$ aqueous $\mathrm{HCl}(15$ $\mathrm{mL}$ ) were added and the mixture was stirred at room temperature for 16 hours. The solution was extracted with $\mathrm{Et}_{2} \mathrm{O}(3 \times 25 \mathrm{~mL})$, the organic extracts were combined, washed with water $(40 \mathrm{~mL})$, saturated aqueous $\mathrm{NaHCO}_{3}(40 \mathrm{~mL})$ and brine $(40 \mathrm{~mL})$, dried over $\mathrm{Na}_{2} \mathrm{SO}_{4}$ and concentrated in vacuo. The crude mixture was purified by flash column chromatography (35\% EtOAc/hexane) to afford the $N$-cyclopropyl- $N$-(3-oxopropyl)-1 $H$-indole-1-carboxamide (640 $\mathrm{mg}, 59 \%$ ) as a colourless oil; $v_{\max } / \mathrm{cm}^{-1}: 1725(\mathrm{~m}), 1673$ (s), 1453 (s), 1412 (s), 1319 (m), $1210(\mathrm{~m}) ;{ }^{1} \mathrm{H}$ NMR $\left(400 \mathrm{MHz}, \mathrm{CDCl}_{3}\right): \delta 9.86(1 \mathrm{H}, \mathrm{t}, J=1.0 \mathrm{~Hz}), 7.74(1 \mathrm{H}$, ddd, $J=8.3,0.9$, $0.9 \mathrm{~Hz}), 7.58(1 \mathrm{H}$, ddd, $J=7.7,0.9,0.9 \mathrm{~Hz}), 7.40(1 \mathrm{H}, \mathrm{d}, J=3.6 \mathrm{~Hz}), 7.29-7.25(1 \mathrm{H}, \mathrm{m})$, $7.19(1 \mathrm{H}, \mathrm{ddd}, J=8.3,7.2,0.9 \mathrm{~Hz}), 6.57(1 \mathrm{H}, \mathrm{dd}, J=3.6,0.9 \mathrm{~Hz}), 3.86(2 \mathrm{H}, \mathrm{t}, J=6.4 \mathrm{~Hz})$, $2.97-2.91(3 \mathrm{H}, \mathrm{m}), 0.72-0.65(2 \mathrm{H}, \mathrm{m}), 0.59-0.51(2 \mathrm{H}, \mathrm{m}) ;{ }^{13} \mathrm{C} \mathrm{NMR}\left(101 \mathrm{MHz}, \mathrm{CDCl}_{3}\right)$ : $\delta 200.3,154.8,135.6,129.5,125.9,123.6,122.0,120.8,113.9,105.8,43.3,42.3$, 32.6, 9.3; $\mathrm{m} / \mathrm{z}\left(\mathrm{ESI}^{+}\right) \mathrm{HRMS}: \mathrm{C}_{15} \mathrm{H}_{17} \mathrm{~N}_{2} \mathrm{O}_{2}: 257.1285$. Found $[\mathrm{M}+\mathrm{H}]^{+}: 257.1288$.

(E)- $N$-Cyclopropyl- $N$-(4-phenylbut-3-en-1-yl)-1H-indole-1-carboxamide $3 r$ and $(Z)-N$ Cyclopropyl- $N$-(4-phenylbut-3-en-1-yl)-1H-indole-1-carboxamide 3r'
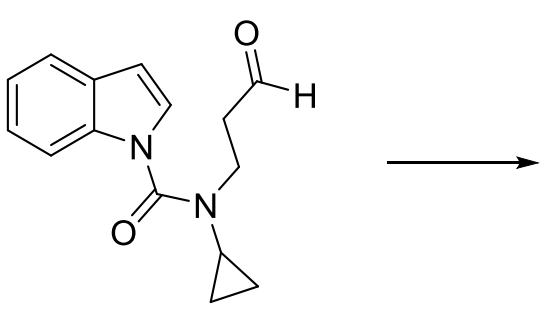

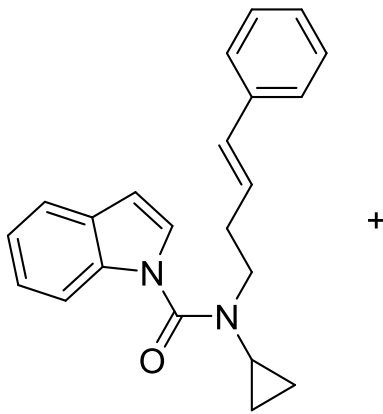

$3 r$

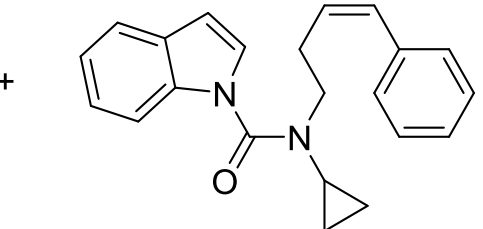

$3 r^{\prime}$

Benzyltriphenylphosphonium bromide $(1.30 \mathrm{~g}, 3.00 \mathrm{mmol})$ was dissolved in dry THF $(15 \mathrm{~mL})$ and potassium tert-butoxide $(336 \mathrm{mg}, 3.00 \mathrm{mmol}$ ) was added. The mixture was stirred for 1 
hour at room temperature before a solution of $N$-cyclopropyl- $N$-(3-oxopropyl)-1H-indole-1carboxamide $(386 \mathrm{mg}, 1.50 \mathrm{mmol})$ in dry THF $(5.00 \mathrm{~mL})$ was added slowly over 5 minutes. The reaction mixture was stirred at room temperature for $16 \mathrm{~h}$. The reaction was quenched by the addition of $2 \mathrm{M}$ aqueous $\mathrm{HCl}(15 \mathrm{~mL})$ and extracted with $\mathrm{Et}_{2} \mathrm{O}(3 \times 25 \mathrm{~mL})$. The organic extracts were combined, washed with brine $(40 \mathrm{~mL})$, dried over $\mathrm{Na}_{2} \mathrm{SO}_{4}$ and concentrated in vacuo. The crude mixture was purified by flash column chromatography (10\% EtOAc/hexane) to yield (E)- $N$-cyclopropyl- $N$-(4-phenylbut-3-en-1-yl)-1H-indole-1-carboxamide 3r (223 mg, $45 \%)$ as a colourless solid and (Z)- $N$-cyclopropyl- $N$-(4-phenylbut-3-en-1-yl)-1H-indole-1carboxamide 3r' (111 mg, 27\%) as a colourless oil.

3r: m.p. 76-78 ${ }^{\circ} \mathrm{C}\left(\mathrm{CH}_{2} \mathrm{Cl}_{2}\right) ; v_{\max } / \mathrm{cm}^{-1}: 2925$ (m), 1673 (s), 1452 (m), 1407 (m), 1317 (m), $1210(\mathrm{~m}) ;{ }^{1} \mathrm{H}$ NMR (400 MHz, $\left.\mathrm{CDCl}_{3}\right): \delta 7.64(1 \mathrm{H}, \mathrm{dd}, J=6.3,3.5 \mathrm{~Hz}), 7.51(1 \mathrm{H}, \mathrm{ddd}, J=$ 6.9, 2.5, $2.5 \mathrm{~Hz}), 7.31-7.25(4 \mathrm{H}, \mathrm{m}), 7.21-7.09(4 \mathrm{H}, \mathrm{m}), 6.51-6.46(2 \mathrm{H}, \mathrm{m}), 6.15(1 \mathrm{H}$, dddd, $J=13.6,7.5,6.1,1.8 \mathrm{~Hz}), 3.67-3.63(2 \mathrm{H}, \mathrm{m}), 2.84-2.80(1 \mathrm{H}, \mathrm{m}), 2.64-2.59(2 \mathrm{H}$, $\mathrm{m}), 0.66(2 \mathrm{H}, \mathrm{dt}, J=6.1,3.0 \mathrm{~Hz}), 0.53-0.49(2 \mathrm{H}, \mathrm{m}) ;{ }^{13} \mathrm{C} \mathrm{NMR}\left(101 \mathrm{MHz}, \mathrm{CDCl}_{3}\right): \delta 155.2$, 137.1, 135.4, 132.8, 129.4, 128.6, 127.4, 126.7, 126.1, 126.0, 123.4, 121.8, 120.8, 113.7, 105.5, 49.0, 31.7, 31.4, 9.0; m/z $\left(\mathrm{ESI}^{+}\right)$HRMS: $\mathrm{C}_{22} \mathrm{H}_{23} \mathrm{~N}_{2} \mathrm{O}: 331.1805$. Found $[\mathrm{M}+\mathrm{H}]^{+}$: 331.1809 .

3r': $v_{\max } / \mathrm{cm}^{-1}: 2928$ (m), 1673 (s), 1452 (s), 1406(s), 1319 (s), 747 (s); ${ }^{1} \mathrm{H}$ NMR (400 MHz, $\left.\mathrm{CDCl}_{3}\right): 7.66(1 \mathrm{H}, \mathrm{dd}, J=8.2,1.9 \mathrm{~Hz}), 7.53(1 \mathrm{H}, \mathrm{d}, J=7.9 \mathrm{~Hz}), 7.32-7.26(2 \mathrm{H}, \mathrm{m}), 7.25-$ $7.11(6 \mathrm{H}, \mathrm{m}), 6.55-6.50(2 \mathrm{H}, \mathrm{m}), 5.63(1 \mathrm{H}, \mathrm{ddd}, J=7.6,7.6,3.9 \mathrm{~Hz}), 3.61(2 \mathrm{H}, \mathrm{td}, J=7.0$, $2.1 \mathrm{~Hz}), 2.77-2.74(2 \mathrm{H}, \mathrm{m}), 2.67-2.61(1 \mathrm{H}, \mathrm{m}), 0.61-0.55(2 \mathrm{H}, \mathrm{m}), 0.47-0.42(2 \mathrm{H}, \mathrm{m})$; ${ }^{13} \mathrm{C} \mathrm{NMR}\left(101 \mathrm{MHz}, \mathrm{CDCl}_{3}\right): \delta 155.2,137.0,135.5,131.6,129.4,128.6,128.4,128.3,126.9$, 126.0, 123.5, 121.8, 120.8, 113.8, 105.5, 49.0, 30.9, 27.2, 9.0; m/z (ESI $\left.{ }^{+}\right)$HRMS: $\mathrm{C}_{22} \mathrm{H}_{23} \mathrm{~N}_{2} \mathrm{O}$ : 331.1805. Found $[\mathrm{M}+\mathrm{H}]^{+}: 331.1807$. 


\section{imidazo[1,5- $a$ ]indole-1,5-dione $4 r$}

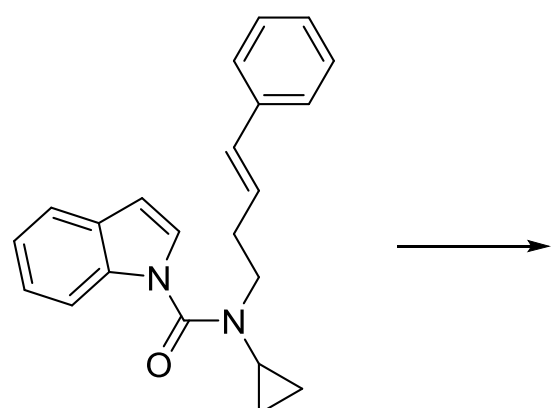

$3 r$

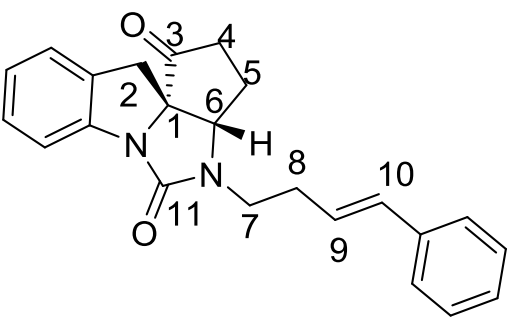

$4 r$

General procedure D: Compound $3 \mathbf{r}(33.0 \mathrm{mg}, 0.10 \mathrm{mmol}),\left[\mathrm{Rh}(\mathrm{cod})_{2}\right] \mathrm{OTf}(3.52 \mathrm{mg}, 7.5$ $\mathrm{mol} \%)$ and anhydrous 1,2-DCB $(1.00 \mathrm{~mL})$ were employed and the reaction was stirred for 72 hours at $130{ }^{\circ} \mathrm{C}$. The crude mixture was purified by column chromatography $(5 \%$ EtOAc/toluene) to yield the title compound $4 \mathbf{r}(15.0 \mathrm{mg}, 42 \%)$ as a colourless oil; $v_{\max } / \mathrm{cm}^{-1}$ : 2923 (m), 1750 (s), 1707 (s), 1480 (s), 1409 (s), 1292 (s); ${ }^{1} \mathrm{H}$ NMR (400 MHz, CDCl 3 ) $\delta 7.45$ - $7.43(1 \mathrm{H}, \mathrm{m}, 1 \times \mathrm{ArC} \underline{\mathrm{H}}), 7.25-7.15(6 \mathrm{H}, \mathrm{m}, 6 \times \mathrm{ArC} \underline{\mathrm{H}}), 7.11(1 \mathrm{H}, \mathrm{d}, J=7.4 \mathrm{~Hz}, 1 \times$ $\operatorname{ArC} \underline{H}), 7.01(1 \mathrm{H}, \mathrm{ddd}, J=7.5,7.5,1.1 \mathrm{~Hz}, 1 \times \mathrm{ArC} \underline{\mathrm{H}}), 6.42-6.37(1 \mathrm{H}, \mathrm{m}, 1 \times \mathrm{C10}-\underline{\mathrm{H}}), 6.12$ $(1 \mathrm{H}, \mathrm{dt}, J=15.8,7.2 \mathrm{~Hz}, 1 \times \mathrm{C} 9-\underline{\mathrm{H}}), 4.25(1 \mathrm{H}, \mathrm{d}, J=5.3 \mathrm{~Hz}, 1 \times \mathrm{C6}-\underline{\mathrm{H}}), 3.57(1 \mathrm{H}, \mathrm{ddd}, J=$ 14.6, 7.5, $\left.7.5 \mathrm{~Hz}, 1 \times \mathrm{C} 7-\underline{\mathrm{H}}_{2}\right), 3.37-3.28\left(2 \mathrm{H}, \mathrm{m}, 1 \times \mathrm{C} 7-\underline{\mathrm{H}}_{2}, 1 \times \mathrm{C} 2-\underline{\mathrm{H}}_{2}\right), 3.05(1 \mathrm{H}, \mathrm{d}, J=$ 16.1 Hz, $\left.1 \times \mathrm{C} 2-\underline{\mathrm{H}}_{2}\right), 2.67\left(1 \mathrm{H}, \mathrm{ddd}, J=17.7,13.7,9.2 \mathrm{~Hz}, 1 \times \mathrm{C} 4-\underline{\mathrm{H}}_{2}\right), 2.60-2.44(2 \mathrm{H}, \mathrm{m}, 2$ $\left.\times \mathrm{C} 8-\underline{\mathrm{H}}_{2}\right), 2.36-2.28\left(2 \mathrm{H}, \mathrm{m}, 1 \times \mathrm{C} 4-\underline{\mathrm{H}}_{2}, 1 \times \mathrm{C} 5-\underline{\mathrm{H}}_{2}\right), 2.03-1.92\left(1 \mathrm{H}, \mathrm{m}, 1 \times \mathrm{C} 5-\underline{\mathrm{H}}_{2}\right) ;{ }^{13} \mathrm{C}$ NMR (101 MHz, $\mathrm{CDCl}_{3}$ ) $\delta 212.1$ (C3), 159.3 (C11), 142.3 (CAr), 136.8 (CAr), 132.6 (C10), 129.8 (CAr), 128.5 (CAr), 127.9 (CAr), 127.3 (CAr), 126.2 (C9), 125.9 (CAr), 124.6 (CAr), 124.2 (CAr), 115.9 (CAr), 70.0 (C1), 64.4 (C6), 41.6 (C7), 35.8 (C2), 33.1 (C4), 31.2 (C8), 23.9 (C5); HRMS: $\left(\mathrm{ESI}^{+}\right)$Calculated for $\mathrm{C}_{23} \mathrm{H}_{23} \mathrm{~N}_{2} \mathrm{O}_{2}: 359.1754$. Found $[\mathrm{M}+\mathrm{H}]^{+}: 359.1759$.

When the above reaction was run in the presence of $\mathrm{P}\left(4-\mathrm{FC}_{6} \mathrm{H}_{4}\right)_{3}(4.47 \mathrm{mg}, 15.0 \mathrm{~mol} \%)$, compound $\mathbf{4 r}(9.7 \mathrm{mg}, 27 \%)$ was obtained.

$N$-(4-Phenylbut-3-yn-1-yl)cyclopropanamine

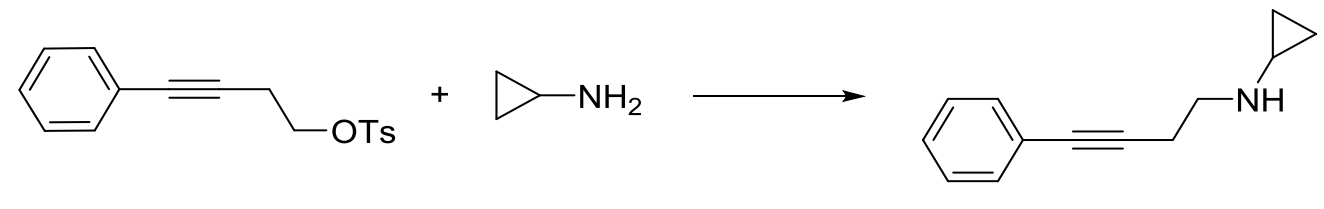


To a flask containing 4-phenylbut-3-yn-1-yl 4-methylbenzenesulfonate (2.13g $7.10 \mathrm{mmol})$ (prepared according to the literature procedure) ${ }^{7}$ in $\mathrm{MeCN}(17.5 \mathrm{~mL}$ ) was added cyclopropylamine $(1.96 \mathrm{~mL}, 28.4 \mathrm{mmol})$. The flask was sealed and heated at $90{ }^{0} \mathrm{C}$ for 16 hours. The reaction mixture was cooled to room temperature and concentrated in vacuo. The residue was dissolved in $\mathrm{CH}_{2} \mathrm{Cl}_{2}(35 \mathrm{~mL})$ and washed with saturated aqueous $\mathrm{NaHCO}_{3}$ (50 $\mathrm{mL})$. The aqueous portion was washed with $\mathrm{CH}_{2} \mathrm{Cl}_{2}(2 \times 30 \mathrm{~mL})$ and the organic extracts were combined, washed with brine $(35 \mathrm{~mL})$, dried over $\mathrm{Na}_{2} \mathrm{SO}_{4}$ and concentrated in vacuo. The crude mixture was purified by flash column chromatography (35\% EtOAc/hexane) to afford the title compound $(1.12 \mathrm{~g}, 85 \%)$ as a yellow oil; $v_{\max } / \mathrm{cm}^{-1}: 3749(\mathrm{~m}), 2926(\mathrm{M}), 1490$ (s), 1441 (s), 1346 (s), 755 (s); ${ }^{1} \mathrm{H}$ NMR (400 MHz, $\left.\mathrm{CDCl}_{3}\right): \delta 7.41-7.37$ (2H, m), 7.29 $7.24(3 \mathrm{H}, \mathrm{m}), 2.92(2 \mathrm{H}, \mathrm{t}, J=6.6 \mathrm{~Hz}), 2.61(2 \mathrm{H}, \mathrm{t}, J=6.6 \mathrm{~Hz}), 2.21-2.16(1 \mathrm{H}, \mathrm{m}), 0.47-$ $0.34(4 \mathrm{H}, \mathrm{m}) ;{ }^{13} \mathrm{C} \mathrm{NMR}\left(101 \mathrm{MHz}, \mathrm{CDCl}_{3}\right): \delta 131.6,128.2,127.7,123.6,88.0,81.7,47.9$, 29.6, 20.5, 6.3; m/z $\left(\mathrm{ESI}^{+}\right)$HRMS: $\mathrm{C}_{13} \mathrm{H}_{16} \mathrm{~N}: 186.1277$. Found $[\mathrm{M}+\mathrm{H}]^{+}: 186.1274$.

\section{$N$-Cyclopropyl- $N$-(4-phenylbut-3-yn-1-yl)-1H-indole-1-carboxamide 5a}
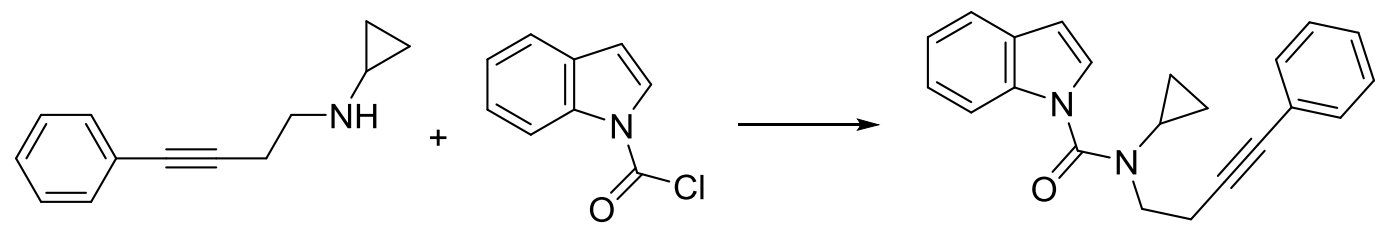

$5 \mathbf{a}$

Triethylamine $(0.69 \mathrm{~mL} 5.00 \mathrm{mmol})$ was added to a solution of $N$-(4-phenylbut-3-yn-1yl)cyclopropanamine $(370 \mathrm{mg}, 2.00 \mathrm{mmol})$ in $\mathrm{CH}_{2} \mathrm{Cl}_{2}(10 \mathrm{~mL})$ at $0{ }^{\circ} \mathrm{C} .1 H$-Indole-1-carbonyl chloride (430 mg, $2.40 \mathrm{mmol})$ in $\mathrm{CH}_{2} \mathrm{Cl}_{2}(3.00 \mathrm{~mL})$ was added dropwise over 5 minutes. The reaction mixture was warmed to room temperature and stirred for 18 hours. Water $(10 \mathrm{~mL})$ was added and the mixture was extracted with $\mathrm{CH}_{2} \mathrm{Cl}_{2}(2 \times 10 \mathrm{~mL})$. The organic extracts were combined, washed with brine $(10 \mathrm{~mL})$, dried over $\mathrm{Na}_{2} \mathrm{SO}_{4}$ and concentrated in vacuo. The crude mixture was purified by flash column chromatography (5\% EtOAc/hexane) to afford the title compound $\mathbf{5 a}(533 \mathrm{mg}, 81 \%)$ as a colourless solid; m.p. $67-69{ }^{\circ} \mathrm{C}\left(\mathrm{CH}_{2} \mathrm{Cl}_{2}\right)$; $v_{\max } / \mathrm{cm}^{-1}: 2937$ (m), 1672 (s), 1452 (m), 1405 (s), 1029 (s), 746 (s); ${ }^{1} \mathrm{H}$ NMR (400 MHz, $\left.\mathrm{CDCl}_{3}\right): \delta 7.76(1 \mathrm{H}, \mathrm{d}, J=7.6 \mathrm{~Hz}), 7.57-7.55(1 \mathrm{H}, \mathrm{m}), 7.44-7.37(3 \mathrm{H}, \mathrm{m}), 7.29-7.14$ $(5 \mathrm{H}, \mathrm{m}), 6.54(1 \mathrm{H}, \mathrm{d}, J=3.6 \mathrm{~Hz}), 3.77(2 \mathrm{H}, \mathrm{t}, J=6.5 \mathrm{~Hz}), 3.02(1 \mathrm{H}, \mathrm{tt}, J=7.1,3.9 \mathrm{~Hz}), 2.85$ $(2 \mathrm{H}, \mathrm{t}, J=6.5 \mathrm{~Hz}), 0.73-0.66(2 \mathrm{H}, \mathrm{m}), 0.61-0.53(2 \mathrm{H}, \mathrm{m}) ;{ }^{13} \mathrm{C} \mathrm{NMR}\left(101 \mathrm{MHz}, \mathrm{CDCl}_{3}\right): \delta$ 
155.1, 135.4, 131.6, 129.5, 128.3, 128.0, 126.2, 123.5, 123.2, 121.8, 120.8, 113.7, 105.6, 86.7, 82.5, 48.4, 31.5, 18.9, 9.0; m/z (ESI $\left.{ }^{+}\right)$HRMS: $\mathrm{C}_{22} \mathrm{H}_{20} \mathrm{~N}_{2} \mathrm{NaO}: 351.1468$. Found $[\mathrm{M}+\mathrm{Na}]^{+}$: 351.1466 .

(E)-3-Benzylidene-1,2,3,3a,4,5-hexahydro-6H,13H-pyrrolo[1',2':3,4][1,3]diazocino[1,8a]indole-6,13-dione $6 \mathrm{a}$

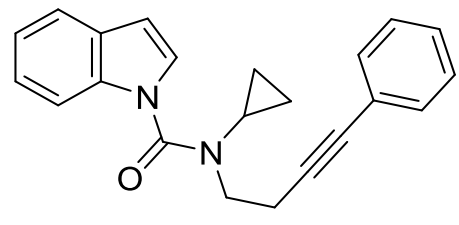

$5 a$

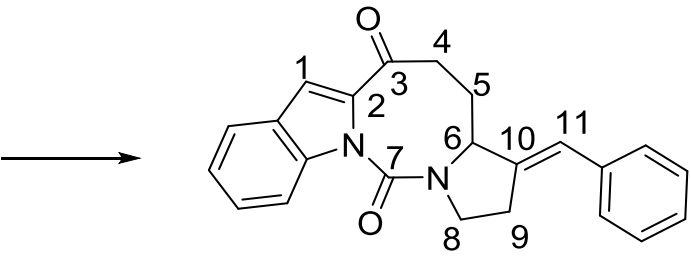

$6 a$

General procedure E: Compound $5 \mathbf{a}(32.8 \mathrm{mg}, 0.10 \mathrm{mmol})$ was employed and the reaction was stirred for 96 hours at $120{ }^{\circ} \mathrm{C}$. The crude mixture was purified by column chromatography (5\% EtOAc/toluene) to yield the title compound $\mathbf{6 a}(19.6 \mathrm{mg}, 55 \%)$ as a yellow oil; $v_{\max } / \mathrm{cm}^{-1}: 2924$ (m), 1741 (s), 1667 (s), 1515 (s), 1405 (s), 1337 (s); ${ }^{1} \mathrm{H}$ NMR $\left(400 \mathrm{MHz}, \mathrm{CDCl}_{3}\right) \delta 7.94(1 \mathrm{H}, \mathrm{d}, J=8.6 \mathrm{~Hz}, 1 \times \mathrm{ArC} \underline{\mathrm{H}}), 7.68(1 \mathrm{H}, \mathrm{d}, J=8.0 \mathrm{~Hz}, 1 \times \mathrm{ArC} \underline{\mathrm{H}})$, $7.57(1 \mathrm{H}, \mathrm{s}, 1 \times \mathrm{C} 1-\underline{\mathrm{H}}), 7.42(1 \mathrm{H}, \mathrm{ddd}, J=8.5,7.0,1.2 \mathrm{~Hz}, 1 \times \operatorname{ArC} \underline{\mathrm{H}}), 7.34-7.30(2 \mathrm{H}, \mathrm{m}, 2$ $\times \operatorname{ArC} \underline{H}), 7.25-7.20(4 \mathrm{H}, \mathrm{m}, 1 \times \mathrm{ArC} \underline{\mathrm{H}}), 6.28(1 \mathrm{H}, \mathrm{d}, J=2.7 \mathrm{~Hz}, 1 \times \mathrm{C} 11-\underline{\mathrm{H}}), 4.55(1 \mathrm{H}, \mathrm{dd}$, $J=12.8,4.9 \mathrm{~Hz}, 1 \times \mathrm{C} 6-\underline{\mathrm{H}}), 4.40\left(1 \mathrm{H}, \mathrm{ddd}, J=12.4,8.6,5.4 \mathrm{~Hz}, 1 \times \mathrm{C} 8-\underline{\mathrm{H}}_{2}\right), 3.81-3.76$ $\left(1 \mathrm{H}, \mathrm{m}, 1 \times \mathrm{C} 8-\underline{\mathrm{H}}_{2}\right), 3.12-2.93\left(3 \mathrm{H}, \mathrm{m}, 1 \times \mathrm{C} 4-\underline{\mathrm{H}}_{2}, 2 \times \mathrm{C} 9-\underline{\mathrm{H}}_{2}\right), 2.74(1 \mathrm{H}, \mathrm{ddd}, J=12.3,3.7$, $\left.3.7 \mathrm{~Hz}, 1 \times \mathrm{C} 4-\underline{\mathrm{H}}_{2}\right), 2.22-2.13\left(1 \mathrm{H}, \mathrm{m}, 1 \times \mathrm{C} 5-\underline{\mathrm{H}}_{2}\right), 2.10-2.01\left(1 \mathrm{H}, \mathrm{m}, 1 \times \mathrm{C} 5-\underline{\mathrm{H}}_{2}\right) ;{ }^{13} \mathrm{C}$ NMR (101 MHz, $\mathrm{CDCl}_{3}$ ) $\delta 191.0$ (C3), 150.6 (C7), 140 (C10), 139.5 (CAr), 137.6 (C2), 136.3 (CAr), 128.5 (CAr), 128.3 (CAr), 127.6 (CAr), 127.3 (CAr), 126.5 (CAr), 123.3 (C11), 122.9 (CAr), 122.8 (CAr), 115.4 (CAr), 114.4 (C1), 61.5 (C6), 45.6 (C8), 37.4 (C4), 33.5 (C9), 26.9 (C5); HRMS: (ESI ${ }^{+}$) Calculated for $\mathrm{C}_{23} \mathrm{H}_{20} \mathrm{~N}_{2} \mathrm{NaO}_{2}$ : 379.1417. Found $[\mathrm{M}+\mathrm{Na}]^{+}: 379.1432$.

When the above reaction was run with $\mathrm{P}\left(4-\mathrm{FC}_{6} \mathrm{H}_{4}\right)_{3}(4.47 \mathrm{mg}, 15.0 \mathrm{~mol} \%)$ as the ligand, compound $\mathbf{6} \mathbf{a}(9.3 \mathrm{mg}, 26 \%)$ was obtained. When the above reaction was run without any phosphine ligand, compound $\mathbf{6} \mathbf{a}$ was not detected. No dearomatisation product was detected under all these above conditions. 


\section{$N$-Cyclopropyl- $N$-(4-(4-(trifluoromethyl)phenyl)but-3-yn-1-yl)-1H-indole-1-}

\section{carboxamide $\mathbf{5 b}$}
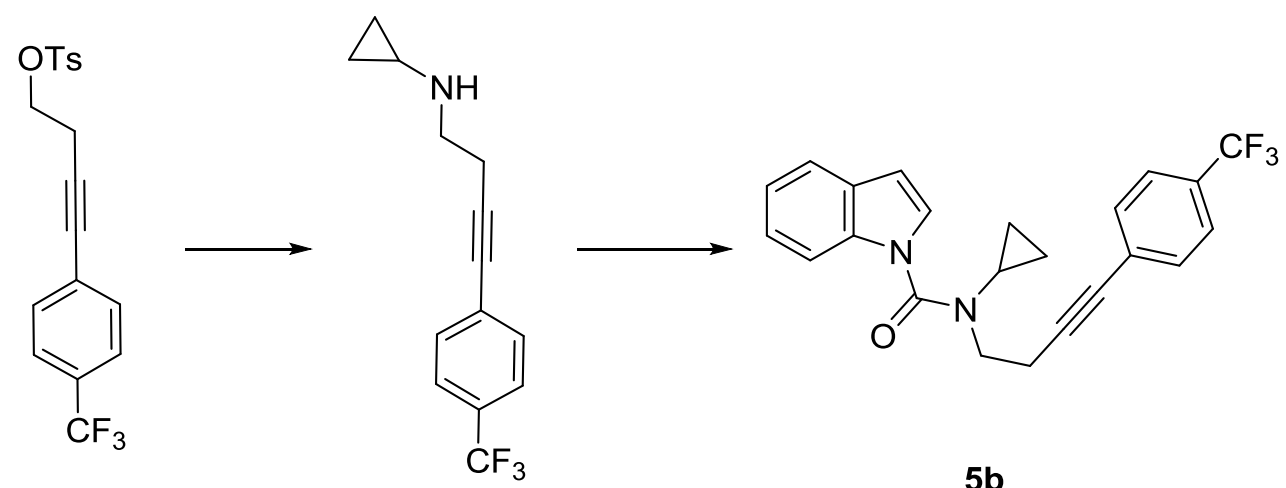

5b

To a flask containing 4-(4-(trifluoromethyl)phenyl)but-3-yn-1-yl 4-methylbenzenesulfonate (prepared according to the literature procedure $)^{8}(555 \mathrm{mg}, 1.52 \mathrm{mmol})$ in $\mathrm{MeCN}(8.00 \mathrm{~mL})$ was added cyclopropylamine $(0.42 \mathrm{~mL}, 6.00 \mathrm{mmol})$. The flask was sealed and heated at 90 ${ }^{0} \mathrm{C}$ for 16 hours. The reaction mixture was cooled to room temperature, dissolved in $\mathrm{CH}_{2} \mathrm{Cl}_{2}$ $(20 \mathrm{~mL})$ and washed with saturated aqueous $\mathrm{NaHCO}_{3}(20 \mathrm{~mL})$. The aqueous portion was extracted with $\mathrm{CH}_{2} \mathrm{Cl}_{2}(2 \times 15 \mathrm{~mL})$, the organic extracts were combined, washed with brine (35 mL), dried over $\mathrm{Na}_{2} \mathrm{SO}_{4}$ and concentrated in vacuo. The residue was dissolved in acetone $(5.00 \mathrm{~mL})$ and water $(0.90 \mathrm{~mL})$ and cooled to $0{ }^{\circ} \mathrm{C} . \mathrm{K}_{2} \mathrm{CO}_{3}(414 \mathrm{mg}, 3.00 \mathrm{mmol})$ and $1 \mathrm{H}$ indole-1-carbonyl chloride $(671 \mathrm{mg}, 3.75 \mathrm{mmol})$ were added. The reaction mixture was warmed to room temperature and stirred for 16 hours. The reaction mixture was concentrated in vacuo to remove acetone and water $(10 \mathrm{~mL})$ was then added. The solution was extracted with $\mathrm{CH}_{2} \mathrm{Cl}_{2}(3 \times 10 \mathrm{~mL})$ and the organic extracts were combined, washed with brine $(20$ $\mathrm{mL}$ ), dried over $\mathrm{Na}_{2} \mathrm{SO}_{4}$ and concentrated in vacuo. The crude mixture was purified by flash column chromatography (6\% EtOAc/hexane) to afford the title compound $\mathbf{5 b}$ (303 $\mathrm{mg}, 51 \%$ ) as a yellow oil; $v_{\max } / \mathrm{cm}^{-1}: 2923$ (m), 1667 (s), 1407 (s), 1322 (s), 1125 (s), 1066 (s); ${ }^{1} \mathrm{H}$ NMR $\left(400 \mathrm{MHz}, \mathrm{CDCl}_{3}\right): \delta 7.75-7.72(1 \mathrm{H}, \mathrm{m}), 7.58-7.56(1 \mathrm{H}, \mathrm{m}), 7.54-7.52(2 \mathrm{H}, \mathrm{m}), 7.47-$ $7.45(2 \mathrm{H}, \mathrm{m}), 7.42(1 \mathrm{H}, \mathrm{d}, J=3.6 \mathrm{~Hz}), 7.22-7.15(2 \mathrm{H}, \mathrm{m}), 6.55(1 \mathrm{H}, \mathrm{dd}, J=3.6,0.8 \mathrm{~Hz})$, $3.79(2 \mathrm{H}, \mathrm{t}, J=6.5 \mathrm{~Hz}), 3.00(1 \mathrm{H}, \mathrm{tt}, J=6.8,3.9 \mathrm{~Hz}), 2.88(2 \mathrm{H}, \mathrm{t}, J=6.5 \mathrm{~Hz}), 0.76-0.71$ $(2 \mathrm{H}, \mathrm{m}), 0.59-0.55(2 \mathrm{H}, \mathrm{m}) ;{ }^{13} \mathrm{C} \mathrm{NMR}\left(126 \mathrm{MHz}, \mathrm{CDCl}_{3}\right): \delta 155.2,135.4,131.8,129.8$ (q, $J=32.8 \mathrm{~Hz}), 129.5,127.0(\mathrm{q}, J=1.1 \mathrm{~Hz}), 126.0,125.2(\mathrm{q}, J=3.8 \mathrm{~Hz}) 123.9(\mathrm{q}, J=297.3$ $\mathrm{Hz}), 123.6,121.9,120.9$, 113.7, 105.8, 89.5, 81.4, 48.2, 31.4, 19.0, 9.1; ${ }^{19} \mathrm{~F}$ NMR (377 MHz, $\left.\mathrm{CDCl}_{3}\right): \delta-62.8 ; \mathrm{m} / z\left(\mathrm{ESI}^{+}\right) \mathrm{HRMS}: \mathrm{C}_{23} \mathrm{H}_{19} \mathrm{~F}_{3} \mathrm{~N}_{2} \mathrm{NaO}: 419.1342$. Found $[\mathrm{M}+\mathrm{Na}]^{+}: 419.1358$. 
(E)-3-(4-(Trifluoromethyl)benzylidene)-1,2,3,3a,4,5-hexahydro-6H,13Hpyrrolo $\left[1^{\prime}, 2^{\prime}: 3,4\right][1,3]$ diazocino[1,8-a]indole-6,13-dione $6 b$

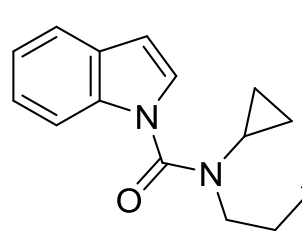

5b

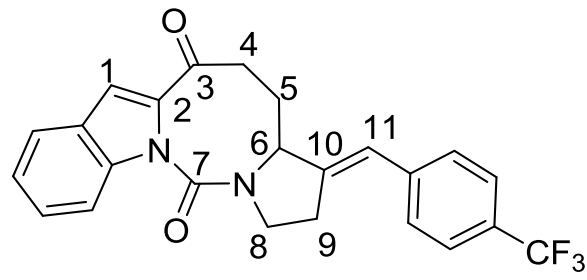

$6 b$
12

General procedure E: Compound $\mathbf{5 b}(39.6 \mathrm{mg}, 0.10 \mathrm{mmol})$ was employed and the reaction was stirred for 96 hours at $120{ }^{\circ} \mathrm{C}$. The crude mixture was purified by column chromatography (5\% EtOAc/toluene) to yield the title compound $\mathbf{6 b}(23.6 \mathrm{mg}, 55 \%)$ as a yellow solid; m.p. $211-213^{\circ} \mathrm{C}\left(\mathrm{CH}_{2} \mathrm{Cl}_{2} /\right.$ hexane); $v_{\max } / \mathrm{cm}^{-1}: 2924$ (m), 1752 (s), 1670 (s), 1408 (s), 1322 (s), 1123 (s); ${ }^{1} \mathrm{H}$ NMR (400 MHz, $\left.\mathrm{CDCl}_{3}\right) \delta 7.88(1 \mathrm{H}, \mathrm{dd}, J=8.7,1.0 \mathrm{~Hz}, 1 \times$ $\operatorname{ArC} \underline{H}), 7.62(1 \mathrm{H}, \mathrm{ddd}, J=7.9,1.1,1.1 \mathrm{~Hz}, 1 \times \mathrm{ArC} \underline{\mathrm{H}}), 7.52-7.50(3 \mathrm{H}, \mathrm{m}, 1 \times \mathrm{C} 1-\underline{\mathrm{H}}, 2 \times$ $\operatorname{ArC} \underline{\mathrm{H}}), 7.36(1 \mathrm{H}, \mathrm{ddd}, J=8.7,7.0,1.1 \mathrm{~Hz}, 1 \times \mathrm{ArC} \underline{\mathrm{H}}), 7.24-7.15(3 \mathrm{H}, \mathrm{m}, 3 \times \operatorname{ArC} \underline{\mathrm{H}}), 6.26$ $(1 \mathrm{H}, \mathrm{d}, J=2.6 \mathrm{~Hz}, 1 \times \mathrm{C} 11-\underline{\mathrm{H}}), 4.52(1 \mathrm{H}, \mathrm{dd}, J=12.8,4.8 \mathrm{~Hz}, 1 \times \mathrm{C6}-\underline{\mathrm{H}}), 4.38(1 \mathrm{H}, \mathrm{ddd}, J=$ 12.3, 9.0, $\left.4.8 \mathrm{~Hz}, 1 \times \mathrm{C} 8-\underline{\mathrm{H}}_{2}\right), 3.74\left(1 \mathrm{H}, \mathrm{ddd}, J=12.3,9.0,7.3 \mathrm{~Hz}, 1 \times \mathrm{C} 8-\underline{\mathrm{H}}_{2}\right), 3.07-2.87$ $\left(3 \mathrm{H}, \mathrm{m}, 1 \times \mathrm{C} 4-\underline{\mathrm{H}}_{2}, 2 \times \mathrm{C} 9-\underline{\mathrm{H}}_{2}\right), 2.70\left(1 \mathrm{H}, \mathrm{ddd}, J=12.3,3.6,3.6 \mathrm{~Hz}, 1 \times \mathrm{C} 4-\underline{\mathrm{H}}_{2}\right), 2.17-2.07$ $\left(1 \mathrm{H}, \mathrm{m}, 1 \times \mathrm{C} 5-\underline{\mathrm{H}}_{2}\right), 2.04-1.96\left(1 \mathrm{H}, \mathrm{m}, 1 \times \mathrm{C} 5-\underline{\mathrm{H}}_{2}\right) ;{ }^{13} \mathrm{C} \mathrm{NMR}\left(101 \mathrm{MHz}, \mathrm{CDCl}_{3}\right) \delta 190.8$ (C3), 150.6 (C7), 142.8 (C10), 139.7 (q, $J=2.0, \mathrm{~Hz}$ ) (CAr), 139.5 (CAr), 137.6 (C2), 129.1 $(\mathrm{q}, J=32.7 \mathrm{~Hz})(\mathrm{CAr}), 128.4(\mathrm{CAr}), 127.6$ (CAr), 126.5 (CAr), 125.4 (q, $J=3.7 \mathrm{~Hz})(\mathrm{CAr})$, 124.0 (q, $J=273.0 \mathrm{~Hz})(\mathrm{C12}), 123.0$ (CAr), 122.8 (C11), 115.4 (CAr), 114.6 (C1), 61.4 (C6), 45.4 (C8), 37.3 (C4), 33.4 (C9), 27.0 (C5); ${ }^{19} \mathrm{~F} \mathrm{NMR} \mathrm{(377} \mathrm{MHz,} \mathrm{CDCl}_{3}$ ): $\delta$-62.6; HRMS: $\left(\mathrm{ESI}^{+}\right)$Calculated for $\mathrm{C}_{24} \mathrm{H}_{20} \mathrm{~F}_{3} \mathrm{~N}_{2} \mathrm{O}_{2}:$ 425.1471. Found $[\mathrm{M}+\mathrm{H}]^{+}:$425.1476. The structure of this compound was determined unambiguously by X-ray crystallography.

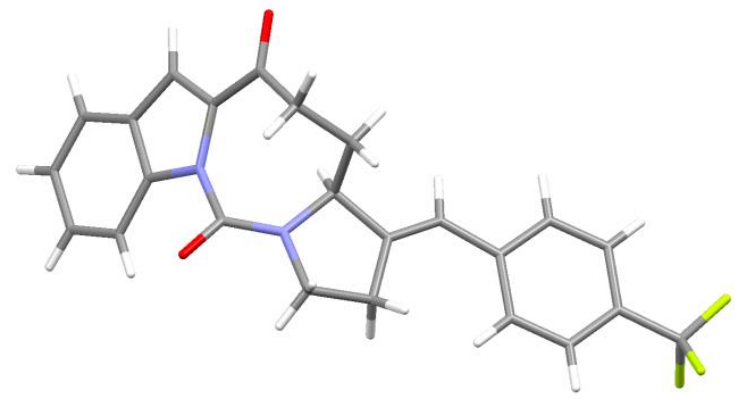




\section{$N$-Cyclopropyl- $N$-(4-(4-methoxyphenyl)but-3-yn-1-yl)-1H-indole-1-carboxamide 5c}

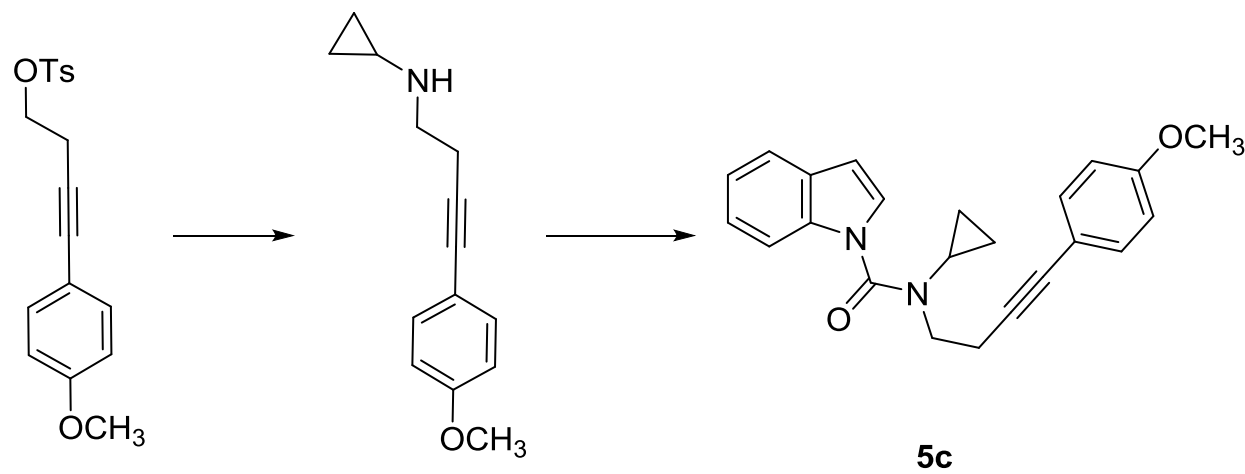

To a flask containing 4-(4-methoxyphenyl)but-3-yn-1-yl 4-methylbenzenesulfonate (prepared according to the literature procedure $)^{8}(500 \mathrm{mg}, 1.50 \mathrm{mmol})$ in $\mathrm{MeCN}(8.00 \mathrm{~mL})$ was added cyclopropylamine $(0.42 \mathrm{~mL}, 6.0 \mathrm{mmol})$. The flask was sealed and heated at $90{ }^{0} \mathrm{C}$ for $16 \mathrm{~h}$. The reaction mixture was cooled to room temperature, diluted with $\mathrm{CH}_{2} \mathrm{Cl}_{2}(20 \mathrm{~mL})$ and washed with saturated aqueous $\mathrm{NaHCO}_{3}(20 \mathrm{~mL})$. The aqueous portion was extracted with $\mathrm{CH}_{2} \mathrm{Cl}_{2}(2 \times 15 \mathrm{~mL})$, the organic extracts were combined, washed with brine $(35 \mathrm{~mL})$, dried over $\mathrm{Na}_{2} \mathrm{SO}_{4}$ and concentrated in vacuo. The residue was dissolved in acetone $(5.00 \mathrm{~mL})$ and water $(0.90 \mathrm{~mL})$ and cooled to $0{ }^{\circ} \mathrm{C} . \mathrm{K}_{2} \mathrm{CO}_{3}(414 \mathrm{mg}, 3.00 \mathrm{mmol})$ and $1 \mathrm{H}$-indole-1-carbonyl chloride (671 mg, $3.75 \mathrm{mmol}$ ) were added. The reaction mixture was warmed to room temperature and stirred for 16 hours. The solution was concentrated in vacuo to remove acetone and then water $(10 \mathrm{~mL})$ was added. The solution was extracted with $\mathrm{CH}_{2} \mathrm{Cl}_{2}(3 \times 10$ $\mathrm{mL})$ and the organic extracts were combined, washed with brine $(20 \mathrm{~mL})$, dried over $\mathrm{Na}_{2} \mathrm{SO}_{4}$ and concentrated in vacuo. The crude mixture was purified by flash column chromatography (6\% EtOAc/hexane) to afford the title compound $\mathbf{5 c}(268 \mathrm{mg}, 50 \%)$ as a yellow oil; $v_{\max } / \mathrm{cm}^{-1}$ : 2922 (m), 1678 (s), 1509 (s), 1408 (s), 1246 (s), 1032 (s); ${ }^{1} \mathrm{H}$ NMR (500 MHz, CDCl $): \delta$ $7.77(1 \mathrm{H}, \mathrm{ddd}, J=8.2,0.9,0.9 \mathrm{~Hz}), 7.59-7.57(1 \mathrm{H}, \mathrm{m}), 7.45(1 \mathrm{H}, \mathrm{d}, J=3.6 \mathrm{~Hz}), 7.34-7.32$ $(2 \mathrm{H}, \mathrm{m}), 7.22-7.16(2 \mathrm{H}, \mathrm{m}), 6.83-6.80(2 \mathrm{H}, \mathrm{m}), 6.55(1 \mathrm{H}, \mathrm{dd}, J=3.6,0.9 \mathrm{~Hz}), 3.81-3.76$ $(5 \mathrm{H}, \mathrm{m}), 3.04(1 \mathrm{H}, \mathrm{tt}, J=6.8,3.9 \mathrm{~Hz}), 2.85(2 \mathrm{H}, \mathrm{t}, J=6.4 \mathrm{~Hz}), 0.74-0.70(2 \mathrm{H}, \mathrm{m}), 0.58-$ $0.55(2 \mathrm{H}, \mathrm{m}) ;{ }^{13} \mathrm{C}$ NMR $\left(126 \mathrm{MHz}, \mathrm{CDCl}_{3}\right): \delta 159.3,155.2,135.4,133.0,129.5,126.2,123.5$, $121.8,120.8,115.4,113.9,113.8,105.6,85.1,82.3,55.3,48.4,31.4,19.0,9.0 ; \mathrm{m} / \mathrm{z}\left(\mathrm{ESI}^{+}\right)$ HRMS: $\mathrm{C}_{23} \mathrm{H}_{22} \mathrm{~N}_{2} \mathrm{NaO}_{2}$ : 381.1589. Found [M+Na] $]^{+}: 381.1574$. 

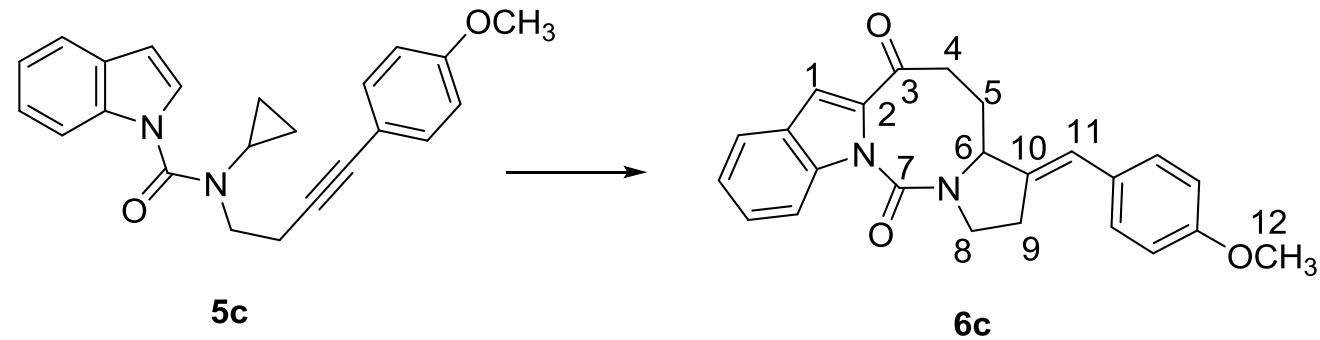

General procedure E: Compound 5c $(35.8 \mathrm{mg}, 0.10 \mathrm{mmol})$ was employed and the reaction was stirred for 96 hours at $110{ }^{\circ} \mathrm{C}$. The crude mixture was purified by column chromatography (5\% EtOAc/toluene) to yield the title compound $\mathbf{6 c}(15.1 \mathrm{mg}, 39 \%)$ as a yellow oil; $v_{\max } / \mathrm{cm}^{-1}: 2924$ (m), 1734 (s), 1667 (s), 1512 (s), 1407 (s), 1249 (s); ${ }^{1} \mathrm{H}$ NMR $\left(400 \mathrm{MHz}, \mathrm{CDCl}_{3}\right) \delta 7.95(1 \mathrm{H}, \mathrm{ddd}, J=8.7,0.9,0.9 \mathrm{~Hz}, 1 \times \mathrm{ArC} \underline{\mathrm{H}}), 7.69(1 \mathrm{H}, \mathrm{ddd}, J=8.0$, $1.2,0.9 \mathrm{~Hz}, 1 \times \operatorname{ArC} \underline{\mathrm{H}}), 7.58(1 \mathrm{H}, \mathrm{d}, J=0.9 \mathrm{~Hz}, 1 \times \mathrm{C} 1-\underline{\mathrm{H}}, 1 \times \mathrm{ArC} \underline{\mathrm{H}}), 7.43(1 \mathrm{H}, \mathrm{ddd}, J=$ 8.5, 7.0, $1.2 \mathrm{~Hz}, 1 \times \mathrm{ArC} \underline{\mathrm{H}}), 7.25-7.21(1 \mathrm{H}, \mathrm{m}, 1 \times \mathrm{ArC} \underline{\mathrm{H}}), 7.20-7.10(2 \mathrm{H}, \mathrm{m}, 2 \times \operatorname{ArC} \underline{\mathrm{H}})$, $6.94-6.83(2 \mathrm{H}, \mathrm{m}, 3 \times \mathrm{ArC} \underline{\mathrm{H}}), 6.23(1 \mathrm{H}, \mathrm{d}, J=2.7 \mathrm{~Hz}, 1 \times \mathrm{C11}-\underline{\mathrm{H}}), 4.54(1 \mathrm{H}, \mathrm{dd}, J=12.8$, $4.9 \mathrm{~Hz}, 1 \times \mathrm{C6}-\underline{\mathrm{H}}), 4.42\left(1 \mathrm{H}, \mathrm{ddd}, J=12.3,8.5,5.6 \mathrm{~Hz}, 1 \times \mathrm{C} 8-\underline{\mathrm{H}}_{2}\right), 3.86-3.74(4 \mathrm{H}, \mathrm{m}, 1 \times$ C8- $\left.\underline{\mathrm{H}}_{2}, 3 \times \mathrm{C} 12-\underline{\mathrm{H}}_{3}\right), 3.13-3.05\left(1 \mathrm{H}, \mathrm{m}, 1 \times \mathrm{C} 4-\underline{\mathrm{H}}_{2}\right), 3.02-2.96\left(2 \mathrm{H}, \mathrm{m}, 2 \times \mathrm{C} 9-\underline{\mathrm{H}}_{2}\right), 2.75$ $\left(1 \mathrm{H}, \mathrm{ddd}, J=12.3,3.7,3.7 \mathrm{~Hz}, 1 \times \mathrm{C} 4-\underline{\mathrm{H}}_{2}\right), 2.22-2.12\left(1 \mathrm{H}, \mathrm{m}, 1 \times \mathrm{C} 5-\underline{\mathrm{H}}_{2}\right), 2.11-2.01(1 \mathrm{H}$, m, $\left.1 \times \mathrm{C} 5-\underline{\mathrm{H}}_{2}\right) ;{ }^{13} \mathrm{C} \mathrm{NMR}\left(101 \mathrm{MHz}, \mathrm{CDCl}_{3}\right) \delta 191.0$ (C3), 158.8 (CAr), 150.6 (C7), 139.5 (CAr), 137.6 (C10), 129.5 (CAr), 129.0 (CAr), 127.5 (CAr), 126.5 (CAr), 122.9 (CAr), 122.8 (CAr), 122.7 (C11), 115.4 (C1), 114.3 (CAr), 113.9 (CAr), 61.5 (C6), 55.3 (C12), 45.6 (C8), 37.5 (C4), 33.6 (C9), 26.7 (C5). C2 can't be assigned confidently; HRMS: (ESI $\left.{ }^{+}\right)$ Calculated for $\mathrm{C}_{24} \mathrm{H}_{23} \mathrm{~N}_{2} \mathrm{O}_{3}: 387.1703$. Found $[\mathrm{M}+\mathrm{H}]^{+}: 387.1706$.

\section{$N$-Cyclopropyl- $N$-(4-phenylbut-3-yn-1-yl)-1H-pyrrole-1-carboxamide 5d}

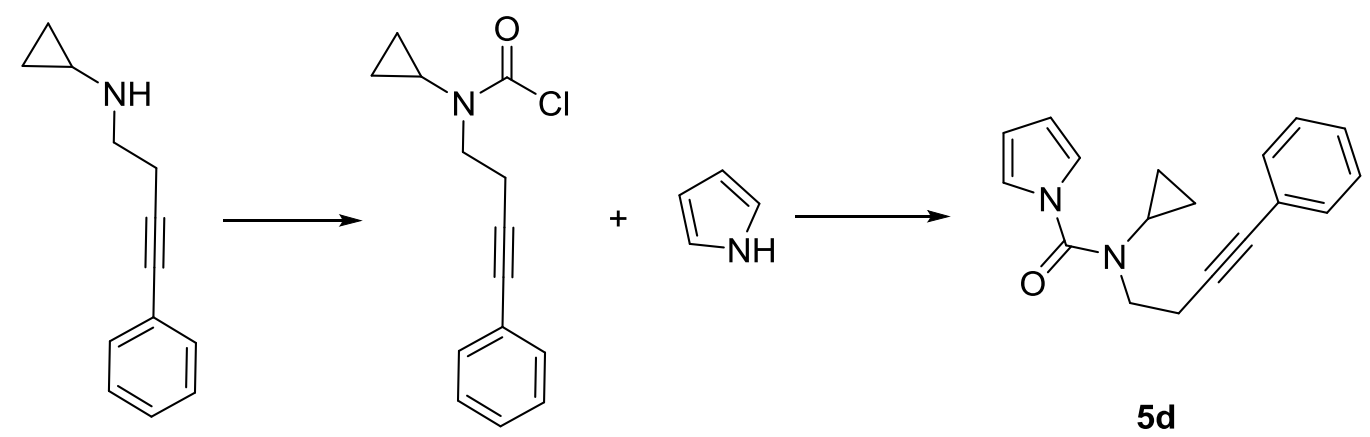


To a solution of triphosgene $(220 \mathrm{mg}, 0.72 \mathrm{mmol})$ and pyridine $(0.19 \mathrm{~mL}, 2.40 \mathrm{mmol})$ in toluene $(15 \mathrm{~mL})$ was added a solution of $N$-(4-phenylbut-3-yn-1-yl)cyclopropanamine (370 $\mathrm{mg}, 2.00 \mathrm{mmol})$ in toluene $(3.00 \mathrm{~mL})$ over 5 minutes at room temperature. The reaction mixture was stirred at room temperature for 16 hours. The reaction was quenched by the addition of saturated aqueous $\mathrm{NaHCO}_{3}(20 \mathrm{~mL})$, extracted with $\mathrm{Et}_{2} \mathrm{O}(3 \times 15 \mathrm{~mL})$. The organic extracts were combined, washed with $0.2 \mathrm{M}$ aqueous $\mathrm{HCl}(20 \mathrm{~mL})$, water $(20 \mathrm{~mL})$ and brine $(20 \mathrm{~mL})$, dried over $\mathrm{Na}_{2} \mathrm{SO}_{4}$ and concentrated in vacuo to yield cyclopropyl(4phenylbut-3-yn-1-yl)carbamic chloride which was used for next step without purification. A flame-dried round-bottomed flask was charged with $\mathrm{NaH}(108 \mathrm{mg}, 2.70 \mathrm{mmol})$ and this was suspended in DMF (7.00 mL) under nitrogen. The suspension was cooled to $0{ }^{\circ} \mathrm{C}$ and $1 \mathrm{H}$ pyrrole (126 uL, $1.80 \mathrm{mmol})$ was added portionwise over 2 minutes. The solution was heated at $70{ }^{\circ} \mathrm{C}$ for 3 hours, then cooled to room temperature followed by dropwise addition of above cyclopropyl(4-phenylbut-3-yn-1-yl)carbamic chloride in DMF (1.00 mL) over 5 minutes. The resulting solution was warmed to $70{ }^{\circ} \mathrm{C}$ and stirred for 18 hours. The reaction mixture was cooled to room temperature and quenched by the saturated aqueous $\mathrm{NH}_{4} \mathrm{Cl}(10$ $\mathrm{mL})$. The mixture was extracted with EtOAc $(3 \times 10 \mathrm{~mL})$, the organic extracts were combined, washed with brine $(15 \mathrm{~mL})$, dried over $\mathrm{Na}_{2} \mathrm{SO}_{4}$, filtered and concentrated in vacuo. The crude mixture was purified by flash column chromatography (6\% EtOAc/hexane) to afford the title compound $\mathbf{5 d}(314 \mathrm{mg}, 62 \%)$ as a yellow oil; $v_{\max } / \mathrm{cm}^{-1}: 2937(\mathrm{~m}), 1675(\mathrm{~s})$, 1490 (m), 1415 (s), 1286 (m), 1032 (s); ${ }^{1} \mathrm{H}$ NMR (500 MHz, $\mathrm{CDCl}_{3}$ ): $\delta 7.40-7.37$ (2H, m), $7.32-7.29(3 \mathrm{H}, \mathrm{m}), 7.22-7.21(2 \mathrm{H}, \mathrm{m}), 6.24-6.23(2 \mathrm{H}, \mathrm{m}), 3.79(2 \mathrm{H}, \mathrm{t}, J=6.7 \mathrm{~Hz}), 3.04$ $(1 \mathrm{H}, \mathrm{tt}, J=6.8,3.9 \mathrm{~Hz}), 2.85(2 \mathrm{H}, \mathrm{t}, J=6.7 \mathrm{~Hz}), 0.85-0.81(2 \mathrm{H}, \mathrm{m}), 0.65-0.61(2 \mathrm{H}, \mathrm{m})$; ${ }^{13} \mathrm{C}$ NMR (126 MHz, $\left.\mathrm{CDCl}_{3}\right): \delta 154.6,131.5,128.3,127.9,123.3,120.6,110.4,86.6,82.3$, 48.5, 32.0, 18.9, 9.6; m/z (ESI $\left.{ }^{+}\right)$HRMS: $\mathrm{C}_{18} \mathrm{H}_{19} \mathrm{~N}_{2} \mathrm{O}: 279.1492$. Found $[\mathrm{M}+\mathrm{H}]^{+}: 279.1491$

\section{(E)-7-Benzylidene-5,6,6a,7,8,9-hexahydro-4H,11H-dipyrrolo[1,2-c:2',1'-}

\section{$h][1,3]$ diazocine-4,11-dione $6 \mathrm{~d}$}

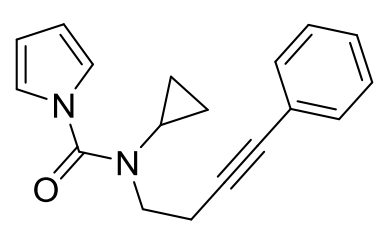

$5 d$

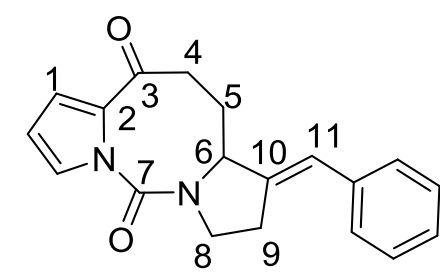

6d 
General procedure E: Compound $5 \mathbf{d}(27.8 \mathrm{mg}, 0.10 \mathrm{mmol})$ was employed and the reaction was stirred for 72 hours at $130{ }^{\circ} \mathrm{C}$. The crude mixture was purified by column chromatography $(10 \% \mathrm{EtOAc} / \mathrm{Hex})$ to yield the title compound $\mathbf{6 d}(25.9 \mathrm{mg}, 82 \%)$ as a colourless oil; $v_{\max } / \mathrm{cm}^{-1}: 2923$ (m), 1681 (s), 1654 (s), 1434 (s), 1398 (s), 1068 (m); ${ }^{1} \mathrm{H}$ NMR $\left(400 \mathrm{MHz}, \mathrm{CDCl}_{3}\right) \delta 7.44(1 \mathrm{H}, \mathrm{dd}, J=3.0,2.0 \mathrm{~Hz}, 1 \times \mathrm{ArC} \underline{\mathrm{H}}), 7.29-7.27(2 \mathrm{H}, \mathrm{m}, 1 \times \mathrm{C} \mathbf{1}-\underline{\mathrm{H}}$, $1 \times \operatorname{ArC} \underline{\mathrm{H}}), 7.19-7.15(4 \mathrm{H}, \mathrm{m}, 4 \times \operatorname{ArC} \underline{\mathrm{H}}), 6.27-6.23(2 \mathrm{H}, \mathrm{m}, 1 \times \mathrm{C11}-\underline{\mathrm{H}}, 1 \times \operatorname{ArC} \underline{\mathrm{H}}), 4.52$ $(1 \mathrm{H}, \mathrm{dd}, J=13.0,4.6 \mathrm{~Hz}, 1 \times \mathrm{C} 6-\underline{\mathrm{H}}), 4.23\left(1 \mathrm{H}, \mathrm{ddd}, J=12.3,8.5,6.1 \mathrm{~Hz}, 1 \times \mathrm{C} 8-\underline{\mathrm{H}}_{2}\right), 3.71-$ $3.64\left(1 \mathrm{H}, \mathrm{m}, 1 \times \mathrm{C} 8-\underline{\mathrm{H}}_{2}\right), 2.94-2.85\left(3 \mathrm{H}, \mathrm{m}, 1 \times \mathrm{C} 4-\underline{\mathrm{H}}_{2}, 2 \times \mathrm{C} 9-\underline{\mathrm{H}}_{2}\right), 2.56(1 \mathrm{H}, \mathrm{ddd}, J=12.5$, 4.2, $\left.3.1 \mathrm{~Hz}, 1 \times \mathrm{C} 4-\underline{\mathrm{H}}_{2}\right), 2.14-2.05\left(1 \mathrm{H}, \mathrm{m}, 1 \times \mathrm{C} 5-\underline{\mathrm{H}}_{2}\right), 1.98-1.89\left(1 \mathrm{H}, \mathrm{m}, 1 \times \mathrm{C} 5-\underline{\mathrm{H}}_{2}\right) ;{ }^{13} \mathrm{C}$ NMR (101 MHz, $\left.\mathrm{CDCl}_{3}\right) \delta 188.8$ (C3), 150.6 (C7), 139.8 (C10), 136.2 (CAr), 134.4 (C2) 130.1 (CAr), 128.5 (CAr), 128.3 (CAr), 127.3 (CAr), 123.3 (C11), 121.0 (C1), 110.7 (CAr), 61.8 (C6), 46.1 (C8), 37.1 (C4), 33.9 (C5), 26.7 (C9); HRMS: (ESI ${ }^{+}$) Calculated for $\mathrm{C}_{19} \mathrm{H}_{19} \mathrm{~N}_{2} \mathrm{O}_{2}: 307.1441$. Found $[\mathrm{M}+\mathrm{H}]^{+}: 307.1441$.

\section{$N$-Cyclopropyl- $N$-(pent-3-yn-1-yl)-1H-pyrrole-1-carboxamide 5 e}

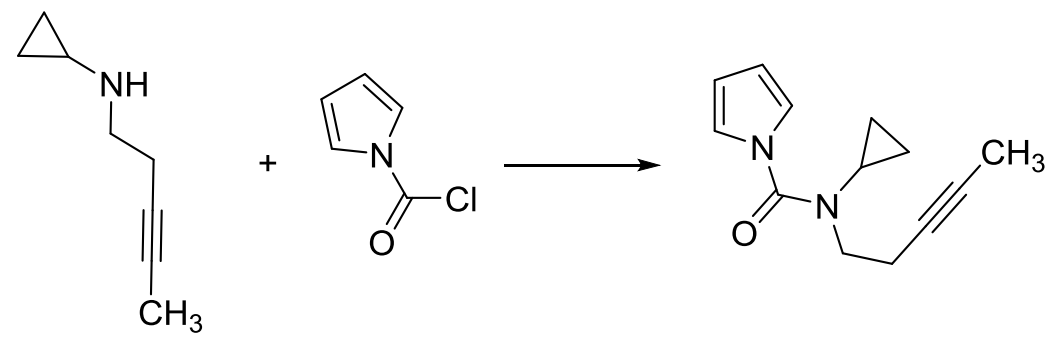

$5 e$

General procedure B: 1H-pyrrole-1-carbonyl chloride (516 mg, $4.00 \mathrm{mmol}$ ) (prepared according to the literature procedure $)^{9}$ and $N$-(4-phenylbut-3-yn-1-yl)cyclopropanamine (246 $\mathrm{mg}, 2.00 \mathrm{mmol}$ ) (prepared according to the literature procedure) ${ }^{10}$ were employed. The crude mixture was purified by flash column chromatography (10\% EtOAc/hexane) to afford the title compound 5e $(62.0 \mathrm{mg}, 15 \%)$ as a yellow oil; $v_{\max } / \mathrm{cm}^{-1}: 2921(\mathrm{~m}), 1670(\mathrm{~s}), 1634(\mathrm{~m})$, 1054 (s), 1032 (s); ${ }^{1} \mathrm{H}$ NMR (500 MHz, $\left.\mathrm{CDCl}_{3}\right): \delta 7.20-7.19(2 \mathrm{H}, \mathrm{m}), 6.25-6.23(2 \mathrm{H}, \mathrm{m})$, $3.65(2 \mathrm{H}, \mathrm{t}, J=6.8 \mathrm{~Hz}), 2.95(1 \mathrm{H}, \mathrm{tt}, J=6.8,3.9 \mathrm{~Hz}), 2.55(2 \mathrm{H}, \mathrm{tq}, J=6.8,2.5 \mathrm{~Hz}), 1.79(3 \mathrm{H}$, $\mathrm{t}, J=2.5 \mathrm{~Hz}), 0.83-0.79(2 \mathrm{H}, \mathrm{m}), 0.60-0.57(2 \mathrm{H}, \mathrm{m}) ;{ }^{13} \mathrm{C} \mathrm{NMR}\left(126 \mathrm{MHz}, \mathrm{CDCl}_{3}\right): \delta$ 154.7, 120.6, 110.3, 77.7, 75.8, 48.6, 31.6, 18.1, 9.4, 3.4; m/z $\left(\mathrm{ESI}^{+}\right) \mathrm{HRMS}: \mathrm{C}_{13} \mathrm{H}_{17} \mathrm{~N}_{2} \mathrm{O}$ : 217.1335. Found $[\mathrm{M}+\mathrm{H}]^{+}: 217.1333$. 


\section{4,11-dione 6e}

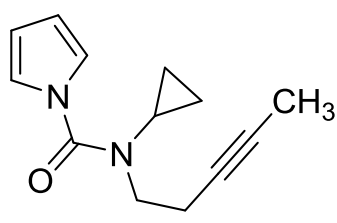

5e

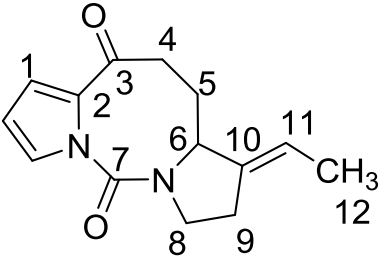

$6 e$

General procedure E: Compound 5 e $(21.4 \mathrm{mg}, 0.10 \mathrm{mmol})$ was employed and the reaction was stirred for 48 hours at $130{ }^{\circ} \mathrm{C}$. The crude mixture was purified by column chromatography (10\% EtOAc/hexane) to yield the title compound $6 \mathbf{e}(9.6 \mathrm{mg}, 40 \%)$ as a colourless oil; $v_{\max } / \mathrm{cm}^{-1}: 2922$ (m), 1680 (s), 1655 (s), 1434 (s), 1064 (s), 747 (s); ${ }^{1} \mathrm{H}$ NMR $\left(500 \mathrm{MHz}, \mathrm{CDCl}_{3}\right) \delta 7.49(1 \mathrm{H}, \mathrm{dd}, J=2.5,2.5 \mathrm{~Hz}, 1 \times \mathrm{ArC} \underline{\mathrm{H}}), 7.23-7.22(1 \mathrm{H}, \mathrm{m}, 1 \times \mathrm{C1}-$ $\underline{\mathrm{H}}), 6.29(1 \mathrm{H}, \mathrm{dd}, J=3.6,2.5 \mathrm{~Hz}, 1 \times \mathrm{ArC} \underline{\mathrm{H}}), 5.40-5.35(1 \mathrm{H}, \mathrm{m}, 1 \times \mathrm{C11}-\underline{\mathrm{H}}), 4.38(1 \mathrm{H}, \mathrm{dd}, J$ $=13.5,4.8 \mathrm{~Hz}, 1 \times \mathrm{C6}-\underline{\mathrm{H}}), 4.26\left(1 \mathrm{H}, \mathrm{ddd}, J=12.1,9.0,5.5 \mathrm{~Hz}, 1 \times \mathrm{C} 8-\underline{\mathrm{H}}_{2}\right), 3.66(1 \mathrm{H}, \mathrm{ddd}, J$ = 12.1, 9.0, 7.2 Hz, $\left.1 \times \mathrm{C} 8-\underline{\mathrm{H}}_{2}\right), 2.95-2.68\left(1 \mathrm{H}, \mathrm{m}, 1 \times \mathrm{C} 4-\underline{\mathrm{H}}_{2}\right), 2.67-2.57(3 \mathrm{H}, \mathrm{m}, 1 \times \mathrm{C} 4-$ $\left.\underline{\mathrm{H}}_{2}, 2 \times \mathrm{C} 9-\underline{\mathrm{H}}_{2}\right), 2.06-1.99\left(1 \mathrm{H}, \mathrm{m}, 1 \times \mathrm{C} 5-\underline{\mathrm{H}}_{2}\right), 1.91-1.84\left(1 \mathrm{H}, \mathrm{m}, 1 \times \mathrm{C} 5-\underline{\mathrm{H}}_{2}\right), 1.62(3 \mathrm{H}, \mathrm{d}$, $\left.J=7.2 \mathrm{~Hz}, 3 \times \mathrm{C12}-\underline{\mathrm{H}}_{3}\right) ;{ }^{13} \mathrm{C} \mathrm{NMR}\left(126 \mathrm{MHz}, \mathrm{CDCl}_{3}\right) \delta 188.9(\mathrm{C} 3), 150.8(\mathrm{C} 7), 139.2$ (C10), 134.6 (C2), 130 (CAr), 120.8 (C1), 117.9 (C11), 110.6 (CAr), 60.5 (C6), 45.6 (C8), 37.3 (C4), 34.0 (C5), 24.7 (C9), 14.2 (C12); HRMS: (ESI ${ }^{+}$) Calculated for $\mathrm{C}_{14} \mathrm{H}_{17} \mathrm{~N}_{2} \mathrm{O}_{2}$ : 245.1285. Found $[\mathrm{M}+\mathrm{H}]^{+}: 245.1285$.

\section{$N$-Cyclopropyl- $N$-(4-phenylbut-3-yn-1-yl)-1H-indole-2-carboxamide $5 f$}

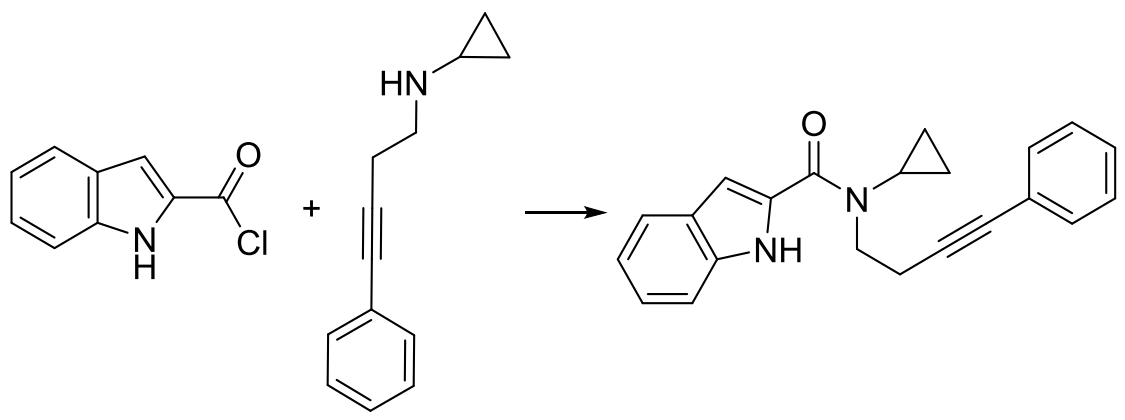

$5 \mathbf{f}$

General procedure B: $1 H$-Indole-2-carbonyl chloride (prepared according to the literature report) ${ }^{11}(537 \mathrm{mg}, 3.00 \mathrm{mmol})$ and $N$-(4-phenylbut-3-yn-1-yl)cyclopropanamine $(325 \mathrm{mg}$, $1.75 \mathrm{mmol}$ ) were employed. The crude mixture was purified by flash column 
chromatography (10\% EtOAc/hexane) to afford the title compound $\mathbf{5 f}(299 \mathrm{mg}, 52 \%)$ as a yellow solid; m.p. $144-146{ }^{\circ} \mathrm{C}\left(\mathrm{CH}_{2} \mathrm{Cl}_{2}\right) ; v_{\max } / \mathrm{cm}^{-1}$ : 3285 (m), 2922 (m), 1598 (s), 1520 (s), 1054 (s), 1032 (s); ${ }^{1} \mathrm{H}$ NMR (400 MHz, $\left.\mathrm{CDCl}_{3}\right): \delta 9.36(1 \mathrm{H}, \mathrm{s}, b r), 7.66(1 \mathrm{H}, \mathrm{ddd}, J=8.0,1.0$ $\mathrm{Hz}), 7.42(1 \mathrm{H}, \mathrm{ddd}, J=8.3,1.0,1.0 \mathrm{~Hz}), 7.34-7.22(6 \mathrm{H}, \mathrm{m}), 7.18(1 \mathrm{H}, \mathrm{d}, J=1.9 \mathrm{~Hz}), 7.12$ $(1 \mathrm{H}, \mathrm{ddd}, J=8.0,7.0,1.0 \mathrm{~Hz}), 3.89(2 \mathrm{H}, \mathrm{t}, J=7.0 \mathrm{~Hz}), 3.22(1 \mathrm{H}, \mathrm{tt}, J=7.0,3.8 \mathrm{~Hz}), 2.83$ $(2 \mathrm{H}, \mathrm{t}, J=7.0 \mathrm{~Hz}), 1.09-1.04(2 \mathrm{H}, \mathrm{m}), 0.92-0.87(2 \mathrm{H}, \mathrm{m}) ;{ }^{13} \mathrm{C} \mathrm{NMR}\left(101 \mathrm{MHz}, \mathrm{CDCl}_{3}\right): \delta$ 164.5, 135.5, 131.5, 130.1, 128.2, 127.9, 127.8, 124.6, 123.5, 122.2, 120.3, 111.7, 107.1, 87.3, 82.1 , 47.6, 31.5, 19.1, 11.2; m/z (ESI $\left.{ }^{+}\right)$HRMS: $\mathrm{C}_{22} \mathrm{H}_{20} \mathrm{~N}_{2} \mathrm{NaO}: 351.1468$. Found $[\mathrm{M}+\mathrm{Na}]^{+}$: 351.1469 .

(E)-3-Benzylidene-1,2,3,3a,4,5-hexahydro-6H-pyrrolo[1',2':1,8]azocino[3,4-b]indole6,12(11H)-dione $6 f$

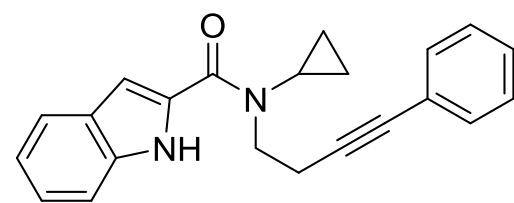

$5 \mathbf{f}$

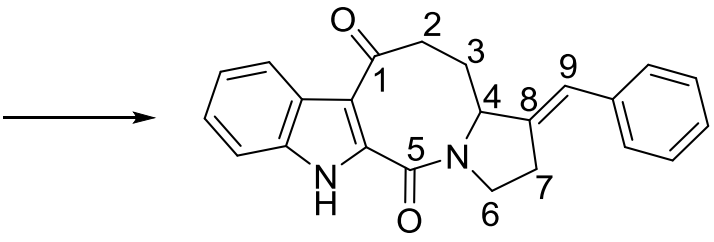

$6 f$

General procedure F: Compound $\mathbf{5 f}(32.8 \mathrm{mg}, 0.10 \mathrm{mmol})$ and anhydrous benzonitrile $(1.00$ $\mathrm{mL}$ ) were employed and the reaction was stirred for 72 hours at $140{ }^{\circ} \mathrm{C}$. The crude mixture was purified by column chromatography (35\% EtOAc/hexane) to yield the title compound $6 \mathbf{f}$ (23.8 mg, 67\%) as a yellow solid; m.p. $259-261{ }^{\circ} \mathrm{C}\left(\mathrm{CH}_{2} \mathrm{Cl}_{2} / \mathrm{hexane}\right) ; v_{\max } / \mathrm{cm}^{-1}: 3222(\mathrm{~m})$, 2922 (m), 1640 (s), 1604 (s), 1422 (s), 1054 (s); ${ }^{1} \mathrm{H}$ NMR (500 MHz, CDCl 3 ) $\delta 10.37$ (1H, s, $b r, \underline{\mathrm{H}}), 8.45-8.43(1 \mathrm{H}, \mathrm{m}, 1 \times \mathrm{ArC} \underline{\mathrm{H}}), 7.43-7.41(1 \mathrm{H}, \mathrm{m}, 1 \times \mathrm{ArC} \underline{\mathrm{H}}), 7.32-7.24(4 \mathrm{H}, \mathrm{m}$, $4 \times \operatorname{ArC} \underline{\mathrm{H}}), 7.18-7.14(3 \mathrm{H}, \mathrm{m}, 3 \times \mathrm{ArC} \underline{\mathrm{H}}), 6.28(1 \mathrm{H}, \mathrm{d}, J=2.9 \mathrm{~Hz}, 1 \times \mathrm{C} 9-\underline{\mathrm{H}}), 4.79(1 \mathrm{H}, \mathrm{dd}$, $J=13.3,4.3 \mathrm{~Hz}, 1 \times \mathrm{C} 4-\underline{\mathrm{H}}), 4.30\left(1 \mathrm{H}, \mathrm{ddd}, J=12.8,9.2,6.2 \mathrm{~Hz}, 1 \times \mathrm{C} 6-\underline{\mathrm{H}}_{2}\right), 3.76(1 \mathrm{H}, \mathrm{ddd}$, $\left.J=12.8,9.2,5.8 \mathrm{~Hz}, 1 \times \mathrm{C} 6-\underline{\mathrm{H}}_{2}\right), 3.14\left(1 \mathrm{H}, \mathrm{ddd}, J=13.8,12.0,5.3 \mathrm{~Hz}, 1 \times \mathrm{C} 2-\underline{\mathrm{H}}_{2}\right), 3.03-$ $2.86\left(2 \mathrm{H}, \mathrm{m}, 2 \times \mathrm{C} 7-\underline{\mathrm{H}}_{2}\right), 2.68-2.63\left(1 \mathrm{H}, \mathrm{m}, 1 \times \mathrm{C} 2-\underline{\mathrm{H}}_{2}\right), 2.33-2.24\left(1 \mathrm{H}, \mathrm{m}, 1 \times \mathrm{C} 3-\underline{\mathrm{H}}_{2}\right)$, $2.09-2.00\left(1 \mathrm{H}, \mathrm{m}, 1 \times \mathrm{C} 3-\underline{\mathrm{H}}_{2}\right) ;{ }^{13} \mathrm{C} \mathrm{NMR}\left(126 \mathrm{MHz}, \mathrm{CDCl}_{3}\right) \delta 196.5$ (C1), 162.0 (C5), 140.1 (C8), 136.4 (CAr), 135.1 (CAr), 134.7 (CAr), 128.5 (CAr), 128.3 (CAr), 127.2 (CAr), 126.1 (CAr), 125.4 (C9), 123.6 (CAr), 123.5 (CAr), 123.3 (CAr), 117.6 (CAr), 111.8 (CAr), 63.6 (C4), 45.3 (C6), 39.7 (C2), 36.6 (C3), 26.8 (C7). HRMS: (ESI ${ }^{+}$) Calculated for $\mathrm{C}_{23} \mathrm{H}_{20} \mathrm{~N}_{2} \mathrm{NaO}_{2}: 379.1417$. Found [M+Na] $]^{+}: 379.1422$.

The structure of this compound was determined unambiguously by X-ray crystallography. 


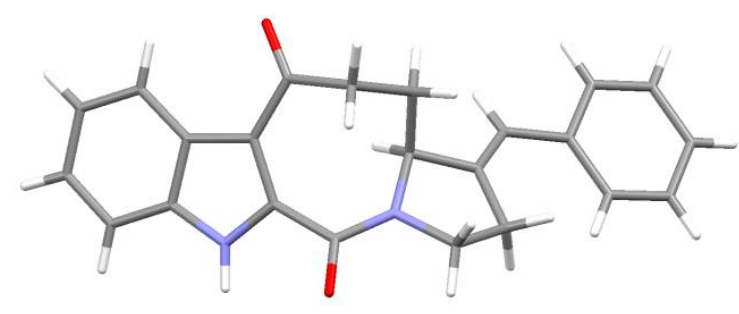

$N$-Cyclopropyl- $N$-(pent-3-yn-1-yl)-1H-indole-2-carboxamide 5g

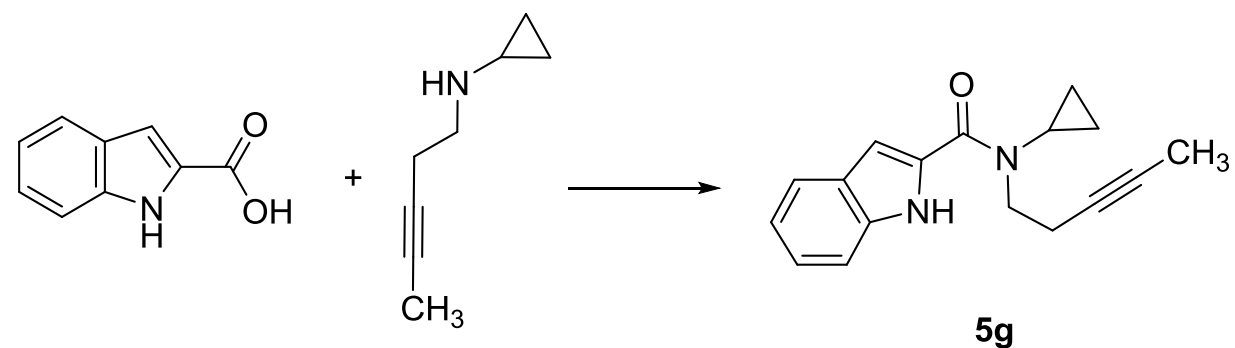

General procedure C: $1 H$-Indole-2-carboxylic (354 mg, $2.20 \mathrm{mmol})$ and $N$-(pent-3-yn-1-yl) cyclopropanamine (246 mg, $2.00 \mathrm{mmol}$ ) were employed. The crude mixture was purified by flash column chromatography (10\% EtOAc/hexane) to afford the title compound $\mathbf{5 g}$ (348 $\mathrm{mg}$, $65 \%)$ as a white solid; m.p. $130-132{ }^{\circ} \mathrm{C}\left(\mathrm{CH}_{2} \mathrm{Cl}_{2}\right) ; v_{\max } / \mathrm{cm}^{-1}: 3281(\mathrm{~m}), 2921(\mathrm{~m}), 1596(\mathrm{~s})$, 1520 (s), 1417 (s), 1346 (m); ${ }^{1} \mathrm{H}$ NMR (500 MHz, $\left.\mathrm{CDCl}_{3}\right): \delta 9.74(1 \mathrm{H}, \mathrm{s}, b r), 7.70(1 \mathrm{H}$, ddd, $J=8.2,0.9,0.9 \mathrm{~Hz}), 7.48(1 \mathrm{H}, \mathrm{ddd}, J=8.2,0.9,0.9 \mathrm{~Hz}), 7.31(1 \mathrm{H}, \mathrm{ddd}, J=8.2,7.0,0.9 \mathrm{~Hz})$, $7.19-7.14(2 \mathrm{H}, \mathrm{m}), 3.82(2 \mathrm{H}, \mathrm{t}, J=7.2 \mathrm{~Hz}), 3.19(1 \mathrm{H}, \mathrm{tt}, J=7.3,4.0 \mathrm{~Hz}), 2.59(2 \mathrm{H}, \mathrm{td}, J=$ 7.2, 2.5), $1.79(3 \mathrm{H}, \mathrm{t}, J=2.5 \mathrm{~Hz}), 1.09-1.05(2 \mathrm{H}, \mathrm{m}), 0.90-0.86(2 \mathrm{H}, \mathrm{m}) ;{ }^{13} \mathrm{C} \mathrm{NMR}(126$ $\left.\mathrm{MHz}, \mathrm{CDCl}_{3}\right): \delta 164.4,135.5,130.2,127.9,124.4,122.1,120.2,111.7,106.9,77.3,76.2$, 47.8, 31.2, 18.3, 10.9, 3.5; m/z $\left(\mathrm{ESI}^{+}\right)$HRMS: $\mathrm{C}_{17} \mathrm{H}_{18} \mathrm{~N}_{2} \mathrm{NaO}: 289.1311$. Found $[\mathrm{M}+\mathrm{Na}]^{+}$: 289.1307 .

(E)-3-Ethylidene-1,2,3,3a,4,5-hexahydro-6H-pyrrolo[1',2':1,8]azocino[3,4-b]indole6,12(11H)-dione $6 \mathrm{~g}$<smiles>CC#CCCN(C(=O)c1cc2ccccc2[nH]1)C1CC1</smiles>

$5 g$

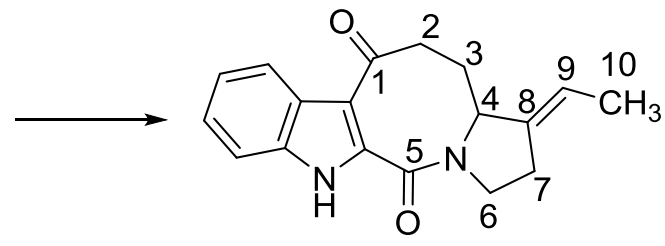

$6 \mathrm{~g}$ 
General procedure F: Compound $5 \mathrm{~g}(26.6 \mathrm{mg}, 0.10 \mathrm{mmol})$ and anhydrous benzonitrile $(1.00 \mathrm{~mL})$ were employed and the reaction was stirred for 72 hours at $140{ }^{\circ} \mathrm{C}$. The crude mixture was purified by column chromatography ( $8 \%$ acetone/toluene) to yield the title compound 6g (12.6 mg, 43\%) as a yellow solid; m.p. 208-210 ${ }^{\circ} \mathrm{C}\left(\mathrm{CDCl}_{3}\right) ; v_{\max } / \mathrm{cm}^{-1}: 3217$ (m), 2922 (m), 1644 (s), 1602 (s), 1420 (s), 1032 (s); ${ }^{1} \mathrm{H}$ NMR (400 MHz, CDCl $) \delta 10.51$ $(1 \mathrm{H}, \mathrm{s}, b r, \underline{\mathrm{H}}), 8.49(1 \mathrm{H}, \mathrm{dd}, J=7.0,2.0 \mathrm{~Hz}, 1 \times \mathrm{ArC} \underline{\mathrm{H}}), 7.49-7.47(1 \mathrm{H}, \mathrm{m}, 1 \times \operatorname{ArC} \underline{\mathrm{H}})$, $7.36-7.29(2 \mathrm{H}, \mathrm{m}, 2 \times \mathrm{ArC} \underline{\mathrm{H}}), 5.31-5.25(1 \mathrm{H}, \mathrm{m}, 1 \times \mathrm{C} 9-\underline{\mathrm{H}}), 4.62(1 \mathrm{H}, \mathrm{dd}, J=13.3,4.2$ $\mathrm{Hz}, 1 \times \mathrm{C} 4-\underline{\mathrm{H}}), 4.33\left(1 \mathrm{H}, \mathrm{ddd}, J=12.8,8.4,7.1 \mathrm{~Hz}, 1 \times \mathrm{C} 6-\underline{\mathrm{H}}_{2}\right), 3.73(1 \mathrm{H}, \mathrm{ddd}, J=12.8,8.4$, $\left.7.7 \mathrm{~Hz}, 1 \times \mathrm{C6}-\underline{\mathrm{H}}_{2}\right), 3.14\left(1 \mathrm{H}, \mathrm{ddd}, J=13.6,12.0,5.3 \mathrm{~Hz}, 1 \times \mathrm{C} 2-\underline{\mathrm{H}}_{2}\right), 2.68-2.63(3 \mathrm{H}, \mathrm{m}, 1$ $\left.\times \mathrm{C} 2-\underline{\mathrm{H}}_{2}, 2 \times \mathrm{C} 7-\underline{\mathrm{H}}_{2}\right), 2.25-2.15\left(1 \mathrm{H}, \mathrm{m}, 1 \times \mathrm{C} 3-\underline{\mathrm{H}}_{2}\right), 1.97(1 \mathrm{H}, \mathrm{ddd}, J=13.6,5.4,3.4 \mathrm{~Hz}, 1$ $\left.\times \mathrm{C} 3-\underline{\mathrm{H}}_{2}\right), 1.61\left(3 \mathrm{H}, \mathrm{d}, J=6.7 \mathrm{~Hz}, 3 \times \mathrm{C10}-\underline{\mathrm{H}}_{3}\right) ;{ }^{13} \mathrm{C} \mathrm{NMR}\left(101 \mathrm{MHz}, \mathrm{CDCl}_{3}\right) \delta 196.6(\mathrm{C1})$, 162.0 (C5), 138.9 (C8), 135.0 (CAr), 134.9 (CAr), 126.06 (CAr), 125.3 (CAr), 123.5 (CAr), 123.5 (CAr), 117.8 (C9), 117.5 (CAr), 111.8 (CAr), 62.1 (C4), 44.7 (C6), 39.7 (C2), 36.5 (C3), 24.7 (C7), 14.4 (C10); HRMS: (ESI ${ }^{+}$) Calculated for $\mathrm{C}_{18} \mathrm{H}_{18} \mathrm{~N}_{2} \mathrm{NaO}_{2}$ : 317.1260. Found $[\mathrm{M}+\mathrm{Na}]^{+}: 317.1266$.

\section{$N$-Cyclopropyl- $N$-(4-phenylbut-3-yn-1-yl)-1H-pyrrole-2-carboxamide 5 h}

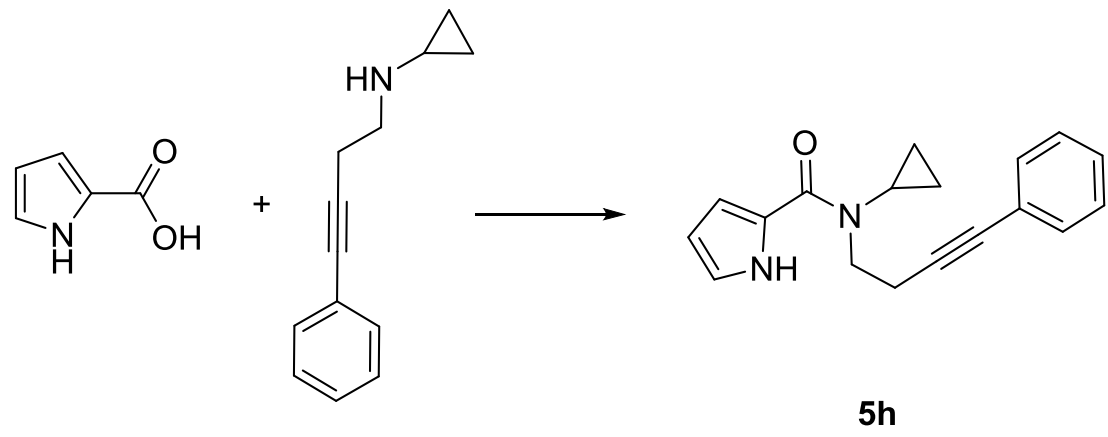

General procedure C: 1H-Pyrrole-2-carboxylic acid (244 $\mathrm{mg}, 2.20 \mathrm{mmol})$ and $\mathrm{N}$-(4phenylbut-3-yn-1-yl)cyclopropanamine (370 mg, $2.00 \mathrm{mmol}$ ) were employed. The crude mixture was purified by flash column chromatography (15\% EtOAc/hexane) to afford the title compound $\mathbf{5 h}(288 \mathrm{mg}, 52 \%)$ as a yellow solid; m.p. $98-100{ }^{\circ} \mathrm{C}\left(\mathrm{CH}_{2} \mathrm{Cl}_{2}\right) ; v_{\max } / \mathrm{cm}^{-1}$ : 3260 (m), 2967 (m), 1588 (s), 1420 (s), 1367 (s), 1053 (s); ${ }^{1} \mathrm{H}$ NMR (400 MHz, CDCl $): \delta$ $9.69(1 \mathrm{H}, \mathrm{s}, b r), 7.31-7.29(2 \mathrm{H}, \mathrm{m}),, 7.23-7.21(3 \mathrm{H}, \mathrm{m}),, 6.91-6.88(2 \mathrm{H}, \mathrm{m}),, 6.29-6.18$ $(1 \mathrm{H}, \mathrm{m}, b r), 3.81-3.77(2 \mathrm{H}, \mathrm{m}, b r), 3.09-3.04(1 \mathrm{H}, \mathrm{m}), 2.76-2.72(2 \mathrm{H}, \mathrm{m}, b r), 1.00-0.95$ $(2 \mathrm{H}, \mathrm{m}), 0.87-0.83(2 \mathrm{H}, \mathrm{m}) ;{ }^{13} \mathrm{C}$ NMR $\left(101 \mathrm{MHz}, \mathrm{CDCl}_{3}\right): \delta 163.7,131.5,128.2,127.7$, 
125.6, 123.6, 121.1, 113.9, 109.8, 87.5, 81.9, 47.3, 31.2, 19.1, 11.0; m/z (ESI $\left.{ }^{+}\right)$HRMS: $\mathrm{C}_{18} \mathrm{H}_{18} \mathrm{~N}_{2} \mathrm{NaO}: 301.1311$. Found [M+Na] ${ }^{+}: 301.1302$.

(E)-7-Benzylidene-5,6,6a,7,8,9-hexahydro-1H-dipyrrolo[1,2-a:3',2'-f]azocine-4,11-dione $6 \mathrm{~g}$

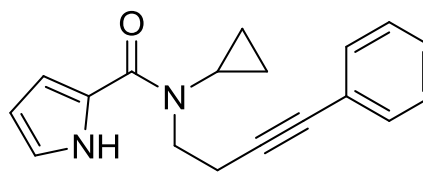

$5 \mathrm{~g}$

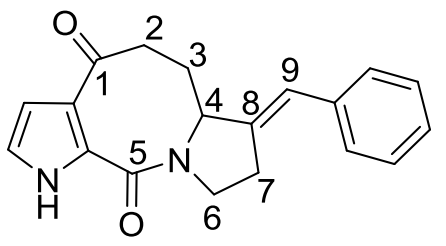

$6 \mathrm{~g}$

General procedure F: Compound $5 \mathrm{~g}(27.6 \mathrm{mg}, 0.10 \mathrm{mmol})$ and anhydrous benzonitrile $(1.80 \mathrm{~mL})$ were employed and the reaction was stirred for 72 hours at $150{ }^{\circ} \mathrm{C}$. The crude mixture was purified by column chromatography (10\% acetone/hexane) to yield the title compound $6 \mathrm{~g}$ (13.3 mg, 44\%) as an off-white solid; m.p. $241-243{ }^{\circ} \mathrm{C}\left(\mathrm{CH}_{2} \mathrm{Cl}_{2} /\right.$ hexane); $v_{\max } /$ $\mathrm{cm}^{-1}: 3230$ (m), 2922 (m), 1662 (s), 1596 (s), 1454 (s), 1054 (s); ${ }^{1} \mathrm{H}$ NMR (400 MHz, CDCl 3 ) $\delta 10.2(1 \mathrm{H}, \mathrm{s}, \mathrm{N} \underline{\mathrm{H}}), 7.29-7.25(2 \mathrm{H}, \mathrm{m}, 2 \times \mathrm{ArC} \underline{\mathrm{H}}), 7.19-7.16(3 \mathrm{H}, \mathrm{m}, 3 \times \mathrm{ArC} \underline{\mathrm{H}}), 6.81-$ $6.80(1 \mathrm{H}, \mathrm{m}, 1 \times \mathrm{ArC} \underline{\mathrm{H}}), 6.69-6.67(1 \mathrm{H}, \mathrm{m}, 1 \times \mathrm{ArC} \underline{\mathrm{H}}), 6.29(1 \mathrm{H}, \mathrm{d}, J=2.5 \mathrm{~Hz}, 1 \times \mathrm{C} 9-\underline{\mathrm{H}})$, $4.66(1 \mathrm{H}, \mathrm{dd}, J=13.4,4.2 \mathrm{~Hz}, 1 \times \mathrm{C} 4-\underline{\mathrm{H}}), 4.26\left(1 \mathrm{H}, \mathrm{ddd}, J=12.5,8.9,6.4 \mathrm{~Hz}, 1 \times \mathrm{C6}-\underline{\mathrm{H}}_{2}\right)$, $3.63\left(1 \mathrm{H}, \mathrm{ddd}, J=12.5,8.9,6.0 \mathrm{~Hz}, 1 \times \mathrm{C} 6-\underline{\mathrm{H}}_{2}\right), 3.03(1 \mathrm{H}, \mathrm{ddd}, J=13.4,11.7,5.5 \mathrm{~Hz}, 1 \times$ C2 $\left.-\underline{\mathrm{H}}_{2}\right), 2.96-2.83\left(2 \mathrm{H}, \mathrm{m}, 2 \times \mathrm{C} 7-\underline{\mathrm{H}}_{2}\right), 2.58-2.53\left(1 \mathrm{H}, \mathrm{m}, 1 \times \mathrm{C} 2-\underline{\mathrm{H}}_{2}\right), 2.24-2.15(1 \mathrm{H}, \mathrm{m}$, $\left.1 \times \mathrm{C} 3-\underline{\mathrm{H}}_{2}\right), 2.05-1.96\left(1 \mathrm{H}, \mathrm{m}, 1 \times \mathrm{C} 3-\underline{\mathrm{H}}_{2}\right) ;{ }^{13} \mathrm{C} \mathrm{NMR}\left(101 \mathrm{MHz}, \mathrm{CDCl}_{3}\right) \delta 196.2(\mathrm{C1})$, 161.8 (C5), 140.6 (C8), 136.5 (CAr), 128.5 (CAr), 128.4 (CAr), 128.3 (CAr), 127.2 (CAr), 127.0 (CAr), 123.1 (C9), 120.8 (CAr), 110.4 (CAr), 63.6 (C4), 44.9 (C6), 39.2 (C2), 36.7 (C3), 26.8 (C7). HRMS: $\left(\mathrm{ESI}^{+} \text {) Calculated for } \mathrm{C}_{19} \mathrm{H}_{18} \mathrm{~N}_{2} \mathrm{NaO}_{2} \text { : 329.1260. Found [M+Na] }\right]^{+}$: 329.1244.

$N$-Cyclopropyl- $N$-(4-phenylbut-3-yn-1-yl)benzofuran-2-carboxamide $5 \mathrm{i}$

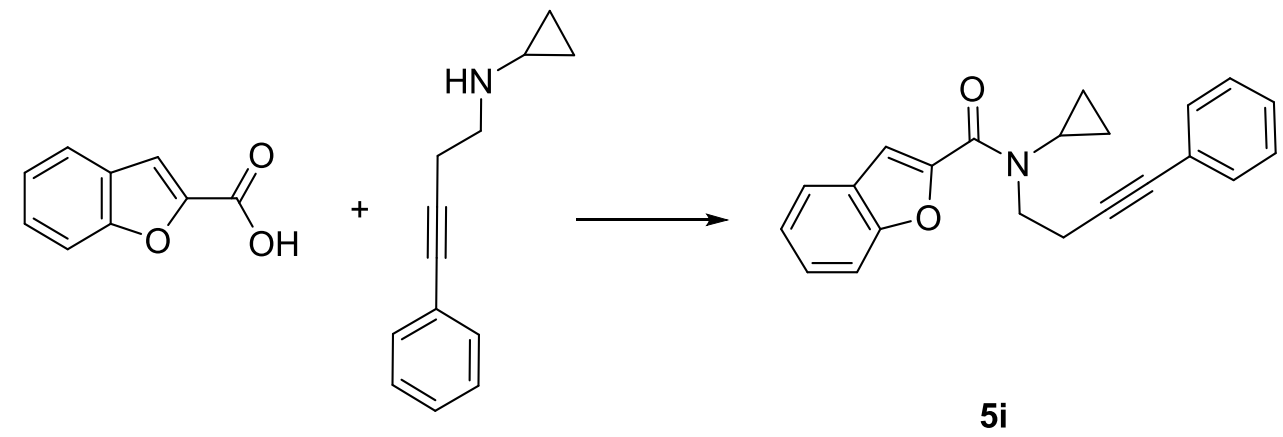


General procedure C: Benzofuran-2-carboxylic acid (356 mg, $2.20 \mathrm{mmol})$ and $\mathrm{N}$-(4phenylbut-3-yn-1-yl) cyclopropanamine (370 mg, $2.00 \mathrm{mmol})$ were employed. The crude mixture was purified by flash column chromatography ( $8 \%$ EtOAc/hexane) to afford the title compound 5i (392 mg, 60\%) as a yellow oil; $v_{\max } / \mathrm{cm}^{-1}: 2938(\mathrm{~m}), 1635$ (s), 1403 (s), 1258 (m), 1054 (s), 747 (s); ${ }^{1} \mathrm{H}$ NMR (400 MHz, $\left.\mathrm{CDCl}_{3}\right): \delta 7.62(1 \mathrm{H}, \mathrm{d}, J=7.8 \mathrm{~Hz}), 7.51(1 \mathrm{H}, \mathrm{d}, J$ $=8.4 \mathrm{~Hz}), 7.39-7.33(4 \mathrm{H}, \mathrm{m}), 7.27-7.23(4 \mathrm{H}, \mathrm{m}), 3.85(2 \mathrm{H}, \mathrm{t}, J=6.9 \mathrm{~Hz}), 3.21(1 \mathrm{H}, \mathrm{s}, b r)$, $2.82(2 \mathrm{H}, \mathrm{t}, J=6.9 \mathrm{~Hz}), 0.89-0.84(2 \mathrm{H}, \mathrm{m}), 0.73-0.69(2 \mathrm{H}, \mathrm{m}) ;{ }^{13} \mathrm{C}$ NMR $(101 \mathrm{MHz}$, $\left.\mathrm{CDCl}_{3}\right): \delta 162.6,154.7,149.5,131.5,128.2,127.8,127.1,126.4,123.5,123.4,122.3,111.8$, 111.6, 87.1, 82.2, 47.2, 31.3, 19.0, 9.6.; m/z (ESI $\left.{ }^{+}\right)$HRMS: $\mathrm{C}_{22} \mathrm{H}_{19} \mathrm{NNaO}_{2}$ : 352.1308. Found $[\mathrm{M}+\mathrm{Na}]^{+}: 352.1293$.

\section{(E)-3-Benzylidene-1,2,3,3a,4,5-hexahydrobenzofuro[3,2-f]pyrrolo[1,2-a]azocine-6,12-}

\section{dione $6 \mathbf{i}$}

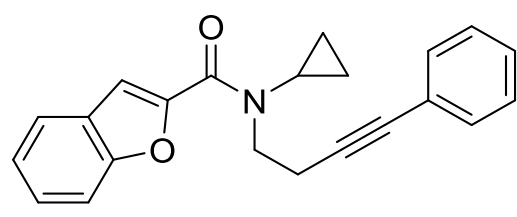

$5 \mathbf{i}$

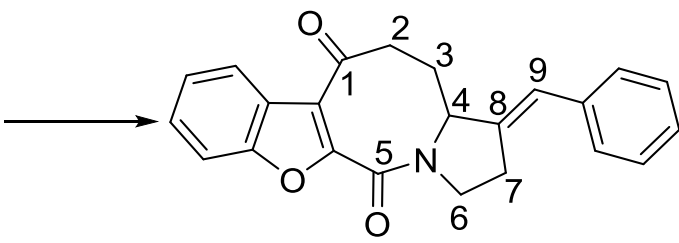

$6 \mathbf{i}$

General procedure F: Compound $\mathbf{5 i}(32.7 \mathrm{mg}, 0.10 \mathrm{mmol})$ and anhydrous benzonitrile (1.80 $\mathrm{mL}$ ) were employed and the reaction was stirred for 72 hours at $150{ }^{\circ} \mathrm{C}$. The crude mixture was purified by column chromatography (35\% EtOAc/hexane then $2 \%$ acetone/toluene) to yield the title compound $\mathbf{6 i}(15.9 \mathrm{mg}, 45 \%)$ as a yellow oil. $v_{\max } / \mathrm{cm}^{-1}: 2922(\mathrm{~m}), 1665(\mathrm{~s})$, 1636 (s), 1550 (m), 1425 (s), 1032 (s); ${ }^{1} \mathrm{H}$ NMR (400 MHz, $\left.\mathrm{CDCl}_{3}\right) \delta 8.31-8.28(1 \mathrm{H}, \mathrm{m}, 1$ $\times \operatorname{ArC} \underline{\mathrm{H}}), 7.52(1 \mathrm{H}, \mathrm{d}, J=8.1 \mathrm{~Hz}, 1 \times \mathrm{ArC} \underline{\mathrm{H}}), 7.41-7.33(2 \mathrm{H}, \mathrm{m}, 2 \times \mathrm{ArC} \underline{\mathrm{H}}), 7.30-7.26$ $(2 \mathrm{H}, \mathrm{m}, 2 \times \mathrm{ArC} \underline{\mathrm{H}}), 7.20-7.16(3 \mathrm{H}, \mathrm{m}, 3 \times \mathrm{ArC} \underline{\mathrm{H}}), 6.29(1 \mathrm{H}, \mathrm{s}, 1 \times \mathrm{C} 9-\underline{\mathrm{H}}), 4.70(1 \mathrm{H}, \mathrm{dd}, J=$ $13.3,4.5 \mathrm{~Hz}, 1 \times \mathrm{C} 4-\underline{\mathrm{H}}), 4.35\left(1 \mathrm{H}, \mathrm{ddd}, J=12.9,9.0,5.8 \mathrm{~Hz}, 1 \times \mathrm{C} 6-\underline{\mathrm{H}}_{2}\right), 3.70(1 \mathrm{H}, \mathrm{ddd}, J=$ 12.9, 9.0, $\left.6.5 \mathrm{~Hz}, 1 \times \mathrm{C} 6-\underline{\mathrm{H}}_{2}\right), 3.11\left(1 \mathrm{H}, \mathrm{ddd}, J=13.0,12.2,5.4 \mathrm{~Hz}, 1 \times \mathrm{C}^{2}-\underline{\mathrm{H}}_{2}\right), 3.02-2.88$ $\left(2 \mathrm{H}, \mathrm{m}, 1 \times \mathrm{C} 7-\underline{\mathrm{H}}_{2}\right), 2.74-2.69\left(1 \mathrm{H}, \mathrm{m}, 1 \times \mathrm{C} 2-\underline{\mathrm{H}}_{2}\right), 2.30-2.21\left(1 \mathrm{H}, \mathrm{m}, 1 \times \mathrm{C} 3-\underline{\mathrm{H}}_{2}\right), 2.10-$ $2.01\left(1 \mathrm{H}, \mathrm{m}, 1 \times \mathrm{C} 3-\underline{\mathrm{H}}_{2}\right) ;{ }^{13} \mathrm{C} \mathrm{NMR}\left(101 \mathrm{MHz}, \mathrm{CDCl}_{3}\right) \delta 195.8$ (C1), 159.0 (C5), 154.2 (CAr), 151.5 (CAr), 139.8 (C8), 136.3 (CAr), 128.5 (CAr), 128.3 (CAr), 127.4 (CAr), 127.3 (CAr), 125.3 (CAr), 124.4 (CAr), 124.0 (CAr), 123.6 (C9), 121.7 (CAr), 111.8 (CAr), 63.0 (C4), 44.8 (C6), 39.6 (C2) 35.9 (C3), 26.9 (C7); HRMS: (ESI ${ }^{+}$) Calculated for $\mathrm{C}_{23} \mathrm{H}_{19} \mathrm{NNaO}_{3}$ : 380.1257. Found $[\mathrm{M}+\mathrm{Na}]^{+}: 380.1273$. 


\section{$N$-Cyclopropyl- $N$-(4-phenylbut-3-yn-1-yl)-1H-indole-3-carboxamide $5 \mathbf{j}$}

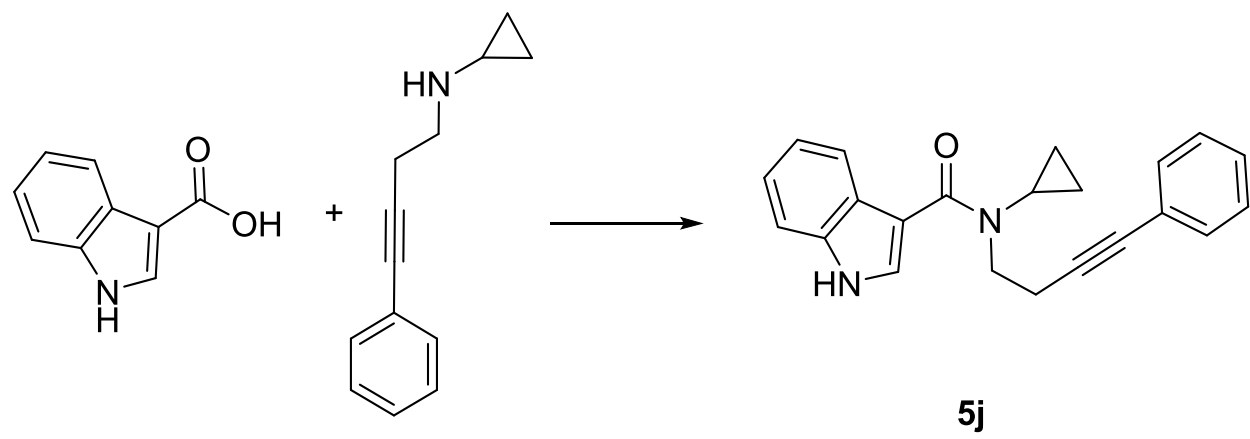

General procedure C: $1 H$-Indole-3-carboxylic acid $(152 \mathrm{mg}, 0.95 \mathrm{mmol})$ and $N$-(4phenylbut-3-yn-1-yl) cyclopropanamine (160 mg, $0.86 \mathrm{mmol})$ were employed. The crude mixture was purified by flash column chromatography (35\% EtOAc/hexane) to afford the title compound 5j (253 mg, 89\%) as a colourless solid; m.p. $143-145{ }^{\circ} \mathrm{C}\left(\mathrm{CH}_{2} \mathrm{Cl}_{2}\right) ; v_{\max } / \mathrm{cm}^{-}$ 1: 3180 (m), 2924 (m), 1592 (s), 1441 (s), 1230 (s), 749 (s); ${ }^{1} \mathrm{H}$ NMR (400 MHz, CDCl 3 ): $\delta$ $8.89(1 \mathrm{H}, \mathrm{s}, b r), 7.92(1 \mathrm{H}, \mathrm{d}, J=7.8 \mathrm{~Hz}), 7.38(1 \mathrm{H}, \mathrm{d}, J=2.8 \mathrm{~Hz}), 7.32-7.30(2 \mathrm{H}, \mathrm{m}), 7.25$ $(1 \mathrm{H}, \mathrm{d}, J=8.0 \mathrm{~Hz}), 7.21-7.17(3 \mathrm{H}, \mathrm{m}), 7.21-7.17(2 \mathrm{H}, \mathrm{m}), 3.79(2 \mathrm{H}, \mathrm{t}, J=6.8 \mathrm{~Hz}), 2.94$ $(1 \mathrm{H}, \mathrm{tt}, J=6.9,4.0 \mathrm{~Hz}), 2.77(2 \mathrm{H}, \mathrm{t}, J=6.8 \mathrm{~Hz}), 0.70-0.56(4 \mathrm{H}, \mathrm{m}) ;{ }^{13} \mathrm{C} \mathrm{NMR}(101 \mathrm{MHz}$, $\left.\mathrm{CDCl}_{3}\right): \delta 168.8,135.4,131.5,128.2,127.8,127.3,126.5,123.6,122.7,121.3,121.1,112.4$, 111.3, 87.7, 82.0, 47.3, 31.1, 19.1, 10.3; m/z (ESI $\left.{ }^{+}\right)$HRMS: $\mathrm{C}_{22} \mathrm{H}_{20} \mathrm{~N}_{2} \mathrm{NaO}$ : 351.1468. Found $[\mathrm{M}+\mathrm{Na}]^{+}: 351.1465$.

(E)-3-Benzylidene-1,2,3,3a,4,5-hexahydro-6H-pyrrolo[1',2':1,8]azocino[4,3-b]indole-

\section{6,12(7H)-dione $6 \mathbf{j}$}

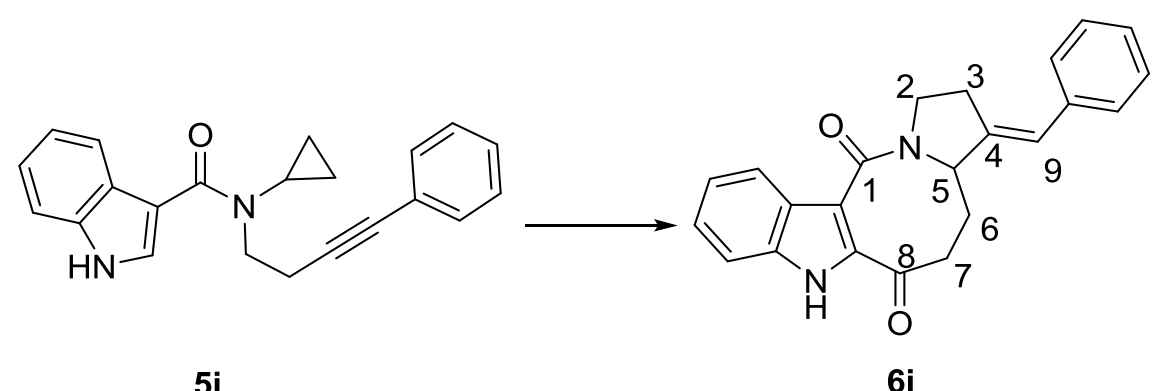

General procedure F: Compound $\mathbf{5 j}(32.8 \mathrm{mg}, 0.10 \mathrm{mmol})$ and anhydrous benzonitrile (1.00 $\mathrm{mL}$ ) were employed and the reaction was stirred for 72 hours at $140{ }^{\circ} \mathrm{C}$. The crude mixture was purified by column chromatography ( $8 \%$ acetone/toluene) to yield the title compound $\mathbf{6 j}$ (22.1 mg, 62\%) as a yellow solid; m.p. $253-255^{\circ} \mathrm{C}\left(\mathrm{CDCl}_{3}\right) ; v_{\max } / \mathrm{cm}^{-1}: 3288(\mathrm{~m}), 1657$ (s),

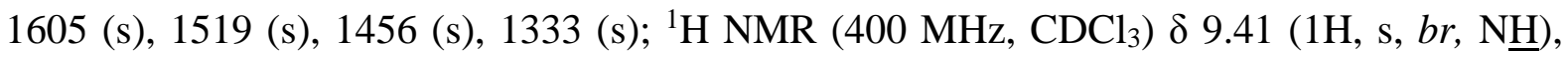


$8.05(1 \mathrm{H}, \mathrm{d}, J=8.2 \mathrm{~Hz}, 1 \times \operatorname{ArC} \underline{\mathrm{H}}), 7.38-7.24(4 \mathrm{H}, \mathrm{m}, 4 \times \operatorname{ArC} \underline{\mathrm{H}}), 7.18-7.14(4 \mathrm{H}, \mathrm{m}, 4 \times$ $\operatorname{ArC} \underline{\mathrm{H}}), 6.22(1 \mathrm{H}, \mathrm{d}, J=2.8 \mathrm{~Hz}, 1 \times \mathrm{C} 9-\underline{\mathrm{H}}), 4.60(1 \mathrm{H}, \mathrm{dd}, J=13.0,4.8 \mathrm{~Hz}, 1 \times \mathrm{C5}-\underline{\mathrm{H}}), 4.40$ $\left(1 \mathrm{H}, \mathrm{ddd}, J=12.6,9.0,5.7 \mathrm{~Hz}, 1 \times \mathrm{C} 2-\underline{\mathrm{H}}_{2}\right), 3.68\left(1 \mathrm{H}, \mathrm{ddd}, J=12.6,9.0,6.6 \mathrm{~Hz}, 1 \times \mathrm{C} 2-\underline{\mathrm{H}}_{2}\right)$, $3.20\left(1 \mathrm{H}\right.$, ddd $\left., J=12.9,12.9,5.6 \mathrm{~Hz}, 1 \times \mathrm{C} 7-\underline{\mathrm{H}_{2}}\right), 3.01-2.86\left(2 \mathrm{H}, \mathrm{m}, 2 \times \mathrm{C} 3-\underline{\mathrm{H}_{2}}\right), 2.71-$ $2.66\left(1 \mathrm{H}, \mathrm{m}, 1 \times \mathrm{C} 7-\underline{\mathrm{H}}_{2}\right), 2.22-2.01\left(2 \mathrm{H}, \mathrm{m}, 2 \times \mathrm{C} 6-\underline{\mathrm{H}}_{2}\right) ;{ }^{13} \mathrm{C} \mathrm{NMR}\left(101 \mathrm{MHz}, \mathrm{CDCl}_{3}\right) \delta$ 192.4 (C8), 163.9 (C1), 141.0 (C4), 136.6 (CAr), 136.0 (CAr), 133.3 (CAr), 128.4 (CAr), 128.2 (CAr), 127.7 (CAr), 127.2 (CAr), 127.1 (CAr), 123.8 (CAr), 122.8 (C9), 122.3 (CAr), 116.2 (CAr), 111.9 (CAr), 62.7 (C5), 44.2 (C2), 37.7 (C7), 35.9 (C6), 27.3 (C3); HRMS: $\left(\mathrm{ESI}^{+}\right)$Calculated for $\mathrm{C}_{23} \mathrm{H}_{21} \mathrm{~N}_{2} \mathrm{O}_{2}: 357.1598$. Found $[\mathrm{M}+\mathrm{H}]^{+}: 357.1593$.

When the above reaction was run with tris(3,5-dimethylphenyl)phosphine (5.19 $\mathrm{mg}, 15.0$ mol\%) as the ligand, compound $6 j(22.0 \mathrm{mg}, 62 \%)$ was obtained. When the above reaction was run with 20 mol\% of 2-nitrobenzoic acid (instead of $100 \mathrm{~mol} \%$ in the standard conditions), compound $\mathbf{6 j}$ (16.8 mg, 47\%) was obtained.

\section{$N$-Cyclopropyl- $N$-(4-phenylbut-3-yn-1-yl)-1H-pyrrole-3-carboxamide 5k}

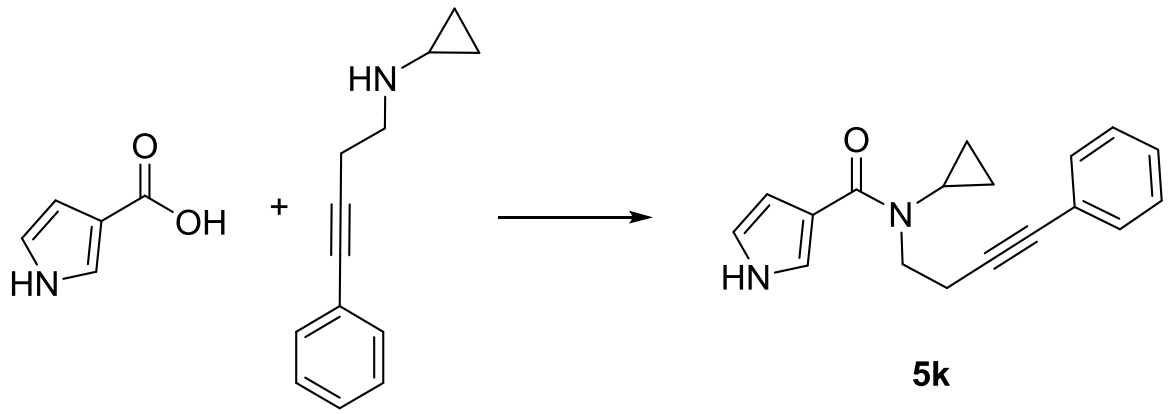

General procedure C: 1H-Pyrrole-3-carboxylic acid (244 mg, $2.20 \mathrm{mmol})$ and $\mathrm{N}$-(4phenylbut-3-yn-1-yl) cyclopropanamine $(370 \mathrm{mg}, 2.00 \mathrm{mmol})$ were employed. The crude mixture was purified by flash column chromatography (50\% EtOAc/hexane) to afford the title compound 5k (323 mg, 58\%) as a colourless solid; m.p. $95-97{ }^{\circ} \mathrm{C}\left(\mathrm{CH}_{2} \mathrm{Cl}_{2}\right) ; v_{\max } / \mathrm{cm}^{-1}$ : 3221 (m), 2923 (m), 1591 (s), 1415 (s), 1033 (s), 755 (s); ${ }^{1} \mathrm{H}$ NMR (400 MHz, $\mathrm{CDCl}_{3}$ ): $\delta 8.74$ $(1 \mathrm{H}, \mathrm{s}, b r), 7.37-7.34(2 \mathrm{H}, \mathrm{m}), 7.30(1 \mathrm{H}, \mathrm{d}, J=2.6 \mathrm{~Hz}), 7.26-7.24(3 \mathrm{H}, \mathrm{m}), 6.71-6.69$ $(1 \mathrm{H}, \mathrm{m}, b r), 6.63-6.62(1 \mathrm{H}, \mathrm{m}, b r), 3.79(2 \mathrm{H}, \mathrm{t}, J=6.9 \mathrm{~Hz}), 3.03(1 \mathrm{H}, \mathrm{tt}, J=6.9,3.9 \mathrm{~Hz})$, $2.78(2 \mathrm{H}, \mathrm{t}, J=6.9 \mathrm{~Hz}), 0.87-0.82(2 \mathrm{H}, \mathrm{m}), 0.74-0.70(2 \mathrm{H}, \mathrm{m}) ;{ }^{13} \mathrm{C}$ NMR $(126 \mathrm{MHz}$, $\left.\mathrm{CDCl}_{3}\right): \delta 168.4,131.5,128.2,127.7,123.7,122.2,120.0,117.5,110.1,87.8,81.7,47.2,31.5$, 19.1, 10.7; m/z $\left(\mathrm{ESI}^{+}\right)$HRMS: $\mathrm{C}_{18} \mathrm{H}_{18} \mathrm{~N}_{2} \mathrm{NaO}: 301.1311$. Found $[\mathrm{M}+\mathrm{Na}]^{+}: 301.1310$. 
6k

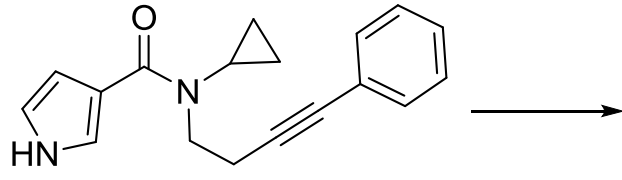

$5 \mathbf{k}$

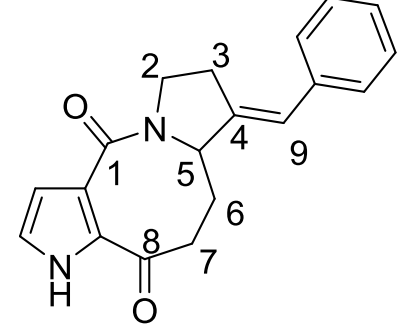

$6 k$

General procedure F: Compound $5 \mathbf{k}(27.6 \mathrm{mg}, 0.10 \mathrm{mmol})$ and anhydrous benzonitrile $(1.00 \mathrm{~mL})$ were employed and the reaction was stirred for 48 hours at $130{ }^{\circ} \mathrm{C}$. The crude mixture was purified by column chromatography (65\% EtOAc/hexane) to yield the title compound 6k $(21.0 \mathrm{mg}, 69 \%)$ as a colourless solid; m.p. 263-265 ${ }^{\circ} \mathrm{C}\left(\mathrm{CDCl}_{3}\right) ; v_{\max } / \mathrm{cm}^{-1}$ : 3237 (m), 2924 (m), 1648 (s), 1606 (s), 1485 (s), 1402 (s); ${ }^{1} \mathrm{H}$ NMR (400 MHz, CDCl 3 ) $\delta$ $9.74(1 \mathrm{H}, \mathrm{s}, b r, \underline{\mathrm{H}}), 7.29-7.25(2 \mathrm{H}, \mathrm{m}, 2 \times \mathrm{ArC} \underline{\mathrm{H}}), 7.19-7.14(3 \mathrm{H}, \mathrm{m}, 3 \times \mathrm{ArC} \underline{\mathrm{H}}), 6.97-$ $6.95(1 \mathrm{H}, \mathrm{m}, 1 \times \mathrm{ArC} \underline{\mathrm{H}}), 6.62(1 \mathrm{H}, \mathrm{dd}, J=2.8,2.8 \mathrm{~Hz}, 1 \times \mathrm{ArC} \underline{\mathrm{H}}), 6.24(1 \mathrm{H}, \mathrm{d}, J=2.6 \mathrm{~Hz}, 1$ $\times$ C9- $\underline{\mathrm{H}}), 4.55(1 \mathrm{H}, \mathrm{dd}, J=13.0,4.8 \mathrm{~Hz}, 1 \times \mathrm{C} 5-\underline{\mathrm{H}}), 4.30(1 \mathrm{H}, \mathrm{ddd}, J=12.5,8.9,5.8 \mathrm{~Hz}, 1 \times$ C2 $\left.-\underline{\mathrm{H}}_{2}\right), 3.59\left(1 \mathrm{H}, \mathrm{ddd}, J=12.5,8.9,6.6 \mathrm{~Hz}, 1 \times \mathrm{C} 2-\underline{\mathrm{H}}_{2}\right), 3.06-2.83\left(3 \mathrm{H}, \mathrm{m}, 1 \times \mathrm{C} 7-\underline{\mathrm{H}}_{2}, 2 \times\right.$ C3- $\left.\underline{\mathrm{H}}_{2}\right), 2.55\left(1 \mathrm{H}, \mathrm{ddd}, J=12.3,3.8,3.8 \mathrm{~Hz}, 1 \times \mathrm{C} 7-\underline{\mathrm{H}}_{2}\right), 2.16-2.07\left(1 \mathrm{H}, \mathrm{m}, 1 \times \mathrm{C6}-\underline{\mathrm{H}}_{2}\right)$, $2.02-1.93\left(1 \mathrm{H}, \mathrm{m}, 1 \times \mathrm{C} 6-\underline{\mathrm{H}}_{2}\right) ;{ }^{13} \mathrm{C}$ NMR $\left(101 \mathrm{MHz}, \mathrm{CDCl}_{3}\right) \delta 189.9$ (C8), 164.7 (C1), 141.2 (C4), 136.6 (CAr), 130.1 (CAr), 128.4 (CAr), 128.2 (CAr), 127.0 (CAr), 125.2 (CAr), 123.6 (CAr), 122.7 (C9), 113.9 (CAr), 62.9 (C5), 44.2 (C2), 37.3 (C7), 35.6 (C6), 27.3 (C3); HRMS: $\left(\mathrm{ESI}^{+}\right)$Calculated for $\mathrm{C}_{19} \mathrm{H}_{19} \mathrm{~N}_{2} \mathrm{O}_{2}$ : 307.1441. Found $[\mathrm{M}+\mathrm{H}]^{+}$: 307.1436. The structure of this compound was determined unambiguously by X-ray crystallography.

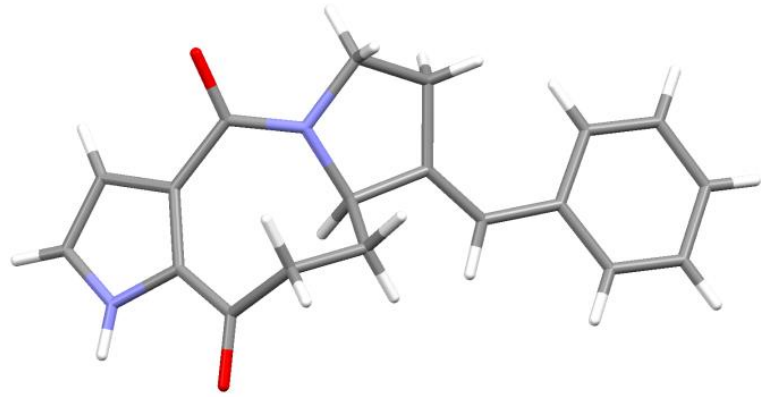




\section{$N$-Cyclopropyl- $N$-(pent-3-yn-1-yl)-1H-pyrrole-3-carboxamide 51}
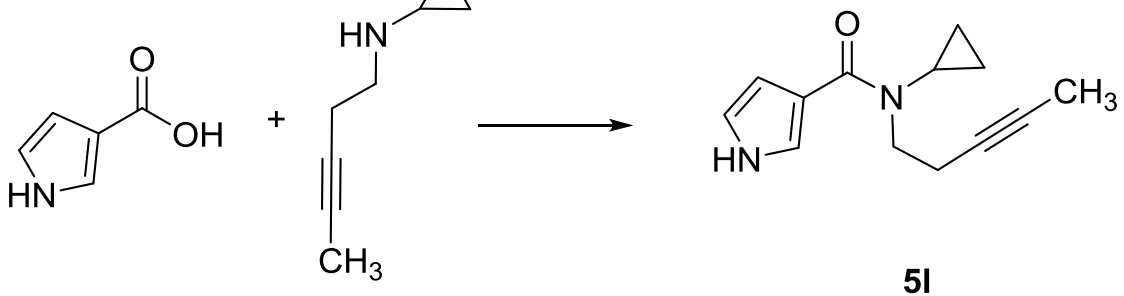

General procedure C: $1 H$-Pyrrole-3-carboxylic acid (244 mg, $2.20 \mathrm{mmol})$ and $\mathrm{N}$-(pent-3yn-1-yl) cyclopropanamine (246 mg, $2.00 \mathrm{mmol}$ ) were employed. The crude mixture was purified by flash column chromatography (60\% EtOAc/hexane) to afford the title compound $5 \mathbf{l}\left(248\right.$ mg, 58\%) as a yellow oil; $v_{\max } / \mathrm{cm}^{-1}: 3216(\mathrm{~m}), 2920$ (m), 1585 (s), 1500 (s), 1414 (s), 1355 (s); ${ }^{1} \mathrm{H}$ NMR (400 MHz, $\left.\mathrm{CDCl}_{3}\right): \delta 9.60(1 \mathrm{H}, \mathrm{s}, b r), 7.21-7.20(1 \mathrm{H}, \mathrm{m}), 6.65(1 \mathrm{H}, \mathrm{d}, J$ $=2.5 \mathrm{~Hz}), 6.57-6.55(1 \mathrm{H}, \mathrm{m}), 3.66(2 \mathrm{H}, \mathrm{t}, J=7.1 \mathrm{~Hz}), 2.94(1 \mathrm{H}, \mathrm{tt}, J=7.1,4.0 \mathrm{~Hz}), 2.50-$ $2.46(2 \mathrm{H}, \mathrm{m}), 1.73(3 \mathrm{H}, \mathrm{t}, J=2.6 \mathrm{~Hz}), 0.84-0.77(2 \mathrm{H}, \mathrm{m}), 0.68-0.64(2 \mathrm{H}, \mathrm{m}) ;{ }^{13} \mathrm{C} \mathrm{NMR}$ $\left(126 \mathrm{MHz}, \mathrm{CDCl}_{3}\right): \delta 168.9,122.4,119.4,117.7,109.6,77.0,76.6,47.4,31.0,18.3,10.4,3.4$; m/z $\left(\mathrm{ESI}^{+}\right)$HRMS: $\mathrm{C}_{13} \mathrm{H}_{16} \mathrm{~N}_{2} \mathrm{NaO}: 239.1155$. Found $[\mathrm{M}+\mathrm{Na}]^{+}: 239.1154$.

(E)-7-Ethylidene-5,6,6a,7,8,9-hexahydro-3H-dipyrrolo[1,2-a:2',3'-f]azocine-4,11-dione 61

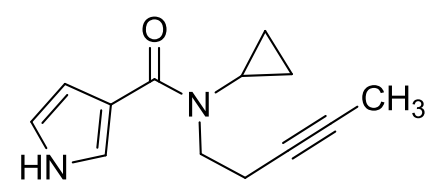

5!

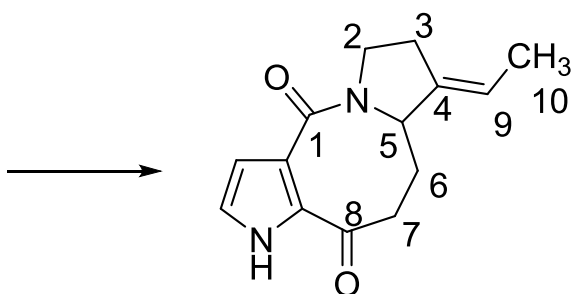

6I

General procedure F: Compound $\mathbf{5 l}(21.4 \mathrm{mg}, 0.10 \mathrm{mmol})$ and anhydrous benzonitrile (1.00 $\mathrm{mL}$ ) were employed and the reaction was stirred for 72 hours at $140{ }^{\circ} \mathrm{C}$. The crude mixture was purified by column chromatography (15\% acetone/toluene) to yield the title compound $\mathbf{6 l}$ (12.6 mg, 52\%) as a colourless solid. m.p. $185-187{ }^{\circ} \mathrm{C}\left(\mathrm{CDCl}_{3}\right) ; v_{\max } / \mathrm{cm}^{-1}: 3240(\mathrm{~m}), 2922$

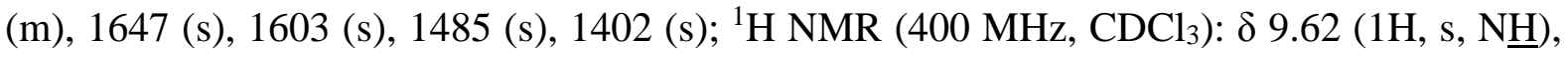
$7.00-6.99(1 \mathrm{H}, \mathrm{m}, 1 \times \mathrm{ArC} \underline{\mathrm{H}}), 6.66(1 \mathrm{H}, \mathrm{dd}, J=2.8,2.8 \mathrm{~Hz}, 1 \times \operatorname{ArC} \underline{\mathrm{H}}), 5.34-5.27(1 \mathrm{H}, \mathrm{m}$, $1 \times \mathrm{C} 9-\underline{\mathrm{H}}), 4.38(1 \mathrm{H}, \mathrm{dd}, J=12.9,4.8 \mathrm{~Hz}, 1 \times \mathrm{C} 5-\underline{\mathrm{H}}), 4.31(1 \mathrm{H}, \mathrm{ddd}, J=12.6,7.4,7.4 \mathrm{~Hz}, 1$ $\left.\times \mathrm{C} 2-\underline{\mathrm{H}}_{2}\right), 3.55\left(1 \mathrm{H}, \mathrm{ddd}, J=12.6,7.9,7.9 \mathrm{~Hz}, 1 \times \mathrm{C} 2-\underline{\mathrm{H}}_{2}\right), 3.02(1 \mathrm{H}, \mathrm{ddd}, J=13.6,12.2,5.7$ $\left.\mathrm{Hz}, 1 \times \mathrm{C} 7-\underline{\mathrm{H}}_{2}\right), 2.63-2.52\left(3 \mathrm{H}, \mathrm{m}, 1 \times \mathrm{C} 7-\underline{\mathrm{H}}_{2}, 2 \times \mathrm{C} 3-\underline{\mathrm{H}}_{2}\right), 2.06-1.97\left(1 \mathrm{H}, \mathrm{m}, 1 \times \mathrm{C} 6-\underline{\mathrm{H}}_{2}\right)$, 
$1.95-1.86\left(1 \mathrm{H}, \mathrm{m}, 1 \times \mathrm{C} 6-\underline{\mathrm{H}}_{2}\right), 1.60\left(3 \mathrm{H}, \mathrm{d}, J=6.0, \mathrm{~Hz}, 3 \times \mathrm{C} \mathbf{1 0}-\underline{\mathrm{H}}_{3}\right) ;{ }^{13} \mathrm{C} \mathrm{NMR}(101 \mathrm{MHz}$, $\left.\mathrm{CDCl}_{3}\right): \delta 190.1$ (C8), 164.7 (C1), 138.0 (C4), 130.1 (CAr), 125.3 (CAr), 123.4 (CAr), 117.0 (C9), 113.8 (CAr), 61.4 (C5), 43.6 (C2), 37.4 (C7), 35.6 (C6), 25.2 (C3), 14.4 (C10); HRMS: $\left(\mathrm{ESI}^{+} \text {) Calculated for } \mathrm{C}_{14} \mathrm{H}_{16} \mathrm{~N}_{2} \mathrm{NaO}_{2} \text { : 267.1104. Found [M+Na] }\right]^{+}: 267.1094$.

\section{$N$-Cyclopropyl- $N$-(4-phenylbut-3-yn-1-yl)furan-3-carboxamide $5 \mathrm{~m}$}

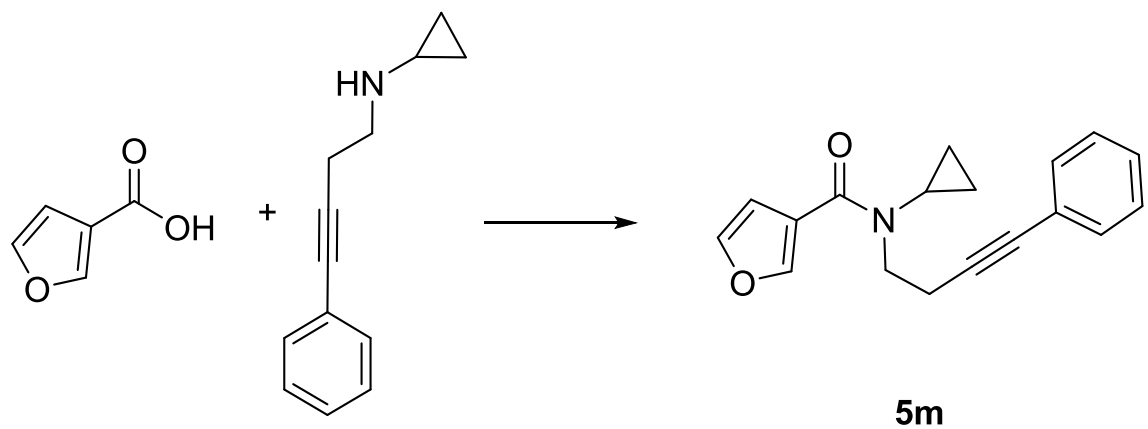

General procedure C: Furan-3-carboxylic acid (246 mg, $2.20 \mathrm{mmol})$ and $\mathrm{N}$-(4-phenylbut-3yn-1-yl) cyclopropanamine $(370 \mathrm{mg}, 2.00 \mathrm{mmol}$ ) were employed. The crude mixture was purified by flash column chromatography (20\% EtOAc/hexane) to afford the title compound 5m (315 mg, 57\%) as a yellow oil; $v_{\max } / \mathrm{cm}^{-1}: 2922(\mathrm{~m}), 1621(\mathrm{~s}), 1411(\mathrm{~s}), 1167$ (s), 875 (m), 749 (s); ${ }^{1} \mathrm{H}$ NMR (500 MHz, $\left.\mathrm{CDCl}_{3}\right): \delta 7.91-7.90(1 \mathrm{H}, \mathrm{m}), 7.42(1 \mathrm{H}, \mathrm{dd}, J=1.7,1.7 \mathrm{~Hz})$, $7.40-7.37(2 \mathrm{H}, \mathrm{m}), 7.31-7.28(3 \mathrm{H}, \mathrm{m}), 6.79-6.78(1 \mathrm{H}, \mathrm{m}), 3.80(2 \mathrm{H}, \mathrm{t}, J=6.8 \mathrm{~Hz}), 3.01$ $(1 \mathrm{H}, \mathrm{tt}, J=7.0,3.9 \mathrm{~Hz}), 2.82(2 \mathrm{H}, \mathrm{t}, J=6.8 \mathrm{~Hz}), 0.93-0.89(2 \mathrm{H}, \mathrm{m}), 0.80-0.76(2 \mathrm{H}, \mathrm{m})$; ${ }^{13} \mathrm{C}$ NMR $\left(126 \mathrm{MHz}, \mathrm{CDCl}_{3}\right): \delta 166.0,144.8,142.2,131.5,128.3,127.8,123.5,122.4,110.9$, 87.4, 81.9, 47.0, 31.2, 18.9, 10.7; m/z (ESI $\left.{ }^{+}\right)$HRMS: $\mathrm{C}_{18} \mathrm{H}_{17} \mathrm{NNaO}_{2}$ : 302.1511. Found $[\mathrm{M}+\mathrm{Na}]^{+}: 302.1143$.

(E)-7-Benzylidene-5,6,6a,7,8,9-hexahydrofuro[2,3-f]pyrrolo[1,2-a]azocine-4,11-dione 6m

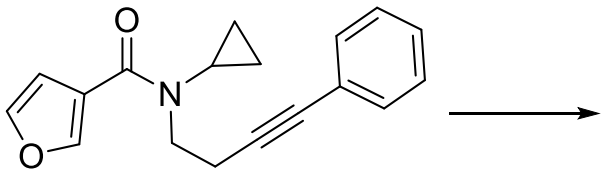

$5 \mathrm{~m}$

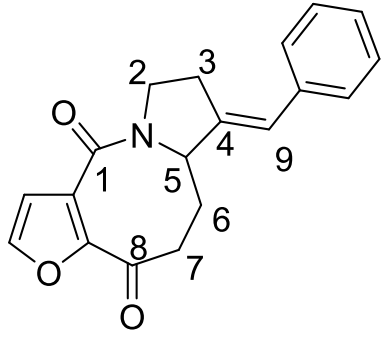

$6 m$

General procedure F: Compound $\mathbf{5 m}(27.7 \mathrm{mg}, 0.10 \mathrm{mmol})$ and anhydrous benzonitrile (1.0 $\mathrm{mL}$ ) were employed and the reaction was stirred for 48 hours at $140{ }^{\circ} \mathrm{C}$. The crude mixture 
was purified by column chromatography ( $8 \%$ acetone/toluene) to yield the title compound 6m (20.2 mg, 66\%) as a colourless oil; $v_{\max } / \mathrm{cm}^{-1}: 2930(\mathrm{~m}), 1672(\mathrm{~s}), 1623$ (s), 1488 (s),

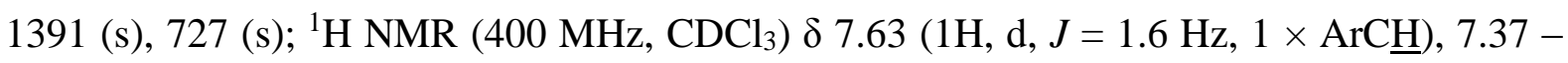
$7.33(2 \mathrm{H}, \mathrm{m}, 2 \times \mathrm{ArC} \underline{\mathrm{H}}), 7.26-7.24(3 \mathrm{H}, \mathrm{m}, 3 \times \mathrm{ArC} \underline{\mathrm{H}}), 6.84(1 \mathrm{H}, \mathrm{d}, J=1.6 \mathrm{~Hz}, 1 \times \mathrm{ArC} \underline{\mathrm{H}})$, $6.35(1 \mathrm{H}, \mathrm{d}, J=2.6 \mathrm{~Hz}, 1 \times \mathrm{C} 9-\underline{\mathrm{H}}), 4.58(1 \mathrm{H}, \mathrm{dd}, J=13.1,4.7 \mathrm{~Hz}, 1 \times \mathrm{C} 5-\underline{\mathrm{H}}), 4.31(1 \mathrm{H}$, ddd, $\left.J=12.7,9.0,5.7 \mathrm{~Hz}, 1 \times \mathrm{C} 2-\underline{\mathrm{H}}_{2}\right), 3.68\left(1 \mathrm{H}, \mathrm{ddd}, J=12.7,9.0,6.7 \mathrm{~Hz}, 1 \times \mathrm{C} 2-\underline{\mathrm{H}}_{2}\right), 3.10-$ $2.93\left(3 \mathrm{H}, \mathrm{m}, 1 \times \mathrm{C} 7-\underline{\mathrm{H}}_{2}, 2 \times \mathrm{C} 3-\underline{\mathrm{H}}_{2}\right), 2.71\left(1 \mathrm{H}, \mathrm{ddd}, J=12.7,3.8,3.8 \mathrm{~Hz}, 1 \times \mathrm{C} 7-\underline{\mathrm{H}}_{2}\right), 2.28-$ $2.19\left(1 \mathrm{H}, \mathrm{m}, 1 \times \mathrm{C} 6-\underline{\mathrm{H}}_{2}\right), 2.12-2.03\left(1 \mathrm{H}, \mathrm{m}, 1 \times \mathrm{C} 6-\underline{\mathrm{H}}_{2}\right) ;{ }^{13} \mathrm{C} \mathrm{NMR}\left(101 \mathrm{MHz}, \mathrm{CDCl}_{3}\right) \delta$ 187.5 (C8), 161.9 (C1), 148.8 (CAr), 146.4 (CAr), 140.2 (C4), 136.4 (CAr), 128.5 (CAr), 128.3 (CAr), 128.2 (CAr), 127.3 (CAr), 123.3 (CAr), 114.2 (CAr), 63.0 (C5), 44.5 (C2), 37.6 (C7), 35.5 (C6), 27.2 (C3); HRMS: $\left(\mathrm{ESI}^{+}\right)$Calculated for $\mathrm{C}_{19} \mathrm{H}_{18} \mathrm{NO}_{3}$ : 308.1281. Found $[\mathrm{M}+\mathrm{H}]^{+}: 308.1288$.

\section{$N$-Cyclopropyl- $N$-(4-phenylbut-3-yn-1-yl)thiophene-3-carboxamide 5n}

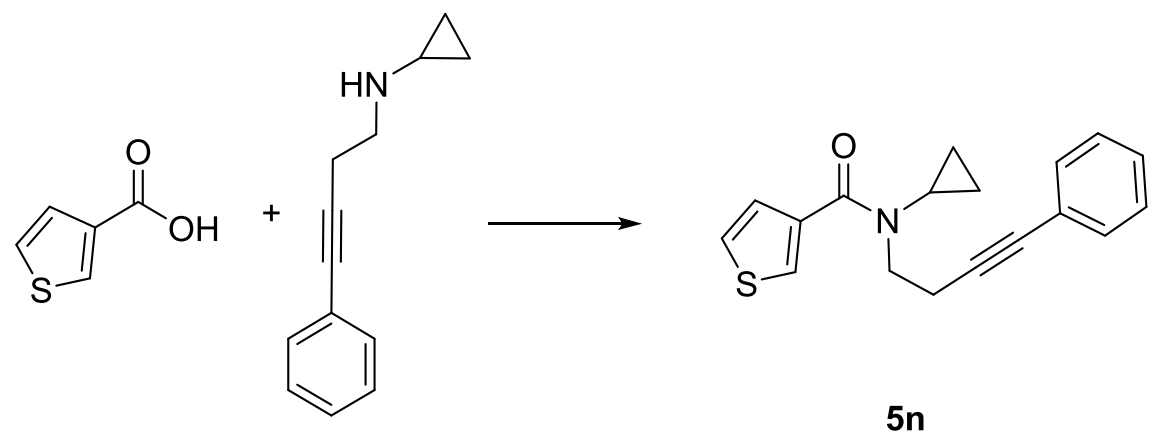

General procedure C: Thiophene-3-carboxylic acid (282 $\mathrm{mg}, 2.20 \mathrm{mmol})$ and $\mathrm{N}$-(4phenylbut-3-yn-1-yl) cyclopropanamine $(370 \mathrm{mg}, 2.00 \mathrm{mmol})$ were employed. The crude mixture was purified by flash column chromatography (15\% EtOAc/hexane) to afford the title compound 5n (337 mg, 57\%) as a colourless oil; $v_{\max } / \mathrm{cm}^{-1}: 2937$ (m), 1624 (s), 1418 (s), 1365 (s), 1033 (s), 757 (s); ${ }^{1} \mathrm{H}$ NMR (500 MHz, $\left.\mathrm{CDCl}_{3}\right): \delta 7.70-7.69$ (1H, m), $7.41-7.36$ $(2 \mathrm{H}, \mathrm{m}), 7.37-7.36(1 \mathrm{H}, \mathrm{m}), 7.31-7.28(4 \mathrm{H}, \mathrm{m}), 3.81(2 \mathrm{H}, \mathrm{t}, J=6.8 \mathrm{~Hz}), 3.01(1 \mathrm{H}, \mathrm{tt}, J=$ 7.1, 3.9 Hz), $2.85(2 \mathrm{H}, \mathrm{t}, J=6.8 \mathrm{~Hz}), 0.81-0.75(2 \mathrm{H}, \mathrm{m}), 0.63-0.57(2 \mathrm{H}, \mathrm{m}) ;{ }^{13} \mathrm{C} \mathrm{NMR}$ $\left(126 \mathrm{MHz}, \mathrm{CDCl}_{3}\right): \delta 167.8,137.8,131.5,128.2,127.8,127.6,127.4,124.8,123.5,87.3$, 82.0, 46.9, 31.7, 18.8, 10.2; m/z $\left(\mathrm{ESI}^{+}\right)$HRMS: $\mathrm{C}_{18} \mathrm{H}_{17} \mathrm{NNaOS}: 318.0923$. Found $[\mathrm{M}+\mathrm{Na}]^{+}$: 318.0911 . 
6n

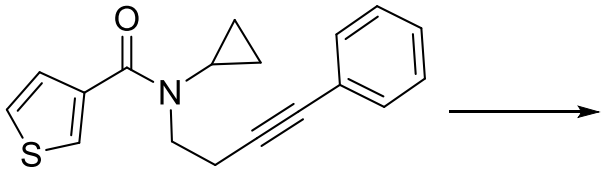

$5 n$

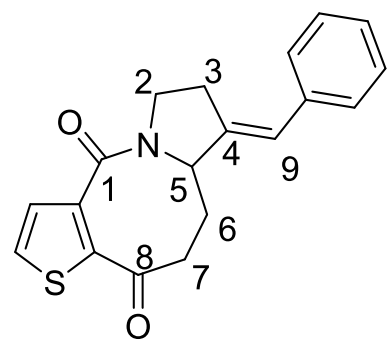

$6 n$

General procedure F: Compound 5 n $(30.9 \mathrm{mg}, 0.10 \mathrm{mmol})$ and anhydrous benzonitrile $(1.00 \mathrm{~mL})$ were employed and the reaction was stirred for 72 hours at $140{ }^{\circ} \mathrm{C}$. The crude mixture was purified by column chromatography ( $8 \%$ acetone/toluene) to yield the title compound 6n (21.6 mg, 64\%) as a colourless solid; m.p. 190-192 ${ }^{\circ} \mathrm{C}\left(\mathrm{CDCl}_{3}\right) ; v_{\max } / \mathrm{cm}^{-1}$ : 2922 (m), 1654 (s), 1618 (s), 1434 (s), 1405 (s), 729 (s); ${ }^{1} \mathrm{H}$ NMR (400 MHz, $\mathrm{CDCl}_{3}$ ) $\delta 7.54$ $(1 \mathrm{H}, \mathrm{d}, J=5.1 \mathrm{~Hz}, 1 \times \mathrm{ArC} \underline{\mathrm{H}}), 7.35(1 \mathrm{H}, \mathrm{d}, J=5.1 \mathrm{~Hz}, 1 \times \mathrm{ArC} \underline{\mathrm{H}}), 7.29-7.25(2 \mathrm{H}, \mathrm{m}, 2 \times$ $\operatorname{ArC} \underline{\mathrm{H}}), 7.19-7.15(3 \mathrm{H}, \mathrm{m}, 3 \times \mathrm{ArC} \underline{\mathrm{H}}), 6.27(1 \mathrm{H}, \mathrm{d}, J=2.6 \mathrm{~Hz}, 1 \times \mathrm{C} 9-\underline{\mathrm{H}}), 4.53(1 \mathrm{H}, \mathrm{dd}, J=$ 13.0, $4.7 \mathrm{~Hz}, 1 \times \mathrm{C} 5-\underline{\mathrm{H}}), 4.24\left(1 \mathrm{H}, \mathrm{ddd}, J=12.7,9.0,5.2 \mathrm{~Hz}, 1 \times \mathrm{C} 2-\underline{\mathrm{H}}_{2}\right), 3.63(1 \mathrm{H}, \mathrm{ddd}, J=$ 12.7, 9.0, $\left.6.9 \mathrm{~Hz}, 1 \times \mathrm{C} 2-\underline{\mathrm{H}}_{2}\right), 3.09-2.85\left(3 \mathrm{H}, \mathrm{m}, 1 \times \mathrm{C} 7-\underline{\mathrm{H}}_{2}, 2 \times \mathrm{C} 3-\underline{\mathrm{H}}_{2}\right), 2.65(1 \mathrm{H}, \mathrm{ddd}, J=$ 12.3, 3.7, 3.7 Hz, $\left.1 \times \mathrm{C} 7-\underline{\mathrm{H}}_{2}\right), 2.19-2.10\left(1 \mathrm{H}, \mathrm{m}, 1 \times \mathrm{C} 6-\underline{\mathrm{H}}_{2}\right), 2.05-1.96(1 \mathrm{H}, \mathrm{m}, 1 \times \mathrm{C} 6-$ $\left.\underline{\mathrm{H}}_{2}\right) ;{ }^{13} \mathrm{C}$ NMR (101 MHz, $\mathrm{CDCl}_{3}$ ) $\delta 192.7$ (C8), 163.1 (C1), 143.3 (C4), 140.5 (CAr), 139.7 (CAr), 136.4 (CAr), 133.0 (CAr), 132.1 (CAr), 128.5 (CAr), 128.2 (CAr), 127.2 (CAr), 123.2 (C9), 62.5 (C5), 44.3 (C2), 37.7 (C7), 35.0 (C6), 27.2 (C3); HRMS: (ESI ${ }^{+}$) Calculated for $\mathrm{C}_{19} \mathrm{H}_{17} \mathrm{NNaO}_{2} \mathrm{~S}: 346.0872$. Found $[\mathrm{M}+\mathrm{Na}]^{+}: 346.0869$.

\section{$N$-Cyclopropyl-2-methyl- $N$-(4-phenylbut-3-yn-1-yl)-1H-pyrrole-3-carboxamide 50}

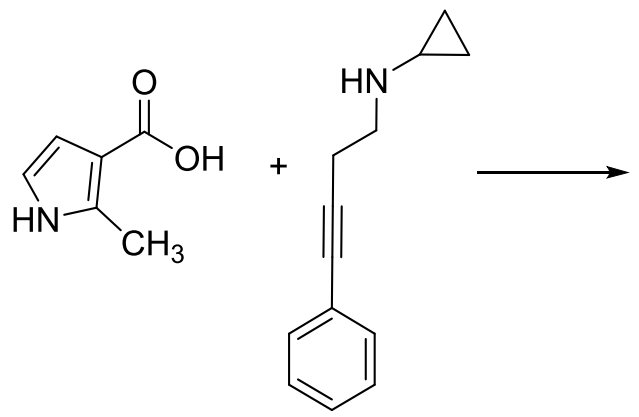

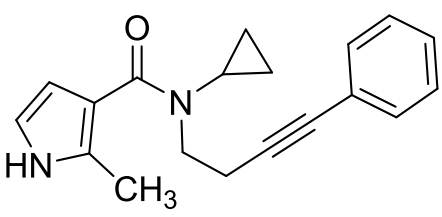

50

General procedure C: 2-Methyl-1 $H$-pyrrole-3-carboxylic acid (273 mg, $2.20 \mathrm{mmol})$ and $\mathrm{N}$ (4-phenylbut-3-yn-1-yl) cyclopropanamine (370 mg, $2.00 \mathrm{mmol}$ ) were employed. The crude 
mixture was purified by flash column chromatography $(50 \%$ EtOAc/hexane to $20 \%$ acetone/hexane) to afford the title compound 50 (300 mg, 52\%) as a yellow solid; m.p. 102$104{ }^{\circ} \mathrm{C}\left(\mathrm{CDCl}_{3}\right) ; v_{\max } / \mathrm{cm}^{-1}: 3237$ (m), 2922 (m), 1595 (s), 1441 (s), 1032 (s), 755 (s); ${ }^{1} \mathrm{H}$ NMR (400 MHz, $\left.\mathrm{CDCl}_{3}\right): 8.72(1 \mathrm{H}, \mathrm{s}), 7.31$ - 7.29 (2H, m), $7.19-7.17$ (3H, m), $6.41-6.40$ $(1 \mathrm{H}, \mathrm{m}), 6.26-6.24(1 \mathrm{H}, \mathrm{m}), 3.70(2 \mathrm{H}, \mathrm{t}, J=6.9 \mathrm{~Hz}), 2.88(1 \mathrm{H}, \mathrm{tt}, J=7.2,4.0 \mathrm{~Hz}), 2.70(2 \mathrm{H}$, $\mathrm{t}, J=6.9 \mathrm{~Hz}), 0.73-0.68(2 \mathrm{H}, \mathrm{m}), 0.54-0.51(2 \mathrm{H}, \mathrm{m}) ;{ }^{13} \mathrm{C} \mathrm{NMR}\left(101 \mathrm{MHz}, \mathrm{CDCl}_{3}\right): \delta$ $170.3,131.8,131.5,128.1,127.6,123.6,115.5,115.0,109.2,87.7,81.7,47.1,30.9,19.0$, 12.5, 9.8; m/z $\left(\mathrm{ESI}^{+}\right) \mathrm{HRMS}: \mathrm{C}_{19} \mathrm{H}_{20} \mathrm{~N}_{2} \mathrm{NaO}: 315.1468$. Found $[\mathrm{M}+\mathrm{Na}]^{+}: 315.1458$.

(E)-7-Benzylidene-1-methyl-5,6,6a,7,8,9-hexahydro-4H-dipyrrolo[1,2-a:3',4'-f]azocine4,11(2H)-dione 60

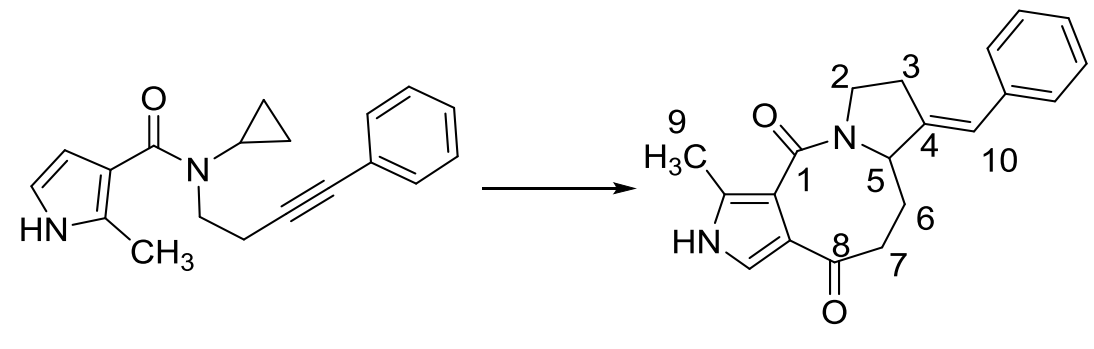

50

60

General procedure F: Compound $50(29.0 \mathrm{mg}, 0.10 \mathrm{mmol})$ and anhydrous benzonitrile $(1.00 \mathrm{~mL})$ were employed and the reaction was stirred for 72 hours at $130{ }^{\circ} \mathrm{C}$. The crude mixture was purified by column chromatography (25\% acetone/toluene) to yield the title compound $60(12.8 \mathrm{mg}, 40 \%)$ as a colourless oil. $v_{\max } / \mathrm{cm}^{-1}: 3226(\mathrm{~m}), 2923(\mathrm{~m}), 1722(\mathrm{~s})$, 1597 (s), 1447 (s), 1033 (s); ${ }^{1} \mathrm{H}$ NMR (400 MHz, $\left.\mathrm{CDCl}_{3}\right) \delta 9.78(1 \mathrm{H}, \mathrm{s}, b r, \mathrm{NH}), 7.28-7.25$ $(3 \mathrm{H}, \mathrm{m}, 3 \times \mathrm{ArC} \underline{\mathrm{H}}), 7.19-7.14(3 \mathrm{H}, \mathrm{m}, 3 \times \mathrm{ArC} \underline{\mathrm{H}}), 6.25(1 \mathrm{H}, \mathrm{d}, J=2.6 \mathrm{~Hz}, 1 \times \mathrm{C10}-\underline{\mathrm{H}})$, $4.56(1 \mathrm{H}, \mathrm{dd}, J=12.9,4.5 \mathrm{~Hz}, 1 \times \mathrm{C} 5-\underline{\mathrm{H}}), 4.27\left(1 \mathrm{H}, \mathrm{ddd}, J=12.5,8.9,5.6 \mathrm{~Hz}, 1 \times \mathrm{C} 2^{-} \underline{\mathrm{H}}_{2}\right)$, $3.60-3.53\left(1 \mathrm{H}, \mathrm{m}, 1 \times \mathrm{C} 2-\underline{\mathrm{H}}_{2}\right), 2.96-2.82\left(3 \mathrm{H}, \mathrm{m}, 1 \times \mathrm{C} 7-\underline{\mathrm{H}}_{2}, 2 \times \mathrm{C} 3-\underline{\mathrm{H}}_{2}\right), 2.44$ (1H, ddd, $J$ = 11.7, 3.7, $\left.3.7 \mathrm{~Hz}, 1 \times \mathrm{C} 7-\underline{\mathrm{H}}_{2}\right), 2.29\left(3 \mathrm{H}, \mathrm{s}, 3 \times \mathrm{C} 9-\underline{\mathrm{H}}_{3}\right), 2.11-1.99\left(1 \mathrm{H}, \mathrm{m}, 1 \times \mathrm{C} 6-\underline{\mathrm{H}}_{2}\right)$, $1.96-1.87\left(1 \mathrm{H}, \mathrm{m}, 1 \times \mathrm{C6}-\underline{\mathrm{H}}_{2}\right) ;{ }^{13} \mathrm{C} \mathrm{NMR}\left(101 \mathrm{MHz}, \mathrm{CDCl}_{3}\right): \delta 196.5$ (C8), $165.4(\mathrm{C1})$, 141.7 (C4), 136.8 (CAr), 134.5 (CAr), 128.4 (CAr), 128.2 (CAr), 127.0 (CAr), 125.5 (CAr), 122.5 (C10), 121.5 (CAr), 114.8 (CAr), 62.3 (C5), 43.9 (C2), 38.4 (C7), 34.8 (C6), 27.2 (C3), 12.7 (C9); HRMS: (ESI ${ }^{+}$) Calculated for $\mathrm{C}_{20} \mathrm{H}_{21} \mathrm{~N}_{2} \mathrm{O}_{2}$ : 321.1598. Found $[\mathrm{M}+\mathrm{H}]^{+}$: 321.1621 . 


\section{3-Benzyl-1-cyclopropyl-1-(4-phenylbut-3-yn-1-yl)urea 5p}

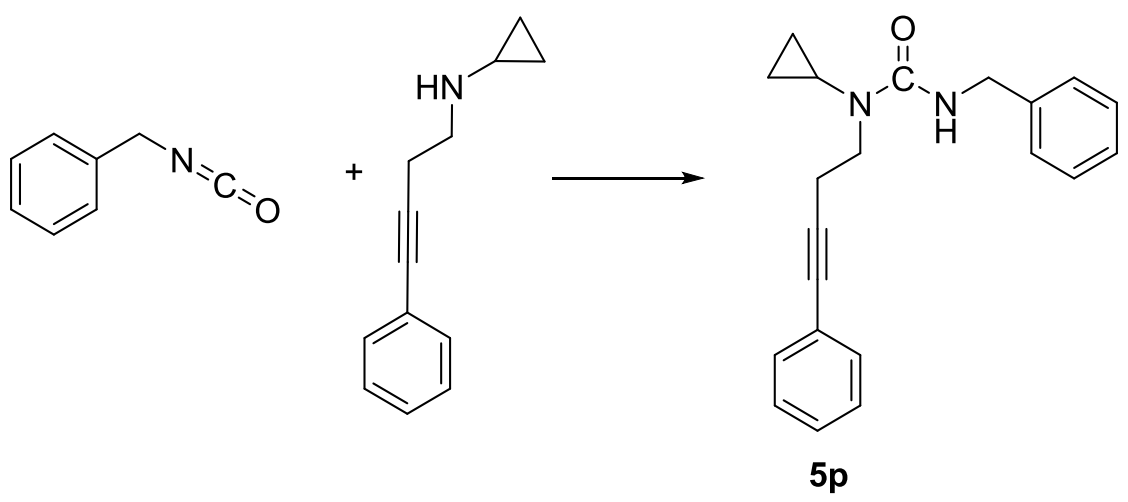

To a solution of $N$-(4-phenylbut-3-yn-1-yl)cyclopropanamine (635 mg, $3.40 \mathrm{mmol}$ ) and $\mathrm{Et}_{3} \mathrm{~N}$ $(0.92 \mathrm{~mL}, 6.20 \mathrm{mmol})$ in $\mathrm{CH}_{2} \mathrm{Cl}_{2}(10 \mathrm{~mL})$ at $0{ }^{\circ} \mathrm{C}$ was added (isocyanatomethyl)benzene $(0.38 \mathrm{~mL}, 3.10 \mathrm{mmol})$. The reaction mixture was warmed to room temperature and stirred for 18 hours. The solution was diluted with $\mathrm{CH}_{2} \mathrm{Cl}_{2}(10 \mathrm{~mL})$, washed with water $(15 \mathrm{~mL}), 1 \mathrm{M}$ aqueous $\mathrm{HCl}(15 \mathrm{~mL})$, saturated aqueous $\mathrm{NaHCO}_{3}(15 \mathrm{~mL})$ and brine $(15 \mathrm{~mL})$. The organic layer was dried over $\mathrm{Na}_{2} \mathrm{SO}_{4}$ and concentrated in vacuo. The crude mixture was purified by column chromatography (20\% EtOAc/hexane) to yield the title compound 5p (525 mg, 54\%) as a yellow oil. $v_{\max } / \mathrm{cm}^{-1}: 3373(\mathrm{~m}), 2922(\mathrm{~m}), 1644$ (s), 1513 (s), 1287 (s), 1032 (s); ${ }^{1} \mathrm{H}$ NMR (400 MHz, $\left.\mathrm{CDCl}_{3}\right): 7.32-7.29(2 \mathrm{H}, \mathrm{m}), 7.26-7.16(8 \mathrm{H}, \mathrm{m}), 5.52(1 \mathrm{H}, \mathrm{t}, J=5.7 \mathrm{~Hz})$, $4.40(2 \mathrm{H}, \mathrm{d}, J=5.7 \mathrm{~Hz}), 3.55(2 \mathrm{H}, \mathrm{t}, J=6.8 \mathrm{~Hz}), 2.65(2 \mathrm{H}, \mathrm{t}, J=6.8 \mathrm{~Hz}), 2.57(1 \mathrm{H}, \mathrm{tt}, J=$ 6.4, $4.1 \mathrm{~Hz}), 0.78-0.73(4 \mathrm{H}, \mathrm{m}) ;{ }^{13} \mathrm{C} \mathrm{NMR}\left(101 \mathrm{MHz}, \mathrm{CDCl}_{3}\right): \delta 158.6,139.6,131.5,128.6$, 128.2, 127.6, 127.4, 127.2, 123.8, 88.2, 81.6, 46.5, 44.6, 28.3, 19.4, 8.9; m/z (ESI $\left.{ }^{+}\right) \mathrm{HRMS}$ : $\mathrm{C}_{21} \mathrm{H}_{22} \mathrm{~N}_{2} \mathrm{NaO}$ : 341.1624. Found [M+Na] $]^{+}$341.1618.

(E)-2-Benzyl-6-benzylidenehexahydro-1H-pyrrolo[1,2-c][1,3]diazepine-1,3(2H)-dione 6p
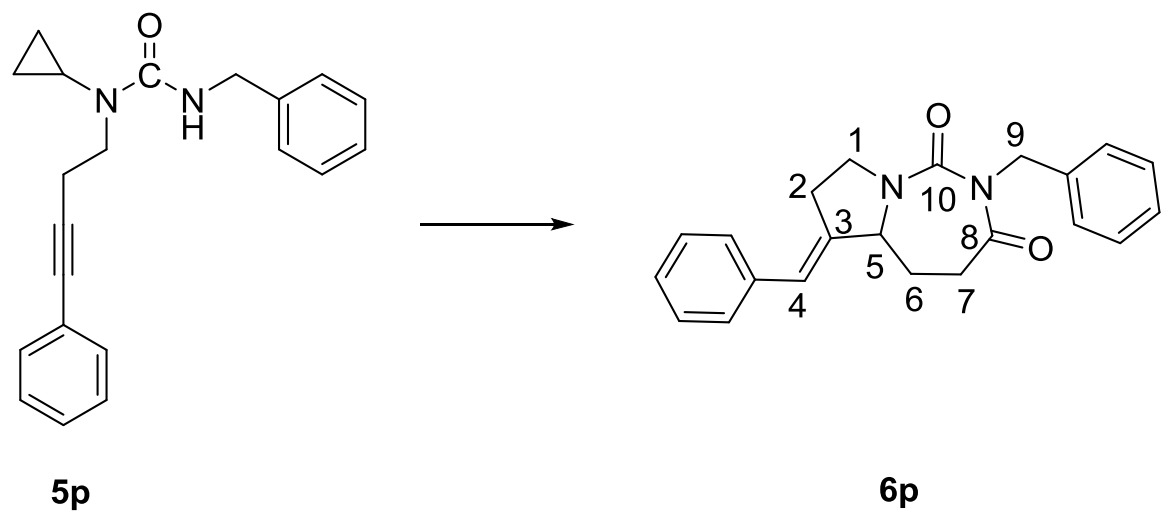

$6 p$ 
An oven dried reaction tube, fitted with a magnetic stirrer, was charged with $\left[\mathrm{Rh}(\operatorname{cod})_{2}\right] \mathrm{OTf}$ (3.52 mg, $7.5 \mu \mathrm{mol})$, 4-(dimethylamino)benzoic acid $(2.48 \mathrm{mg}, 15.0 \mu \mathrm{mol})$, tris(4fluorophenyl)phosphine (4.47mg, $15.0 \mu \mathrm{mol}), \mathrm{Na}_{2} \mathrm{SO}_{4}(14.2 \mathrm{mg}, 0.10 \mathrm{mmol}$ ) and 3-benzyl-1cyclopropyl-1-(4-phenylbut-3-yn-1-yl)urea (31.8 mg, $0.10 \mathrm{mmol})$. The tube was fitted with a rubber septum and purged with argon. Anhydrous 1,2-DCB $(1.00 \mathrm{~mL})$ was added by syringe. The reaction vessel was purged with $\mathrm{CO}$ for 10 minutes and the solution was subsequently sparged with CO for approximately 20 seconds. The mixture was then heated at $130{ }^{\circ} \mathrm{C}$, under a $\mathrm{CO}$ atmosphere (with $\mathrm{CO}$ balloon attached) (1 atm) for 20 hours. The mixture was cooled to room temperature, concentrated in vacuo. The crude mixture was purified by column chromatography ( $8 \%$ toluene/hexane) to yield the title compound $\mathbf{6 p}(25.3 \mathrm{mg}, 73 \%$ ) as a colourless solid; m.p. $152-154{ }^{\circ} \mathrm{C}\left(\mathrm{CDCl}_{3}\right) ; v_{\max } / \mathrm{cm}^{-1}: 2950(\mathrm{~m}), 1694$ (s), 1652 (s), 1414 (s), 1351 (s), 699 (s); ${ }^{1} \mathrm{H}$ NMR (400 MHz, $\left.\mathrm{CDCl}_{3}\right): 7.32$ - 7.15 (10H, m, $\left.10 \times \mathrm{ArCH}\right)$, $6.25(1 \mathrm{H}, \mathrm{dd}, J=2.1,2.1 \mathrm{~Hz}, 1 \times \mathrm{C} 4-\underline{\mathrm{H}}), 4.89\left(1 \mathrm{H}, \mathrm{d}, J=14.4 \mathrm{~Hz}, 1 \times \mathrm{C} 9-\underline{\mathrm{H}}_{2}\right), 4.78(1 \mathrm{H}, \mathrm{d}, J$ $\left.=14.4 \mathrm{~Hz}, 1 \times \mathrm{C} 9-\underline{\mathrm{H}}_{2}\right), 4.28(1 \mathrm{H}, \mathrm{dd}, J=12.5,6.4 \mathrm{~Hz}, 1 \times \mathrm{C} 5-\underline{\mathrm{H}}), 3.74(1 \mathrm{H}, \mathrm{ddd}, J=11.6,8.1$, $\left.5.4 \mathrm{~Hz}, 1 \times \mathrm{C} 1-\underline{\mathrm{H}}_{2}\right), 3.62\left(1 \mathrm{H}, \mathrm{ddd}, J=11.6,8.1,6.4 \mathrm{~Hz}, 1 \times \mathrm{C} 1-\underline{\mathrm{H}}_{2}\right), 2.86-2.82(2 \mathrm{H}, \mathrm{m}, 2$ $\left.\times \mathrm{C} 2-\underline{\mathrm{H}}_{2}\right), 2.73-2.65\left(1 \mathrm{H}, \mathrm{m}, 1 \times \mathrm{C} 7-\underline{\mathrm{H}}_{2}\right), 2.58\left(1 \mathrm{H}, \mathrm{ddd}, J=12.5,6.5,1.3 \mathrm{~Hz}, 1 \times \mathrm{C} 7-\underline{\mathrm{H}}_{2}\right)$, $2.41-2.33\left(1 \mathrm{H}, \mathrm{m}, 1 \times \mathrm{C6}-\underline{\mathrm{H}}_{2}\right), 1.94\left(1 \mathrm{H}, \mathrm{dddd}, J=13.4,12.5,8.6,1.4 \mathrm{~Hz}, 1 \times \mathrm{C6}-\underline{\mathrm{H}}_{2}\right) ;{ }^{13} \mathrm{C}$ NMR (101 MHz, $\mathrm{CDCl}_{3}$ ): $\delta 172.2$ (C8), 155.8 (C10), 140.8 (C3), 138.0 (CAr), 136.3 (CAr), 128.5 (CAr), 128.4 (CAr), 128.3 (CAr), 127.3 (CAr), 127.2 (CAr), 123.0 (C4), 61.5 (C5), 46.8 (C9), 46.5 (C1), 34.7 (C6), 34.3 (C7), 27.5 (C2); One aromatic carbon signal is overlapped; $m / z\left(\mathrm{ESI}^{+}\right) \mathrm{HRMS}: \mathrm{C}_{22} \mathrm{H}_{22} \mathrm{~N}_{2} \mathrm{NaO}_{2}$ : 369.1573. Found [M+Na] $]^{+}: 369.1561$.

(E)-N-Cyclopropyl- $N$-(hexa-3,5-dien-1-yl)-1H-pyrrole-3-carboxamide 5q

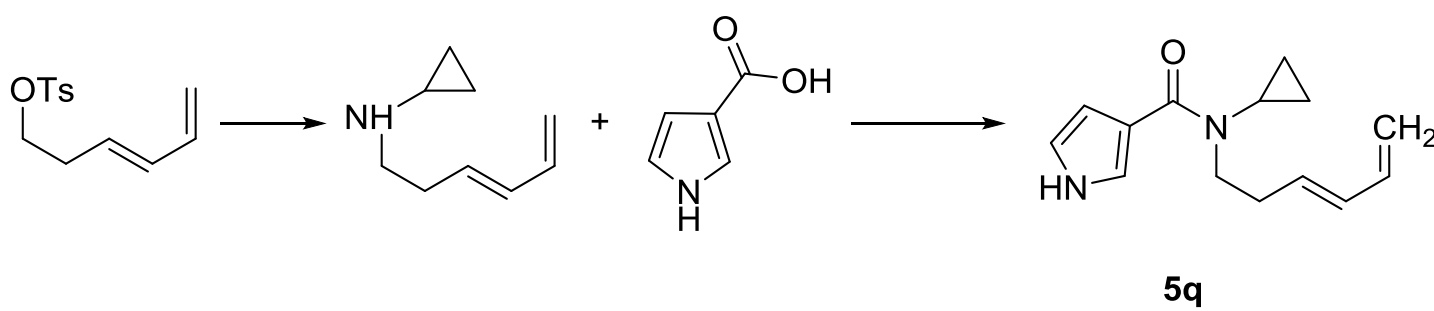

To a flask containing (E)-hexa-3,5-dien-1-yl 4-methylbenzenesulfonate (prepared according to the literature report $)^{12}(504 \mathrm{mg}, 2.00 \mathrm{mmol})$ in $\mathrm{MeCN}(8.0 \mathrm{~mL})$ was added cyclopropylamine $(0.55 \mathrm{~mL}, 8.00 \mathrm{mmol})$. The flask sealed and heated at $90{ }^{\circ} \mathrm{C}$ for $16 \mathrm{~h}$. The reaction mixture was cooled to room temperature and trifluoroacetic acid $(0.23 \mathrm{ml}, 3.00 \mathrm{mmol})$ was added and the reaction mixture was stirred for 10 minutes. Then the mixture was 
concentrated in vacuo and dissolved in $\mathrm{CH}_{2} \mathrm{Cl}_{2}(20 \mathrm{~mL})$, washed with saturated aqueous $\mathrm{NaHCO}_{3}(20 \mathrm{~mL})$. The aqueous portion was extracted with $\mathrm{CH}_{2} \mathrm{Cl}_{2}(2 \times 15 \mathrm{~mL})$ and the organic extracts were combined, washed with brine $(35 \mathrm{~mL})$, dried over $\mathrm{Na}_{2} \mathrm{SO}_{4}$ and concentrated in vacuo. The residue was dissolved in $\mathrm{CH}_{2} \mathrm{Cl}_{2}(8.0 \mathrm{~mL})$ and under an atmosphere of nitrogen, EDCI (458 mg, $2.40 \mathrm{mmol}$ ) and DMAP (24.4 mg, $0.20 \mathrm{mmol}$ ) were added. $1 \mathrm{H}$-pyrrole-3-carboxylic acid was then added $(244 \mathrm{mg}, 2.20 \mathrm{mmol})$ portionwise over 5 minutes. The mixture was stirred at room temperature for 18 hours. Then the mixture was concentrated in vacuo and suspended in $1 \mathrm{M}$ aqueous $\mathrm{NaOH}(10 \mathrm{~mL})$ and extracted with $\mathrm{CH}_{2} \mathrm{Cl}_{2}(2 \times 10 \mathrm{~mL})$. The organic extracts were combined and washed with $1 \mathrm{M}$ aqueous $\mathrm{HCl}$ $(10 \mathrm{~mL})$, brine $(20 \mathrm{~mL})$, dried over $\mathrm{Na}_{2} \mathrm{SO}_{4}$ and concentrated in vacuo. The residue was purified by flash column chromatography (60\% EtOAc/hexane) to afford the title compound 5q $(267 \mathrm{mg}, 58 \%)$ as an off-white solid; m.p. 56-58 ${ }^{\circ} \mathrm{C}$ (EtOAc/hexane); $v_{\max } / \mathrm{cm}^{-1}: 3214(\mathrm{~m})$, 2972 (m), 1588 (s), 1415 (s), 1054 (s), 755 (s); ${ }^{1} \mathrm{H}$ NMR (400 MHz, CDCl 3 ): 9.00 (1H, s, br), $7.20(1 \mathrm{H}, \mathrm{s}, b r), 6.65(1 \mathrm{H}, \mathrm{s}, b r), 6.53(1 \mathrm{H}, \mathrm{s}, b r), 6.26(1 \mathrm{H}, \mathrm{ddd}, J=17.0,10.4,10.4 \mathrm{~Hz})$, $6.09(1 \mathrm{H}, \mathrm{dd}, J=15.0,10.4 \mathrm{~Hz}), 5.67(1 \mathrm{H}, \mathrm{dt}, J=15.0,7.2 \mathrm{~Hz}), 5.06(1 \mathrm{H}, \mathrm{dd}, J=17.0,1.7$ $\mathrm{Hz}), 5.02-4.82(1 \mathrm{H}, \mathrm{m}), 3.58(2 \mathrm{H}, \mathrm{t}, J=7.2 \mathrm{~Hz}), 2.83(1 \mathrm{H}, \mathrm{tt}, J=7.0,4.0 \mathrm{~Hz}), 2.45-2.40$ $(2 \mathrm{H}, \mathrm{m}), 0.80-0.75(2 \mathrm{H}, \mathrm{m}), 0.63-0.59(2 \mathrm{H}, \mathrm{m}) ;{ }^{13} \mathrm{C} \mathrm{NMR}\left(101 \mathrm{MHz}, \mathrm{CDCl}_{3}\right): \delta 168.5$, 137.0, 132.8, 131.8, 122.1, 120.0, 117.5, 115.5, 109.8, 47.2, 31.5, 31.0, 10.4; m/z $\left(\mathrm{ESI}^{+}\right)$ HRMS: $\mathrm{C}_{14} \mathrm{H}_{18} \mathrm{~N}_{2} \mathrm{NaO}: 253.1311$. Found $[\mathrm{M}+\mathrm{Na}]^{+}: 253.1305$.

\section{7-Methyl-5,6,6a,7,8,9,10,11-octahydro-3H-azepino[1,2- $a]$ pyrrolo[2,3-f]azocine-4,13-}

\section{dione 6q}

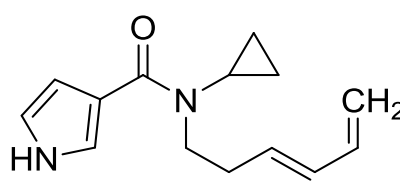

$5 q$

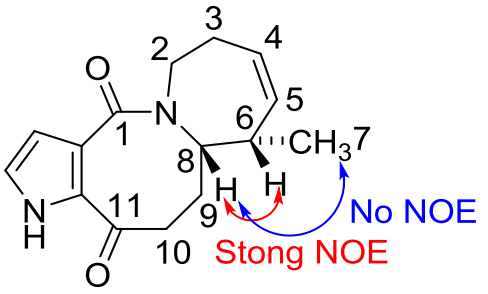

$6 q$

An oven dried reaction tube, fitted with a magnetic stirrer, was charged with $\left[\mathrm{Rh}(\mathrm{cod})_{2}\right]$ BARF $(8.87 \mathrm{mg}, 7.5 \mu \mathrm{mol}), 2$-nitrobenzoic acid $(3.34 \mathrm{mg}, 20.0 \mu \mathrm{mol})$, tris(3,5dimethylphenyl)phosphine $(5.19 \mathrm{mg}, 15.0 \mu \mathrm{mol})$ and $(E)-N$-cyclopropyl- $N$-(hexa-3,5-dien-1yl)-1H-pyrrole-3-carboxamide 5q $(22.8 \mathrm{mg}, 0.10 \mathrm{mmol})$. The tube was fitted with a rubber septum and purged with argon. Anhydrous benzonitrile $(1.00 \mathrm{~mL})$ was added by syringe. The 
reaction vessel was purged with $\mathrm{CO}$ for 10 minutes and the solution was subsequently sparged with CO for approximately 20 seconds. The mixture was then heated at $130{ }^{\circ} \mathrm{C}$, under a $\mathrm{CO}$ atmosphere (with $\mathrm{CO}$ balloon attached) (1 atm) for 72 hours. The mixture was cooled to room temperature, concentrated in vacuo. The crude mixture was purified by column chromatography ( $20 \%$ actone/hexane) to yield the title compound $\mathbf{6 q}(9.1 \mathrm{mg}, 36 \%)$ as a colourless oil;

The relative stereochemistry of product $\mathbf{6 q}$ was corroborated by nOe experiments (as indicated on the compound structure). A strong nOe correlations was observed between C6$H$ and C8-H and no nOe correlations was observed between $C 7-H$ and $C 8-H$.

$v_{\max } / \mathrm{cm}^{-1}: 3223$ (m), 2923 (m), 1645 (s), 1600 (s), 1484 (s), 1400 (s); ${ }^{1} \mathrm{H}$ NMR (500 MHz, $\left.\mathrm{CDCl}_{3}, 50{ }^{\circ} \mathrm{C}\right): 9.31(0.5 \mathrm{H}, \mathrm{s}, b r, \underline{\mathrm{H}}), 6.97-6.96(1 \mathrm{H}, \mathrm{m}, 1 \times \mathrm{ArC} \underline{\mathrm{H}}), 6.72-6.70(1 \mathrm{H}, \mathrm{m}, 1$ $\times \mathrm{ArCH}), 5.41-5.31(2 \mathrm{H}, \mathrm{m}, 1 \times \mathrm{C} 4-\underline{\mathrm{H}}, 1 \times \mathrm{C} 5-\underline{\mathrm{H}}), 3.98-3.92\left(1 \mathrm{H}, \mathrm{m}, 1 \times \mathrm{C} 2-\underline{\mathrm{H}}_{2}\right), 3.81$ $(1 \mathrm{H}, \mathrm{dd}, J=13.1,4.5 \mathrm{~Hz}, 1 \times \mathrm{C} 8-\underline{\mathrm{H}}), 3.58\left(1 \mathrm{H}, \mathrm{ddd}, J=12.2,8.9,2.9 \mathrm{~Hz}, 1 \times \mathrm{C} 2-\underline{\mathrm{H}}_{2}\right), 3.02$ $\left(1 \mathrm{H}, \mathrm{ddd}, J=13.8,12.2,5.3 \mathrm{~Hz}, 1 \times \mathrm{C10}-\underline{\mathrm{H}}_{2}\right), 2.60-2.47\left(2 \mathrm{H}, \mathrm{m}, 1 \times \mathrm{C} \mathbf{1 0}-\underline{\mathrm{H}}_{2}, 1 \times \mathrm{C6}-\underline{\mathrm{H}}\right)$, $2.33-2.15\left(1 \mathrm{H}, \mathrm{m}, 1 \times \mathrm{C} 3-\underline{\mathrm{H}}_{2}\right), 2.02-1.99\left(1 \mathrm{H}, \mathrm{m}, 1 \times \mathrm{C} 9-\underline{\mathrm{H}}_{2}\right), 1.92-1.73(2 \mathrm{H}, \mathrm{m}, 1 \times \mathrm{C} 9-$ $\left.\underline{\mathrm{H}}_{2}, 1 \times \mathrm{C} 3-\underline{\mathrm{H}}_{2}\right), 1.56\left(3 \mathrm{H}, \mathrm{d}, J=5.2 \mathrm{~Hz}, 3 \times \mathrm{C} 7-\underline{\mathrm{H}}_{3}\right) ;{ }^{13} \mathrm{C} \mathrm{NMR}\left(101 \mathrm{MHz}, \mathrm{CDCl}_{3}\right): \delta 190.3$ (C11), 165.3 (C1), 131.4 (C5), 130 (CAr), 125.5 (C4), 125.4 (CAr), 123.5 (CAr), 113.9 (CAr), 62.94 (C8), 46.5 (C6), 44.6 (C2), 37.3 (C10), 34.2 (C9), 28.4 (C3), 17.8 (C7); m/z $\left(\mathrm{ESI}^{+}\right)$HRMS: $\mathrm{C}_{15} \mathrm{H}_{18} \mathrm{~N}_{2} \mathrm{NaO}_{2}: 281.1260$. Found $[\mathrm{M}+\mathrm{Na}]^{+}: 281.1248$.

\section{$N$-Benzyl- $N$-ethyl-1H-indole-1-carboxamide ethyl-3a}

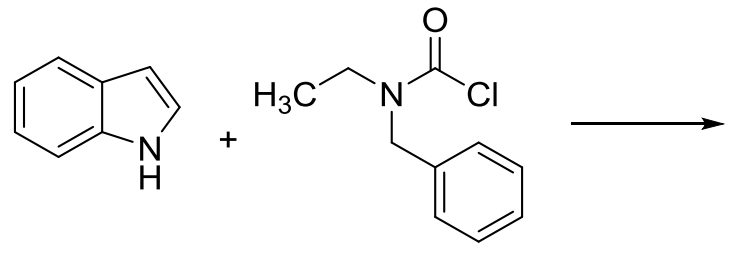

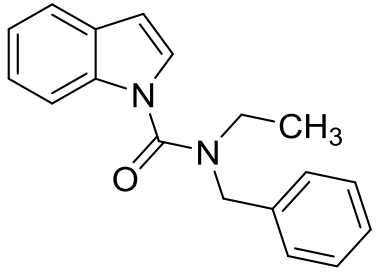

ethyl-3a

General procedure A: Indole $(292 \mathrm{mg}, 2.50 \mathrm{mmol})$ and benzyl(ethyl)carbamic chloride (prepared according to the literature procedure $)^{13}(1.00 \mathrm{~g}, 5.10 \mathrm{mmol})$ were employed. The crude mixture was purified by flash column chromatography ( $80 \%$ toluene/hexane) to afford the title compound ethyl-3a (536 mg, 77\%) as a yellow oil; $v_{\max } / \mathrm{cm}^{-1}: 1674$ (s), 1451 (s), 1416 (s), 1318 (s), 1210 (s), 747 (s); ${ }^{1} \mathrm{H}$ NMR (400 MHz, $\mathrm{CDCl}_{3}$ ): $\delta 7.75-7.73$ (1H, m), $7.62-7.59(1 \mathrm{H}, \mathrm{m}), 7.41-7.36(2 \mathrm{H}, \mathrm{m}), 7.34-7.29(5 \mathrm{H}, \mathrm{m}), 7.21(1 \mathrm{H}, \mathrm{ddd}, J=8.1,7.2,1.0$ 
Hz), $6.58(1 \mathrm{H}, \mathrm{dd}, J=3.5,0.8 \mathrm{~Hz}), 4.67(2 \mathrm{H}, \mathrm{s}), 3.44(2 \mathrm{H}, \mathrm{q}, J=7.1 \mathrm{~Hz}), 1.24(3 \mathrm{H}, \mathrm{t}, J=7.1$ $\mathrm{Hz}) ;{ }^{13} \mathrm{C} \mathrm{NMR}\left(101 \mathrm{MHz}, \mathrm{CDCl}_{3}\right): \delta 155.0,136.6,135.7,129.4,128.8,127.7,127.6,125.8$, 123.6, 121.8, 121.0, 113.2, 105.8, 51.0, 42.5, 12.9; m/z (ESI $\left.{ }^{+}\right)$HRMS: Calculated for $\mathrm{C}_{18} \mathrm{H}_{18} \mathrm{~N}_{2} \mathrm{NaO}: 301.1311$. Found [M+Na] $]^{+}: 301.1317$.

\section{Mechanistic experiments}

\section{Equation 1:}

(1)

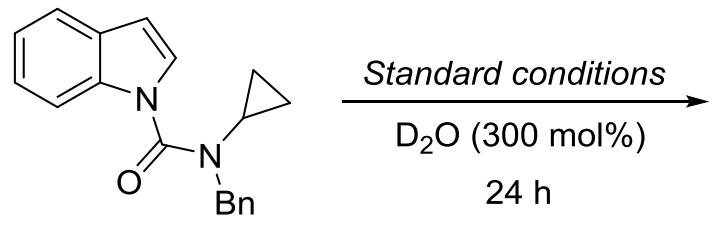

$3 a$

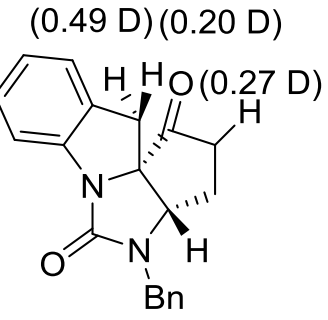

deuterio-4aa $42 \%$ Yield<smiles>O=C(N(Cc1ccccc1)c1ccccc1)N(Cc1ccccc1)C1CC1</smiles>

deuterio-3aa $32 \%$ Yield

An oven dried reaction tube, fitted with a magnetic stirrer, was charged with $\left[\mathrm{Rh}(\operatorname{cod})_{2}\right] \mathrm{OTf}$ (5.28 mg, $11.2 \mu \mathrm{mol})$, 4-(dimethylamino)benzoic acid (7.43 mg, $45.0 \mu \mathrm{mol})$ and substrate $\mathbf{3 a}$ (43.5 $\mathrm{mg}, 0.15 \mathrm{mmol}$ ). The tube was fitted with a rubber septum and purged with argon. Anhydrous 1,2-DCB (1.50 mL) was added by syringe. The reaction vessel was purged with $\mathrm{CO}$ for 10 minutes and the solution was subsequently sparged with $\mathrm{CO}$ for approximately 20 seconds. Then the $\mathrm{CO}$ balloon was removed and $\mathrm{D}_{2} \mathrm{O}(5.40 \mathrm{uL}, 0.30 \mathrm{mmol})$ was added. The tube was sealed and the reaction was heated at $130{ }^{\circ} \mathrm{C}$ for 24 hours. The mixture was cooled to room temperature and concentrated in vacuo. The residue was purified by flash column chromatography (10\% EtOAc/hexane then 5\% EtOAc/toluene) to afford deuterio-3aa (13.8 $\mathrm{mg}, 32 \%)$ and deuterio-4aa (19.9 mg, 42\%). The percentage of deuterium incorporation was measured by ${ }^{1} \mathrm{H}$ NMR. 

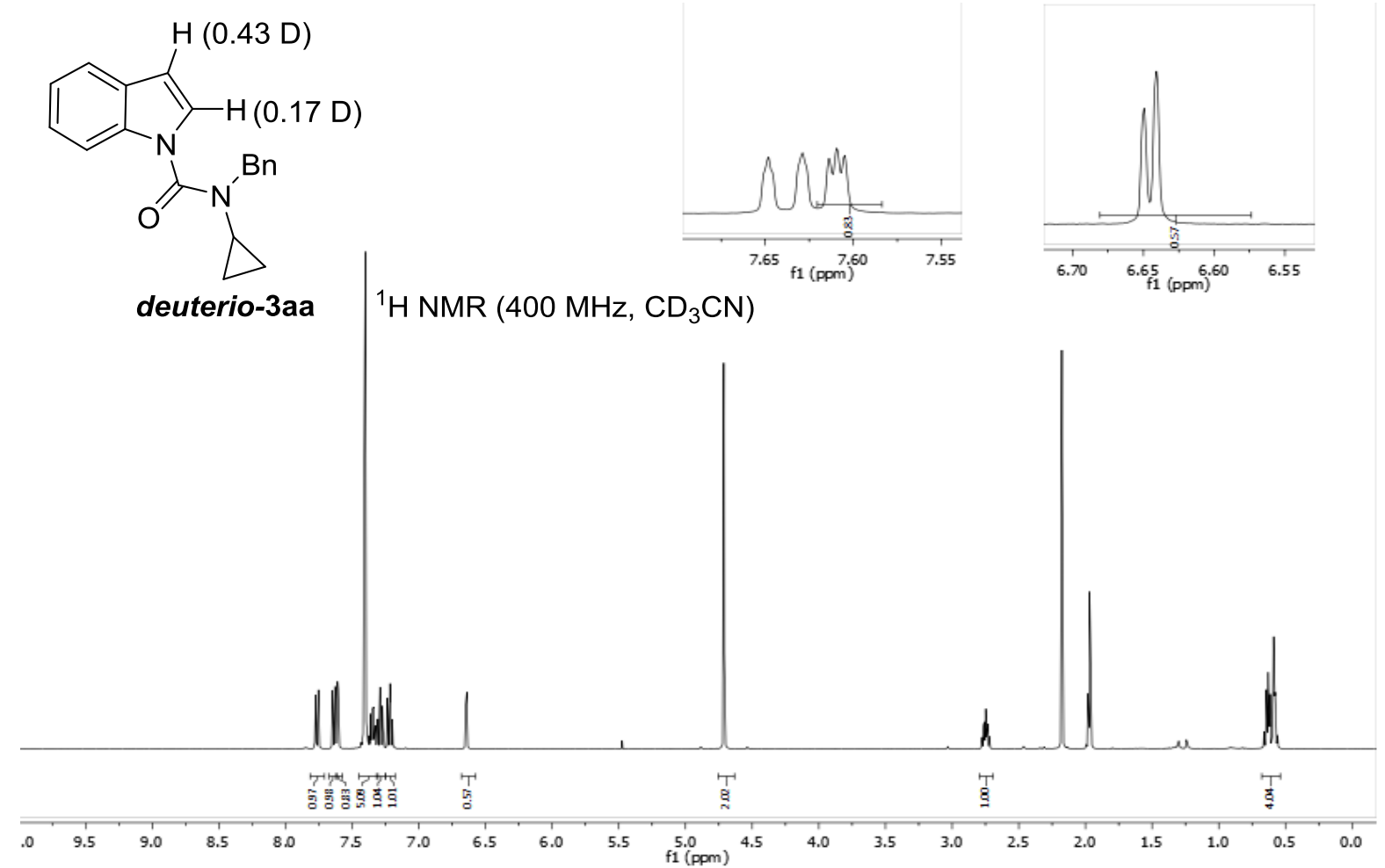

(0.49 D) (0.20 D)
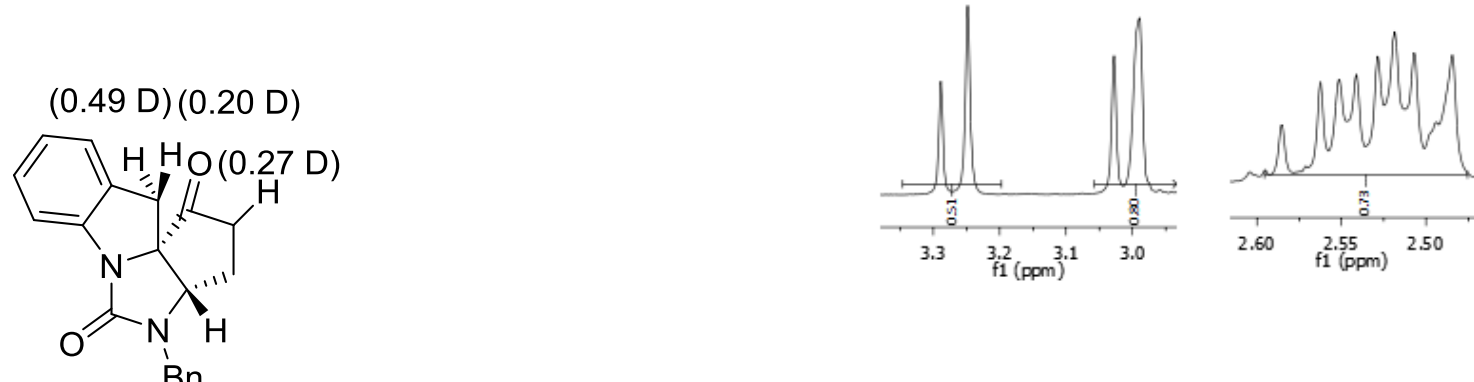

deuterio-4aa $\quad{ }^{1} \mathrm{H}$ NMR $\left(400 \mathrm{MHz}, \mathrm{CDCl}_{3}\right)$

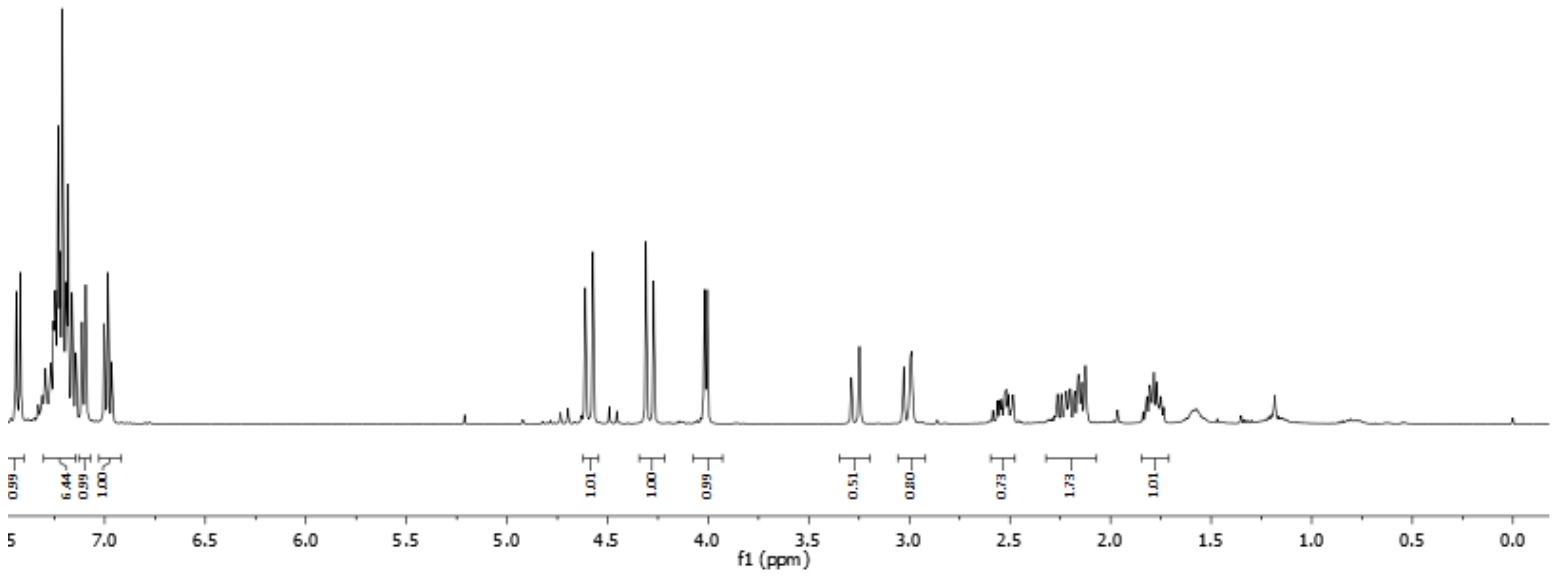




\section{Equation 2:}

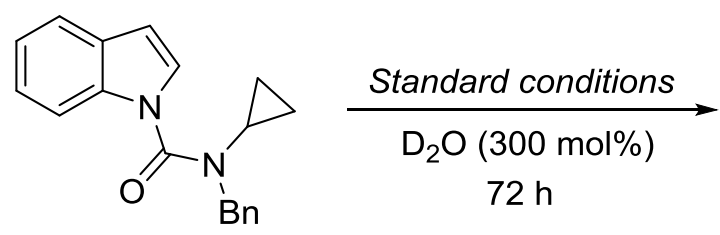

$3 a$
(0.39 D) (0.14 D)

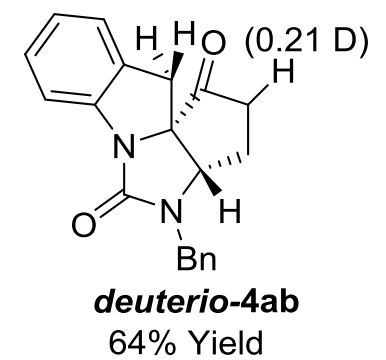

An oven dried reaction tube, fitted with a magnetic stirrer, was charged with $\left[\mathrm{Rh}(\operatorname{cod})_{2}\right] \mathrm{OTf}$ (5.28 mg, $11.2 \mu \mathrm{mol})$, 4-(dimethylamino)benzoic acid $(7.43 \mathrm{mg}, 45.0 \mu \mathrm{mol})$ and substrate $\mathbf{3 a}$ (43.5 $\mathrm{mg}, 0.15 \mathrm{mmol}$ ). The tube was fitted with a rubber septum and purged with argon. Anhydrous 1,2-DCB (1.50 mL) was added by syringe. The reaction vessel was purged with $\mathrm{CO}$ for 10 minutes and the solution was subsequently sparged with $\mathrm{CO}$ for approximately 20 seconds. Then the $\mathrm{CO}$ balloon was removed and $\mathrm{D}_{2} \mathrm{O}(5.40 \mathrm{uL}, 0.30 \mathrm{mmol})$ was added. The tube was sealed and the reaction was heated at $130{ }^{\circ} \mathrm{C}$ for 72 hours. The mixture was cooled to room temperature and concentrated in vacuo. The residue was purified by flash column chromatography (5\% EtOAc/toluene) to afford deuterio-4ab (30.5 mg, 64\%). The percentage of deuterium incorporation was measured by ${ }^{1} \mathrm{H}$ NMR.

(0.39 D) (0.14 D)

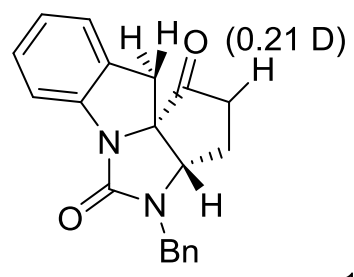
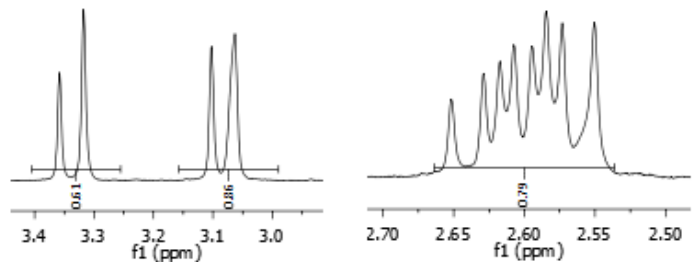
deuterio-4ab $\quad{ }^{1} \mathrm{H}$ NMR $\left(400 \mathrm{MHz}, \mathrm{CDCl}_{3}\right)$

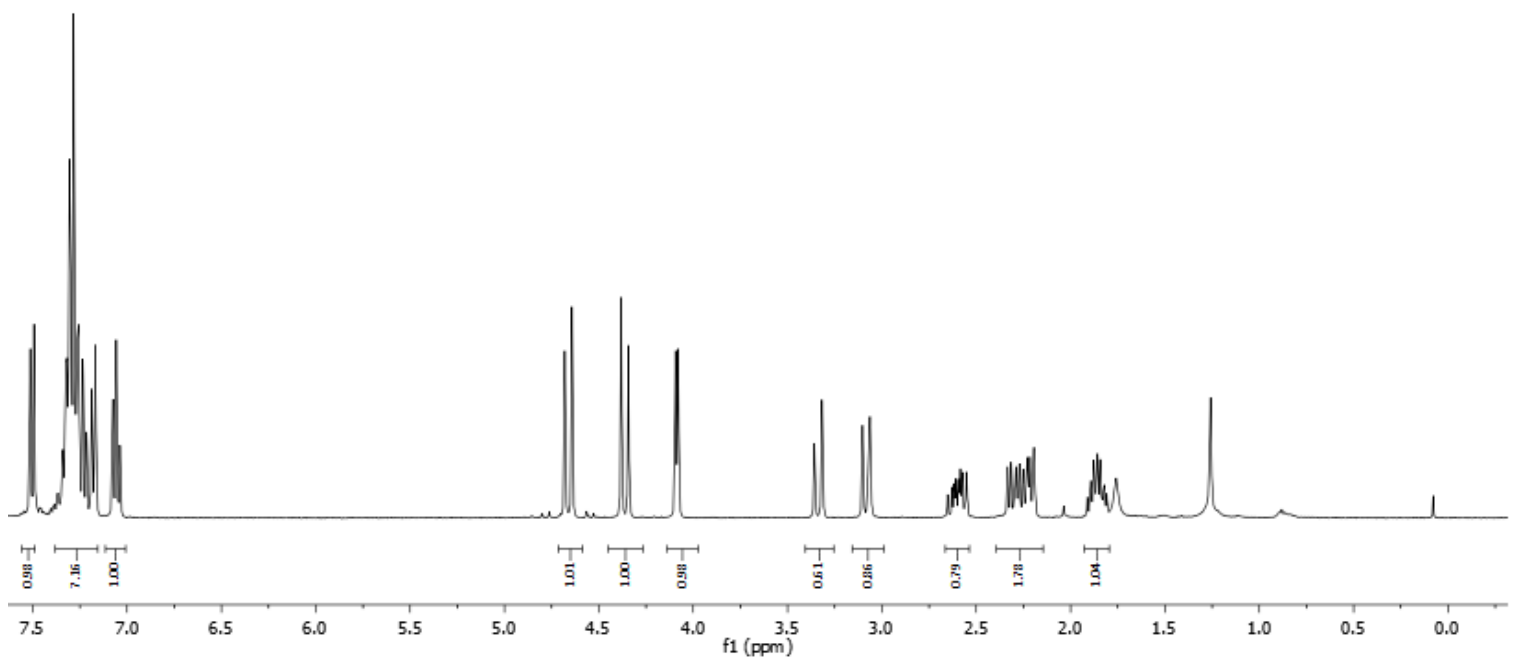




\section{Equation 3:}

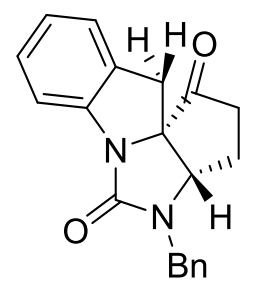

$4 a$

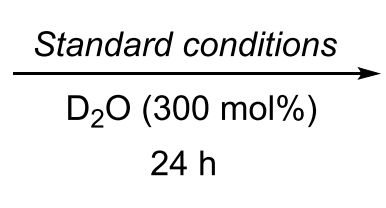

deuterio-4ac, 98\% Yield

An oven dried reaction tube, fitted with a magnetic stirrer, was charged with $\left[\mathrm{Rh}(\mathrm{cod})_{2}\right] \mathrm{OTf}$ (5.28 mg, $11.2 \mu \mathrm{mol})$, 4-(dimethylamino)benzoic acid (7.43 mg, $45.0 \mu \mathrm{mol})$ and compound 4a $(47.7 \mathrm{mg}, 0.15 \mathrm{mmol})$. The tube was fitted with a rubber septum and purged with argon. Anhydrous 1,2-DCB (1.50 mL) was added by syringe. The reaction vessel was purged with CO for 10 minutes and the solution was subsequently sparged with CO for approximately 20 seconds. Then the $\mathrm{CO}$ balloon was removed and $\mathrm{D}_{2} \mathrm{O}(5.40 \mathrm{uL}, 0.30 \mathrm{mmol})$ was added. The tube was sealed and the reaction was heated at $130{ }^{\circ} \mathrm{C}$ for 24 hours. The mixture was cooled to room temperature and concentrated in vacuo. The residue was purified by flash column chromatography (5\% EtOAc/toluene) to afford deuterio-4ac $(47.1 \mathrm{mg}, 98 \%)$ The percentage of deuterium incorporation was measured by ${ }^{1} \mathrm{H}$ NMR.

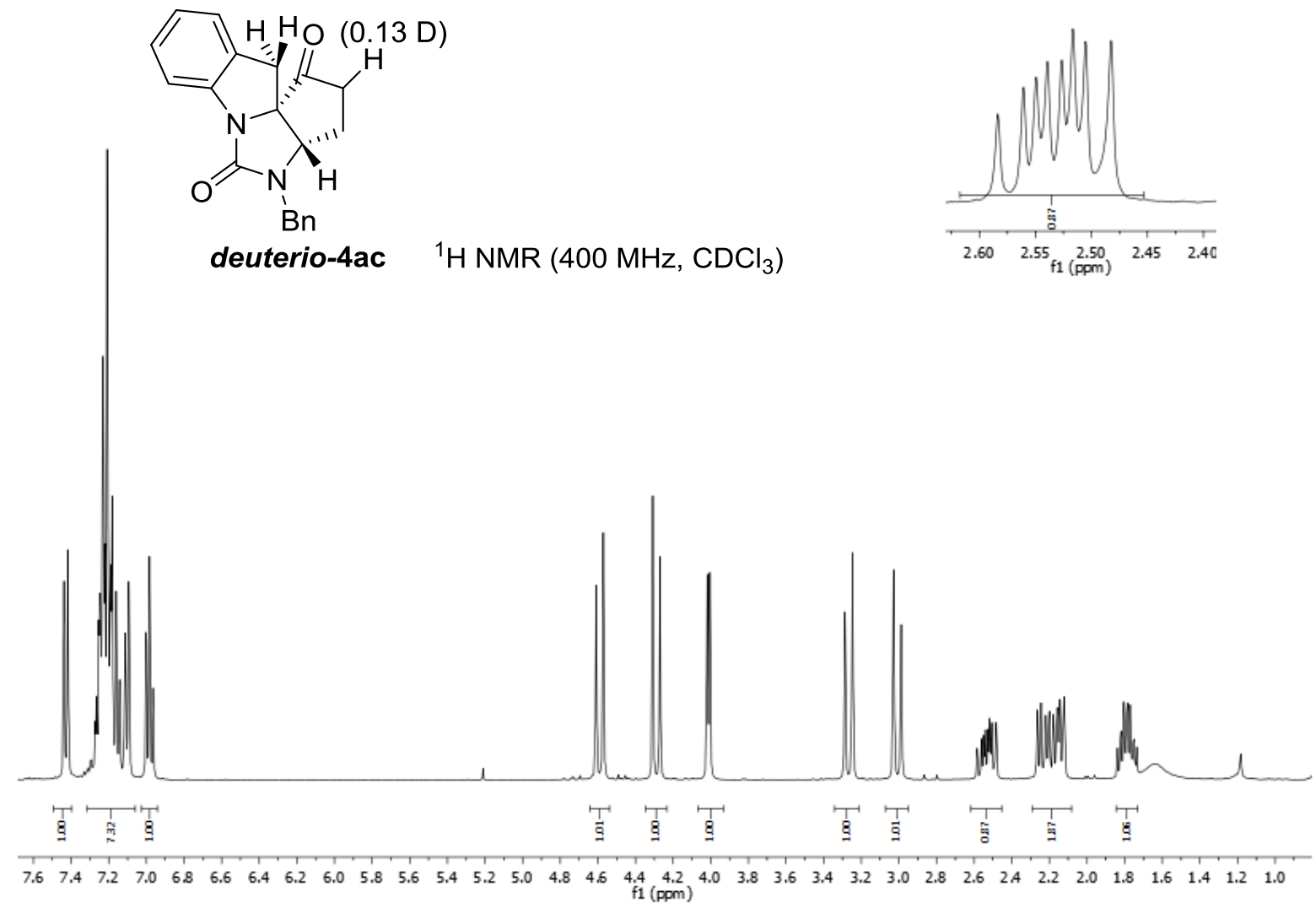




\section{Equation 4:}

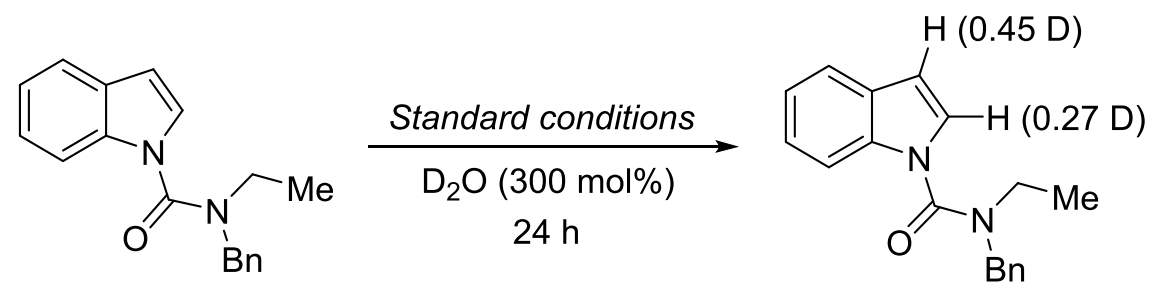

ethyl-3a

deuterio-ethyl-3a, 97\% Yield

An oven dried reaction tube, fitted with a magnetic stirrer, was charged with $\left[\mathrm{Rh}(\operatorname{cod})_{2}\right] \mathrm{OTf}$ (5.28 mg, $11.2 \mu \mathrm{mol})$, 4-(dimethylamino)benzoic acid (7.43 mg, $45.0 \mu \mathrm{mol})$ and substrate ethyl-3a (41.7 mg, $0.15 \mathrm{mmol})$. The tube was fitted with a rubber septum and purged with argon. Anhydrous 1,2-DCB (1.50 mL) was added by syringe. The reaction vessel was purged with $\mathrm{CO}$ for 10 minutes and the solution was subsequently sparged with $\mathrm{CO}$ for approximately 20 seconds. Then the $\mathrm{CO}$ balloon was removed and $\mathrm{D}_{2} \mathrm{O}(5.40 \mathrm{uL}, 0.30 \mathrm{mmol})$ was added. The tube was sealed and the reaction was heated at $130{ }^{\circ} \mathrm{C}$ for 24 hours. The mixture was cooled to room temperature and concentrated in vacuo. The residue was purified by flash column chromatography (3\% EtOAc/toluene) to afford deuterio-ethyl-3a (40.3 mg, 97\%) The percentage of deuterium incorporation was measured by ${ }^{1} \mathrm{H}$ NMR.<smiles></smiles>

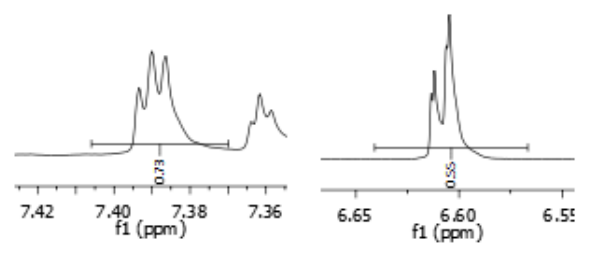

deuterio-ethyl-3a ${ }^{1} \mathrm{H}$ NMR $\left(400 \mathrm{MHz}, \mathrm{CD}_{3} \mathrm{OD}\right)$

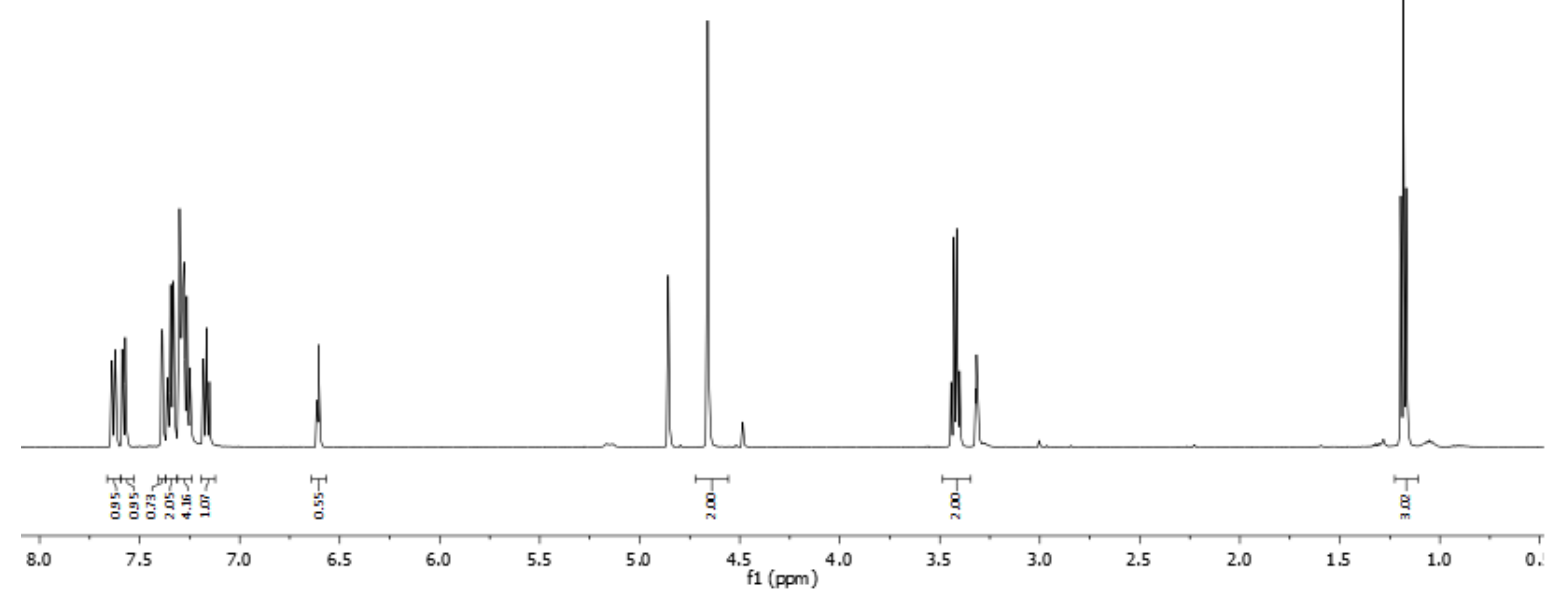


<smiles>O=C(N(Cc1ccccc1)C1CC1)n1ccc2ccccc21</smiles>

$3 a$

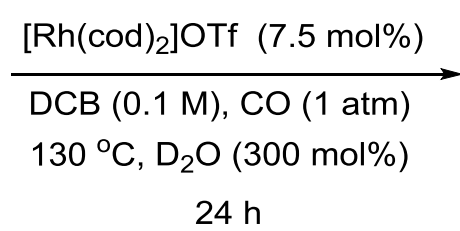

$24 \mathrm{~h}$

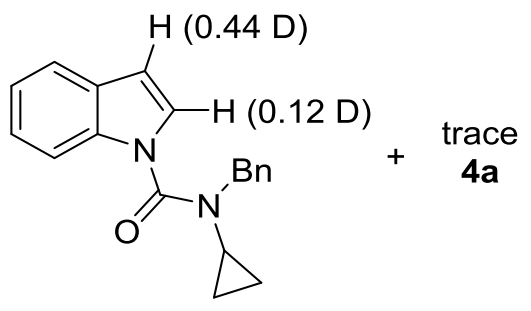

deuterio-3a', 56\% Yield

An oven dried reaction tube, fitted with a magnetic stirrer, was charged with $\left[\mathrm{Rh}(\operatorname{cod})_{2}\right] \mathrm{OTf}$ $(5.28 \mathrm{mg}, 11.2 \mu \mathrm{mol})$ and substrate $\mathbf{3 a}(43.5 \mathrm{mg}, 0.15 \mathrm{mmol})$. The tube was fitted with a rubber septum and purged with argon. Anhydrous 1,2-DCB $(1.50 \mathrm{~mL})$ was added by syringe. The reaction vessel was purged with $\mathrm{CO}$ for 10 minutes and the solution was subsequently sparged with $\mathrm{CO}$ for approximately 20 seconds. Then the $\mathrm{CO}$ balloon was removed and $\mathrm{D}_{2} \mathrm{O}$ (5.40 uL, $0.30 \mathrm{mmol}$ ) was added. The tube was sealed and the reaction was heated at $130{ }^{\circ} \mathrm{C}$ for 24 hours. The mixture was cooled to room temperature and concentrated in vacuo. The residue was purified by flash column chromatography (10\% EtOAc/hexane) to afford deuterio-3a' $(24.3 \mathrm{mg}, 56 \%)$ and trace amount of product $4 \mathbf{a}$. The percentage of deuterium incorporation was measured by ${ }^{1} \mathrm{H}$ NMR.<smiles>O=C(O[Na])c1c(C=[18O])n(C(=O)N(Cc2ccccc2)C2CC2)c2ccccc12</smiles>

deuterio-3a' ${ }^{1} \mathrm{H}$ NMR $\left(400 \mathrm{MHz}, \mathrm{CD}_{3} \mathrm{CN}\right)$
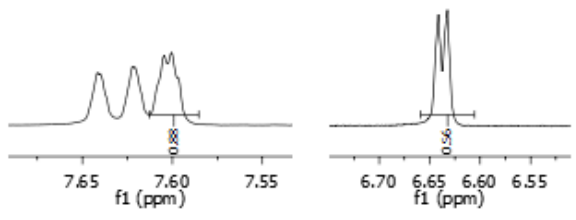

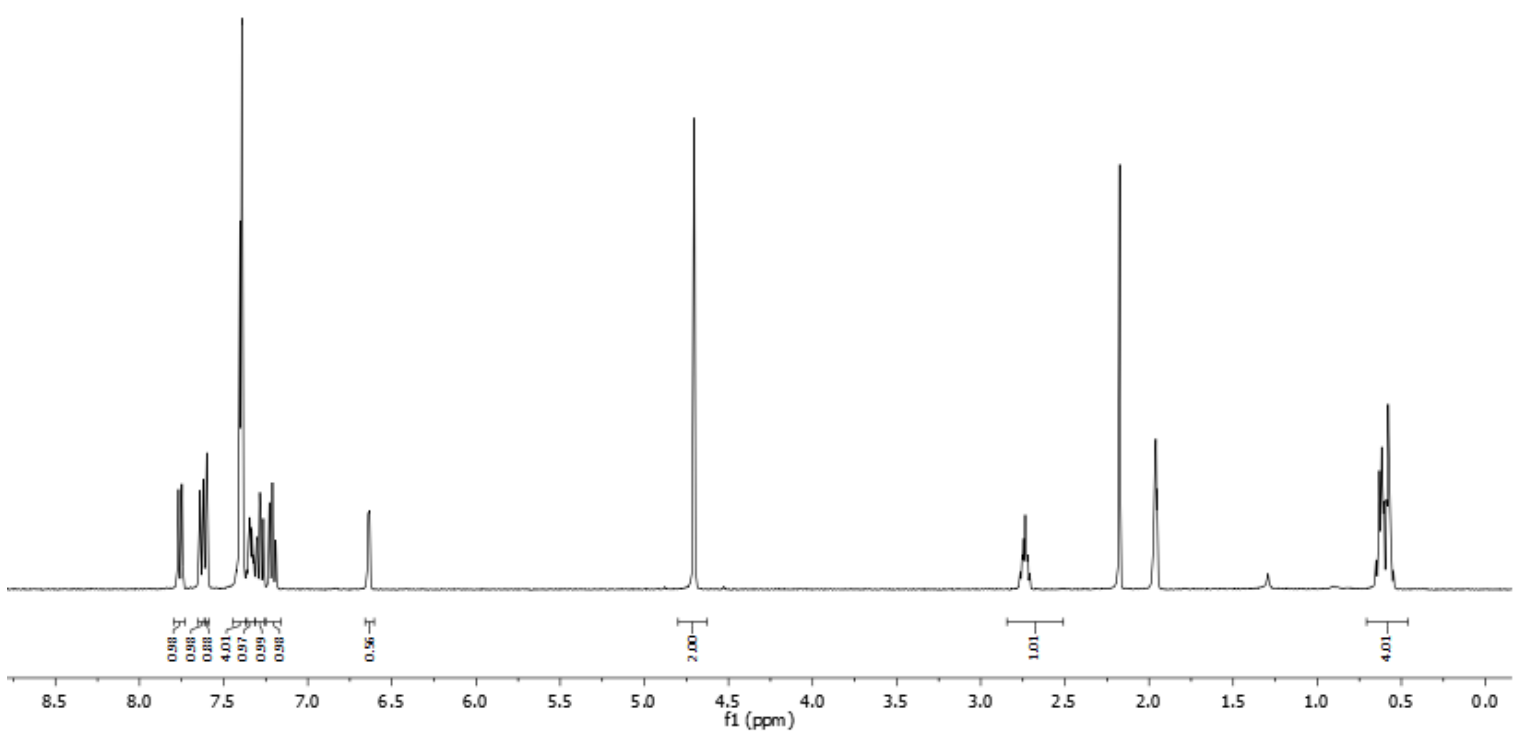


<smiles>O=C(N(Cc1ccccc1)C1CC1)n1ccc2ccccc21</smiles>

$3 a$

$$
\begin{gathered}
\underset{\text { DCB }(0.1 \mathrm{M}), \mathrm{CO}(1 \mathrm{~atm})}{\stackrel{\mathrm{N}(\mathrm{Me}){ }_{2} \mathrm{C}_{6} \mathrm{H}_{4} \mathrm{CO}_{2} \mathrm{H}(30 \%)}{\longrightarrow}} \\
\begin{array}{c}
{ }^{\circ} \mathrm{C}, \mathrm{D}_{2} \mathrm{O}(300 \mathrm{~mol} \%) \\
24 \mathrm{~h}
\end{array}
\end{gathered}
$$<smiles></smiles>

deuterio-3a", 99\% Yield

An oven dried reaction tube, fitted with a magnetic stirrer, was charged with 4(dimethylamino)benzoic acid $(7.43 \mathrm{mg}, 45.0 \mu \mathrm{mol})$ and substrate $\mathbf{3 a}(43.5 \mathrm{mg}, 0.15 \mathrm{mmol})$. The tube was fitted with a rubber septum and purged with argon. Anhydrous 1,2-DCB (1.50 $\mathrm{mL}$ ) was added by syringe. The reaction vessel was purged with $\mathrm{CO}$ for 10 minutes and the solution was subsequently sparged with $\mathrm{CO}$ for approximately 20 seconds. Then the $\mathrm{CO}$ balloon was removed and $\mathrm{D}_{2} \mathrm{O}(5.40 \mathrm{uL}, 0.30 \mathrm{mmol})$ was added. The tube was sealed and the reaction was heated at $130{ }^{\circ} \mathrm{C}$ for 24 hours. The mixture was cooled to room temperature and concentrated in vacuo. The residue was purified by flash column chromatography $(10 \%$ EtOAc/hexane) to afford deuterio-3a", (43.1 mg, 99\%). There is no deuterium incorporation was found by ${ }^{1} \mathrm{H}$ NMR.<smiles>O=Cc1c(CO[N+](=O)[O-])c2ccccc2n1C(=O)N(Cc1ccccc1)C1CC1</smiles>
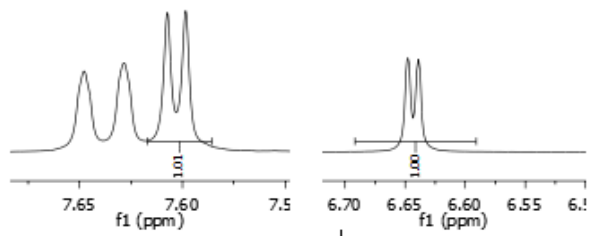

deuterio-3a" $\quad{ }^{1} \mathrm{H}$ NMR $\left(400 \mathrm{MHz}, \mathrm{CD}_{3} \mathrm{CN}\right)$

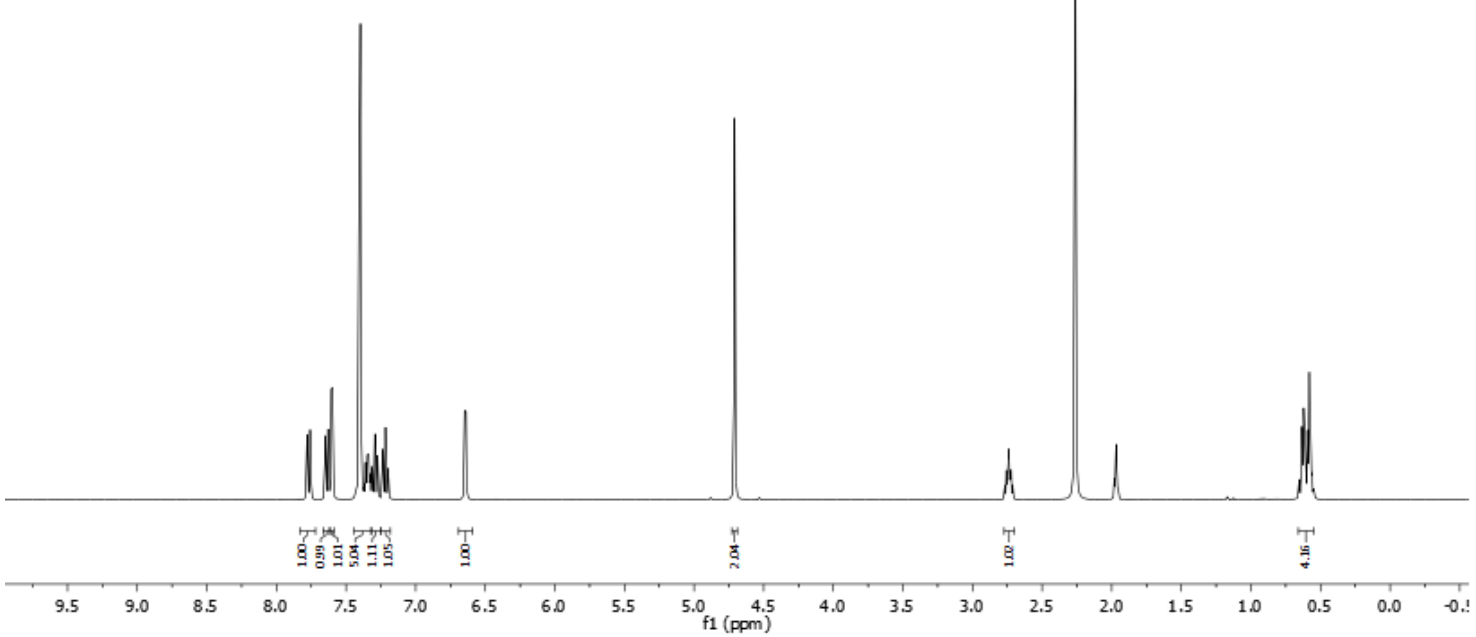




\section{Equation 5:}

$N$-Benzyl- $N$-cyclopropyl-1H-indole-1-carboxamide-3d deuterio-3ab<smiles>[2H]c1c[nH]c2ccccc12</smiles><smiles>O=C(Cl)N(Cc1ccccc1)C1CC1</smiles><smiles>O=C(N(Cc1ccccc1)C1CC1)n1cc([O-])c2ccccc21</smiles>

deuterio-3ab

General procedure A: $1 H$-indole- $3-d(177 \mathrm{mg}, 1.50 \mathrm{mmol})$ (prepared according to the literature procedure) ${ }^{14}$ and benzyl(cyclopropyl)carbamic chloride (470 $\mathrm{mg}, 2.25 \mathrm{mmol}$ ) were employed. The crude mixture was purified by flash column chromatography $(65 \%$ toluene/hexane then $10 \%$ EtOAc/hexane) to afford the title compound deuterio-3ab (425 mg, 97\%) as a colourless solid; $m / z\left(\mathrm{ESI}^{+}\right)$HRMS: Calculated for $\mathrm{C}_{19} \mathrm{H}_{18} \mathrm{~N}_{2} \mathrm{OD}$ : 292.1555. Found $[\mathrm{M}+\mathrm{H}]^{+}: 292.1550$. The percentage of deuterium incorporation was measured by ${ }^{1} \mathrm{H} \mathrm{NMR}$.

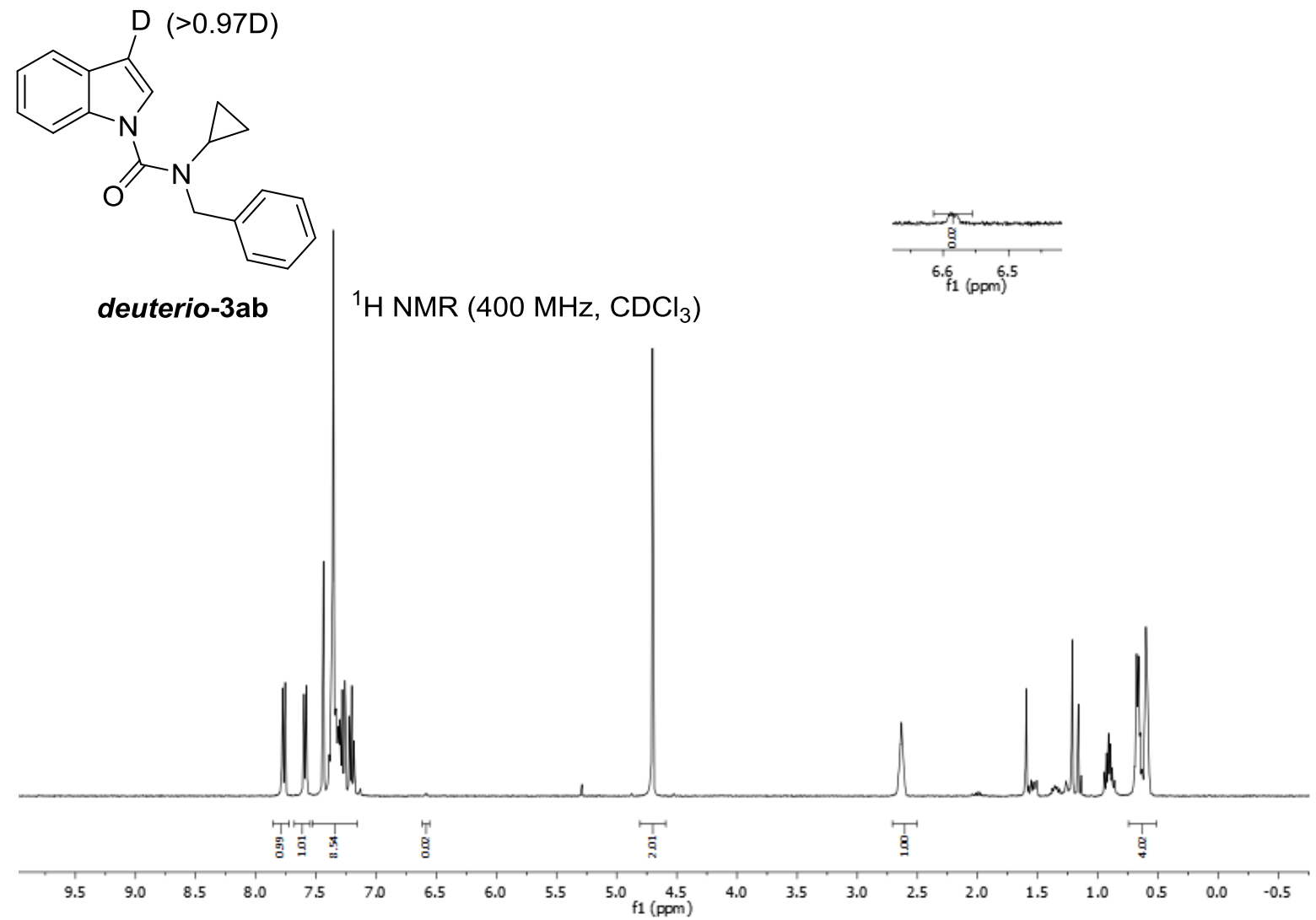


<smiles>O=C(N(Cc1ccccc1)C1CC1)n1cc([Ge]O[O-])c2ccccc21</smiles>

deuterio-3ab

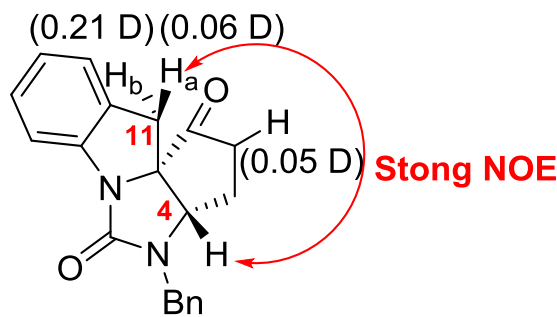

deuterio-4ad, $71 \%$ Yield

General procedure D: Compound deuterio-3ab $(43.5 \mathrm{mg}, 0.15 \mathrm{mmol}),\left[\mathrm{Rh}(\operatorname{cod})_{2}\right] \mathrm{OTf}(5.28$ $\mathrm{mg}, 7.5 \mathrm{~mol} \%)$ and anhydrous 1,2-DCB $(1.50 \mathrm{~mL})$ were employed and the reaction was stirred for 72 hours at $130{ }^{\circ} \mathrm{C}$. The crude mixture was purified by flash column chromatography (5\% EtOAc/toluene) to yield the title compound deuterio-4ad (33.7 $\mathrm{mg}$, $71 \%$ ) as a colourless solid. The percentage of deuterium incorporation was measured by ${ }^{1} \mathrm{H}$ NMR.

A strong nOe correlation was observed between $C \mathrm{C1}_{\mathrm{a}}-\mathrm{H}$ and $\mathrm{C4}-\mathrm{H}$; this result supports a mechanism were alkyl-Rh(I) species (VII in main paper) undergoes syn-stereospecific carbometallation of the C2-C3 $\pi$-system of the indole unit prior to protodemetallation.

$(0.21 \mathrm{D})(0.06 \mathrm{D})$<smiles></smiles>

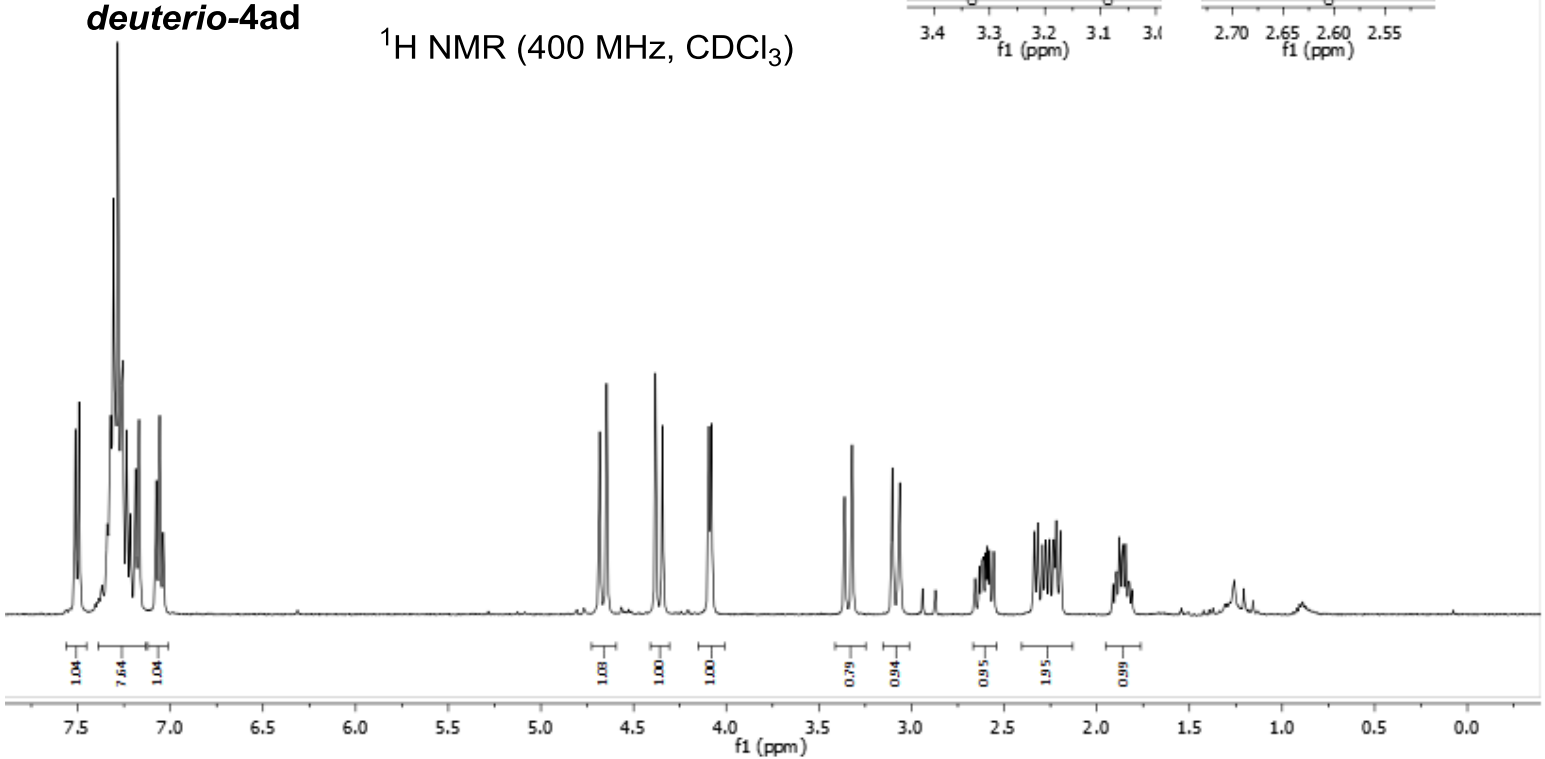




\section{Equation 6:}

$N$-Benzyl- $N$-cyclopropyl-1H-indole-1-carboxamide-2d deuterio-3ac<smiles>[2H]c1cc2ccccc2[nH]1</smiles>

General procedure A: $1 H$-Indole-2- $d(236 \mathrm{mg}, 2.00 \mathrm{mmol})$ (prepared according to the literature procedure) ${ }^{15}$ and benzyl(cyclopropyl)carbamic chloride (543 mg, $2.60 \mathrm{mmol}$ ) were employed. The crude mixture was purified by flash column chromatography (65\% toluene/hexane then $1 \%$ EtOAc/hexane) to afford the title compound deuterio-3ac (501 mg, $86 \%$ ) as a colourless solid; $m / z\left(\mathrm{ESI}^{+}\right)$HRMS: Calculated for $\mathrm{C}_{19} \mathrm{H}_{18} \mathrm{~N}_{2} \mathrm{OD}$ : 292.1555. Found $[\mathrm{M}+\mathrm{H}]^{+}: 292.1547$. The percentage of deuterium incorporation was measured by ${ }^{1} \mathrm{H}$ NMR.<smiles>[2H]c1cc2ccccc2n1C(=O)N(Cc1ccccc1)C1CC1</smiles>

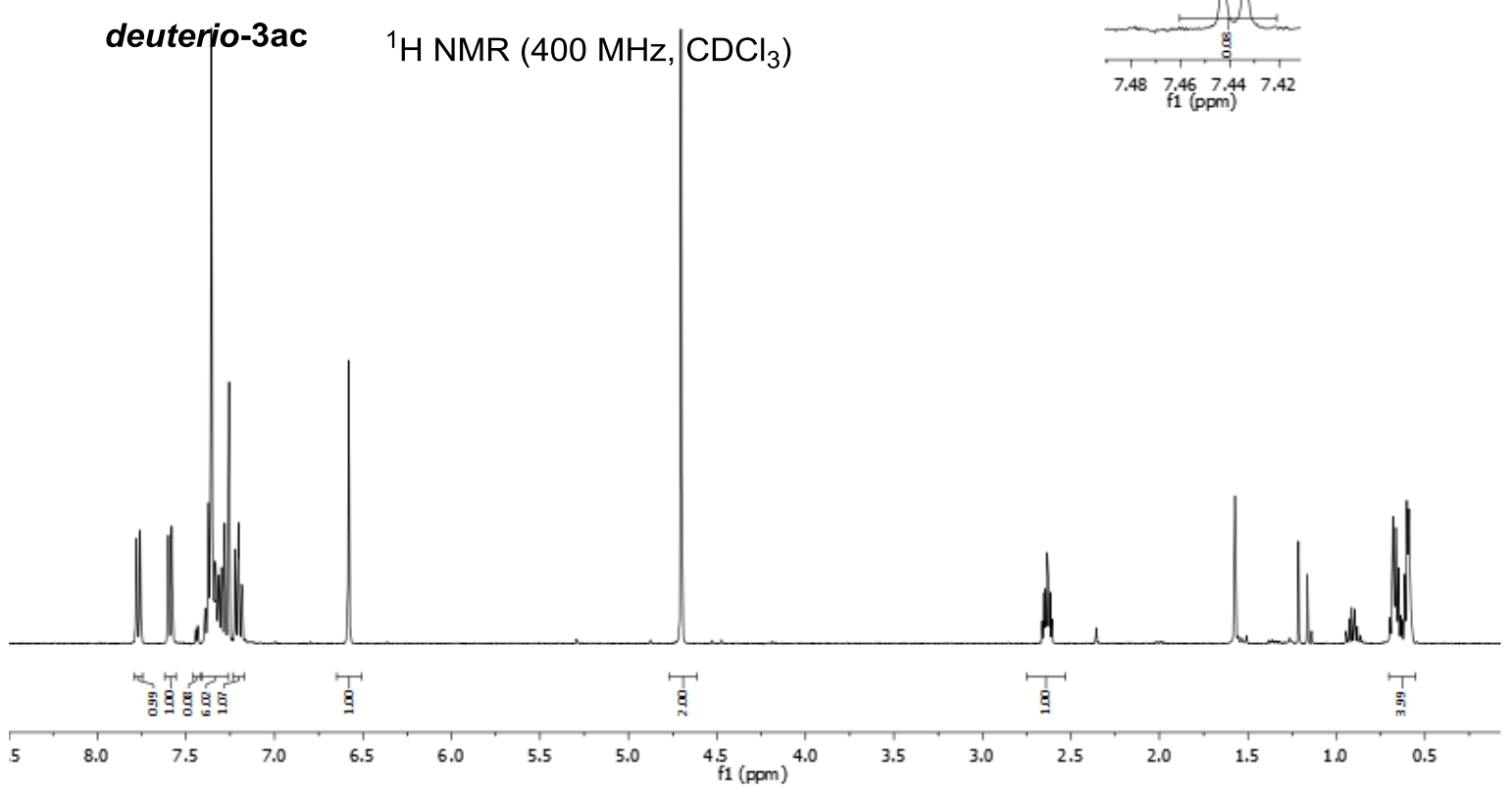


<smiles>O=C(N(Cc1ccccc1)C1CC1)n1ccc2ccccc21</smiles>

$3 a$

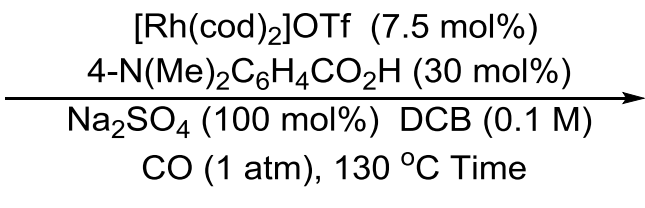

$\mathrm{CO}(1 \mathrm{~atm}), 130^{\circ} \mathrm{C}$ Time

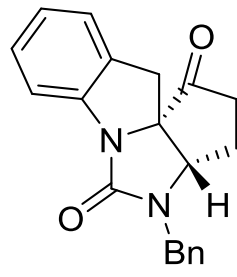

$4 a$

Five identical, oven dried reaction tubes were fitted with a magnetic stirrer and charged with $\left[\operatorname{Rh}(\operatorname{cod})_{2}\right]$ OTf $(5.28 \mathrm{mg}, 11.2 \mu \mathrm{mol}), 4$-(dimethylamino)benzoic acid $(7.43 \mathrm{mg}, 45.0 \mu \mathrm{mol})$ and substrate $3 \mathbf{a}(43.5 \mathrm{mg}, 0.15 \mathrm{mmol})$. Each tube was fitted with a rubber septum and purged with argon. Anhydrous 1,2-DCB $(1.50 \mathrm{~mL})$ was added by syringe. The reaction vessel was purged with $\mathrm{CO}$ for 10 minutes and the solution was subsequently sparged with $\mathrm{CO}$ for approximately 20 seconds. These reaction tubes were heated at $130{ }^{\circ} \mathrm{C}$ under a $\mathrm{CO}$ atmosphere (with CO balloon attached) and stopped at $2.5 \mathrm{~h}, 5.0 \mathrm{~h}, 7.5 \mathrm{~h}, 10.0 \mathrm{~h}$ and $12.5 \mathrm{~h}$, respectively. The in-situ yield of product $4 \mathbf{a}$ was determined by ${ }^{1} \mathrm{H}$ NMR analysis by using 1,4-dinitrobenzene as an internal standard.

\begin{tabular}{|l|c|c|c|c|c|}
\hline Reaction time (h) & 2.5 & 5.0 & 7.5 & 10.0 & 12.5 \\
\hline In-situ yield of 4a (\%) & 10.9 & 20.6 & 28.3 & 33.4 & 37.3 \\
\hline
\end{tabular}<smiles>[2H]c1cc2ccccc2n1C(=O)N(Cc1ccccc1)C1CC1</smiles>

deuterio-3ac

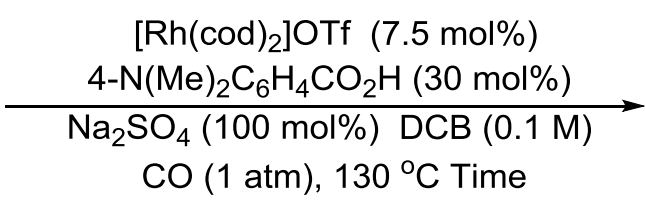

$\mathrm{CO}(1 \mathrm{~atm}), 130{ }^{\circ} \mathrm{C}$ Time

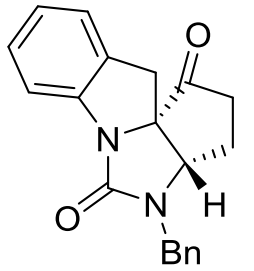

$4 a$

Five identical, oven dried reaction tubes were fitted with a magnetic stirrer and charged with $\left[\mathrm{Rh}(\mathrm{cod})_{2}\right] \mathrm{OTf}(5.28 \mathrm{mg}, 11.2 \mu \mathrm{mol}), 4$-(dimethylamino)benzoic acid $(7.43 \mathrm{mg}, 45.0 \mu \mathrm{mol})$ and substrate deuterio-3ac $(43.5 \mathrm{mg}, 0.15 \mathrm{mmol})$. Each tube was fitted with a rubber septum and purged with argon. Anhydrous 1,2-DCB $(1.50 \mathrm{~mL})$ was added by syringe. The reaction vessel was purged with $\mathrm{CO}$ for 10 minutes and the solution was subsequently sparged with $\mathrm{CO}$ for approximately 20 seconds. These reaction tubes were heated at $130{ }^{\circ} \mathrm{C}$ under a $\mathrm{CO}$ atmosphere (CO balloon) and stopped at $2.5 \mathrm{~h}, 5.0 \mathrm{~h}, 7.5 \mathrm{~h}, 10.0 \mathrm{~h}$ and $12.5 \mathrm{~h}$, respectively. The in-situ yield of product $4 \mathbf{a}$ was determined by ${ }^{1} \mathrm{H}$ NMR analysis by using 1,4dinitrobenzene as an internal standard. 


\begin{tabular}{|l|c|c|c|c|c|}
\hline Reaction time (h) & 2.5 & 5.0 & 7.5 & 10.0 & 12.5 \\
\hline In-situ yield of $\mathbf{4 a}(\%)$ & 6.5 & 12.8 & 18.1 & 22.9 & 28.5 \\
\hline
\end{tabular}

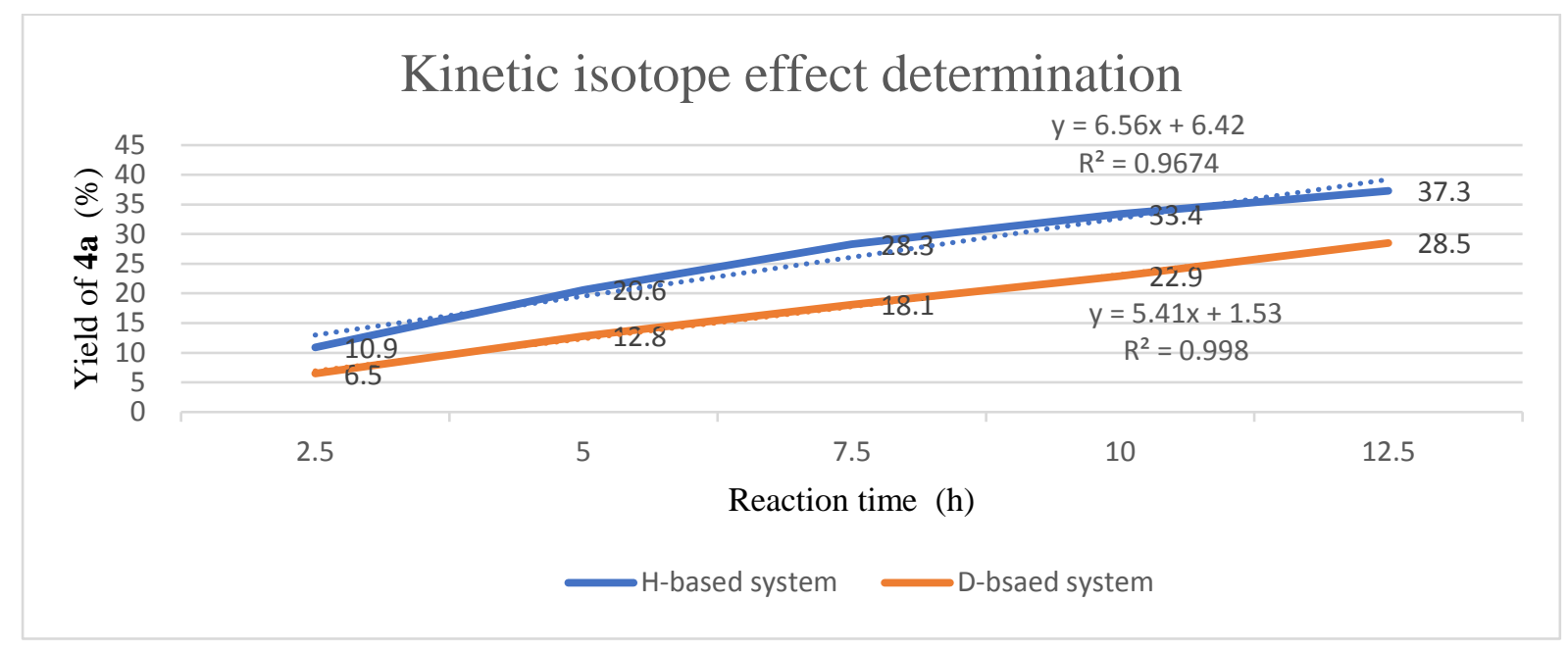

\section{$k_{\mathrm{H}} / k_{\mathrm{D}}=6.56 / 5.41=1.21$}

\section{Deuterium exchange experiment for $5 \mathrm{~d}$ to deuterio-6d}

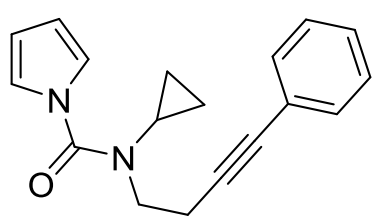

5d

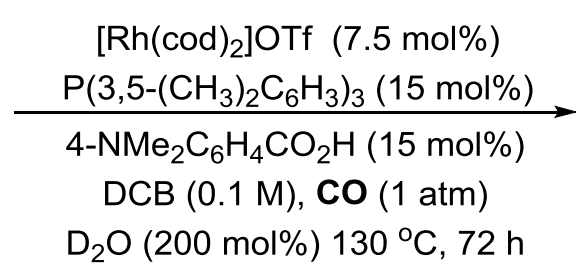

$\mathrm{D}_{2} \mathrm{O}(200 \mathrm{~mol} \%) 130^{\circ} \mathrm{C}, 72 \mathrm{~h}$<smiles>O=C1CC([18OH])C2/C(=C(/[18OH])c3ccccc3)CCN2C(=O)n2cccc21</smiles>

deuterio-6d, $71 \%$ Yield

An oven dried reaction tube, fitted with a magnetic stirrer, was charged with $\left[\mathrm{Rh}(\operatorname{cod})_{2}\right] \mathrm{OTf}$ (3.52 mg, $7.5 \mu \mathrm{mol})$, 4-(dimethylamino)benzoic acid (2.48 mg, $15.0 \mu \mathrm{mol})$, tris(3,5dimethylphenyl)phosphine (5.19 mg, $15 \mu \mathrm{mol})$ and $N$-cyclopropyl- $N$-(4-phenylbut-3-yn-1yl)-1H-pyrrole-1-carboxamide $\mathbf{5 d}(27.8 \mathrm{mg}, 0.10 \mathrm{mmol})$. The tube was fitted with a rubber septum and purged with argon. Anhydrous 1,2-DCB $(1.00 \mathrm{~mL})$ was added by syringe. The reaction vessel was purged with $\mathrm{CO}$ for 10 minutes and the solution was subsequently sparged with $\mathrm{CO}$ for approximately 20 seconds. Then the $\mathrm{CO}$ balloon was removed and $\mathrm{D}_{2} \mathrm{O}$ (3.6 $\mathrm{uL}, 0.20 \mathrm{mmol}$ ) was added. The tube was sealed and the reaction was heated at $130{ }^{\circ} \mathrm{C}$ for 72 hours. The mixture was cooled to room temperature and concentrated in vacuo. The crude mixture was purified by column chromatography $(10 \% \mathrm{EtOAc/Hex})$ to yield the title compound deuterio-6d $(21.8 \mathrm{mg}, 71 \%)$ as a colourless oil. The percentage of deuterium incorporation was measured by ${ }^{1} \mathrm{H}$ NMR analysis. 
<smiles>O=C1CC([O+])c2cccn2C(=O)N2CC/C(=C\c3ccccc3)C12</smiles>

deuterio-6d $\quad{ }^{1} \mathrm{H}$ NMR (400 MHz, $d_{6}$-DMSO)
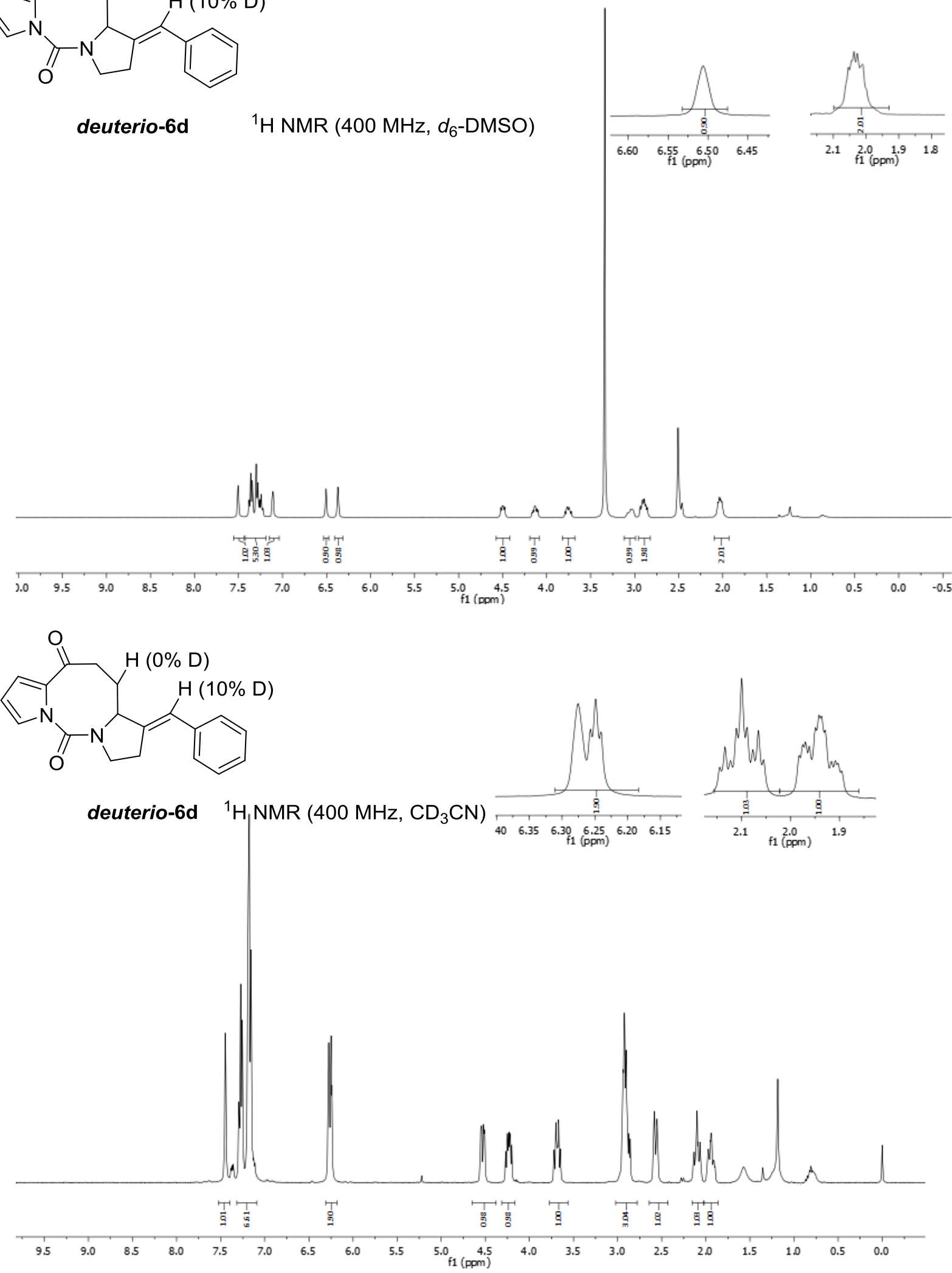

S76 


\section{Additional deuterium labelling studies}

1.

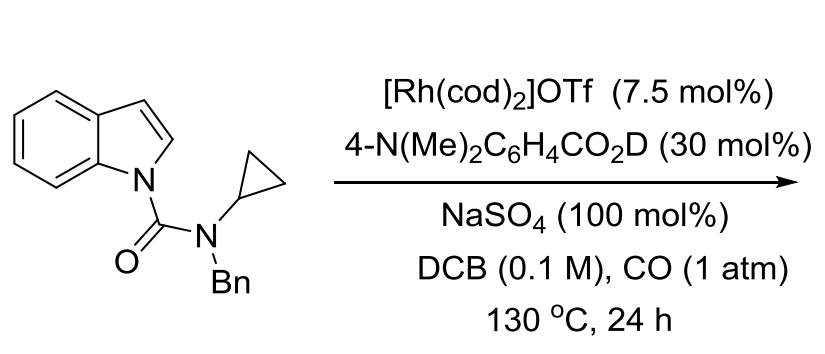

3a

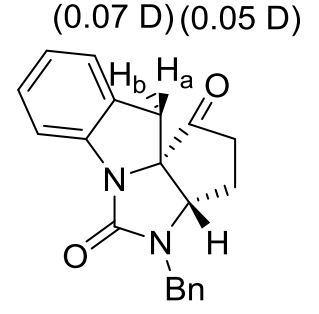

deuterio-4ae $54 \%$ Yield<smiles></smiles>

deuterio-3ad $27 \%$ Yield

p-NMe $\mathrm{C}_{6} \mathrm{C}_{4} \mathrm{CO}_{2} \mathrm{D}$ was prepared by repeatedly dissolving $p-\mathrm{NMe}_{2} \mathrm{C}_{6} \mathrm{H}_{4} \mathrm{CO}_{2} \mathrm{H}$ in $\mathrm{MeOD}-d_{4}$ and concentrating the resulting solution in vacuo.

An oven dried reaction tube, fitted with a magnetic stirrer, was charged with $\left[\mathrm{Rh}(\operatorname{cod})_{2}\right] \mathrm{OTf}$ (5.28 mg, $11.2 \mu \mathrm{mol}), p-\mathrm{NMe}_{2} \mathrm{C}_{6} \mathrm{H}_{4} \mathrm{CO}_{2} \mathrm{D}(7.47 \mathrm{mg}, 45 \mu \mathrm{mol})$ and substrate $\mathbf{3 a}(43.5 \mathrm{mg}, 0.15$ mmol). The tube was fitted with a rubber septum and purged with argon. Anhydrous 1,2DCB $(1.50 \mathrm{~mL})$ was added by syringe. The reaction vessel was purged with CO for 10 minutes and the solution was subsequently sparged with $\mathrm{CO}$ for approximately 20 seconds. The reaction mixture was heated at $130{ }^{\circ} \mathrm{C}$ for 24 hours. The mixture was cooled to room temperature and concentrated in vacuo. The residue was purified by flash column chromatography (10\% EtOAc/Hexane then 5\% EtOAc/Toluene) to afford deuterio-3ad (11.7 $\mathrm{mg}, 27 \%)$ and deuterio-4ae $(25.8 \mathrm{mg}, 54 \%)$. The percentage of deuterium incorporation was measured by ${ }^{1} \mathrm{H}$ NMR. 

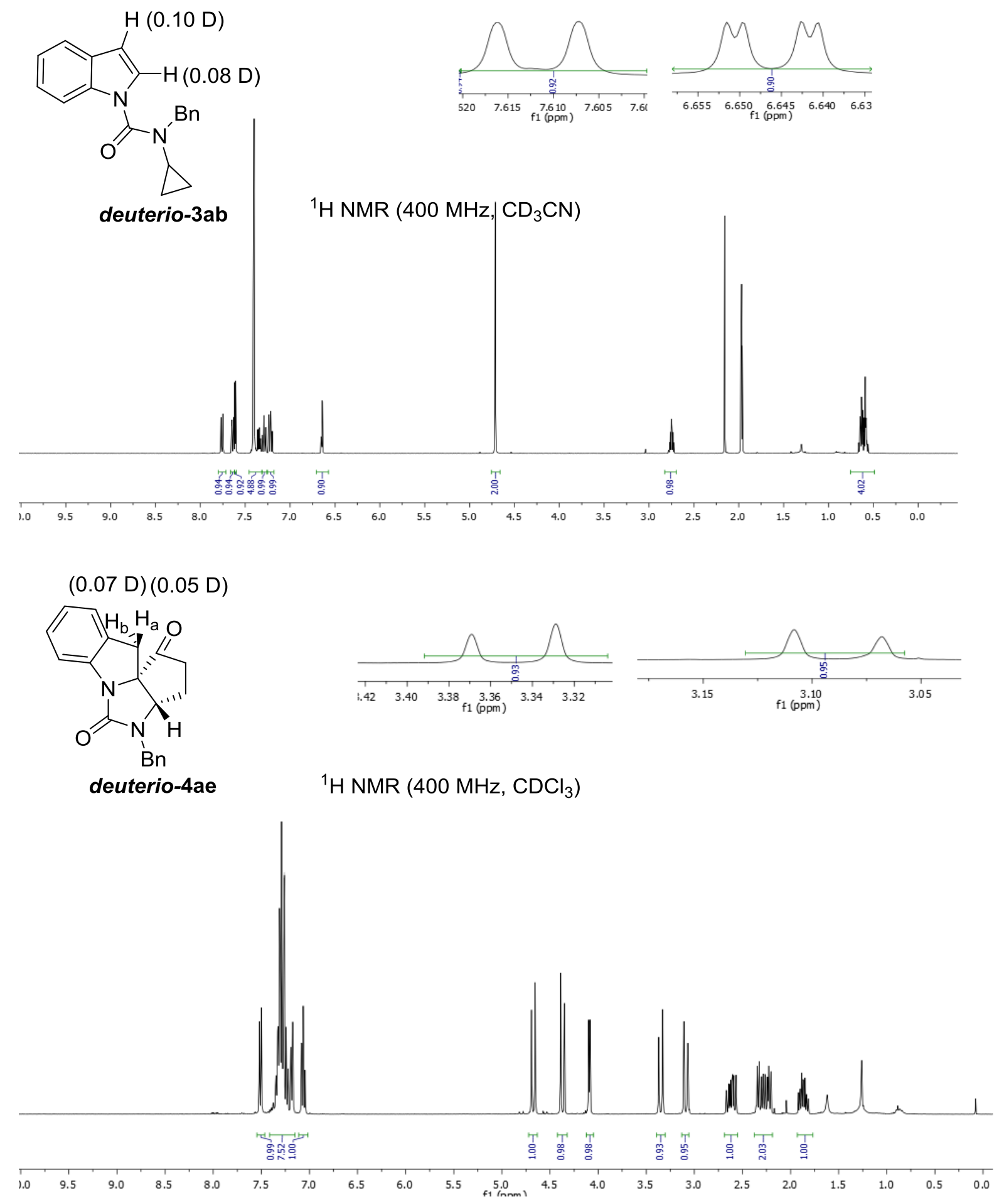
<smiles>O=C(N(CCC#Cc1ccccc1)C1CC1)n1cccc1</smiles>

$5 d$<smiles>O=C1CC(C=[18O])C2/C(=C/c3ccccc3)CCN2C(=O)n2cccc21</smiles>

deuterio-6 da, $70 \%$ Yield

An oven dried reaction tube, fitted with a magnetic stirrer, was charged with $\left[\mathrm{Rh}(\operatorname{cod})_{2}\right] \mathrm{OTf}$ (3.52 mg, $7.0 \mu \mathrm{mol}), \quad p-\mathrm{NMe}_{2} \mathrm{C}_{6} \mathrm{H}_{4} \mathrm{CO}_{2} \mathrm{D} \quad(2.30 \quad \mathrm{mg}, \quad 14 \mu \mathrm{mol}), \quad$ tris(3,5dimethylphenyl)phosphine $(4.83 \mathrm{mg}, 14 \mu \mathrm{mol})$ and $N$-cyclopropyl- $N$-(4-phenylbut-3-yn-1yl)-1H-pyrrole-1-carboxamide $\mathbf{5 d}(25.8 \mathrm{mg}, 0.093 \mathrm{mmol})$. The tube was fitted with a rubber septum and purged with argon. Anhydrous 1,2-DCB $(0.93 \mathrm{~mL})$ was added by syringe. The reaction vessel was purged with $\mathrm{CO}$ for 10 minutes and the solution was subsequently sparged with $\mathrm{CO}$ for approximately 20 seconds. The reaction mixture was heated at $130{ }^{\circ} \mathrm{C}$ for 72 hours. The mixture was cooled to room temperature and concentrated in vacuo. The crude mixture was purified by column chromatography (5\% EtOAc/Toluene) to yield the title compound deuterio-6da $(19.7 \mathrm{mg}, 70 \%)$ as a colourless oil. The percentage of deuterium incorporation was measured by ${ }^{1} \mathrm{H}$ NMR.<smiles>O=C1CC(C=[18O])C2/C(=C/c3ccccc3)CCN2C(=O)n2cccc21</smiles>

deuterio-6da
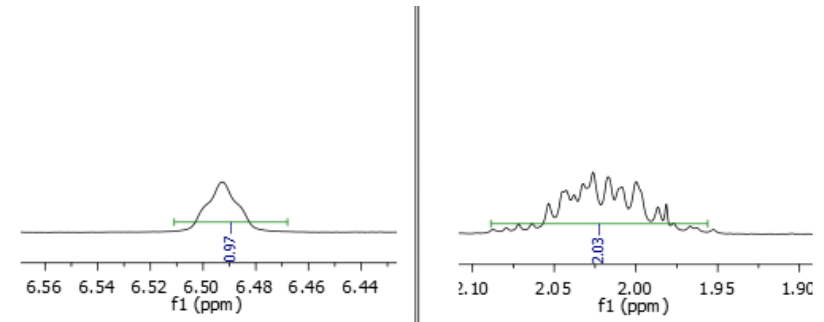

${ }^{1} \mathrm{H}$ NMR $\left(400 \mathrm{MHz}, d_{6}-\mathrm{MSO}\right)$

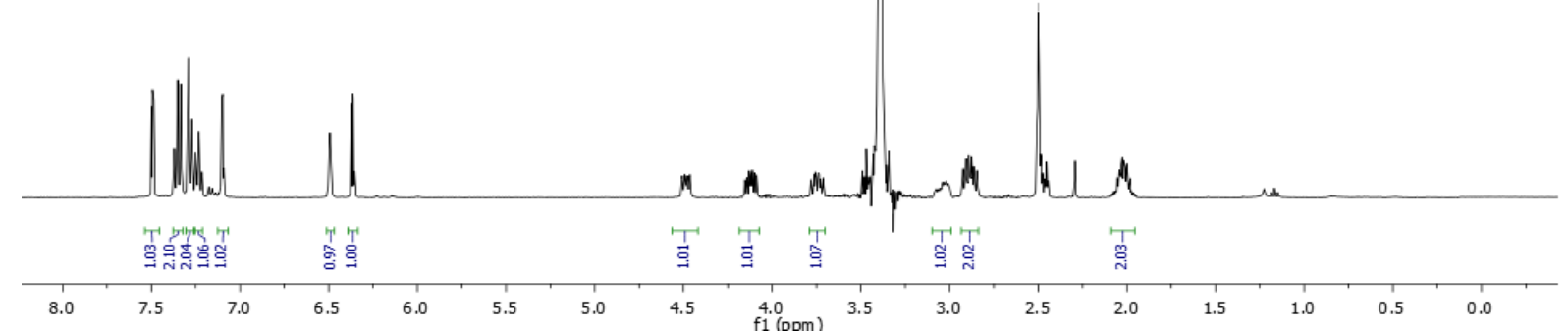


3.<smiles>O=C1CCC2/C(=C\c3ccccc3)CCN2C(=O)n2cccc21</smiles>

6d
$\left[\mathrm{Rh}(\mathrm{cod})_{2}\right] \mathrm{OTf}(7.5 \mathrm{~mol} \%)$

$$
\begin{gathered}
\underset{4-\mathrm{NMe}_{2} \mathrm{C}_{6} \mathrm{H}_{4} \mathrm{CO}_{2} \mathrm{H}(15 \mathrm{~mol} \%)}{\mathrm{P}\left(3,5-\left(\mathrm{CH}_{3} \mathrm{C}_{6} \mathrm{H}_{3}\right)_{3}(15 \mathrm{~mol} \%)\right.} \\
\mathrm{DCB}(0.1 \mathrm{M}), \mathrm{CO}(1 \mathrm{~atm}) \\
\mathrm{D}_{2} \mathrm{O}(200 \mathrm{~mol} \%) 130^{\circ} \mathrm{C}, 72 \mathrm{~h}
\end{gathered}
$$

$(17 \%$ D) D

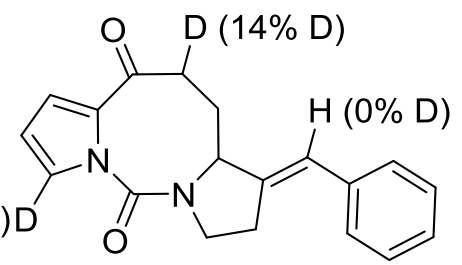

deuterio- $6 \mathrm{db}, 82 \%$ Yield

An oven dried reaction tube, fitted with a magnetic stirrer, was charged with $\left[\mathrm{Rh}(\operatorname{cod})_{2}\right] \mathrm{OTf}$ (2.48 mg, $5.2 \mu \mathrm{mol})$, 4-(dimethylamino)benzoic acid (1.77 mg, $10.5 \mu \mathrm{mol})$, tris(3,5dimethylphenyl)phosphine (3.68 $\mathrm{mg}, 10.5 \mu \mathrm{mol})$ and $\mathbf{6 d}(21.6 \mathrm{mg}, 0.071 \mathrm{mmol})$. The tube was fitted with a rubber septum and purged with argon. Anhydrous 1,2-DCB $(0.71 \mathrm{~mL})$ was added by syringe. The reaction vessel was purged with $\mathrm{CO}$ for 10 minutes and the solution was subsequently sparged with $\mathrm{CO}$ for approximately 20 seconds. Then the $\mathrm{CO}$ balloon was removed and $\mathrm{D}_{2} \mathrm{O}(2.5 \mathrm{uL}, 0.14 \mathrm{mmol})$ was added. The tube was sealed and the reaction was heated at $130{ }^{\circ} \mathrm{C}$ for 72 hours. The mixture was cooled to room temperature and concentrated in vacuo. The crude mixture was purified by column chromatography (5\% EtOAc/Toluene) to yield the title compound deuterio-6db $(17.9 \mathrm{mg}, 82 \%)$ as a colourless oil. The percentage of deuterium incorporation was measured by ${ }^{1} \mathrm{H}$ NMR. No exchange at the vinylic position of the product was observed.

$(17 \%$ D)D<smiles></smiles>

deuterio-6 db

${ }^{1} \mathrm{H}$ NMR $\left(400 \mathrm{MHz}, d_{6}-\mathrm{MSO}\right)$

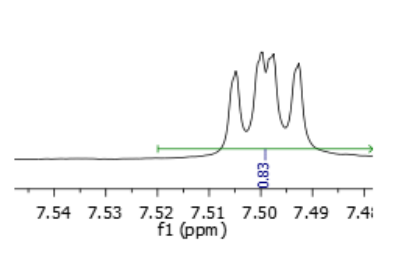

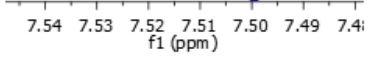

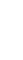

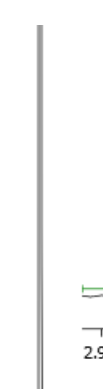

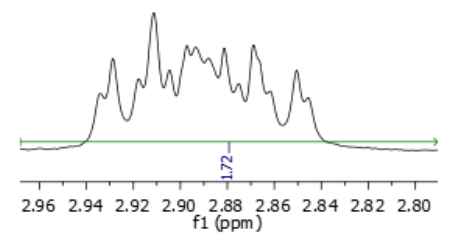

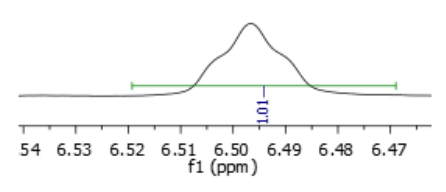

\begin{tabular}{|c|c|c|c|c|c|c|c|c|c|c|c|c|c|c|}
\hline & & & ${ }_{0}^{T}$ & & & & $\stackrel{\square}{\vec{\Xi}}$ & 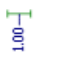 & 'T' & 菅罢 & & 'T' & & \\
\hline 8.0 & 7.5 & 7.0 & 6.5 & 6.0 & 5.5 & 5.0 & $\begin{array}{l}4.5 \\
\text { f1 } 1(\mathrm{ppm})\end{array}$ & 4.0 & 3.5 & 3.0 & 2.5 & 2.0 & 1.5 & 1.0 \\
\hline
\end{tabular}
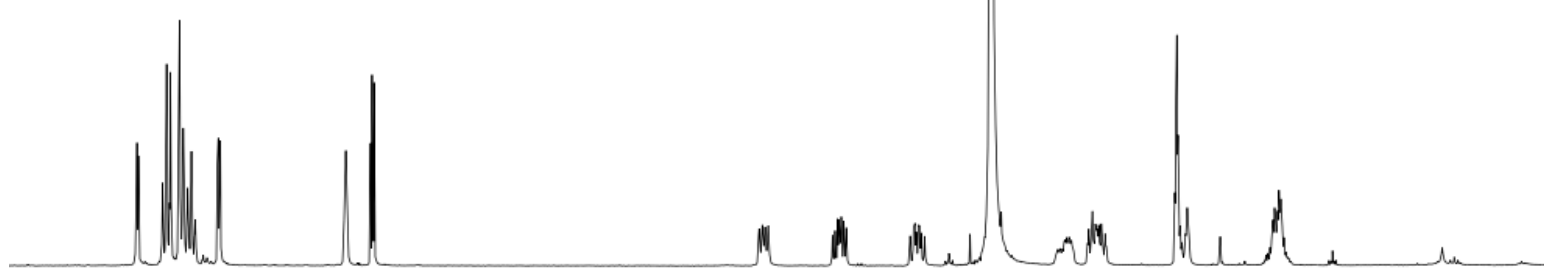


\section{Computational Details}

With limited structural data in hand, a range of conformers were explored using DFT (B3PW91-D3BJ/LACVP* in Jaguar v 8.5, denoted M1) to identify the most likely low energy species. The LACVP* basis set uses the Pople 6-31G(d) basis with five Cartesian $d$ functions on all non-Rh atoms and LACVP* with a Hay-Wadt effective core potential (ECP) on $\mathrm{Rh}$. For all intermediates and transition states, the relative stability of 14,16 and 18 electron complexes, corresponding to differing Rh-coordination numbers, were evaluated at the same level. Species within $\Delta \mathrm{E}<5 \mathrm{kcal} \mathrm{mol}^{-1}$ of the lowest energy conformer were then optimized with Gaussian 09. Gaussian 09 geometry optimisations were performed at the B3PW91-D3BJ/6-31G(d),Rh(MWBja28) level of theory (denoted M2), with ultrafine integration grids and a basis set containing five spherical harmonic components of the dpolarisation functions. Analytical frequency calculations were used to assign stationary points as minima or transition states (TSs) by the presence of zero or one imaginary frequencies respectively. Thermochemical contributions (enthalpy and Gibbs free energies) were calculated at $T=403 \mathrm{~K}\left(130^{\circ} \mathrm{C}\right)$. A correction to account for the differing standard states of gas phase $(1 \mathrm{~atm})$ and solution phase $(1 \mathrm{M})$ reactions was added, appropriate for $T=403 \mathrm{~K}$ $\left(\Delta \mathrm{G}_{\mathrm{g} \rightarrow \mathrm{s}}=+\mathrm{RT} \ln \left(1 / C_{\mathrm{g}}\right)\right.$ where $\left.C_{\mathrm{g}}=0.0302 \mathrm{~mol} / \mathrm{dm}^{3}\right)$. Single point energies at M2 geometries were computed using a larger, triple- $\zeta$ basis set with diffuse functions on non-hydrogen atoms $(6-311+\mathrm{G}(2 \mathrm{~d}, \mathrm{p}))$ and f-polarisation functions on Rh [LANTZ(f)] with the same B3PW91-D3BJ functional. o-dichlorobenzene (DCB) solvent effects were accounted for implicitly using the SMD solvent model implemented in Gaussian 09 during the single point energy calculation. Together this method is B3PW91-D3BJ/6311+G(2d,p),Rh(LANTZ(f))/SMD(DCB)//B3PW91-D3BJ/6-31G(d),Rh(MWB28) or M3.

Solvated free energies quoted in the manuscript are calculated using the following

$$
G_{M 3}=E_{M 3}+\left(G_{M 2}-E_{M 2}\right)+R T \ln \left(\frac{R T}{101325}\right)
$$

\section{Model System}

To generate a computationally tractable model, the N-benzyl substrate $\mathbf{3 a}$ was reduced to its $\mathrm{N}-\mathrm{H}$ counterpart. While this simplification alters the electronic character and steric properties of the amide, incomplete sampling of the large conformational freedom introduced is likely to 
outweigh the small accuracy gained in binding energies of the substrate and perhaps small differences in $\mathrm{C}-\mathrm{C}$ reductive elimination barriers.

Additionally, the $\mathrm{Rh}$ complexes throughout the catalytic cycle are assumed to be cationic and fully solvated, with free triflate present in solution. Deprotonated base is likewise assumed not to be coordinated. These assumptions are made based on the affinity of late-TMs for relatively soft ligands (e.g. CO) and required due to the error associated with calculating relative free energies of differently charged complexes, which can be unacceptably large in the absence of an explicit treatment of solvation. Indeed, previous studies have noted errors in excess of $15 \mathrm{kcal} \mathrm{mol}^{-1}$ for solvation energies of charged species using the implicit model used here.

The equilibrium $\left[\mathrm{Rh}(\mathrm{COD})_{2}\right]^{+} \rightleftharpoons\left[\mathrm{Rh}(\mathrm{CO})_{4}\right]^{+}$between the identically charged complexes was found to lie to the right, with the square planar tetra-carbonyl complex $5.9 \mathrm{kcal} \mathrm{mol}^{-1}$ lower in free energy (at M3). As such, the sterically less demanding and stronger binding CO ligands are assumed to be dominant co-ligands throughout the catalytic cycle. Furthermore, species A is calculated to be $2.0 \mathrm{kcal} \mathrm{mol}^{-1}$ (at M3) higher in free energy than $\left[\mathrm{Rh}(\mathrm{CO})_{4}\right]^{+}$, which we find to be the lowest energy cationic $[\mathrm{Rh}]$ species.

Figure S1 DFT analysis of (a) C-C bond activation and (b) C-C bond forming pathways. ${ }^{a}$
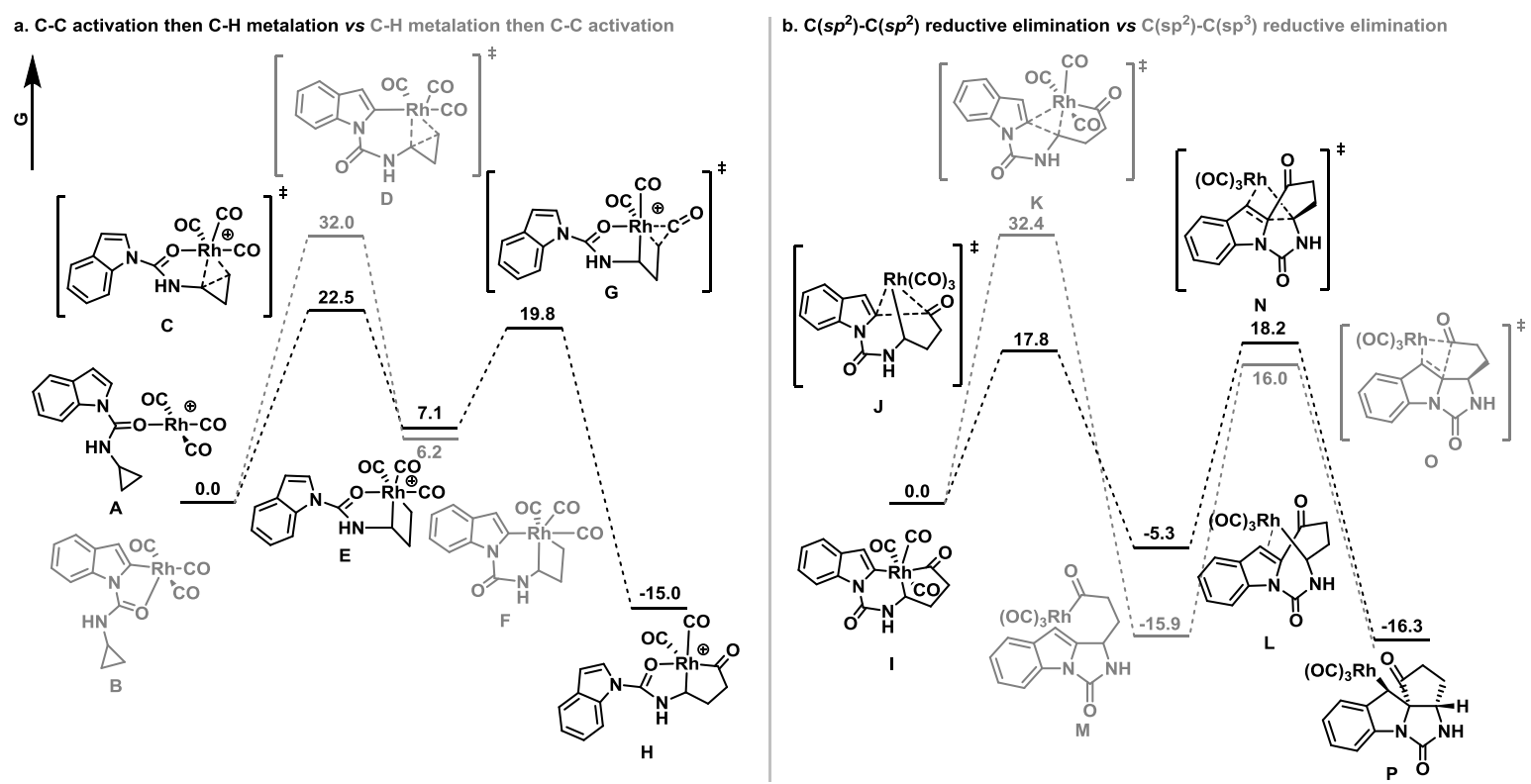

a Calculations performed at the B3PW91-D3BJ/6-311+G(2d,p),Rh(LANTZ(f))/SMD(DCB)//B3PW91$\mathrm{D} 3 \mathrm{BJ} / 6 \neg 31 \mathrm{G}(\mathrm{d}), \mathrm{Rh}(\mathrm{MWB} 28)$ level of theory, with thermochemical corrections calculated at $T=403 \mathrm{~K}$ and a 1 M standard state. 


\section{Geometries}

Shown in Figure S2 are geometries of intermediates and transition states outlined in the manuscript with key bond distances highlighted.
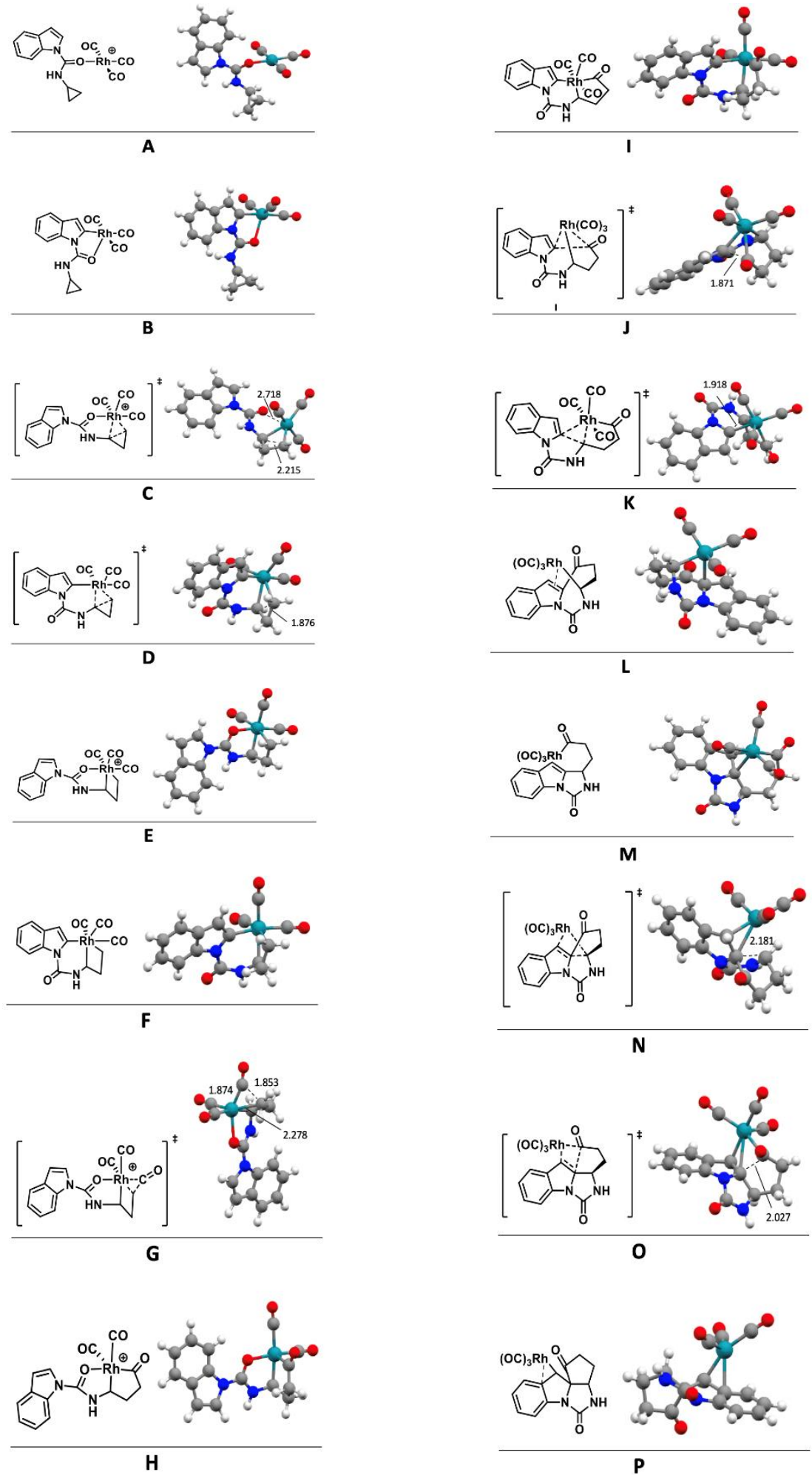

Figure S2. Optimized geometries calculated at the M2 level. Bond distances are shown in Angstroms. 


\section{C-H activation}

Different mechanisms of $\mathrm{C}-\mathrm{H}$ activation were considered using both computational means and experimental evidence. Direct $\mathrm{C}-\mathrm{H}$ oxidative addition from $\mathbf{A}$ is a high energy process (TSA-CHoa, $\Delta \mathrm{G}^{*}=28.1 \mathrm{kcal} \mathrm{mol}^{-1}$ at M3) and considerably less favorable than the alternative direct $\mathrm{C}-\mathrm{C}$ oxidative addition $\left(\mathbf{C}, \Delta \mathrm{G}^{+}=22.5 \mathrm{kcal} \mathrm{mol}^{-1}\right.$ at $\left.\mathrm{M} 3\right)$. Likewise, $\mathrm{C}-\mathrm{H}$ oxidative addition from $\mathbf{H}$ was determined to be energetically infeasible, with the resultant $\mathrm{Rh}(\mathrm{V})$ complex $32.7 \mathrm{kcal} \mathrm{mol}^{-1}$ (at M1) less stable than the reactant (the TS then lies out of energetic reach at $130^{\circ} \mathrm{C}$ for $\left.\Delta \mathrm{G}^{\ddagger}>35 \mathrm{kcal} \mathrm{mol}^{-1}\right)$.

$\mathrm{C}-\mathrm{H}$ activation is therefore proposed to occur via either an electrophilic substitution or a concerted deprotonation-metalation pathway. The error propagation from solvation free energies to $\mathrm{pKa}$ values makes computational prediction of the base involved in the deprotonation difficult. Experimental evidence is therefore used to determine whether or not the $\mathrm{C}-\mathrm{H}$ metalation is a low energy, reversible process. Previous work has shown where the indole moiety is replaced by an aryl equivalent the $\mathrm{C}-\mathrm{H}$ metalation is reversible. ${ }^{16}$ By analogy one would therefore expect the metalation considered here to also be reversible. Indeed, deuterium incorporation at $\mathrm{C} 2$ for a similar substrate 7 (cyclopropyl $\rightarrow$ ethyl) under standard conditions in the presence of $\mathrm{D}_{2} \mathrm{O}$ (Scheme 2, Equation 4) strongly imply that this reaction is reversible and there exists an equilibrium between $\mathbf{A}$ and $\mathbf{B}$. Similar levels of deuterium incorporation are seen when $\mathbf{3 a}$ is subjected to standard conditions in the presence of $\mathrm{D}_{2} \mathrm{O}$ and quenched before completion. This suggests two possibilities (1) $\mathrm{C}-\mathrm{H}$ metalation is reversible or (2) deuterium incorporation into $\mathbf{I}$ is achieved and reverse $\mathrm{CO}$ insertion occurs to regenerate deuterio-3aa. To regenerate deuterated substrate (C2-D) from I would require either (1) protonation, generating what is known to be an unstable $\mathrm{Rh}(\mathrm{V}) \operatorname{species}^{17}$ (vide supra) or (2) $\sigma$-bond metathesis between the $\mathrm{Rh}-\mathrm{C}(2)$ bond and that of a Rh-coordinated $\mathrm{D}_{2} \mathrm{O}$ molecule. As the $\sigma$-bond metathesis involves no change in charge, the free energy of activation is likely to be representative. Computing this at M3 suggests that this reaction is not energetically viable (TSJ-D2O-sbm, $\Delta \mathrm{G}^{\ddagger}=38.7 \mathrm{kcal} \mathrm{mol}^{-1}$, Scheme S1). 


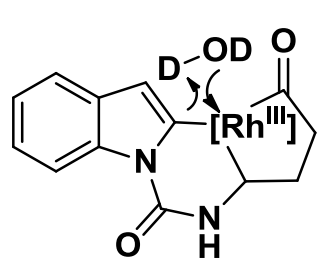

I
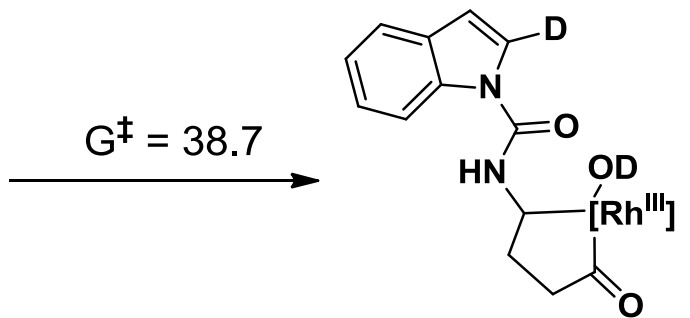

Scheme S1. $\sigma$-bond metathesis from $\mathbf{I}-\mathbf{D}_{2} \mathbf{O}$ generating C2-D substrate. Energy in $\mathrm{kcal}^{\mathrm{mol}}{ }^{-1}$ calculated at $\mathrm{M} 3$ and $[\mathrm{Rh}]=\mathrm{Rh}(\mathrm{CO})_{2}$.

From these studies the $\mathrm{C} 2-\mathrm{H}$ bond activation is considered to be reversible and non-turnover limiting. Therefore, in the mechanism outlined here we assume such and, due to the inaccuracy in computed values, do not attempt to calculate a complete catalytic cycle.

\section{Alternate Reaction Pathways}

Given reversible formation of $\mathbf{B}$, the energy barrier for aryl-directed $\mathbf{C}-\mathrm{C}$ oxidative addition must be compared to the alternative direct $\mathrm{C}-\mathrm{C}$ oxidative addition from $\mathbf{A}$ (and subsequent steps, should that process be reversible). As shown in Figure S1 C-C oxidative addition from B directly is disfavored $\left(\mathbf{D}, \Delta \mathrm{G}^{+}=32.0 \mathrm{kcal} \mathrm{mol}^{-1}\right.$, at M3). Dissociation of a carbonyl ligand and $\mathrm{C}-\mathrm{C}$ oxidative addition is a slightly lower energy process $\left(\mathbf{D}^{\prime}, \Delta \mathrm{G}^{*}=29.6 \mathrm{kcal} \mathrm{mol}^{-1}\right.$, at M3) as shown in Scheme S2, but is still strongly disfavored when compared to $\mathrm{C}-\mathrm{C}$ activation from $\mathbf{A}$.
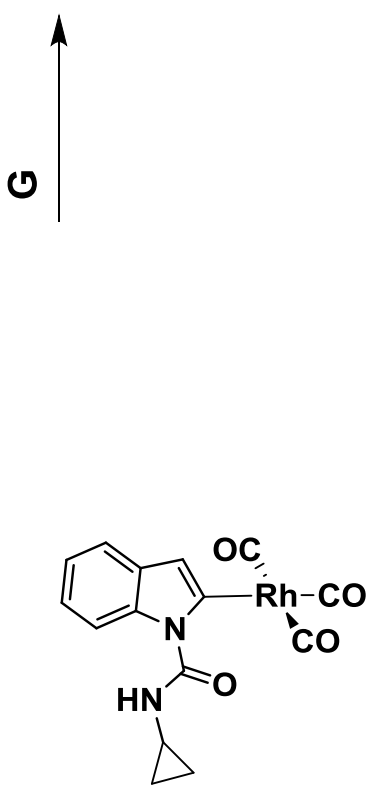

B

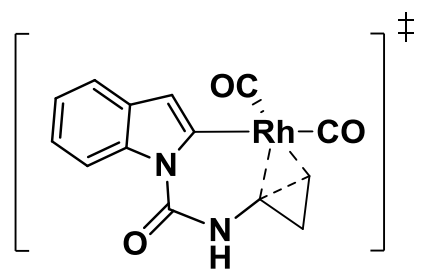

D'

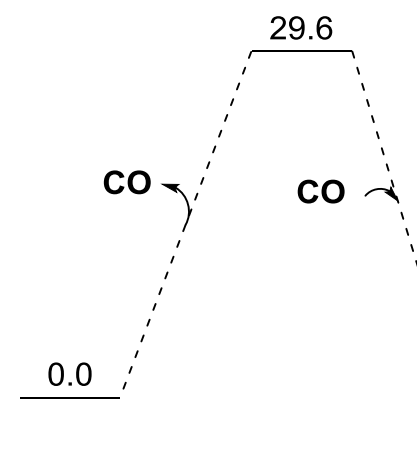

6.2<smiles>C[R](C)([O-])c1cc2ccccc2n1C(=O)NC1CCCCC1</smiles>

F 
Scheme S2. Reaction profile in $\mathrm{kcal} \mathrm{mol}^{-1}$ calculated at M3 for aryl-directed C-C oxidative addition. The energy barriers $\mathrm{CO}$ (dis)association are assumed to be negligible compared to the bond making/breaking step.

As carbonylation of $\mathbf{E}$ is calculated to be facile $\left(\mathbf{G}, \Delta \mathrm{G}^{\dagger}=12.7 \mathrm{kcal} \mathrm{mol}^{-1}\right.$, at $\left.\mathrm{M} 3\right)$ and irreversible $\left(\Delta \mathrm{G}^{\dagger}\right.$ rev $\left.=34.8 \mathrm{kcal} \mathrm{mol}^{-1}\right)$, the key $\mathrm{C}-\mathrm{C}$ oxidative addition step occurs directly from $\mathbf{A}$.

Subsequent C(2)- $\mathrm{H}$ metalation from $\mathbf{H}$ to $\mathbf{I}$ once again occurs with loss of $\mathrm{H}^{+}$and is therefore subject to an unacceptable error in calculating the relative energies. Therefore, by analogy to metalation of $\mathbf{A}$, this process is suggested to be facile and a new zero of energy set at $\mathbf{I}$.

To afford the product, two $\mathrm{C}-\mathrm{C}$ reductive elimination steps must occur from $\mathbf{I}$ to regenerate the catalyst. As outlined in the main text, $\mathrm{C}\left(\mathrm{sp}^{2}\right)-\mathrm{C}\left(\mathrm{sp}^{2}\right)$ reductive elimination to generate an intermediate with an 8-membered ring $(\mathbf{L})$ is strongly favoured over the alternative elimination of a 5-membered ring $\left(\mathbf{J}, \Delta \mathrm{G}^{\dagger}=17.8 \mathrm{kcal} \mathrm{mol}^{-1} c f . \mathbf{K}, \Delta \mathrm{G}^{\dagger}=32.4 \mathrm{kcal} \mathrm{mol}^{-1}\right.$, both at M3). Formation of $\mathbf{L}$ is calculated to be reversible $\left(\Delta \mathrm{G}^{\dagger}\right.$ rev $\left.=22.9 \mathrm{kcal} \mathrm{mol}^{-1}\right)$, thus the final $\mathrm{C}-\mathrm{C}$ reductive elimination to $\mathbf{P}$ must be considered and compared to that of the irreversible $\mathrm{C}-\mathrm{C}$ reductive elimination to $\mathbf{K}$. Calculated free energy barriers indicate that transition over $\mathbf{N}\left(\Delta \mathrm{G}^{\ddagger}=23.5 \mathrm{kcal} \mathrm{mol}^{-1}\right.$, at $\left.\mathrm{M} 3\right)$ to $\mathbf{P}$ proceeds faster than the reverse reaction and subsequent $\mathrm{sp}^{2}-\mathrm{sp}^{3}$ reductive elimination $\left(\mathbf{O}, \Delta \mathrm{G}^{\ddagger}=31.9 \mathrm{kcal} \mathrm{mol}^{-1}\right.$, at M3) to generate $\mathbf{M}$. Energy profiles for both possibilities are shown in Figure S1.

\section{Method test}

We have considered two common and generally accurate hybrid functionals (namely PBE0 and B3LYP) in combination with Grimme's damped dispersion correction (D3BJ) ${ }^{18}$ to evaluate the functional dependence of the relative energies quoted and ensure the conclusions are robust to the choice of functional. At M2 geometries single point energies were computed using PBE0-D3BJ and B3LYP-D3BJ functionals, using the 6-311+G(2d,p),Rh(LANTZ(f) basis set with DCB solvent modeled implicitly within SMD and compared to M3 (identical basis set and solvent model, but using the B3PW91-D3BJ functional). Table S1 outlines these data. Crucial $\Delta \mathrm{E}^{\ddagger}$ values differ little between functionals $\left(\mathrm{MAD}(\mathrm{PBE} 0-\mathrm{D} 3 \mathrm{BJ})=2.0 \mathrm{kcal} \mathrm{mol}^{-}\right.$ ${ }^{-1}, \operatorname{MAD}(\mathrm{B} 3 \mathrm{LYP}-\mathrm{D} 3 \mathrm{BJ})=2.5 \mathrm{kcal} \mathrm{mol}^{-1}$ from M3 values $)$. 


\begin{tabular}{|c|c|c|c|c|c|}
\hline \multirow[b]{2}{*}{ Species } & \multirow{2}{*}{$\begin{array}{c}\text { B3PW91- } \\
\text { D3BJ } \\
\Delta \mathrm{E}\end{array}$} & \multicolumn{2}{|c|}{ PBE0-D3BJ } & \multicolumn{2}{|c|}{ B3LYP-D3BJ } \\
\hline & & $\Delta \mathrm{E}$ & $\mathrm{AD}$ & $\Delta \mathrm{E}$ & $\mathrm{AD}$ \\
\hline$\left[\mathrm{Rh}(\mathrm{CO})_{4}\right]^{+}$ & 0.0 & 0.0 & 0.0 & 0.0 & 0.0 \\
\hline A & 0.2 & 2.5 & 2.3 & -0.6 & 0.8 \\
\hline $\mathrm{C}$ & 22.2 & 26.9 & 4.7 & 23.3 & 1.1 \\
\hline $\mathbf{E}$ & 3.5 & 7.7 & 4.2 & 6.3 & 2.8 \\
\hline G & 16.0 & 19.8 & 3.8 & 20.8 & 4.9 \\
\hline H & -20.3 & -16.9 & 3.4 & -16.8 & 3.6 \\
\hline I & 0.0 & 0.0 & 0.0 & 0.0 & 0.0 \\
\hline $\mathbf{J}$ & 17.5 & 17.2 & 0.3 & 19.2 & 1.7 \\
\hline $\mathbf{K}$ & 43.7 & 43.3 & 0.5 & 40.9 & 2.8 \\
\hline $\mathbf{L}$ & -7.0 & -6.9 & 0.1 & -7.1 & 0.0 \\
\hline $\mathbf{N}$ & 18.1 & 17.2 & 0.9 & 20.0 & 1.9 \\
\hline $\mathbf{P}$ & -17.3 & -19.6 & 2.2 & -14.2 & 3.2 \\
\hline
\end{tabular}

Table S1. Functional dependence of potential energy differences $(\Delta \mathrm{E})$. All energies are quoted in $\mathrm{kcal} \mathrm{mol}^{-1}$ and calculated with the $6-311+\mathrm{G}(2 \mathrm{~d}, \mathrm{p}), \operatorname{Rh}(\operatorname{LANTZ}(\mathrm{f}))$ basis set and $\mathrm{SMD}(\mathrm{DCB})$ implicit solvation model. Absolute deviations (AD) are quoted against the M3 level of theory.

\section{Geometries and Absolute Energies}

Free energy and enthalpy corrections have been computed at $403 \mathrm{~K}$. Gibbs free energies quoted here are without the $1 \mathrm{M}$ standard state correction. All energies are in Hartrees.

Species: $\left[\mathrm{Rh}(\mathrm{COD})_{2}\right]^{+}$

$\mathrm{E}(\mathrm{B} 3 \mathrm{PW} 91-\mathrm{D} 3 \mathrm{BJ} / 6-31 \mathrm{G}(\mathrm{d}))=-734.4458601$

$\mathrm{H}(\mathrm{B} 3 \mathrm{PW} 91-\mathrm{D} 3 \mathrm{BJ} / 6-31 \mathrm{G}(\mathrm{d}))=-734.045928$

G(B3PW91-D3BJ/6-31G(d)) = -734.142368

$\mathrm{E}(\mathrm{B} 3 \mathrm{PW} 91-\mathrm{D} 3 \mathrm{BJ} / 6-311+\mathrm{G}(2 \mathrm{~d}, \mathrm{p}), \mathrm{Rh}(\mathrm{LANTZ}(\mathrm{f})))=-733.634548$

Rh $\quad 0.00005200 \quad 0.00059700 \quad-0.00000200$ 


\begin{tabular}{|c|c|c|c|}
\hline $\mathrm{C}$ & -1.47819500 & 0.49993600 & -1.57906900 \\
\hline $\mathrm{C}$ & -1.63429900 & -0.84542700 & -1.28690300 \\
\hline $\mathrm{C}$ & -2.29697600 & 1.64066300 & -1.03387200 \\
\hline $\mathrm{C}$ & -2.72218200 & 1.45768300 & 0.43214600 \\
\hline $\mathrm{H}$ & -1.69608100 & 2.55292700 & -1.12265500 \\
\hline $\mathrm{H}$ & -3.18210700 & 1.79851000 & -1.66712300 \\
\hline $\mathrm{C}$ & -1.63497000 & 0.84522900 & 1.28699000 \\
\hline $\mathrm{H}$ & -2.97320800 & 2.43834400 & 0.84785800 \\
\hline $\mathrm{H}$ & -3.63120700 & 0.85638500 & 0.50387100 \\
\hline $\mathrm{C}$ & -1.47802800 & -0.49998300 & 1.57921100 \\
\hline $\mathrm{C}$ & -2.29593700 & -1.64127900 & 1.03394500 \\
\hline $\mathrm{C}$ & -2.72116800 & -1.45855200 & -0.43208800 \\
\hline $\mathrm{H}$ & -3.18099400 & -1.79975600 & 1.66714500 \\
\hline $\mathrm{H}$ & -1.69439800 & -2.55311400 & 1.12274400 \\
\hline $\mathrm{H}$ & -3.63055500 & -0.85780400 & -0.50384500 \\
\hline $\mathrm{H}$ & -2.97158300 & -2.43936500 & -0.84780800 \\
\hline $\mathrm{H}$ & -0.89336700 & 0.74544300 & -2.46519500 \\
\hline $\mathrm{H}$ & -1.09079400 & -1.54967700 & -1.91541000 \\
\hline $\mathrm{H}$ & -1.09171900 & 1.54980800 & 1.91537300 \\
\hline $\mathrm{H}$ & -0.89293800 & -0.74506700 & 2.46527200 \\
\hline $\mathrm{C}$ & 1.47816600 & 0.50000200 & 1.57897900 \\
\hline $\mathrm{C}$ & 1.63425700 & -0.84538400 & 1.28682400 \\
\hline $\mathrm{C}$ & 2.29603900 & -1.64122600 & -1.03391000 \\
\hline $\mathrm{C}$ & 1.47776200 & -0.50010400 & -1.57902100 \\
\hline $\mathrm{C}$ & 1.63461200 & 0.84518500 & -1.28684800 \\
\hline $\mathrm{C}$ & 2.72202800 & 1.45770600 & -0.43227600 \\
\hline $\mathrm{C}$ & 2.29717800 & 1.64060900 & 1.03386100 \\
\hline $\mathrm{H}$ & 3.63106200 & 0.85644500 & -0.50426100 \\
\hline $\mathrm{H}$ & 2.97289500 & 2.43838300 & -0.84804100 \\
\hline $\mathrm{H}$ & 3.18247300 & 1.79808000 & 1.66697500 \\
\hline
\end{tabular}




$\begin{array}{llrr}\mathrm{H} & 1.69656300 & 2.55302700 & 1.12290200 \\ \mathrm{H} & 0.89332800 & 0.74555700 & 2.46508700 \\ \mathrm{H} & 1.09142100 & 1.54971600 & -1.91533700 \\ \mathrm{H} & 0.89281600 & -0.74528900 & -2.46515200 \\ \mathrm{C} & 2.72124500 & -1.45845000 & 0.43211900 \\ \mathrm{H} & 1.69474800 & -2.55322100 & -1.12273900 \\ \mathrm{H} & 3.18110300 & -1.79939600 & -1.66717600 \\ \mathrm{H} & 3.63056200 & -0.85759300 & 0.50389600 \\ \mathrm{H} & 2.97174300 & -2.43921900 & 0.84789100 \\ \mathrm{H} & 1.09071300 & -1.54964700 & 1.91528800\end{array}$

Species: $\left[\mathrm{Rh}(\mathrm{CO})_{4}\right]^{+}$

$\mathrm{E}(\mathrm{B} 3 \mathrm{PW} 91-\mathrm{D} 3 \mathrm{BJ} / 6-31 \mathrm{G}(\mathrm{d}))=-563.5847007$

$\mathrm{H}(\mathrm{B} 3 \mathrm{PW} 91-\mathrm{D} 3 \mathrm{BJ} / 6-31 \mathrm{G}(\mathrm{d}))=-563.534402$

G(B3PW91-D3BJ/6-31G(d)) = -563.608047

$\mathrm{E}(\mathrm{B} 3 \mathrm{PW} 91-\mathrm{D} 3 \mathrm{BJ} / 6-311+\mathrm{G}(2 \mathrm{~d}, \mathrm{p}), \mathrm{Rh}(\mathrm{LANTZ}(\mathrm{f})))=-562.7494198$

$\begin{array}{llll}\text { Rh } & -0.00002000 & -0.00002600 & -0.00001000 \\ \mathrm{C} & 1.38623400 & -1.38535900 & 0.00003900 \\ \mathrm{O} & 2.18667200 & -2.18522700 & 0.00006500 \\ \mathrm{C} & 1.38545400 & 1.38599300 & -0.00003100 \\ \mathrm{O} & 2.18548800 & 2.18627300 & -0.00004200 \\ \mathrm{C} & -1.38607200 & 1.38549800 & 0.00003900 \\ \mathrm{O} & -2.18641800 & 2.18545900 & 0.00006500 \\ \mathrm{C} & -1.38560400 & -1.38611100 & -0.00003100 \\ \mathrm{O} & -2.18563600 & -2.18637800 & -0.00004200\end{array}$

Species: A

$\mathrm{E}(\mathrm{B} 3 \mathrm{PW} 91-\mathrm{D} 3 \mathrm{BJ} / 6-31 \mathrm{G}(\mathrm{d}))=-1099.3779806$

H(B3PW91-D3BJ/6-31G(d)) = -1099.092748 
G(B3PW91-D3BJ/6-31G(d)) = -1099.215452

E(B3PW91-D3BJ/6-311+G(2d,p),Rh(LANTZ(f))) = -1098.6894146

$\begin{array}{llll}\text { C } & 4.14330000 & 0.15640000 & 0.02550000\end{array}$

C $\quad 2.79080000 \quad-0.20460000 \quad 0.20540000$

C $\quad 4.18760000 \quad 1.57670000 \quad-0.23340000$

C $\quad 2.91890000 \quad 2.04450000 \quad-0.23210000$

$\mathrm{N} \quad 2.02780000 \quad 0.97670000 \quad 0.02730000$

C $\quad 0.67160000 \quad 1.04500000 \quad-0.02120000$

O $\quad-0.00410000 \quad 0.13470000 \quad 0.54400000$

$\mathrm{N} \quad 0.11290000 \quad 2.08900000 \quad-0.65720000$

C $\quad-1.27180000 \quad 2.45910000 \quad-0.56510000$

$\begin{array}{llll}\text { C } & -1.85920000 & 2.74750000 & 0.79320000\end{array}$

C $\quad-1.57740000 \quad 3.87320000 \quad-0.15330000$

$\mathrm{H} \quad-1.91550000 \quad 2.00980000 \quad-1.31840000$

$\mathrm{H} \quad-1.20680000 \quad 2.63670000 \quad 1.65440000$

$\begin{array}{llll}\mathrm{H} & -2.89260000 & 2.45950000 & 0.96030000\end{array}$

$\mathrm{H} \quad-2.40860000 \quad 4.36730000 \quad-0.64560000$

$\mathrm{H} \quad-0.73410000 \quad 4.51820000 \quad 0.07700000$

$\mathrm{H} \quad 0.71060000 \quad 2.65230000 \quad-1.24790000$

C $\quad 2.40030000 \quad-1.51310000 \quad 0.47280000$

$\mathrm{H} \quad 5.07730000 \quad 2.17510000 \quad-0.37520000$

Rh $\quad-1.80140000 \quad-0.70370000 \quad-0.01680000$

C $\quad-2.38560000 \quad-0.48520000 \quad 1.83860000$

C $\quad-3.38470000 \quad-1.56400000 \quad-0.45070000$

O $\quad-4.36150000 \quad-2.08860000 \quad-0.72520000$

O $\quad-2.69320000 \quad-0.34850000 \quad 2.92180000$

C $\quad-1.13560000 \quad-0.96820000 \quad-1.81830000$

O $\quad-0.71020000-1.12220000-2.86050000$

$\begin{array}{llll}\mathrm{H} & 2.55490000 & 3.05880000 & -0.30560000\end{array}$ 


$\begin{array}{llll}\mathrm{C} & 3.41050000 & -2.46980000 & 0.55370000 \\ \mathrm{C} & 4.75940000 & -2.13170000 & 0.38030000 \\ \mathrm{C} & 5.13780000 & -0.82070000 & 0.11700000 \\ \mathrm{H} & 5.51720000 & -2.90510000 & 0.45700000 \\ \mathrm{H} & 6.18240000 & -0.55730000 & -0.01710000 \\ \mathrm{H} & 1.36560000 & -1.78090000 & 0.63960000 \\ \mathrm{H} & 3.14210000 & -3.49970000 & 0.76880000\end{array}$

Species: $\mathbf{T S}_{\mathrm{A}-\mathrm{CHoa}}$

$\mathrm{E}(\mathrm{B} 3 \mathrm{PW} 91-\mathrm{D} 3 \mathrm{BJ} / 6-31 \mathrm{G}(\mathrm{d}))=-1099.3381824$

H(B3PW91-D3BJ/6-31G(d)) = -1099.058785

G(B3PW91-D3BJ/6-31G(d)) = -1099.177912

E(B3PW91-D3BJ/6-311+G(2d,p),Rh(LANTZ(f))) = -1098.6424267

Imaginary frequency $=-957.35 \mathrm{~cm}^{-1}$

$\begin{array}{llrr}\mathrm{C} & -1.02954100 & -2.20334700 & -0.63690400 \\ \mathrm{C} & -0.27572400 & -1.09336900 & -0.38190700 \\ \mathrm{C} & -2.40968900 & -1.87281300 & -0.39501800 \\ \mathrm{C} & -2.47357000 & -0.51509800 & 0.00552000 \\ \mathrm{~N} & -1.15129300 & -0.03381200 & -0.01448900 \\ \mathrm{C} & -0.60282500 & 1.20768600 & 0.13529400 \\ \mathrm{O} & 0.66798200 & 1.29782000 & 0.18306400 \\ \mathrm{~N} & -1.36561200 & 2.29316900 & 0.20635100 \\ \mathrm{C} & -0.83186200 & 3.61604700 & 0.40871000 \\ \mathrm{C} & -0.00469100 & 4.24204600 & -0.67765100 \\ \mathrm{C} & -1.40393300 & 4.72308100 & -0.42707100 \\ \mathrm{H} & -0.57278000 & 3.82653500 & 1.44297400 \\ \mathrm{H} & 0.17144700 & 3.65273200 & -1.57210000 \\ \mathrm{H} & 0.83048800 & 4.86239600 & -0.36915000\end{array}$ 


$\begin{array}{llll}\mathrm{H} & -1.55640700 & 5.68304700 & 0.05540300 \\ \mathrm{H} & -2.16025200 & 4.46259400 & -1.16312500 \\ \mathrm{H} & -2.36136900 & 2.18649000 & 0.07432000 \\ \mathrm{H} & -0.65268900 & -3.16429400 & -0.96035800 \\ \mathrm{H} & 0.71739800 & -0.67395400 & -1.41996400 \\ \mathrm{C} & -3.58494700 & -2.62902700 & -0.46732900 \\ \mathrm{Rh} & 1.66368400 & -0.49485400 & -0.09608800 \\ \mathrm{C} & 3.31348700 & 0.49876300 & -0.63492400 \\ \mathrm{C} & 2.39830700 & -2.16713400 & -0.48244000 \\ \mathrm{O} & 2.80514300 & -3.20424300 & -0.72353000 \\ \mathrm{O} & 4.18518600 & 1.12329500 & -1.00899000 \\ \mathrm{C} & 1.90113400 & -0.62752400 & 1.99568500 \\ \mathrm{O} & 1.95130700 & -0.75066300 & 3.12170700 \\ \mathrm{C} & -4.78834300 & -2.02417600 & -0.13091200 \\ \mathrm{C} & -4.82828000 & -0.68923000 & 0.29546100 \\ \mathrm{C} & -3.67298600 & 0.08532900 & 0.37843200 \\ \mathrm{H} & -3.54996900 & -3.67038100 & -0.77190200 \\ \mathrm{H} & -5.71036300 & -2.59438200 & -0.18118500 \\ \mathrm{H} & -5.77730000 & -0.24753100 & 0.58235800 \\ \mathrm{H} & -3.74180800 & 1.09259200 & 0.77595700\end{array}$

Species: B

$\mathrm{E}(\mathrm{B} 3 \mathrm{PW} 91-\mathrm{D} 3 \mathrm{BJ} / 6-31 \mathrm{G}(\mathrm{d}))=-1098.9960392$

H(B3PW91-D3BJ/6-31G(d)) = -1098.724062

G(B3PW91-D3BJ/6-31G(d)) = -1098.843607

E(B3PW91-D3BJ/6-311+G(2d,p),Rh(LANTZ(f))) = -1098.2427801
C $\quad-3.80259800 \quad 2.24838000 \quad-0.12181500$
C $\quad-2.48316600 \quad 2.17219500 \quad-0.40418700$
C $\quad-4.31952900 \quad 0.90126300 \quad-0.02278400$ 


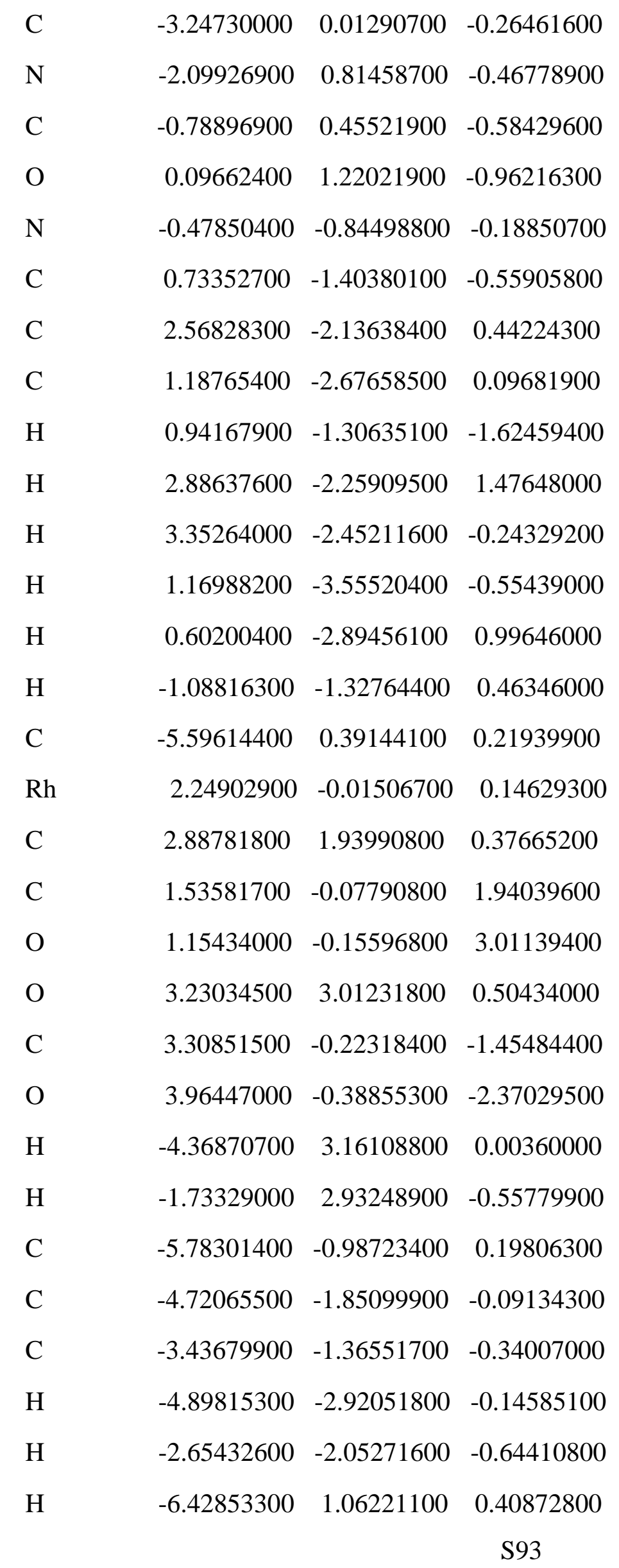




\begin{abstract}
Species: C
$\mathrm{E}(\mathrm{B} 3 \mathrm{PW} 91-\mathrm{D} 3 \mathrm{BJ} / 6-31 \mathrm{G}(\mathrm{d}))=-1099.3419171$

H(B3PW91-D3BJ/6-31G(d)) = -1099.059515

G(B3PW91-D3BJ/6-31G(d)) = -1099.178626
\end{abstract}

E(B3PW91-D3BJ/6-311+G(2d,p),Rh(LANTZ(f))) = -1098.6543639

Imaginary frequency $=-170.84 \mathrm{~cm}^{-1}$

\begin{tabular}{|c|c|c|c|}
\hline $\mathrm{C}$ & -3.80259800 & 2.24838000 & -0.12181500 \\
\hline $\mathrm{C}$ & -2.48316600 & 2.17219500 & -0.40418700 \\
\hline $\mathrm{C}$ & -4.31952900 & 0.90126300 & -0.02278400 \\
\hline $\mathrm{C}$ & -3.24730000 & 0.01290700 & -0.26461600 \\
\hline $\mathrm{N}$ & -2.09926900 & 0.81458700 & -0.46778900 \\
\hline $\mathrm{C}$ & -0.78896900 & 0.45521900 & -0.58429600 \\
\hline $\mathrm{O}$ & 0.09662400 & 1.22021900 & -0.96216300 \\
\hline $\mathrm{N}$ & -0.47850400 & -0.84498800 & -0.18850700 \\
\hline $\mathrm{C}$ & 0.73352700 & -1.40380100 & -0.55905800 \\
\hline $\mathrm{C}$ & 2.56828300 & -2.13638400 & 0.44224300 \\
\hline $\mathrm{C}$ & 1.18765400 & -2.67658500 & 0.09681900 \\
\hline $\mathrm{H}$ & 0.94167900 & -1.30635100 & -1.62459400 \\
\hline $\mathrm{H}$ & 2.88637600 & -2.25909500 & 1.47648000 \\
\hline $\mathrm{H}$ & 3.35264000 & -2.45211600 & -0.24329200 \\
\hline $\mathrm{H}$ & 1.16988200 & -3.55520400 & -0.55439000 \\
\hline $\mathrm{H}$ & 0.60200400 & -2.89456100 & 0.99646000 \\
\hline $\mathrm{H}$ & -1.08816300 & -1.32764400 & 0.46346000 \\
\hline $\mathrm{C}$ & -5.59614400 & 0.39144100 & 0.21939900 \\
\hline $\mathrm{Rh}$ & 2.24902900 & -0.01506700 & 0.14629300 \\
\hline $\mathrm{C}$ & 2.88781800 & 1.93990800 & 0.37665200 \\
\hline $\mathrm{C}$ & 1.53581700 & -0.07790800 & 1.94039600 \\
\hline
\end{tabular}




$\begin{array}{lrrr}\mathrm{O} & 1.15434000 & -0.15596800 & 3.01139400 \\ \mathrm{O} & 3.23034500 & 3.01231800 & 0.50434000 \\ \mathrm{C} & 3.30851500 & -0.22318400 & -1.45484400 \\ \mathrm{O} & 3.96447000 & -0.38855300 & -2.37029500 \\ \mathrm{H} & -4.36870700 & 3.16108800 & 0.00360000 \\ \mathrm{H} & -1.73329000 & 2.93248900 & -0.55779900 \\ \mathrm{C} & -5.78301400 & -0.98723400 & 0.19806300 \\ \mathrm{C} & -4.72065500 & -1.85099900 & -0.09134300 \\ \mathrm{C} & -3.43679900 & -1.36551700 & -0.34007000 \\ \mathrm{H} & -4.89815300 & -2.92051800 & -0.14585100 \\ \mathrm{H} & -2.65432600 & -2.05271600 & -0.64410800 \\ \mathrm{H} & -6.42853300 & 1.06221100 & 0.40872800 \\ \mathrm{H} & -6.77017800 & -1.39908100 & 0.38261800\end{array}$

Species: D

$\mathrm{E}(\mathrm{B} 3 \mathrm{PW} 91-\mathrm{D} 3 \mathrm{BJ} / 6-31 \mathrm{G}(\mathrm{d}))=-1098.9427423$

H(B3PW91-D3BJ/6-31G(d)) = -1098.673082

G(B3PW91-D3BJ/6-31G(d)) = -1098.787524

E(B3PW91-D3BJ/6-311+G(2d,p),Rh(LANTZ(f))) = -1098.1945737

Imaginary frequency $=-389.29 \mathrm{~cm}^{-1}$

$\begin{array}{llll}\mathrm{C} & 2.63763300 & 1.24131500 & -0.09145400 \\ \mathrm{C} & 2.68283100 & -0.16490400 & 0.02693300 \\ \mathrm{C} & 1.25278800 & 1.62277800 & -0.07083200 \\ \mathrm{C} & 0.47657500 & 0.50237800 & 0.03722400 \\ \mathrm{~N} & 1.34671600 & -0.61479000 & 0.09719200 \\ \mathrm{C} & 1.03315800 & -1.95955100 & 0.26932300 \\ \mathrm{O} & 1.88011900 & -2.79581900 & 0.54343200 \\ \mathrm{~N} & -0.28643100 & -2.35373000 & 0.15052200 \\ \mathrm{C} & -1.33335800 & -1.83316400 & -0.68286300\end{array}$




$\begin{array}{lrrr}\mathrm{C} & -1.29074000 & -0.56866300 & -2.06866500 \\ \mathrm{C} & -1.21115700 & -2.05146800 & -2.16262000 \\ \mathrm{H} & -2.30835800 & -2.11430000 & -0.29977900 \\ \mathrm{H} & -2.04583100 & -2.55953700 & -2.64545700 \\ \mathrm{H} & -0.24578900 & -2.44259500 & -2.48128300 \\ \mathrm{H} & -0.28437500 & -3.37116400 & 0.16637100 \\ \mathrm{C} & 3.89056600 & -0.86224200 & 0.03220500 \\ \mathrm{H} & 0.88591400 & 2.63911400 & -0.11324800 \\ \mathrm{Rh} & -1.56571000 & 0.34705200 & 0.11017800 \\ \mathrm{C} & -1.67085000 & 2.19818000 & -0.44768000 \\ \mathrm{C} & -3.48559800 & 0.15992700 & 0.16422000 \\ \mathrm{O} & -1.74298600 & 3.31359000 & -0.71319400 \\ \mathrm{O} & -4.62542500 & 0.03320400 & 0.19465900 \\ \mathrm{H} & -2.19400700 & -0.10671100 & -2.45200600 \\ \mathrm{H} & -0.37233300 & -0.02512900 & -2.25677500 \\ \mathrm{O} & -1.00913400 & -0.21350900 & 3.08868700 \\ \mathrm{C} & -1.25649200 & -0.06567500 & 1.97925900 \\ \mathrm{C} & 5.06028900 & -0.11544100 & -0.08324600 \\ \mathrm{H} & 5.03403200 & 1.28228900 & -0.19848500 \\ \mathrm{H} & 3.82741400 & 1.96937100 & -0.20363500 \\ \mathrm{H} & 3.91168800 & -1.93757200 & 0.13675300 \\ \mathrm{H} & 6.01541300 & -0.63356300 & -0.07713100 \\ \mathrm{H} & 5.96824100 & 1.83109600 & -0.28271300 \\ \mathrm{H} & 3.80125700 & 3.05214600 & -0.29353000\end{array}$

Species: D'

$\mathrm{E}(\mathrm{B} 3 \mathrm{PW} 91-\mathrm{D} 3 \mathrm{BJ} / 6-31 \mathrm{G}(\mathrm{d}))=-985.6597643$

$\mathrm{H}(\mathrm{B} 3 \mathrm{PW} 91-\mathrm{D} 3 \mathrm{BJ} / 6-31 \mathrm{G}(\mathrm{d}))=-985.402947$

G(B3PW91-D3BJ/6-31G(d)) = -985.507204

$\mathrm{E}(\mathrm{B} 3 \mathrm{PW} 91-\mathrm{D} 3 \mathrm{BJ} / 6-311+\mathrm{G}(2 \mathrm{~d}, \mathrm{p}), \mathrm{Rh}(\mathrm{LANTZ}(\mathrm{f})))=-984.8829755$ 
Imaginary frequency $=-302.70 \mathrm{~cm}^{-1}$

\begin{tabular}{|c|c|c|c|}
\hline $\mathrm{C}$ & 2.61523000 & -1.11983400 & 0.31004600 \\
\hline $\mathrm{C}$ & 2.64662400 & 0.22721200 & -0.11625200 \\
\hline $\mathrm{C}$ & 1.23819200 & -1.48802100 & 0.43017700 \\
\hline $\mathrm{C}$ & 0.43447000 & -0.41885900 & 0.10660400 \\
\hline $\mathrm{N}$ & 1.31016900 & 0.65649100 & -0.21874100 \\
\hline $\mathrm{C}$ & 0.99409800 & 1.98644800 & -0.44992500 \\
\hline $\mathrm{O}$ & 1.82969000 & 2.82593300 & -0.74676800 \\
\hline $\mathrm{N}$ & -0.30593400 & 2.39816600 & -0.23464100 \\
\hline $\mathrm{C}$ & -1.55170500 & 1.74824000 & -0.15765900 \\
\hline $\mathrm{C}$ & -1.86592200 & 1.09215900 & 1.60363800 \\
\hline $\mathrm{C}$ & -2.40178300 & 2.31685800 & 0.96130800 \\
\hline $\mathrm{H}$ & -2.06808400 & 1.58932600 & -1.12040600 \\
\hline $\mathrm{H}$ & -0.89104800 & 1.18949900 & 2.07057000 \\
\hline $\mathrm{H}$ & -2.54474100 & 0.39566800 & 2.09187200 \\
\hline $\mathrm{H}$ & -3.47327700 & 2.28259800 & 0.75915800 \\
\hline $\mathrm{H}$ & -2.08665500 & 3.28668200 & 1.34812900 \\
\hline $\mathrm{H}$ & -0.34855300 & 3.40796100 & -0.31517100 \\
\hline $\mathrm{C}$ & 3.85206300 & 0.89460800 & -0.34361100 \\
\hline $\mathrm{H}$ & 0.88457400 & -2.46650900 & 0.72622000 \\
\hline $\mathrm{Rh}$ & -1.58941600 & -0.40613100 & -0.07572200 \\
\hline $\mathrm{C}$ & -1.46959400 & -2.29262900 & -0.31570500 \\
\hline $\mathrm{C}$ & -3.48962400 & -0.46015500 & -0.32409300 \\
\hline $\mathrm{O}$ & -1.42018600 & -3.43175200 & -0.43841100 \\
\hline $\mathrm{O}$ & -4.62400000 & -0.51951000 & -0.50530500 \\
\hline $\mathrm{C}$ & 5.02742400 & 0.18014600 & -0.13449600 \\
\hline $\mathrm{C}$ & 5.01405500 & -1.15892200 & 0.28919900 \\
\hline C & 3.81395400 & -1.81621000 & 0.51438700 \\
\hline \multirow[t]{2}{*}{$\mathrm{H}$} & 5.95432200 & -1.68276200 & 0.43935200 \\
\hline & & & S97 \\
\hline
\end{tabular}




\begin{tabular}{|c|c|c|c|}
\hline $\mathrm{H}$ & 3.79618700 & -2.85239400 & 0.84179000 \\
\hline & 3.86337000 & 1.92421400 & -0.67055000 \\
\hline & 5.97907600 & 0.67537700 & $-0.307 t$ \\
\hline
\end{tabular}

Species: $\mathbf{E}$

$\mathrm{E}(\mathrm{B} 3 \mathrm{PW} 91-\mathrm{D} 3 \mathrm{BJ} / 6-31 \mathrm{G}(\mathrm{d}))=-1099.3818656$

H(B3PW91-D3BJ/6-31G(d)) = -1099.097551

G(B3PW91-D3BJ/6-31G(d)) = -1099.213154

E(B3PW91-D3BJ/6-311+G(2d,p),Rh(LANTZ(f))) = -1098.684231

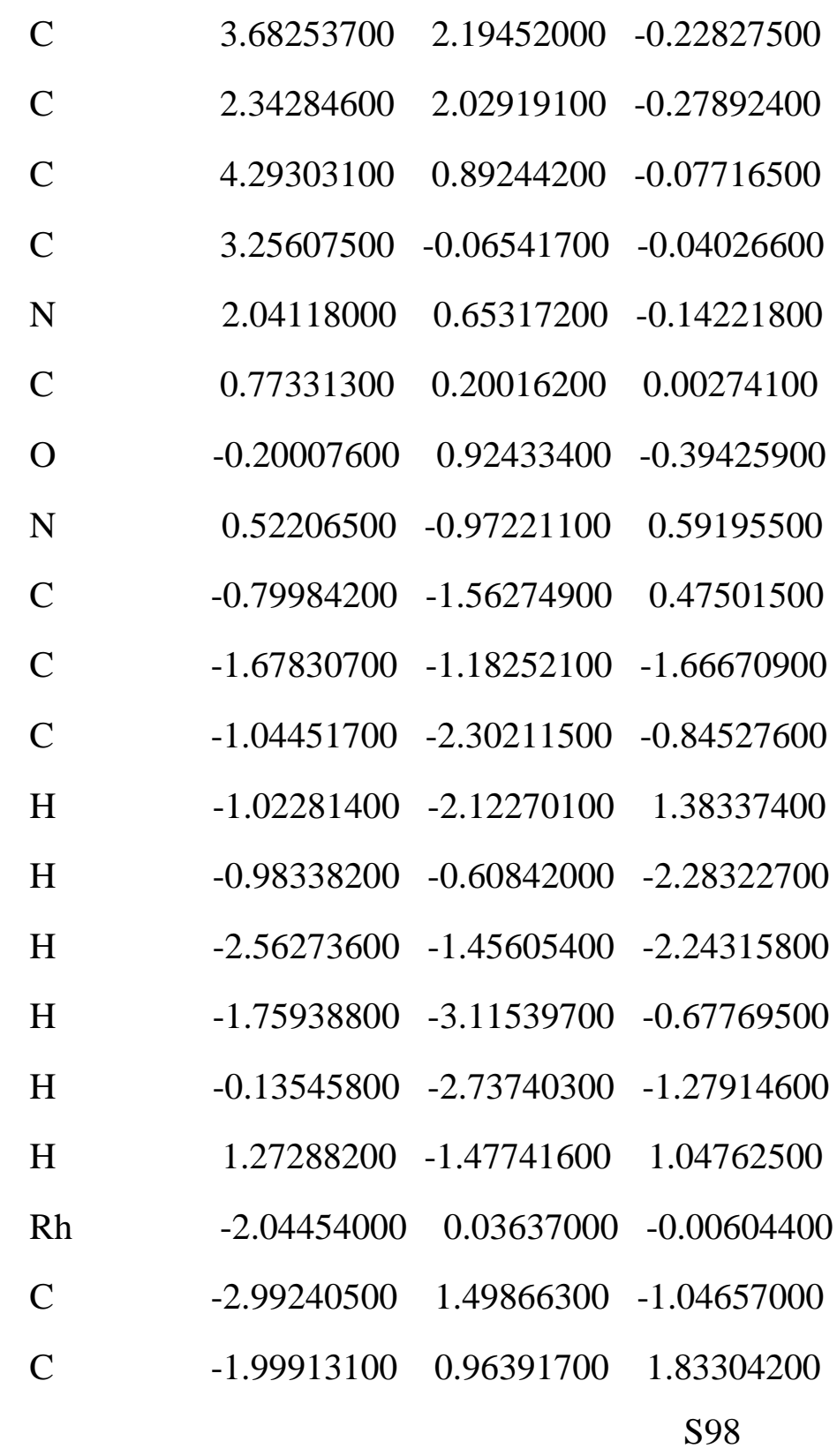




$\begin{array}{llll}\mathrm{O} & -1.89209400 & 1.38495800 & 2.88083200 \\ \mathrm{O} & -3.47090600 & 2.26669100 & -1.73006000 \\ \mathrm{C} & -3.57338500 & -1.00305500 & 0.30911700 \\ \mathrm{O} & -4.49678500 & -1.64312700 & 0.50177300 \\ \mathrm{H} & 4.20237900 & 3.14039300 & -0.29353500 \\ \mathrm{H} & 1.53467500 & 2.73655300 & -0.37919600 \\ \mathrm{C} & 5.62247100 & 0.47099900 & -0.00322800 \\ \mathrm{C} & 5.88925200 & -0.89051700 & 0.08857400 \\ \mathrm{C} & 4.85002900 & -1.82885500 & 0.07823700 \\ \mathrm{C} & 3.51538200 & -1.43309400 & 0.00405500 \\ \mathrm{H} & 5.08324800 & -2.88838200 & 0.11311200 \\ \mathrm{H} & 2.73971600 & -2.18795300 & -0.07561100 \\ \mathrm{H} & 6.43028100 & 1.19581000 & -0.02837500 \\ \mathrm{H} & 6.91697200 & -1.23468300 & 0.14714300\end{array}$

Species: F

$\mathrm{E}(\mathrm{B} 3 \mathrm{PW} 91-\mathrm{D} 3 \mathrm{BJ} / 6-31 \mathrm{G}(\mathrm{d}))=-1098.9900609$

H(B3PW91-D3BJ/6-31G(d)) = -1098.718618

G(B3PW91-D3BJ/6-31G(d)) = -1098.831595

E(B3PW91-D3BJ/6-311+G(2d,p),Rh(LANTZ(f))) = -1098.2388899
$\begin{array}{llll}\text { C } & 2.60840200 & 1.22455700 & -0.12571300\end{array}$
C $\quad 2.68611000 \quad-0.17884800 \quad 0.02098600$
C $\quad 1.21206200 \quad 1.56542700 \quad-0.16138200$
C $\quad 0.46828300 \quad 0.42221500 \quad-0.05624000$
$\mathrm{N} \quad 1.36575500 \quad-0.66412600 \quad 0.04583500$
C $\quad 1.07030500 \quad-2.03263000 \quad 0.08300400$
O $\quad 1.88588100 \quad-2.87196200 \quad 0.43397000$
$\mathrm{N} \quad-0.19466200 \quad-2.38442600-0.29882400$
C $\quad-1.20924800 \quad-1.57993700 \quad-0.90031400$ 


$\begin{array}{lrrr}\mathrm{C} & -1.52485300 & 0.46475000 & -2.01386500 \\ \mathrm{C} & -0.92570400 & -0.92166300 & -2.25474700 \\ \mathrm{H} & -2.11817800 & -2.18289600 & -0.91977900 \\ \mathrm{H} & -1.34499500 & -1.47015800 & -3.10983300 \\ \mathrm{H} & 0.15532800 & -0.84584700 & -2.40283600 \\ \mathrm{H} & -0.36005500 & -3.37442400 & -0.18132800 \\ \mathrm{C} & 3.90770000 & -0.84807500 & 0.08384000 \\ \mathrm{H} & 0.81533000 & 2.56894500 & -0.24542900 \\ \mathrm{Rh} & -1.58821900 & 0.23944700 & 0.08780200 \\ \mathrm{C} & -1.74851000 & 2.18912400 & 0.45057900 \\ \mathrm{C} & -3.49178600 & -0.11520500 & -0.03409100 \\ \mathrm{O} & -1.83047900 & 3.32702200 & 0.50157400 \\ \mathrm{O} & -4.60107200 & -0.36450300 & -0.16524000 \\ \mathrm{H} & -2.52101300 & 0.60201600 & -2.44115100 \\ \mathrm{H} & -0.87993300 & 1.29923600 & -2.28873000 \\ \mathrm{O} & -0.95573300 & -0.89010800 & 2.92473400 \\ \mathrm{C} & -1.25744300 & -0.43046100 & 1.92547900 \\ \mathrm{C} & 5.06248300 & -0.07484300 & 0.00268400 \\ \mathrm{H} & 5.00607700 & 1.32066600 & -0.13635200 \\ \mathrm{H} & 3.78508700 & 1.97891900 & -0.20153800 \\ \mathrm{H} & 5.92957700 & 1.89082000 & -0.19293600 \\ \mathrm{H} & 3.73896800 & 3.05922700 & -0.31209400 \\ \mathrm{H} & 3.94517800 & -1.92203800 & 0.20445200 \\ \mathrm{H} & 6.02947500 & -0.56772200 & 0.05522800\end{array}$

Species: G

$\mathrm{E}(\mathrm{B} 3 \mathrm{PW} 91-\mathrm{D} 3 \mathrm{BJ} / 6-31 \mathrm{G}(\mathrm{d}))=-1099.3540155$

H(B3PW91-D3BJ/6-31G(d)) = -1099.071279

G(B3PW91-D3BJ/6-31G(d)) = -1099.185082

E(B3PW91-D3BJ/6-311+G(2d,p),Rh(LANTZ(f))) = -1098.6642965 
Imaginary frequency $=-338.72 \mathrm{~cm}^{-1}$

\begin{tabular}{|c|c|c|c|}
\hline $\mathrm{C}$ & 3.78379900 & 2.18471400 & -0.39753800 \\
\hline $\mathrm{C}$ & 2.44204400 & 2.03846300 & -0.45473300 \\
\hline $\mathrm{C}$ & 4.36871300 & 0.88603400 & -0.14876200 \\
\hline $\mathrm{C}$ & 3.31377600 & -0.04916000 & -0.06014700 \\
\hline $\mathrm{N}$ & 2.11375000 & 0.68131700 & -0.22896500 \\
\hline $\mathrm{C}$ & 0.83107300 & 0.26491500 & -0.08004300 \\
\hline $\mathrm{O}$ & -0.12469000 & 0.96788600 & -0.51962500 \\
\hline $\mathrm{N}$ & 0.57329700 & -0.88473500 & 0.57152100 \\
\hline $\mathrm{C}$ & -0.75159300 & -1.45625800 & 0.52560700 \\
\hline $\mathrm{C}$ & -2.10039000 & -1.55229200 & -1.52243800 \\
\hline $\mathrm{C}$ & -1.05883800 & -2.32957900 & -0.70938900 \\
\hline $\mathrm{H}$ & -0.94637700 & -1.96536000 & 1.46934900 \\
\hline $\mathrm{H}$ & -1.68420800 & -0.79841000 & -2.20350000 \\
\hline $\mathrm{H}$ & -2.80622000 & -2.14347600 & -2.10697800 \\
\hline $\mathrm{H}$ & -1.46882000 & -3.29354200 & -0.39062200 \\
\hline $\mathrm{H}$ & -0.15865100 & -2.52898300 & -1.30079800 \\
\hline $\mathrm{H}$ & 1.29603900 & -1.30898500 & 1.14054200 \\
\hline $\mathrm{Rh}$ & -2.04015900 & 0.10723900 & 0.03664200 \\
\hline $\mathrm{C}$ & -3.06359700 & 1.69508300 & -0.72854300 \\
\hline $\mathrm{C}$ & -2.12204800 & 0.70169200 & 1.83200600 \\
\hline $\mathrm{O}$ & -2.18789900 & 0.98798500 & 2.93320100 \\
\hline $\mathrm{O}$ & -3.58129100 & 2.61631300 & -1.13848700 \\
\hline $\mathrm{C}$ & -3.39515500 & -1.15254100 & -0.25848500 \\
\hline $\mathrm{O}$ & -4.38981100 & -1.73146100 & -0.11042900 \\
\hline $\mathrm{H}$ & 4.32131400 & 3.11493000 & -0.51978400 \\
\hline $\mathrm{H}$ & 1.64700900 & 2.74998000 & -0.61346400 \\
\hline $\mathrm{C}$ & 5.68928500 & 0.44875400 & -0.02544500 \\
\hline \multirow[t]{2}{*}{$\mathrm{C}$} & 5.93100800 & -0.90704300 & 0.16653200 \\
\hline & & & S101 \\
\hline
\end{tabular}




$\begin{array}{lrrr}\mathrm{C} & 4.87552200 & -1.82598700 & 0.20861600 \\ \mathrm{C} & 3.54907300 & -1.41428800 & 0.08718700 \\ \mathrm{H} & 5.08942500 & -2.88393400 & 0.32390900 \\ \mathrm{H} & 2.76007400 & -2.15835900 & 0.05526800 \\ \mathrm{H} & 6.51004600 & 1.15647700 & -0.08926800 \\ \mathrm{H} & 6.95169700 & -1.26297900 & 0.26472900\end{array}$

Species: $\mathbf{H}$

$\mathrm{E}(\mathrm{B} 3 \mathrm{PW} 91-\mathrm{D} 3 \mathrm{BJ} / 6-31 \mathrm{G}(\mathrm{d}))=-1099.4040168$

H(B3PW91-D3BJ/6-31G(d)) = -1099.118626

$\mathrm{G}(\mathrm{B} 3 \mathrm{PW} 91-\mathrm{D} 3 \mathrm{BJ} / 6-31 \mathrm{G}(\mathrm{d}))=-1099.232549$

E(B3PW91-D3BJ/6-311+G(2d,p),Rh(LANTZ(f))) = -1098.7221837

$\begin{array}{lrrr}\mathrm{C} & 2.66229900 & -1.24178200 & -0.05889900 \\ \mathrm{C} & 2.78526000 & 0.16654800 & -0.04968300 \\ \mathrm{C} & 1.25569400 & -1.54273000 & -0.09976300 \\ \mathrm{C} & 0.55279600 & -0.37066500 & -0.10778900 \\ \mathrm{~N} & 1.48007000 & 0.69366600 & -0.06216500 \\ \mathrm{C} & 1.21446300 & 2.05887200 & 0.10030000 \\ \mathrm{O} & 2.05693700 & 2.92144600 & -0.09276700 \\ \mathrm{~N} & -0.06405700 & 2.37381200 & 0.48156700 \\ \mathrm{C} & -1.04462000 & 1.48288400 & 1.04495200 \\ \mathrm{C} & -1.48184900 & -0.34426500 & 2.68003700 \\ \mathrm{C} & -0.62842400 & 0.87523300 & 2.38876800 \\ \mathrm{H} & -1.95474300 & 2.07591100 & 1.17105800 \\ \mathrm{H} & -0.71588200 & 1.62298400 & 3.18763200 \\ \mathrm{H} & 0.42199000 & 0.57286500 & 2.33690600 \\ \mathrm{H} & -0.18058100 & 3.37156100 & 0.59868600 \\ \mathrm{C} & 4.02548200 & 0.80085100 & -0.00652500 \\ \mathrm{H} & 0.81778300 & -2.53227500 & -0.11603600\end{array}$




$\begin{array}{llll}\mathrm{Rh} & -1.48018900 & -0.08748900 & -0.30402300 \\ \mathrm{C} & -3.38778000 & 0.22701700 & -0.12911400 \\ \mathrm{C} & -1.54660000 & -1.21570000 & 1.43563300 \\ \mathrm{O} & -4.49939200 & 0.44282400 & 0.03802200 \\ \mathrm{O} & -1.65372100 & -2.41309100 & 1.45105000 \\ \mathrm{O} & -0.84248000 & 1.83824800 & -2.74339700 \\ \mathrm{C} & -1.12406400 & 1.14249500 & -1.88711300 \\ \mathrm{C} & 5.15497300 & -0.01291300 & 0.02373300 \\ \mathrm{C} & 5.05468000 & -1.41264000 & 0.01077500 \\ \mathrm{C} & 3.81421100 & -2.03611000 & -0.03024700 \\ \mathrm{H} & 5.95953600 & -2.01409100 & 0.03210600 \\ \mathrm{H} & 3.73403700 & -3.11987400 & -0.03785600 \\ \mathrm{H} & 4.09627200 & 1.88000700 & -0.00827400 \\ \mathrm{H} & 6.13672500 & 0.45194400 & 0.05206800 \\ \mathrm{H} & -2.52258600 & -0.04685600 & 2.88479500 \\ \mathrm{H} & -1.13369900 & -0.93848800 & 3.53111200 \\ \mathrm{C} & -1.58599800 & -1.76645400 & -1.39125500 \\ \mathrm{O} & -1.59704100 & -2.75478600 & -1.95663800\end{array}$

Species: I

$\mathrm{E}(\mathrm{B} 3 \mathrm{PW} 91-\mathrm{D} 3 \mathrm{BJ} / 6-31 \mathrm{G}(\mathrm{d}))=-1212.3067182$

H(B3PW91-D3BJ/6-31G(d)) = -1212.022238

G(B3PW91-D3BJ/6-31G(d)) = -1212.141783

$\mathrm{E}(\mathrm{B} 3 \mathrm{PW} 91-\mathrm{D} 3 \mathrm{BJ} / 6-311+\mathrm{G}(2 \mathrm{~d}, \mathrm{p}), \mathrm{Rh}(\mathrm{LANTZ}(\mathrm{f})))=-1211.5943952$
C $\quad 2.66229900 \quad-1.24178200 \quad-0.05889900$
C $\quad 2.78526000 \quad 0.16654800 \quad-0.04968300$
C $\quad 1.25569400 \quad-1.54273000 \quad-0.09976300$
C $\quad 0.55279600 \quad-0.37066500-0.10778900$
$\mathrm{N} \quad 1.48007000 \quad 0.69366600 \quad-0.06216500$ 


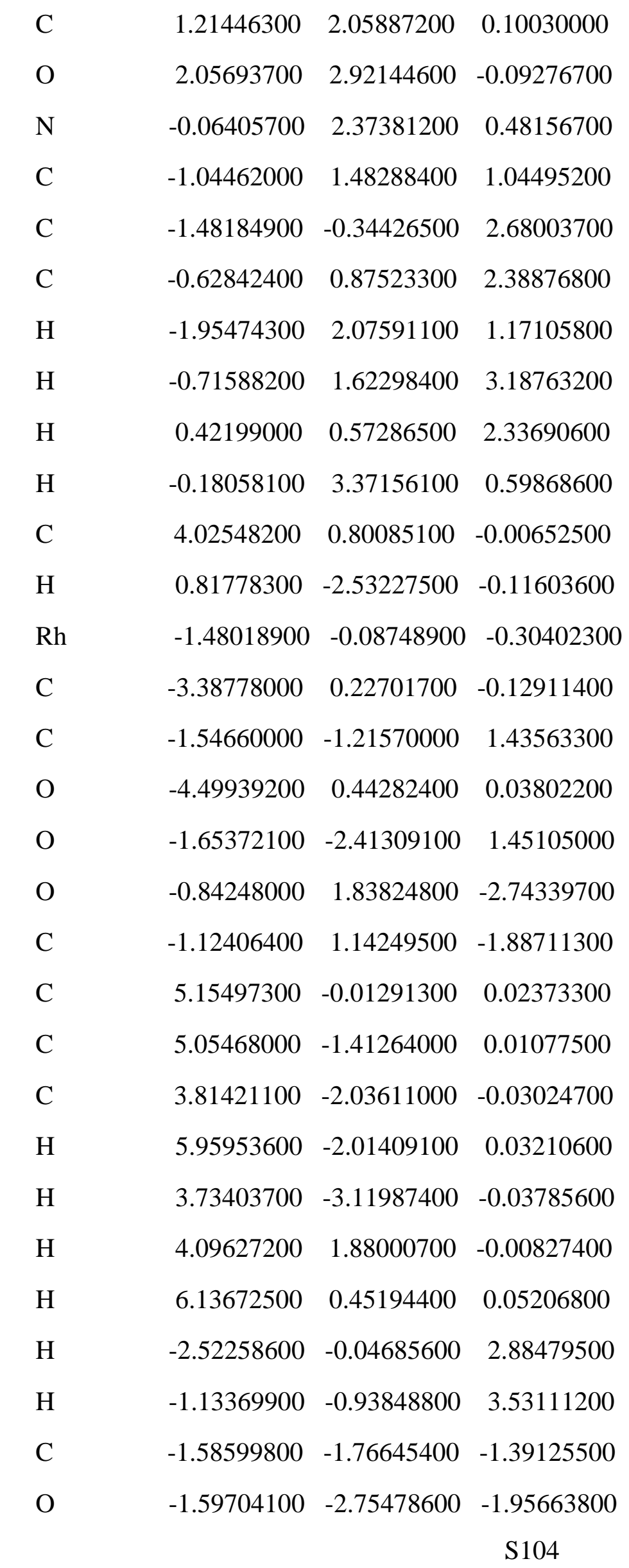




\section{Species: I-D2 $\mathbf{O}$}

$\mathrm{E}(\mathrm{B} 3 \mathrm{PW} 91-\mathrm{D} 3 \mathrm{BJ} / 6-31 \mathrm{G}(\mathrm{d}))=-1175.4313299$

H(B3PW91-D3BJ/6-31G(d)) = -1175.128842

G(B3PW91-D3BJ/6-31G(d)) = -1175.245439

E(B3PW91-D3BJ/6-311+G(2d,p),Rh(LANTZ(f))) = -1174.7211896

$\begin{array}{lrrr}\mathrm{O} & 1.08164400 & -0.90027100 & -2.37463600 \\ \mathrm{C} & -1.25927700 & 1.52856800 & 0.07742600 \\ \mathrm{C} & -0.53880200 & 0.37889100 & -0.10326100 \\ \mathrm{~N} & -1.47571000 & -0.68814800 & -0.19759700 \\ \mathrm{C} & -1.20691200 & -2.04599100 & -0.31056300 \\ \mathrm{O} & -2.06784200 & -2.88415900 & -0.52064600 \\ \mathrm{~N} & 0.13532200 & -2.41018500 & -0.22624800 \\ \mathrm{C} & 1.10812100 & -1.69255700 & 0.59145900 \\ \mathrm{C} & 1.54892000 & -0.32468000 & 2.60118200 \\ \mathrm{C} & 0.69381700 & -1.45312400 & 2.04492100 \\ \mathrm{Rh} & 1.48904800 & 0.16029300 & -0.29614900 \\ \mathrm{C} & 1.60397100 & 1.97246700 & -1.07907900 \\ \mathrm{C} & 1.60819200 & 0.82033500 & 1.59472800 \\ \mathrm{O} & 1.63815500 & 3.03073800 & -1.50511500 \\ \mathrm{O} & 1.73548800 & 1.97741700 & 1.88850900 \\ \mathrm{O} & 4.49240900 & -0.49849500 & -0.38364500 \\ \mathrm{C} & 3.38123400 & -0.22111500 & -0.37268400 \\ \mathrm{H} & 0.71882700 & -1.69188300 & -1.90819600 \\ \mathrm{H} & 0.29971500 & -0.43927000 & -2.71789100 \\ \mathrm{H} & 2.01566800 & -2.30338800 & 0.56136100 \\ \mathrm{H} & 0.79597500 & -2.36165400 & 2.65304500 \\ \mathrm{H} & -0.35780800 & -1.15051000 & 2.08236100 \\ \mathrm{H} & 0.18275100 & -3.42423300 & -0.19130600\end{array}$




$\begin{array}{lrrr}\mathrm{H} & -0.82745700 & 2.51268500 & 0.20753700 \\ \mathrm{H} & 2.59037400 & -0.65773500 & 2.72805300 \\ \mathrm{H} & 1.20528500 & 0.05839900 & 3.56750000 \\ \mathrm{C} & -2.78449100 & -0.17414600 & -0.09514700 \\ \mathrm{C} & -4.02439000 & -0.80857900 & -0.14095400 \\ \mathrm{C} & -2.66399400 & 1.22104900 & 0.09194800 \\ \mathrm{C} & -5.15472200 & -0.00854100 & 0.01101300 \\ \mathrm{C} & -5.05578900 & 1.37810800 & 0.19837300 \\ \mathrm{C} & -3.81546700 & 2.00220200 & 0.23925100 \\ \mathrm{H} & -5.96073000 & 1.96891500 & 0.31157600 \\ \mathrm{H} & -3.73507300 & 3.07616300 & 0.38403200 \\ \mathrm{H} & -4.09766200 & -1.87652600 & -0.29222200 \\ \mathrm{H} & -6.13560400 & -0.47496600 & -0.01962500\end{array}$

Species: TSI-D2O-sbm

$\mathrm{E}(\mathrm{B} 3 \mathrm{PW} 91-\mathrm{D} 3 \mathrm{BJ} / 6-31 \mathrm{G}(\mathrm{d}))=-1175.3768148$

H(B3PW91-D3BJ/6-31G(d)) = -1175.079979

G(B3PW91-D3BJ/6-31G(d)) = -1175.194968

E(B3PW91-D3BJ/6-311+G(2d,p),Rh(LANTZ(f))) = -1174.6688018

Imaginary frequency $=-284.90 \mathrm{~cm}^{-1}$
$\begin{array}{llll}\mathrm{O} & -1.38145900 & 0.77856400 & -2.25881000\end{array}$
C $\quad 1.23591400 \quad-1.49496300 \quad-0.75629700$
C $\quad 0.56869100 \quad-0.27403600 \quad-0.87000000$
$\mathrm{N} \quad \begin{array}{llll}1.49228000 & 0.72776500 & -0.48264800\end{array}$
C $\quad 1.24985200 \quad 2.12044700 \quad-0.60653200$
$\begin{array}{llll}\text { O } & 2.11355600 & 2.89453400 & -0.97630800\end{array}$
$\mathrm{N} \quad \begin{array}{llll}0.01686400 & 2.46755200 & -0.26567200\end{array}$
C $\quad-0.91630300 \quad 1.72456900 \quad 0.57718600$
$\begin{array}{llll}\text { C } & -1.06977700 & 0.29465300 & 2.62954900\end{array}$

S106 


\begin{tabular}{|c|c|c|c|}
\hline $\mathrm{C}$ & -0.31036300 & 1.42680700 & 1.95726300 \\
\hline $\mathrm{Rh}$ & -1.53435100 & -0.11686500 & -0.25952400 \\
\hline $\mathrm{C}$ & -1.96424900 & -1.93762600 & -0.93239200 \\
\hline $\mathrm{C}$ & -1.22975900 & -0.83295200 & 1.61972300 \\
\hline $\mathrm{O}$ & -2.22674600 & -3.00225300 & -1.25057800 \\
\hline $\mathrm{O}$ & -1.15608700 & -2.00315400 & 1.89955100 \\
\hline $\mathrm{O}$ & -4.35491500 & 0.68300300 & 0.45396600 \\
\hline $\mathrm{C}$ & -3.28879200 & 0.35282200 & 0.18571700 \\
\hline $\mathrm{H}$ & -0.14305700 & 0.09662200 & -1.77793600 \\
\hline $\mathrm{H}$ & -1.82914200 & 0.21459400 & -2.90731600 \\
\hline $\mathrm{H}$ & -1.79555000 & 2.36224300 & 0.68879000 \\
\hline $\mathrm{H}$ & -0.30660100 & 2.33270300 & 2.57684100 \\
\hline $\mathrm{H}$ & 0.73292500 & 1.11868800 & 1.83365200 \\
\hline $\mathrm{H}$ & -0.28216700 & 3.39162800 & -0.57451300 \\
\hline $\mathrm{H}$ & 0.80417000 & -2.45999200 & -0.98808900 \\
\hline $\mathrm{H}$ & -2.08881600 & 0.61590600 & 2.89426500 \\
\hline $\mathrm{H}$ & -0.58663600 & -0.07582100 & 3.53978700 \\
\hline $\mathrm{C}$ & 2.69944600 & 0.15429000 & -0.17777500 \\
\hline $\mathrm{C}$ & 3.90697000 & 0.74222100 & 0.22467800 \\
\hline $\mathrm{C}$ & 2.55314700 & -1.25843900 & -0.29932500 \\
\hline $\mathrm{C}$ & 4.95171900 & -0.11449000 & 0.51922500 \\
\hline $\mathrm{C}$ & 4.82544800 & -1.52134400 & 0.41721900 \\
\hline $\mathrm{C}$ & 3.64211900 & -2.10009700 & 0.00998900 \\
\hline $\mathrm{H}$ & 5.67912600 & -2.14712900 & 0.66023400 \\
\hline $\mathrm{H}$ & 3.54216800 & -3.17840500 & -0.07315300 \\
\hline $\mathrm{H}$ & 4.00897900 & 1.81897100 & 0.27837200 \\
\hline $\mathrm{H}$ & 5.90330400 & 0.30496500 & 0.83398000 \\
\hline
\end{tabular}

Species: J

$\mathrm{E}(\mathrm{B} 3 \mathrm{PW} 91-\mathrm{D} 3 \mathrm{BJ} / 6-31 \mathrm{G}(\mathrm{d}))=-1212.2829708$ 
$\mathrm{H}(\mathrm{B} 3 \mathrm{PW} 91-\mathrm{D} 3 \mathrm{BJ} / 6-31 \mathrm{G}(\mathrm{d}))=-1212.000116$

G(B3PW91-D3BJ/6-31G(d)) = -1212.117722

E(B3PW91-D3BJ/6-311+G(2d,p),Rh(LANTZ(f))) = -1211.5665521

Imaginary frequency $=-341.61 \mathrm{~cm}^{-1}$

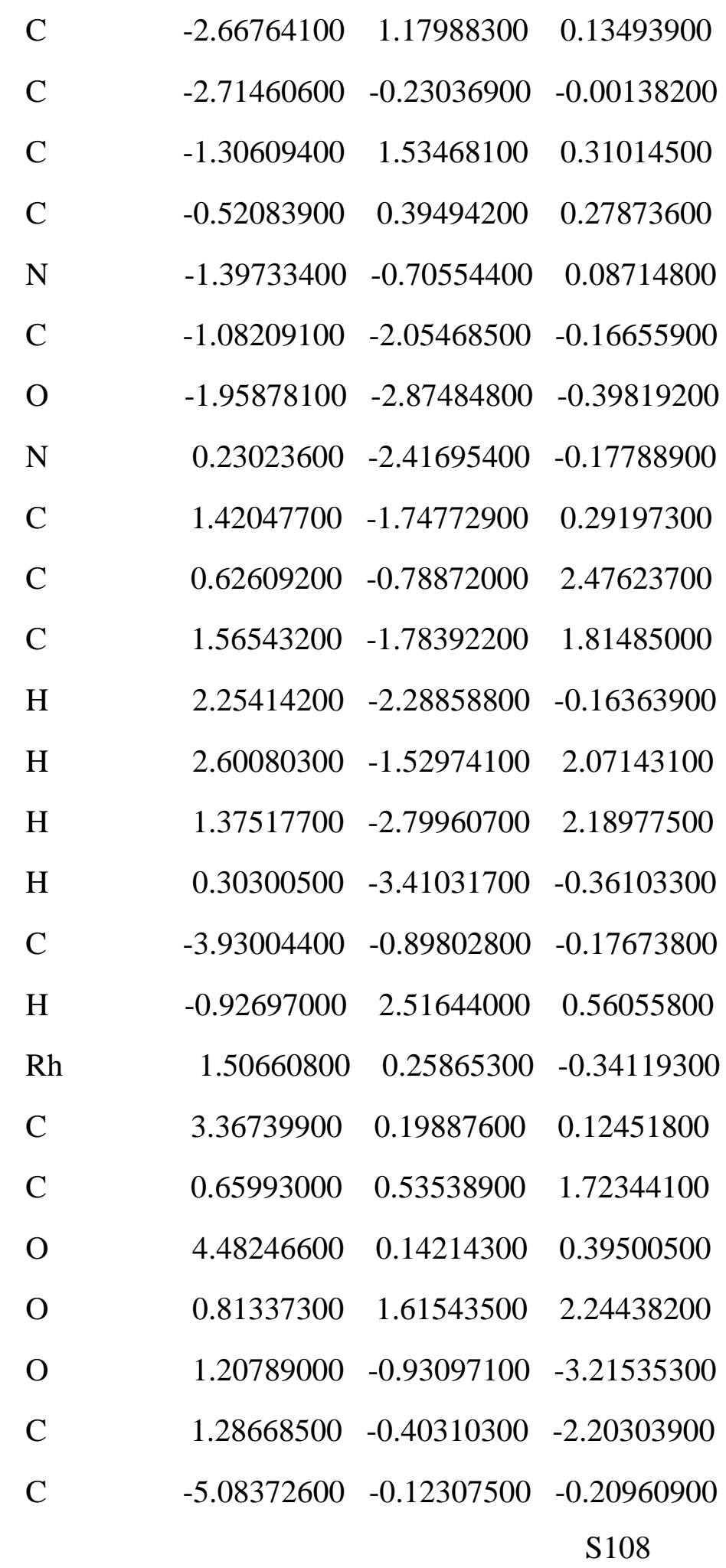




$\begin{array}{llll}\mathrm{C} & -5.05299600 & 1.27674400 & -0.06996900 \\ \mathrm{C} & -3.85023400 & 1.93696300 & 0.10672500 \\ \mathrm{H} & -5.98212200 & 1.83861200 & -0.09984800 \\ \mathrm{H} & -3.81030300 & 3.01686200 & 0.21744200 \\ \mathrm{H} & -3.96135500 & -1.97183600 & -0.28543600 \\ \mathrm{H} & -6.04015800 & -0.62035300 & -0.34622300 \\ \mathrm{H} & 0.90126500 & -0.58154700 & 3.51428000 \\ \mathrm{H} & -0.40494300 & -1.16009200 & 2.47039900 \\ \mathrm{C} & 1.52168100 & 2.17771400 & -0.76340700 \\ \mathrm{O} & 1.53345900 & 3.29510500 & -1.00134200\end{array}$

Species: K

$\mathrm{E}(\mathrm{B} 3 \mathrm{PW}$ 91-D3BJ/6-31G(d)) = -1212.249458

$\mathrm{H}(\mathrm{B} 3 \mathrm{PW} 91-\mathrm{D} 3 \mathrm{BJ} / 6-31 \mathrm{G}(\mathrm{d}))=-1211.967380$

$\mathrm{G}(\mathrm{B} 3 \mathrm{PW} 91-\mathrm{D} 3 \mathrm{BJ} / 6-31 \mathrm{G}(\mathrm{d}))=-1212.087313$

$\mathrm{E}(\mathrm{B} 3 \mathrm{PW} 91-\mathrm{D} 3 \mathrm{BJ} / 6-311+\mathrm{G}(2 \mathrm{~d}, \mathrm{p}), \mathrm{Rh}(\mathrm{LANTZ}(\mathrm{f})))=-1211.539999$

Imaginary frequency $=-482.32 \mathrm{~cm}^{-1}$

$\begin{array}{lrrr}\mathrm{C} & 2.48205100 & -1.22685700 & 0.15034500 \\ \mathrm{C} & 2.75344500 & 0.12566800 & -0.16226800 \\ \mathrm{C} & 1.08472200 & -1.32298500 & 0.46842100 \\ \mathrm{C} & 0.51669900 & -0.07442800 & 0.30294600 \\ \mathrm{~N} & 1.54635900 & 0.82647500 & -0.03515400 \\ \mathrm{C} & 1.48191200 & 2.11560600 & 0.50623700 \\ \mathrm{O} & 2.25626100 & 3.02047600 & 0.28156900 \\ \mathrm{~N} & 0.39926500 & 2.16980800 & 1.35039400 \\ \mathrm{C} & -0.49954600 & 1.06897800 & 1.45933900 \\ \mathrm{C} & -1.64580400 & -0.81713500 & 2.53134100 \\ \mathrm{C} & -0.50546400 & 0.18431700 & 2.69596100 \\ \mathrm{H} & -1.50512400 & 1.50297500 & 1.40140200 \\ & & & \mathrm{~S} 109\end{array}$




\begin{tabular}{lrrr}
$\mathrm{H}$ & -0.68559400 & 0.80899900 & 3.58043400 \\
$\mathrm{H}$ & 0.45571900 & -0.32074700 & 2.81844100 \\
$\mathrm{H}$ & 0.07406900 & 3.09913600 & 1.58143300 \\
$\mathrm{C}$ & 4.02198800 & 0.59204100 & -0.48437600 \\
$\mathrm{H}$ & 0.54777000 & -2.24280800 & 0.65451800 \\
$\mathrm{Rh}$ & -1.51624000 & 0.02611100 & -0.40387300 \\
$\mathrm{C}$ & -3.42709500 & 0.40067100 & -0.28983900 \\
$\mathrm{C}$ & -1.85504000 & -1.36829200 & 1.10578200 \\
$\mathrm{O}$ & -4.56770200 & 0.53079300 & -0.32904300 \\
$\mathrm{O}$ & -2.22589500 & -2.49976100 & 0.93178300 \\
$\mathrm{O}$ & -0.73368400 & 2.32378400 & -2.36995700 \\
$\mathrm{C}$ & -1.04576800 & 1.48272600 & -1.66096400 \\
$\mathrm{C}$ & 5.04506000 & -0.35190100 & -0.52584700 \\
$\mathrm{C}$ & 4.79840600 & -1.70812400 & -0.25345700 \\
$\mathrm{C}$ & 3.52829900 & -2.15829200 & 0.08440200 \\
$\mathrm{H}$ & 5.61985500 & -2.41749300 & -0.30648900 \\
$\mathrm{H}$ & 3.34834800 & -3.20766400 & 0.30084700 \\
$\mathrm{H}$ & 4.19205200 & 1.64170100 & -0.69210800 \\
$\mathrm{H}$ & 6.05130500 & -0.03281300 & -0.78170300 \\
$\mathrm{H}$ & -2.60068600 & -0.31965700 & 2.76157500 \\
$\mathrm{H}$ & -1.55347300 & -1.67226800 & 3.20716100 \\
$\mathrm{O}$ & -1.30527400 & -1.45790600 & -1.69240000 \\
$\mathrm{H}$ & -1.21702500 & -2.36190100 & -2.38580800 \\
\hline & & & \\
$\mathrm{H}$ & & &
\end{tabular}

Species: $\mathbf{L}$

$\mathrm{E}(\mathrm{B} 3 \mathrm{PW} 91-\mathrm{D} 3 \mathrm{BJ} / 6-31 \mathrm{G}(\mathrm{d}))=-1212.320137$

H(B3PW91-D3BJ/6-31G(d)) = -1212.034561

G(B3PW91-D3BJ/6-31G(d)) = -1212.152379

$\mathrm{E}(\mathrm{B} 3 \mathrm{PW} 91-\mathrm{D} 3 \mathrm{BJ} / 6-311+\mathrm{G}(2 \mathrm{~d}, \mathrm{p}), \operatorname{Rh}(\operatorname{LANTZ}(\mathrm{f})))=-1211.6056225$ 


\begin{tabular}{|c|c|c|c|}
\hline $\mathrm{C}$ & -2.15429600 & 0.66454300 & 1.10011700 \\
\hline $\mathrm{C}$ & -2.26944100 & -0.54308200 & 0.36299600 \\
\hline $\mathrm{C}$ & -0.84566500 & 0.69355100 & 1.64840600 \\
\hline $\mathrm{C}$ & -0.16247300 & -0.44457200 & 1.21986100 \\
\hline $\mathrm{N}$ & -1.05186700 & -1.21981200 & 0.43840100 \\
\hline $\mathrm{C}$ & -0.75986600 & -2.24218700 & -0.51257200 \\
\hline $\mathrm{O}$ & -1.59941300 & -3.08534700 & -0.78166000 \\
\hline $\mathrm{N}$ & 0.43580500 & -2.16165800 & -1.14725800 \\
\hline $\mathrm{C}$ & 1.60805900 & -1.33549400 & -0.88666000 \\
\hline $\mathrm{C}$ & 1.66944900 & -2.24210400 & 1.49478300 \\
\hline $\mathrm{C}$ & 2.45496400 & -2.02053200 & 0.19537300 \\
\hline $\mathrm{H}$ & 2.17364400 & -1.32544600 & -1.82204900 \\
\hline $\mathrm{H}$ & 3.35391900 & -1.43440800 & 0.40895600 \\
\hline $\mathrm{H}$ & 2.79409700 & -2.99962200 & -0.17511500 \\
\hline $\mathrm{H}$ & 0.55740400 & -2.95074100 & -1.77214700 \\
\hline $\mathrm{C}$ & -3.43631600 & -0.86659400 & -0.33521100 \\
\hline $\mathrm{H}$ & -0.44081800 & 1.38229000 & 2.37726400 \\
\hline $\mathrm{Rh}$ & 1.10870400 & 0.71449300 & -0.50906000 \\
\hline $\mathrm{C}$ & 2.94917600 & 1.00443300 & -0.15196600 \\
\hline $\mathrm{C}$ & 1.04742700 & -0.94611200 & 1.94204600 \\
\hline $\mathrm{O}$ & 4.07410500 & 1.22213700 & -0.04176900 \\
\hline $\mathrm{O}$ & 1.51509900 & -0.27997300 & 2.85093800 \\
\hline $\mathrm{O}$ & -0.71648500 & 0.50776400 & -2.96297700 \\
\hline $\mathrm{C}$ & -0.08626000 & 0.56959600 & -2.00551500 \\
\hline $\mathrm{C}$ & -4.48873100 & 0.03403600 & -0.25744100 \\
\hline $\mathrm{C}$ & -4.39968100 & 1.22991600 & 0.48468800 \\
\hline $\mathrm{C}$ & -3.24076800 & 1.55479500 & 1.16449000 \\
\hline $\mathrm{H}$ & -5.25374000 & 1.89982400 & 0.51933600 \\
\hline $\mathrm{H}$ & -3.16001100 & 2.47664200 & 1.73317400 \\
\hline $\mathrm{H}$ & -3.50907700 & -1.79212700 & -0.89124300 \\
\hline
\end{tabular}




$\begin{array}{llll}\mathrm{H} & -5.41291800 & -0.19511700 & -0.78043800 \\ \mathrm{H} & 2.33181100 & -2.58789000 & 2.29187000 \\ \mathrm{H} & 0.89552100 & -3.00260000 & 1.33754000 \\ \mathrm{C} & 0.63632500 & 2.55291100 & -0.16063500 \\ \mathrm{O} & 0.34663100 & 3.64400400 & 0.03871900\end{array}$

\section{Species: M}

$\mathrm{E}(\mathrm{B} 3 \mathrm{PW} 91-\mathrm{D} 3 \mathrm{BJ} / 6-31 \mathrm{G}(\mathrm{d}))=-1212.3296853$

$\mathrm{H}(\mathrm{B} 3 \mathrm{PW} 91-\mathrm{D} 3 \mathrm{BJ} / 6-31 \mathrm{G}(\mathrm{d}))=-1212.044877$

G(B3PW91-D3BJ/6-31G(d)) = -1212.165918

$\mathrm{E}(\mathrm{B} 3 \mathrm{PW} 91-\mathrm{D} 3 \mathrm{BJ} / 6-311+\mathrm{G}(2 \mathrm{~d}, \mathrm{p}), \operatorname{Rh}(\mathrm{LANTZ}(\mathrm{f})))=-1211.6184935$

$\begin{array}{lrrr}\mathrm{C} & 1.78033700 & -0.75775600 & -1.04974300 \\ \mathrm{C} & 2.39936400 & 0.21686600 & -0.22919100 \\ \mathrm{C} & 0.42231900 & -0.30049300 & -1.35988400 \\ \mathrm{C} & 0.27196200 & 0.94701800 & -0.69717800 \\ \mathrm{~N} & 1.48033300 & 1.23599200 & -0.03849200 \\ \mathrm{C} & 1.57779300 & 2.57725400 & 0.33867900 \\ \mathrm{O} & 2.51182300 & 3.10767000 & 0.89992400 \\ \mathrm{~N} & 0.38104100 & 3.13645400 & -0.06525400 \\ \mathrm{C} & -0.34954000 & 2.29402500 & -1.00934900 \\ \mathrm{C} & -2.56202000 & 1.28364500 & -1.64356100 \\ \mathrm{C} & -1.85880800 & 2.39494500 & -0.87883100 \\ \mathrm{H} & -0.07364600 & 2.55661800 & -2.04683300 \\ \mathrm{H} & -2.18324300 & 3.36771000 & -1.26773400 \\ \mathrm{H} & -2.12696800 & 2.36796700 & 0.18122800 \\ \mathrm{H} & 0.32676200 & 4.14339100 & -0.10771800 \\ \mathrm{C} & 3.69017600 & 0.07144600 & 0.26759400 \\ \mathrm{H} & -0.11226600 & -0.59908600 & -2.25272800 \\ \mathrm{Rh} & -1.03294400 & -0.58632100 & 0.35186700 \\ \mathrm{C} & -2.11472700 & 0.32767600 & 1.63751200 \\ \mathrm{C} & -2.52488200 & -0.11885200 & -1.01516800\end{array}$




$\begin{array}{lrrr}\mathrm{O} & -2.78796200 & 0.80780000 & 2.43878300 \\ \mathrm{O} & -3.36391300 & -0.92016100 & -1.35473100 \\ \mathrm{O} & -1.78025300 & -3.57008100 & -0.12648400 \\ \mathrm{C} & -1.48797700 & -2.47592000 & 0.04149700 \\ \mathrm{C} & 4.37789800 & -1.08414700 & -0.09426800 \\ \mathrm{C} & 3.78806700 & -2.06179600 & -0.90939600 \\ \mathrm{C} & 2.48916600 & -1.91037200 & -1.38835600 \\ \mathrm{H} & 4.35288000 & -2.95313500 & -1.16669700 \\ \mathrm{H} & 2.03239700 & -2.67575100 & -2.00981900 \\ \mathrm{H} & 4.12968700 & 0.83630400 & 0.89744000 \\ \mathrm{H} & 5.39204100 & -1.23104800 & 0.26589100 \\ \mathrm{H} & -2.13326000 & 1.18358900 & -2.65245000 \\ \mathrm{H} & -3.62297600 & 1.51441400 & -1.78769200 \\ \mathrm{C} & 0.38490400 & -1.02673000 & 1.67334600 \\ \mathrm{O} & 1.14121300 & -1.29954100 & 2.48464300\end{array}$

Species: $\mathbf{N}$

$\mathrm{E}(\mathrm{B} 3 \mathrm{PW} 91-\mathrm{D} 3 \mathrm{BJ} / 6-31 \mathrm{G}(\mathrm{d}))=-1212.2763523$

H(B3PW91-D3BJ/6-31G(d)) = -1211.992946

G(B3PW91-D3BJ/6-31G(d)) = -1212.111275

$\mathrm{E}(\mathrm{B} 3 \mathrm{PW} 91-\mathrm{D} 3 \mathrm{BJ} / 6-311+\mathrm{G}(2 \mathrm{~d}, \mathrm{p}), \operatorname{Rh}(\operatorname{LANTZ}(\mathrm{f})))=-1211.5655846$

Imaginary frequency $=-258.88 \mathrm{~cm}^{-1}$

$\begin{array}{llll}\mathrm{Rh} & -1.22007700 & -0.84184400 & -0.15407100 \\ \mathrm{C} & 1.71315400 & -0.79326800 & 0.95341300 \\ \mathrm{C} & 2.34201300 & 0.21501300 & 0.21196700 \\ \mathrm{C} & 0.33110500 & -0.36675600 & 1.24329700 \\ \mathrm{C} & 0.20368600 & 0.97758600 & 0.67438000 \\ \mathrm{~N} & 1.41980600 & 1.28379400 & 0.01333700 \\ \mathrm{C} & 1.40612100 & 1.93816700 & -1.22853700 \\ \mathrm{O} & 2.39824100 & 2.27676300 & -1.84206800\end{array}$




\begin{tabular}{|c|c|c|c|}
\hline $\mathrm{N}$ & 0.12039600 & 2.05935200 & -1.69291300 \\
\hline $\mathrm{C}$ & -1.03286800 & 1.63217600 & -0.99882400 \\
\hline $\mathrm{C}$ & -0.86770800 & 3.27031800 & 0.87161900 \\
\hline $\mathrm{C}$ & -1.76970600 & 2.71505800 & -0.22709300 \\
\hline $\mathrm{H}$ & -1.70835200 & 1.14883700 & -1.70388500 \\
\hline $\mathrm{H}$ & -2.67411200 & 2.27885900 & 0.21306500 \\
\hline $\mathrm{H}$ & -2.08780300 & 3.50750900 & -0.91937900 \\
\hline $\mathrm{H}$ & 0.07237900 & 2.24616100 & -2.68648100 \\
\hline $\mathrm{C}$ & 3.64618600 & 0.10180600 & -0.24689300 \\
\hline $\mathrm{H}$ & -0.01998700 & -0.46793700 & 2.26548500 \\
\hline $\mathrm{C}$ & -2.66449000 & -1.16342700 & -1.35044700 \\
\hline $\mathrm{C}$ & -0.39342200 & 2.04029600 & 1.58262700 \\
\hline $\mathrm{O}$ & -3.54235300 & -1.42656200 & -2.04495300 \\
\hline $\mathrm{O}$ & -0.55389400 & 1.81921200 & 2.76403700 \\
\hline $\mathrm{O}$ & 0.77522100 & -2.48276000 & -1.82729500 \\
\hline $\mathrm{C}$ & 0.06178200 & -1.82019500 & -1.21936600 \\
\hline $\mathrm{C}$ & 4.33261600 & -1.07050600 & 0.07956700 \\
\hline $\mathrm{C}$ & 3.72993200 & -2.08103900 & 0.83357300 \\
\hline $\mathrm{C}$ & 2.41192200 & -1.95164300 & 1.27332600 \\
\hline $\mathrm{H}$ & 4.29234100 & -2.97756700 & 1.07792600 \\
\hline $\mathrm{H}$ & 1.93201100 & -2.74293000 & 1.84234500 \\
\hline $\mathrm{H}$ & 4.09920000 & 0.89234500 & -0.83147800 \\
\hline $\mathrm{H}$ & 5.35822600 & -1.19118600 & -0.25723900 \\
\hline $\mathrm{H}$ & -1.37832600 & 3.93860300 & 1.56707100 \\
\hline $\mathrm{H}$ & -0.01839600 & 3.80209700 & 0.42278300 \\
\hline $\mathrm{C}$ & -2.28874600 & -1.21449500 & 1.37301100 \\
\hline $\mathrm{O}$ & -2.91653800 & -1.50270000 & 2.29356600 \\
\hline
\end{tabular}

Species: O

$\mathrm{E}(\mathrm{B} 3 \mathrm{PW} 91-\mathrm{D} 3 \mathrm{BJ} / 6-31 \mathrm{G}(\mathrm{d}))=-1212.2777413$ 
$\mathrm{H}(\mathrm{B} 3 \mathrm{PW}$ 91-D3BJ/6-31G(d)) = -1211.996085

G(B3PW91-D3BJ/6-31G(d)) = -1212.111761

E(B3PW91-D3BJ/6-311+G(2d,p),Rh(LANTZ(f))) = -1211.5699798

Imaginary frequency $=-309.18 \mathrm{~cm}^{-1}$

$\begin{array}{lrrr}\mathrm{C} & 1.40882900 & -1.11589400 & -0.84620500 \\ \mathrm{C} & 2.16661300 & -0.52330200 & 0.18197800 \\ \mathrm{C} & 0.34473700 & -0.17457100 & -1.24811900 \\ \mathrm{C} & 0.61300500 & 1.04212500 & -0.47092900 \\ \mathrm{~N} & 1.71327600 & 0.78829600 & 0.37792600 \\ \mathrm{C} & 2.59329800 & 1.89291100 & 0.43936300 \\ \mathrm{O} & 3.60753000 & 1.96073100 & 1.09601500 \\ \mathrm{~N} & 2.08307500 & 2.82049300 & -0.43030800 \\ \mathrm{C} & 0.80722300 & 2.46868900 & -1.02672900 \\ \mathrm{C} & -1.56023000 & 2.70250200 & -0.27142100 \\ \mathrm{C} & -0.30426200 & 3.47846700 & -0.63795500 \\ \mathrm{H} & 0.88936800 & 2.40873600 & -2.11977200 \\ \mathrm{H} & -0.47735900 & 4.19456000 & -1.44676800 \\ \mathrm{H} & 0.03319000 & 4.03298000 & 0.24411300 \\ \mathrm{H} & 2.40800800 & 3.77466200 & -0.37959900 \\ \mathrm{C} & 3.21846000 & -1.17890700 & 0.81027600 \\ \mathrm{H} & 0.05992000 & -0.09604800 & -2.29332200 \\ \mathrm{Rh} & -1.36117500 & -0.52246900 & 0.04345800 \\ \mathrm{C} & -2.59356600 & -0.57658100 & 1.53825800 \\ \mathrm{C} & -1.01364100 & 1.58387500 & 0.60946600 \\ \mathrm{O} & -3.37176300 & -0.64360300 & 2.37741700 \\ \mathrm{O} & -0.81231200 & 1.72549700 & 1.79376100 \\ \mathrm{O} & -3.36651000 & -0.21028700 & -2.24943500 \\ \mathrm{O} & -2.57525900 & -0.20459000 & -1.41224400 \\ \mathrm{O} & 3.52565300 & -2.46077100 & 0.35854600\end{array}$




$\begin{array}{lrrr}\mathrm{C} & 2.80120600 & -3.06008500 & -0.68161100 \\ \mathrm{C} & 1.73854900 & -2.39680600 & -1.28852100 \\ \mathrm{H} & 3.06924000 & -4.05877100 & -1.01383400 \\ \mathrm{H} & 1.16574800 & -2.87176200 & -2.08038900 \\ \mathrm{H} & 3.78456700 & -0.68934400 & 1.59376800 \\ \mathrm{H} & 4.34786900 & -3.00192800 & 0.81771300 \\ \mathrm{H} & -2.05643200 & 2.30735300 & -1.15932900 \\ \mathrm{H} & -2.26885200 & 3.30688100 & 0.30187700 \\ \mathrm{C} & -0.93201200 & -2.39736000 & 0.30236500 \\ \mathrm{O} & -0.77050500 & -3.52433300 & 0.45817800\end{array}$

Species: $\mathbf{P}$

$\mathrm{E}(\mathrm{B} 3 \mathrm{PW}$ 91-D3BJ/6-31G(d)) = -1212.3329429

$\mathrm{H}(\mathrm{B} 3 \mathrm{PW} 91-\mathrm{D} 3 \mathrm{BJ} / 6-31 \mathrm{G}(\mathrm{d}))=-1212.047381$

G(B3PW91-D3BJ/6-31G(d)) = -1212.166312

$\mathrm{E}(\mathrm{B} 3 \mathrm{PW} 91-\mathrm{D} 3 \mathrm{BJ} / 6-311+\mathrm{G}(2 \mathrm{~d}, \mathrm{p}), \mathrm{Rh}(\mathrm{LANTZ}(\mathrm{f})))=-1211.6220031$

$\begin{array}{crrr}\mathrm{Rh} & -1.48658400 & -0.65832800 & 0.11041000 \\ \mathrm{C} & -0.25944500 & 0.90795100 & -1.11490600 \\ \mathrm{C} & 0.49182500 & 1.72845000 & -0.22705400 \\ \mathrm{C} & 0.26656300 & -0.46645700 & -1.07147800 \\ \mathrm{C} & 1.63939400 & -0.30862000 & -0.43675800 \\ \mathrm{~N} & 1.57802200 & 0.99331700 & 0.23139300 \\ \mathrm{C} & 2.09301400 & 0.94376500 & 1.52840600 \\ \mathrm{O} & 2.06891900 & 1.84010900 & 2.34489800 \\ \mathrm{~N} & 2.64678800 & -0.31489900 & 1.65992800 \\ \mathrm{C} & 2.20541000 & -1.26068700 & 0.64208100 \\ \mathrm{C} & 3.97495100 & -0.98677000 & -0.99273000 \\ \mathrm{C} & 3.37667700 & -2.00211000 & -0.01875700 \\ \mathrm{H} & 1.44331600 & -1.94805400 & 1.02621900\end{array}$




$\begin{array}{lrrr}\mathrm{H} & 2.98483100 & -2.86955300 & -0.56437800 \\ \mathrm{H} & 4.09239800 & -2.36989000 & 0.72172200 \\ \mathrm{H} & 2.85040500 & -0.62160300 & 2.60011200 \\ \mathrm{C} & 0.12092300 & 3.02751200 & 0.06256100 \\ \mathrm{H} & 0.25204000 & -1.05347100 & -1.98571800 \\ \mathrm{C} & -3.22092700 & -0.27783500 & 0.82809000 \\ \mathrm{C} & 2.76176800 & -0.24438300 & -1.51793400 \\ \mathrm{O} & -4.26898200 & -0.02288600 & 1.22046300 \\ \mathrm{O} & 2.63847300 & 0.30192000 & -2.58606400 \\ \mathrm{O} & -0.28408500 & -0.76347400 & 2.91798400 \\ \mathrm{C} & -0.63997000 & -0.64062900 & 1.82864000 \\ \mathrm{C} & -1.02973100 & 3.52375100 & -0.56743000 \\ \mathrm{C} & -1.77334600 & 2.75421000 & -1.46011100 \\ \mathrm{C} & -1.40071300 & 1.43428600 & -1.74650800 \\ \mathrm{H} & -2.64398000 & 3.18166800 & -1.94769300 \\ \mathrm{H} & -1.94282500 & 0.84397500 & -2.47889700 \\ \mathrm{H} & 0.70367900 & 3.62784100 & 0.75084100 \\ \mathrm{H} & -1.33774800 & 4.54514100 & -0.36216200 \\ \mathrm{H} & 4.57155500 & -1.40633800 & -1.80581300 \\ \mathrm{H} & 4.59766700 & -0.26174700 & -0.45052500 \\ & -2.04466500 & -2.07406900 & -1.02849600 \\ \mathrm{O} & -2.35671500 & -2.99845700 & -1.64192200\end{array}$


Copies of ${ }^{1} \mathrm{H}$ and ${ }^{13} \mathrm{C}$ NMR for Novel Compounds

\section{$N$-Benzyl- $N$-cyclopropyl-1H-indole-1-carboxamide}

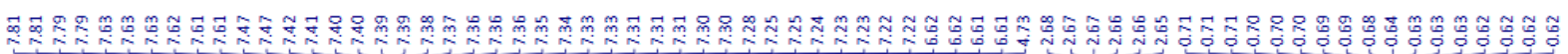

${ }^{1} \mathrm{H}$ NMR $\left(400 \mathrm{MHz}, \mathrm{CDCl}_{3}\right)$

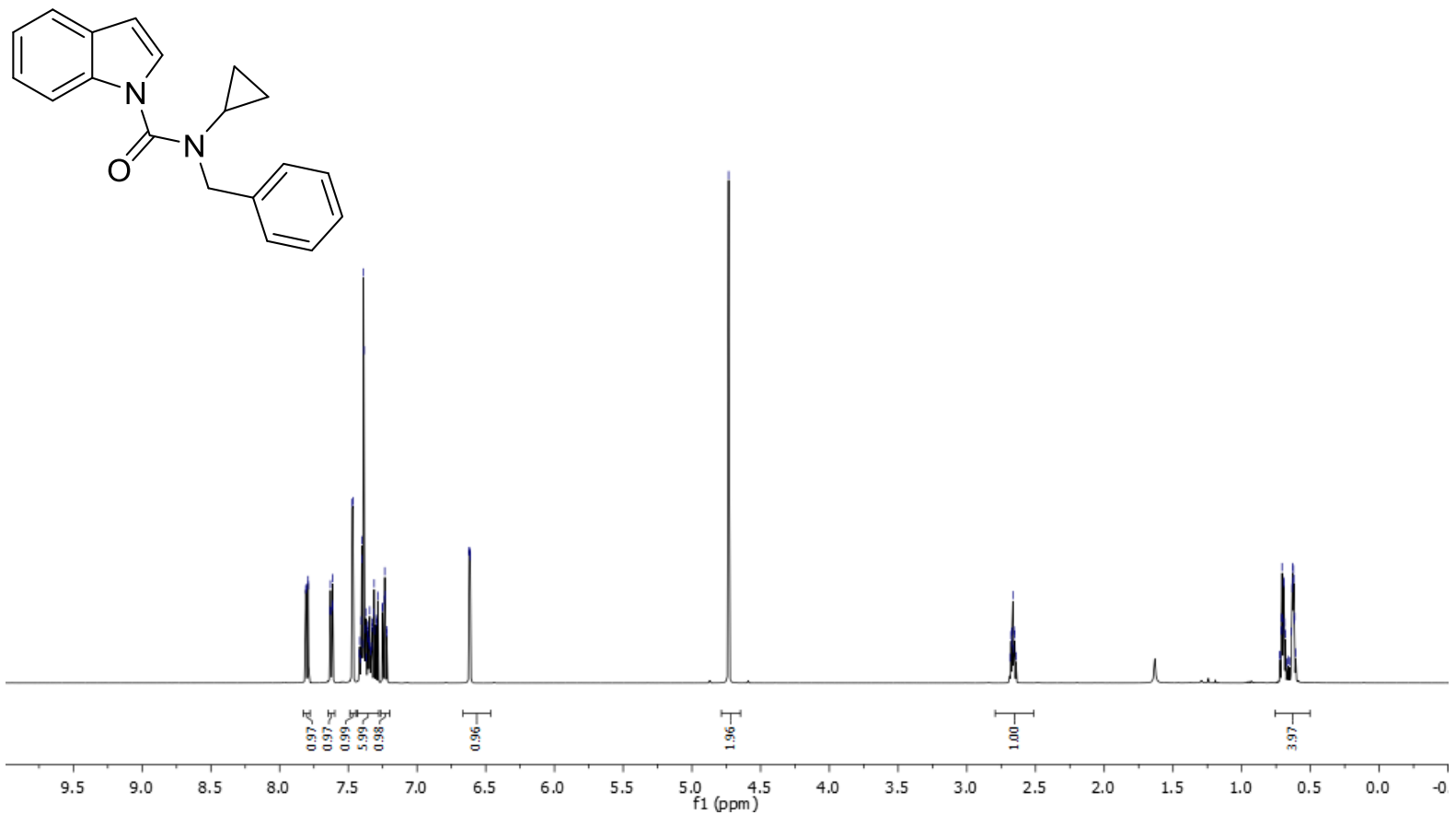

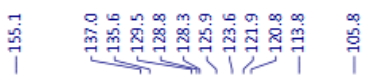

${ }^{13} \mathrm{C} \mathrm{NMR}\left(101 \mathrm{MHz}, \mathrm{CDCl}_{3}\right)$

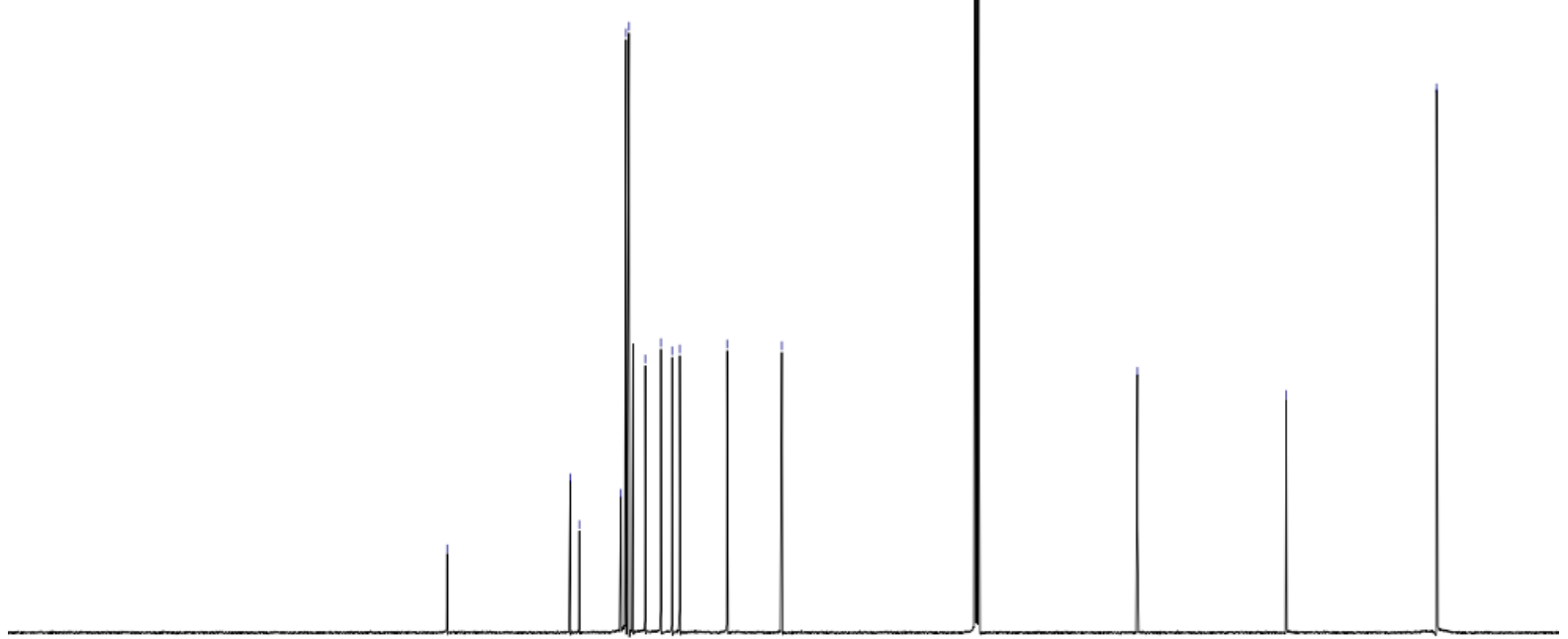

$\begin{array}{lllllllllll}210 & 200 & 190 & 180 & 170 & 160 & 150 & 140 & 130 & 120 & 110 \\ \mathrm{f} 1(\mathrm{ppm}) & 100\end{array}$ 
$\left(3 \mathrm{a} S^{*}, 11 \mathrm{a} S^{*}\right)$-4-Benzyl-2,3,3a,4-tetrahydro-11H-cyclopenta[4,5]imidazo[1,5-a]indole1,5-dione 4a
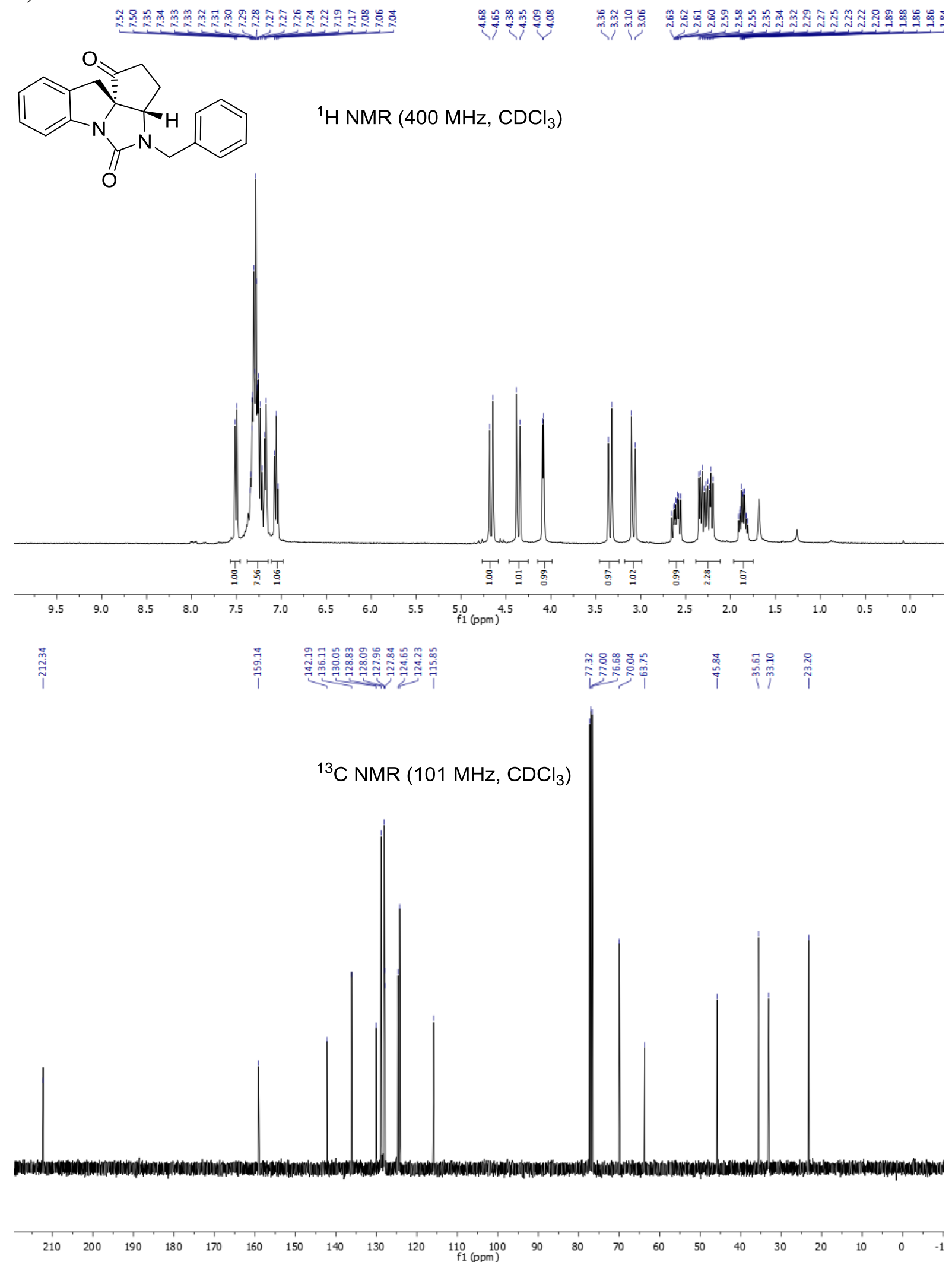
$N$-Butyl- $N$-cyclopropyl-1H-indole-1-carboxamide $3 \mathrm{~b}$

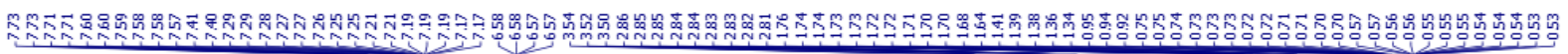<smiles>CCCCN(C(=O)n1ccc2ccccc21)C1CC1</smiles>

${ }^{1} \mathrm{H}$ NMR $\left(400 \mathrm{MHz}, \mathrm{CDCl}_{3}\right)$

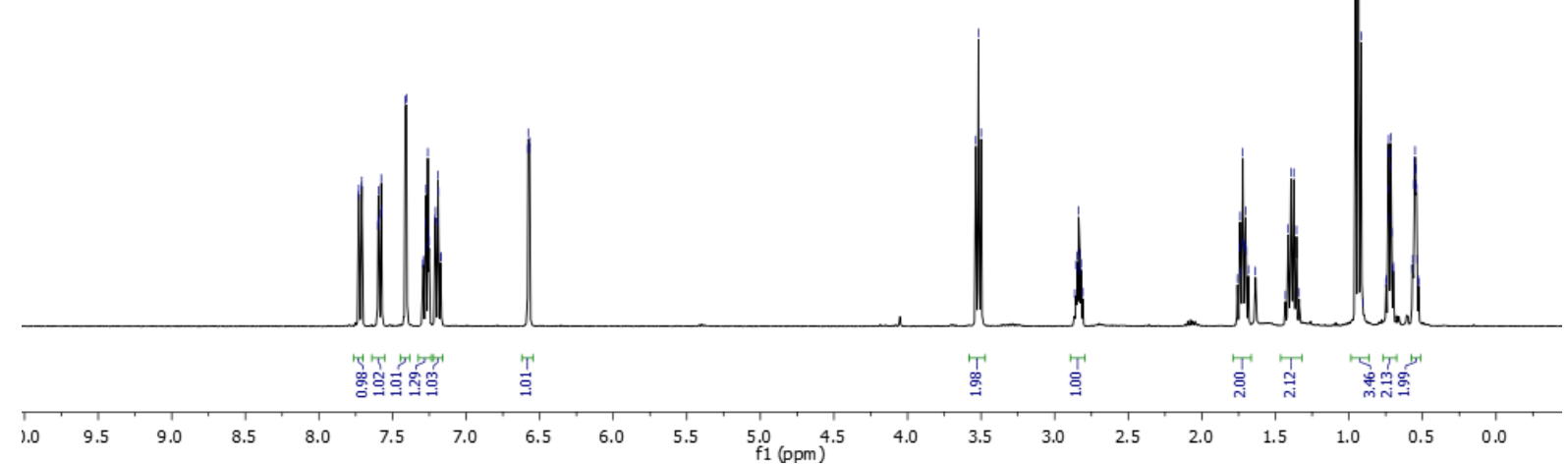

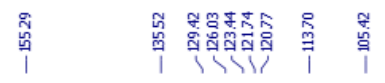

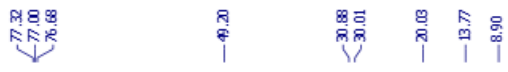

${ }^{13} \mathrm{C}$ NMR $\left(101 \mathrm{MHz}, \mathrm{CDCl}_{3}\right)$

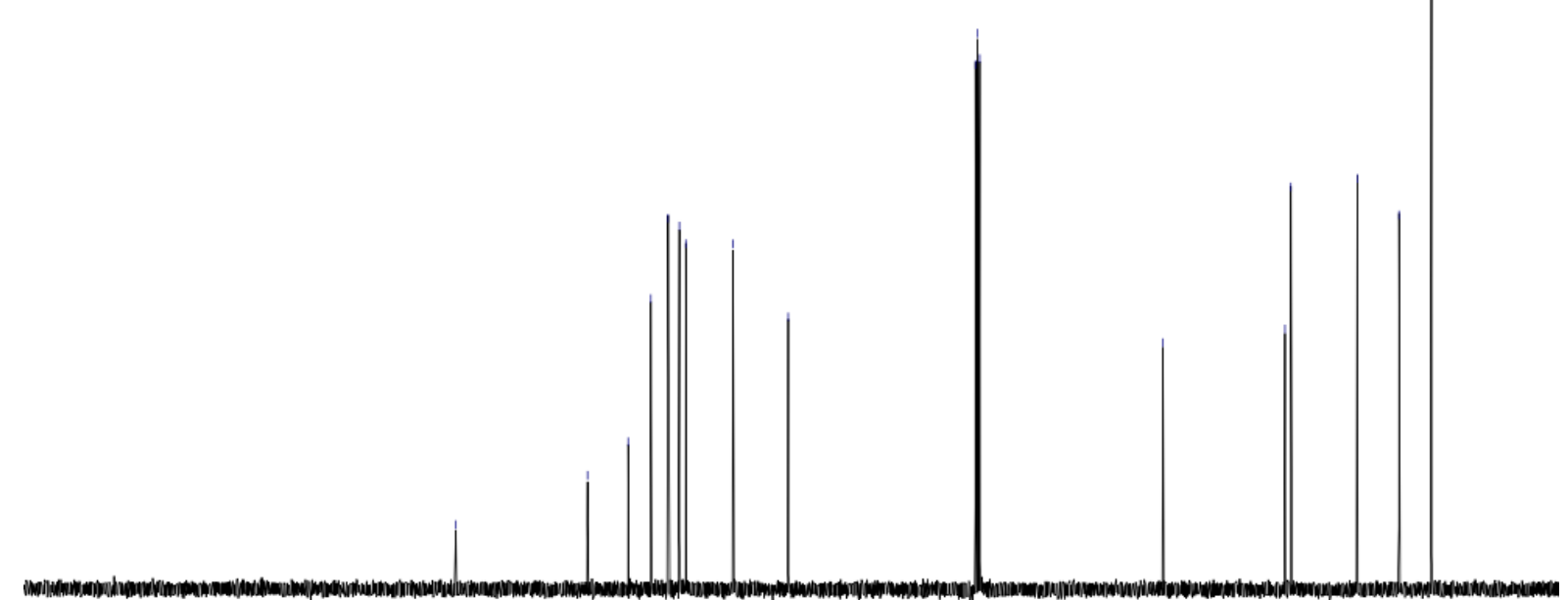

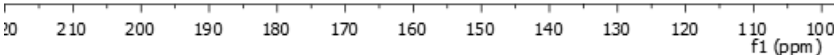


$\left(3 \mathrm{a} S^{*}, 11 \mathrm{a} S^{*}\right)-4-B u t y l-2,3,3 \mathrm{a}, 4-t e t r a h y d r o-11 H$-cyclopenta[4,5]imidazo[1,5-a]indole-1,5dione $4 b$

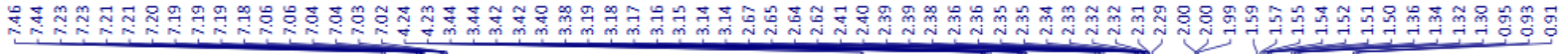<smiles>CCCCN1C(=O)N2c3ccccc3C[C@]23C(=O)CC[C@H]13</smiles>

${ }^{1} \mathrm{H}$ NMR $\left(400 \mathrm{MHz}, \mathrm{CDCl}_{3}\right)$
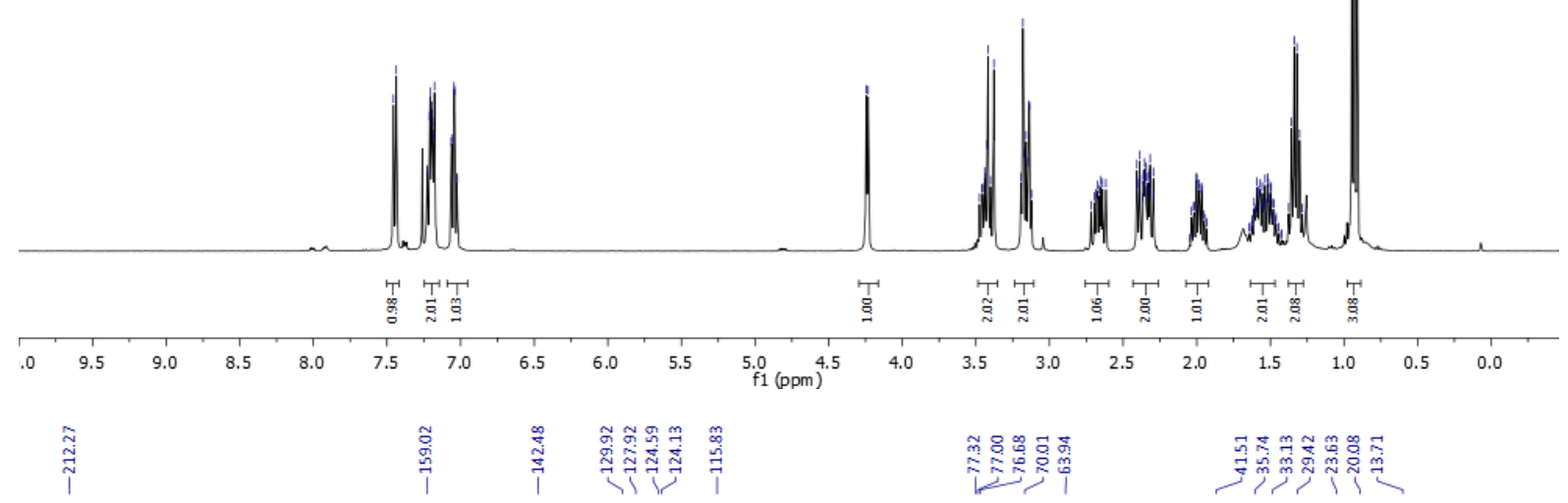

${ }^{13} \mathrm{C}$ NMR $\left(101 \mathrm{MHz}, \mathrm{CDCl}_{3}\right)$

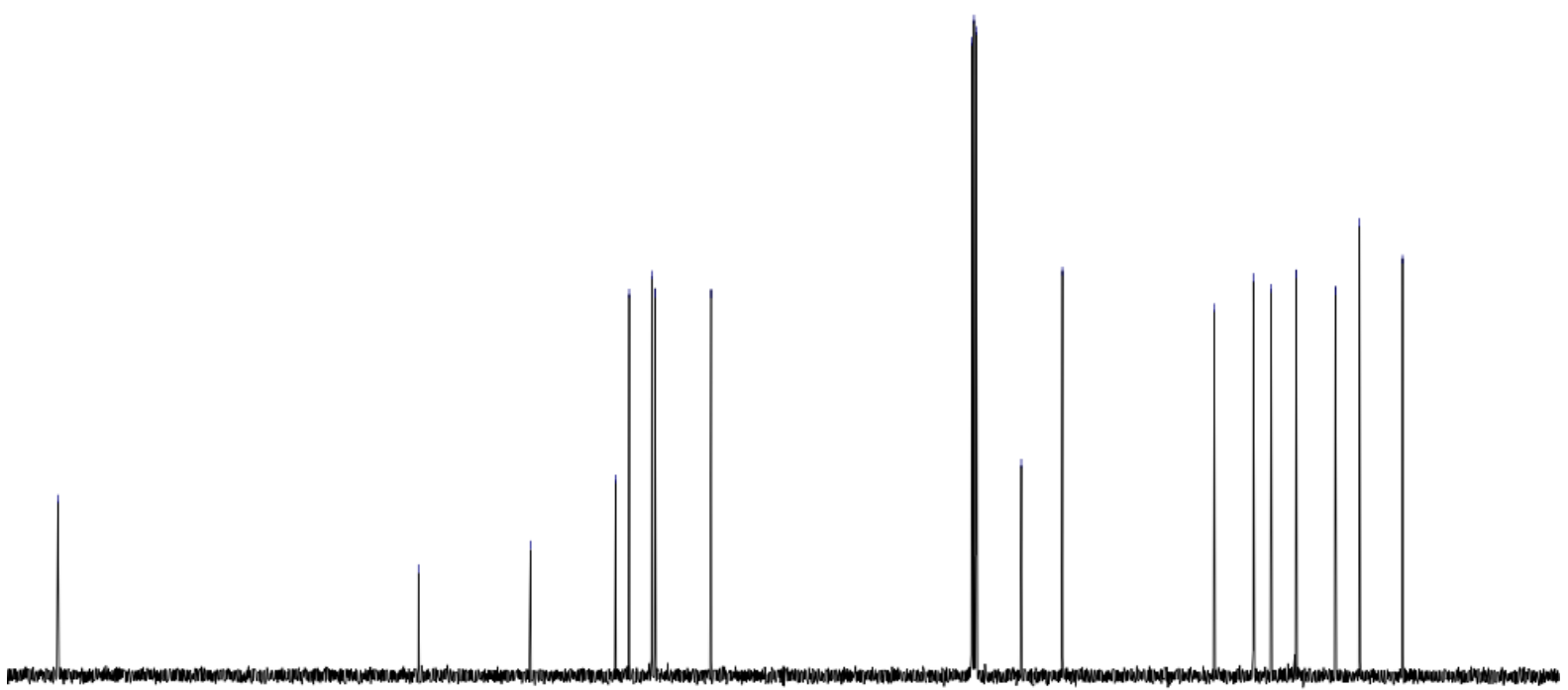

$\underset{f 1(\mathrm{ppm})}{110}$

(20)

70 
$N$-Benzyl- $N$-cyclopropyl-4-fluoro- $1 H$-indole-1-carboxamide $3 \mathrm{c}$

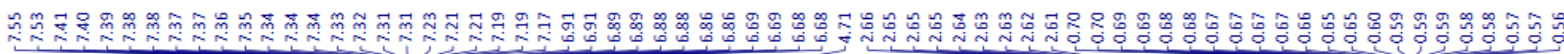

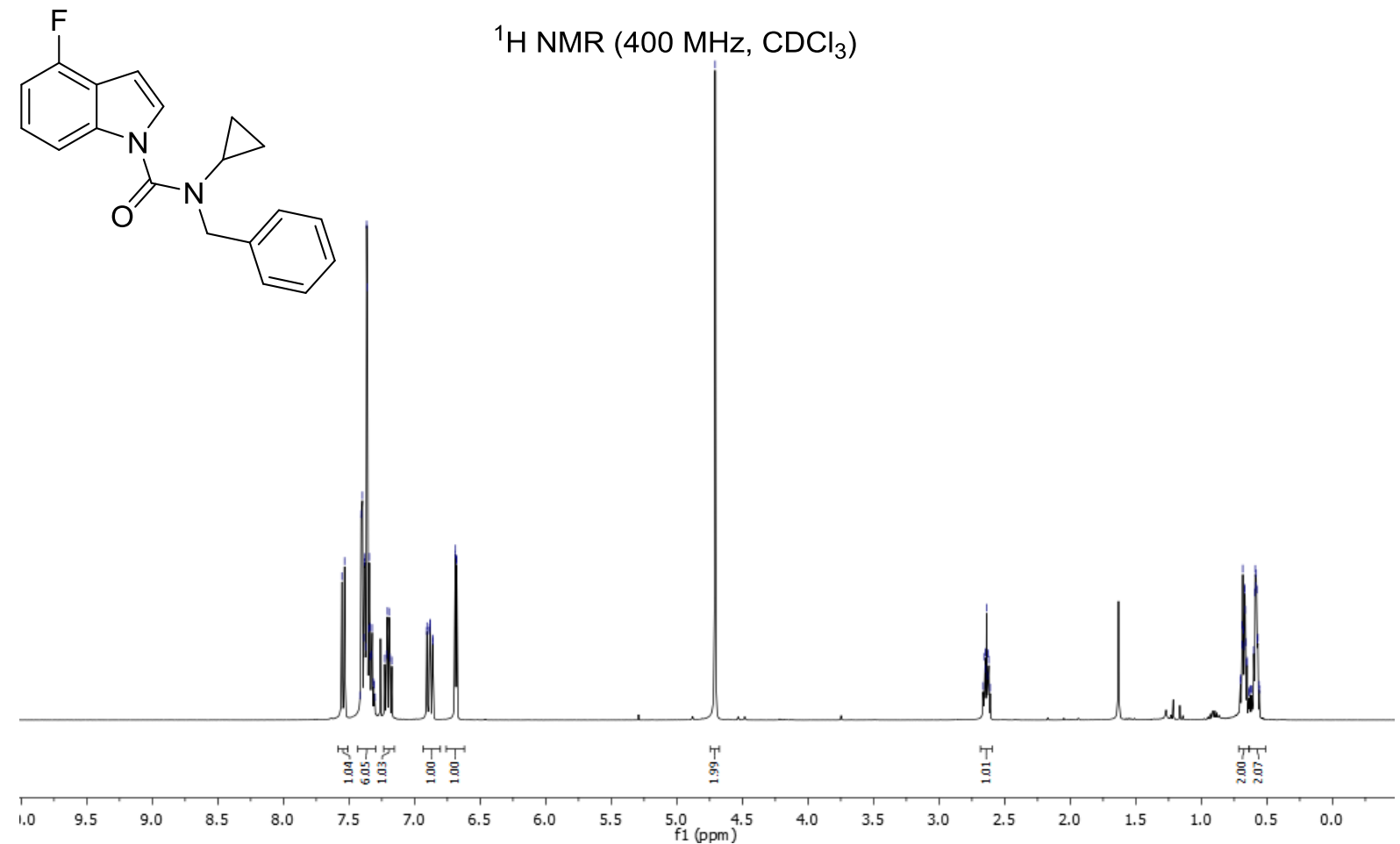

实总

${ }^{13} \mathrm{C}$ NMR $\left(101 \mathrm{MHz}, \mathrm{CDCl}_{3}\right)$

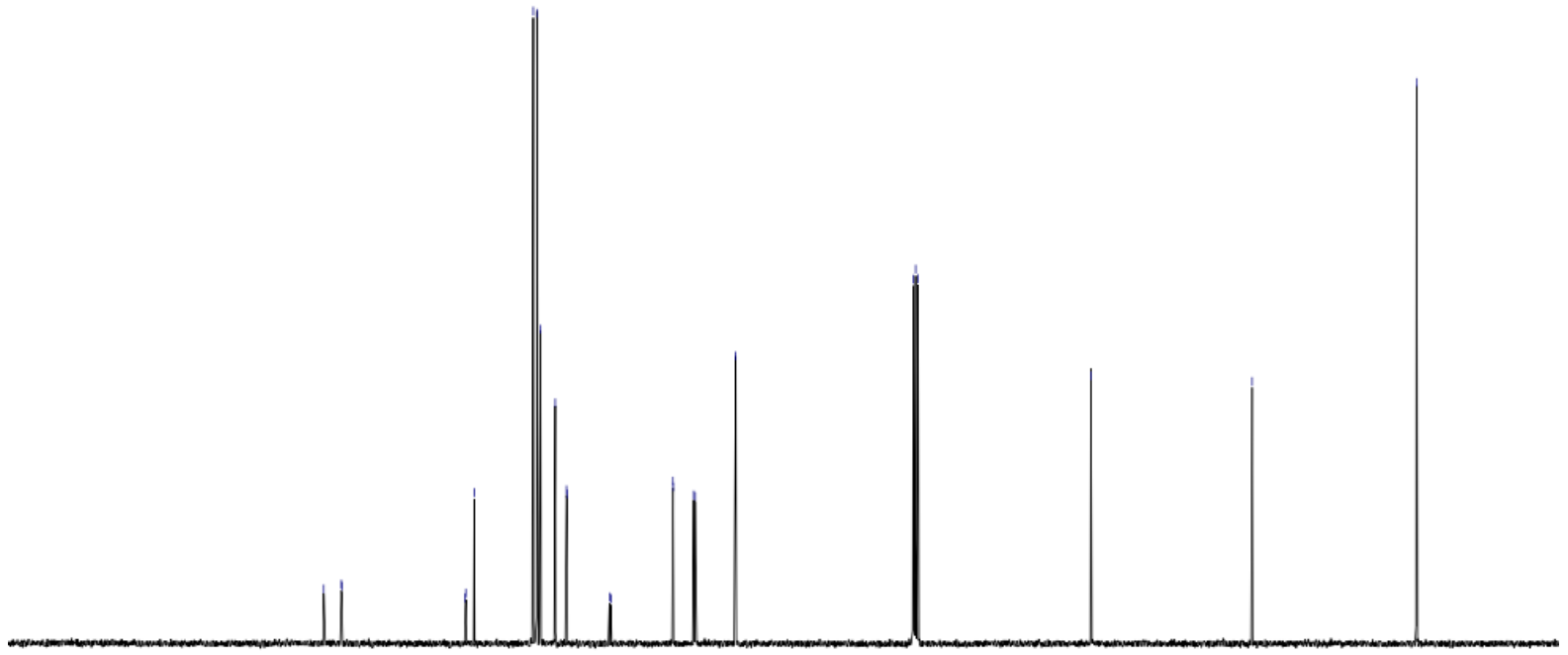

$\begin{array}{llllllllll}190 & 180 & 170 & 160 & 150 & 140 & 130 & 120 & 110 & \begin{array}{c}100 \\ \mathrm{f} 1(\mathrm{ppm})\end{array}\end{array}$ 
${ }^{19} \mathrm{~F} \mathrm{NMR}\left(377 \mathrm{MHz}, \mathrm{CDCl}_{3}\right)$

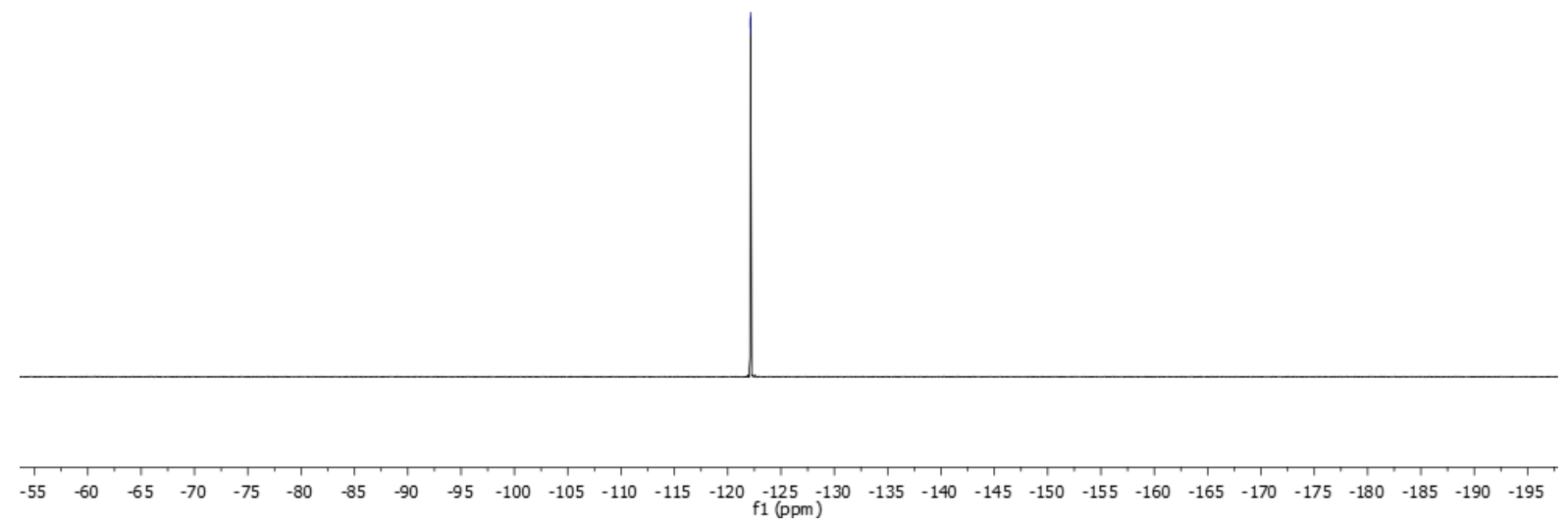

$\left(3 a S^{*}, 11 a S^{*}\right)$-4-Benzyl-10-fluoro-2,3,3a,4-tetrahydro-11H-cyclopenta[4,5]imidazo[1,5-

a]indole-1,5-dione 4c

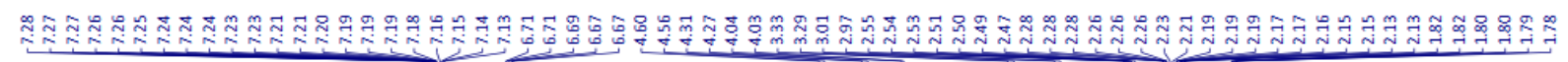<smiles>O=C1Cc2c(F)cccc2N2C(=O)CC[C@H]2C1</smiles>

${ }^{1} \mathrm{H}$ NMR $\left(400 \mathrm{MHz}, \mathrm{CDCl}_{3}\right)$

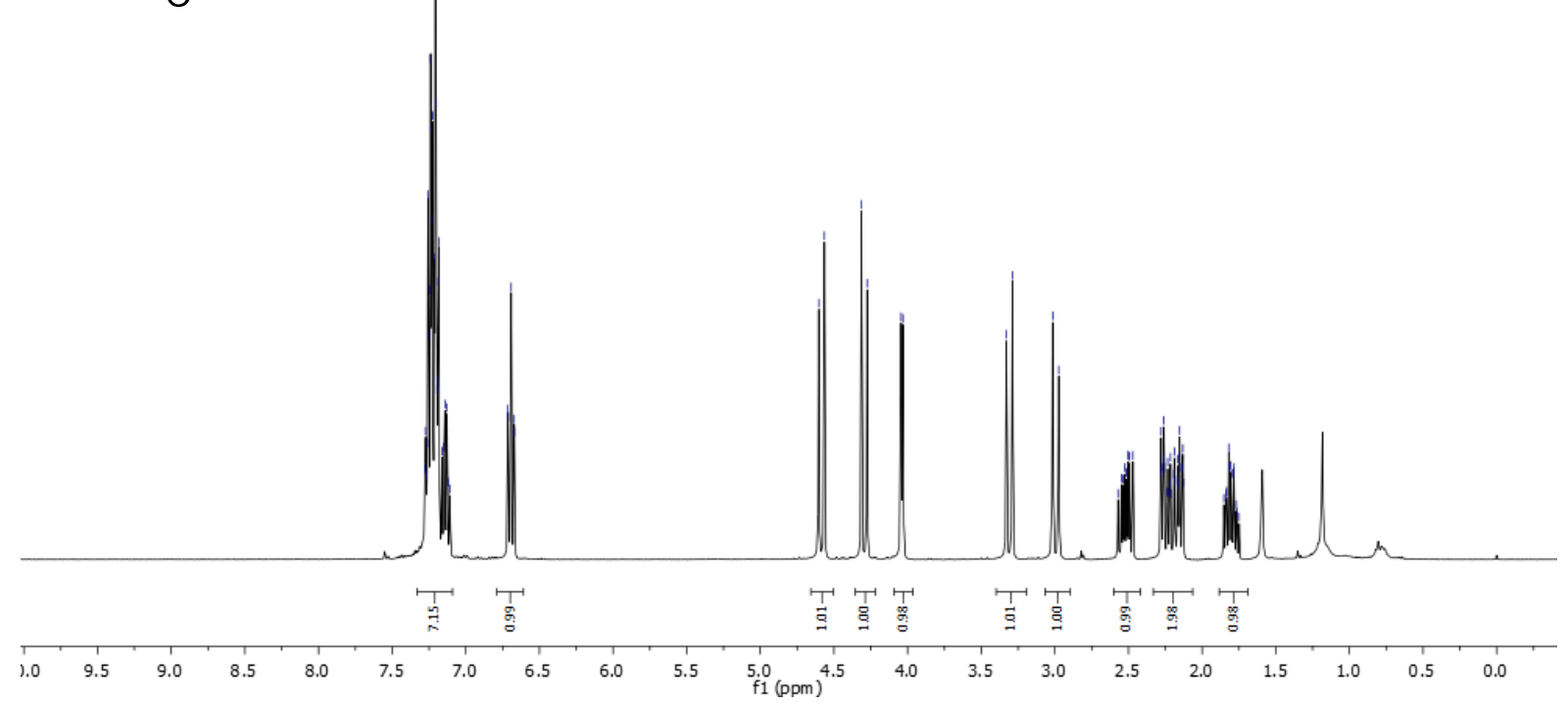



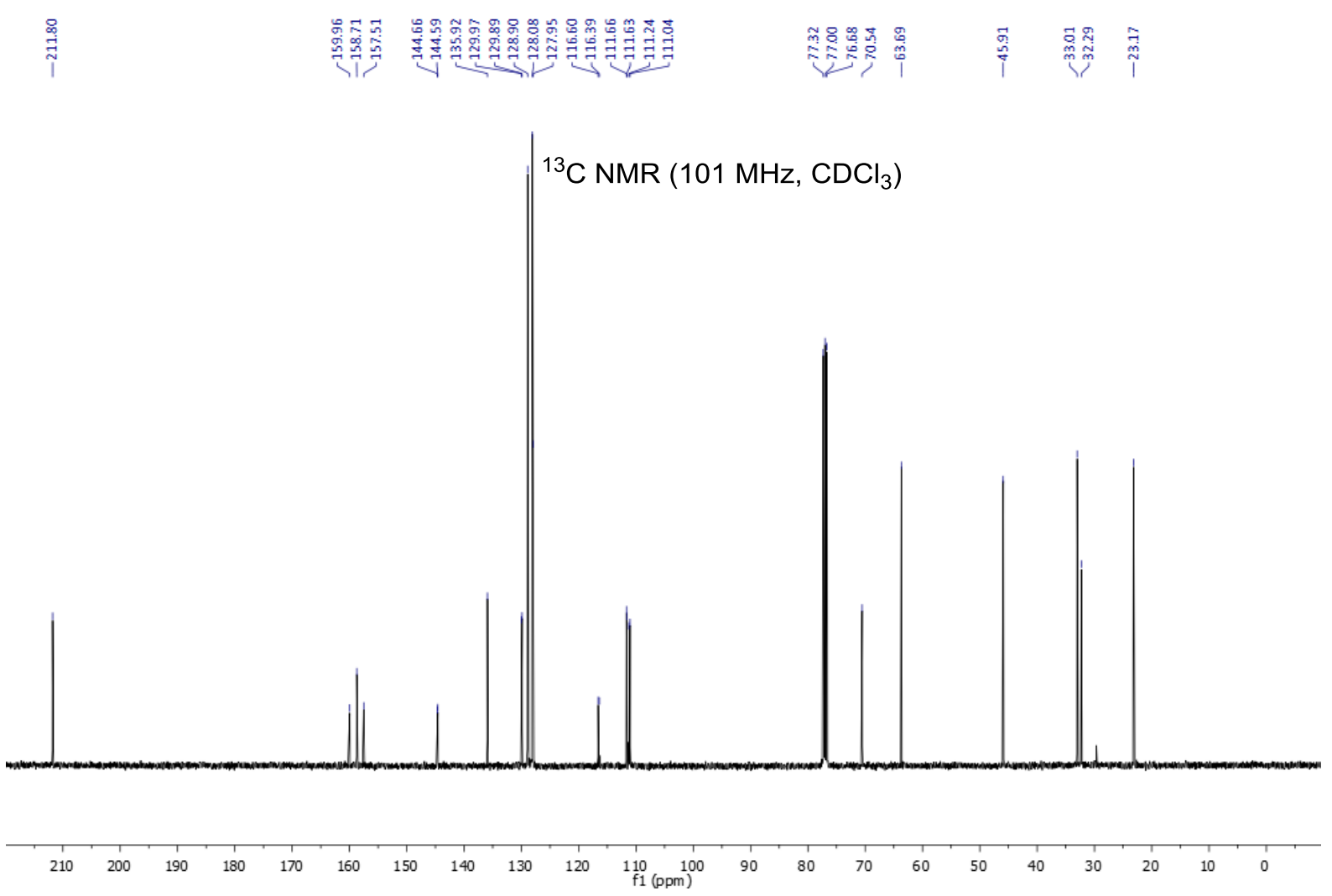

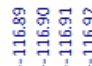

${ }^{19} \mathrm{~F} \mathrm{NMR}\left(377 \mathrm{MHz}, \mathrm{CDCl}_{3}\right)$

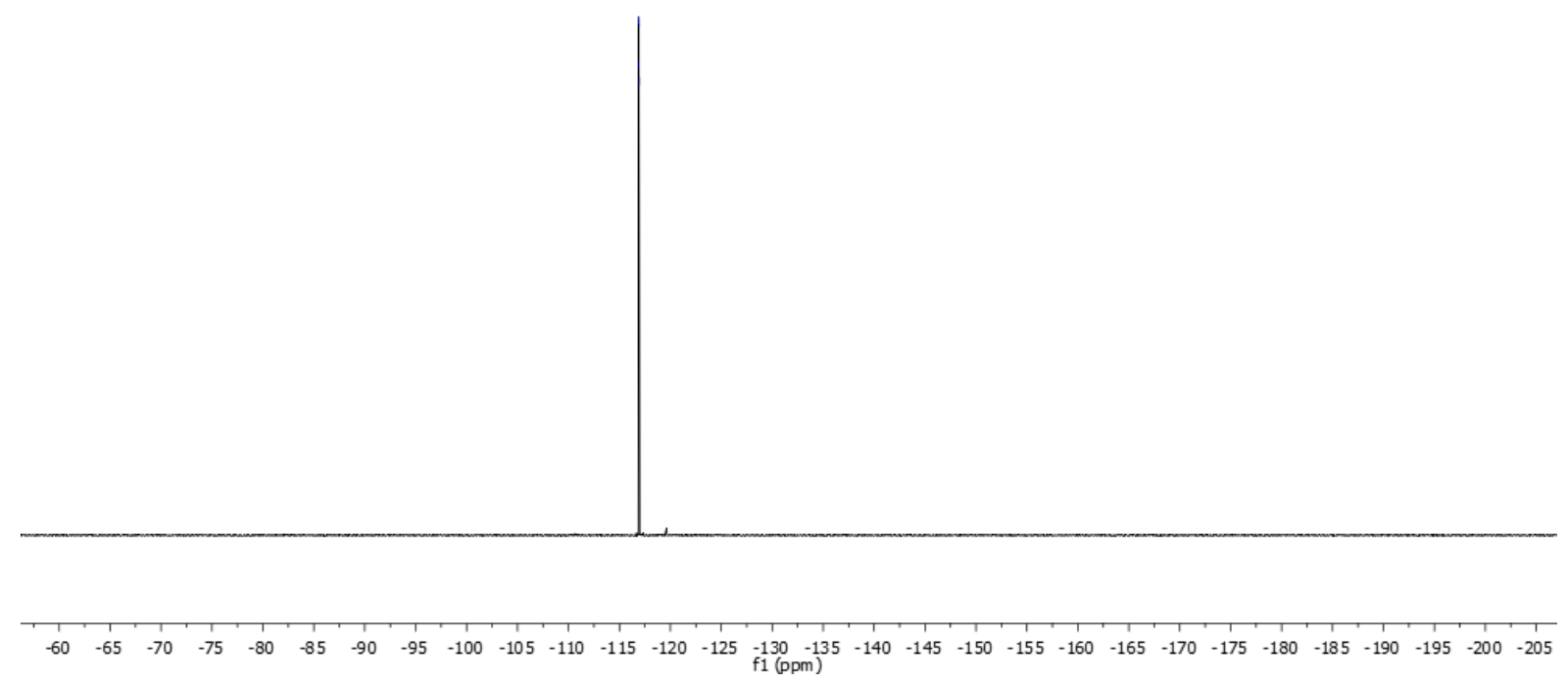


$N$-Benzyl- $N$-cyclopropyl-4-methyl- $1 H$-indole-1-carboxamide 3d

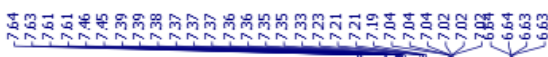

F
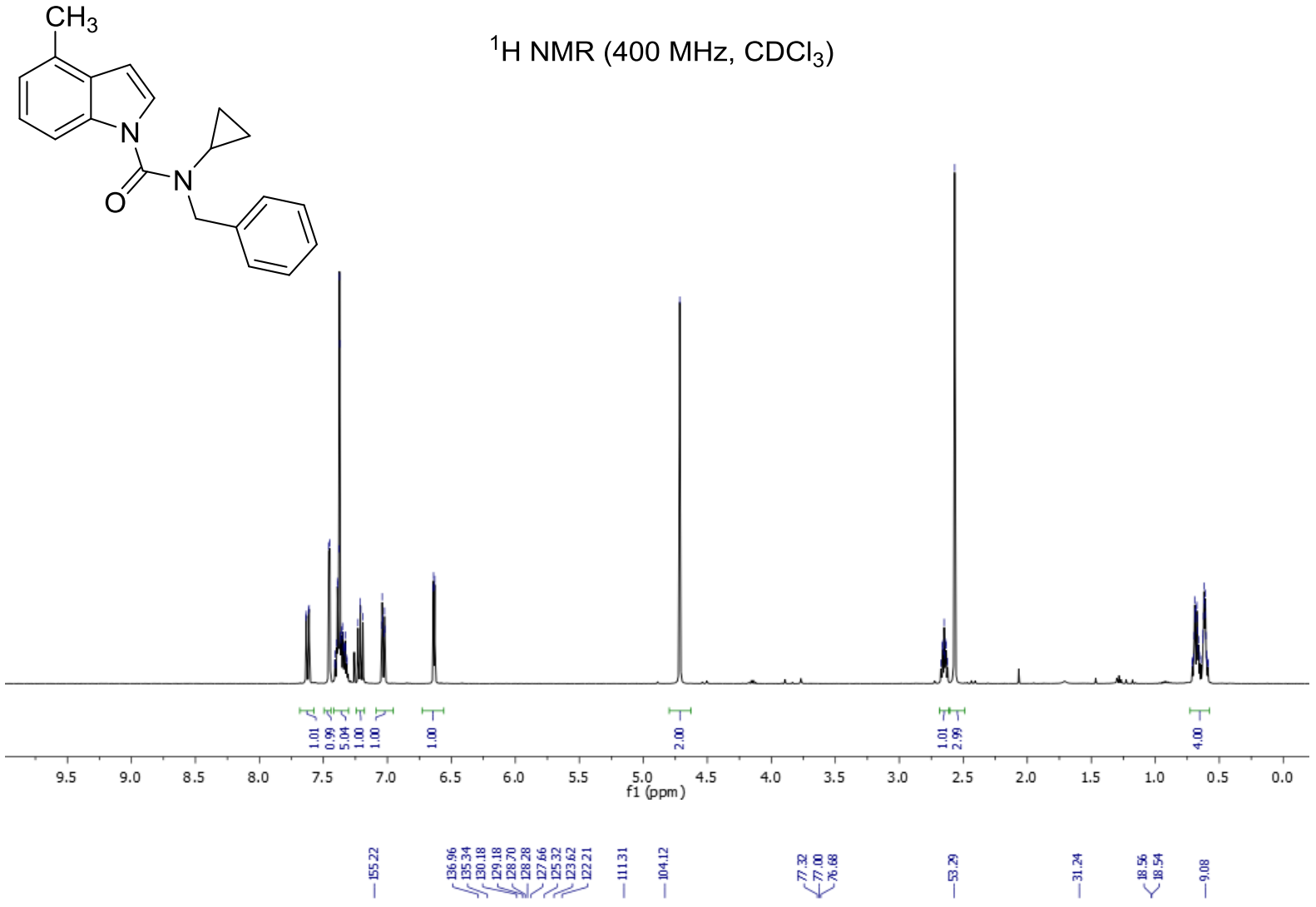

${ }^{13} \mathrm{C}$ NMR $\left(101 \mathrm{MHz}, \mathrm{CDCl}_{3}\right)$

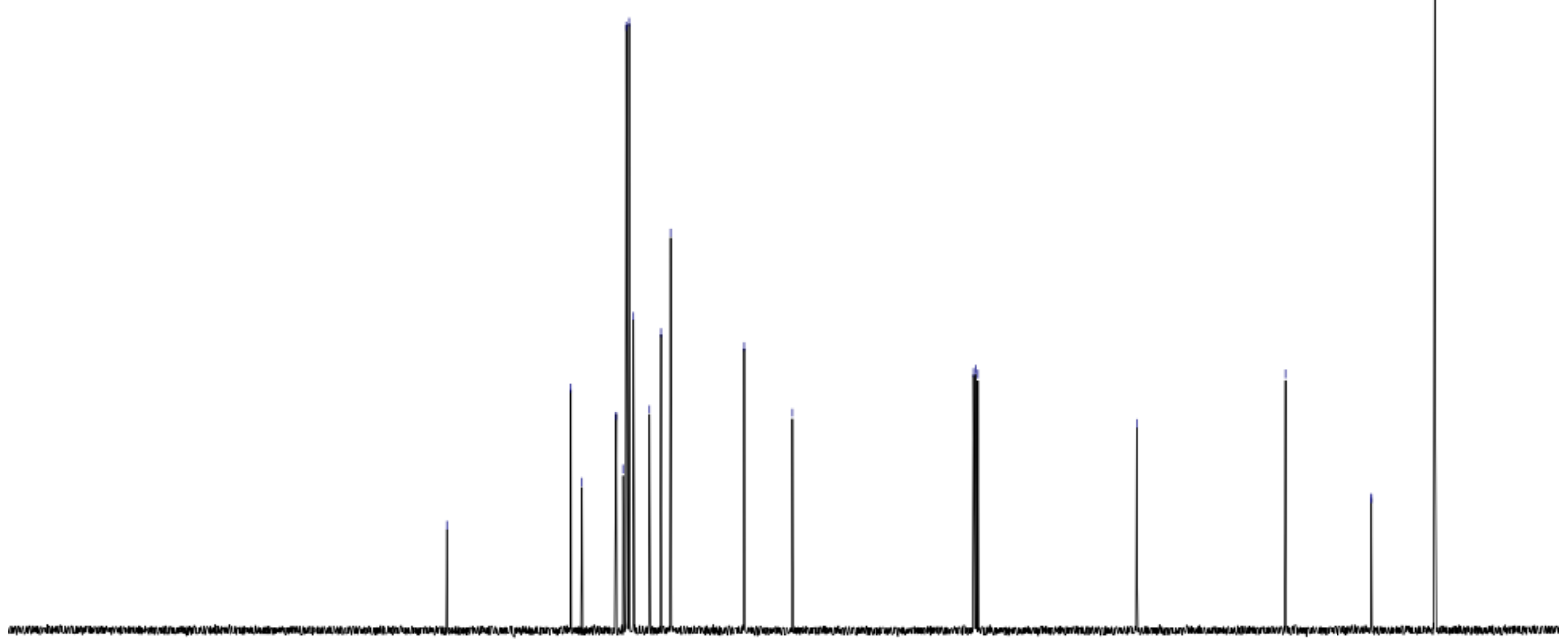

$\begin{array}{llllllllllllllllllllllll}20 & 210 & 200 & 190 & 180 & 170 & 160 & 150 & 140 & 130 & 120 & 110 & 100 & 90 & 80 & 70 & 60 & 50 & 40 & 30 & 20 & 10 & 0 & \end{array}$ 
$\left(3 \mathrm{a} S^{*}, 11 \mathrm{a} S^{*}\right)$-4-Benzyl-10-methyl-2,3,3a,4-tetrahydro-11H-cyclopenta[4,5]imidazo[1,5a]indole-1,5-dione 4d
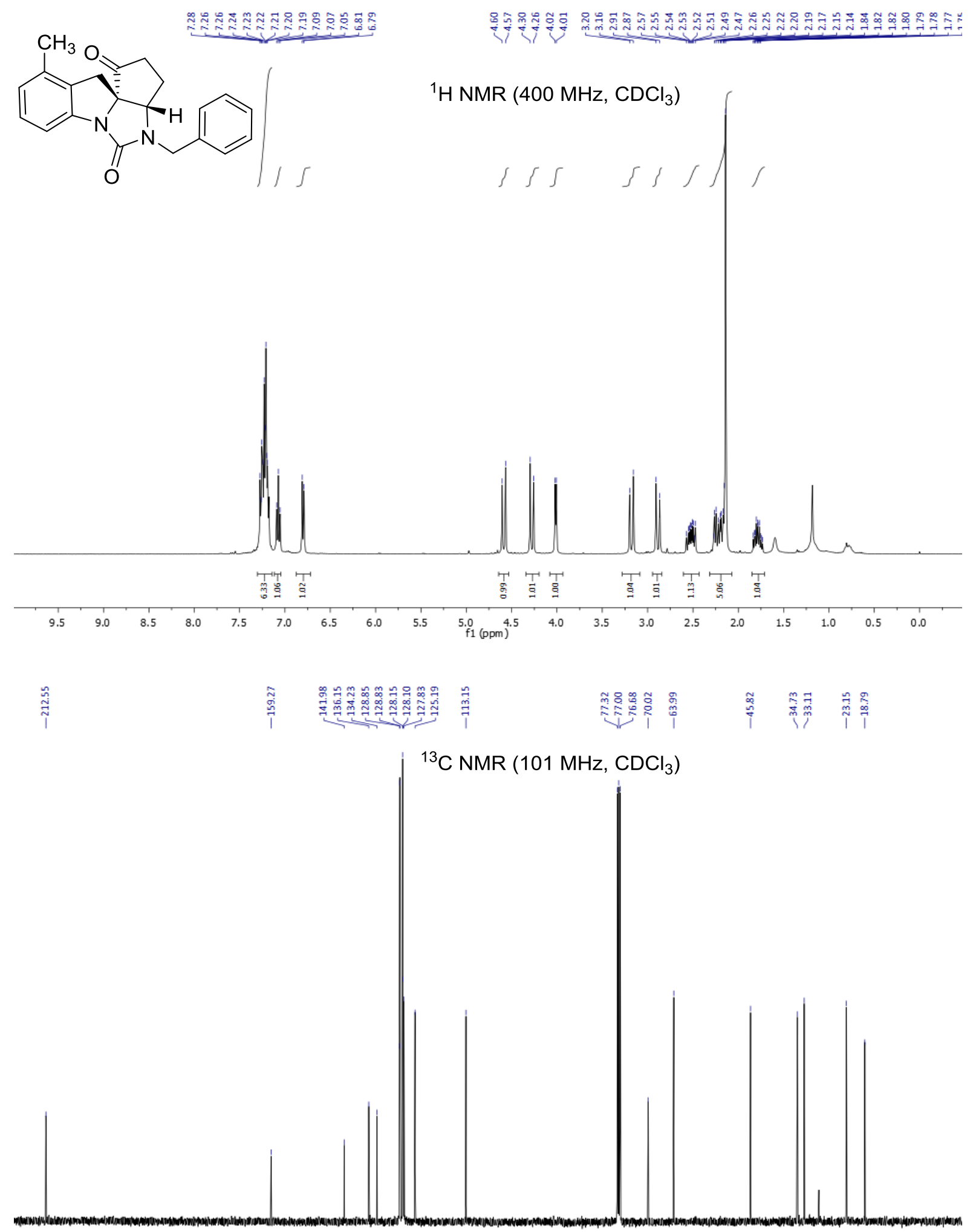

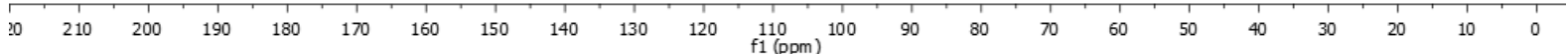


Methyl 1-(benzyl(cyclopropyl)carbamoyl)- $1 H$-indole-4-carboxylate $3 e$

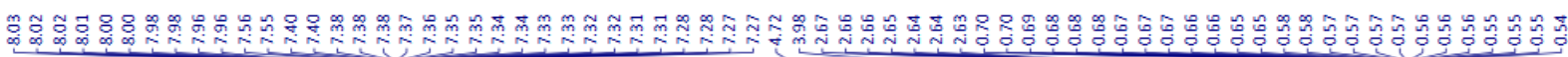<smiles>CC(=O)c1cccc2c1ccn2C(=O)N(Cc1ccccc1)C1CC1</smiles>

${ }^{1} \mathrm{H}$ NMR $\left(400 \mathrm{MHz}, \mathrm{CDCl}_{3}\right)$

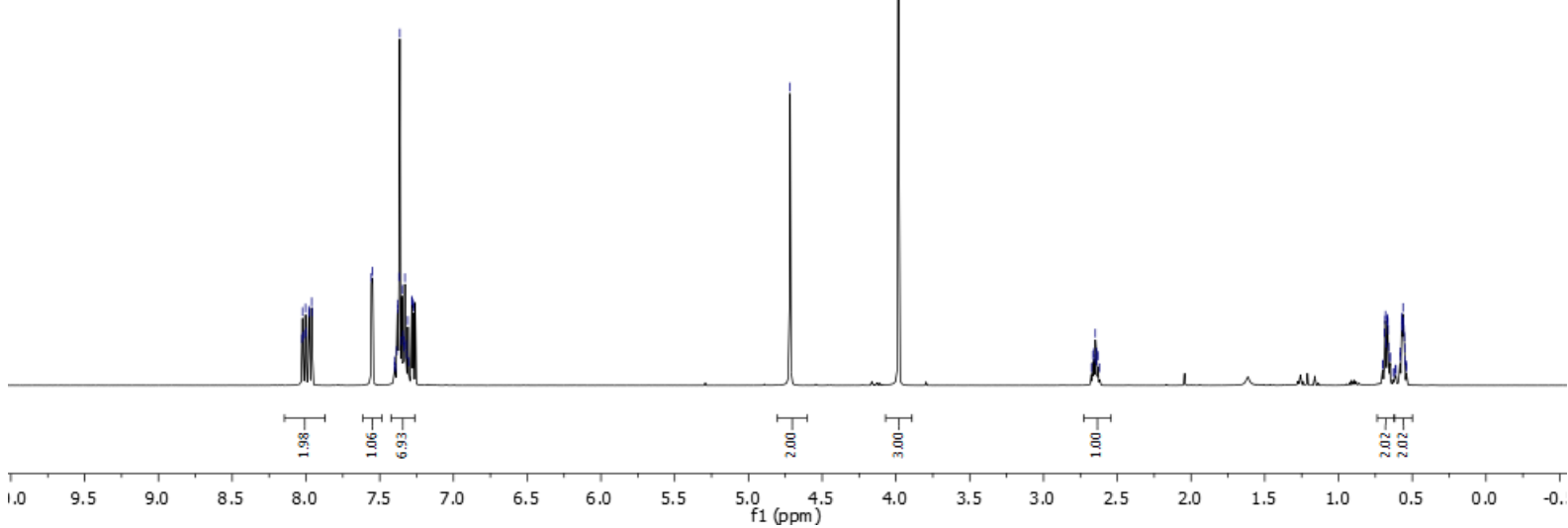

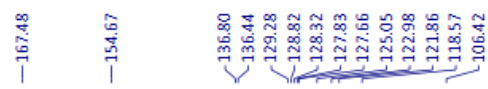

교

तె

${ }^{13} \mathrm{C}$ NMR $\left(101 \mathrm{MHz}, \mathrm{CDCl}_{3}\right)$

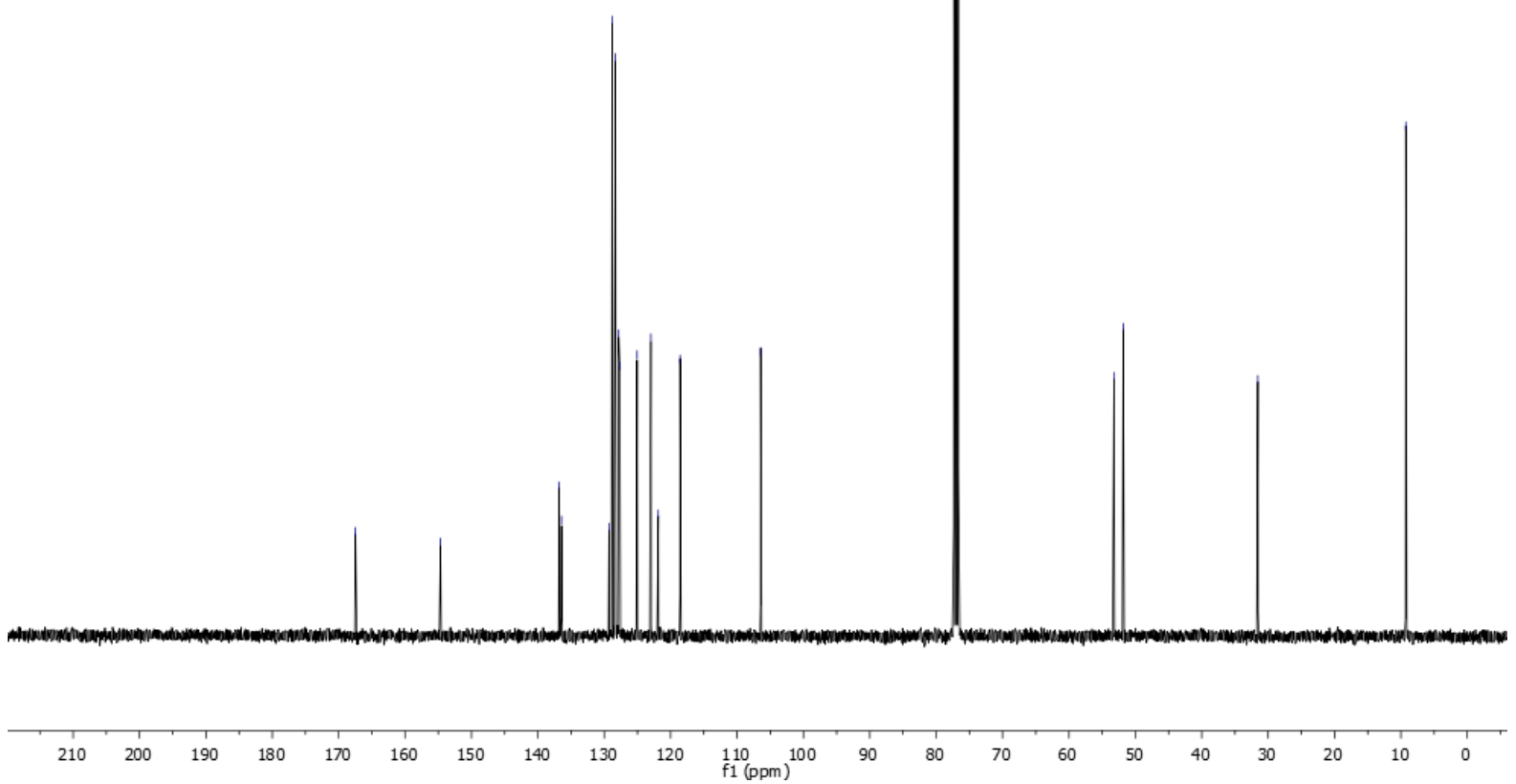


Methyl

$\left(3 \mathrm{a} S^{*}, 11 \mathrm{a} S^{*}\right)$-4-benzyl-1,5-dioxo-1,2,3,3a,4,5-hexahydro-11H-

cyclopenta $[4,5]$ imidazo $[1,5-a]$ indole-10-carboxylate $4 \mathrm{e}$

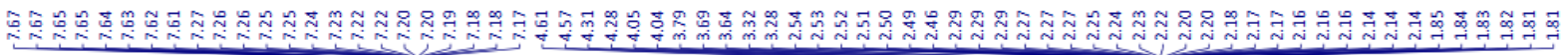<smiles>COC(=O)c1cccc2c1C[C@@]13C(=O)CC[C@H]1[C@H]2N3Cc1ccccc1</smiles>

${ }^{1} \mathrm{H}$ NMR $\left(400 \mathrm{MHz}, \mathrm{CDCl}_{3}\right)$

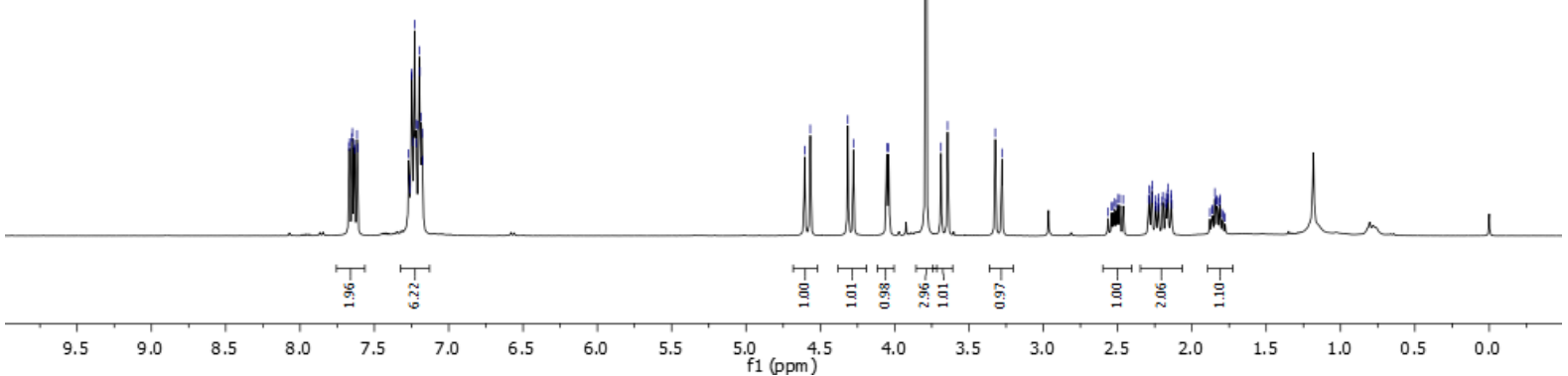

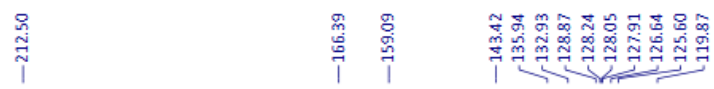

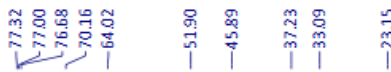

${ }^{13} \mathrm{C}$ NMR $\left(101 \mathrm{MHz}, \mathrm{CDCl}_{3}\right)$

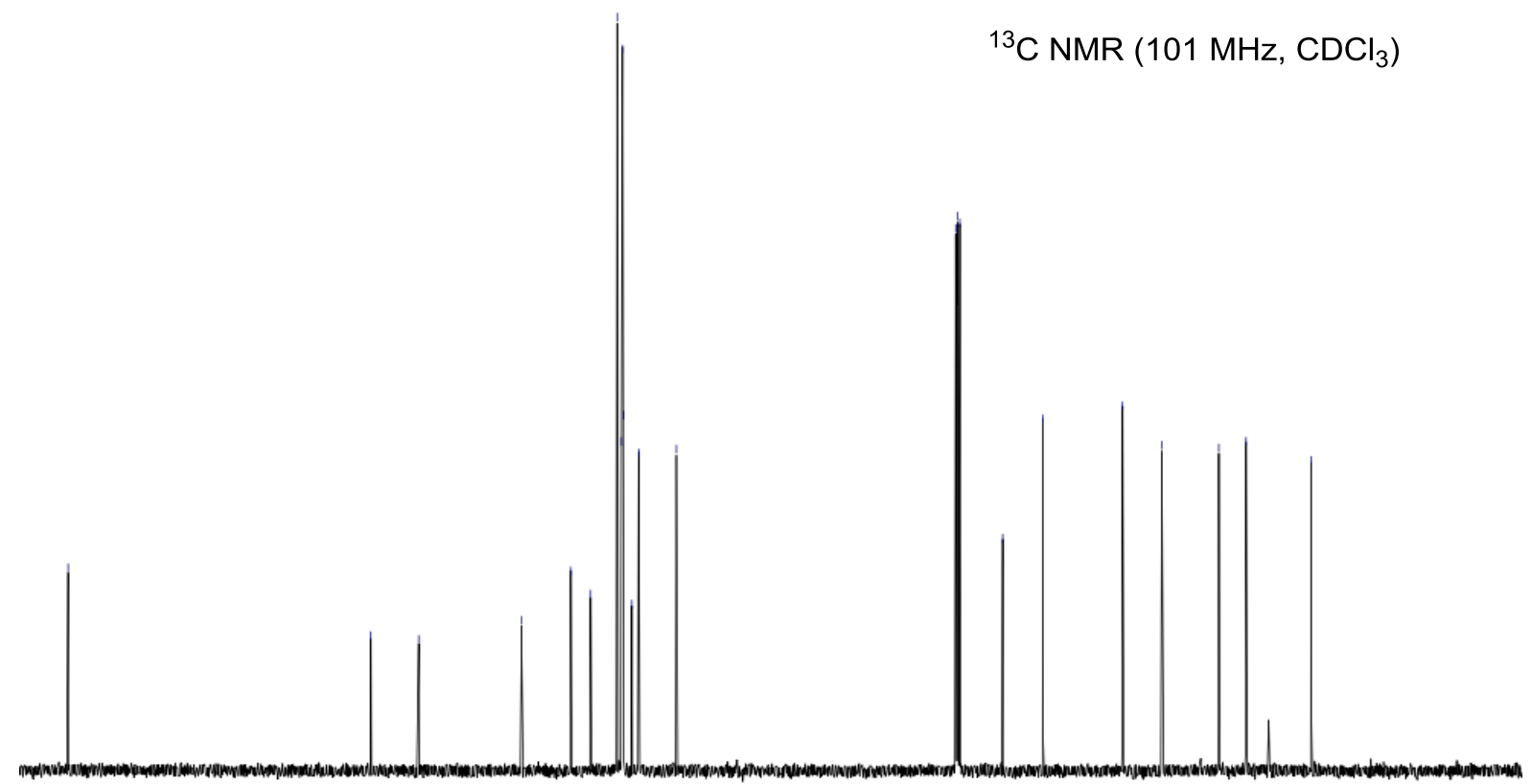

$\begin{array}{lllllllllll}210 & 200 & 190 & 180 & 170 & 160 & 150 & 140 & 130 & 120 & 110 \quad 100\end{array}$ 
$N$-Benzyl-4-((tert-butyldimethylsilyl)oxy)- $N$-cyclopropyl-1H-indole-1-carboxamide $3 f$

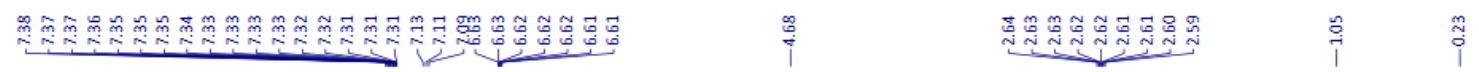<smiles>CC(C)(C)[Si](C)(C)Oc1cccc2c1ccn2C(=O)N(Cc1ccccc1)C1CC1</smiles>

${ }^{1} \mathrm{H}$ NMR $\left(400 \mathrm{MHz}, \mathrm{CDCl}_{3}\right)$

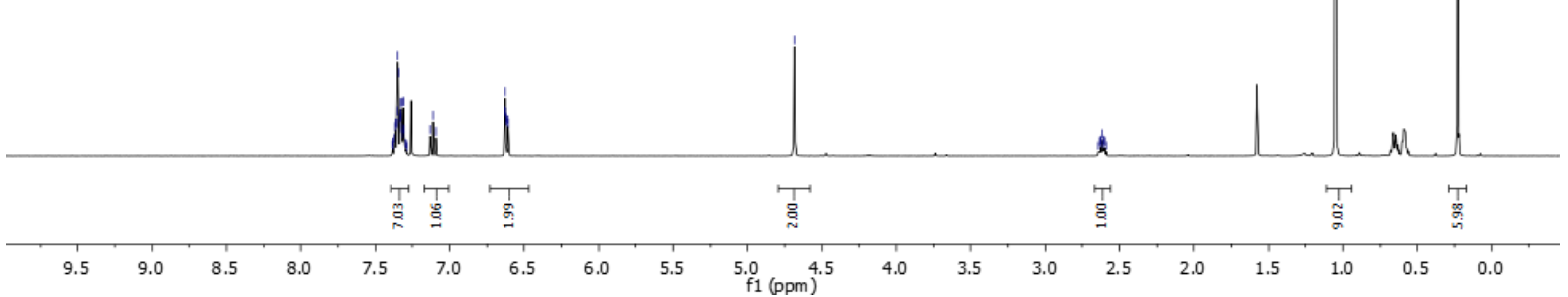

倠全

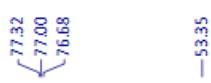

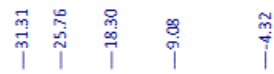

${ }^{13} \mathrm{C}$ NMR $\left(101 \mathrm{MHz}, \mathrm{CDCl}_{3}\right)$

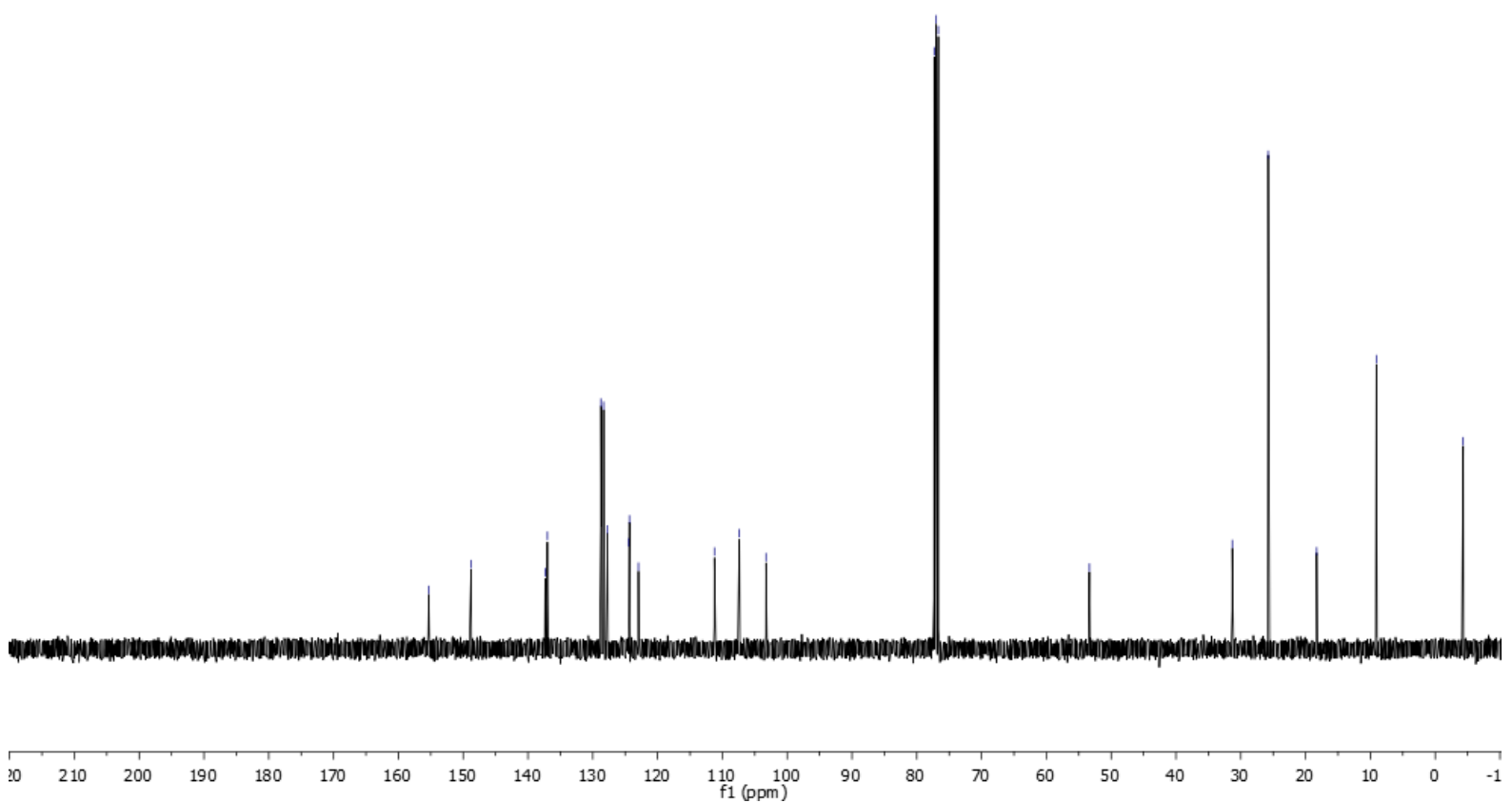


$\left(3 \mathrm{a} S^{*}, 11 \mathrm{a} S^{*}\right)-4-B e n z y l-10$-((tert-butyldimethylsilyl)oxy)-2,3,3a,4-tetrahydro-11Hcyclopenta[4,5]imidazo[1,5-a]indole-1,5-dione $4 \mathrm{f}$

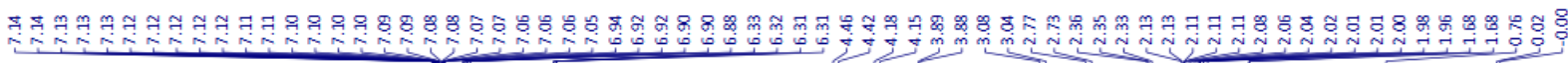<smiles>C[SiH2]NC(=O)ONCC(C)(C)C(C)(C)C</smiles>

$\mathrm{H}_{3} \mathrm{C}-\mathrm{Si}$ $\mathrm{H}_{3} \mathrm{C}$<smiles>COc1cccc2c1C[C@@]13CCC(=O)N1[C@H](Cc1ccccc1)C(=O)N23</smiles>

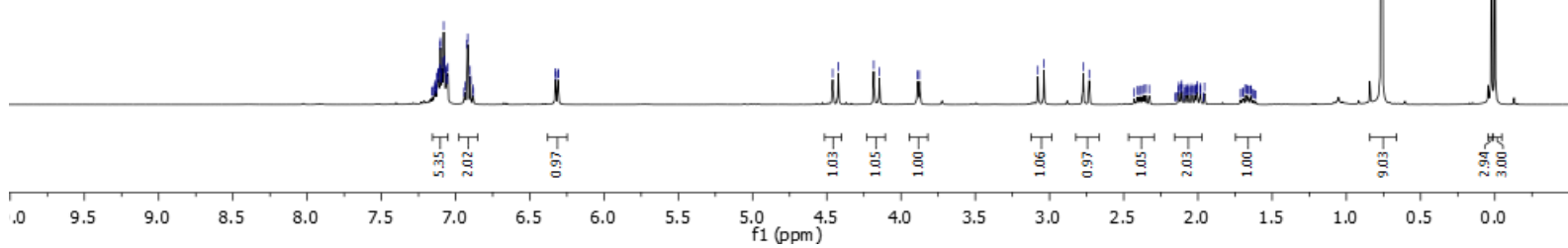

${ }^{13} \mathrm{C} \mathrm{NMR}\left(101 \mathrm{MHz}, \mathrm{CDCl}_{3}\right)$

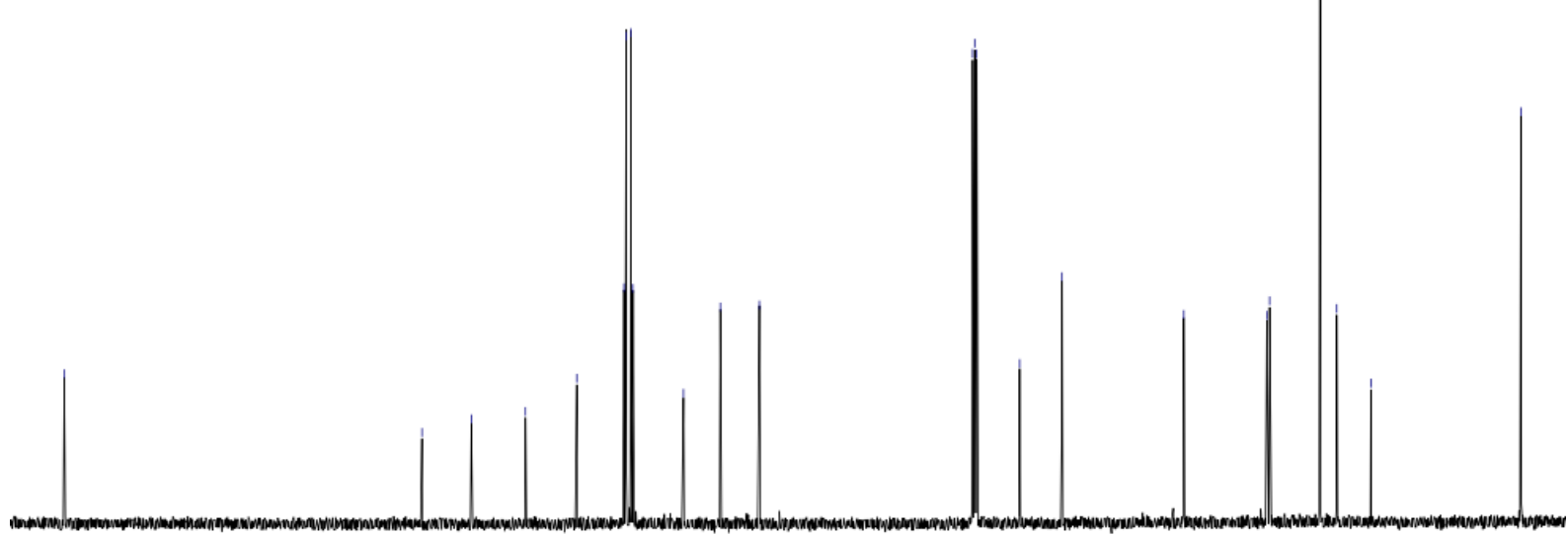

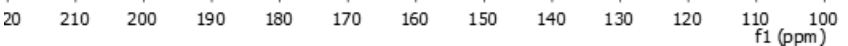


$N$-Benzyl- $N$-cyclopropyl-5-fluoro- $1 H$-indole-1-carboxamide $3 g$

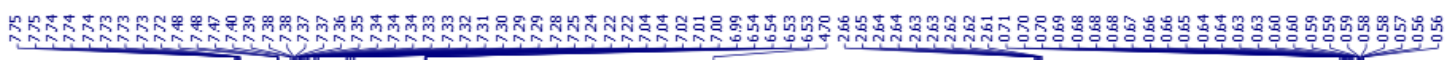<smiles>Fc1ccc2[nH]ccc2c1</smiles>

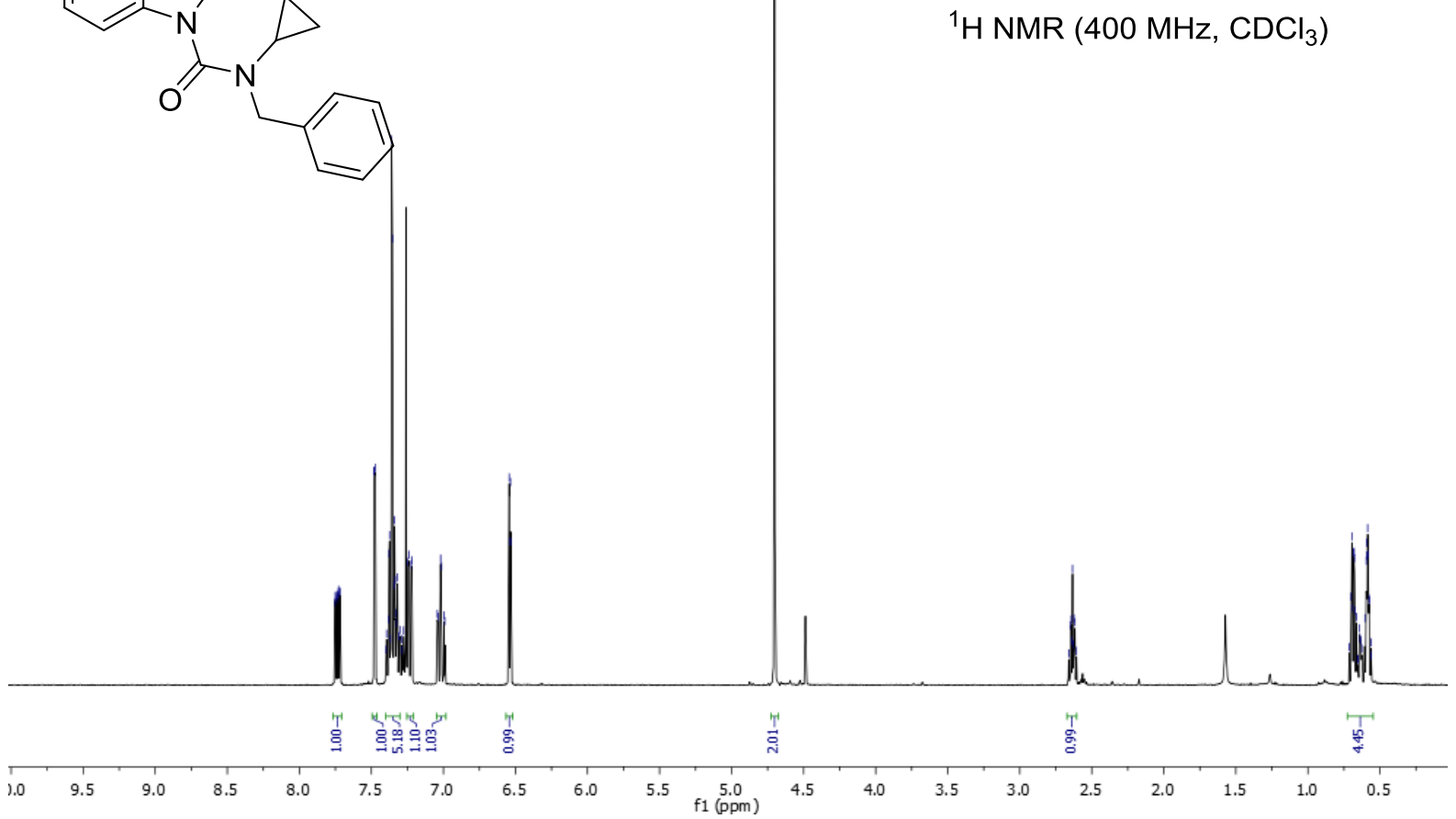

${ }^{1} \mathrm{H}$ NMR $\left(400 \mathrm{MHz}, \mathrm{CDCl}_{3}\right)$
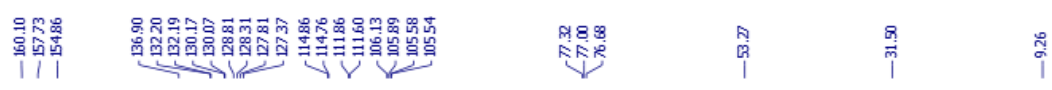

${ }^{13} \mathrm{C}$ NMR $\left(101 \mathrm{MHz}, \mathrm{CDCl}_{3}\right)$

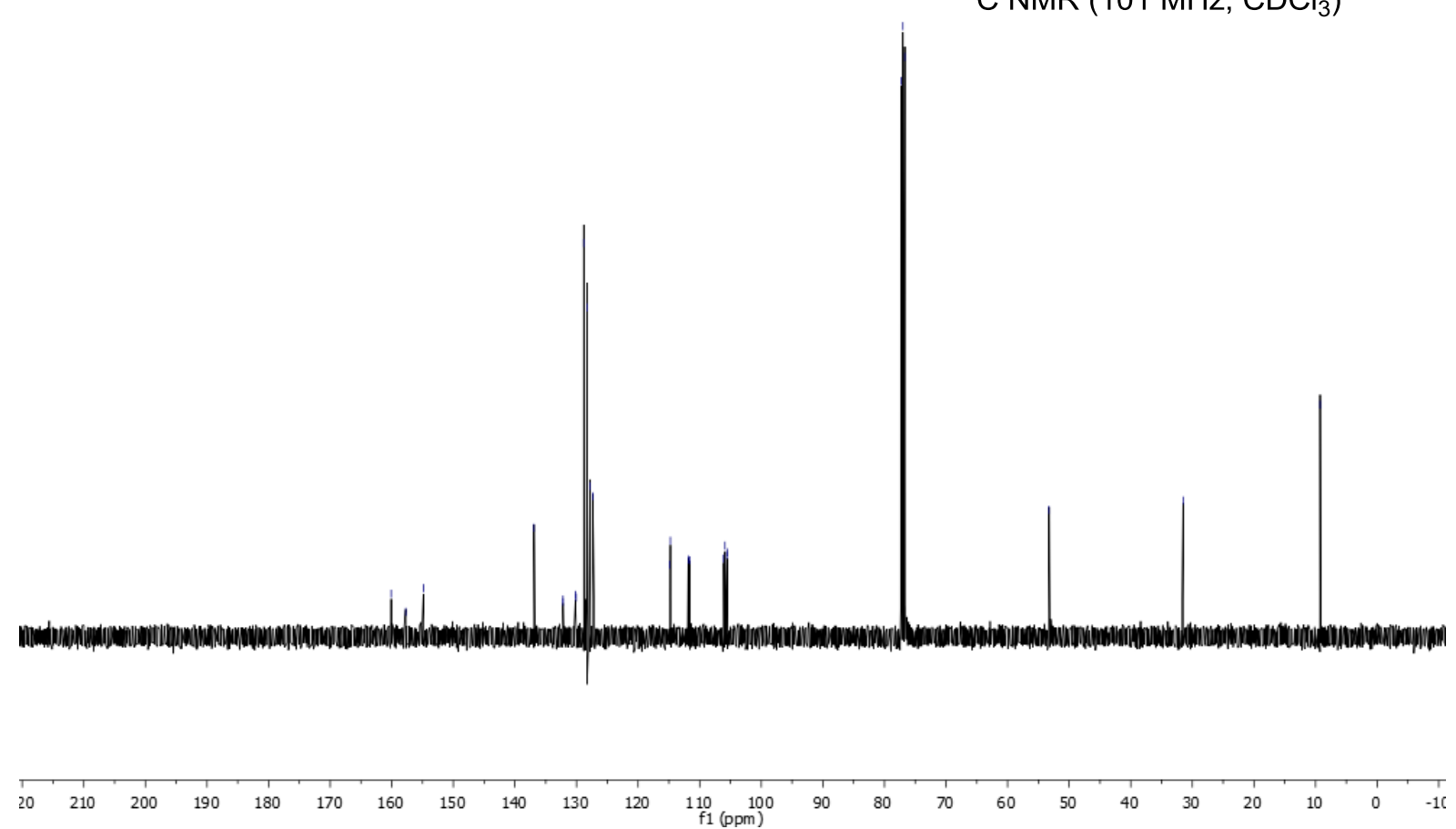


${ }^{19} \mathrm{~F} \mathrm{NMR}\left(377 \mathrm{MHz}, \mathrm{CDCl}_{3}\right)$
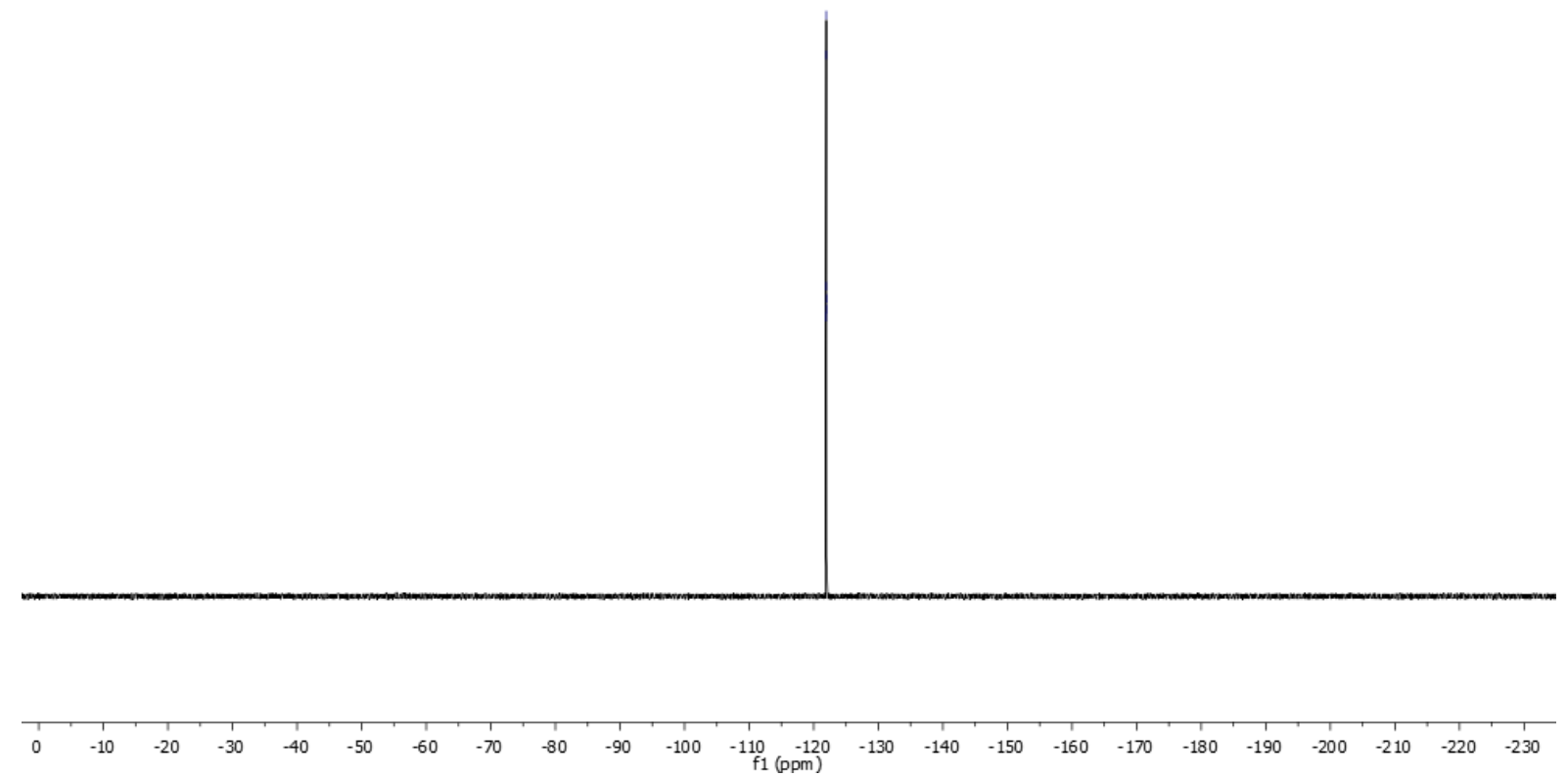

$\left(3 a S^{*}, 11 \mathrm{a} S^{*}\right)$-4-Benzyl-9-fluoro-2,3,3a,4-tetrahydro-11 $H$-cyclopenta[4,5]imidazo[1,5-

a]indole-1,5-dione 4g

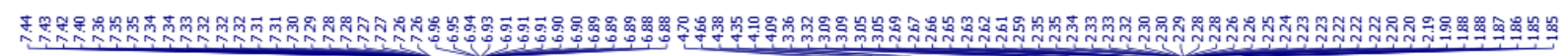

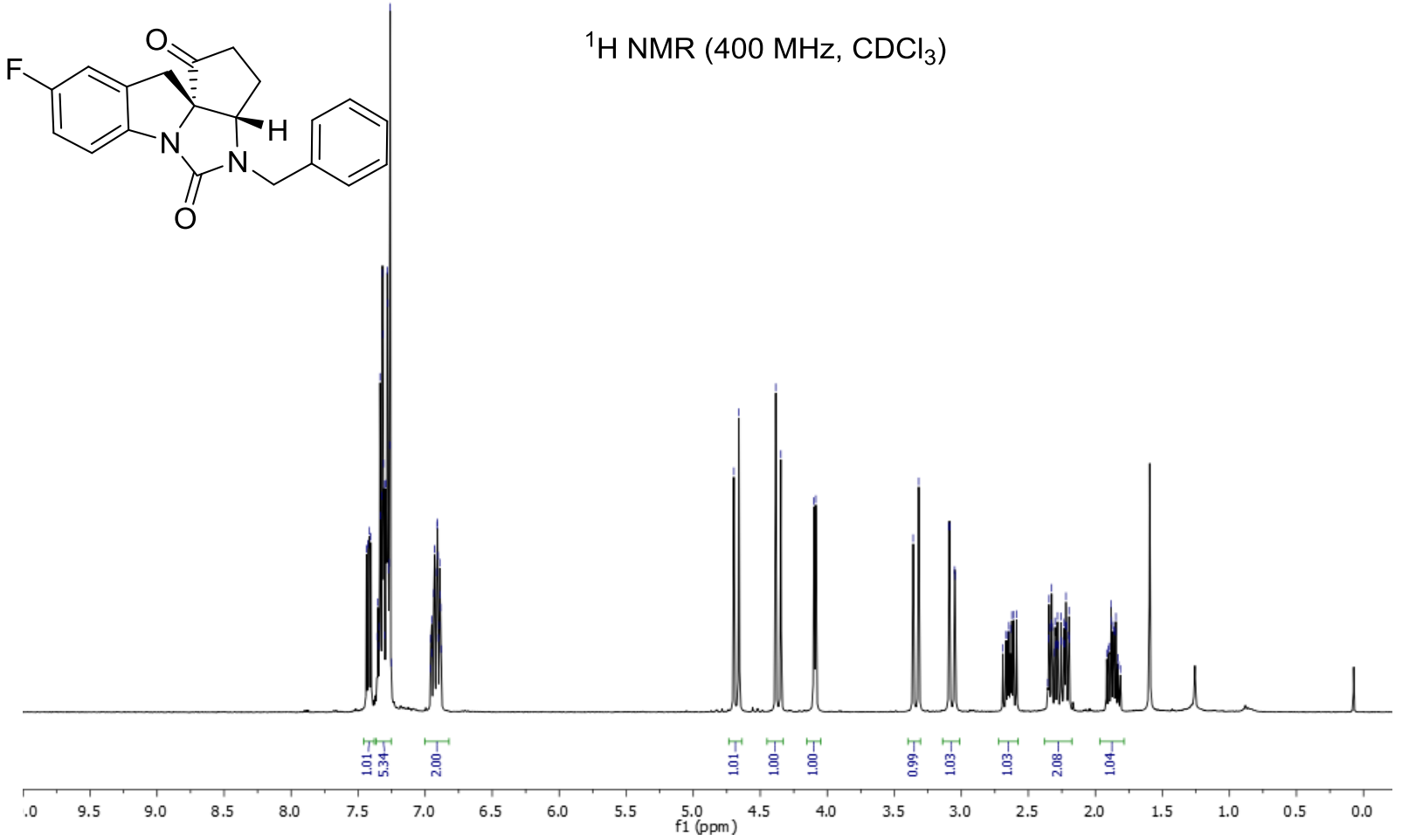



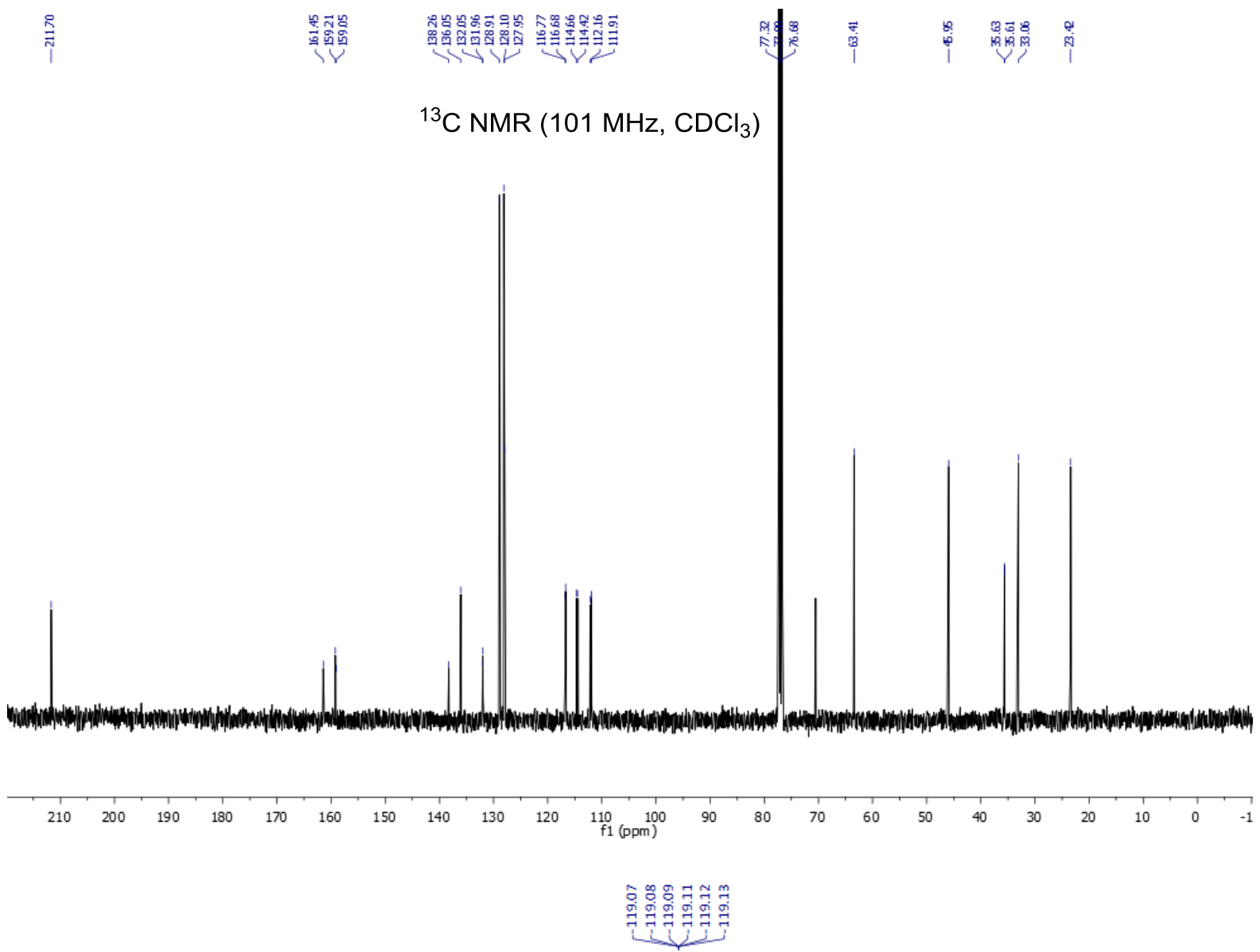

${ }^{19} \mathrm{~F}$ NMR (377 MHz, $\mathrm{CDCl}_{3}$ )

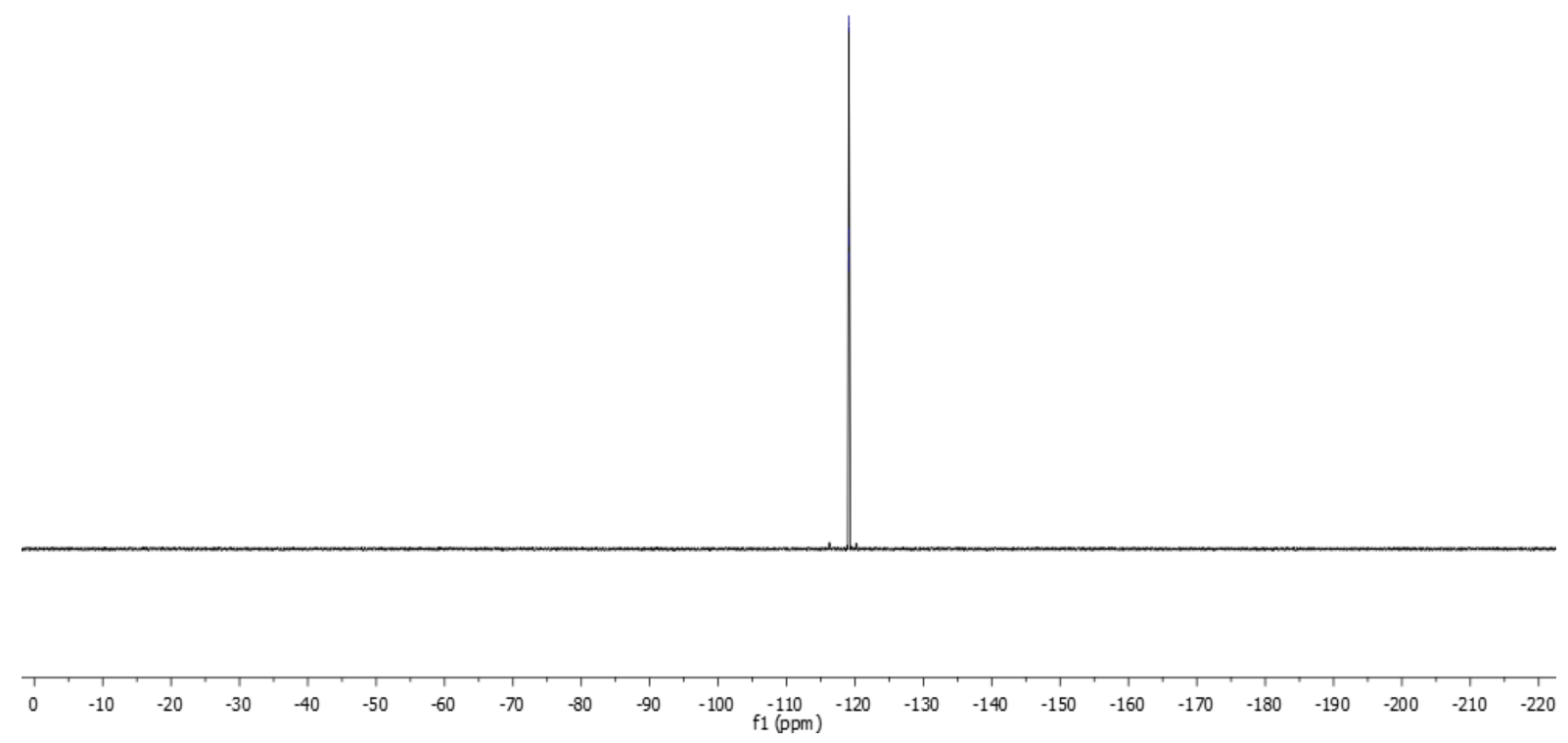


$N$-Benzyl-5-bromo- $N$-cyclopropyl- $1 H$-indole-1-carboxamide $3 \mathrm{~h}$

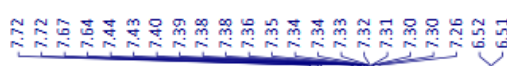<smiles>O=C(N(Cc1ccccc1)C1CC1)n1ccc2cc(Br)ccc21</smiles>

${ }^{1} \mathrm{H} \mathrm{NMR}\left(400 \mathrm{MHz}, \mathrm{CDCl}_{3}\right)$

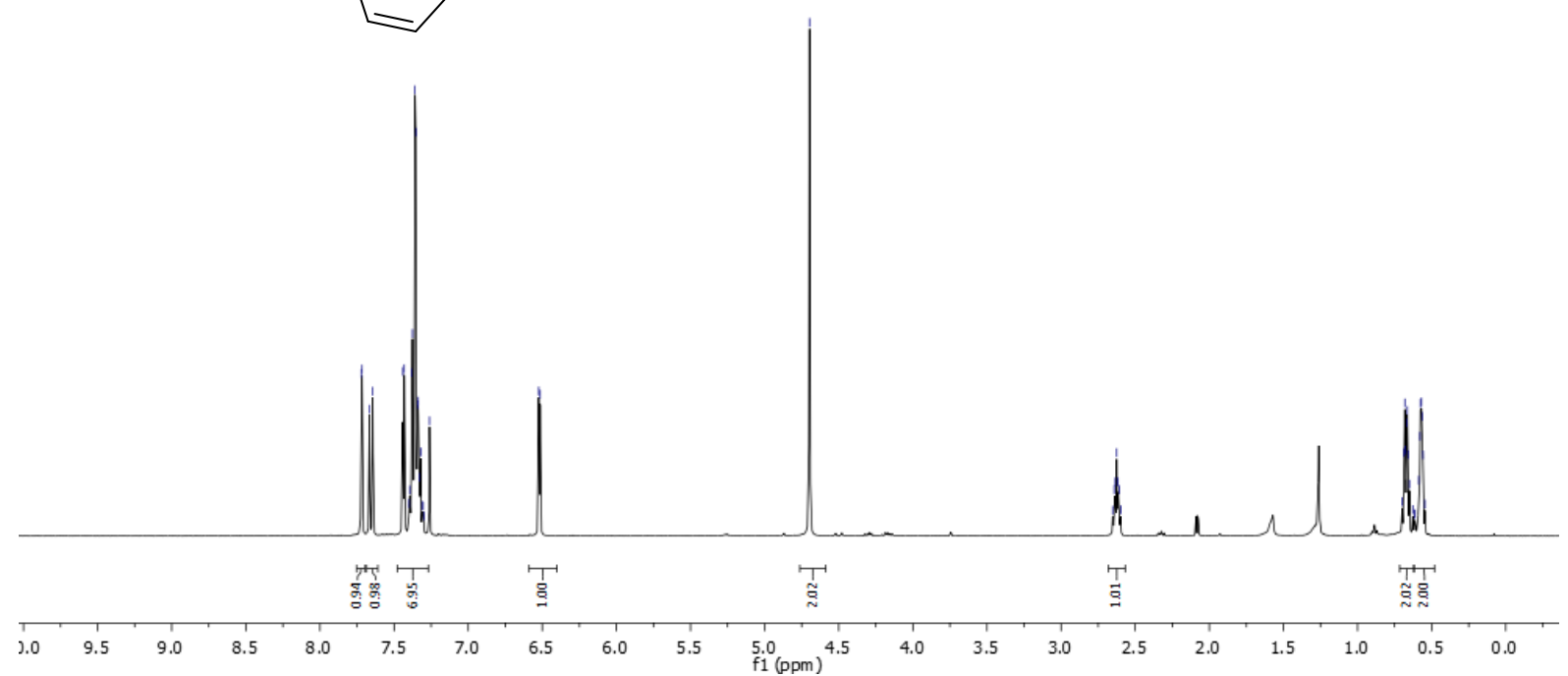

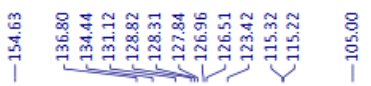

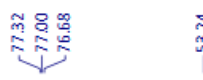

$\underset{\substack{n \\ 1}}{\overrightarrow{0}}$

${ }^{13} \mathrm{C}$ NMR $\left(101 \mathrm{MHz}, \mathrm{CDCl}_{3}\right)$

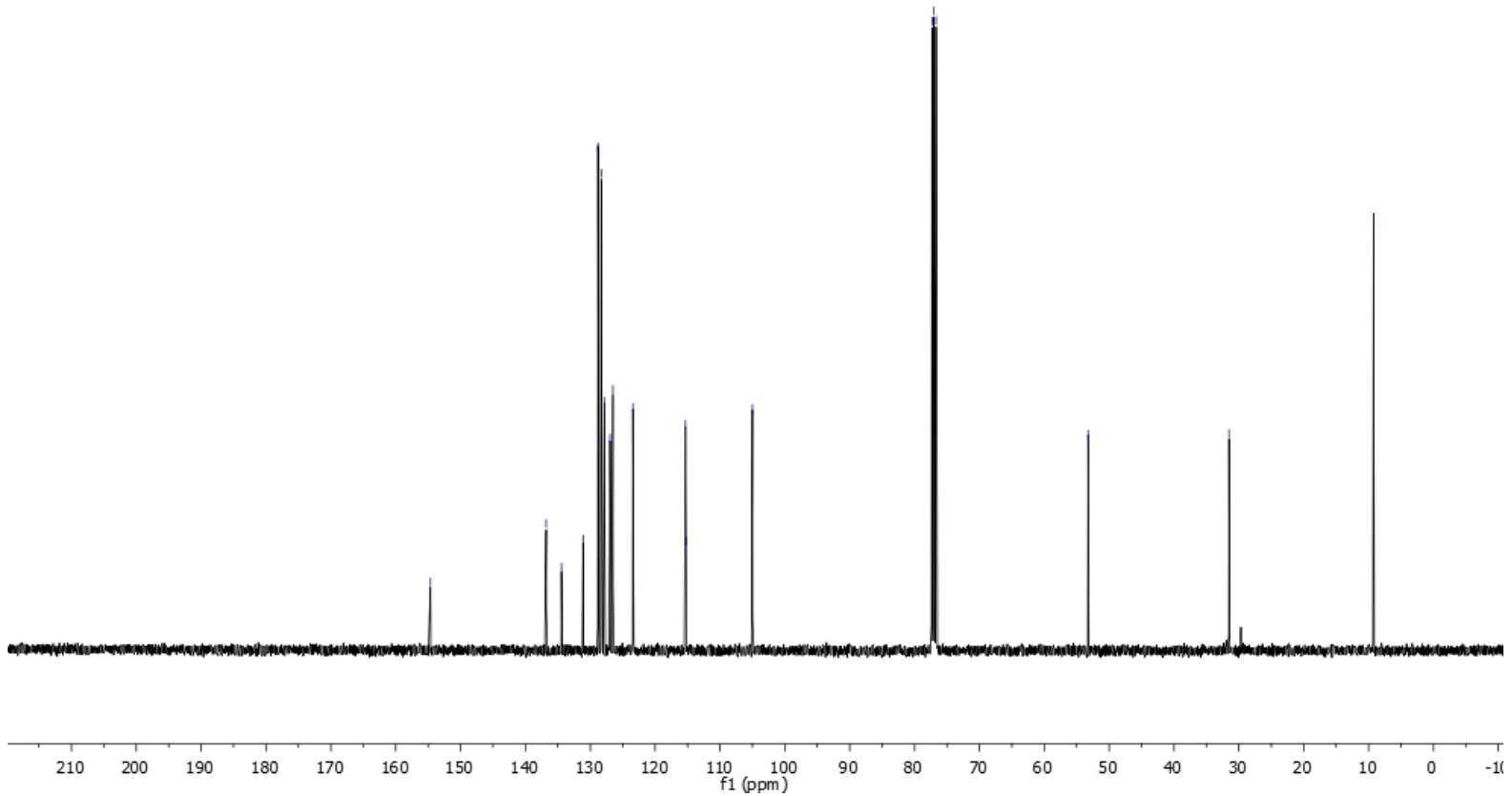


$\left(3 \mathrm{a} S^{*}, 11 \mathrm{a} S^{*}\right)$-4-Benzyl-9-bromo-2,3,3a,4-tetrahydro-11H-cyclopenta[4,5]imidazo[1,5a]indole-1,5-dione $4 \mathrm{~h}$

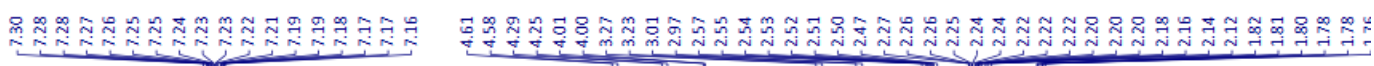
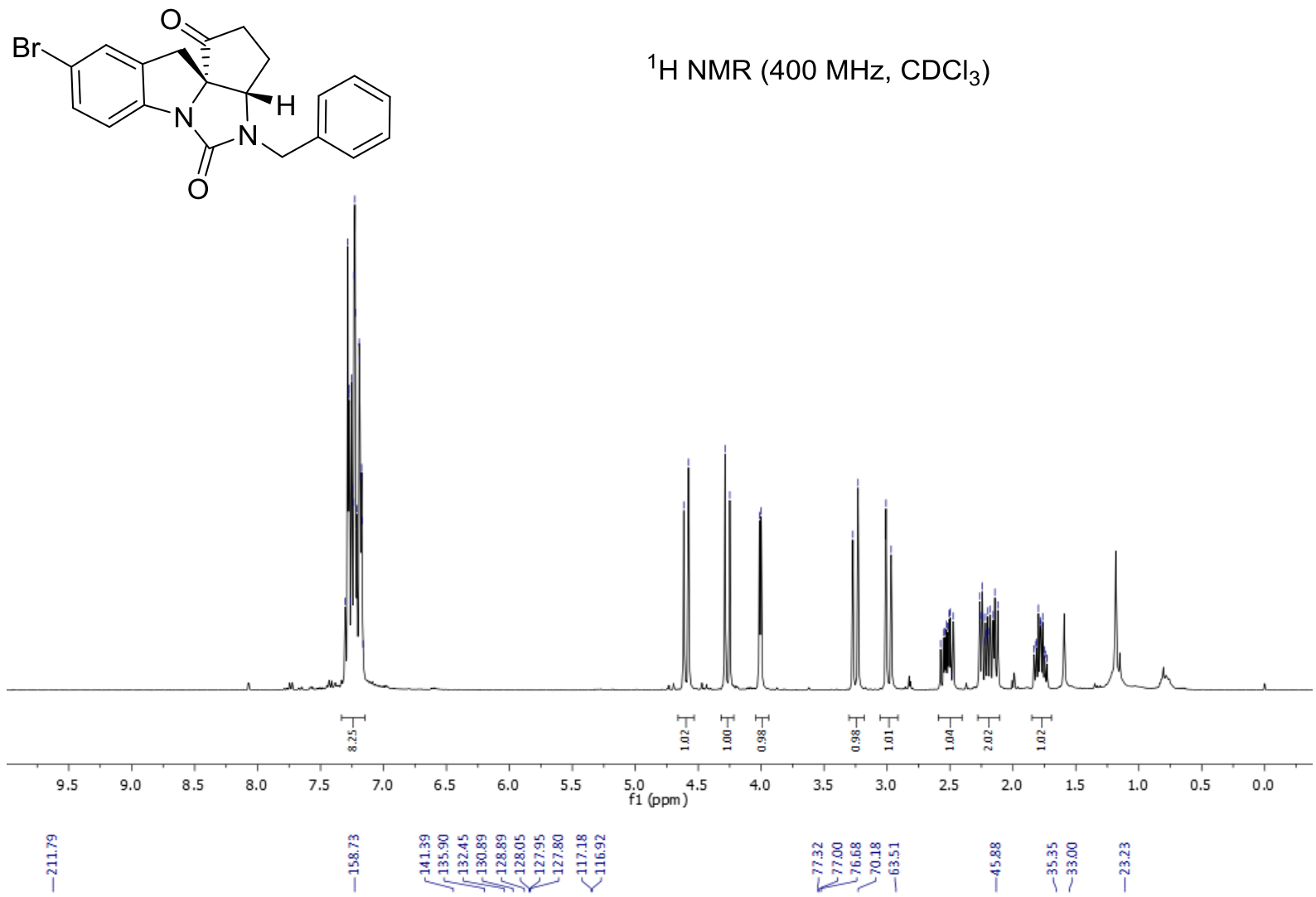

${ }^{13} \mathrm{C}$ NMR $\left(101 \mathrm{MHz}, \mathrm{CDCl}_{3}\right)$

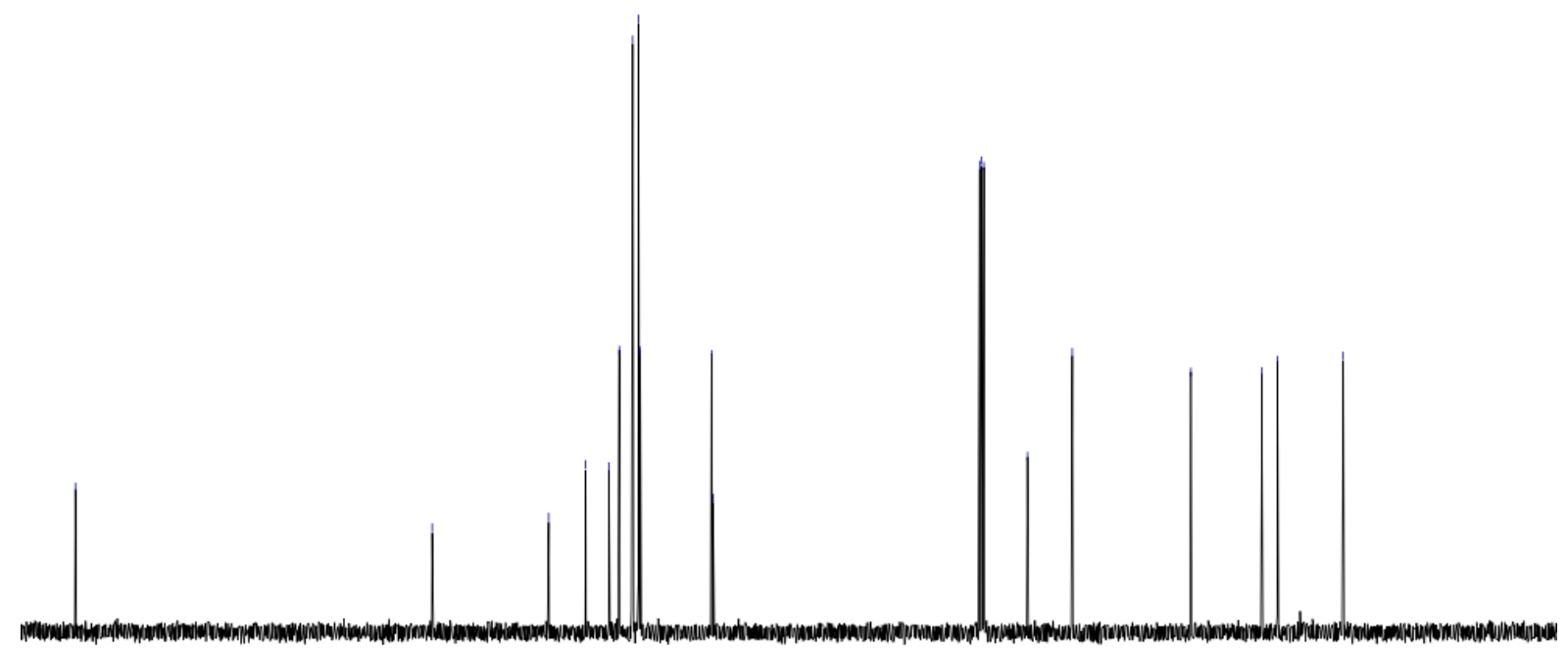

$\begin{array}{llllllllllllllllllllllllllll}210 & 200 & 190 & 180 & 170 & 160 & 150 & 140 & 130 & 120 & 110 & 100 & 90 & 80 & 70 & 60 & 50 & 40 & 30 & 20 & 10 & 0\end{array}$ 
$N$-Benzyl- $N$-cyclopropyl-5-methoxy-1H-indole-1-carboxamide $3 \mathrm{i}$

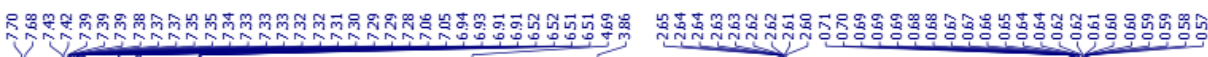

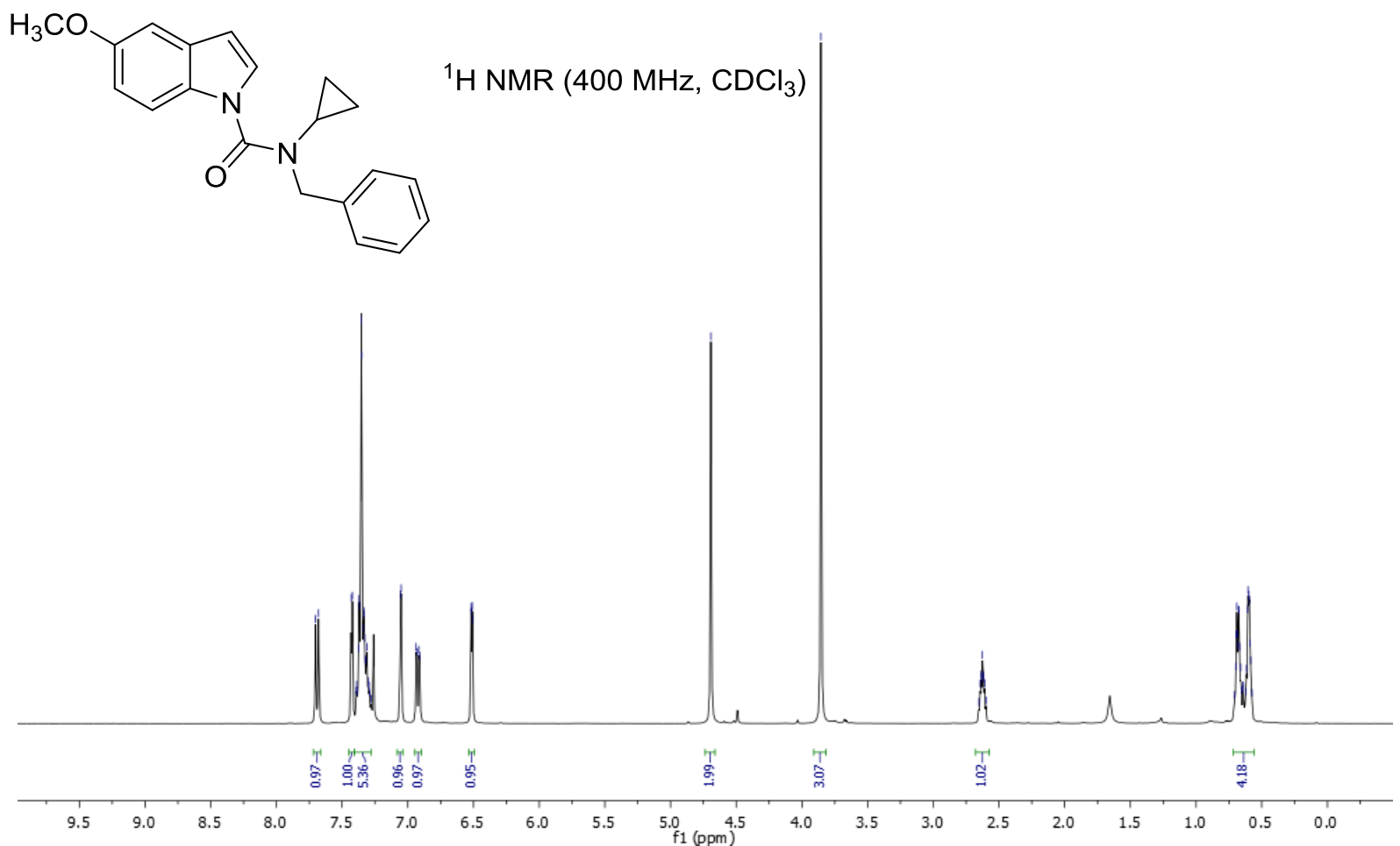

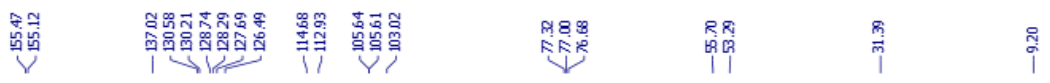

${ }^{13} \mathrm{C}$ NMR $\left(101 \mathrm{MHz}, \mathrm{CDCl}_{3}\right)$

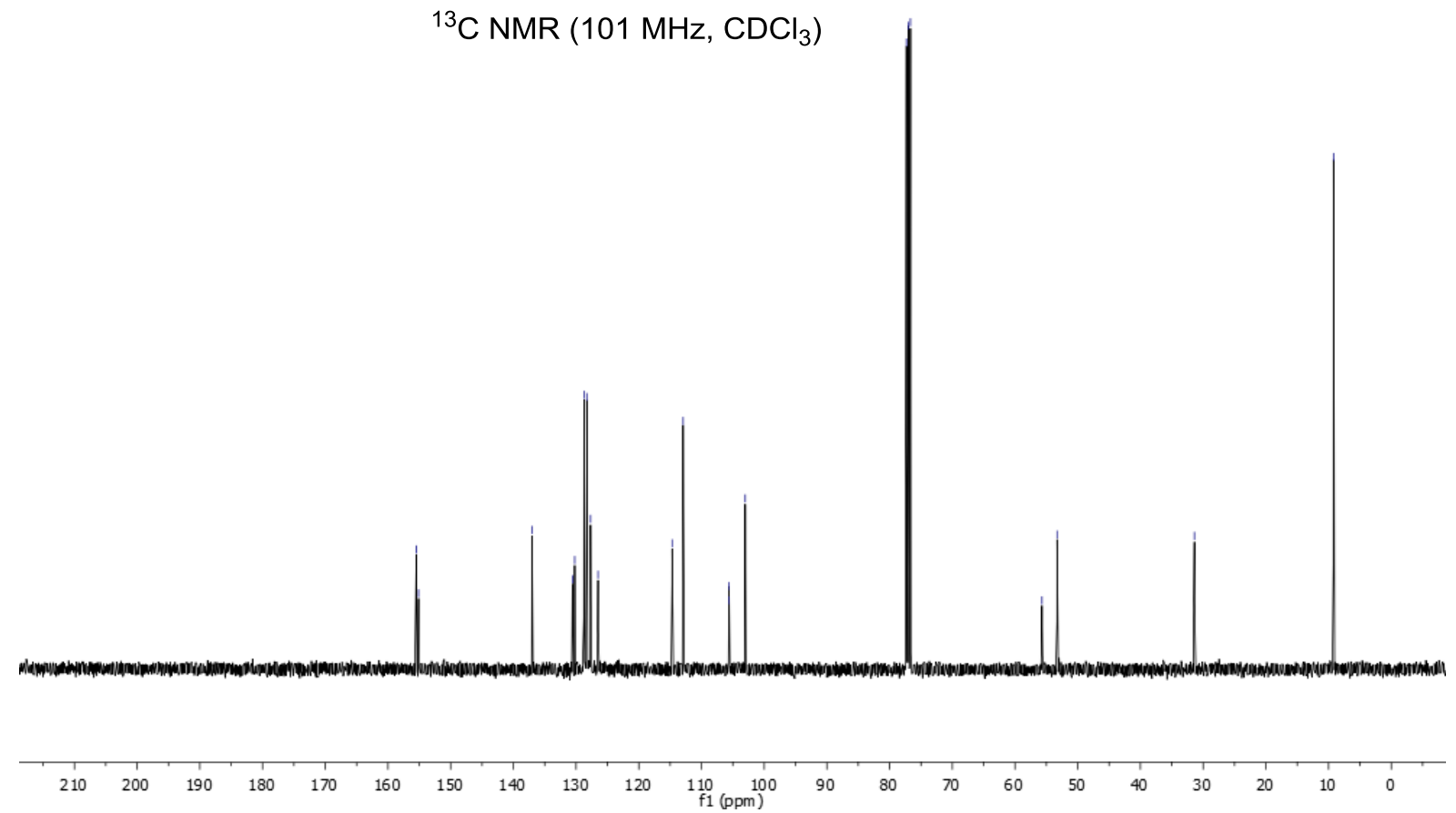


$\left(3 \mathrm{a} S^{*}, 11 \mathrm{a} S^{*}\right)$-4-Benzyl-9-methoxy-2,3,3a,4-tetrahydro-11H-cyclopenta[4,5]imidazo[1,5a]indole-1,5-dione 4i

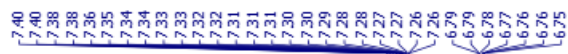

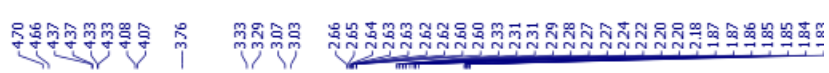<smiles>COc1ccc2c(c1)CC[C@]21C(=O)CC[C@H]1NCc1ccccc1</smiles>

${ }^{1} \mathrm{H}$ NMR (400 MHz, $\left.\mathrm{CDCl}_{3}\right)$

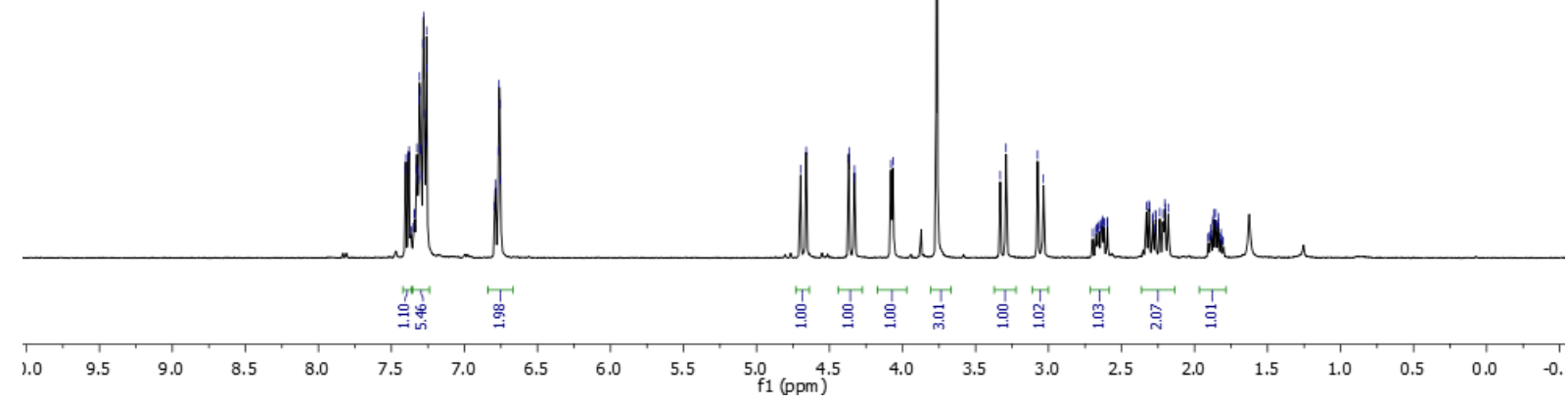

吾

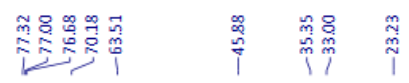

${ }^{13} \mathrm{C}$ NMR $\left(101 \mathrm{MHz}, \mathrm{CDCl}_{3}\right)$

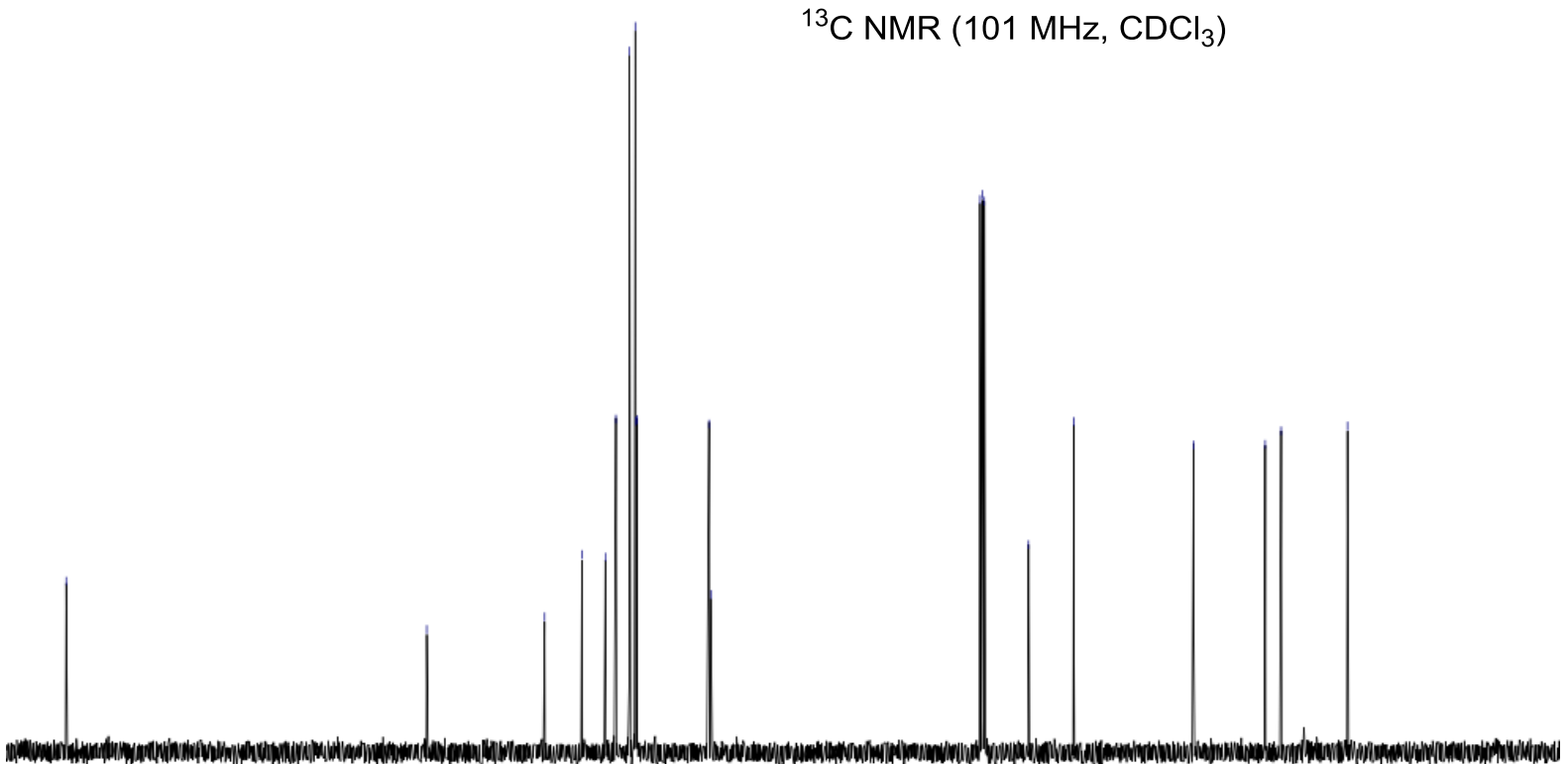

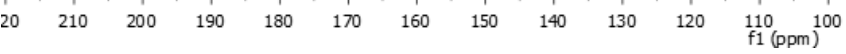


$N$-Benzyl- $N$-cyclopropyl-6-isopropyl- $1 H$-indole-1-carboxamide $3 \mathbf{j}$

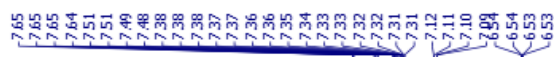

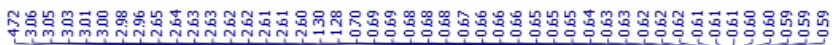<smiles>CC(C)c1ccc2ccn(C(=O)N(Cc3ccccc3)C3CC3)c2c1</smiles>

${ }^{1} \mathrm{H}$ NMR $\left(400 \mathrm{MHz}, \mathrm{CDCl}_{3}\right)$

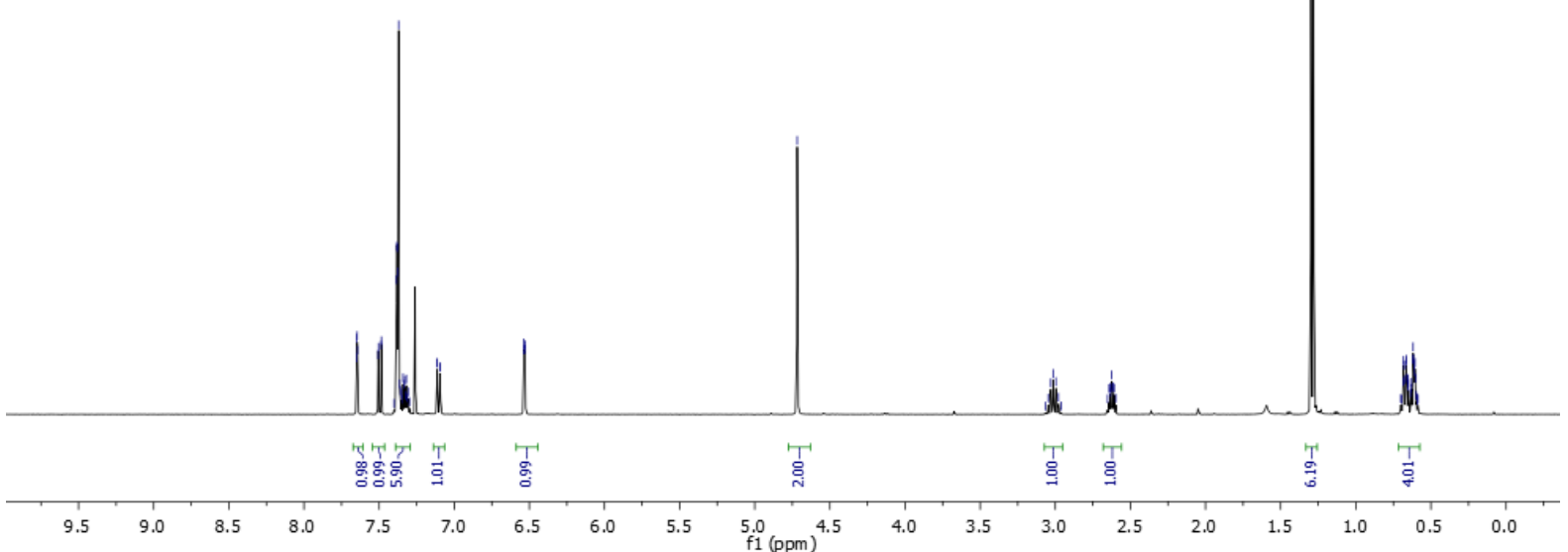

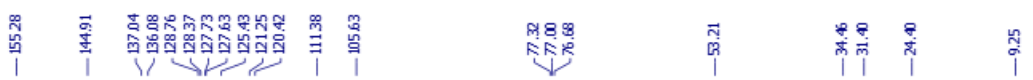

${ }^{13} \mathrm{C}$ NMR $\left(101 \mathrm{MHz}, \mathrm{CDCl}_{3}\right)$

$\begin{array}{lllllllllllll}20 & 210 & 200 & 190 & 180 & 170 & 160 & 150 & 140 & 130 & 120 & 110 & 100\end{array}$
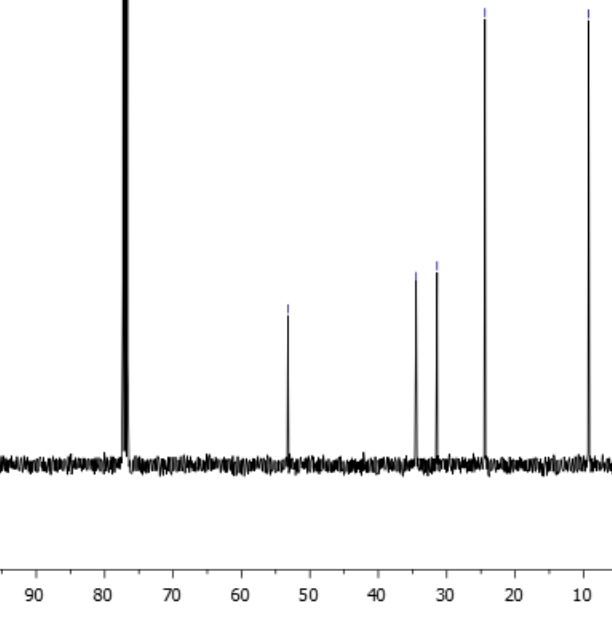
$\left(3 \mathrm{a} S^{*}, 11 \mathrm{a} S^{*}\right)$-4-Benzyl-8-isopropyl-2,3,3a,4-tetrahydro-11H-

cyclopenta[4,5]imidazo[1,5-a]indole-1,5-dione $4 \mathbf{j}$

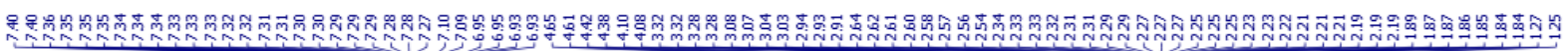<smiles>CC(C)c1ccc2c(c1)N1C(=O)N(Cc3ccccc3)[C@@]13CCCC[C@]23C</smiles>

${ }^{1} \mathrm{H}$ NMR (400 $\left.\mathrm{MHz}, \mathrm{CDCl}_{3}\right)$

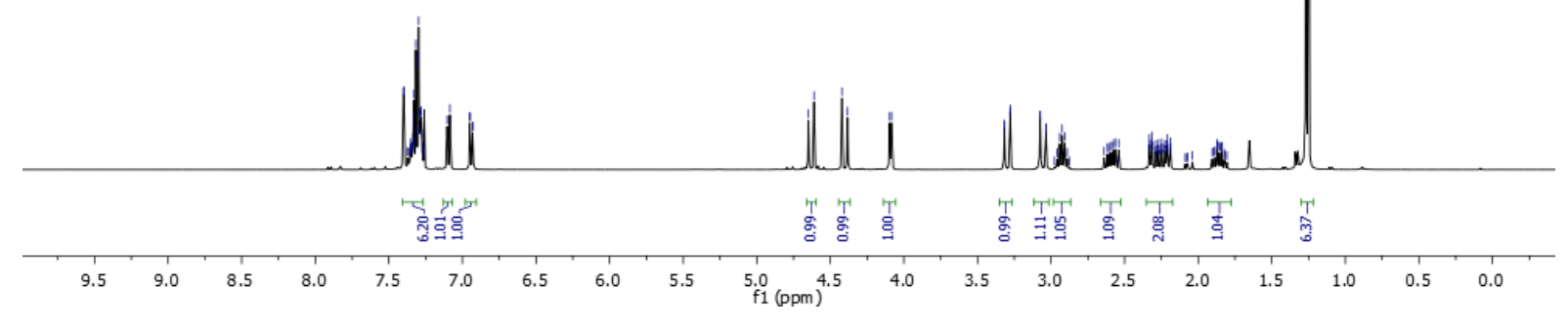

年

${ }^{13} \mathrm{C}$ NMR $\left(101 \mathrm{MHz}, \mathrm{CDCl}_{3}\right)$

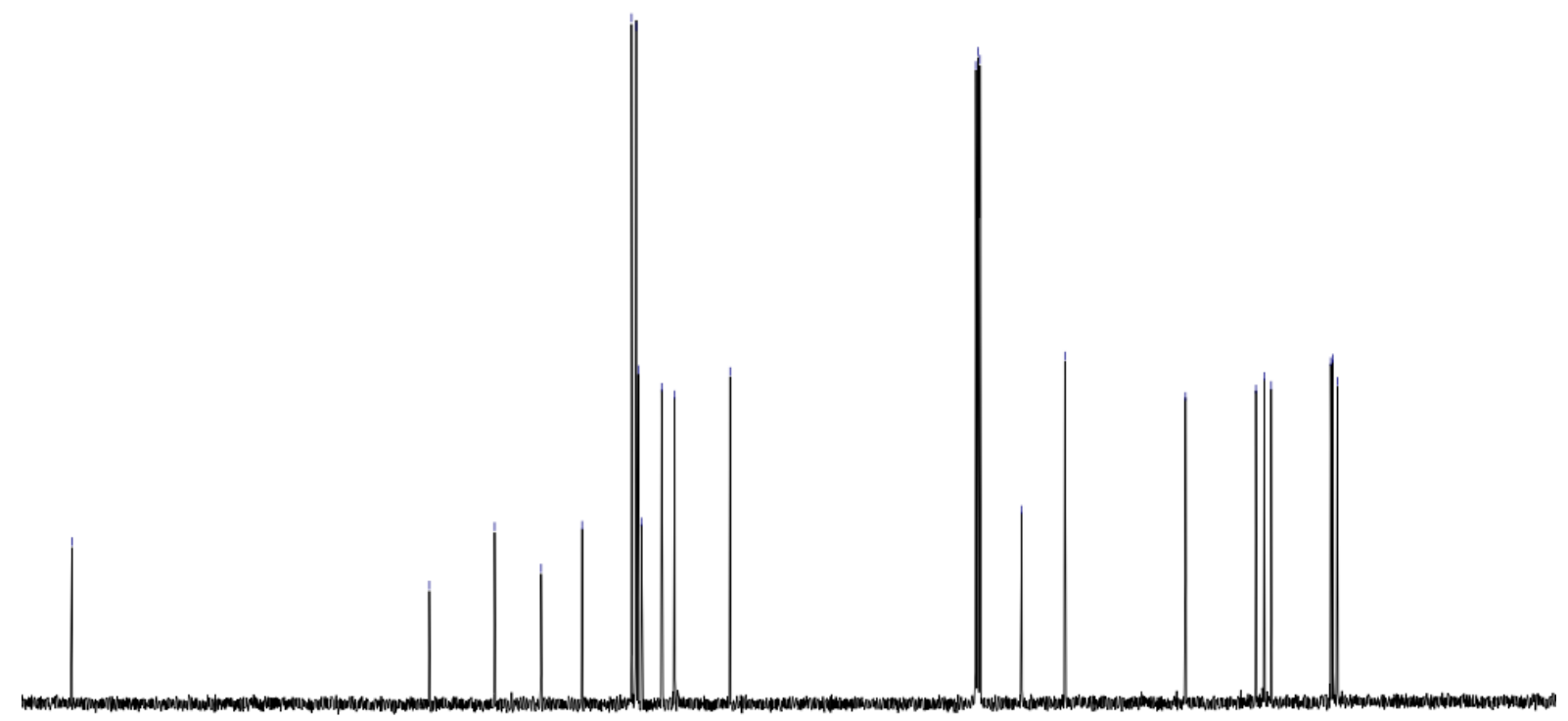

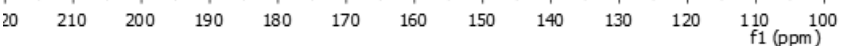


$N$-Benzyl- $N$-cyclopropyl-7-methyl-1H-indole-1-carboxamide $3 \mathrm{k}$
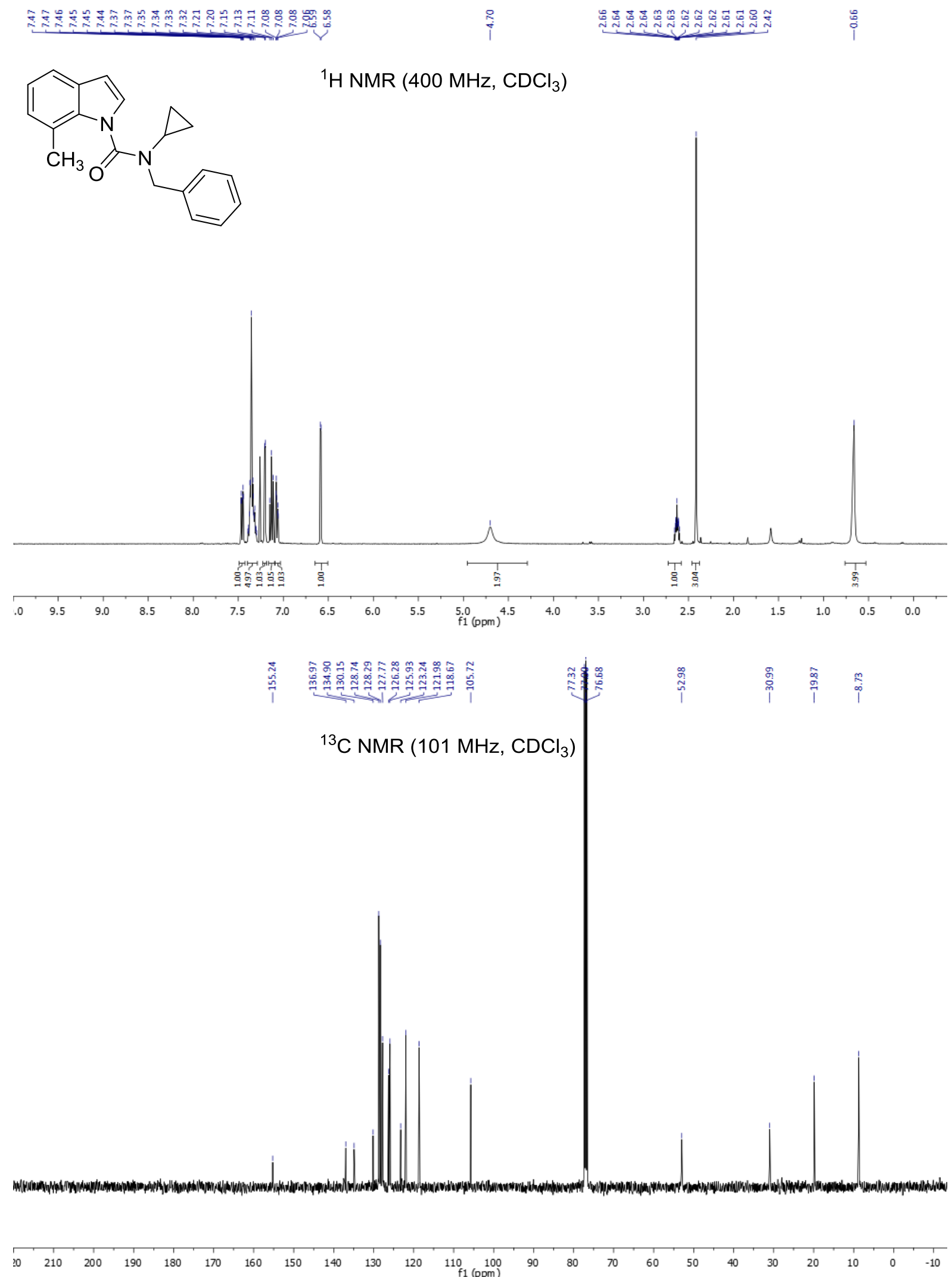
$\left(3 \mathrm{a} S^{*}, 11 \mathrm{a} S^{*}\right)$-4-Benzyl-7-methyl-2,3,3a,4-tetrahydro-11H-cyclopenta[4,5]imidazo[1,5a] indole-1,5-dione $4 \mathrm{k}$

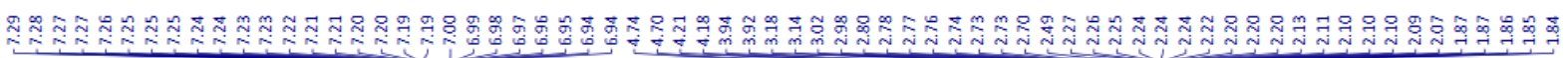<smiles>Cc1cccc2c1N1C(=O)N(Cc3ccccc3)[C@@]3(CCCC[C@@H]13)C2</smiles>

${ }^{1} \mathrm{H} \mathrm{NMR}\left(400 \mathrm{MHz}, \mathrm{CDCl}_{3}\right)$

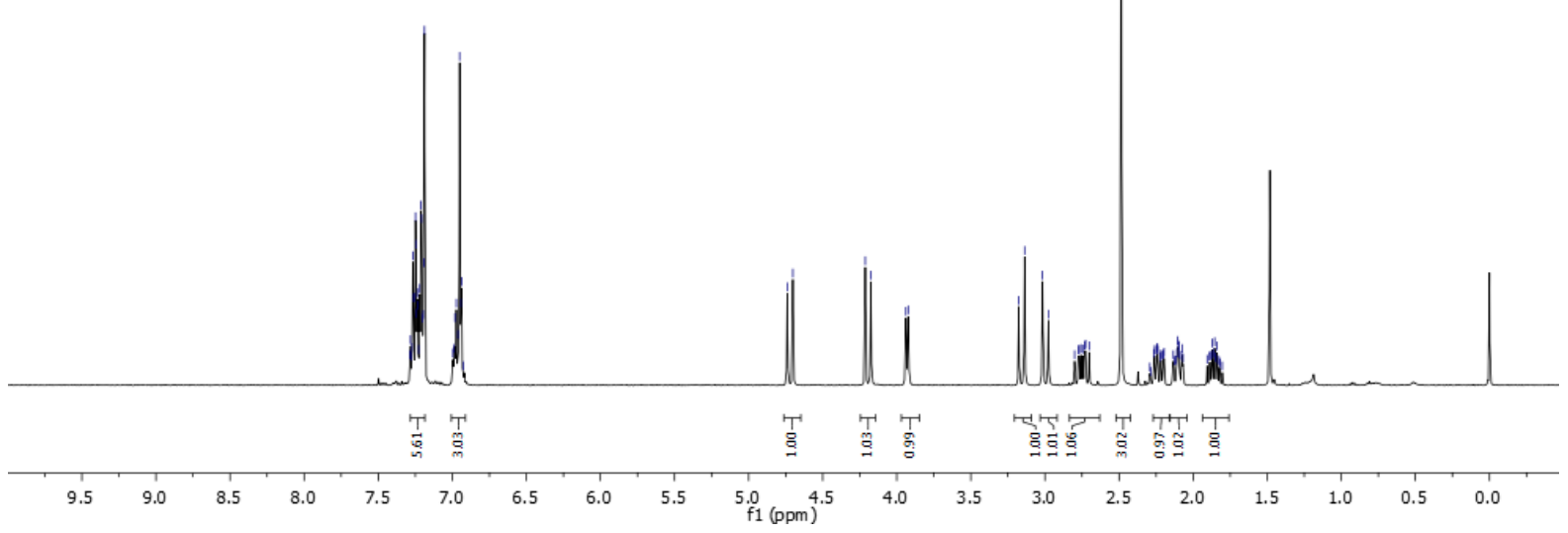

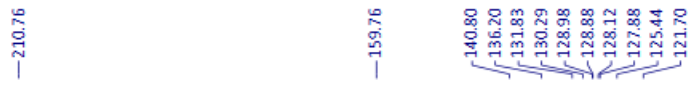

${ }^{13} \mathrm{C} \mathrm{NMR}\left(101 \mathrm{MHz}, \mathrm{CDCl}_{3}\right)$

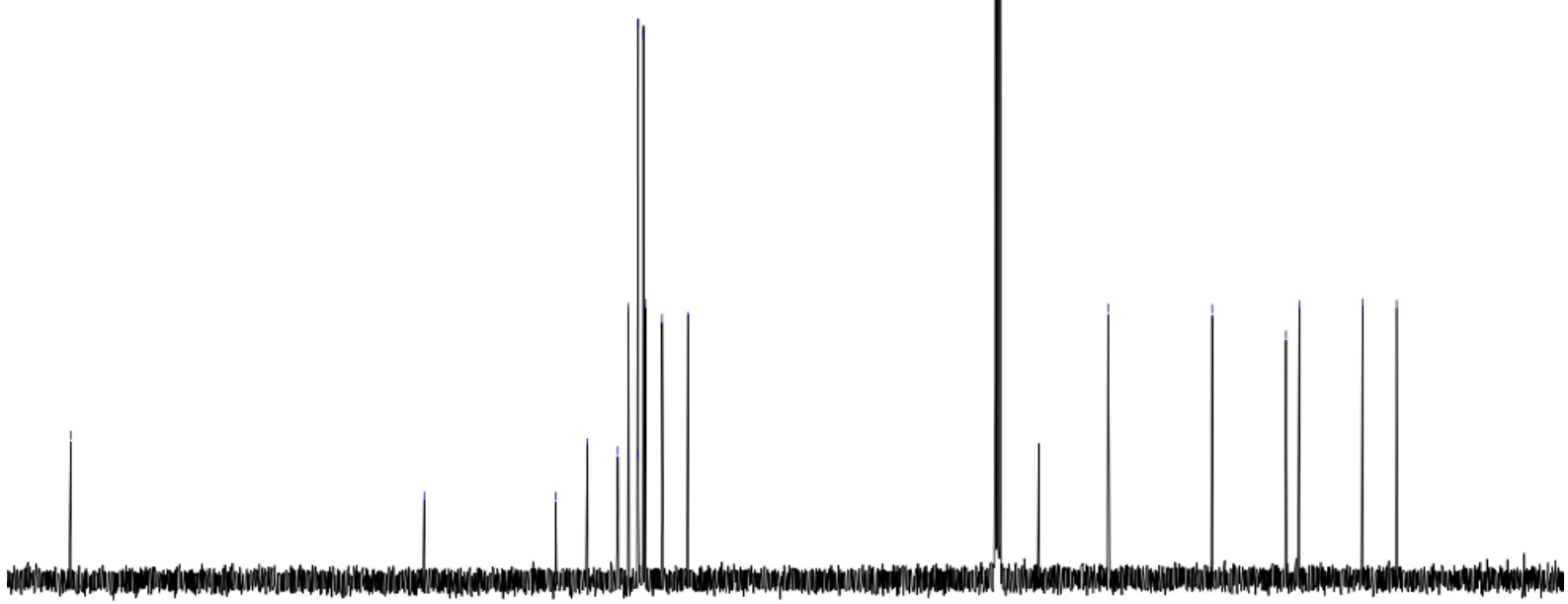

$\begin{array}{llllllllllllllllllllllll}10 & 210 & 200 & 190 & 180 & 170 & 160 & 150 & 140 & 130 & 120 & 110 & 100 & 90 & 80 & 70 & 60 & 50 & 40 & 30 & 20 & 10 & 0\end{array}$ 
$N$-Benzyl- $N$-((1R*,2R*)-2-methylcyclopropyl)-1H-indole-1-carboxamide 31

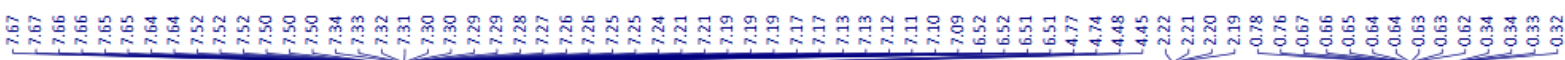

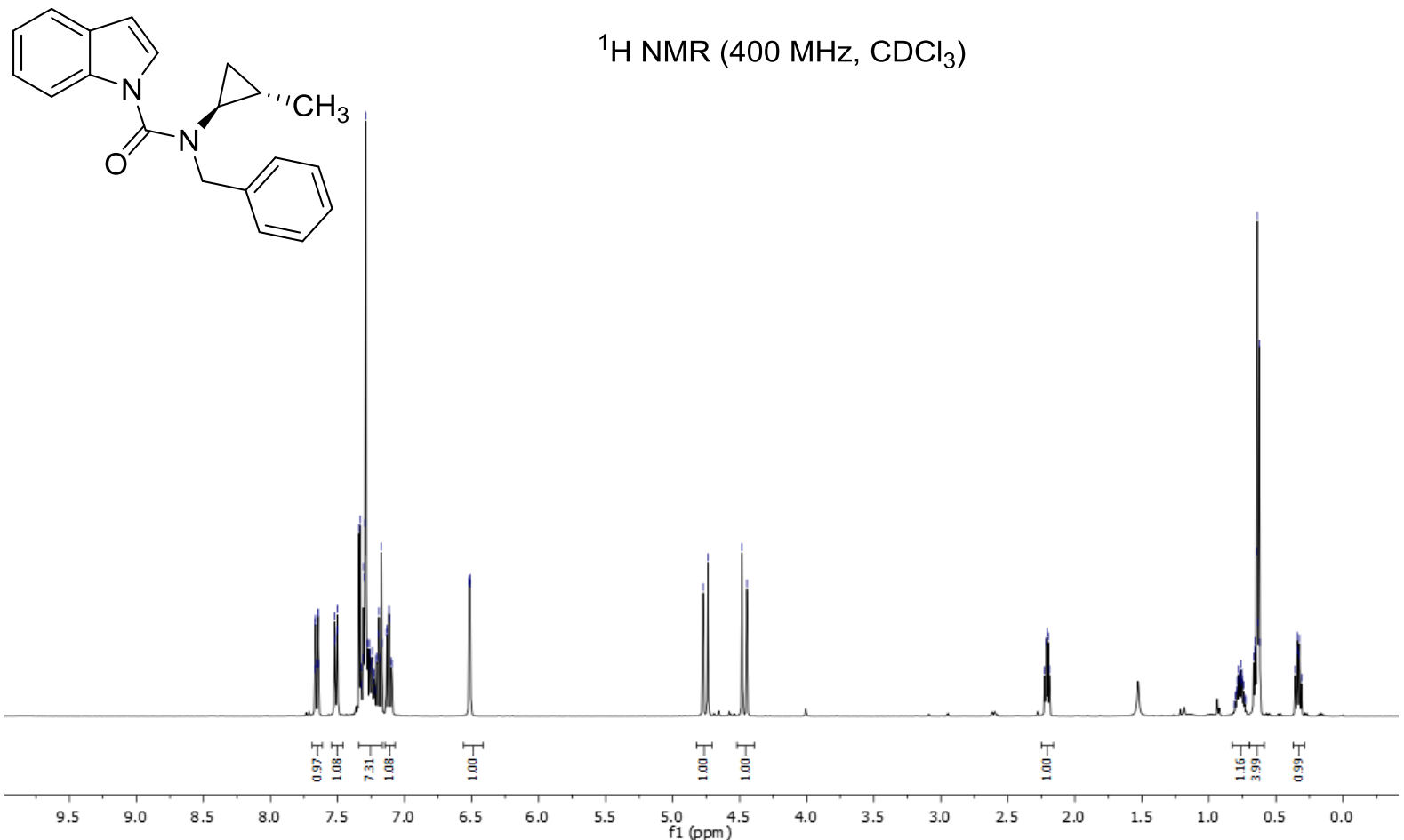

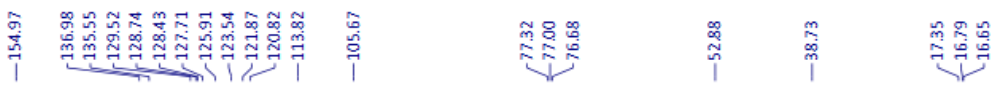

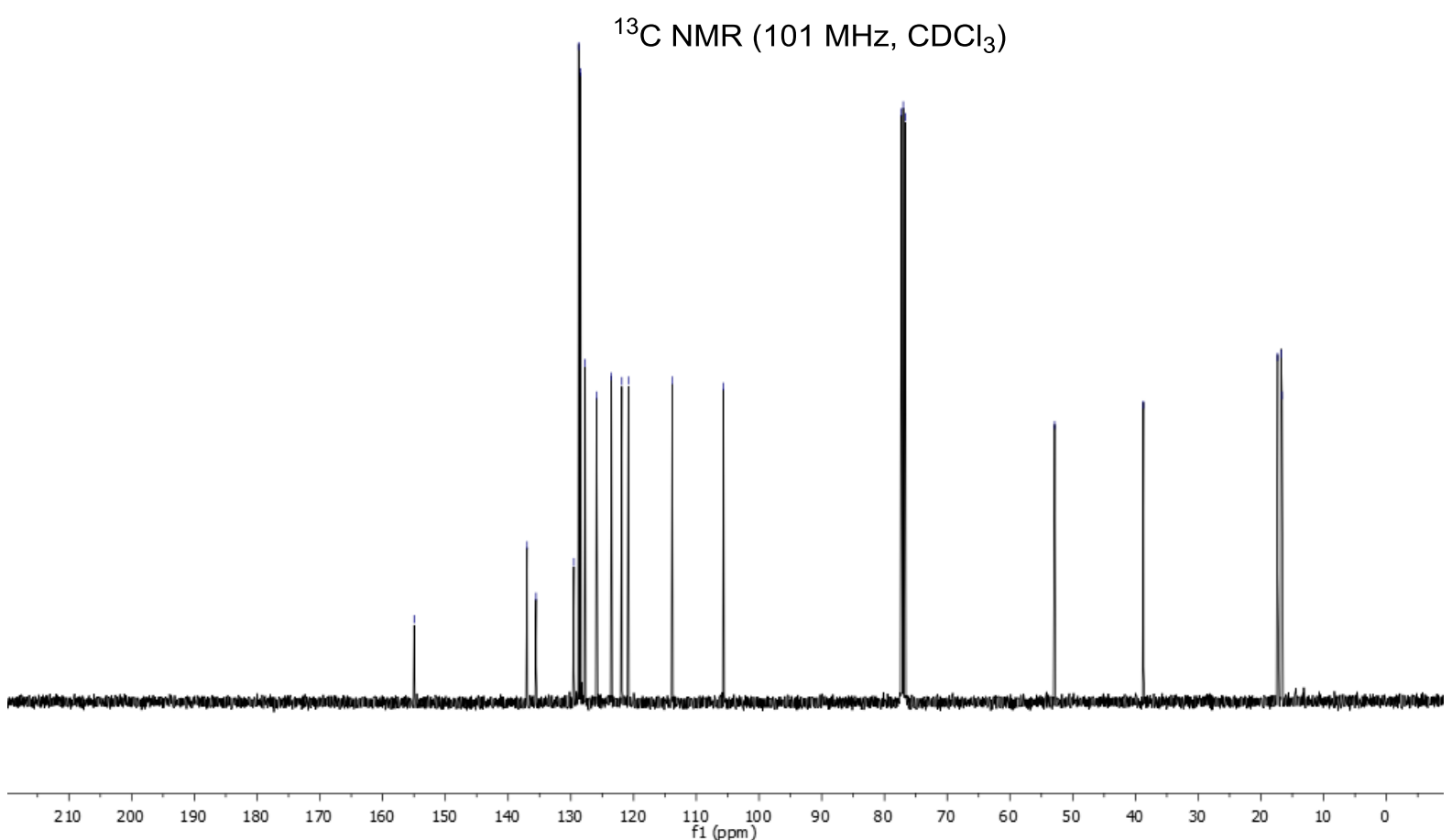


$\left(3 R^{*}, 3 \mathrm{a} S^{*}, 11 \mathrm{a} S^{*}\right)-4-B e n z y l-3-m e t h y l-2,3,3 \mathrm{a}, 4-t e t r a h y d r o-11 H$ -

cyclopenta[4,5]imidazo[1,5- $a]$ indole-1,5-dione 41

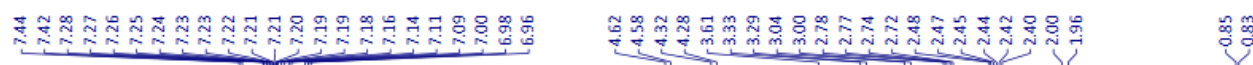

${ }^{1} \mathrm{H}$ NMR $\left(400 \mathrm{MHz}, \mathrm{CDCl}_{3}\right)$<smiles>C[C@@H]1CC(=O)[C@@]23Cc4ccccc4N(C(=O)N2Cc2ccccc2)[C@@H]13</smiles>

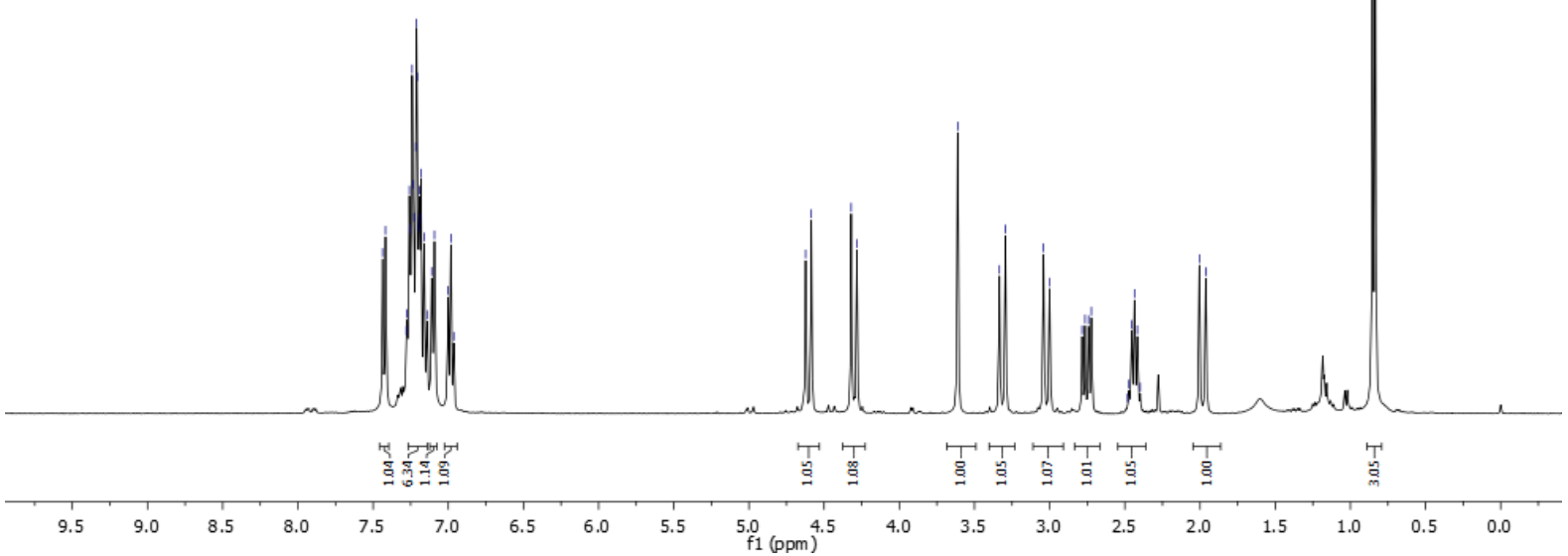

$\underset{3}{3}$

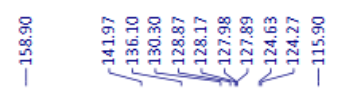

${ }^{13} \mathrm{C}$ NMR $\left(101 \mathrm{MHz}, \mathrm{CDCl}_{3}\right)$

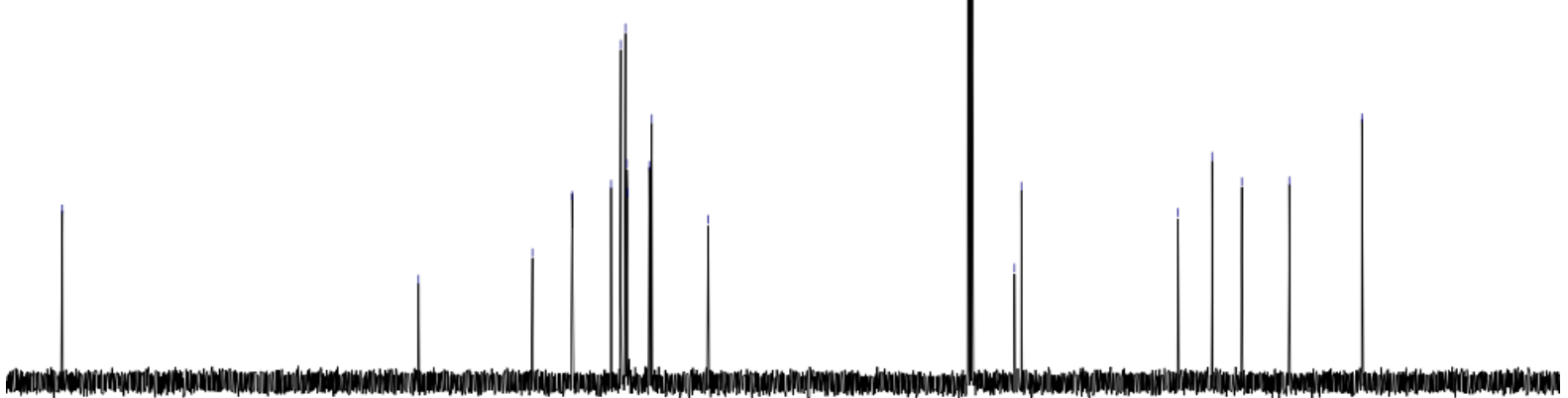

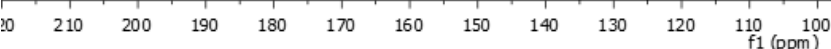


$N$-Benzyl- $N$-((1R*,2R*)-2-benzylcyclopropyl)-1H-indole-1-carboxamide 3m

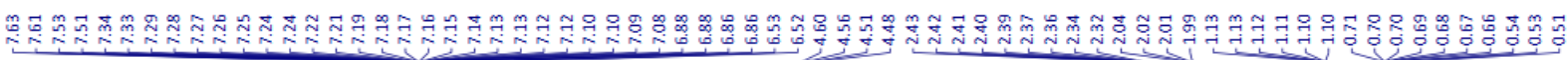
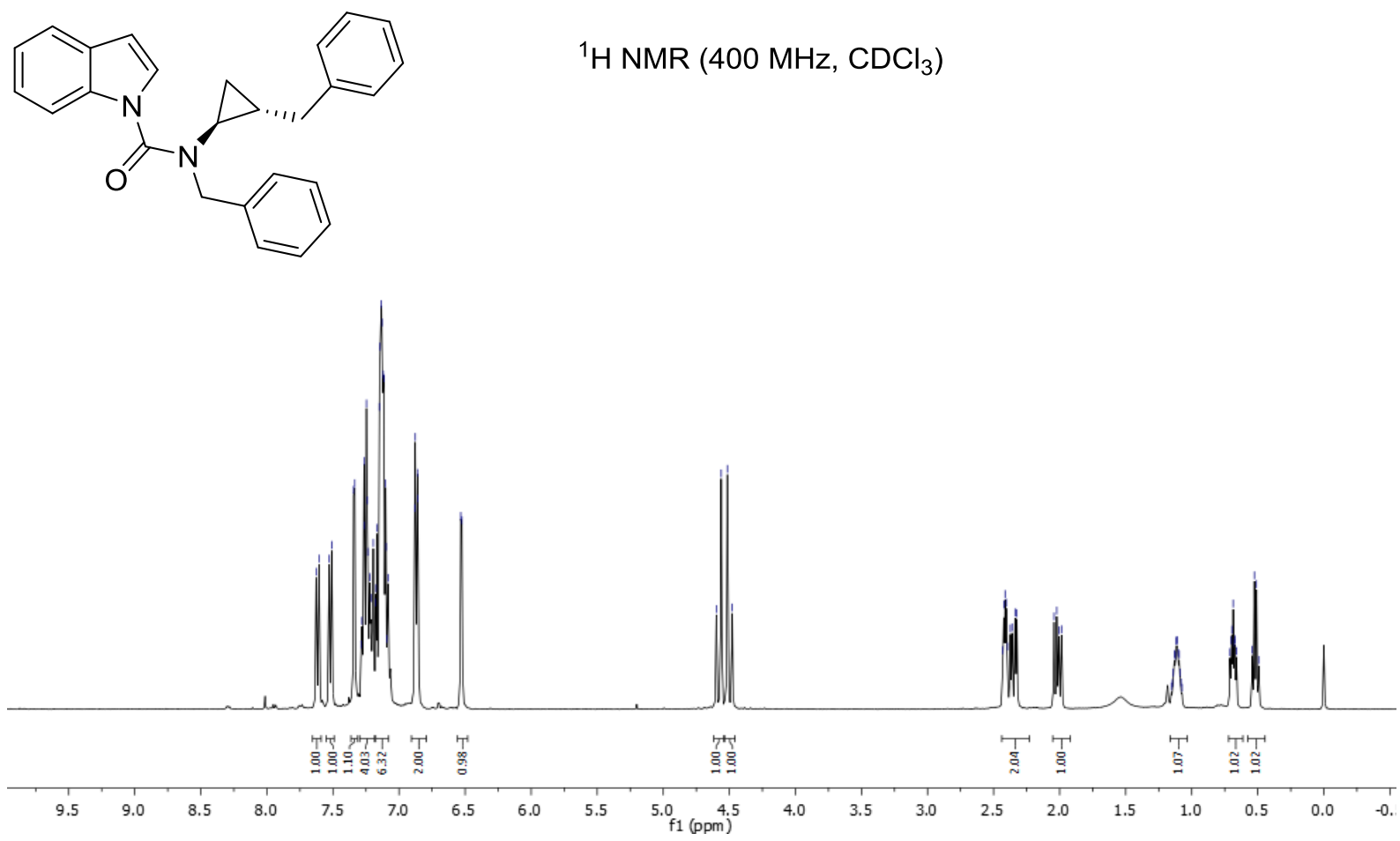

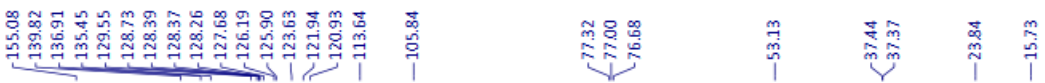

${ }^{13} \mathrm{C}$ NMR $\left(101 \mathrm{MHz}, \mathrm{CDCl}_{3}\right)$

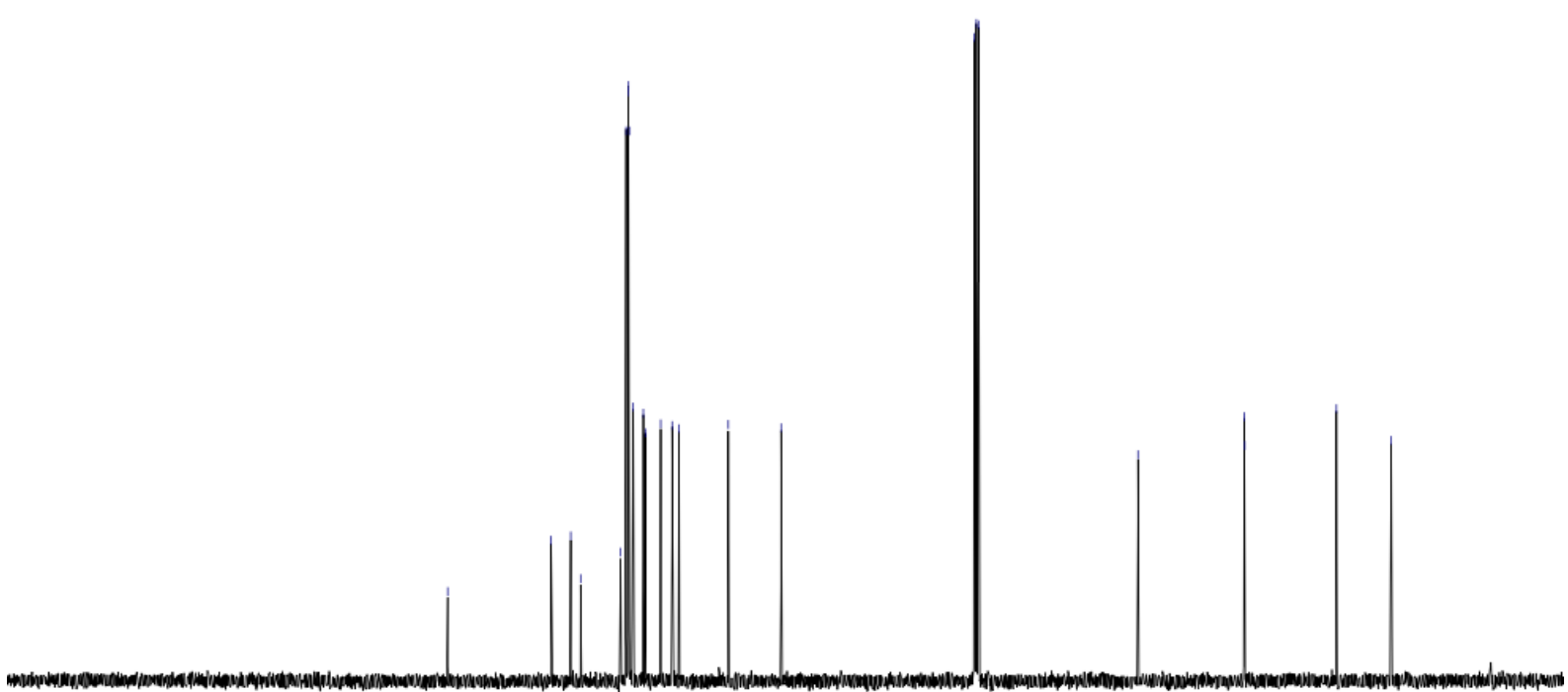

$\begin{array}{llllllllllll}20 & 210 & 200 & 190 & 180 & 170 & 160 & 150 & 140 & 130 & 120 & \begin{array}{c}110 \\ \mathrm{f} 1(\mathrm{ppm})\end{array}\end{array}$ 
$\left(3 R^{*}, 3 \mathrm{a} S^{*}, 11 \mathrm{a} S^{*}\right)-3,4-D i b e n z y l-2,3,3 \mathrm{a}, 4$-tetrahydro-11H-cyclopenta[4,5]imidazo[1,5a] indole-1,5-dione $4 \mathrm{~m}$

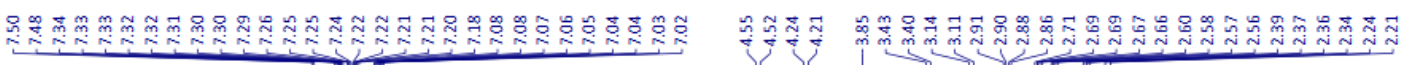
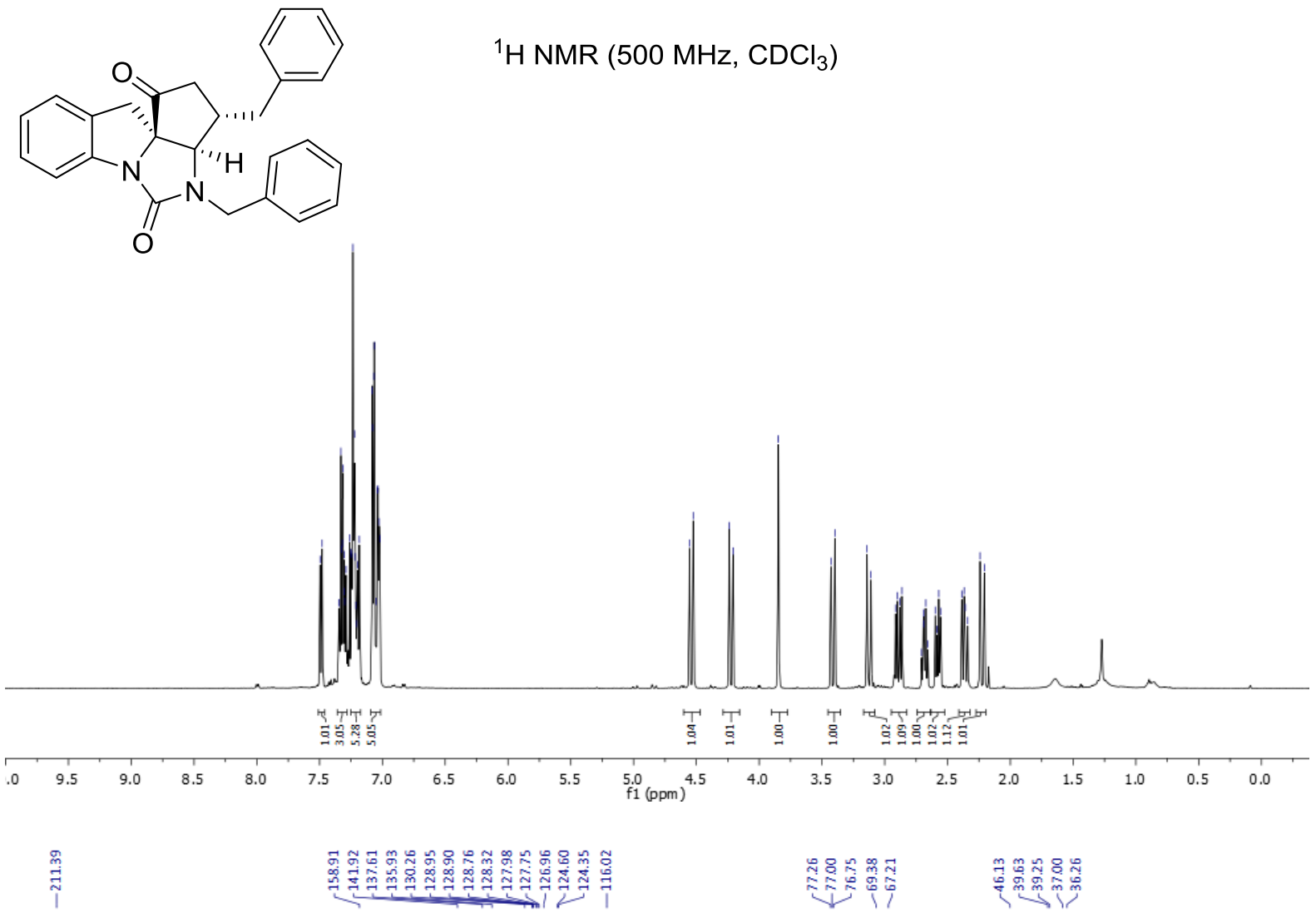

${ }^{13} \mathrm{C} \mathrm{NMR}\left(126 \mathrm{MHz}, \mathrm{CDCl}_{3}\right)$

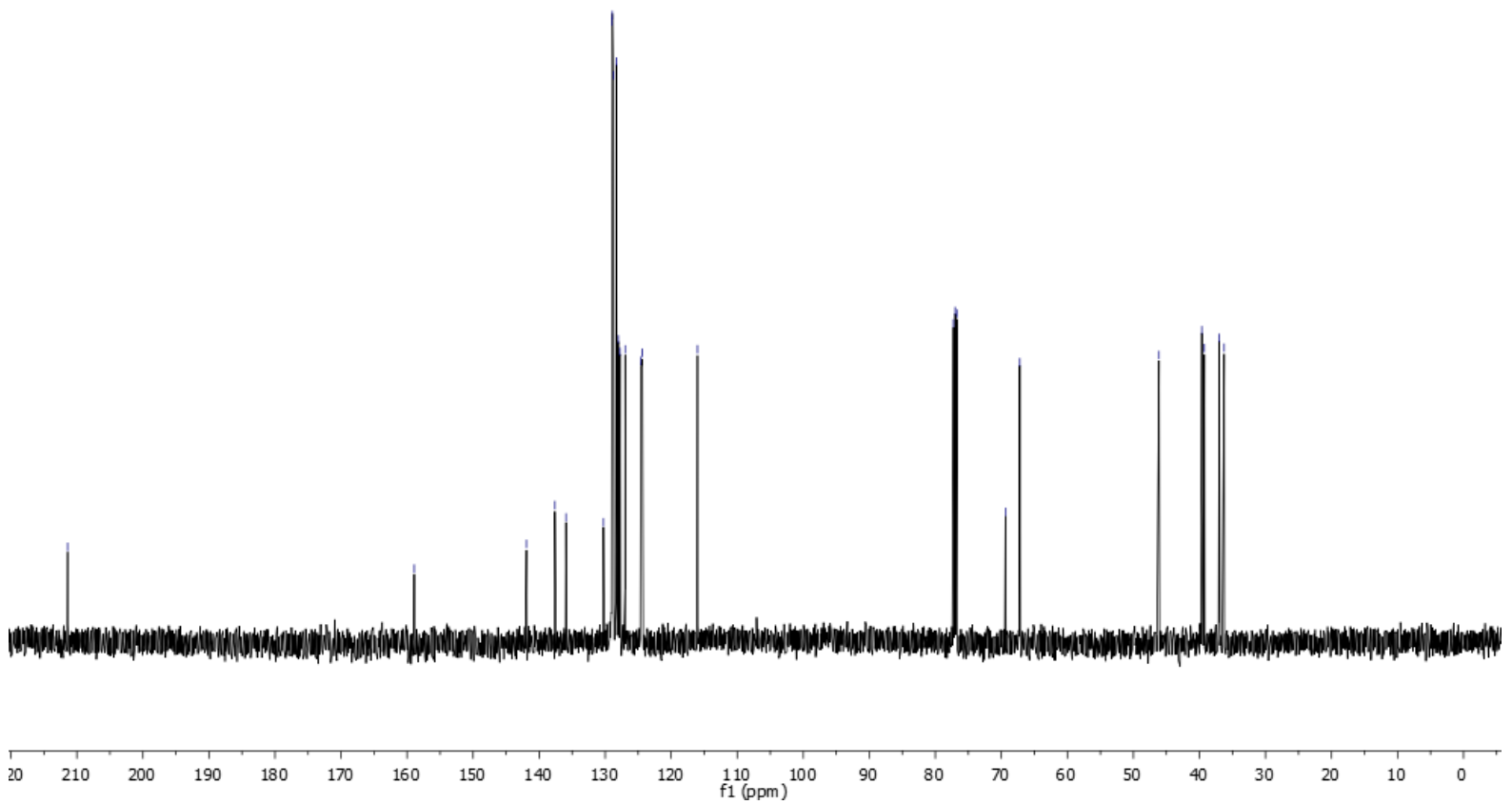


$N$-Benzyl- $N$-((1R*,2R*)-2-butylcyclopropyl)-1H-indole-1-carboxamide 3n

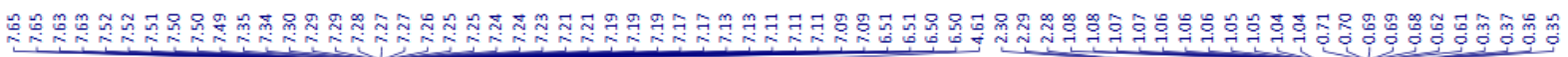
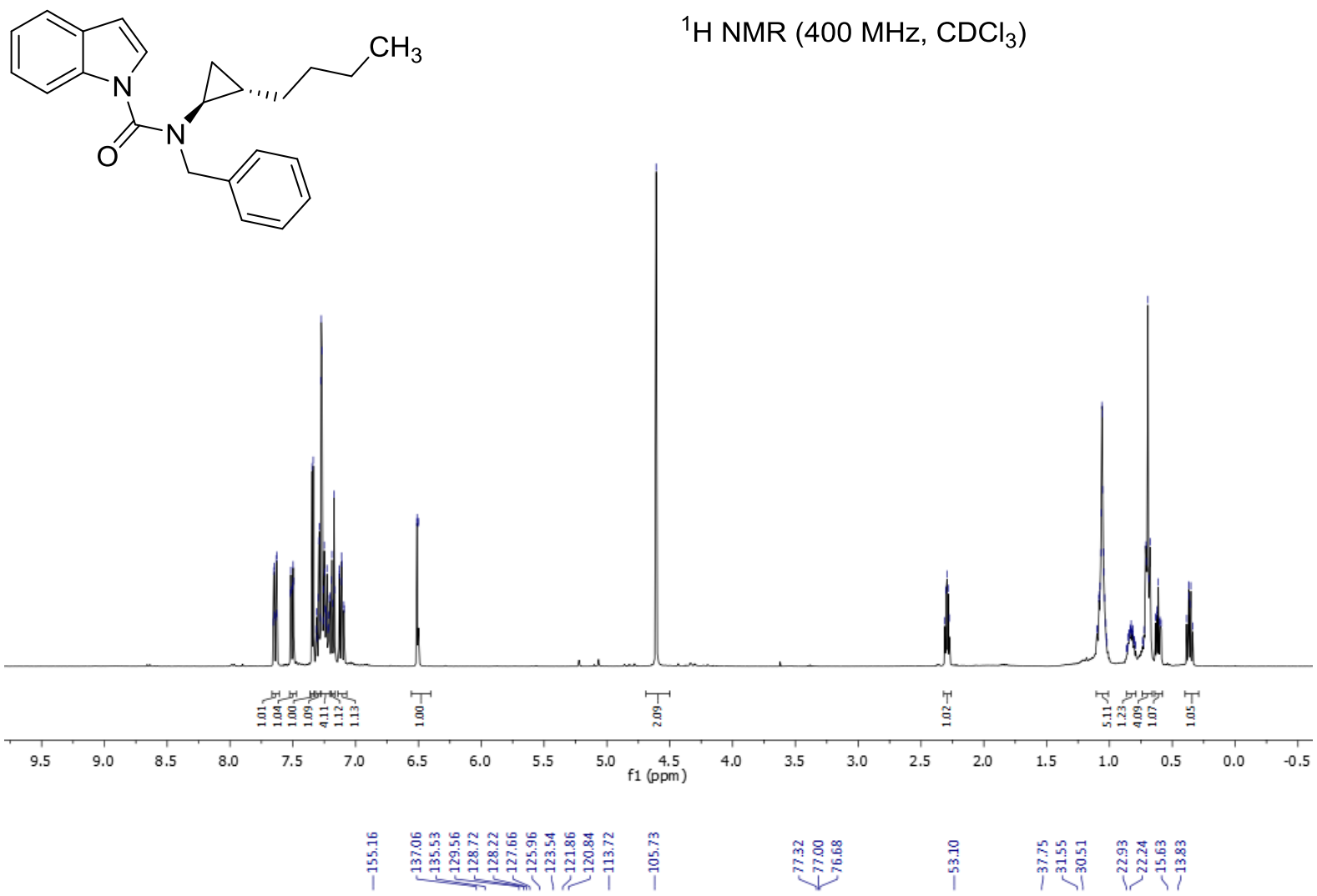

${ }^{13} \mathrm{C} \mathrm{NMR}\left(101 \mathrm{MHz}, \mathrm{CDCl}_{3}\right)$

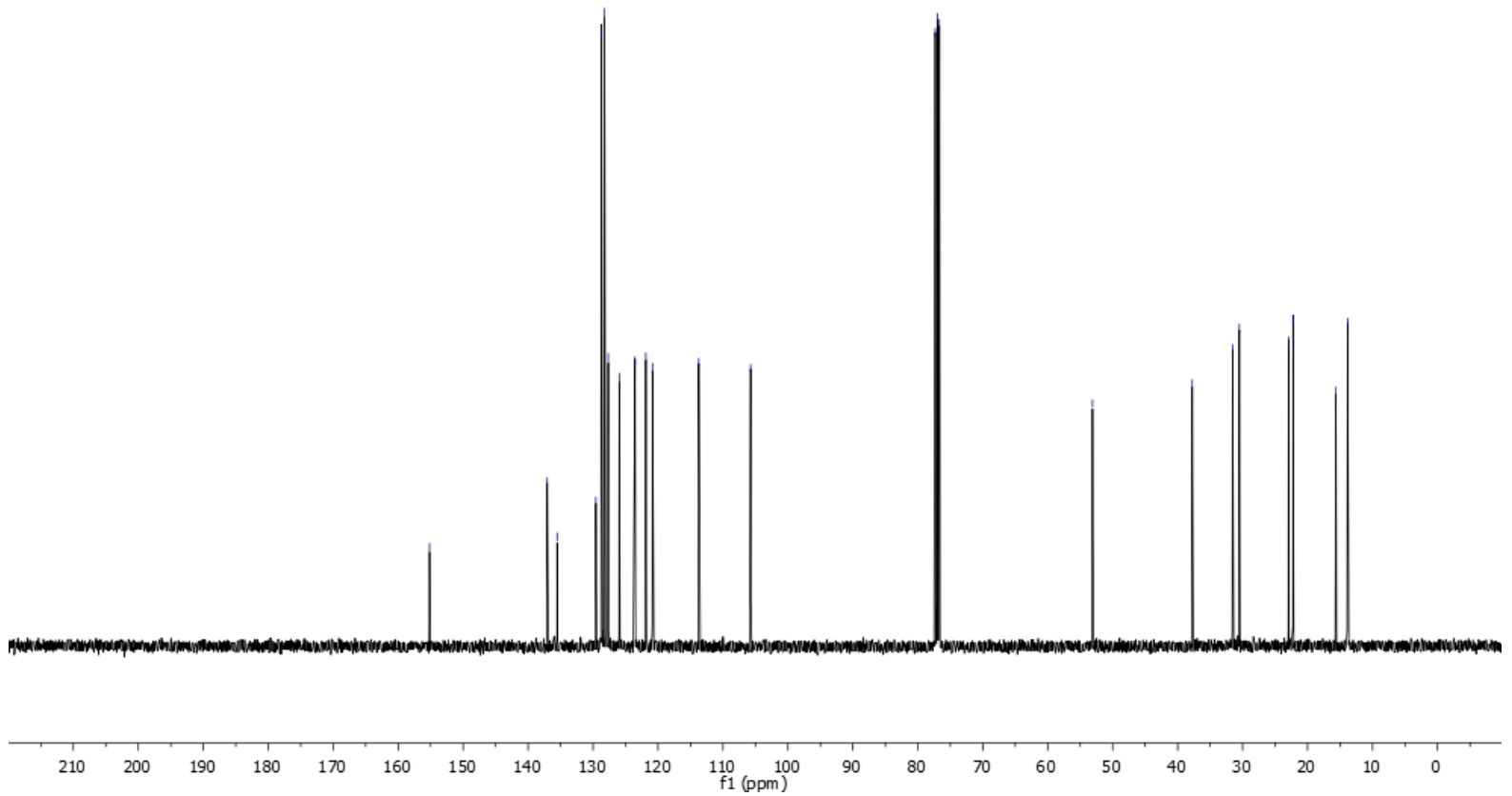


$\left(3 S^{*}, 3 a R^{*}, 11 a R^{*}\right)-4-B e n z y l-3-b u t y l-2,3,3 a, 4-t e t r a h y d r o-11 H-$

cyclopenta[4,5]imidazo[1,5-a]indole-1,5-dione $4 n$

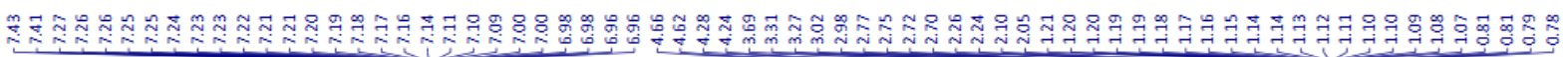<smiles>CCCC[C@@H]1CC(=O)[C@]23Cc4ccccc4N(C(=O)N2Cc2ccccc2)[C@@H]13</smiles>

${ }^{1} \mathrm{H}$ NMR $\left(400 \mathrm{MHz}, \mathrm{CDCl}_{3}\right)$
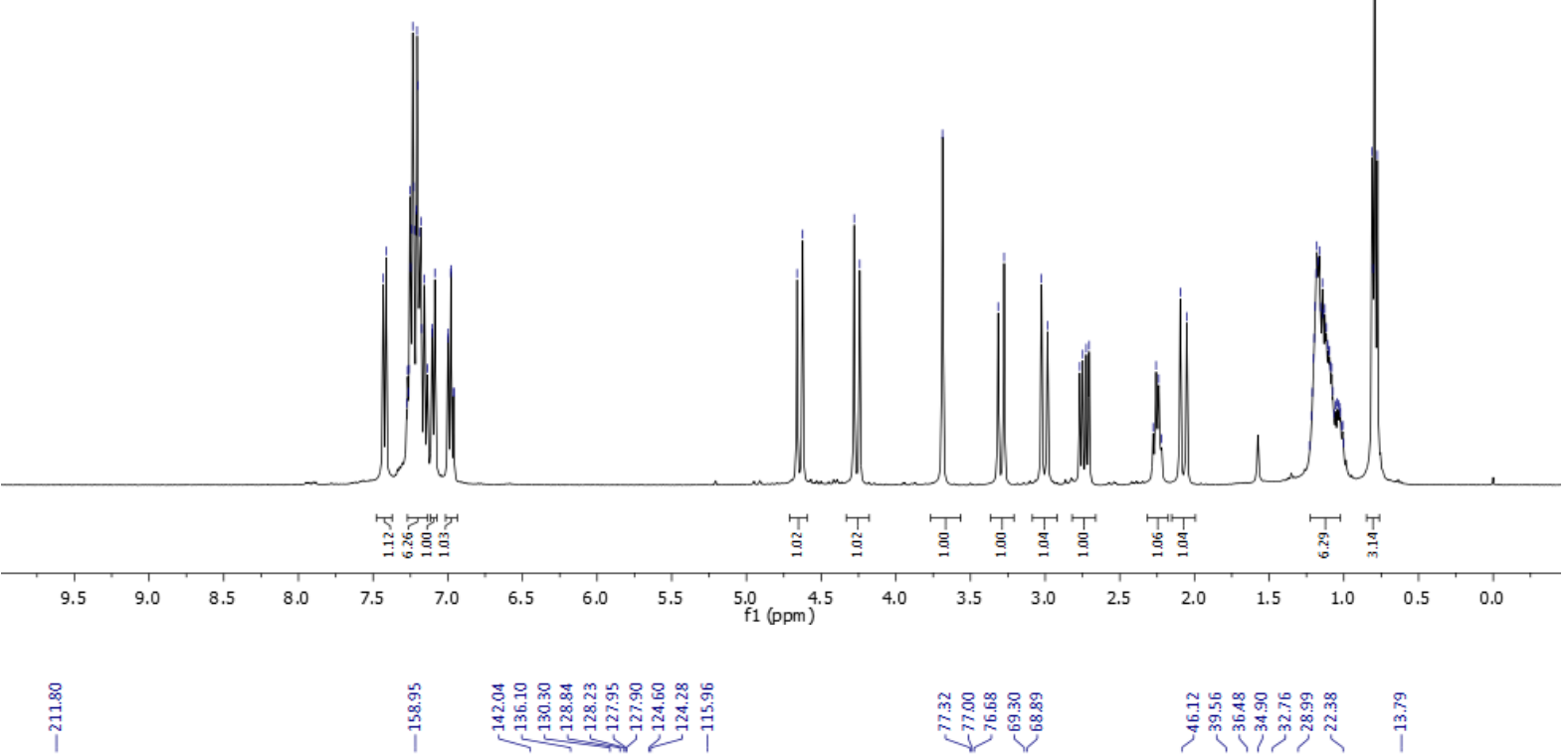

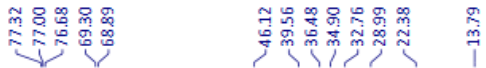

${ }^{13} \mathrm{C}$ NMR (101 MHz, $\left.\mathrm{CDCl}_{3}\right)$

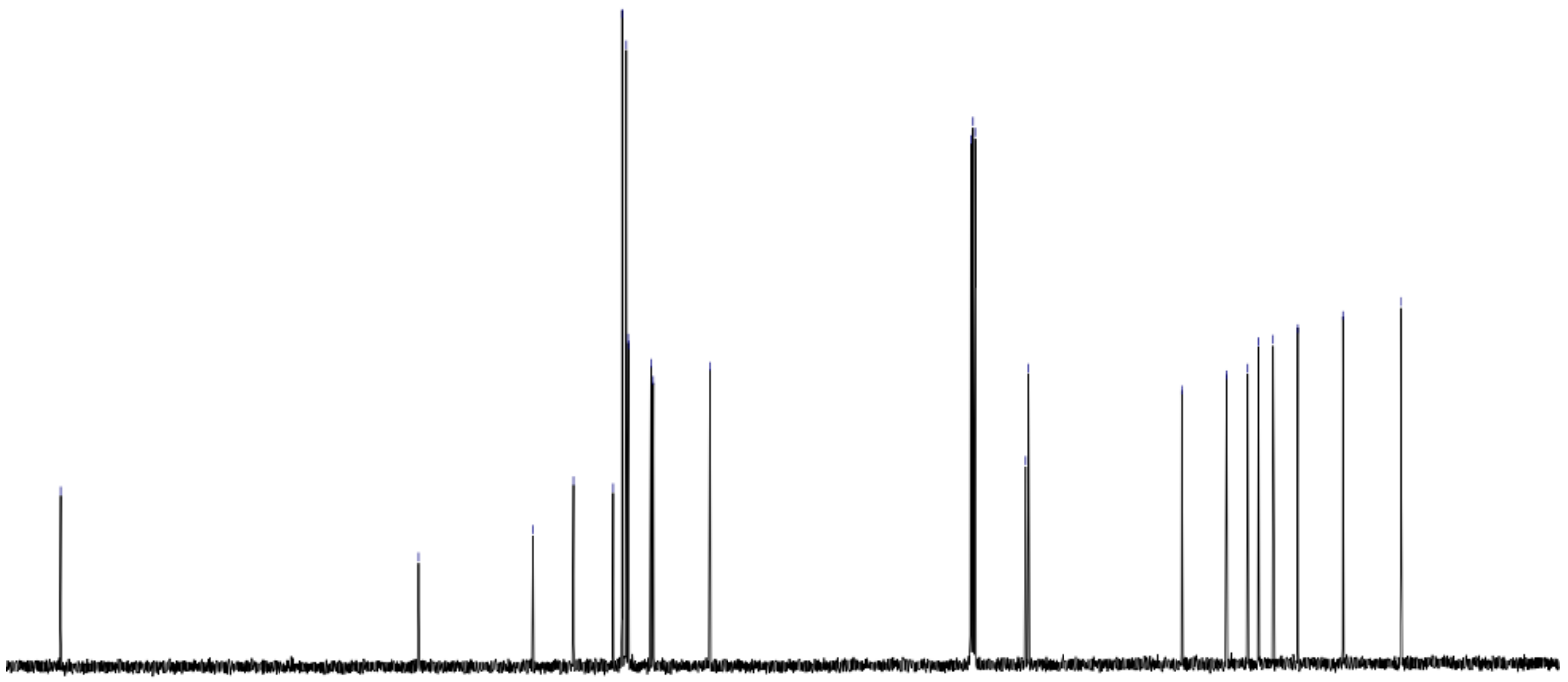

$\begin{array}{lllllllllll}210 & 200 & 190 & 180 & 170 & 160 & 150 & 140 & 130 & 120 & \begin{array}{c}110 \\ \mathrm{f}(\mathrm{ppm})\end{array}\end{array}$ 
$N$-Benzyl- $N$-((1R*,5 $\left.S^{*}, 6 r^{*}\right)$-bicyclo[3.1.0]hexan-6-yl)-1H-indole-1-carboxamide 30

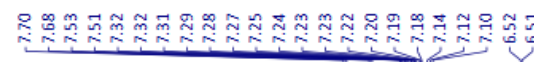

$(>$<smiles>c1ccc(CNC2[C@H]3CCC[C@H]23)cc1</smiles>

${ }^{1} \mathrm{H} \mathrm{NMR}\left(400 \mathrm{MHz}, \mathrm{CDCl}_{3}\right)$

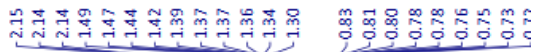

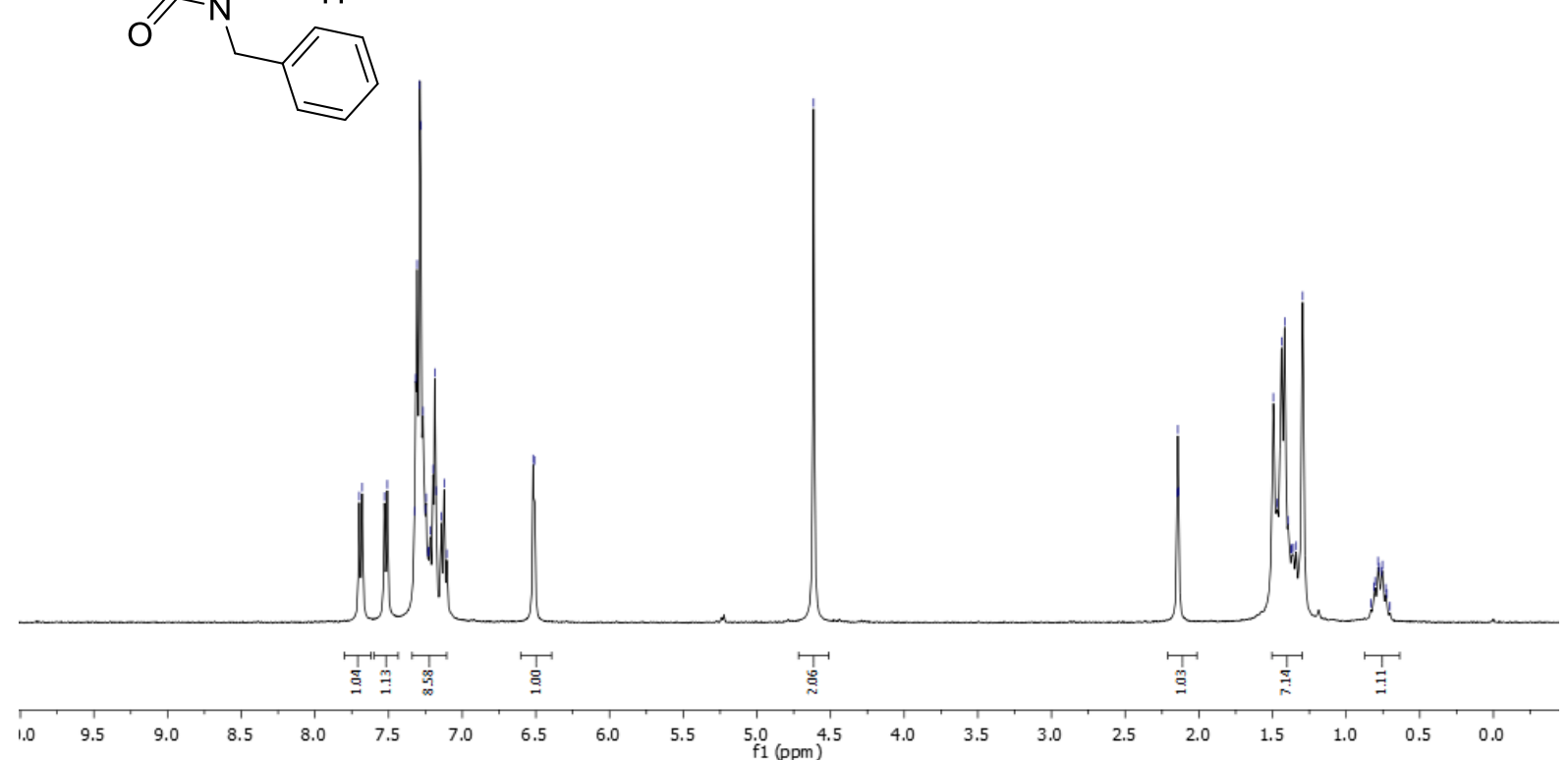

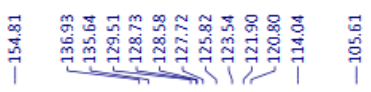

${ }^{13} \mathrm{C}$ NMR $\left(101 \mathrm{MHz}, \mathrm{CDCl}_{3}\right)$
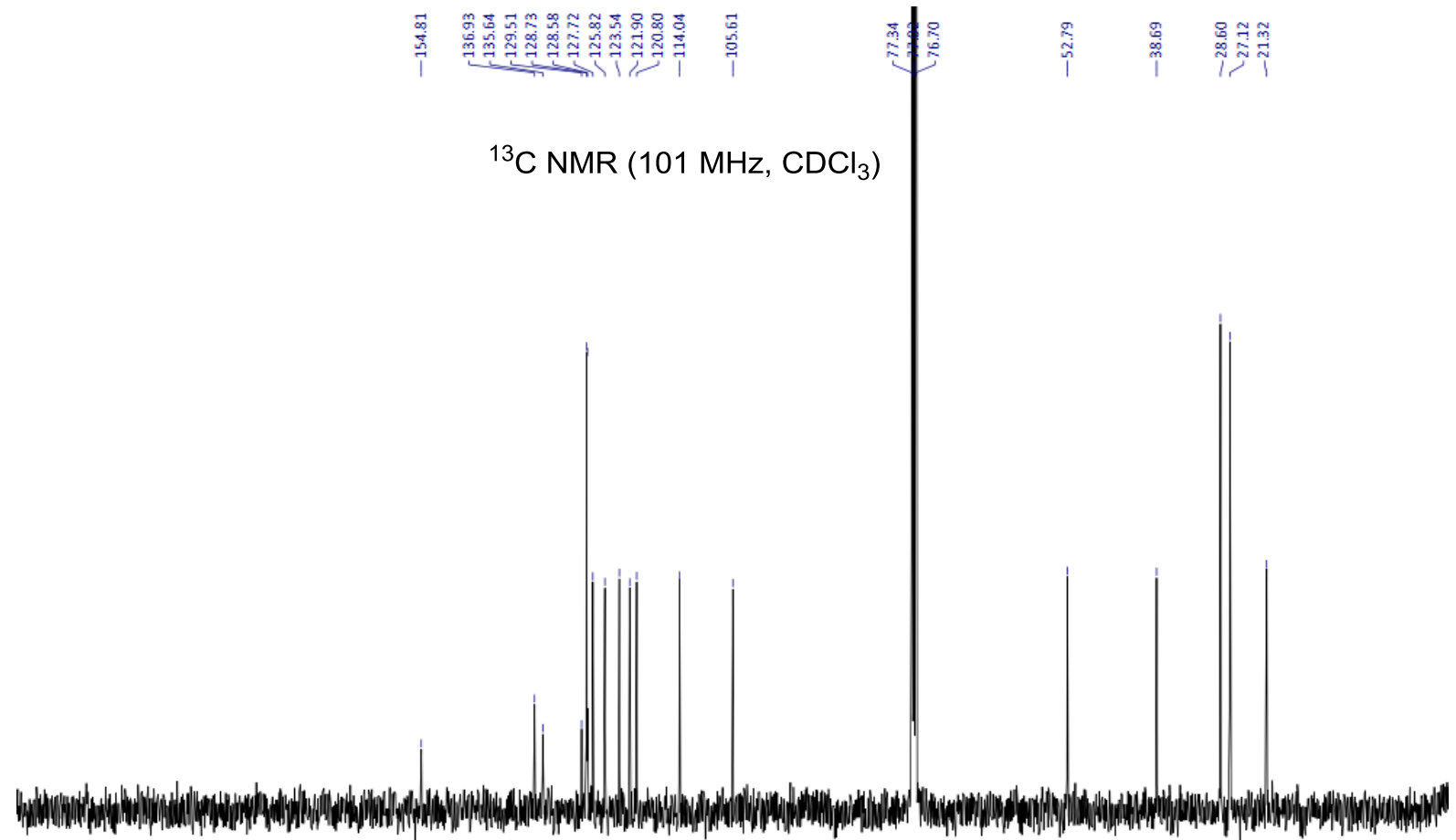

$\begin{array}{lllllllllll}210 & 200 & 190 & 180 & 170 & 160 & 150 & 140 & 130 & 120 & \underset{\mathrm{f} 1}{110}(\mathrm{ppm})\end{array}$ 
$\left(3 \mathrm{a} R^{*}, 3 \mathrm{~b} S^{*}, 11 \mathrm{a} S^{*}, 12 \mathrm{a} S^{*}\right)-4-B e n z y l-2,3,3 \mathrm{a}, 3 \mathrm{~b}, 4,12 \mathrm{a}-h e x a h y d r o-1 H, 11 H-$

pentaleno[1',2':4,5]imidazo[1,5-a]indole-5,12-dione 40

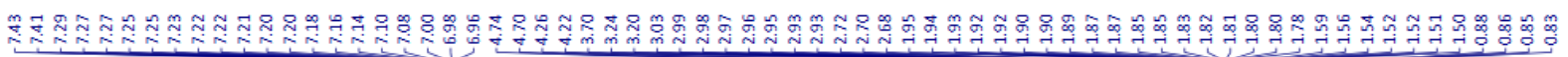

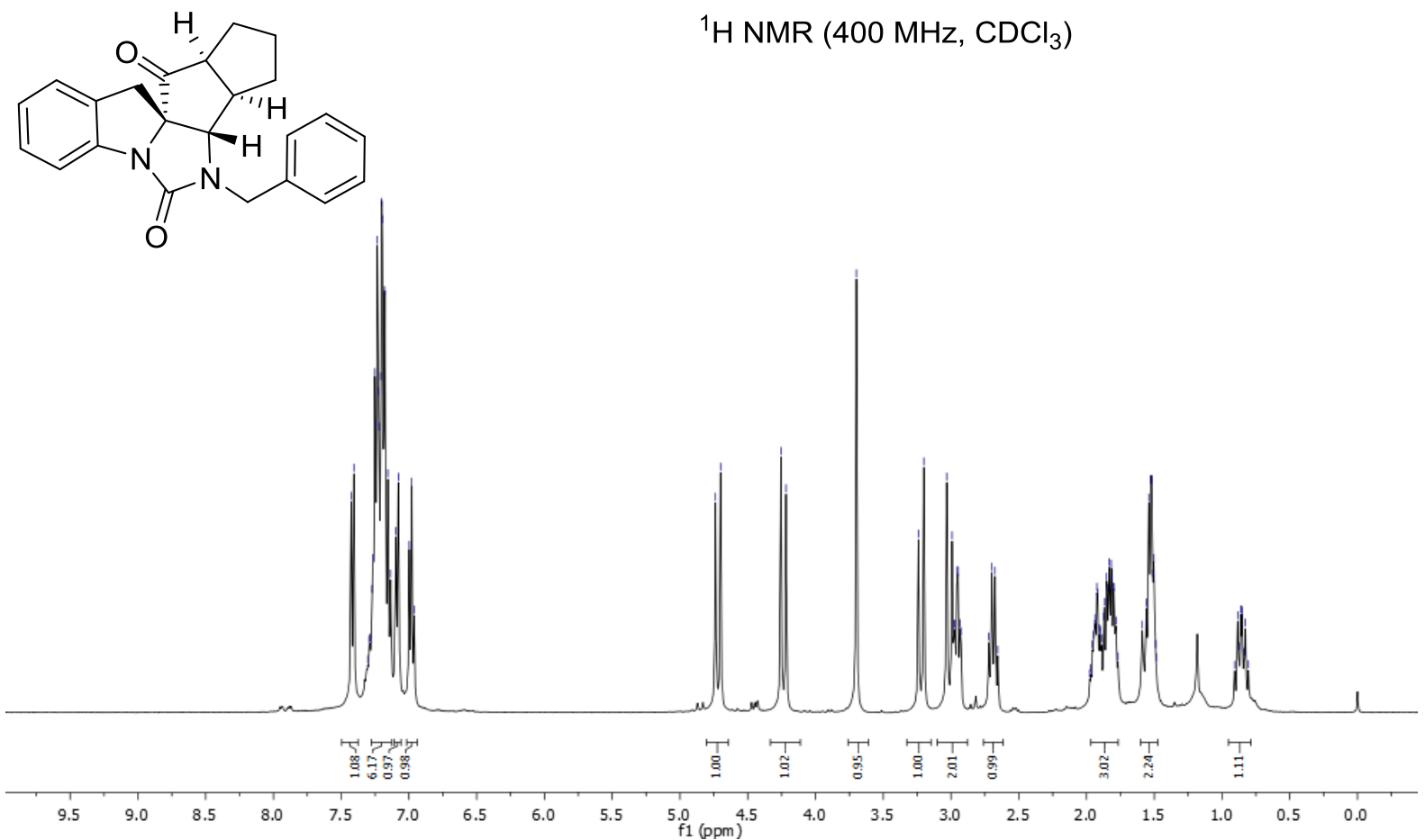

草

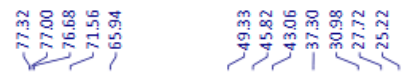

${ }^{13} \mathrm{C}$ NMR $\left(101 \mathrm{MHz}, \mathrm{CDCl}_{3}\right)$

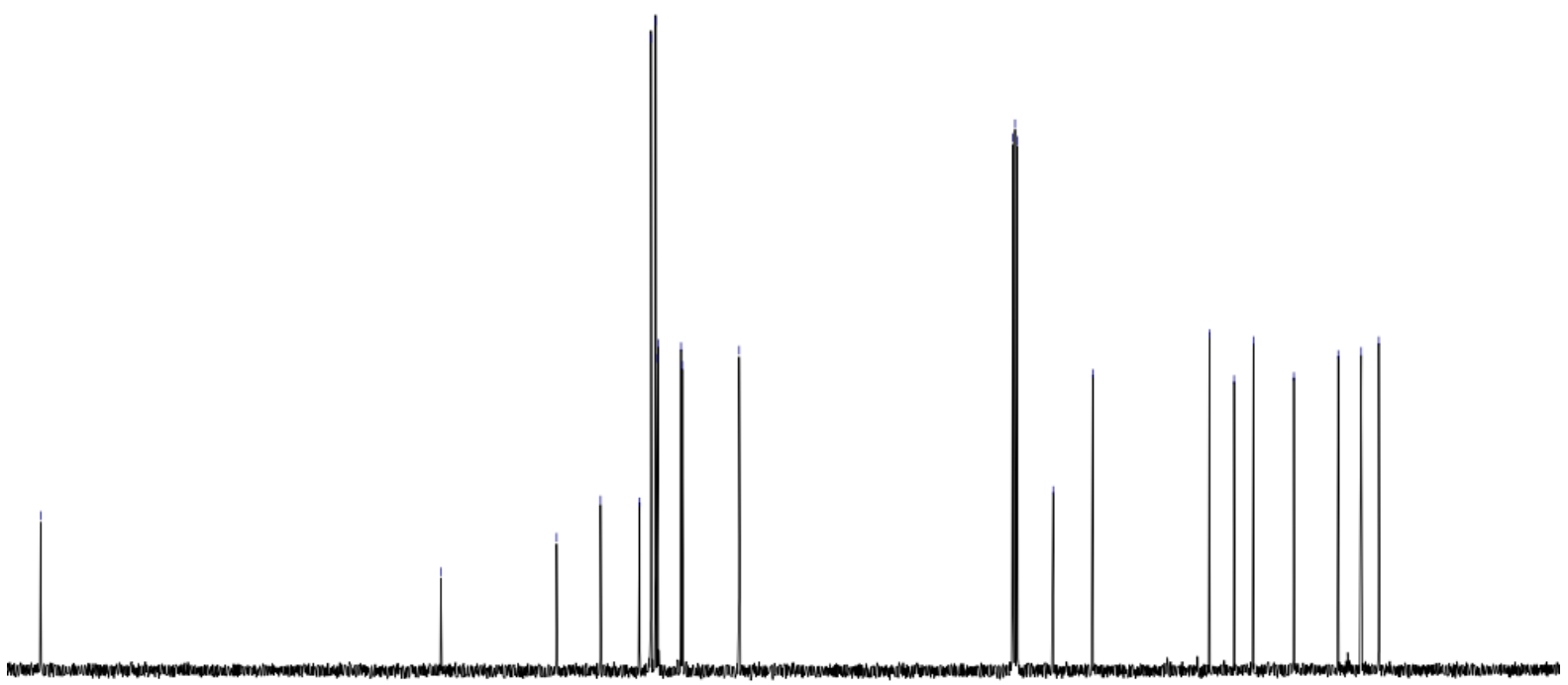

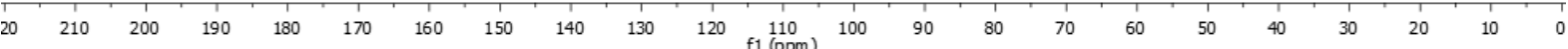


$N$-Benzyl- $N$-((1R*,6S*,7r*)-bicyclo[4.1.0]heptan-7-yl)-1H-indole-1-carboxamide 3p

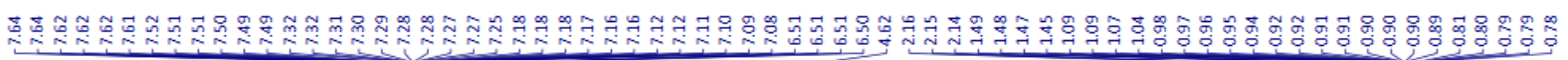<smiles></smiles>

${ }^{1} \mathrm{H}$ NMR $\left(400 \mathrm{MHz}, \mathrm{CDCl}_{3}\right)$

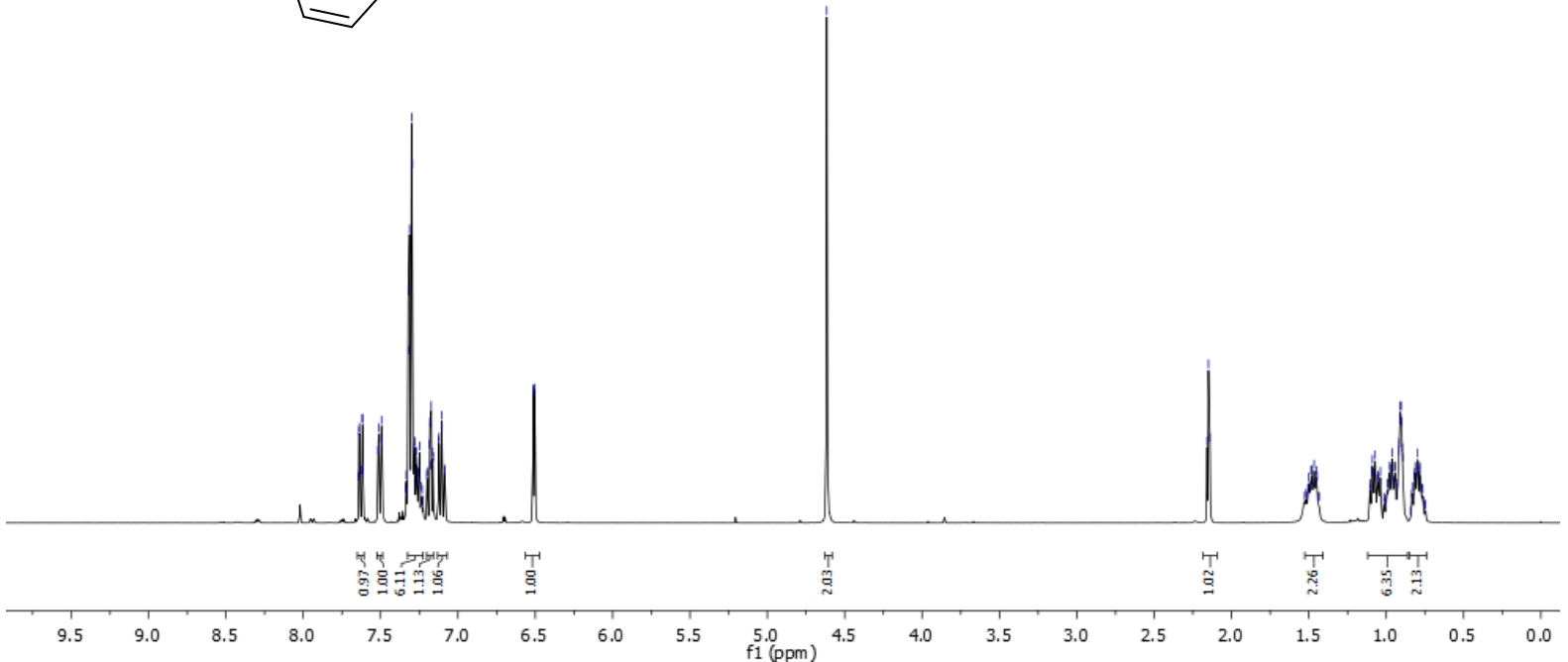

30778 wgw 7-sixfused.11. fid

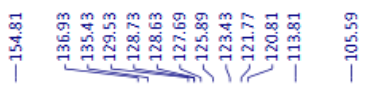

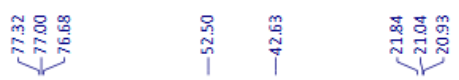

${ }^{13} \mathrm{C} \mathrm{NMR}\left(101 \mathrm{MHz}, \mathrm{CDCl}_{3}\right)$

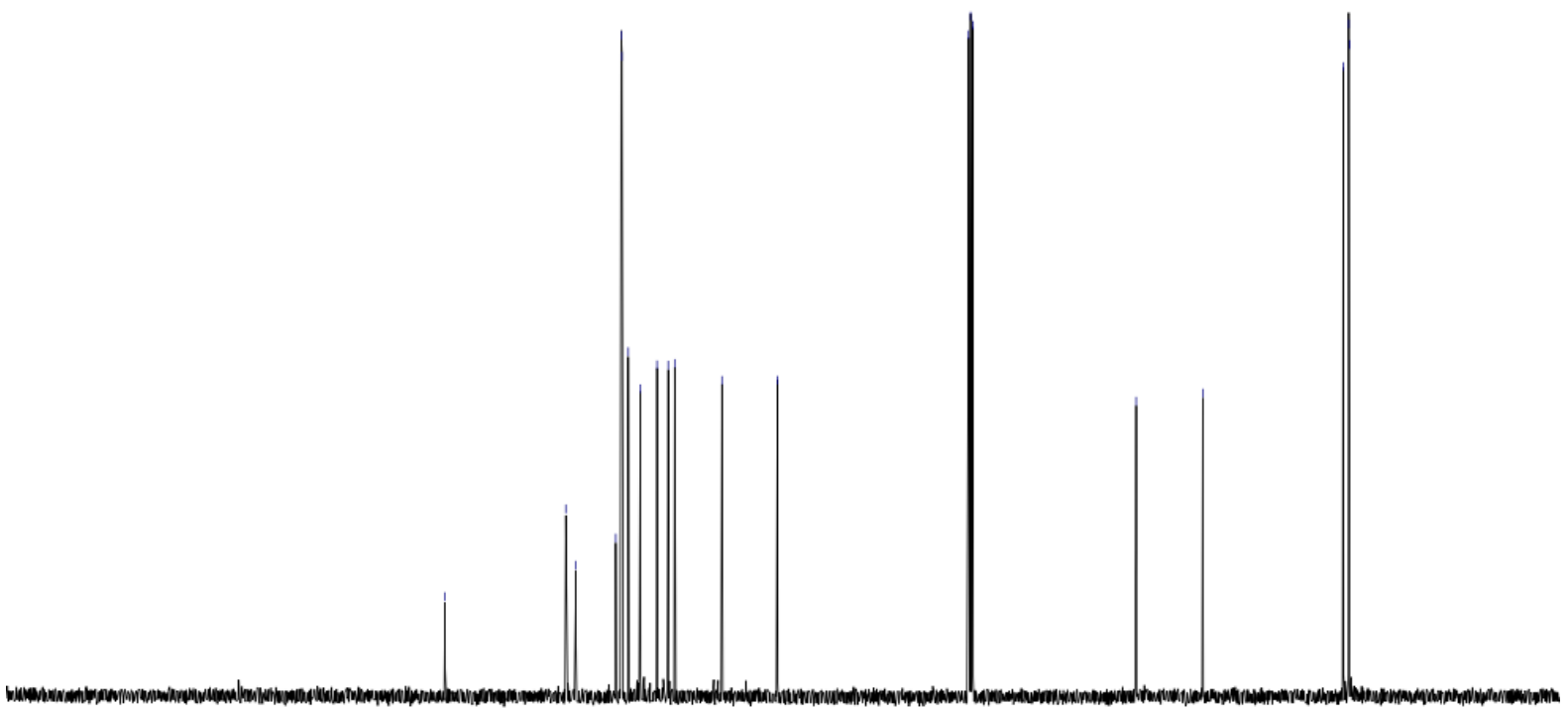

$\begin{array}{lllllllllll}210 & 200 & 190 & 180 & 170 & 160 & 150 & 140 & 130 & 120 & 110 \quad 100 \\ \mathrm{f} 1(\mathrm{ppm})\end{array}$ 
$\left(4 \mathrm{a} R^{*}, 4 \mathrm{~b} S^{*}, 12 \mathrm{a} S^{*}, 13 \mathrm{a} S^{*}\right)-5-B e n z y l-1,2,3,4,4 \mathrm{a}, 4 \mathrm{~b}, 5,13 \mathrm{a}-o c t a h y d r o-12 H-$

indeno $\left[1^{\prime}, 2^{\prime}: 4,5\right]$ imidazo $[1,5-a]$ indole-6,13-dione $4 p$

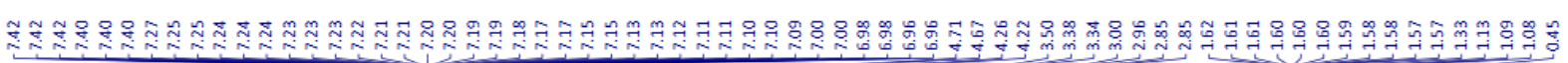<smiles></smiles>

${ }^{1} \mathrm{H}$ NMR $\left(400 \mathrm{MHz}, \mathrm{CDCl}_{3}\right)$

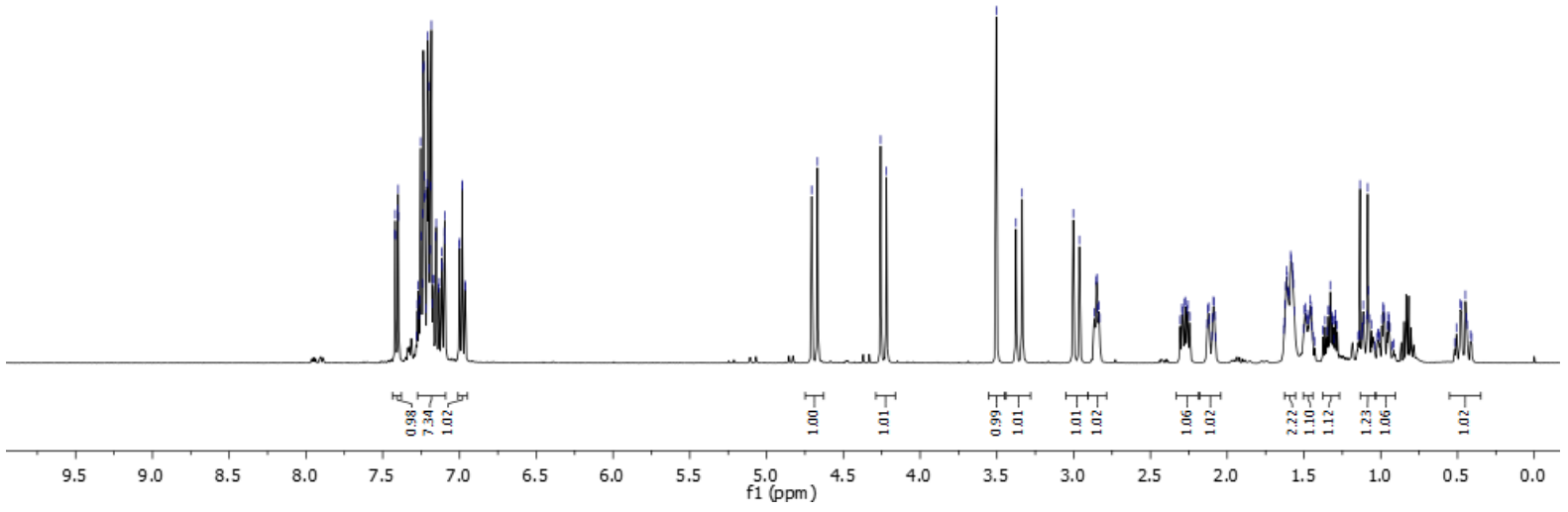

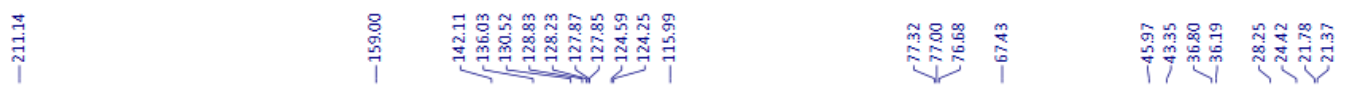

${ }^{13} \mathrm{C}$ NMR $\left(101 \mathrm{MHz}, \mathrm{CDCl}_{3}\right)$

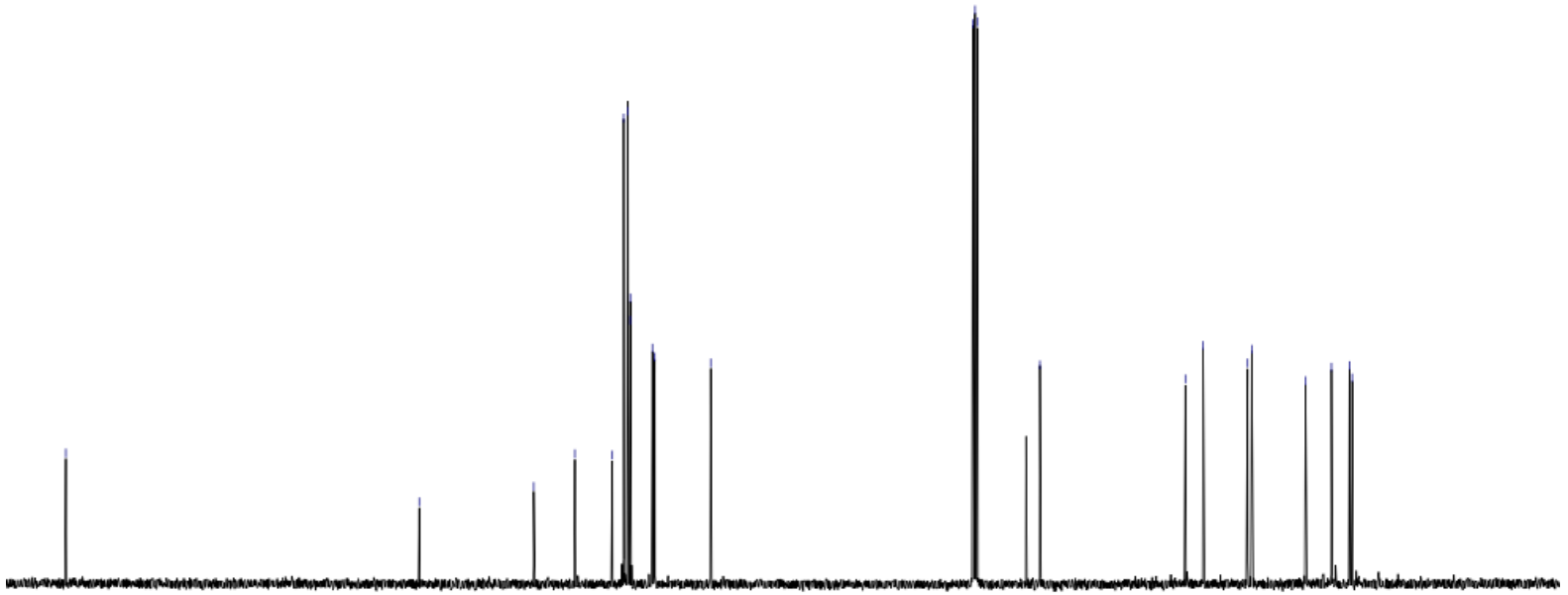

$\begin{array}{lllllllllll}210 & 200 & 190 & 180 & 170 & 160 & 150 & 140 & 130 & 120 & \begin{array}{c}110 \\ \mathrm{f}(\mathrm{ppm})\end{array}\end{array}$ 
$\left(1 S^{*}, 1 \mathrm{a} R^{*}, 6 \mathrm{a} R^{*}\right)-N$-Benzyl-1,1a,6,6a-tetrahydrocyclopropa $[a]$ inden-1-amine

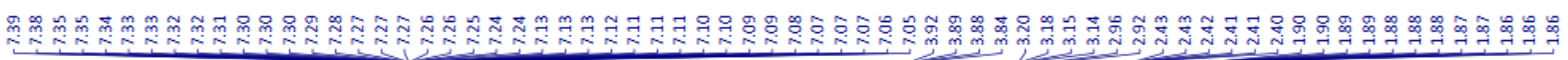<smiles>CC(C)(C)ONC(N)=O</smiles>

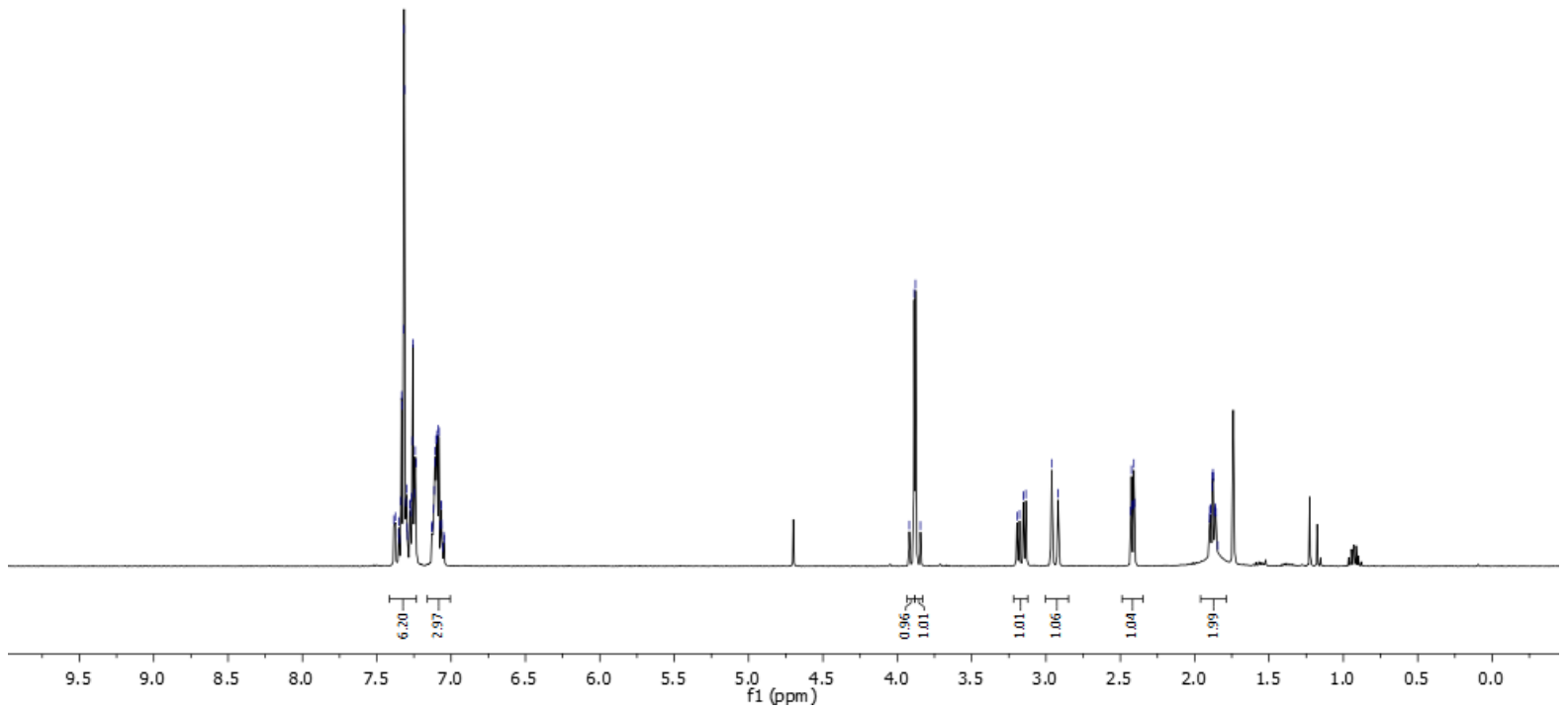

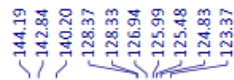

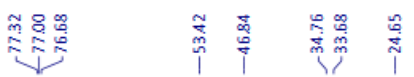

${ }^{13} \mathrm{C}$ NMR $\left(101 \mathrm{MHz}, \mathrm{CDCl}_{3}\right)$

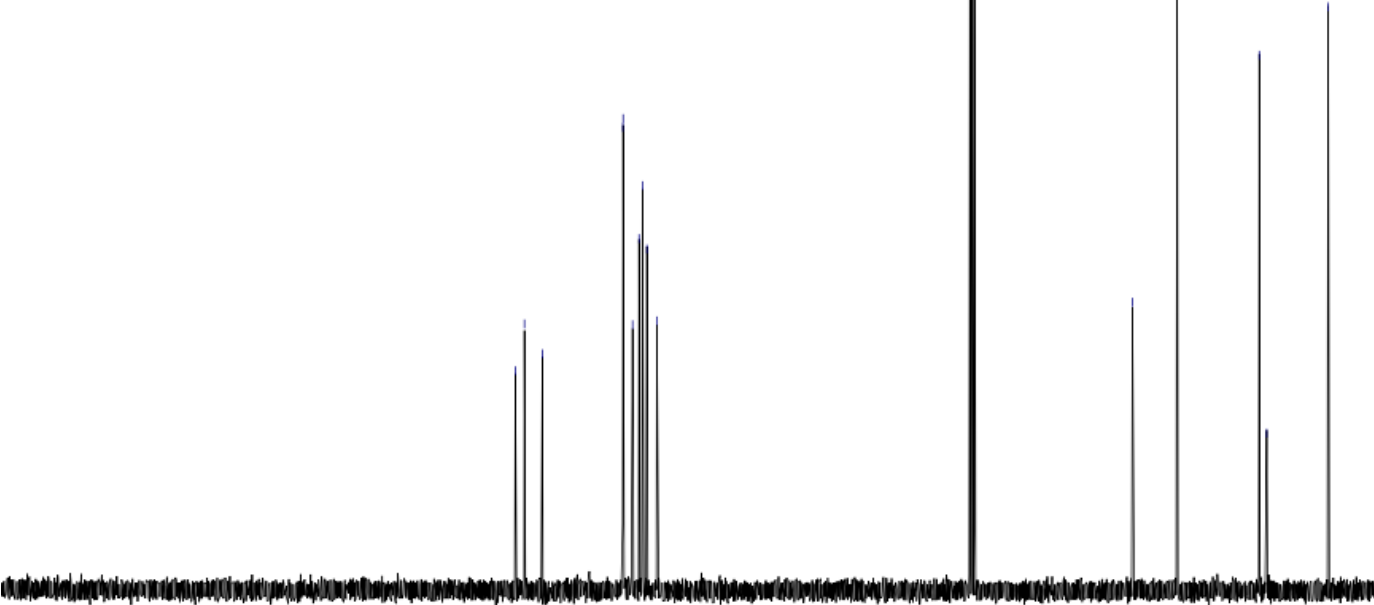

$\begin{array}{llllllllllll}210 & 200 & 190 & 180 & 170 & 160 & 150 & 140 & 130 & 120 & 110 \\ \mathrm{f} 1(\mathrm{ppm}) & 100\end{array}$ 
$N$-Benzyl- $N$-((1S*,1a $\left.R^{*}, 6 \mathrm{a} R^{*}\right)-1,1 \mathrm{a}, 6,6 \mathrm{a}-$ tetrahydrocyclopropa $[a]$ inden-1-yl)-1H-indole1-carboxamide 3q

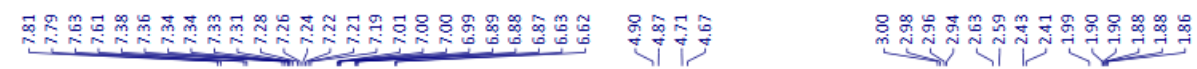
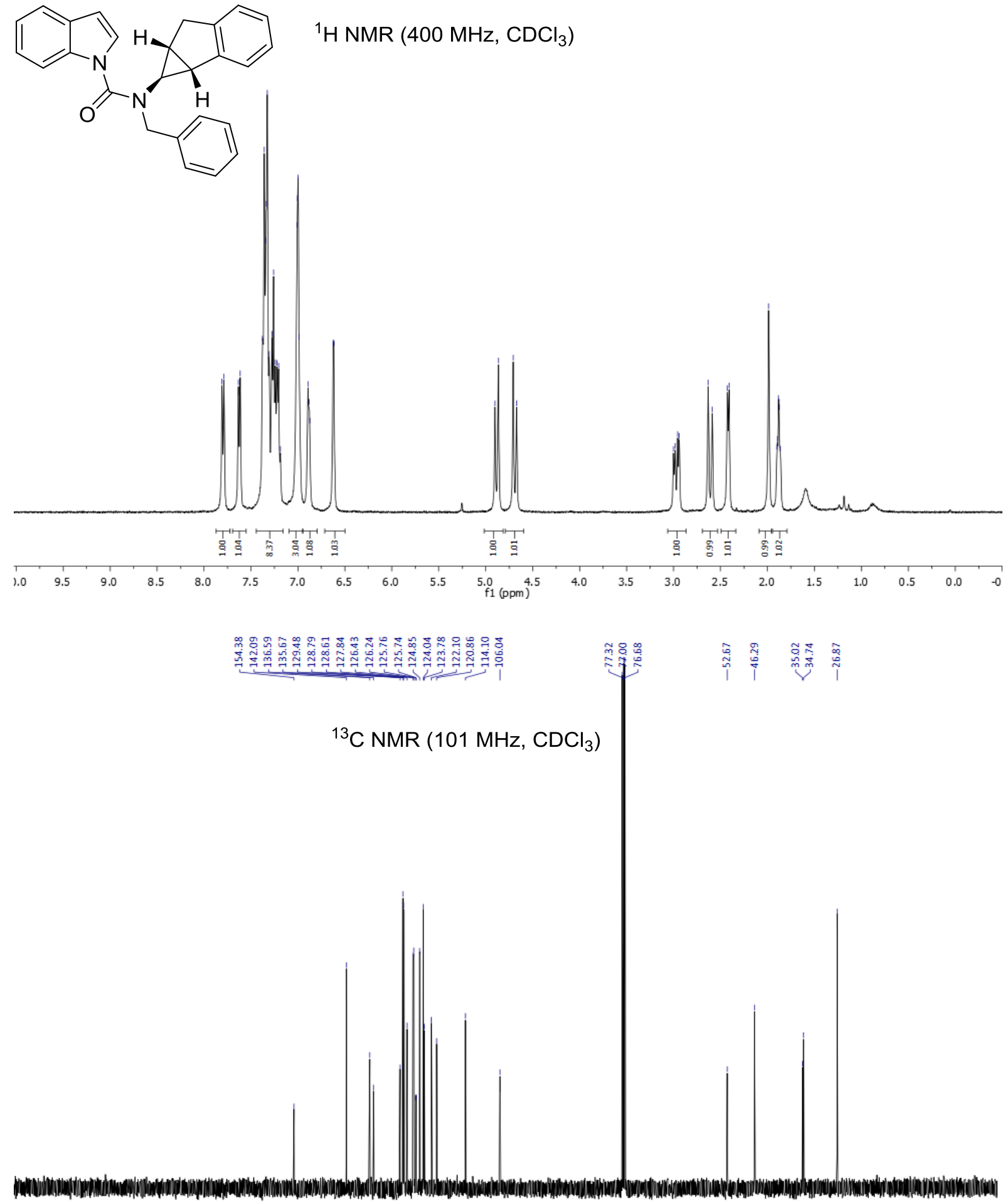

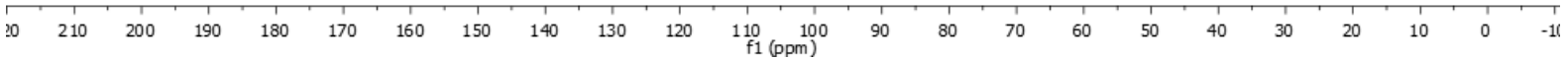


$\left(7 \mathrm{a} S^{*}, 7 \mathrm{~b} R^{*}, 12 \mathrm{~b} R^{*}, 13 \mathrm{a} S^{*}\right)-7-B e n z y l-7 \mathrm{a}, 7 \mathrm{~b}, 8,12 \mathrm{~b}-$ tetrahydro-6H,14Hbenzo[4',5']pentaleno[1',2':4,5]imidazo[1,5-a]indole-6,13(7H)-dione

and $\left(7 \mathrm{a} S^{*}, 7 \mathrm{~b} S^{*}, 12 \mathrm{a} S^{*}, 13 \mathrm{a} S^{*}\right)-7-B e n z y l-7 \mathrm{a}, 7 \mathrm{~b}, 12,12 \mathrm{a}-t e t r a h y d r o-6 H, 14 H$ benzo[5',6']pentaleno[1',2':4,5]imidazo[1,5-a]indole-6,13(7H)-dione 4q',

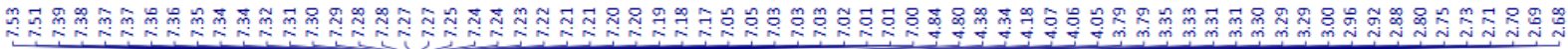<smiles>O=C1[C@H]2c3ccccc3C[C@]23C(=O)N(Cc2ccccc2)[C@@]32Cc3ccccc3N12</smiles><smiles>O=C1[C@H]2C3c4ccccc4[C@H]2[C@H]2C(=O)[C@]3(c3ccccc3)N1c1ccccc12</smiles>
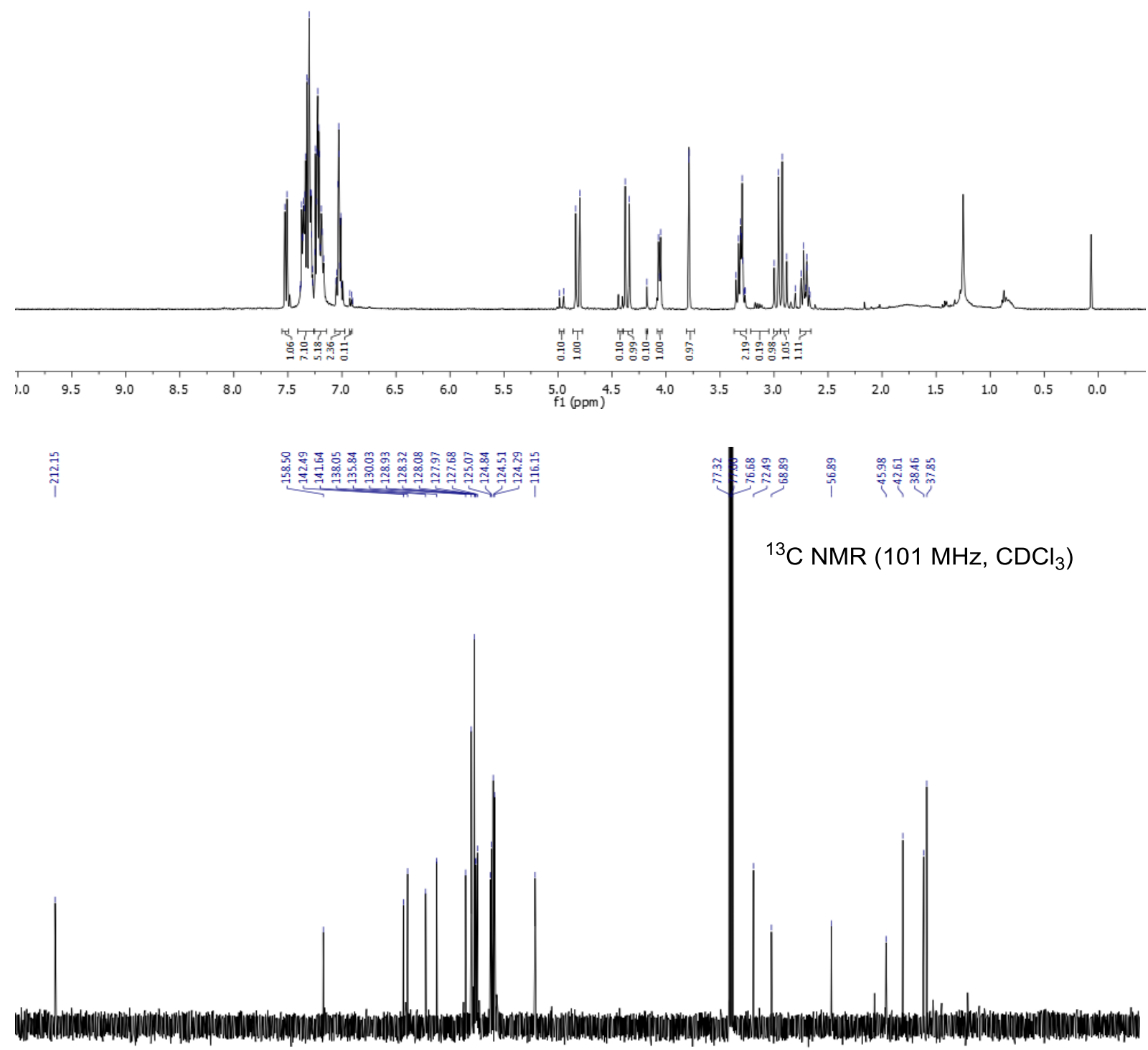

$\begin{array}{lllllllllllllllllllllll}20 & 210 & 200 & 190 & 180 & 170 & 160 & 150 & 140 & 130 & 120 & \begin{array}{c}110 \\ \mathrm{f} 1(\mathrm{ppm})\end{array} & 100 & 90 & 80 & 70 & 60 & 50 & 40 & 30 & 20 & 10 & 0\end{array}$ 


\section{$N$-(2-(1,3-Dioxolan-2-yl)ethyl)- $N$-cyclopropyl-1H-indole-1-carboxamide}

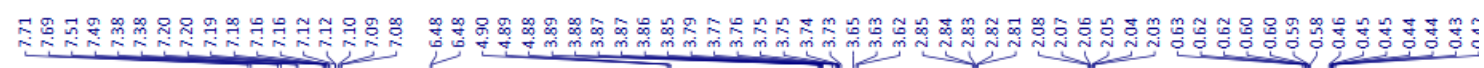
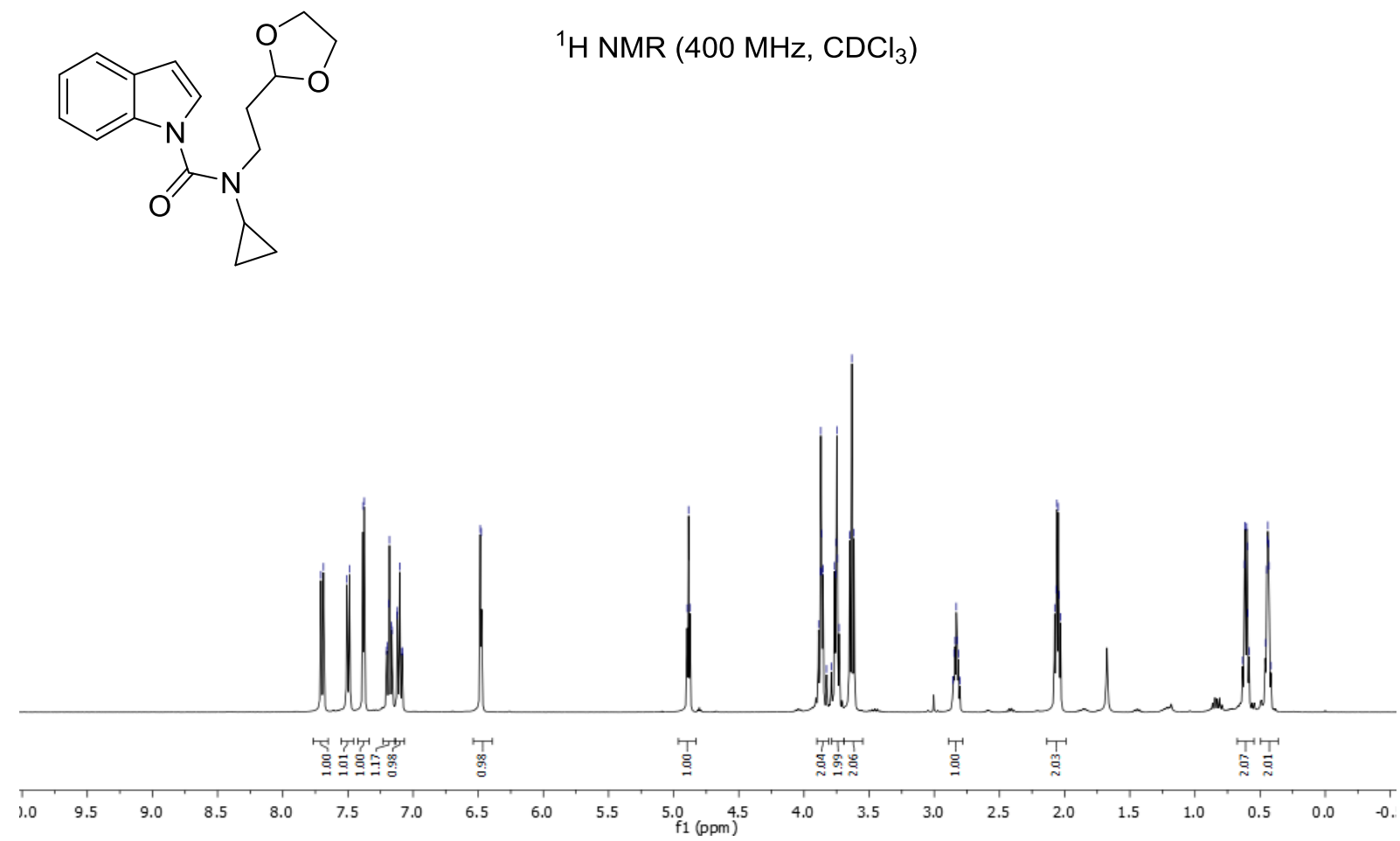

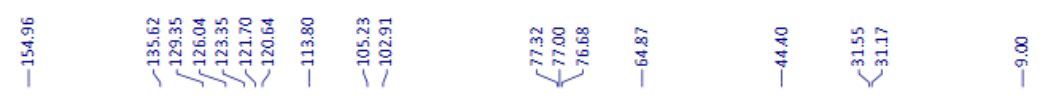

${ }^{13} \mathrm{C}$ NMR $\left(101 \mathrm{MHz}, \mathrm{CDCl}_{3}\right)$

\begin{tabular}{llllllllllll}
\hline 0 & 210 & 200 & 190 & 180 & 170 & 160 & 150 & 140 & 130 & 120 & 110 \\
$\mathrm{f} 1(\mathrm{ppm})$ & 100
\end{tabular} 
<smiles></smiles>

${ }^{1} \mathrm{H}$ NMR $\left(400 \mathrm{MHz}, \mathrm{CDCl}_{3}\right)$
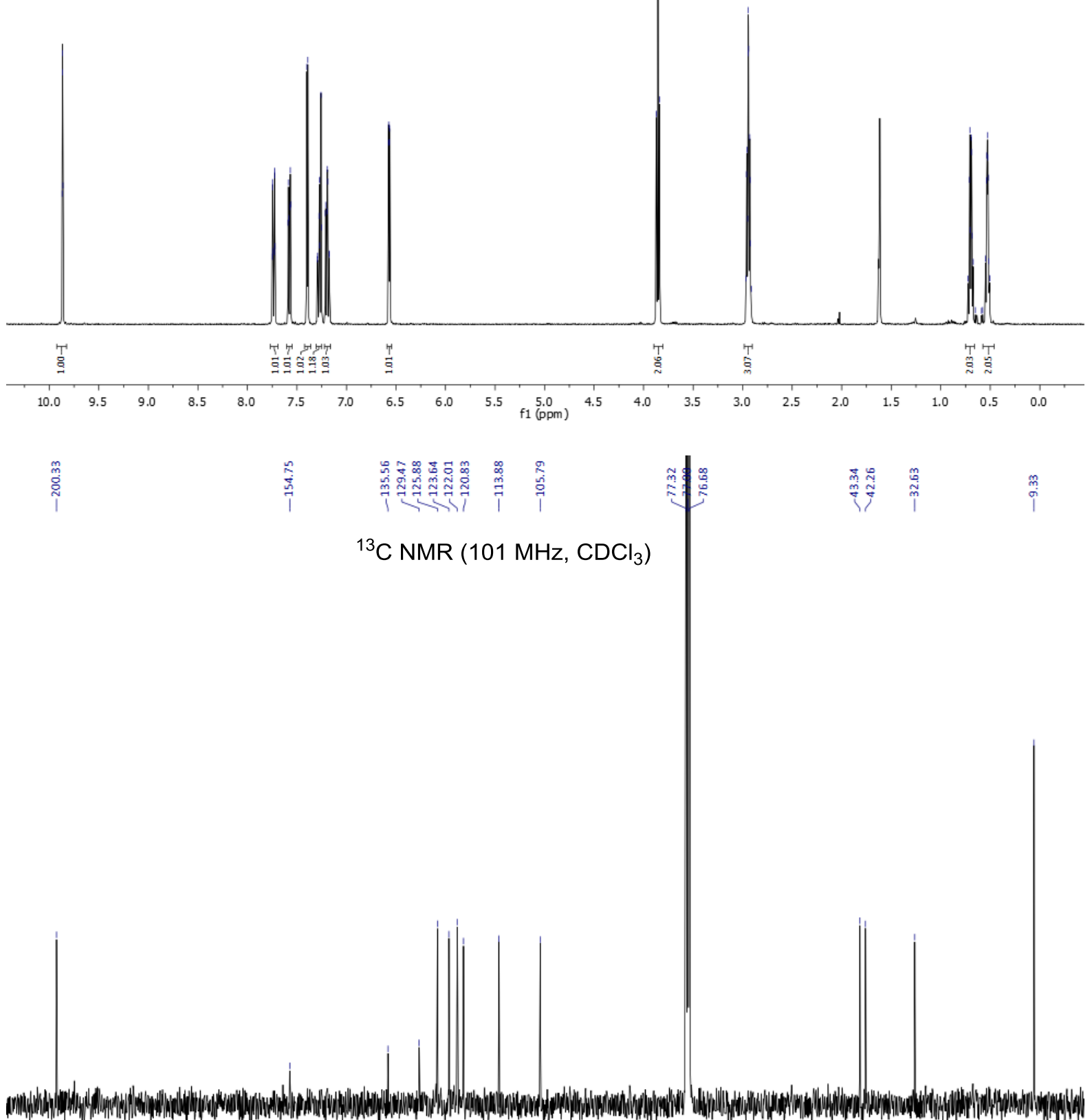

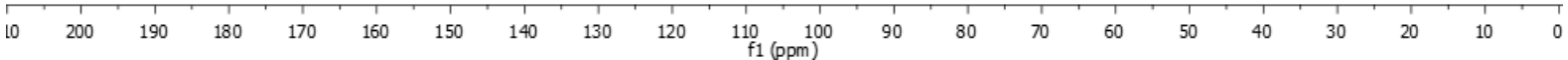


(E)- $N$-Cyclopropyl- $N$-(4-phenylbut-3-en-1-yl)-1H-indole-1-carboxamide $3 r$

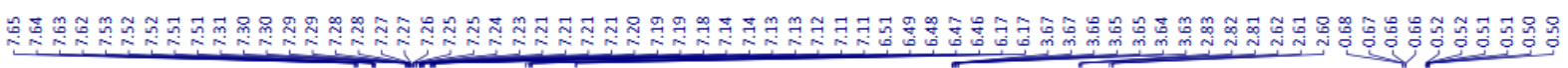

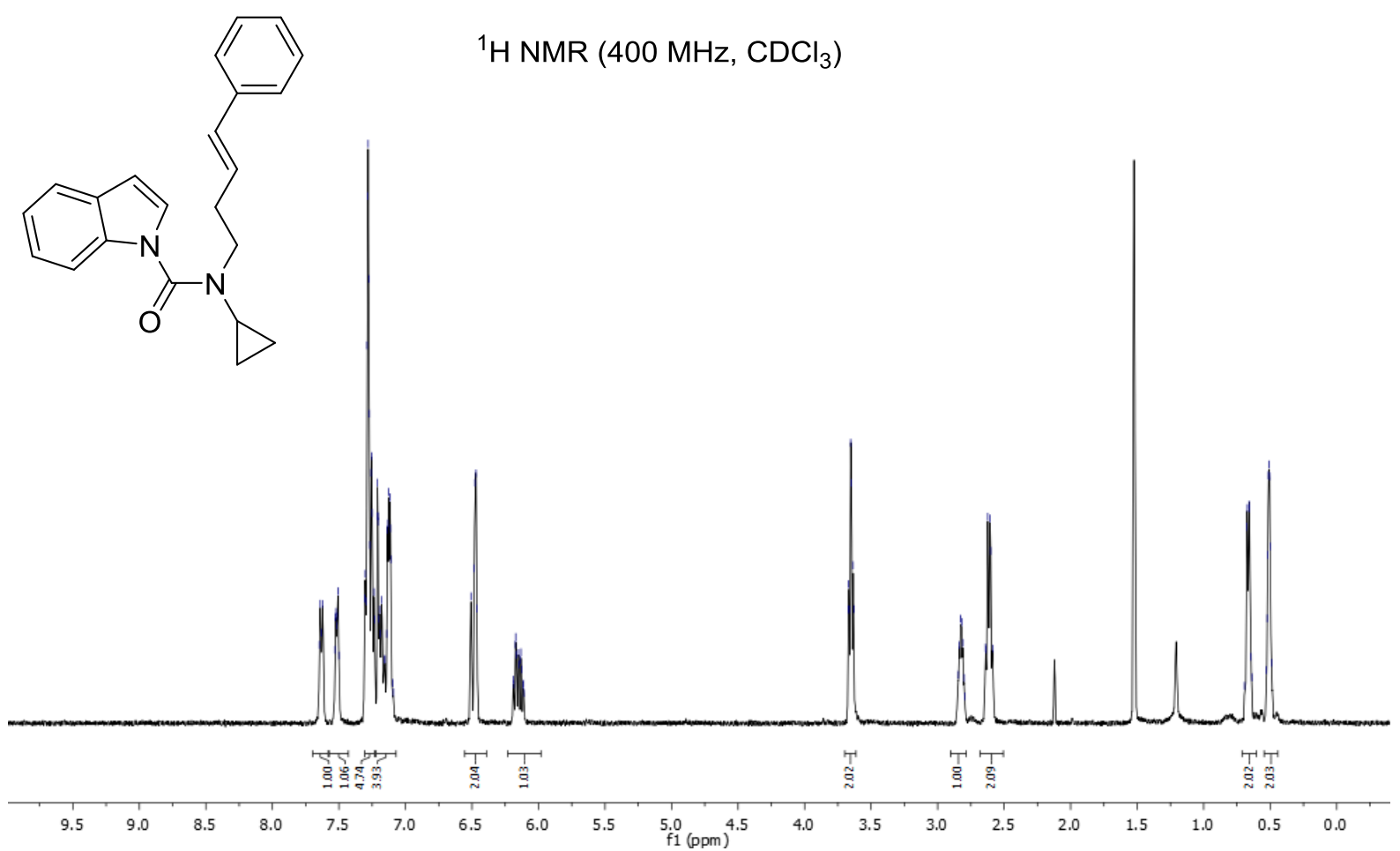

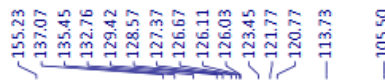

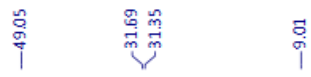

${ }^{13} \mathrm{C}$ NMR $\left(101 \mathrm{MHz}, \mathrm{CDCl}_{3}\right)$

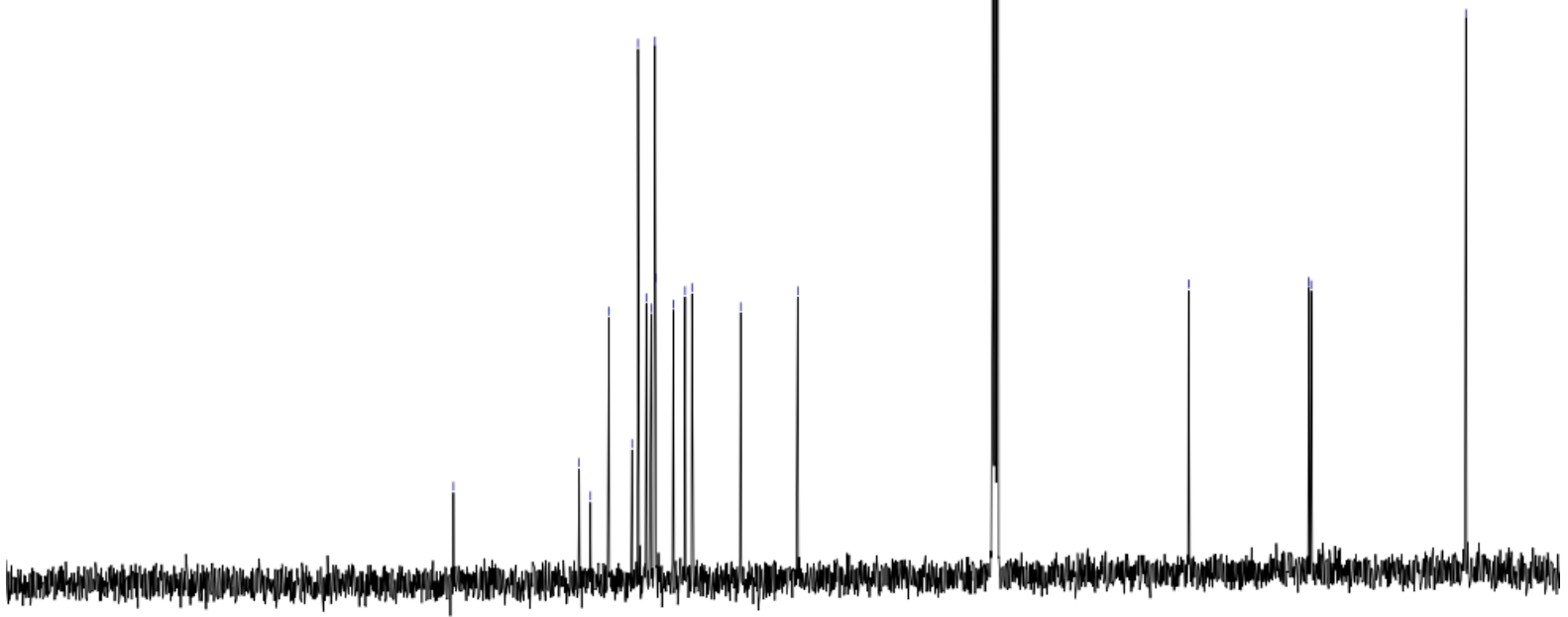

$\begin{array}{lllllllllll}210 & 200 & 190 & 180 & 170 & 160 & 150 & 140 & 130 & 120 & 110 \\ \mathrm{ff}(\mathrm{ppm}) & 100\end{array}$ 
(Z)-N-Cyclopropyl- $N$-(4-phenylbut-3-en-1-yl)-1H-indole-1-carboxamide $3 r^{\prime}$

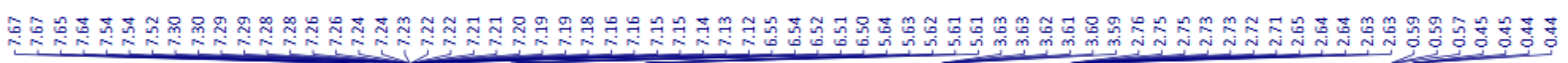
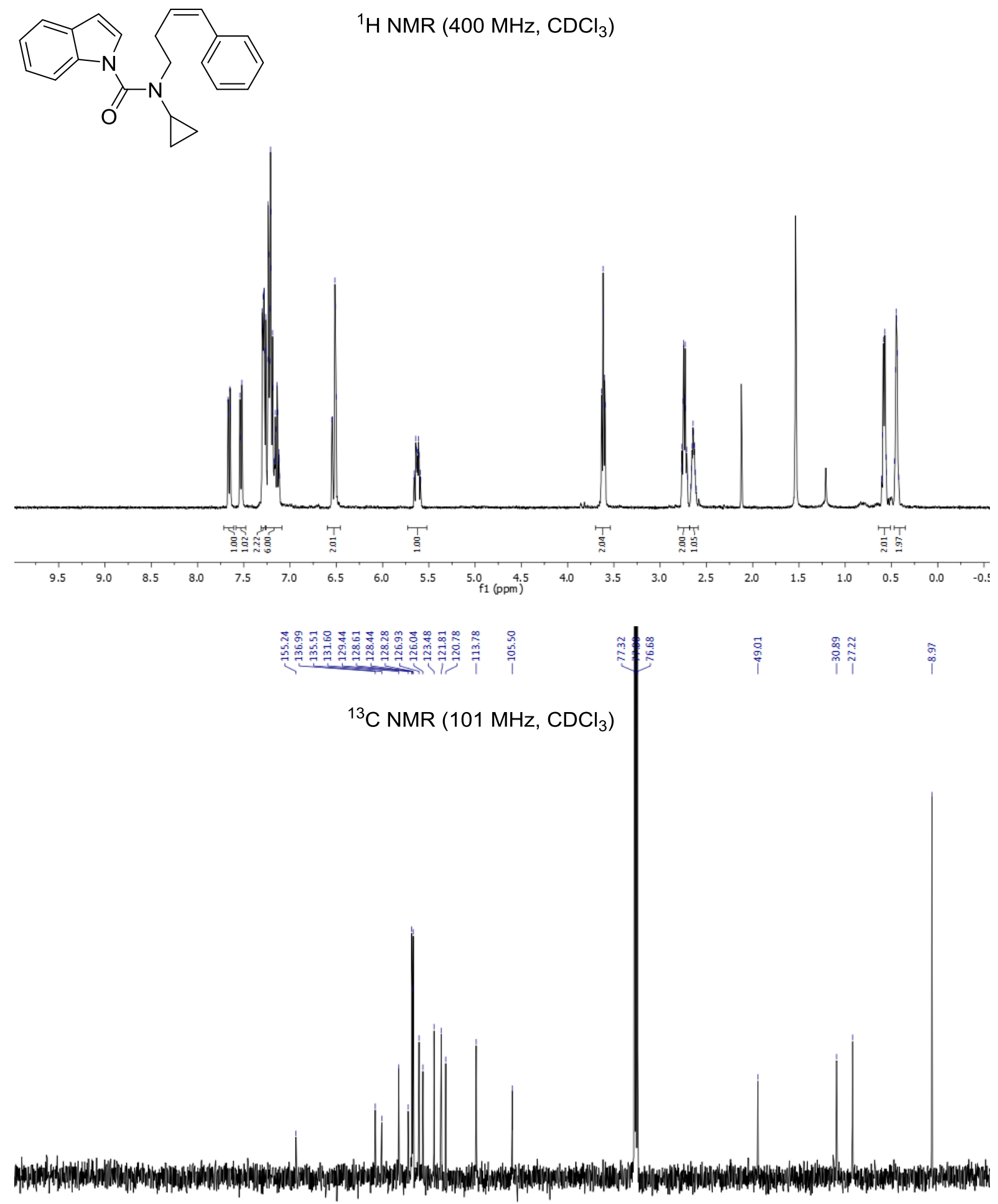

$\begin{array}{lllllllllllllllllllllll}10 & 210 & 200 & 190 & 180 & 170 & 160 & 150 & 140 & 130 & 120 & \begin{array}{l}110 \\ \mathrm{f} 1(\mathrm{ppm})\end{array} & 100 & 90 & 80 & 70 & 60 & 50 & 40 & 30 & 20 & 10 & 0\end{array}$ 
$\left(3 \mathrm{a} S^{*}, 11 \mathrm{a} S^{*}\right)-4-((E)-4-p h e n y l b u t-3-e n-1-y l)-2,3,3 \mathrm{a}, 4-t e t r a h y d r o-11 H-$ cyclopenta[4,5]imidazo[1,5- $a]$ indole-1,5-dione $4 r$

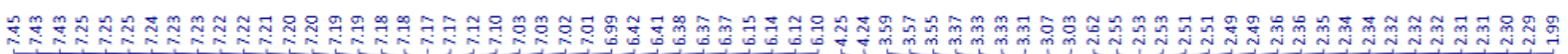

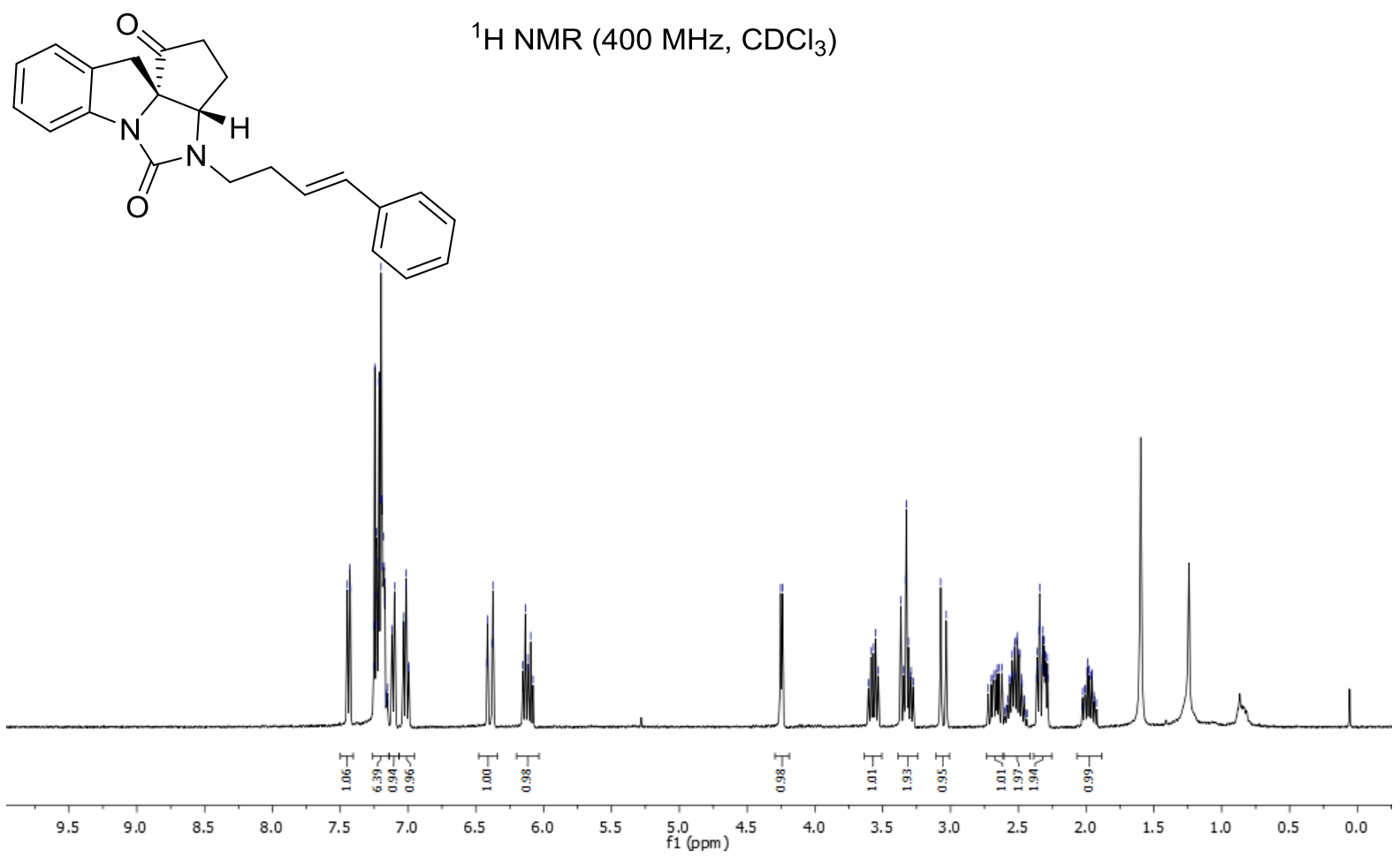

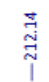

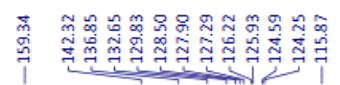

${ }^{13} \mathrm{C}$ NMR $\left(101 \mathrm{MHz}, \mathrm{CDCl}_{3}\right)$

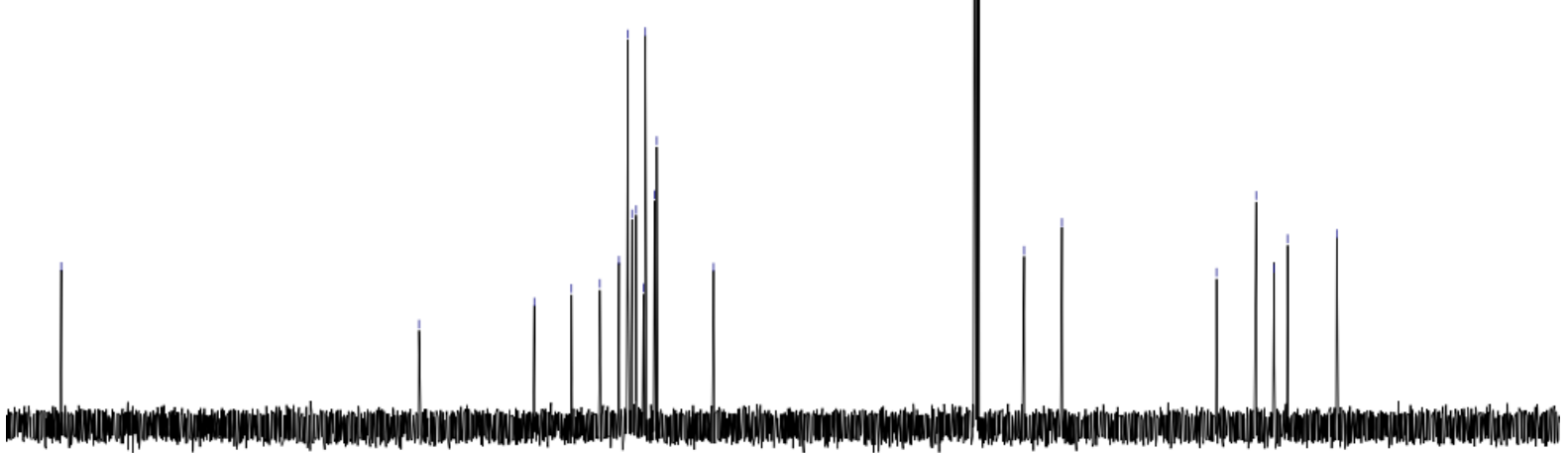

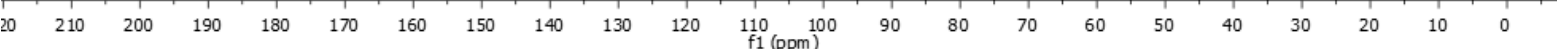


$N$-(4-Phenylbut-3-yn-1-yl)cyclopropanamine

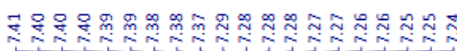

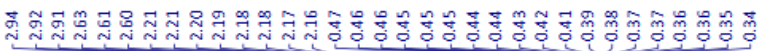

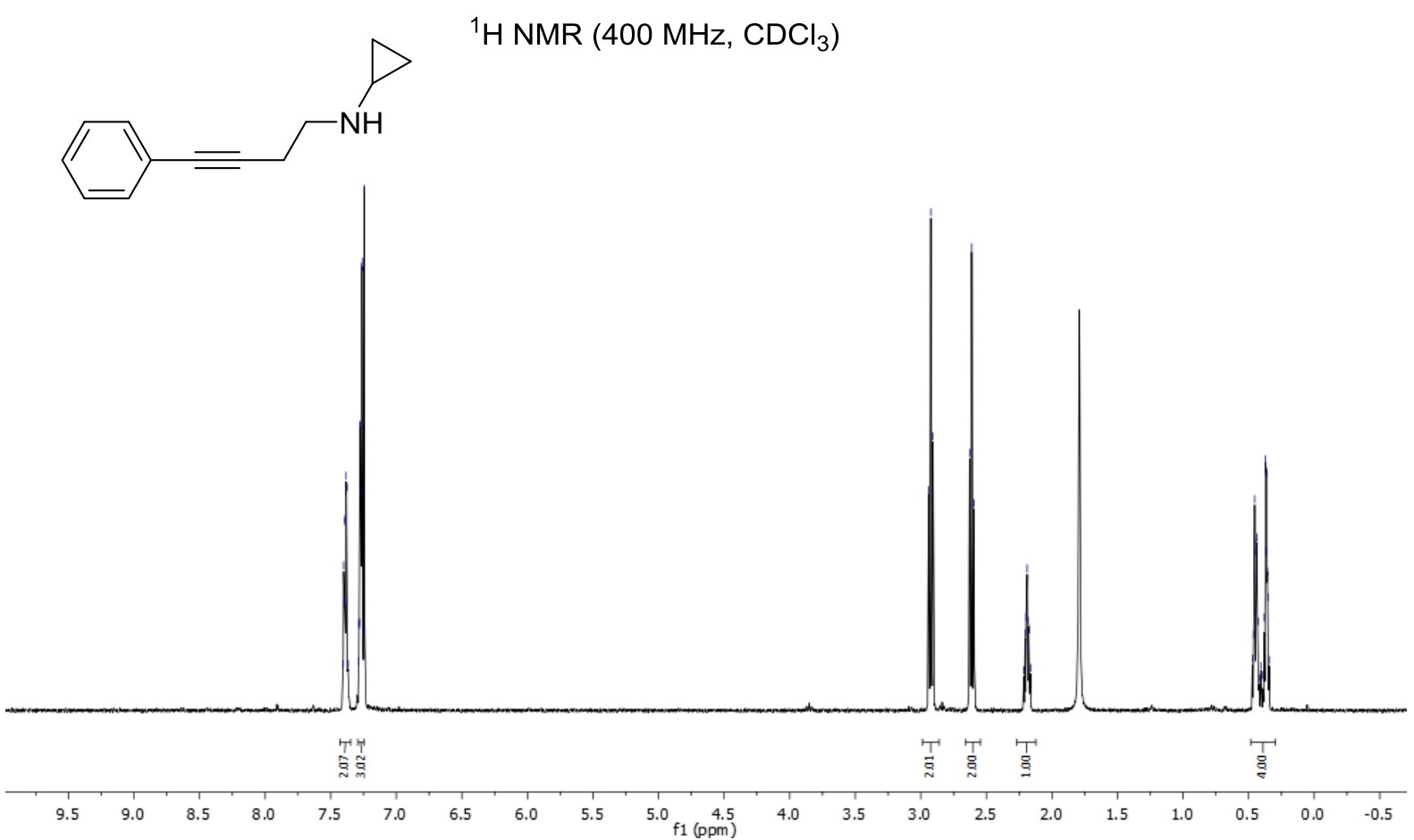

\section{足品芯岕}

가가

mend

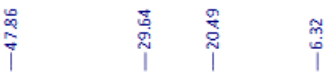

${ }^{13} \mathrm{C}$ NMR $\left(101 \mathrm{MHz}, \mathrm{CDCl}_{3}\right)$

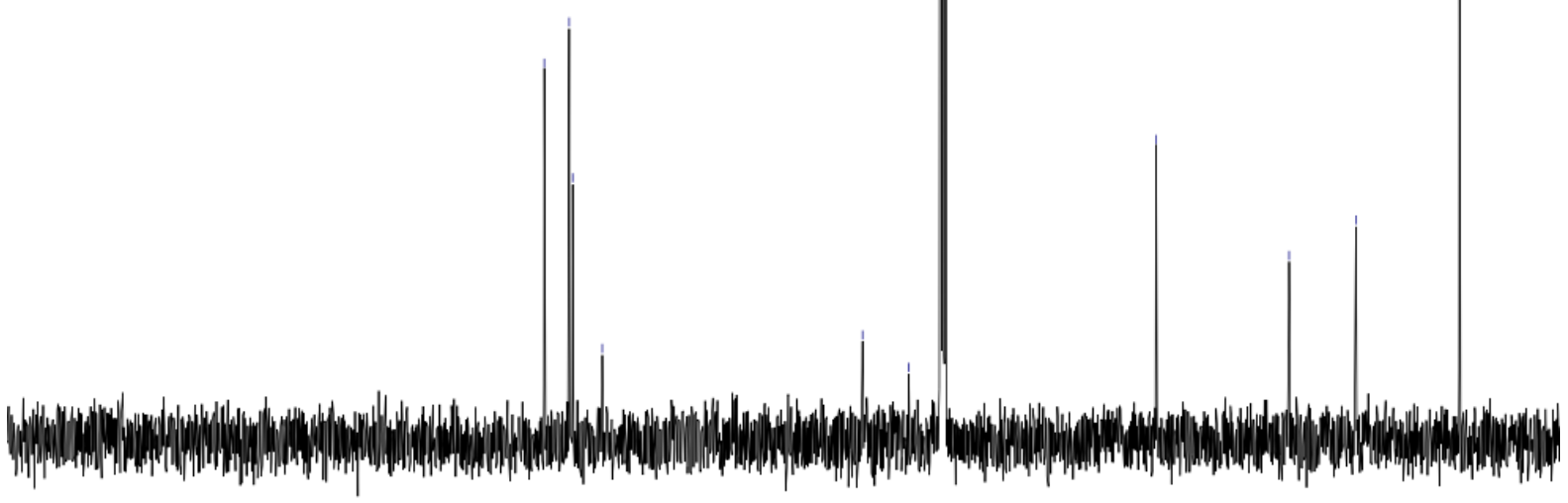

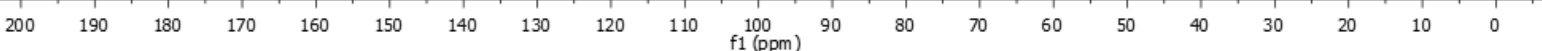


$N$-Cyclopropyl- $N$-(4-phenylbut-3-yn-1-yl)-1H-indole-1-carboxamide 5a

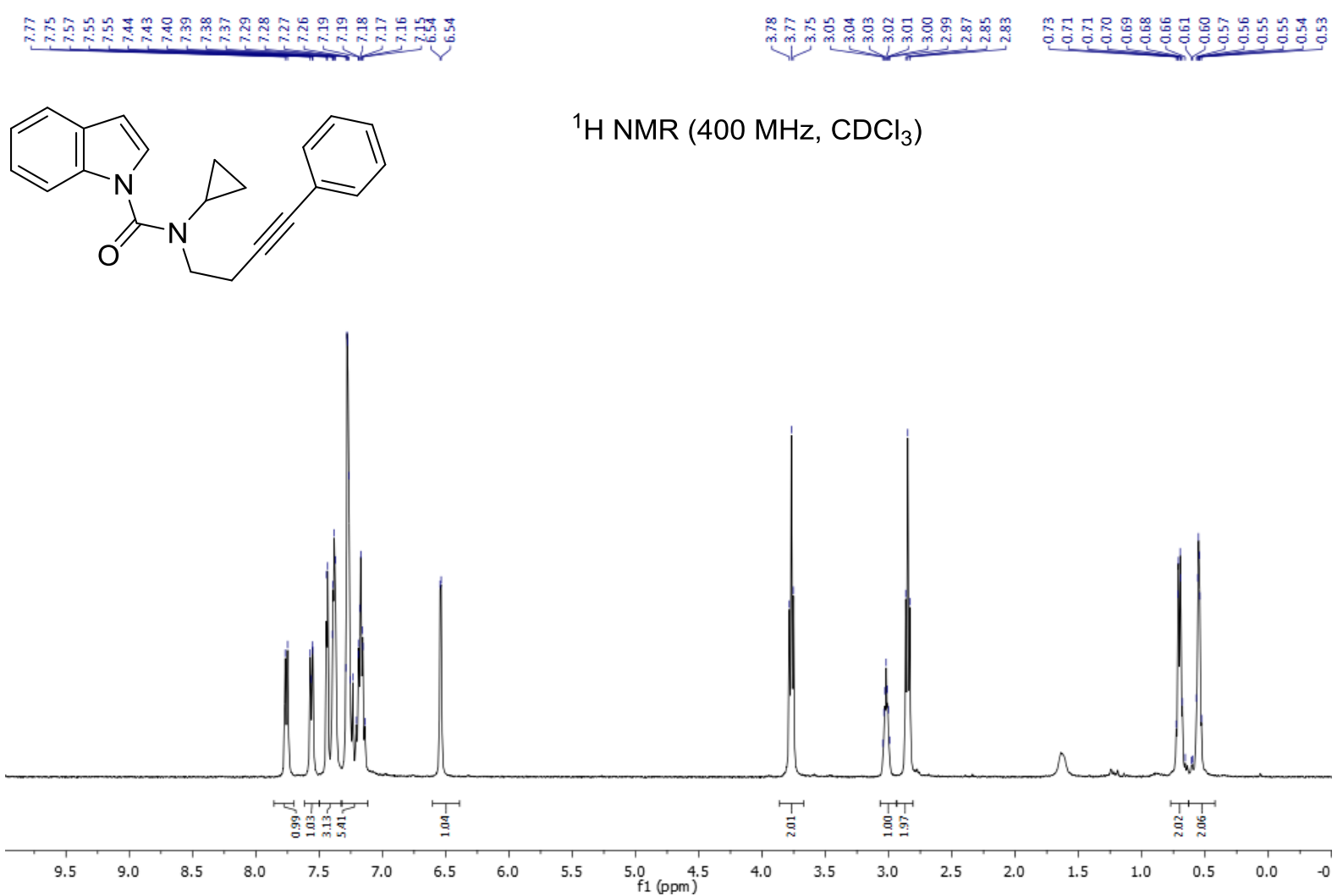

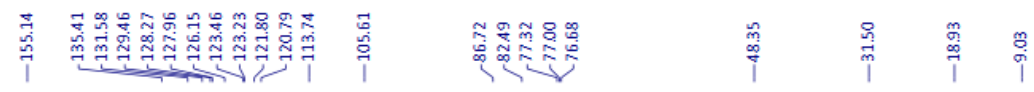

${ }^{13} \mathrm{C}$ NMR $\left(101 \mathrm{MHz}, \mathrm{CDCl}_{3}\right)$

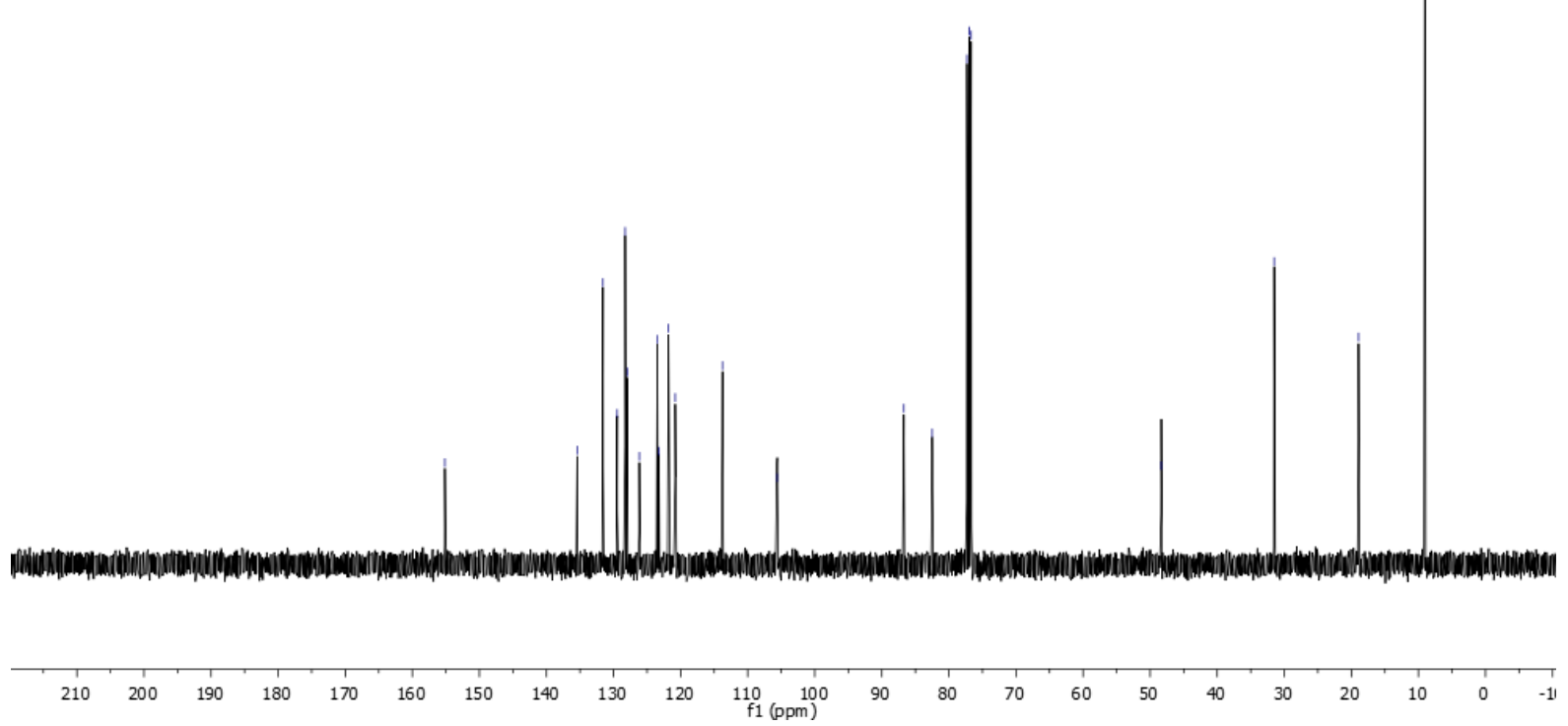


(E)-3-Benzylidene-1,2,3,3a,4,5-hexahydro-6H,13H-pyrrolo[1',2':3,4][1,3]diazocino[1,8a] indole-6,13-dione 6a

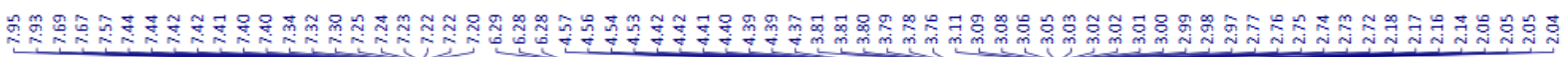<smiles>CC(C)=O</smiles>

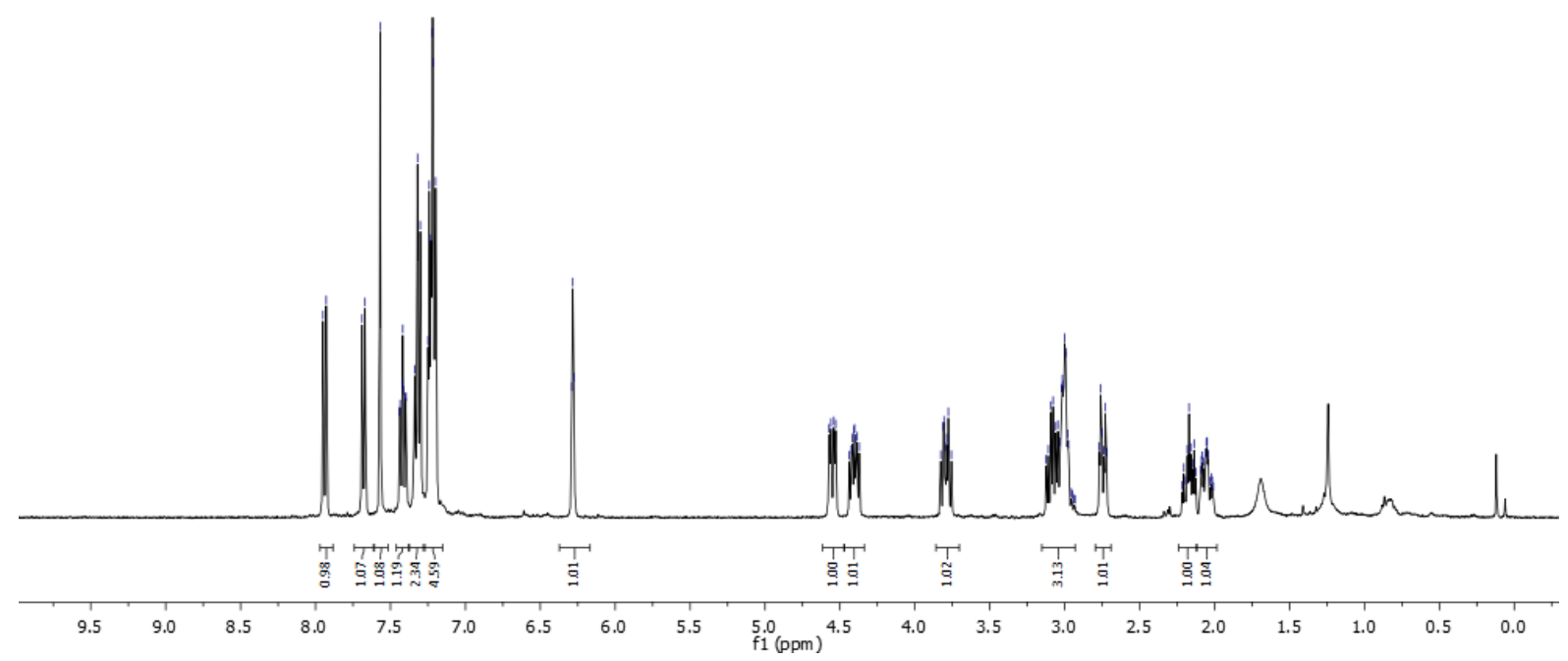

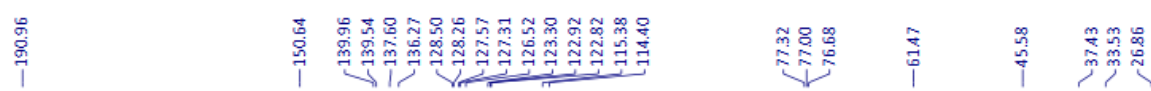

${ }^{13} \mathrm{C}$ NMR $\left(101 \mathrm{MHz}, \mathrm{CDCl}_{3}\right)$

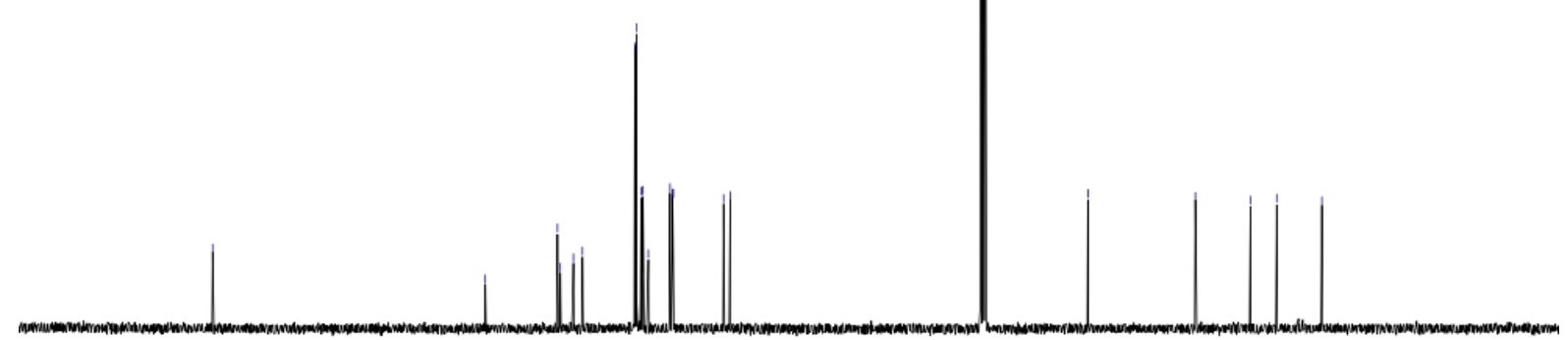

$\begin{array}{lllllllllll}210 & 200 & 190 & 180 & 170 & 160 & 150 & 140 & 130 & 120 & \begin{array}{l}110 \\ \mathrm{f} 1(\mathrm{ppm})\end{array}\end{array}$ 
$N$-Cyclopropyl- $N$-(4-(4-(trifluoromethyl)phenyl)but-3-yn-1-yl)-1H-indole-1carboxamide $5 \mathbf{b}$

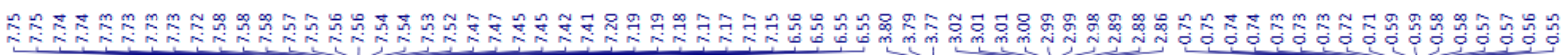<smiles>O=C(N(CCC#Cc1ccc(C(F)(F)F)cc1)C1CC1)n1ccc2ccccc21</smiles>

${ }^{1} \mathrm{H}$ NMR $\left(400 \mathrm{MHz}, \mathrm{CDCl}_{3}\right)$

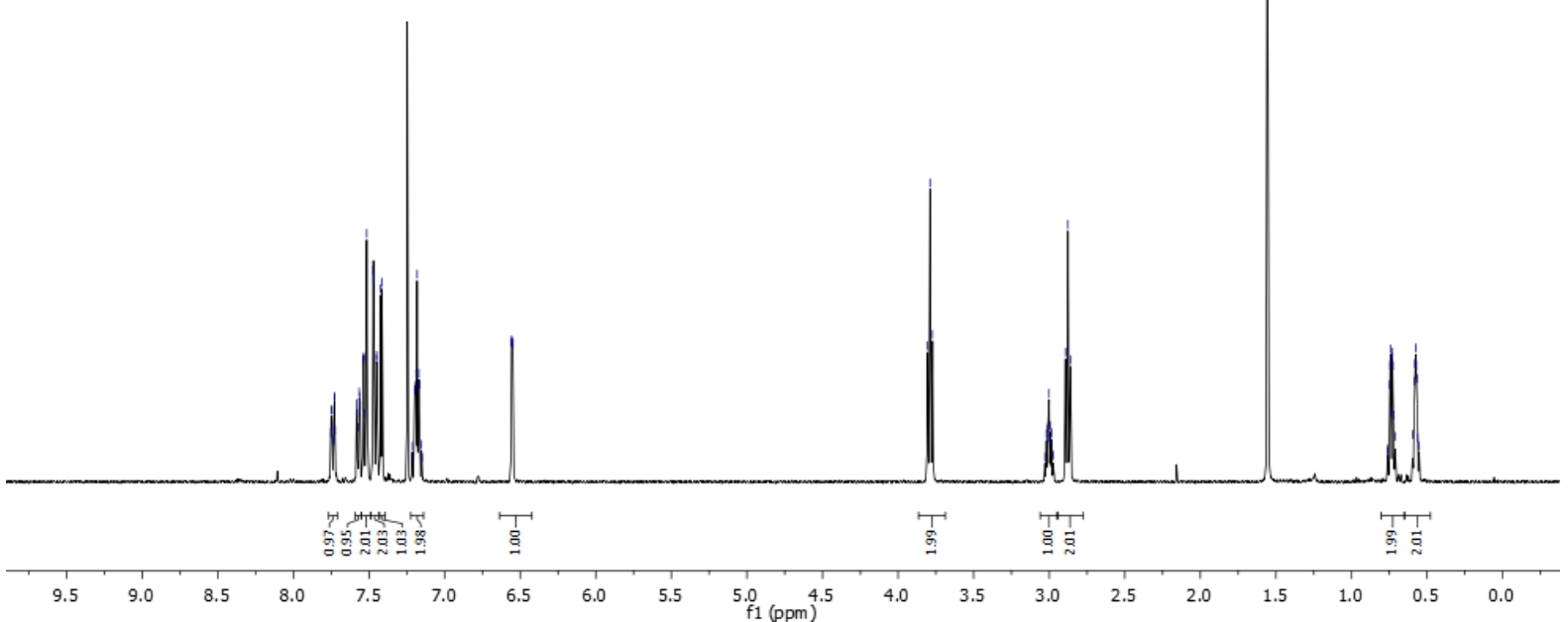

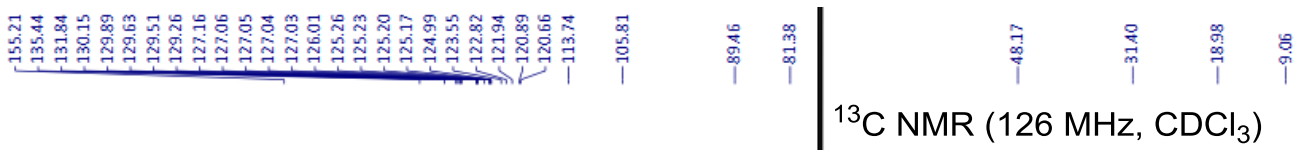

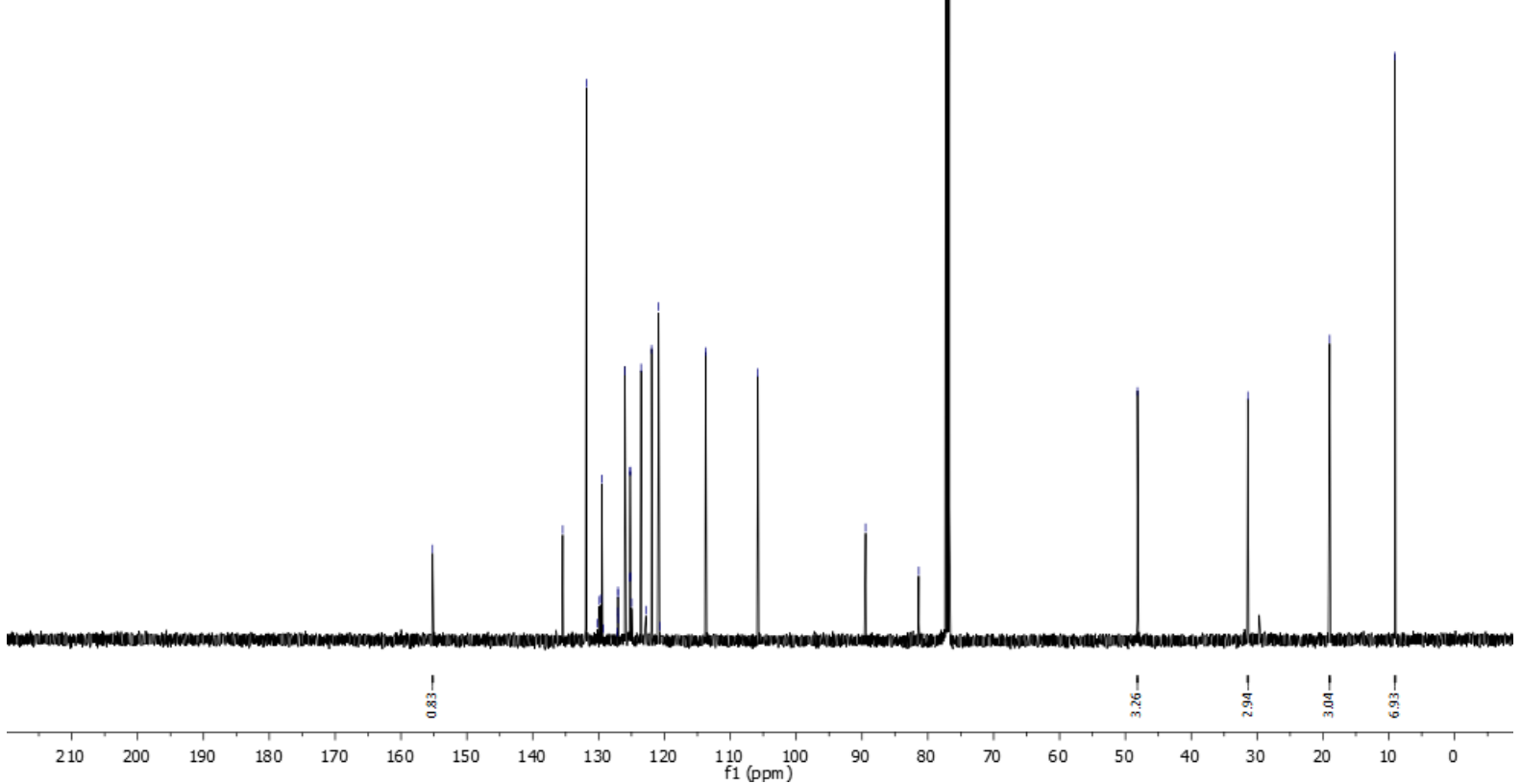



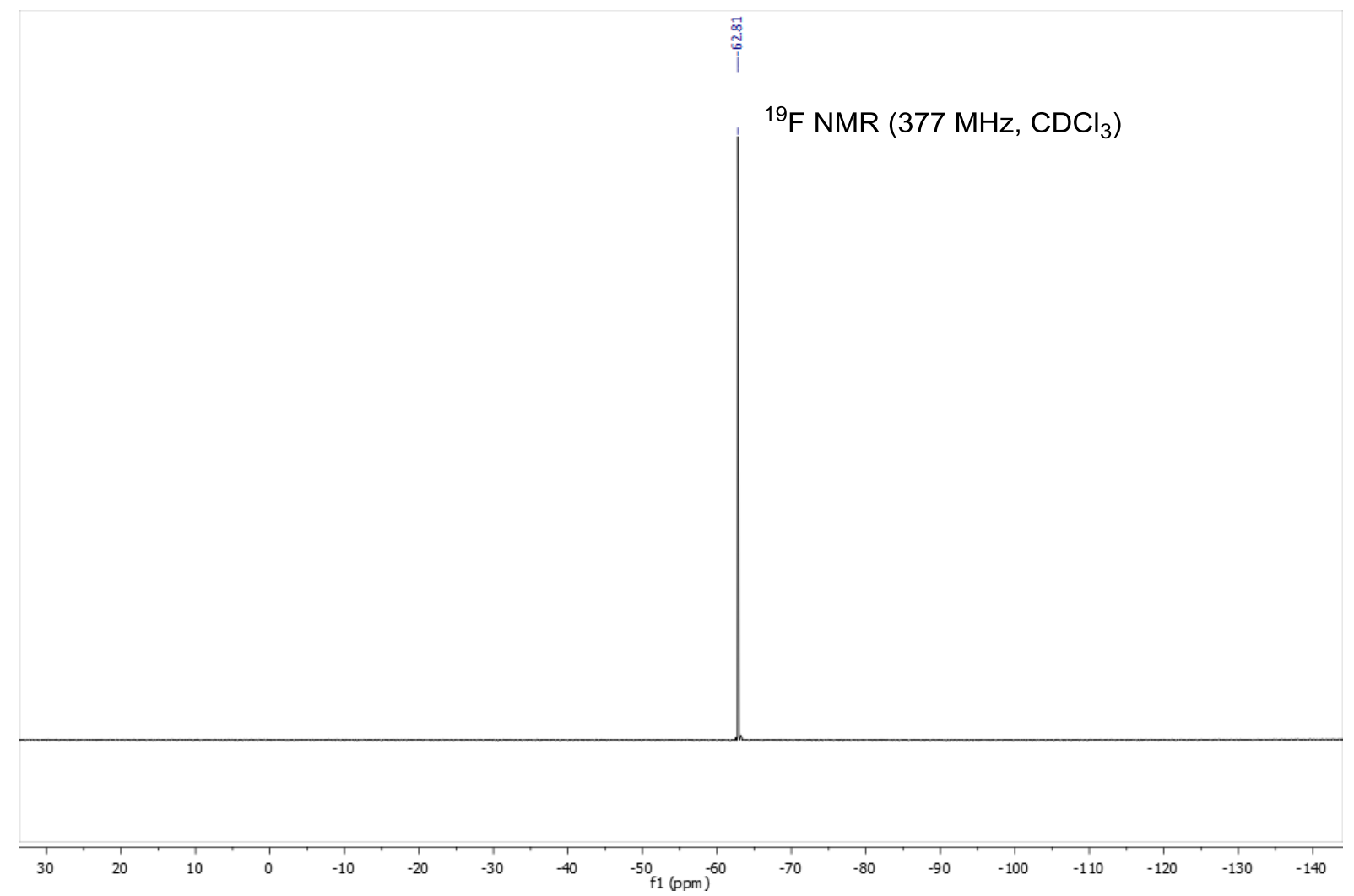

(E)-3-(4-(Trifluoromethyl)benzylidene)-1,2,3,3a,4,5-hexahydro-6H,13H-

pyrrolo[1',2':3,4][1,3]diazocino[1,8-a]indole-6,13-dione $6 \mathrm{~b}$

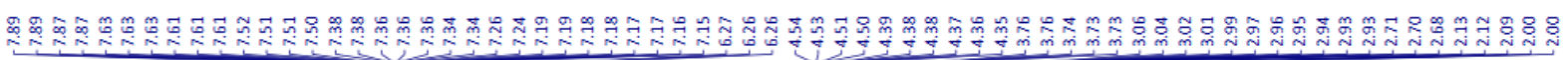<smiles>O=C1CCC2/C(=C/c3ccc(C(F)(F)F)cc3)CCN2C(=O)n2c1cc1ccccc12</smiles>

${ }^{1} \mathrm{H}$ NMR $\left(400 \mathrm{MHz}, \mathrm{CDCl}_{3}\right)$

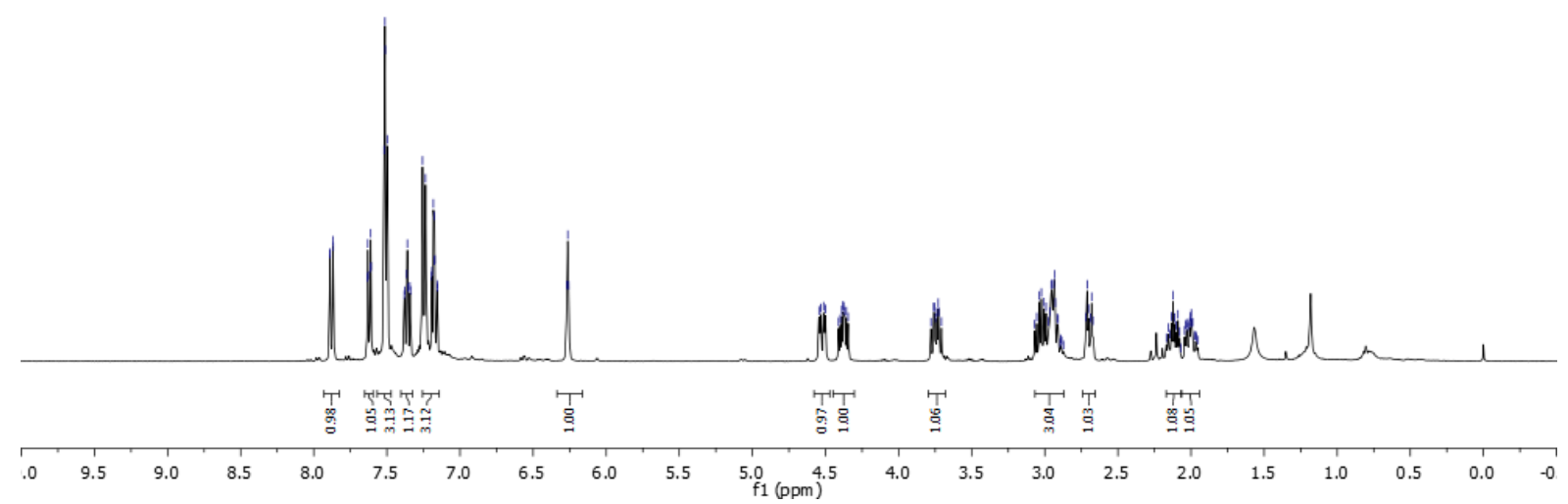



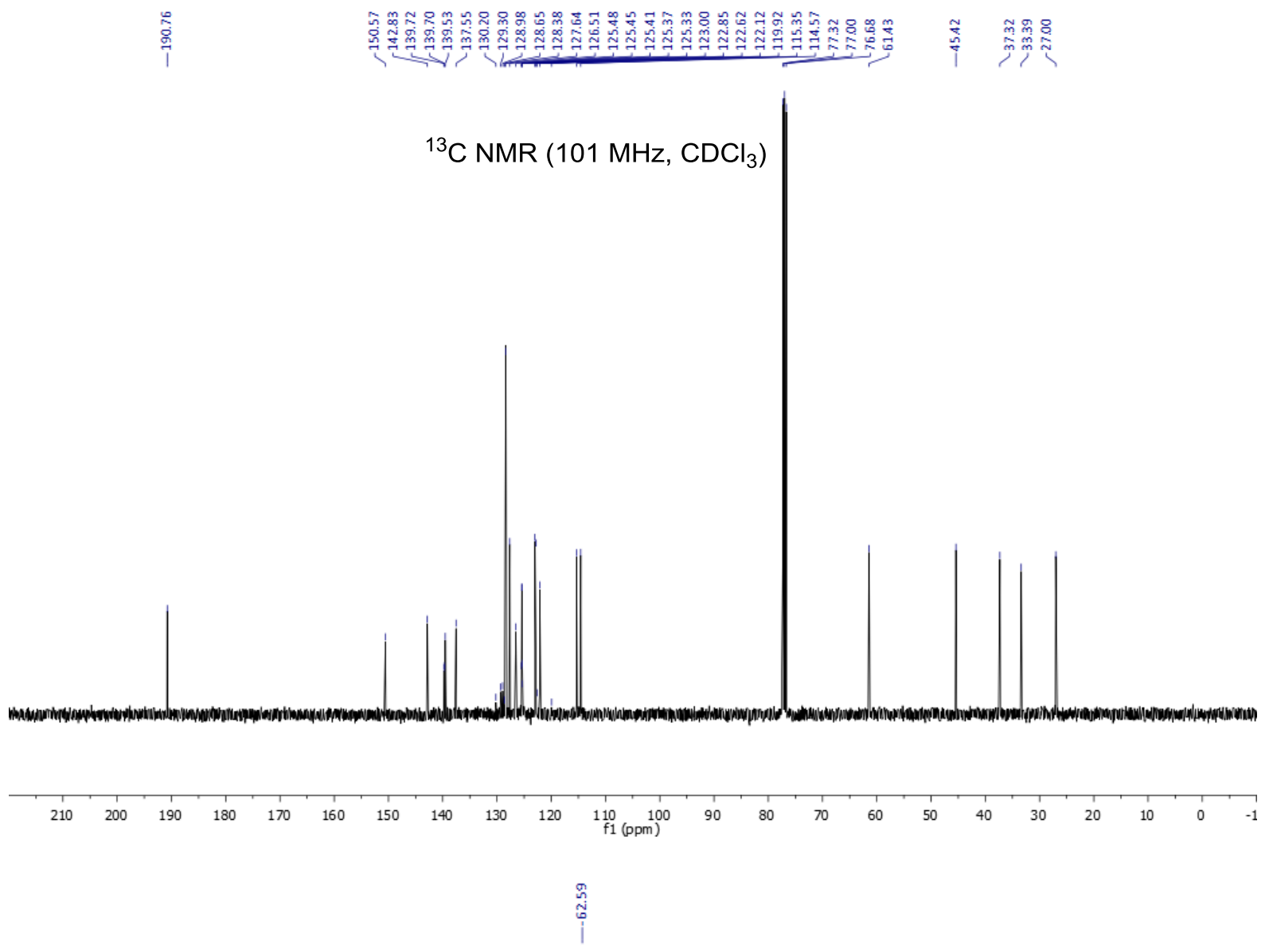

${ }^{19} \mathrm{~F} \mathrm{NMR}\left(377 \mathrm{MHz}, \mathrm{CDCl}_{3}\right)$

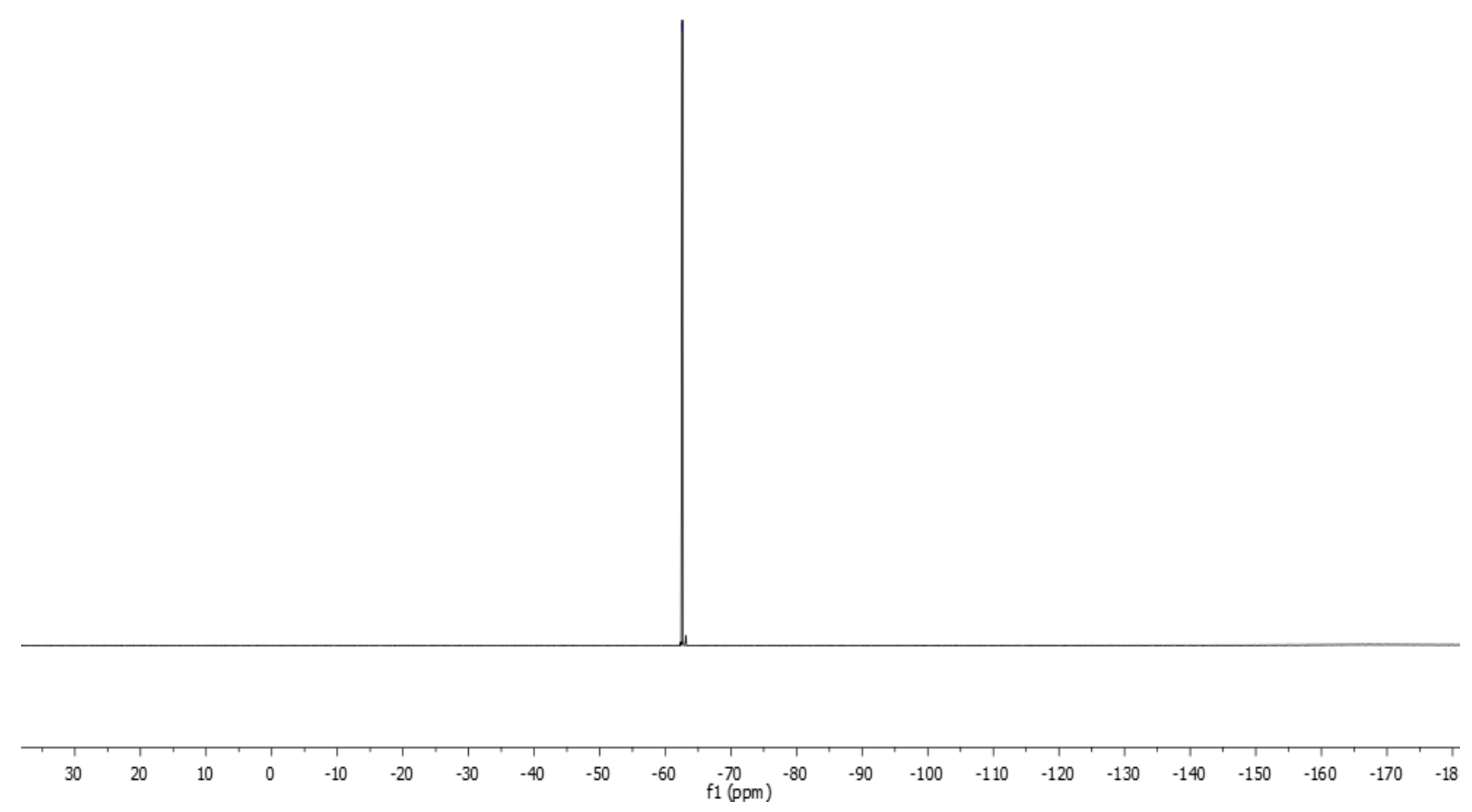


$N$-Cyclopropyl- $N$-(4-(4-methoxyphenyl)but-3-yn-1-yl)-1H-indole-1-carboxamide 5c

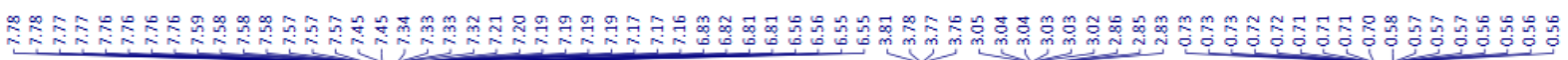

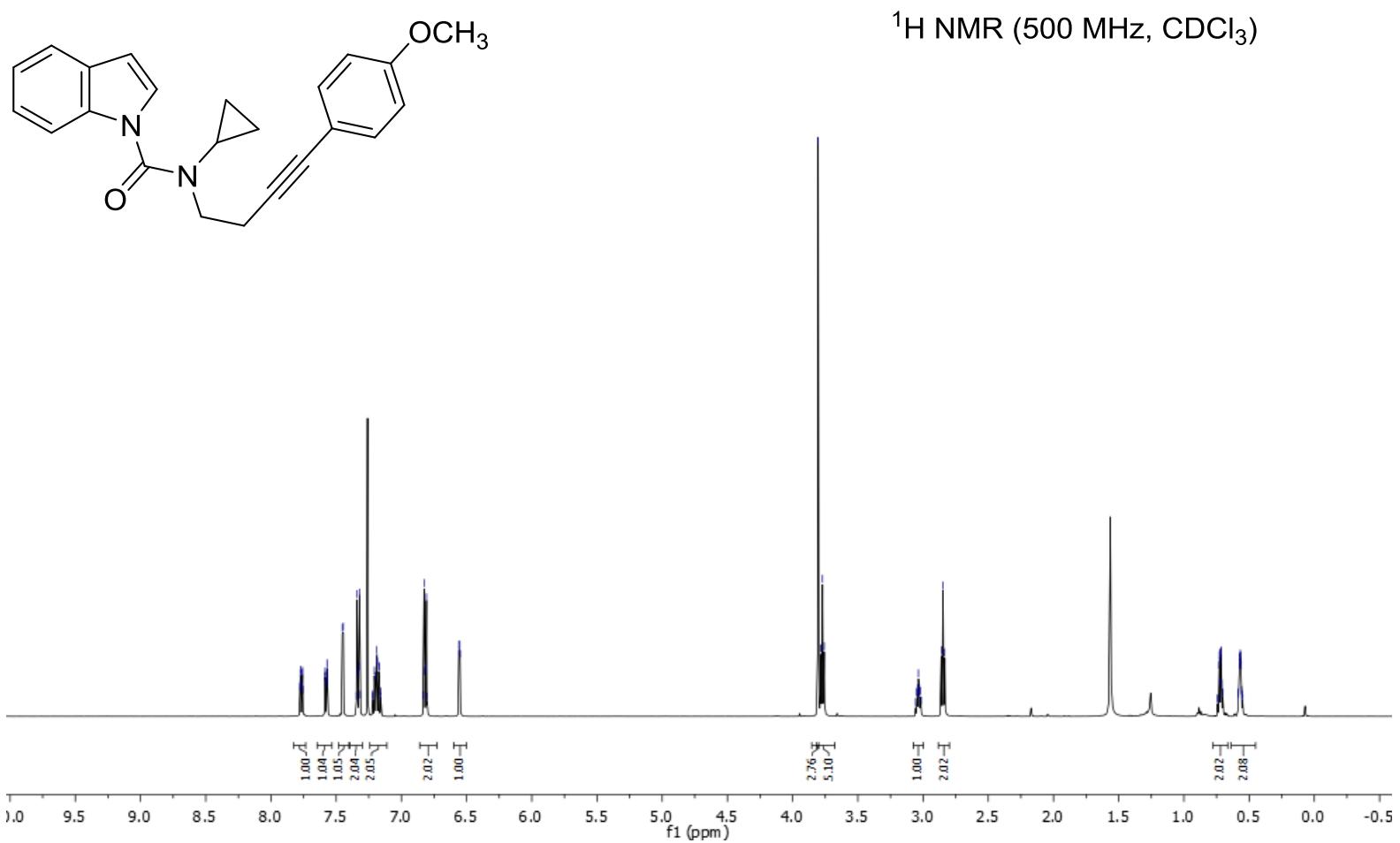

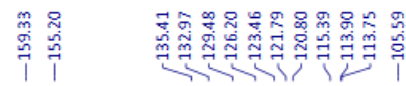
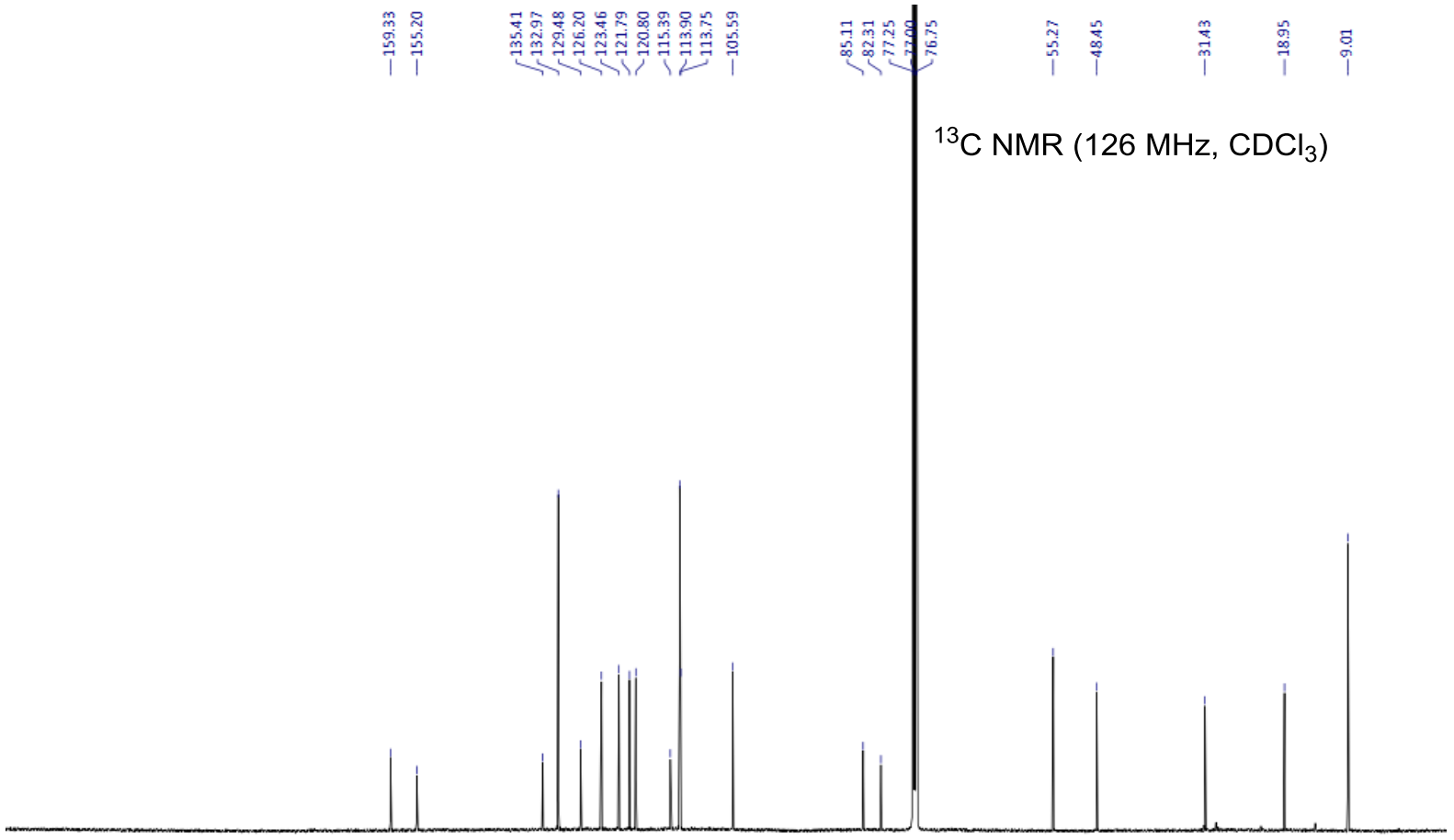

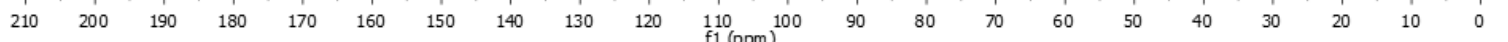


(E)-3-(4-Methoxybenzylidene)-1,2,3,3a,4,5-hexahydro-6H,13Hpyrrolo[1',2':3,4][1,3]diazocino[1,8-a]indole-6,13-dione $6 c$

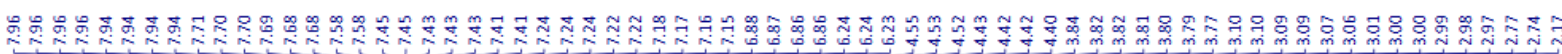<smiles></smiles>

${ }^{1} \mathrm{H} \mathrm{NMR}\left(400 \mathrm{MHz}, \mathrm{CDCl}_{3}\right)$

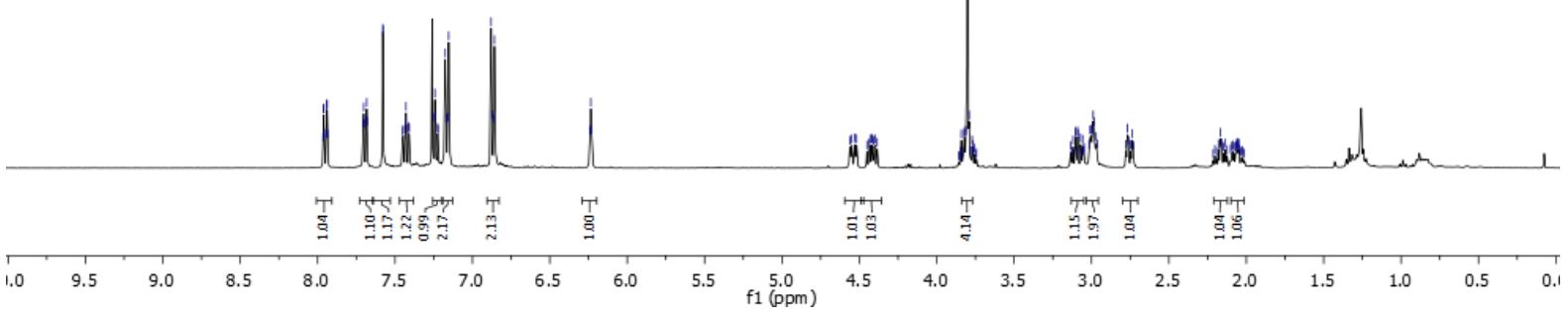

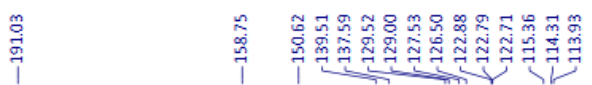

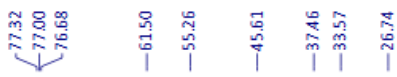

${ }^{3} \mathrm{C}$ NMR $\left(101 \mathrm{MHz}, \mathrm{CDCl}_{3}\right)$

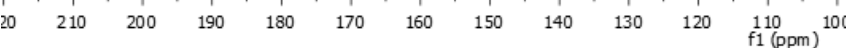


$N$-Cyclopropyl- $N$-(4-phenylbut-3-yn-1-yl)-1H-pyrrole-1-carboxamide 5d

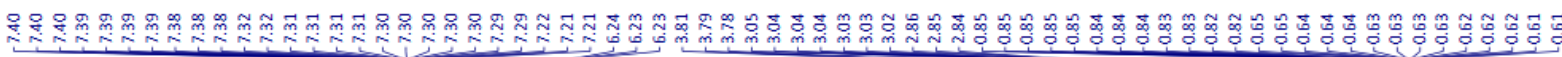<smiles>O=C(N(CCC#Cc1ccccc1)C1CC1)n1cccc1</smiles>

${ }^{1} \mathrm{H}$ NMR $\left(500 \mathrm{MHz}, \mathrm{CDCl}_{3}\right)$
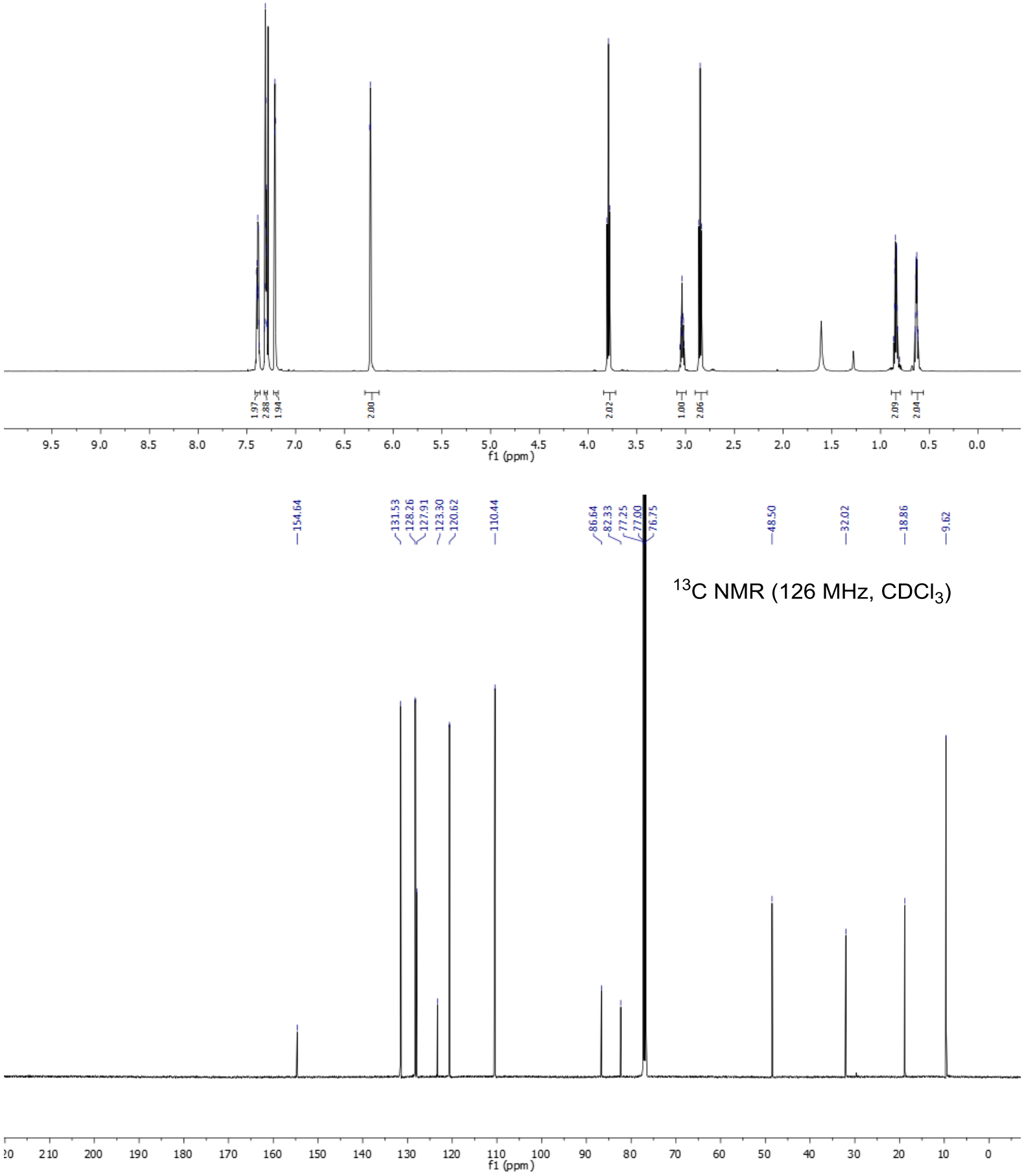
(E)-7-Benzylidene-5,6,6a,7,8,9-hexahydro-4H,11H-dipyrrolo[1,2-c:2',1'-

$h][1,3]$ diazocine-4,11-dione $6 \mathrm{~d}$

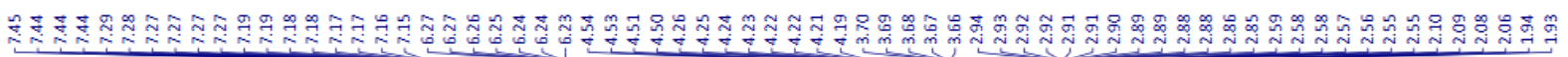
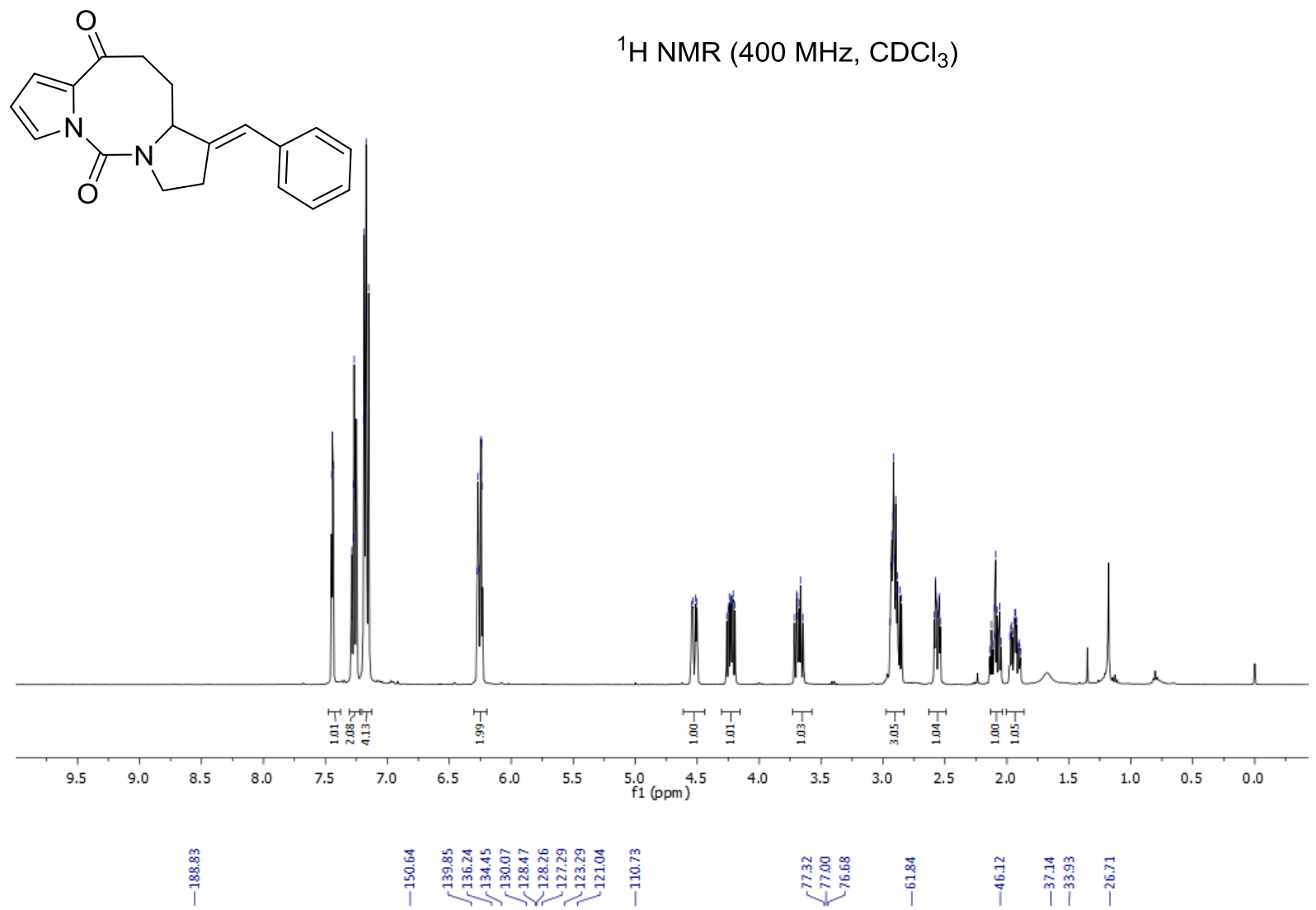

${ }^{13} \mathrm{C}$ NMR $\left(101 \mathrm{MHz}, \mathrm{CDCl}_{3}\right)$

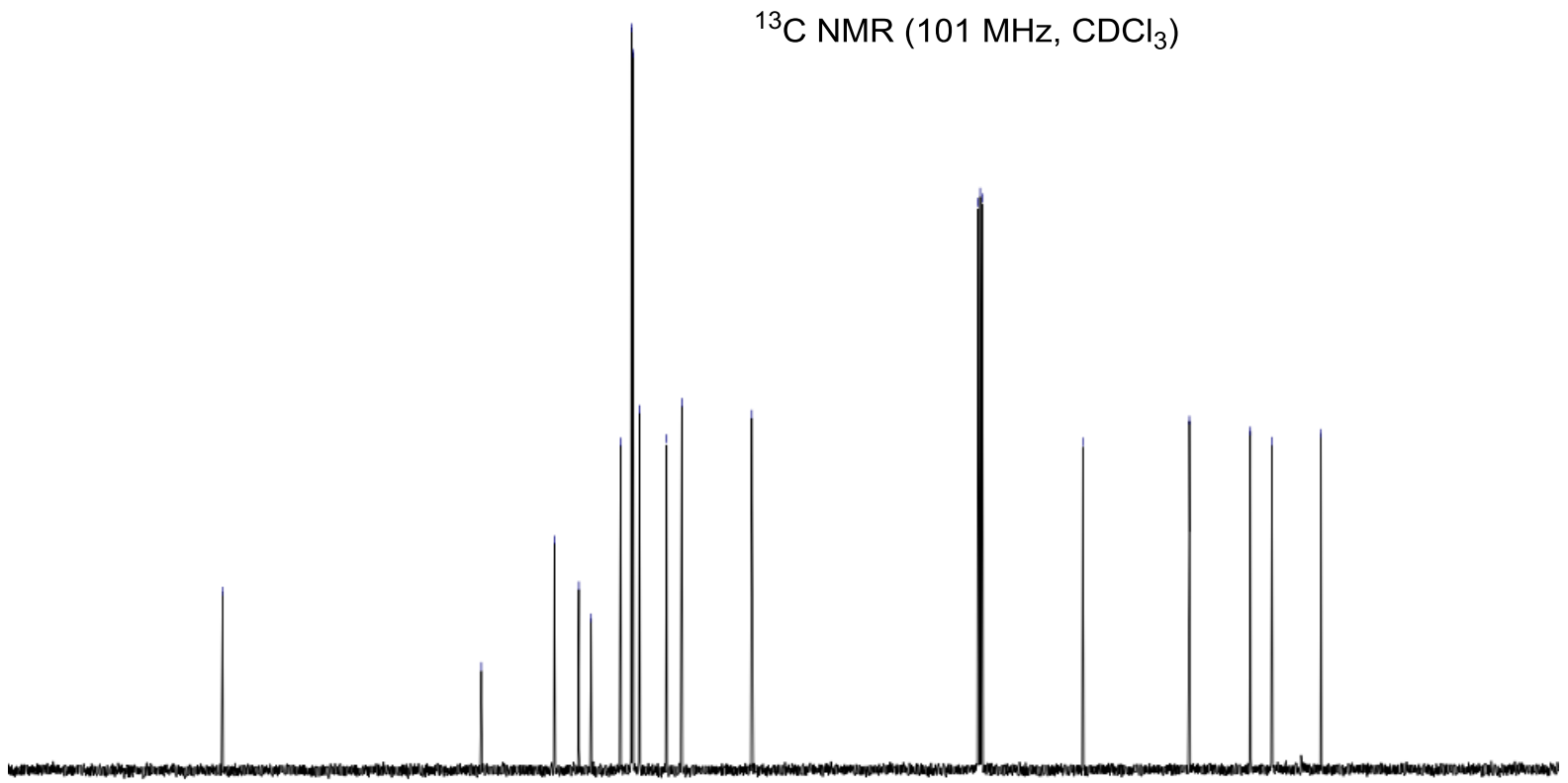

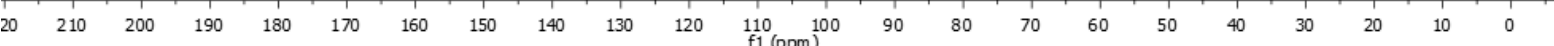


$N$-Cyclopropyl- $N$-(pent-3-yn-1-yl)-1H-pyrrole-1-carboxamide 5e

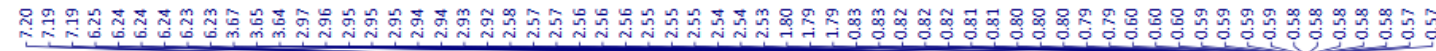
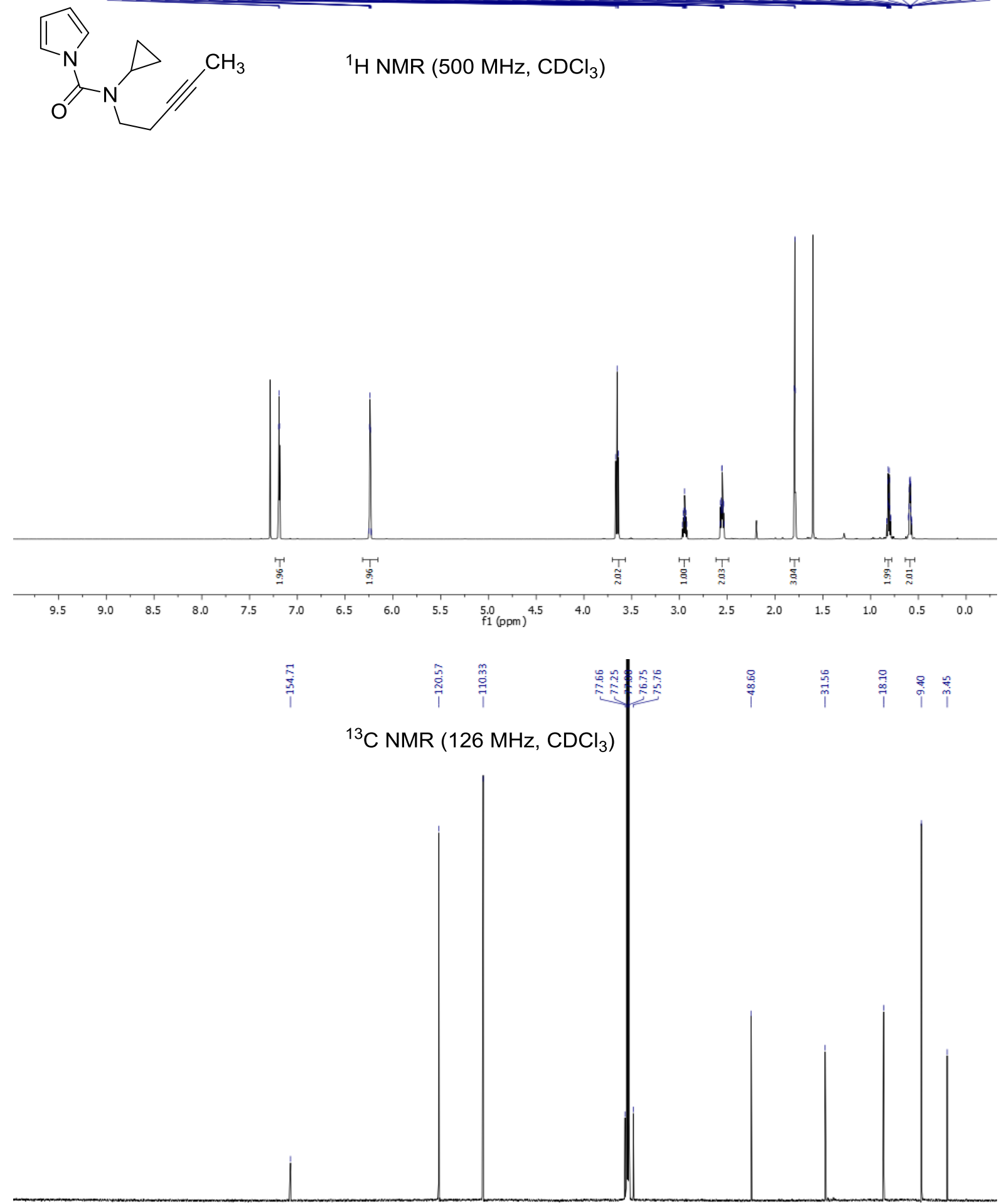

$\begin{array}{lllllllllll}210 & 200 & 190 & 180 & 170 & 160 & 150 & 140 & 130 & 120 & \underset{f 1}{110}(\mathrm{ppm})\end{array}$ 
(E)-7-Ethylidene-5,6,6a,7,8,9-hexahydro-4H,11H-dipyrrolo[1,2-c:2',1'- $h][1,3]$ diazocine-

\section{4,11-dione $6 \mathrm{e}$}

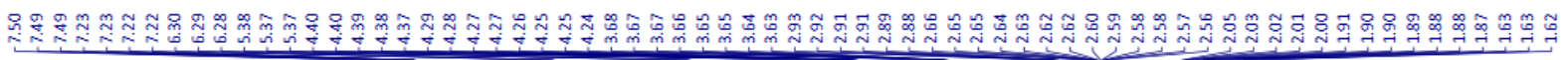
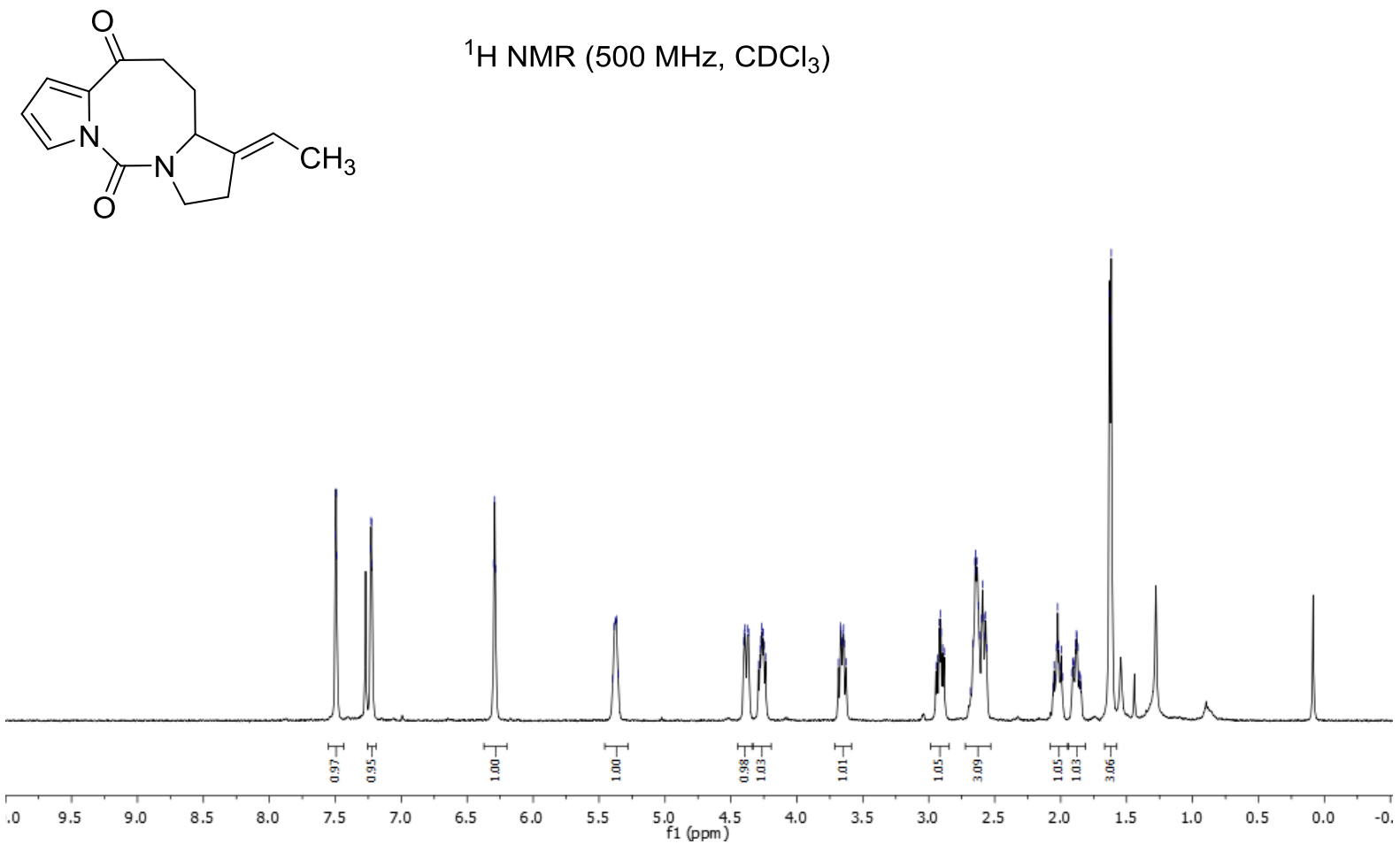

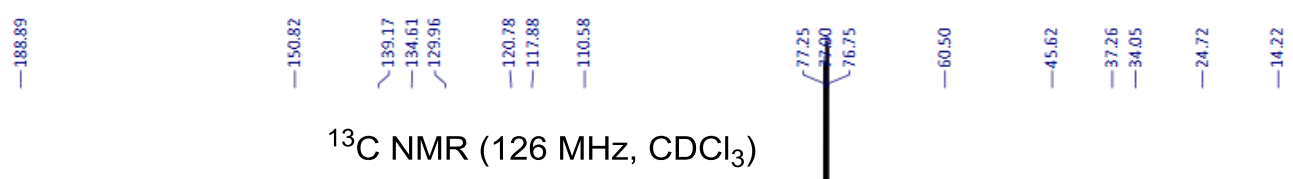

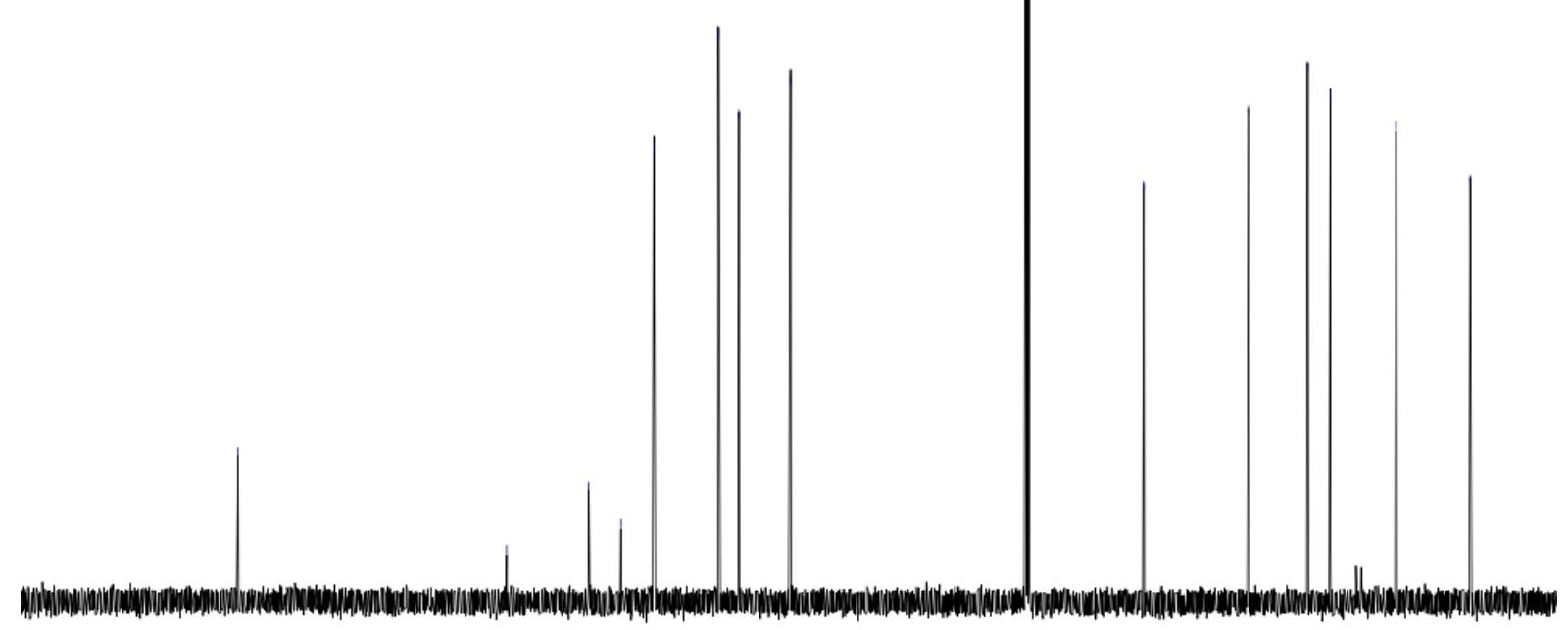

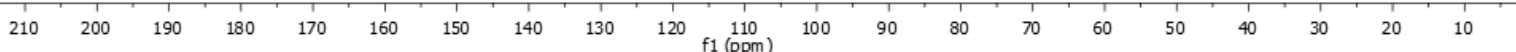


$N$-Cyclopropyl- $N$-(4-phenylbut-3-yn-1-yl)- $1 H$-indole-2-carboxamide $5 f$

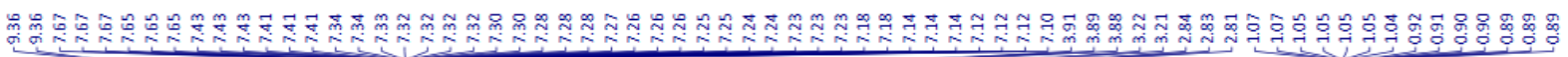

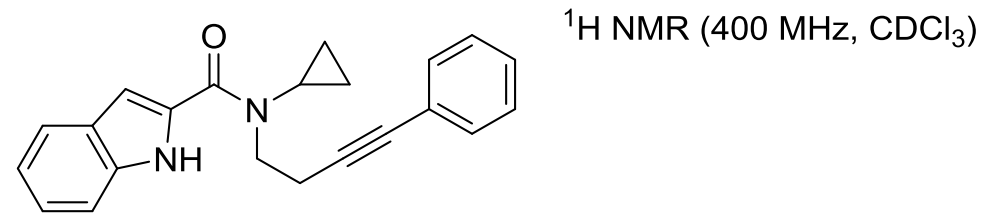

${ }^{1} \mathrm{H} \mathrm{NMR}\left(400 \mathrm{MHz}, \mathrm{CDCl}_{3}\right)$
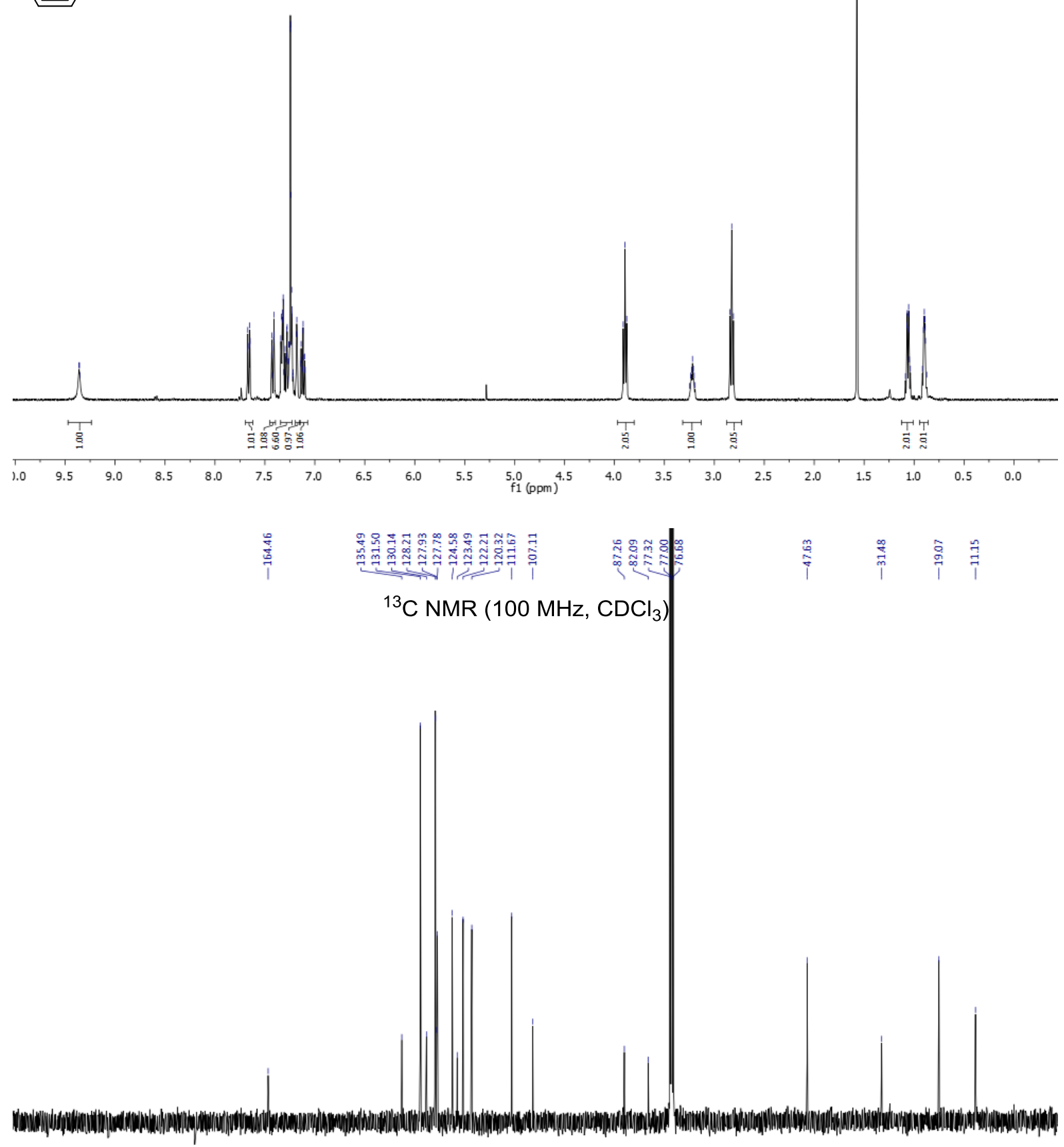

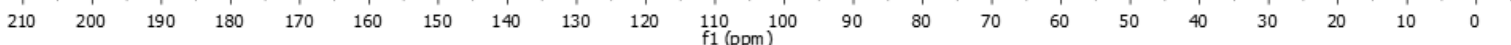


(E)-3-Benzylidene-1,2,3,3a,4,5-hexahydro-6H-pyrrolo[1',2' :1,8]azocino[3,4-b]indole6,12(11H)-dione $6 f$

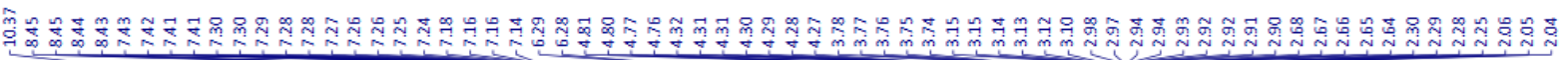<smiles>O=C1CCC2/C(=C/c3ccccc3)CCN2C(=O)c2[nH]c3ccccc3c21</smiles>

${ }^{1} \mathrm{H}$ NMR $\left(500 \mathrm{MHz}, \mathrm{CDCl}_{3}\right)$

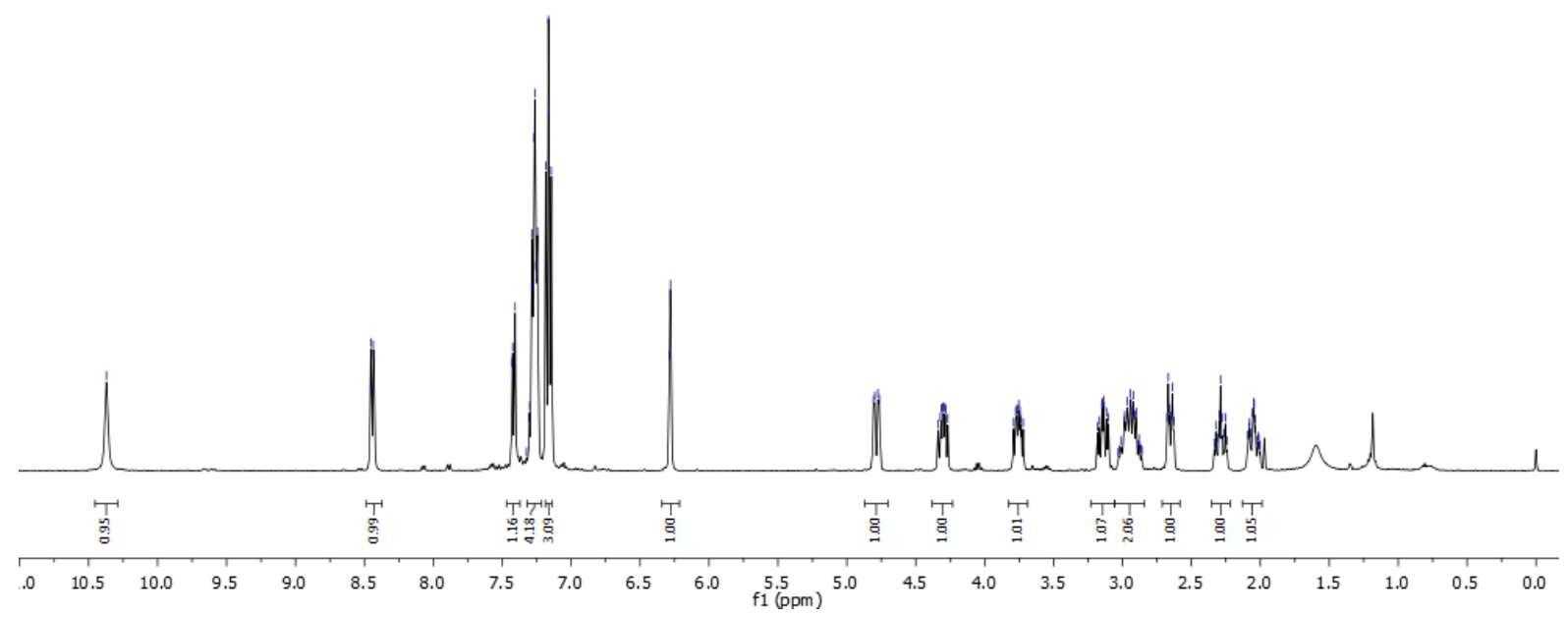

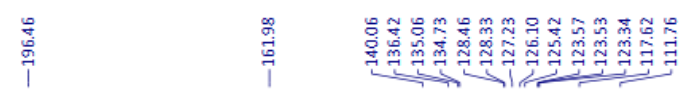

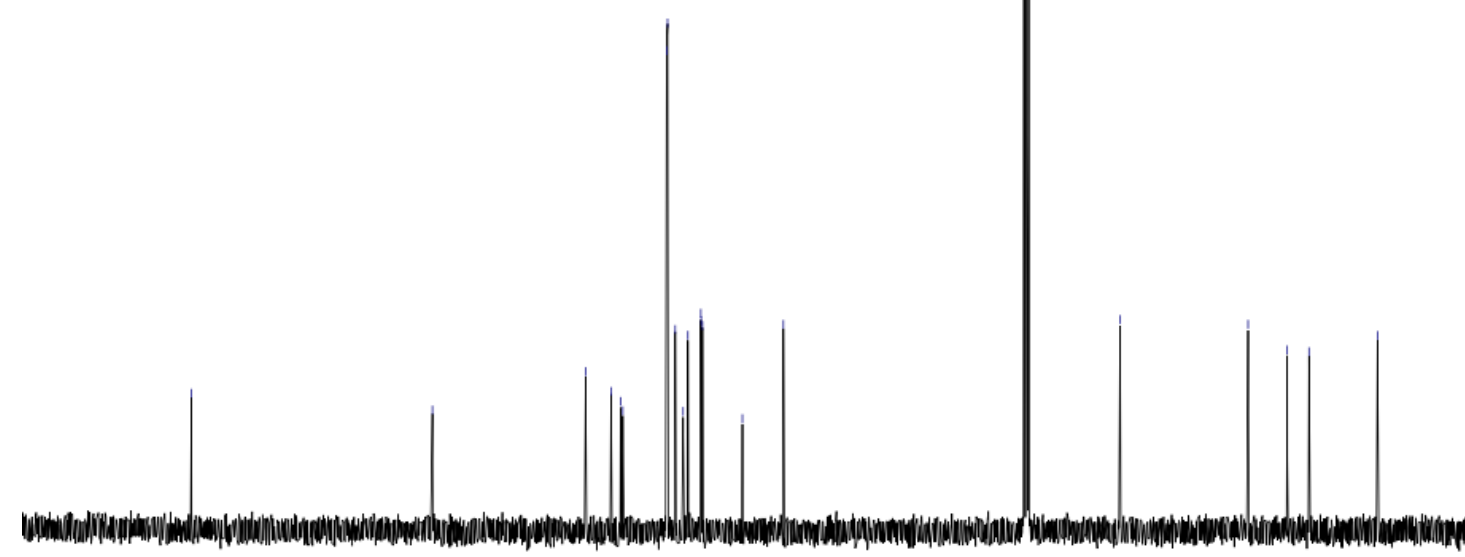

${ }^{13} \mathrm{C} \mathrm{NMR}\left(126 \mathrm{MHz}, \mathrm{CDCl}_{3}\right)$

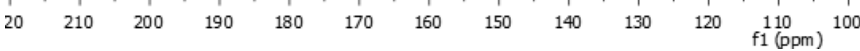




\section{$N$-Cyclopropyl- $N$-(pent-3-yn-1-yl)-1H-indole-2-carboxamide 5g}

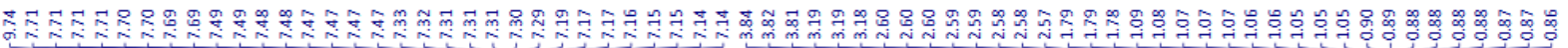<smiles>CC#CCCN(C(=O)c1cc2ccccc2[nH]1)C1CC1</smiles>

${ }^{1} \mathrm{H}$ NMR $\left(400 \mathrm{MHz}, \mathrm{CDCl}_{3}\right)$
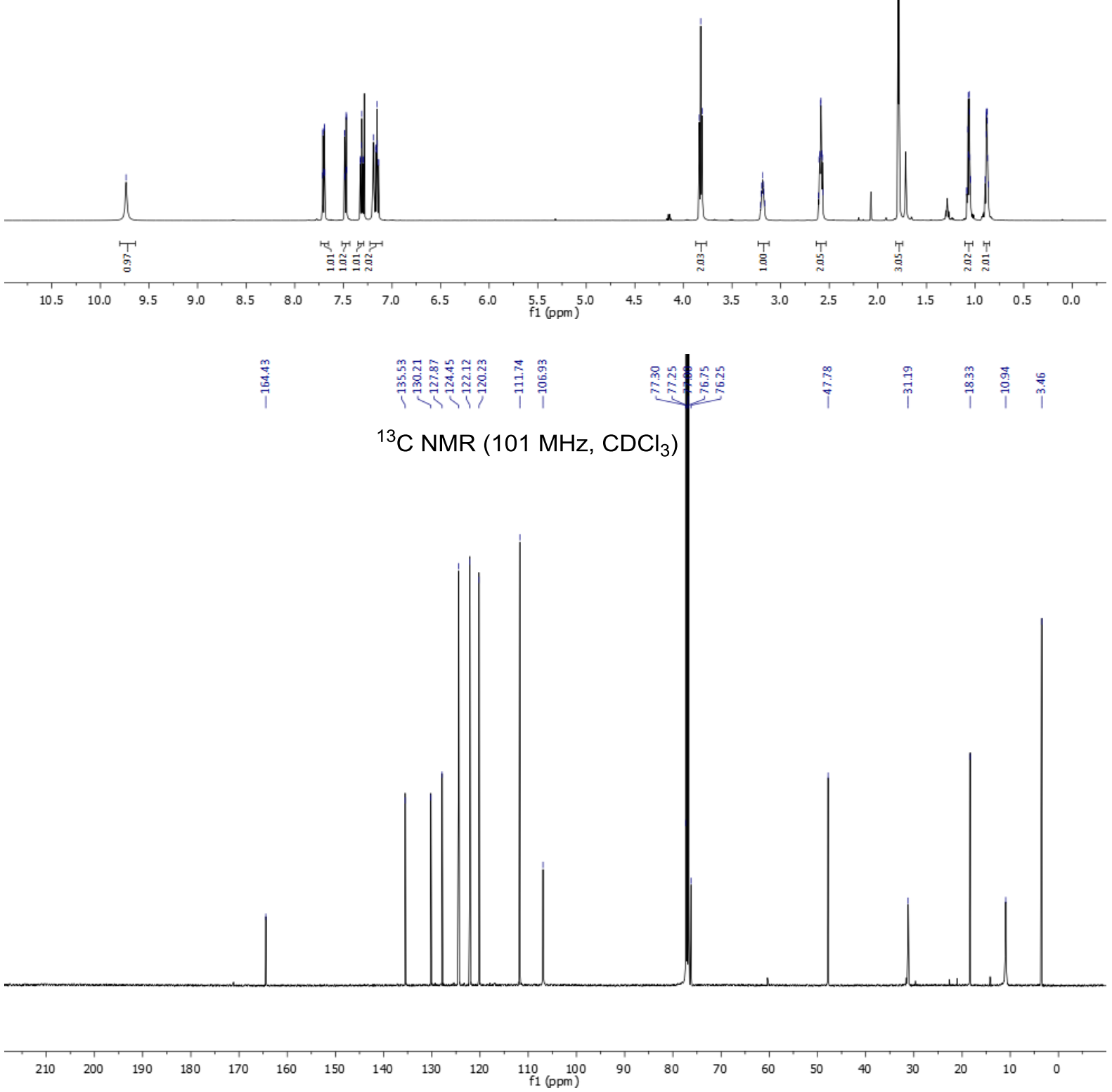
(E)-3-Ethylidene-1,2,3,3a,4,5-hexahydro-6H-pyrrolo[1',2':1,8]azocino[3,4-b]indole6,12(11H)-dione $6 \mathrm{~g}$

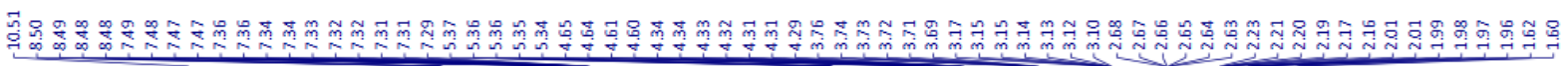<smiles>C/C=C1\CCN2C(=O)c3[nH]c4ccccc4c3C(=O)CCC12</smiles>

${ }^{1} \mathrm{H}$ NMR $\left(500 \mathrm{MHz}, \mathrm{CDCl}_{3}\right)$

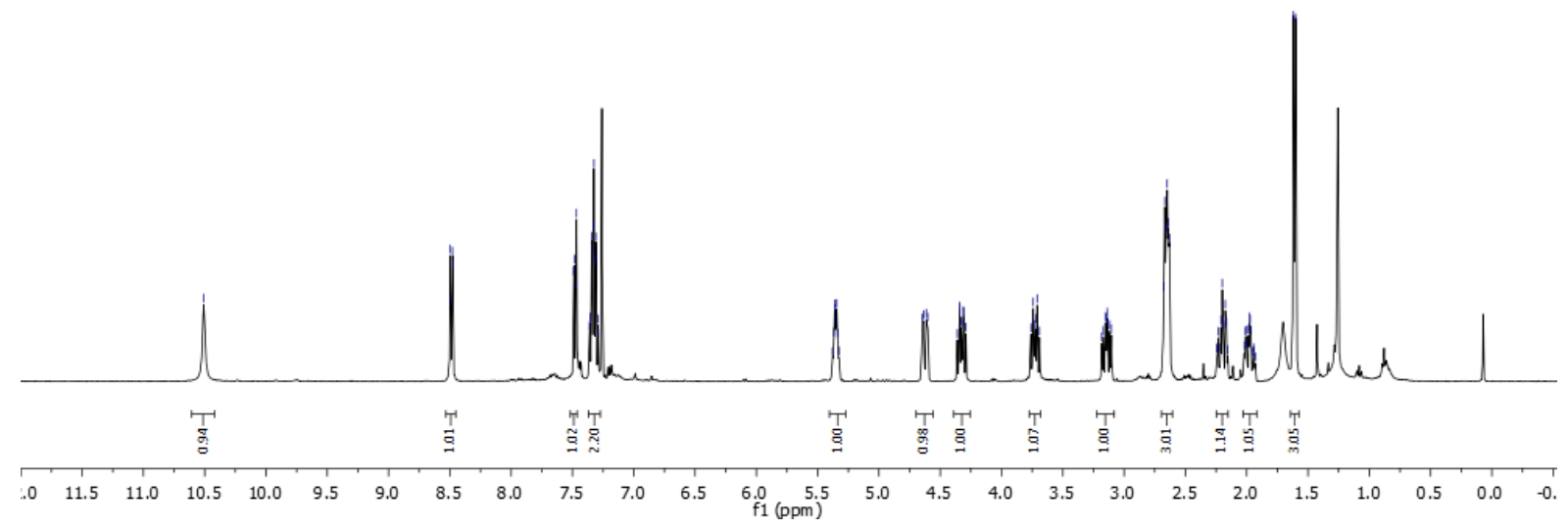

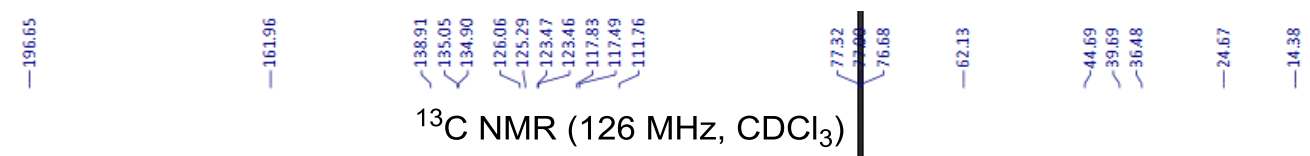

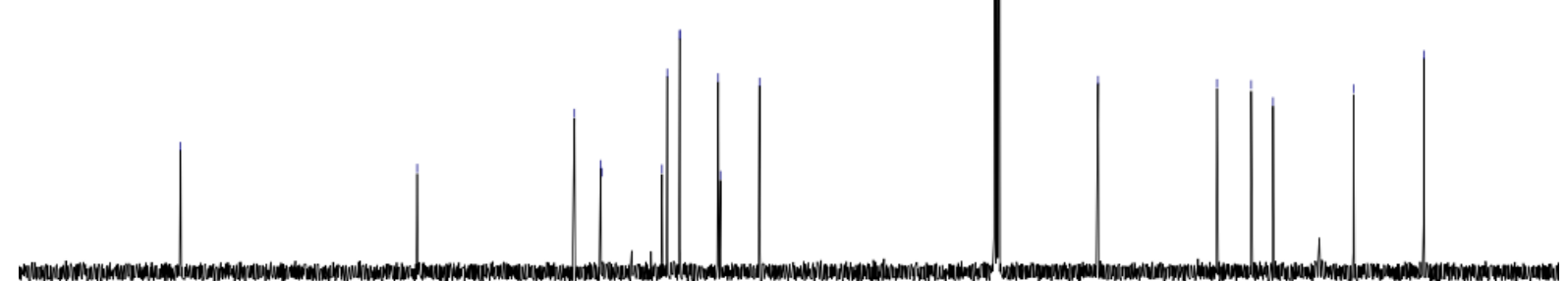

\begin{tabular}{llllllllllllllllllllllllll}
\hline 20 & 210 & 200 & 190 & 180 & 170 & 160 & 150 & 140 & 130 & 120 & 110 & 100 & 90 & 80 & 70 & 60 & 50 & 40 & 30 & 20 & 10 & 0
\end{tabular} 
$N$-Cyclopropyl- $N$-(4-phenylbut-3-yn-1-yl)-1H-pyrrole-2-carboxamide $5 \mathrm{~h}$

$\underbrace{8}$

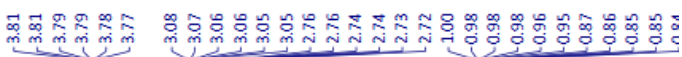
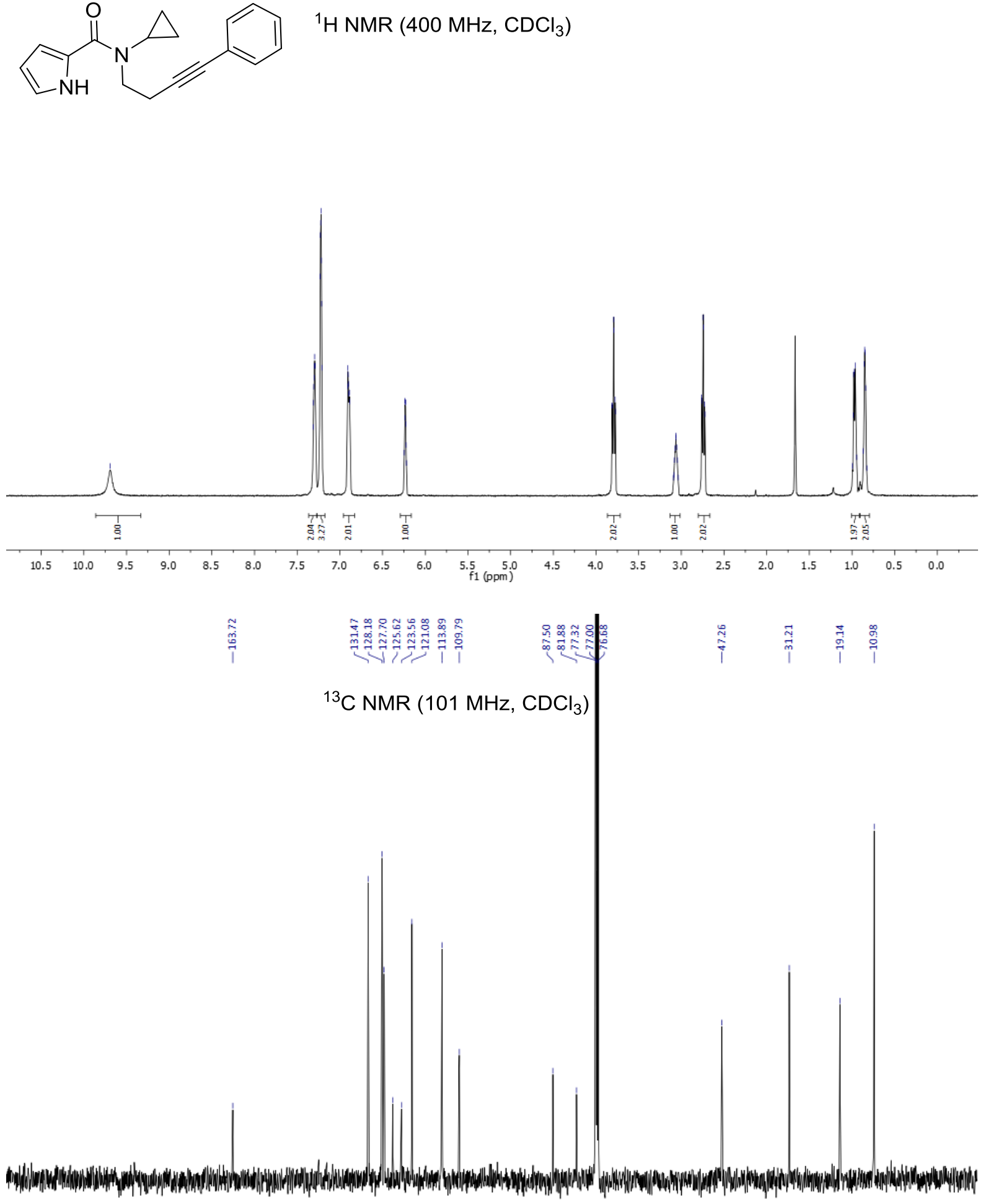

$\begin{array}{lllllllllllllllllllllll}210 & 200 & 190 & 180 & 170 & 160 & 150 & 140 & 130 & 120 & 110 & 100 & 90 & 80 & 70 & 60 & 50 & 40 & 30 & 20 & 10 & 0 & -10\end{array}$ 
(E)-7-Benzylidene-5,6,6a,7,8,9-hexahydro-1H-dipyrrolo[1,2-a:3',2'-f]azocine-4,11-dione 6h

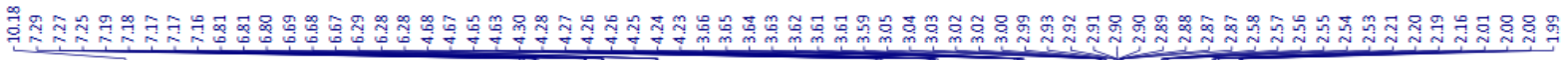

${ }^{1} \mathrm{H}$ NMR $\left(400 \mathrm{MHz}, \mathrm{CDCl}_{3}\right)$<smiles>O=C1CCC2/C(=C/c3ccccc3)CCN2C(=O)c2[nH]ccc21</smiles>
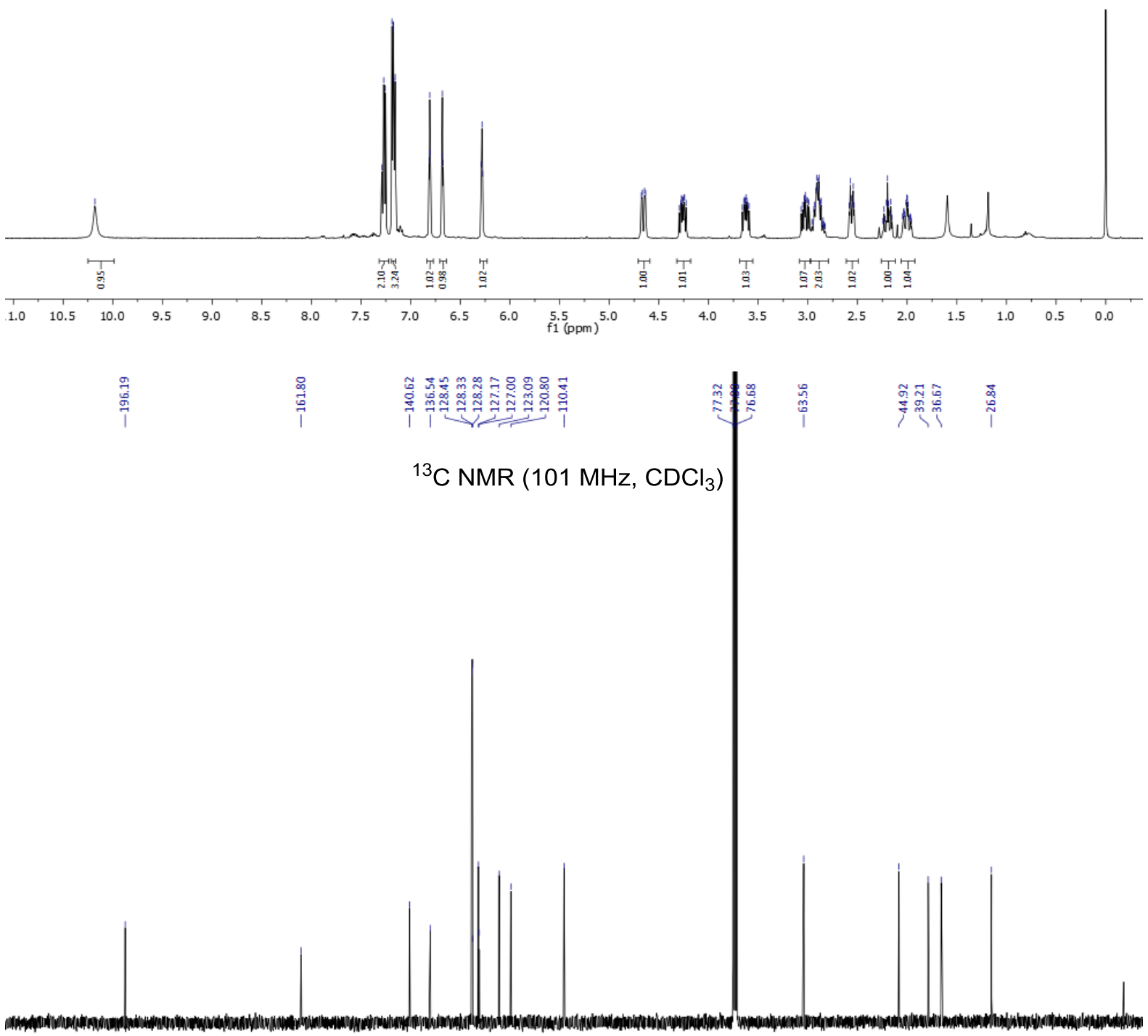

$\begin{array}{lllllllllll}210 & 200 & 190 & 180 & 170 & 160 & 150 & 140 & 130 & 120 & \underset{f 1(\mathrm{ppm})}{110} 100\end{array}$ 
$N$-Cyclopropyl- $N$-(4-phenylbut-3-yn-1-yl)benzofuran-2-carboxamide $5 \mathrm{i}$

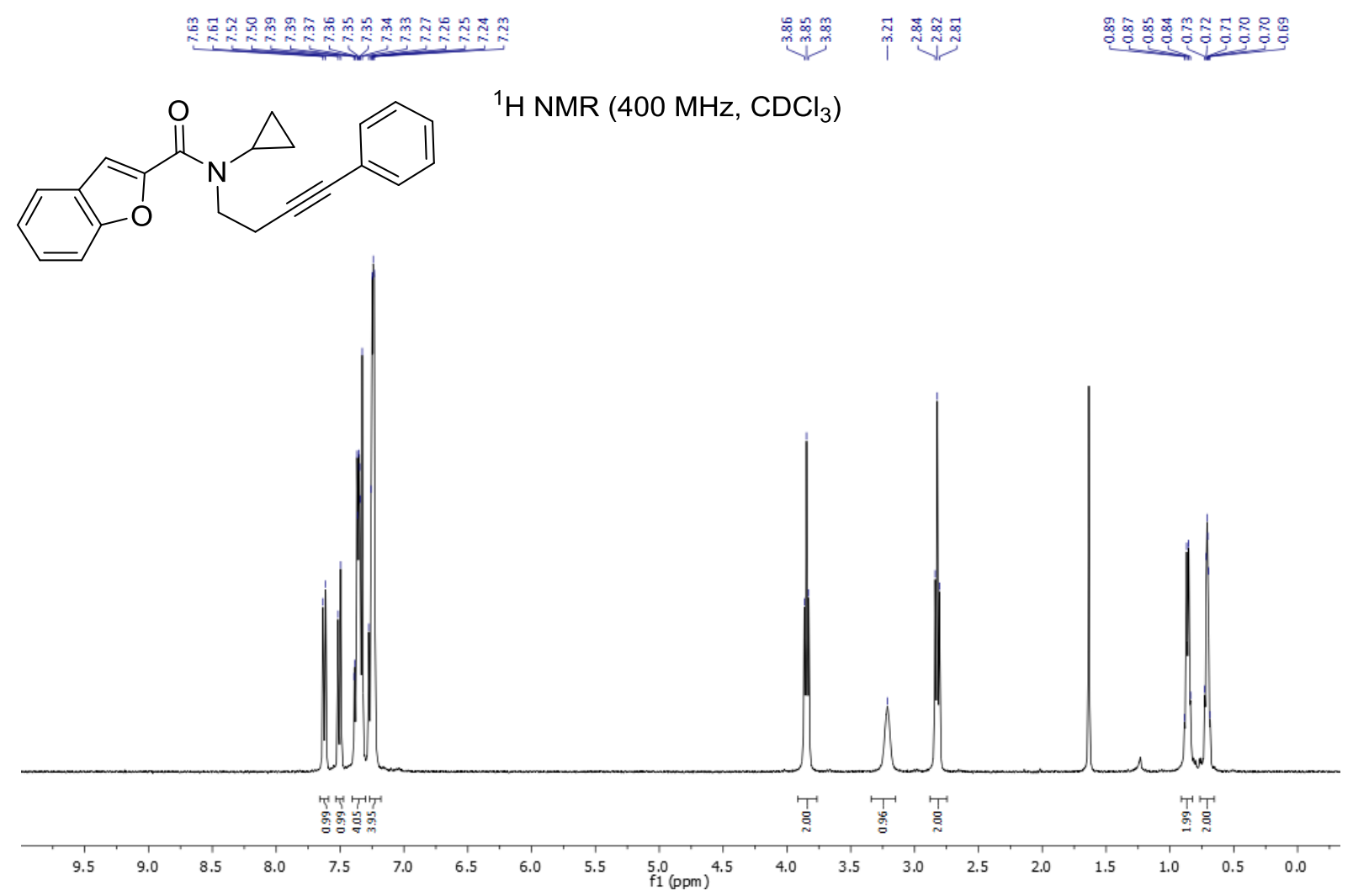

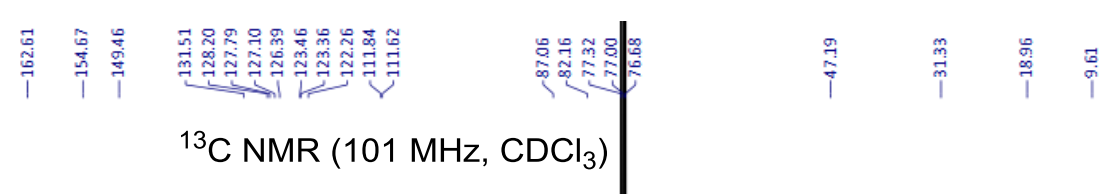

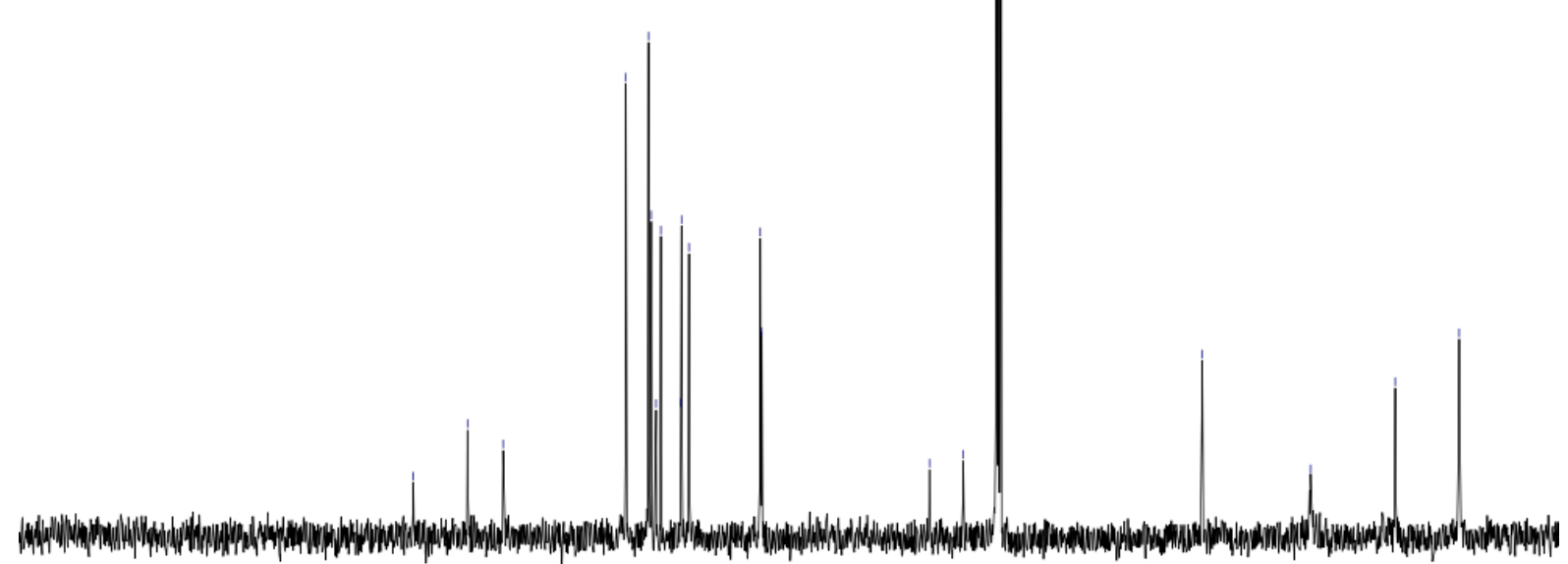

\begin{tabular}{llllllllllllllllllllllllllll}
\hline 20 & 210 & 200 & 190 & 180 & 170 & 160 & 150 & 140 & 130 & 120 & 110 & 100 & 90 & 80 & 70 & 60 & 50 & 40 & 30 & 20 & 10 & 0
\end{tabular} 
(E)-3-Benzylidene-1,2,3,3a,4,5-hexahydrobenzofuro[3,2-f]pyrrolo[1,2-a]azocine-6,12dione $6 \mathbf{i}$

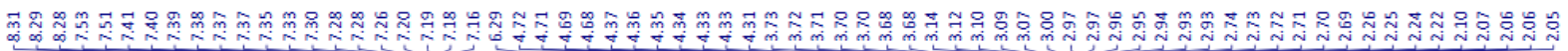<smiles>NC(=O)NNCC(=O)OCc1ccccc1</smiles>
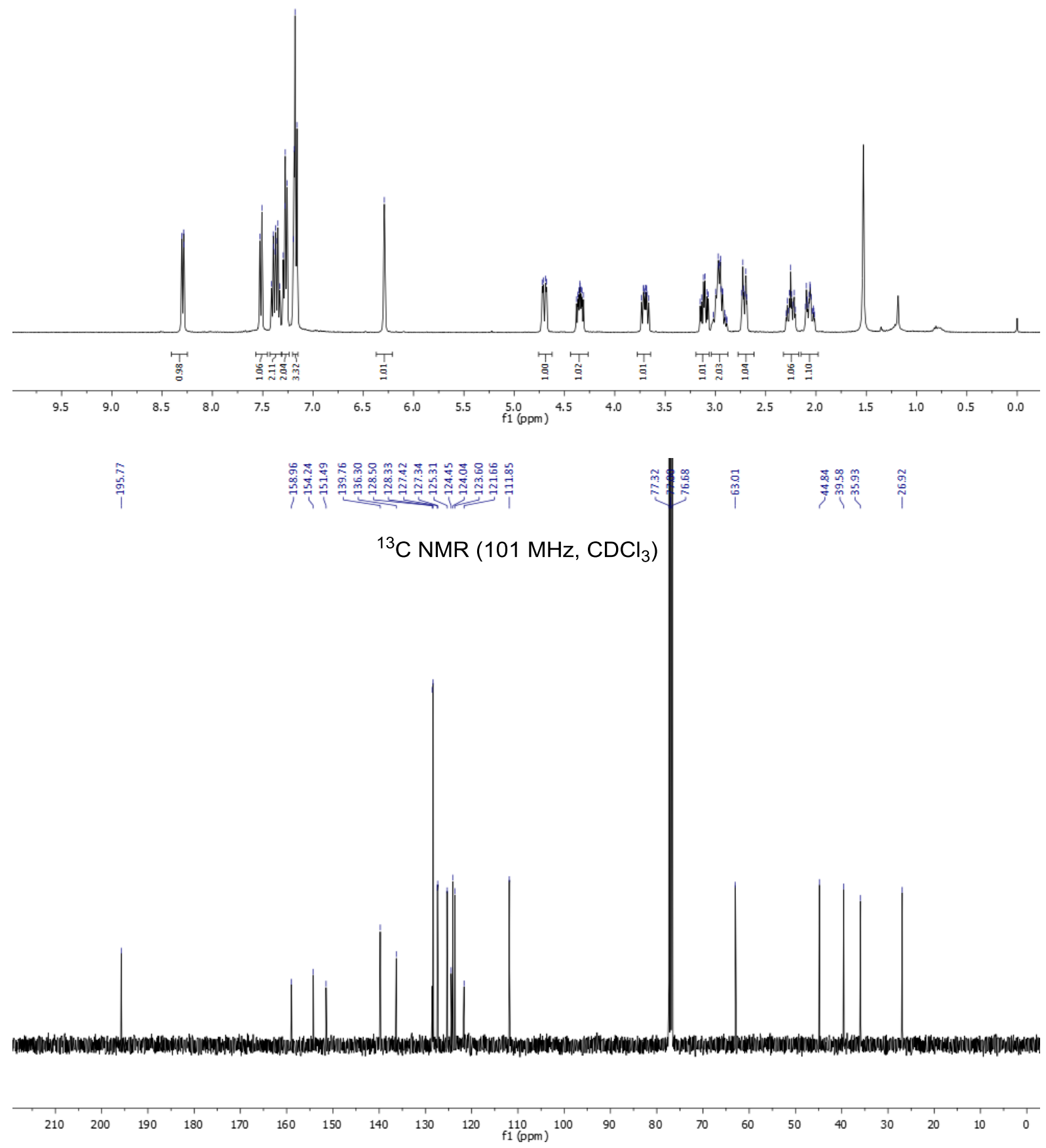
$N$-Cyclopropyl- $N$-(4-phenylbut-3-yn-1-yl)-1H-indole-3-carboxamide $5 \mathrm{j}$

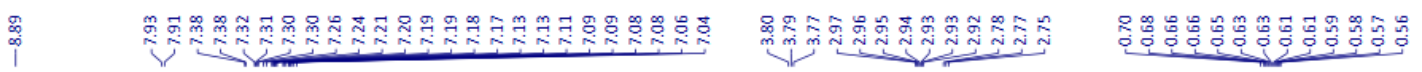
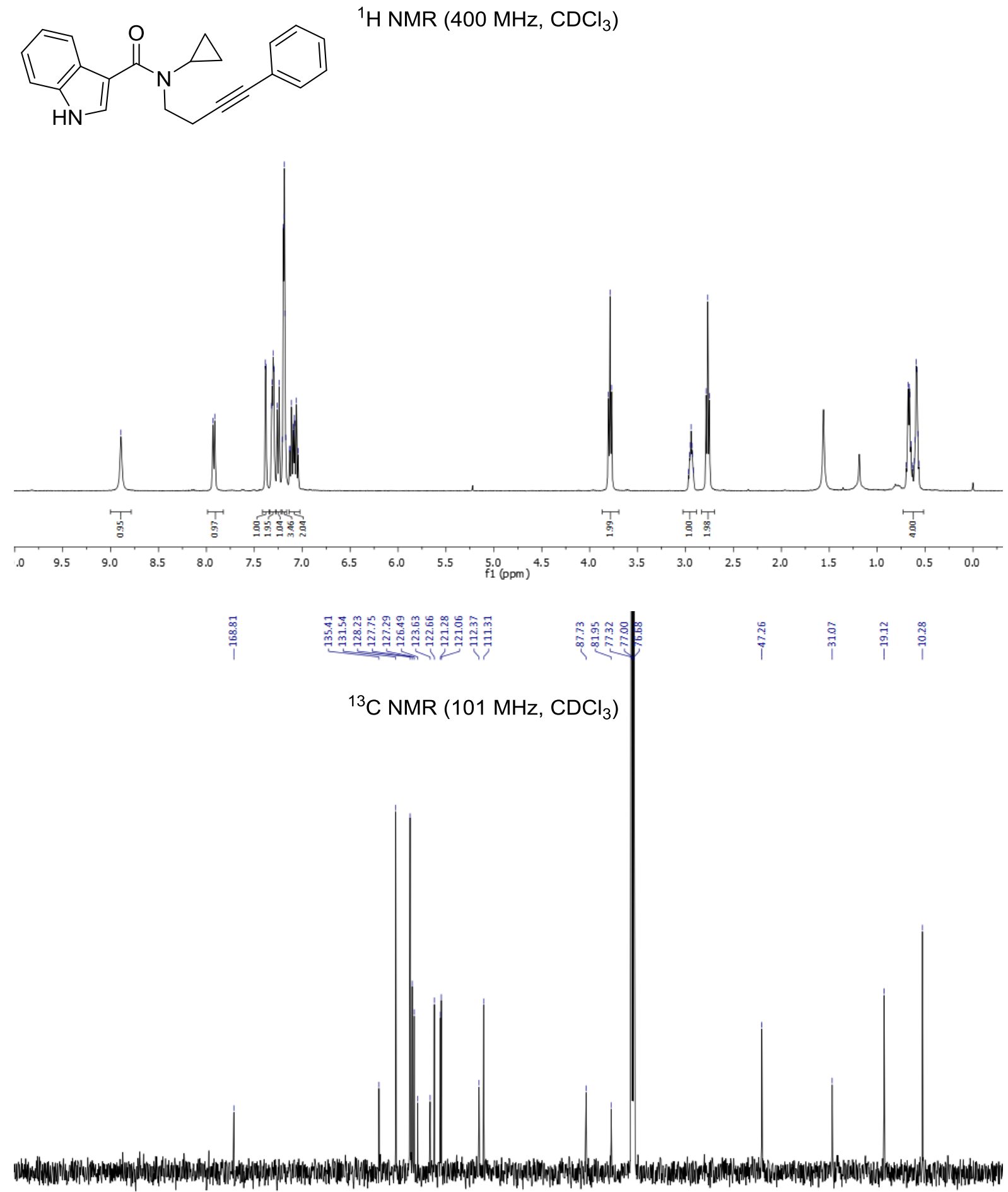

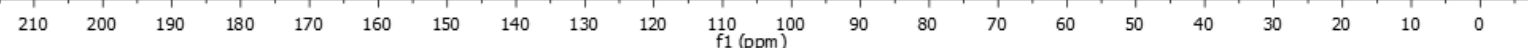


(E)-3-Benzylidene-1,2,3,3a,4,5-hexahydro-6H-pyrrolo[1',2' :1,8]azocino[4,3-b]indole6,12(7H)-dione $6 \mathbf{j}$

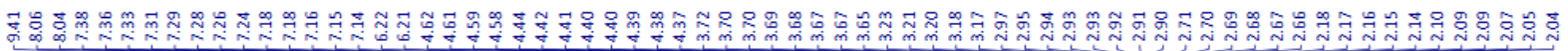
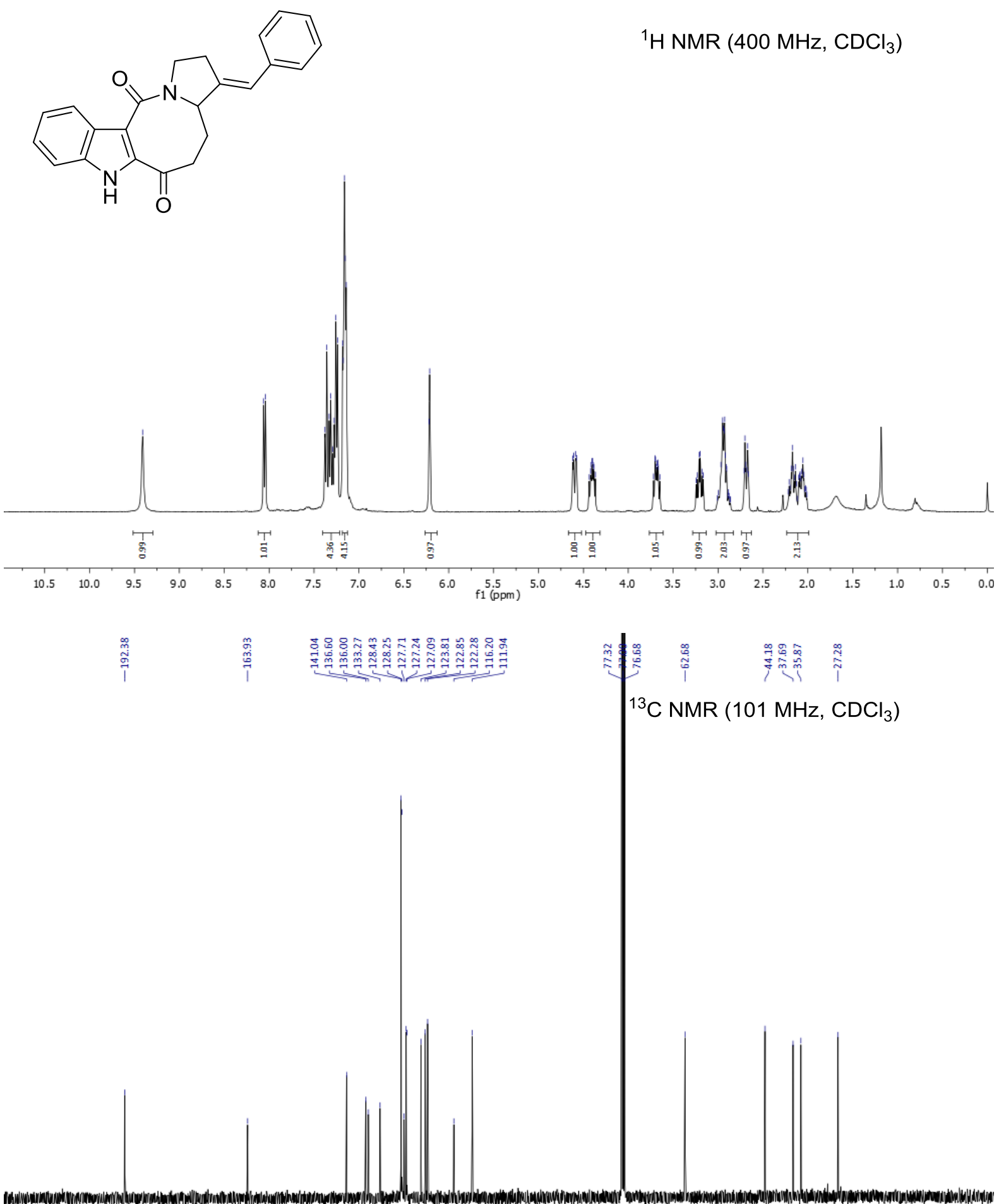

\begin{tabular}{lllllllllllllllllllllllllllllll}
\hline 20 & 210 & 200 & 190 & 180 & 170 & 160 & 150 & 140 & 130 & 120 & 110 & 100 & 90 & 80 & 70 & 60 & 50 & 40 & 30 & 20 & 10 & 0 &
\end{tabular} 
$N$-Cyclopropyl- $N$-(4-phenylbut-3-yn-1-yl)-1H-pyrrole-3-carboxamide 5k

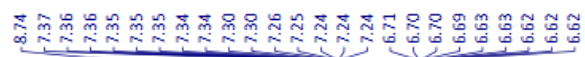

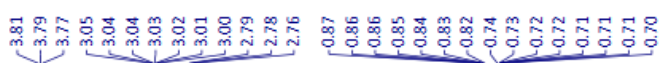
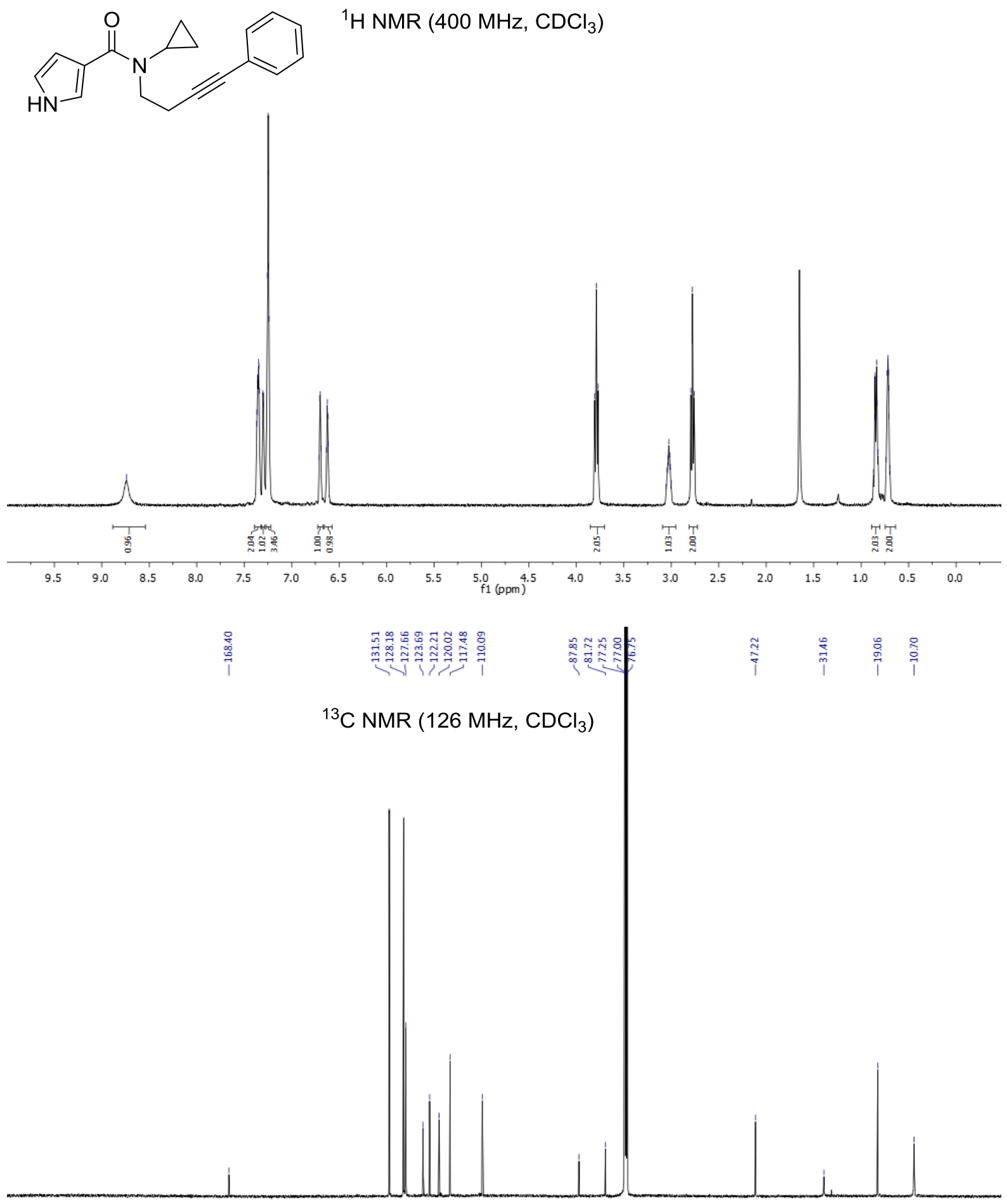

$\begin{array}{lllllllllll}210 & 200 & 190 & 180 & 170 & 160 & 150 & 140 & 130 & 120 & 110 \\ \mathrm{f} 1(\mathrm{ppm}) & 100\end{array}$ 
(E)-7-Benzylidene-5,6,6a,7,8,9-hexahydro-3H-dipyrrolo[1,2-a:2',3'-f]azocine-4,11-dione 6k

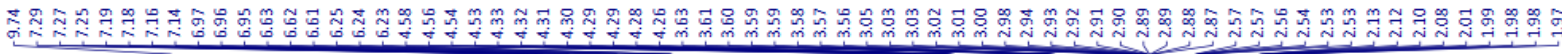<smiles>O=C1CCC2/C(=C/c3ccccc3)CCN2C(=O)c2cc[nH]c21</smiles>

${ }^{1} \mathrm{H} \operatorname{NMR}\left(400 \mathrm{MHz}, \mathrm{CDCl}_{3}\right)$

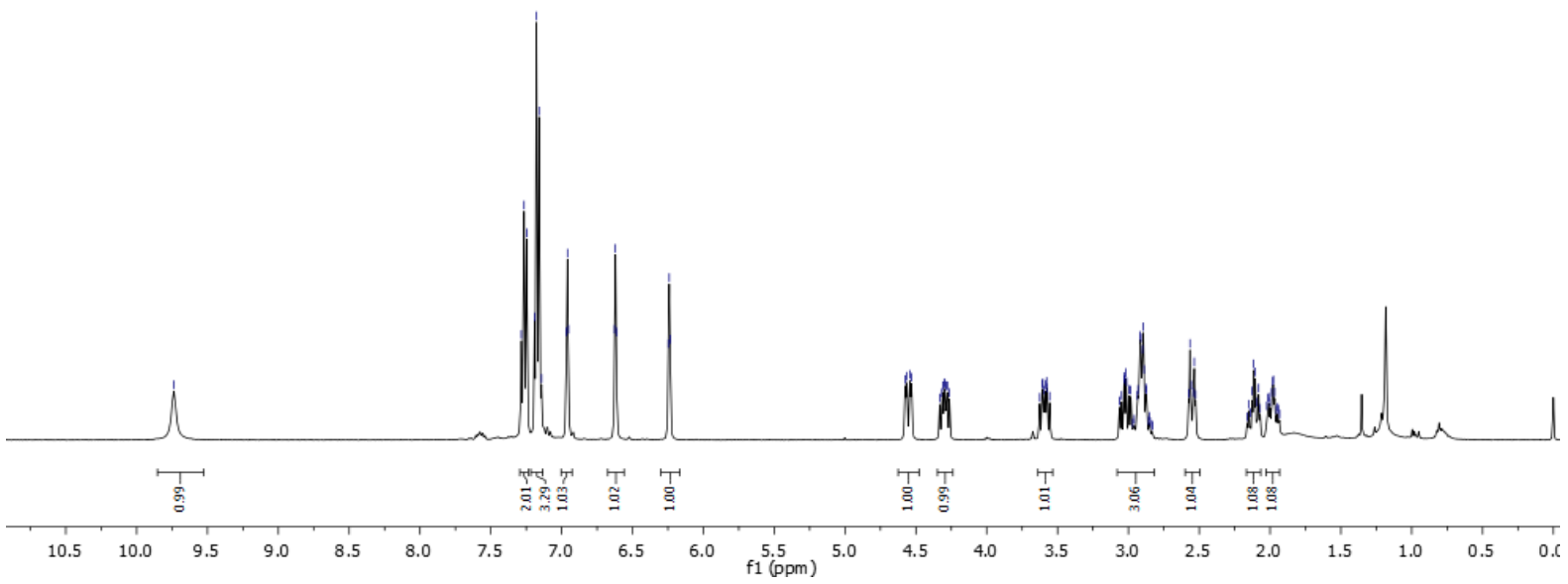

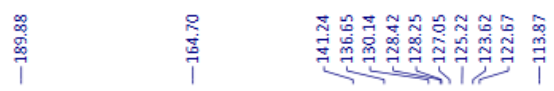

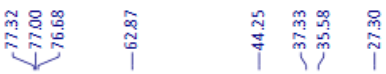

${ }^{13} \mathrm{C}$ NMR $\left(101 \mathrm{MHz}, \mathrm{CDCl}_{3}\right)$

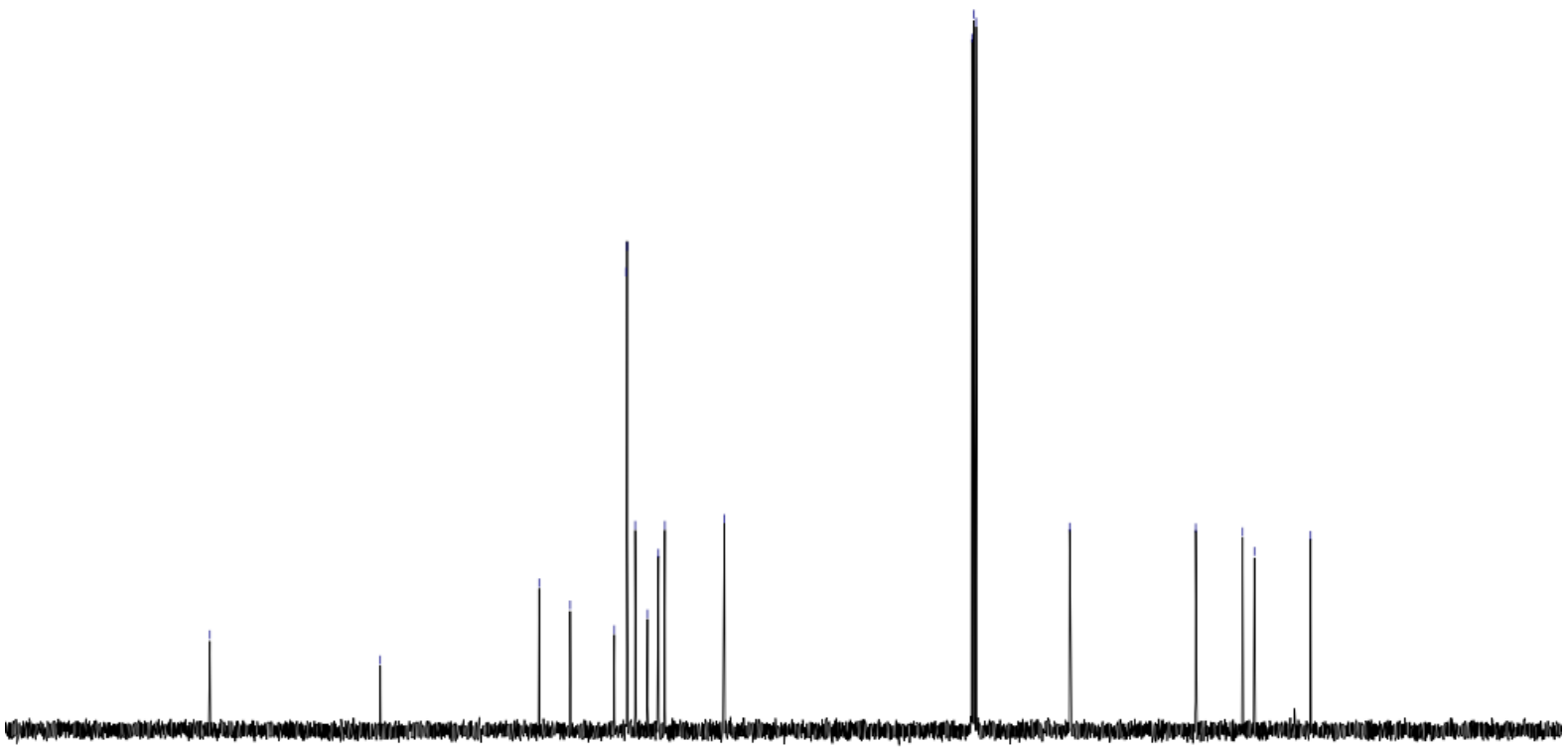

$\begin{array}{llllllllllll}20 & 210 & 200 & 190 & 180 & 170 & 160 & 150 & 140 & 130 & 120 & \begin{array}{l}110 \\ \mathrm{f} 1(\mathrm{ppm})\end{array}\end{array}$ 
$N$-Cyclopropyl- $N$-(pent-3-yn-1-yl)-1H-pyrrole-3-carboxamide 51

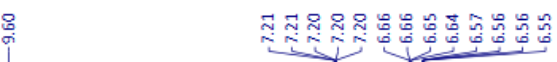

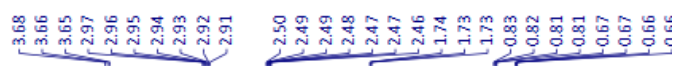<smiles>CC#CCCN(C(=O)c1cc[nH]c1)C1CC1</smiles>

${ }^{1} \mathrm{H} \mathrm{NMR}\left(400 \mathrm{MHz}, \mathrm{CDCl}_{3}\right)$

$\mathrm{HN}$

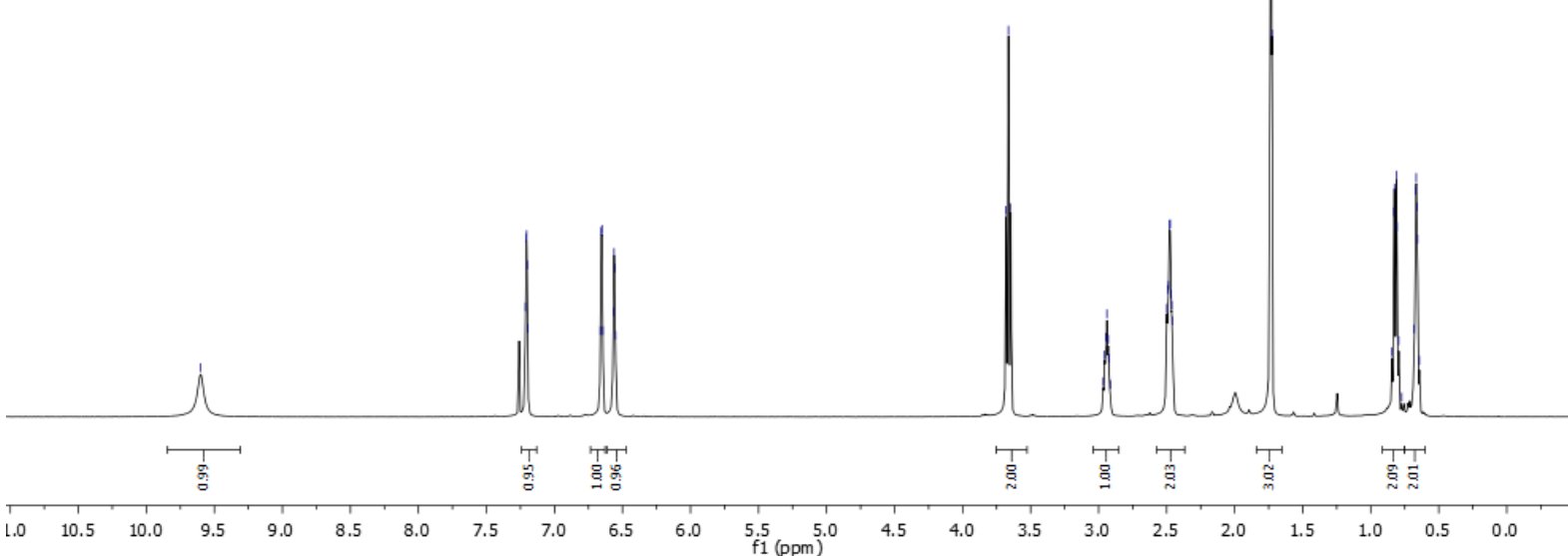

${ }^{13} \mathrm{C} \mathrm{NMR}\left(126 \mathrm{MHz}, \mathrm{CDCl}_{3}\right)$

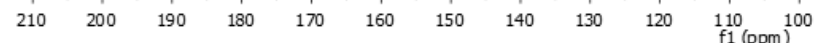

$\left.{ }_{1}^{10}(\mathrm{ppm})\right)^{100}$ 
(E)-7-Ethylidene-5,6,6a,7,8,9-hexahydro-3H-dipyrrolo[1,2-a:2',3'-f]azocine-4,11-dione 61

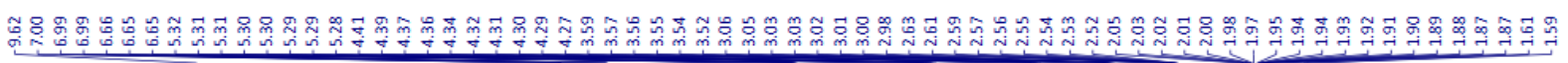<smiles>CC=C1CCN2C(=O)c3cc[nH]c3C(=O)CCC12</smiles>

${ }^{1} \mathrm{H}$ NMR $\left(400 \mathrm{MHz}, \mathrm{CDCl}_{3}\right)$

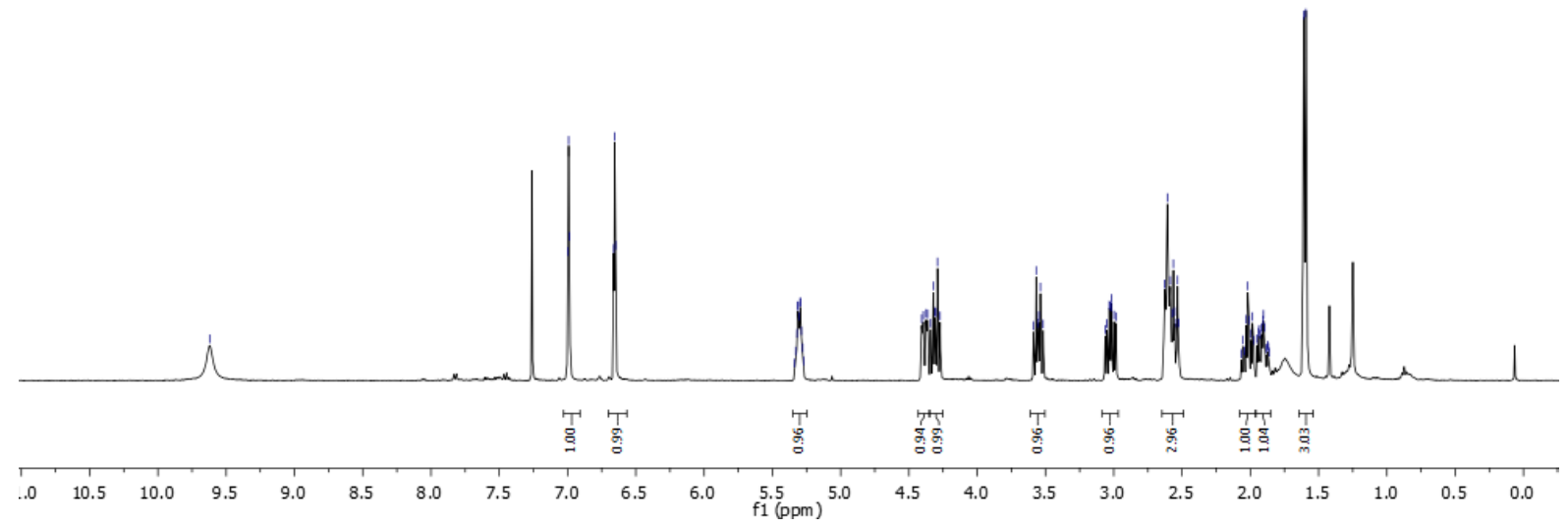

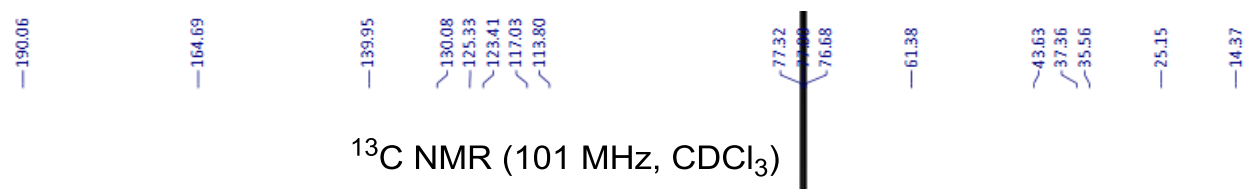

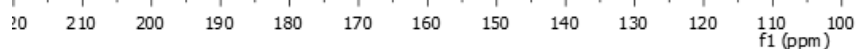


$N$-Cyclopropyl- $N$-(4-phenylbut-3-yn-1-yl)furan-3-carboxamide $5 \mathrm{~m}$

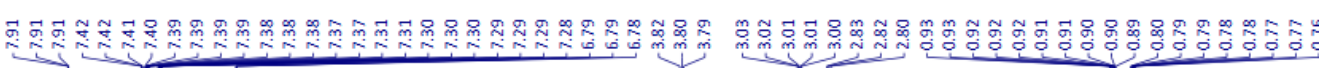

${ }^{1} \mathrm{H} \mathrm{NMR}\left(500 \mathrm{MHz}, \mathrm{CDCl}_{3}\right)$<smiles>O=C(c1ccoc1)N(CCC#Cc1ccccc1)C1CC1</smiles>

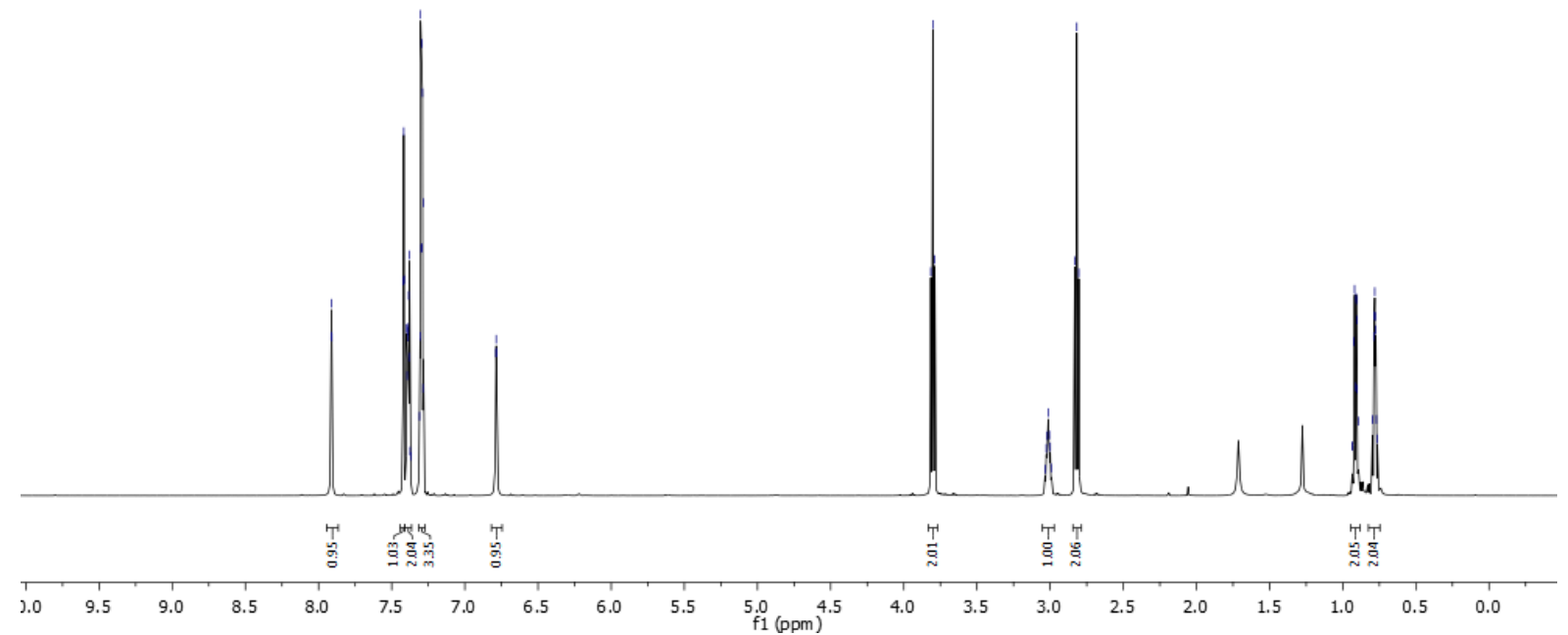
总

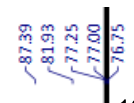

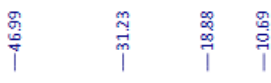

${ }^{13} \mathrm{C} \mathrm{NMR}\left(126 \mathrm{MHz}, \mathrm{CDCl}_{3}\right)$

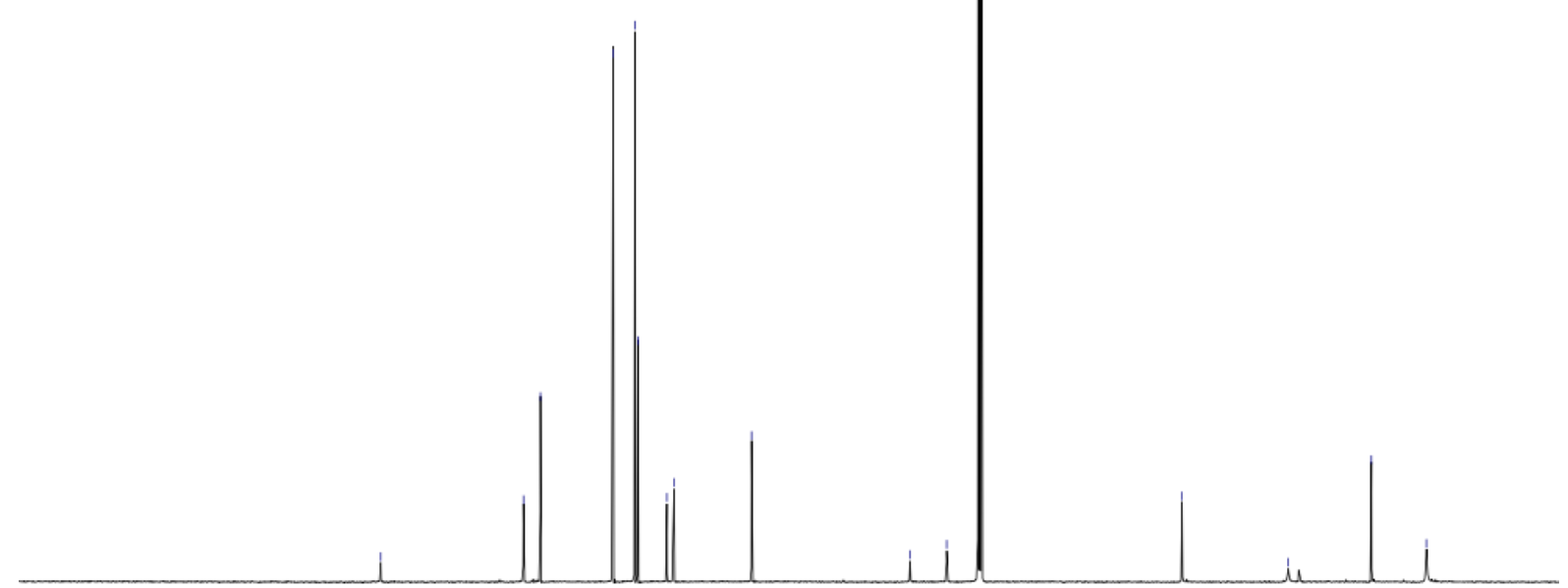

$\begin{array}{llllllllllll}210 & 200 & 190 & 180 & 170 & 160 & 150 & 140 & 130 & 120 & 110 & 100\end{array}$

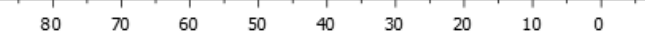


(E)-7-Benzylidene-5,6,6a,7,8,9-hexahydrofuro[2,3-f]pyrrolo[1,2-a]azocine-4,11-dione 6m

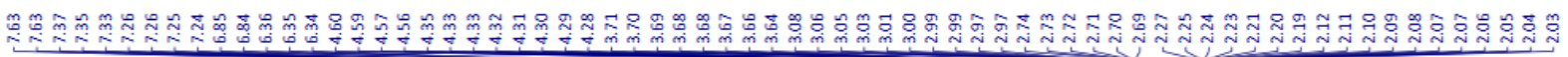
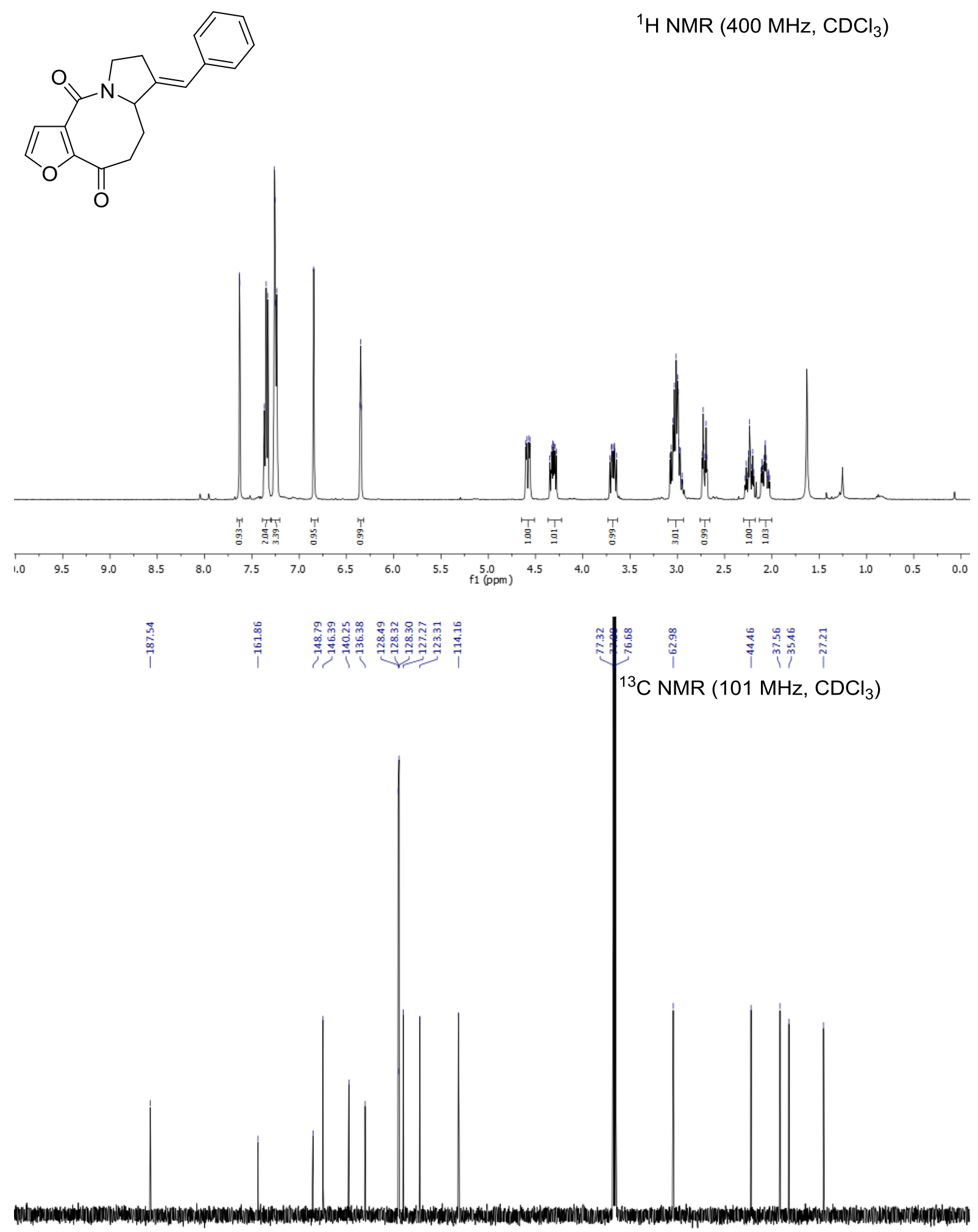

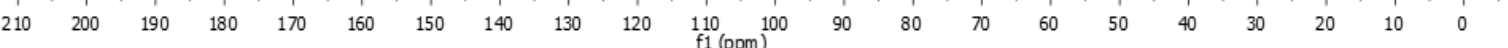


$N$-cyclopropyl- $N$-(4-phenylbut-3-yn-1-yl)thiophene-3-carboxamide 5n

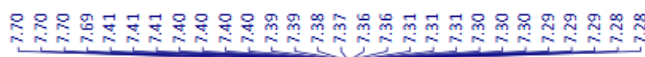<smiles>O=C(c1ccsc1)N(CCC#Cc1ccccc1)C1CC1</smiles>
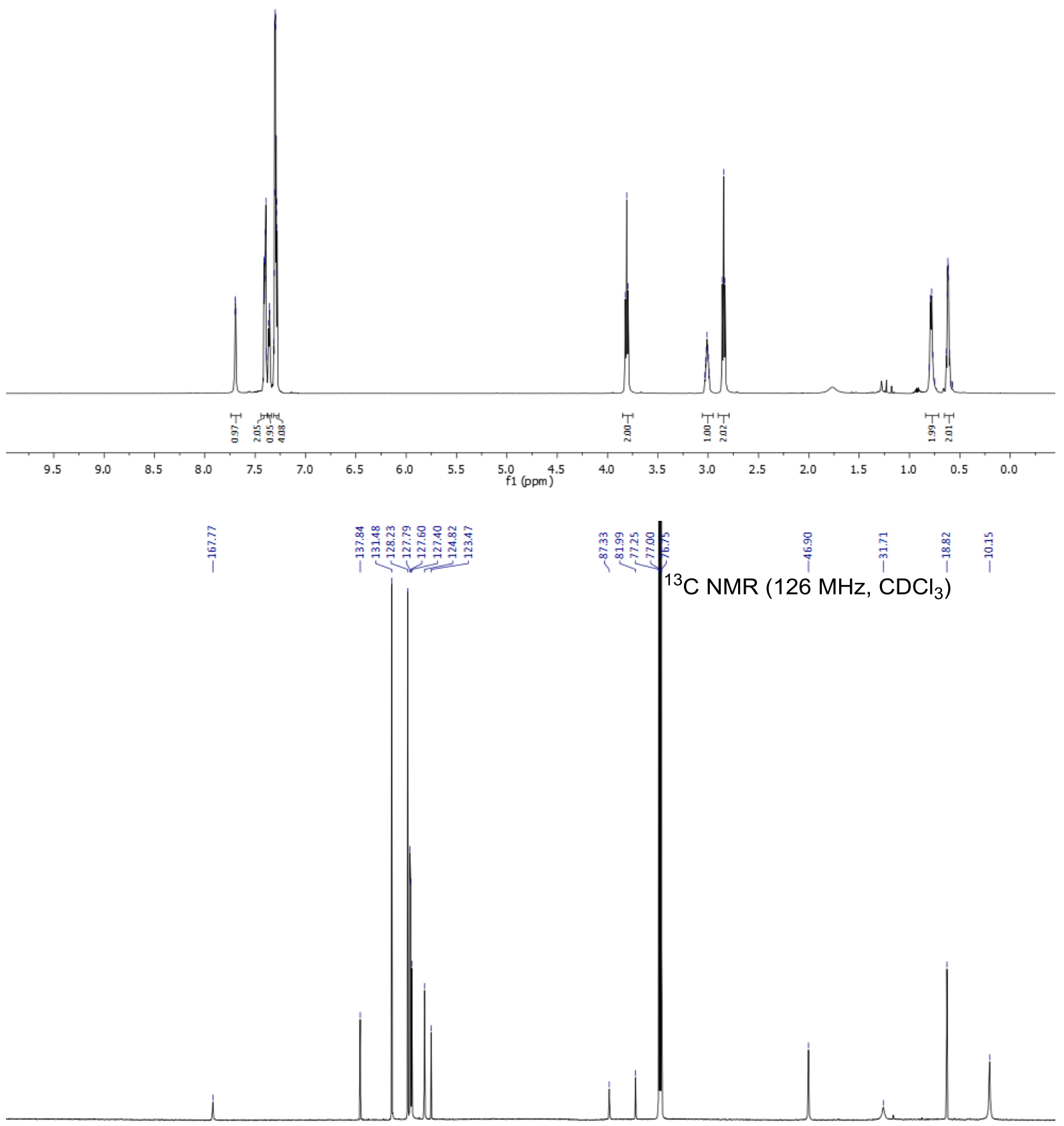

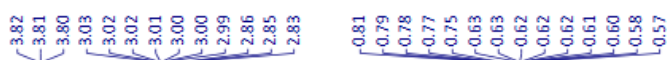

${ }^{1} \mathrm{H}$ NMR (500 $\left.\mathrm{MHz}, \mathrm{CDCl}_{3}\right)$

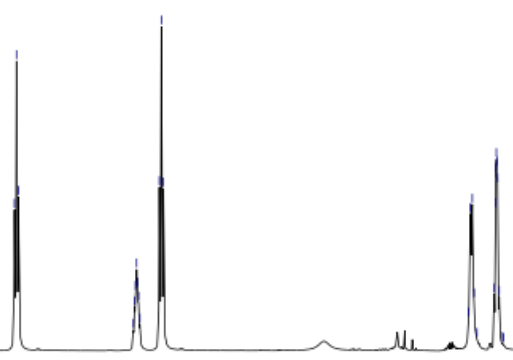

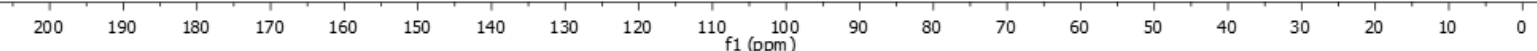


(E)-7-Benzylidene-5,6,6a,7,8,9-hexahydropyrrolo[1,2-a] thieno[2,3-f]azocine-4,11-dione 6n

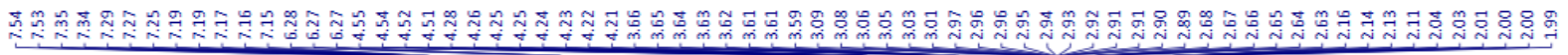

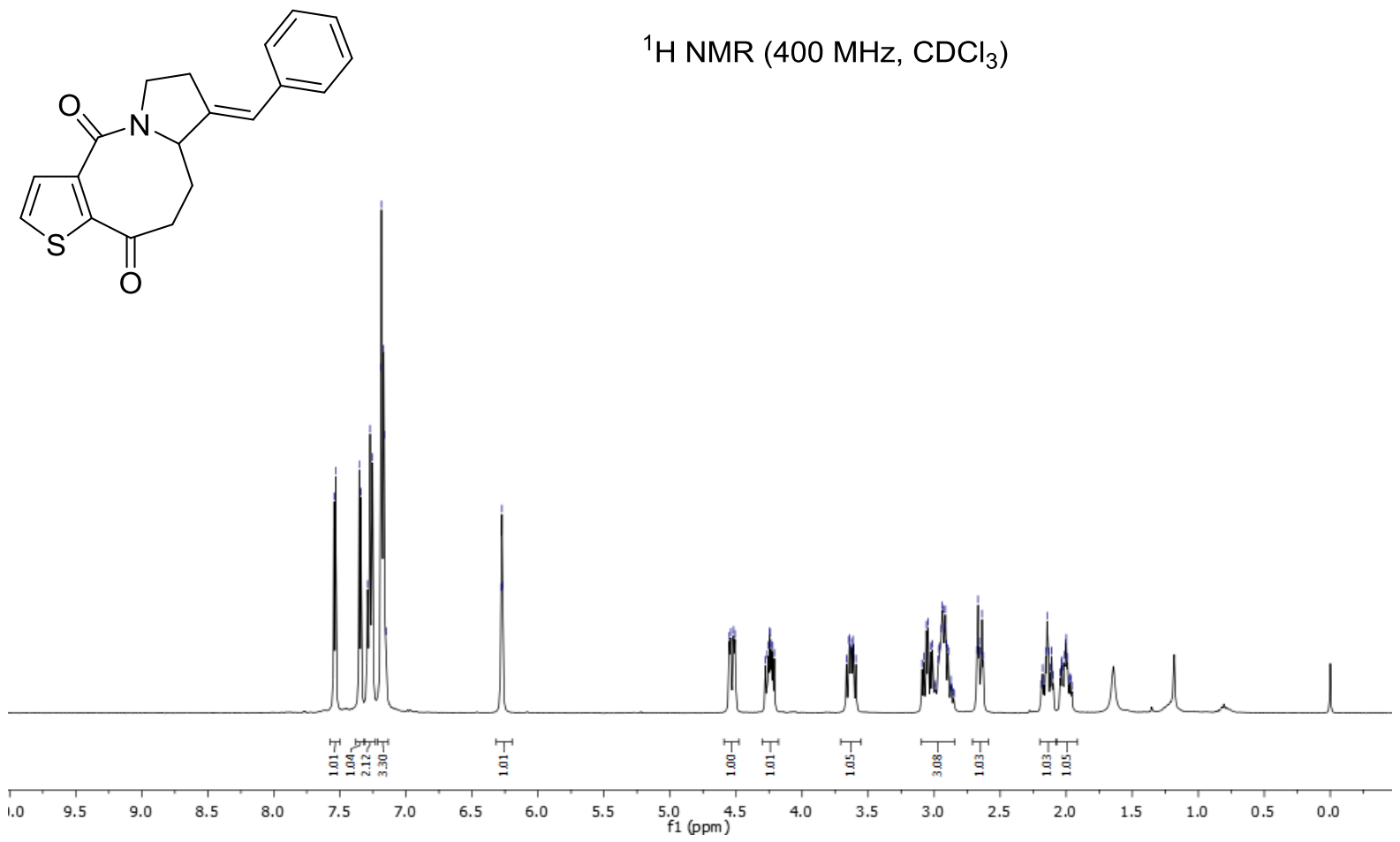

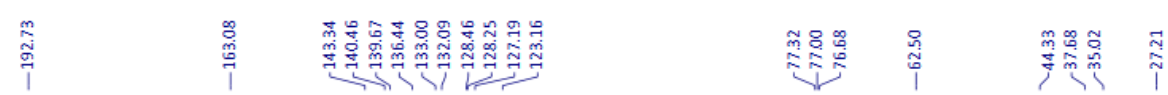

${ }^{13} \mathrm{C}$ NMR $\left(101 \mathrm{MHz}, \mathrm{CDCl}_{3}\right)$

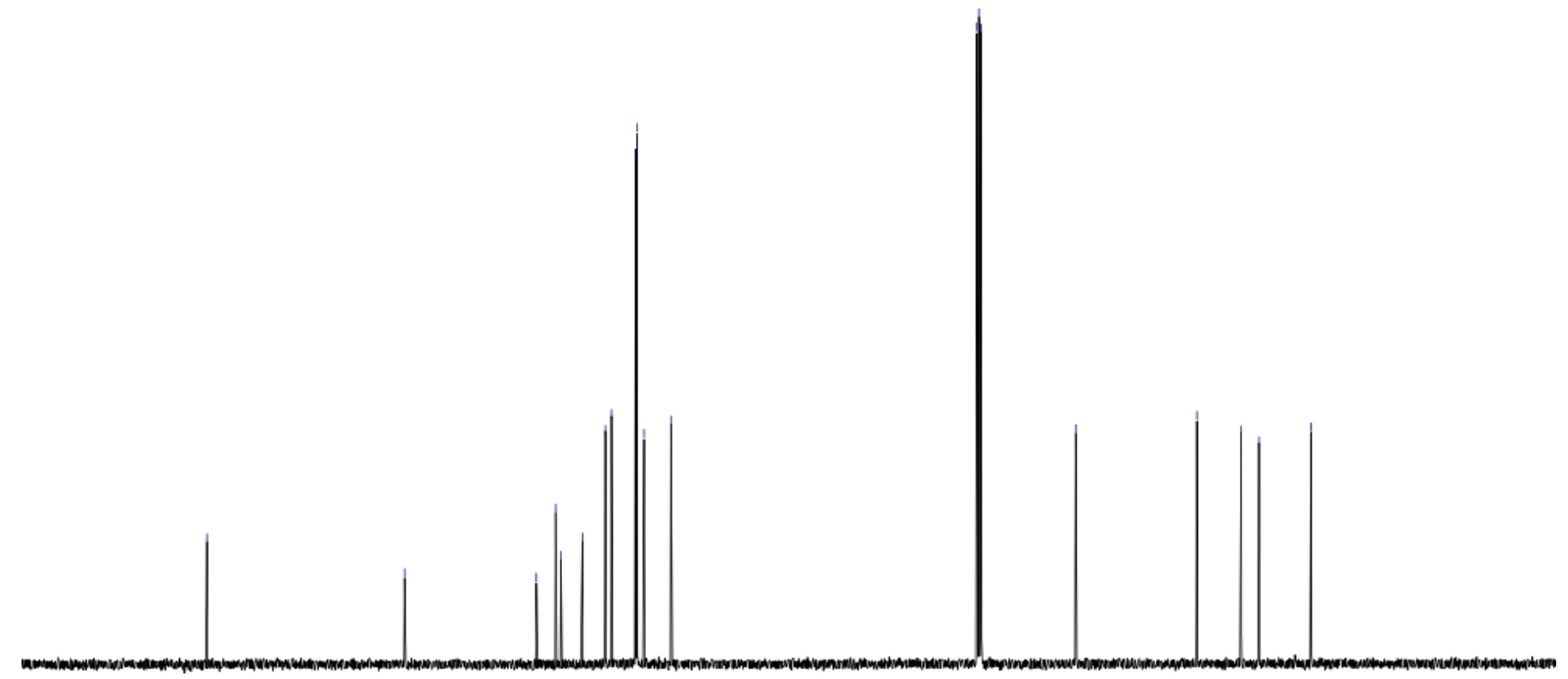

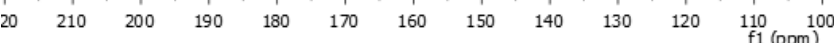


$N$-Cyclopropyl-2-methyl- $N$-(4-phenylbut-3-yn-1-yl)-1H-pyrrole-3-carboxamide 50

管

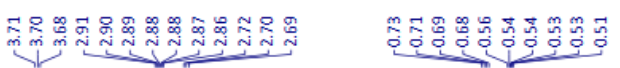

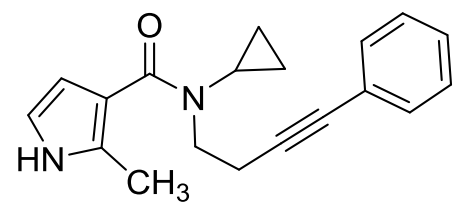

${ }^{1} \mathrm{H}$ NMR $\left(400 \mathrm{MHz}, \mathrm{CDCl}_{3}\right)$

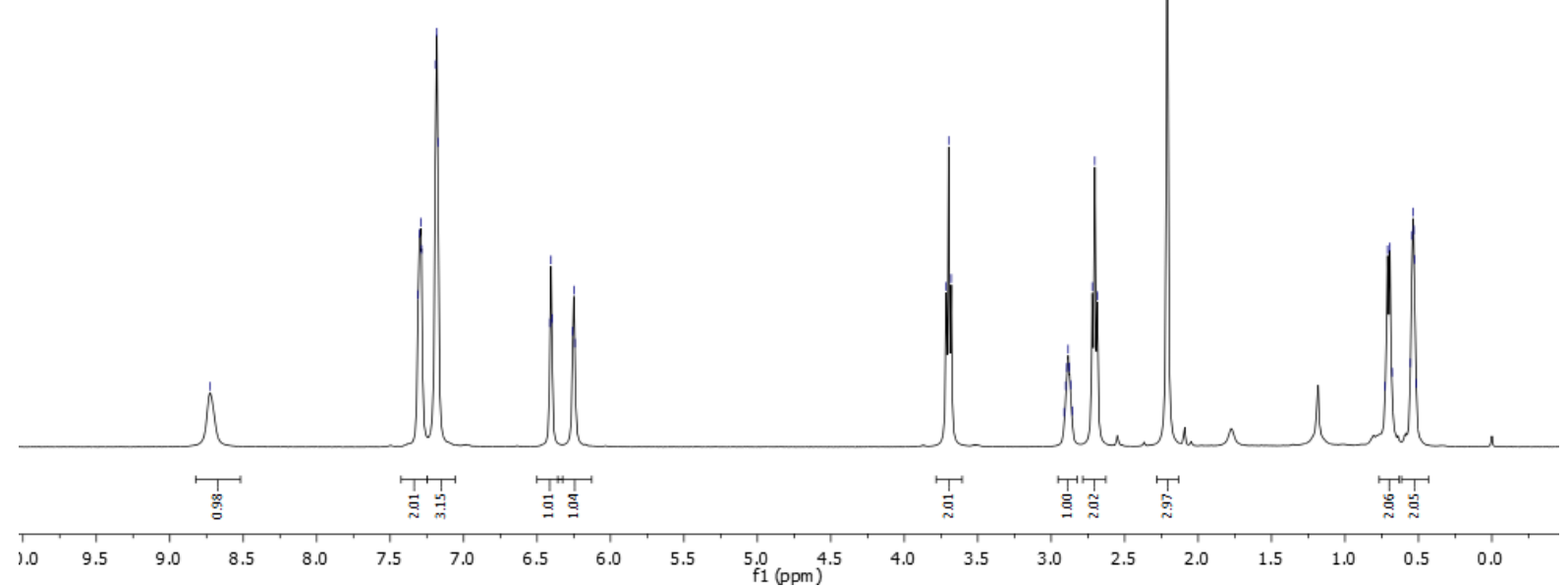

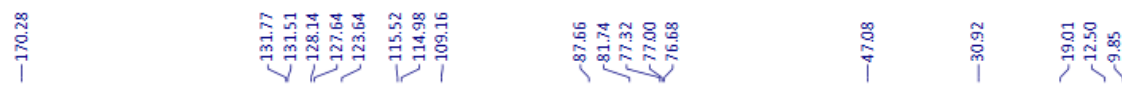

${ }^{13} \mathrm{C}$ NMR $\left(101 \mathrm{MHz}, \mathrm{CDCl}_{3}\right)$

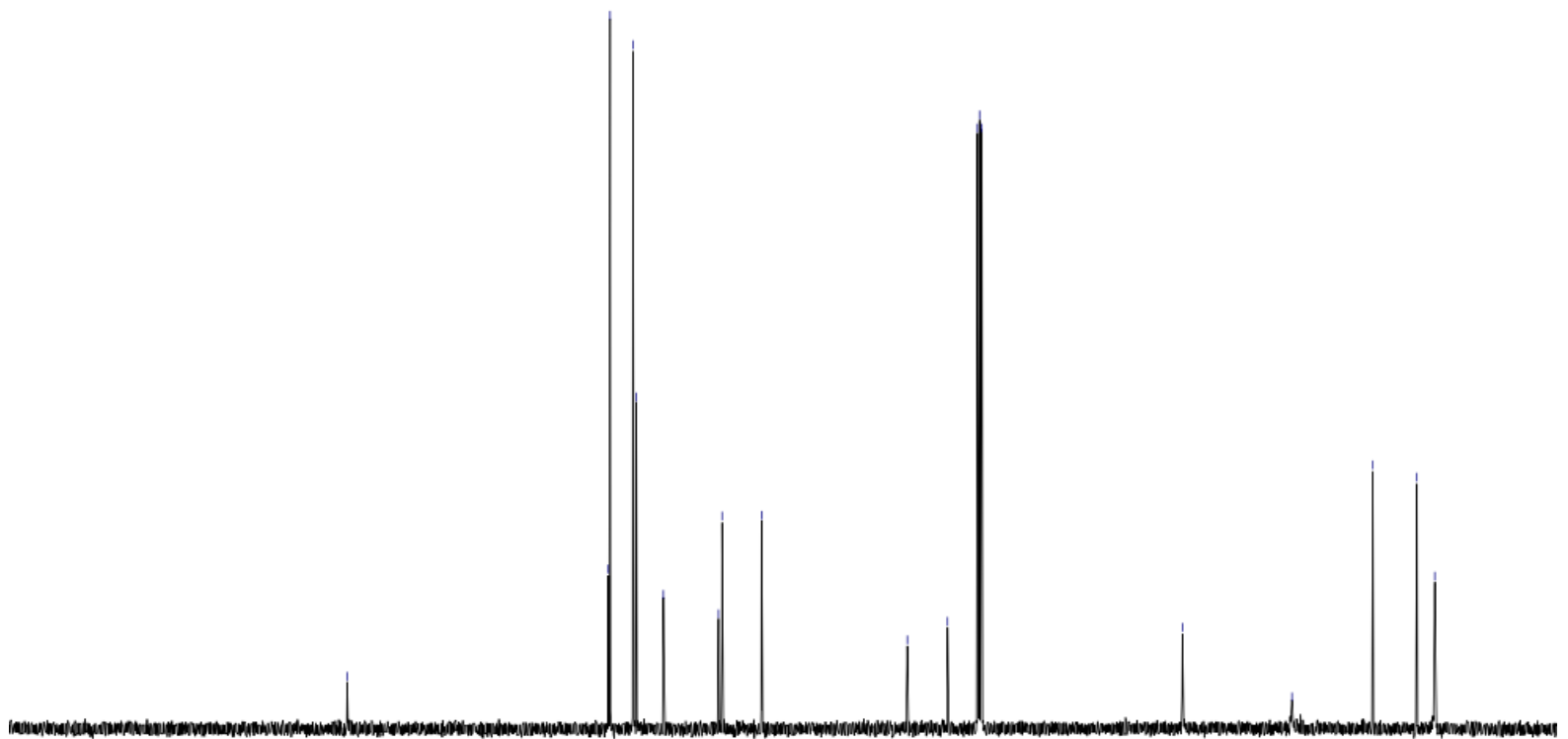

$\begin{array}{lllllllllllllllllllllllll}20 & 210 & 200 & 190 & 180 & 170 & 160 & 150 & 140 & 130 & 120 & 110 & 100 & 90 & 80 & 70 & 60 & 50 & 40 & 30 & 20 & 10 & 0 & 0\end{array}$ 
(E)-7-Benzylidene-1-methyl-5,6,6a,7,8,9-hexahydro-4H-dipyrrolo[1,2-a:3',4'-f]azocine4,11(2H)-dione 60

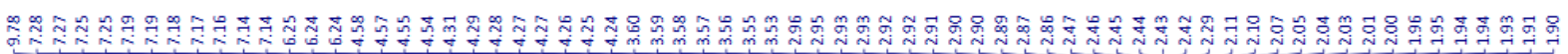<smiles>C[C@@H](O[Na])C(C)(C)C</smiles>
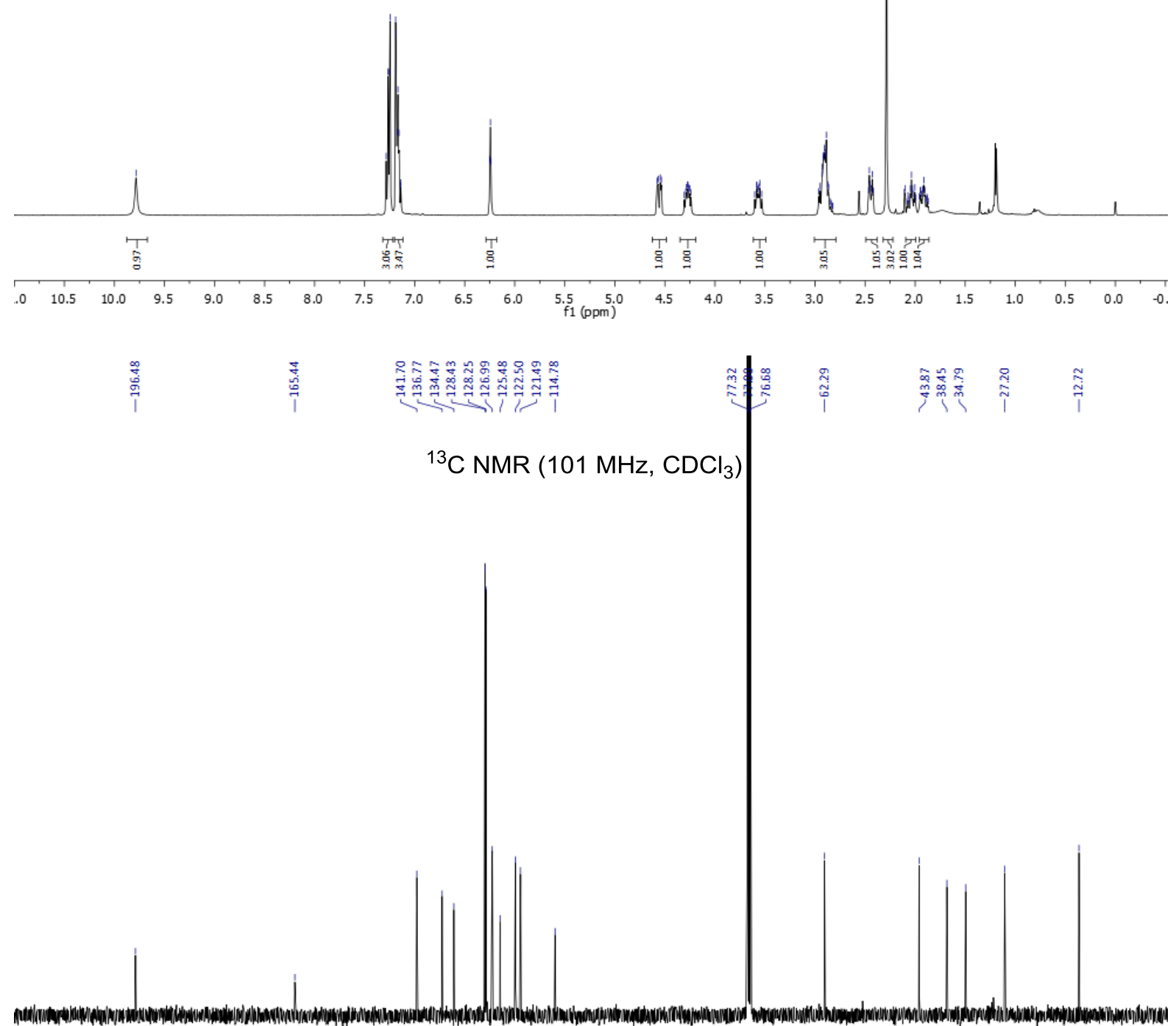

$\begin{array}{lllllllllllllllllllllllllllllllllll}10 & 210 & 200 & 190 & 180 & 170 & 160 & 150 & 140 & 130 & 120 & 10 & 100 & 90 & 80 & 70 & 60 & 50 & 40 & 30 & 20 & 10 & 0\end{array}$ 
3-Benzyl-1-cyclopropyl-1-(4-phenylbut-3-yn-1-yl)urea 5p

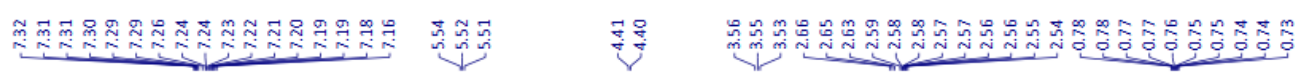
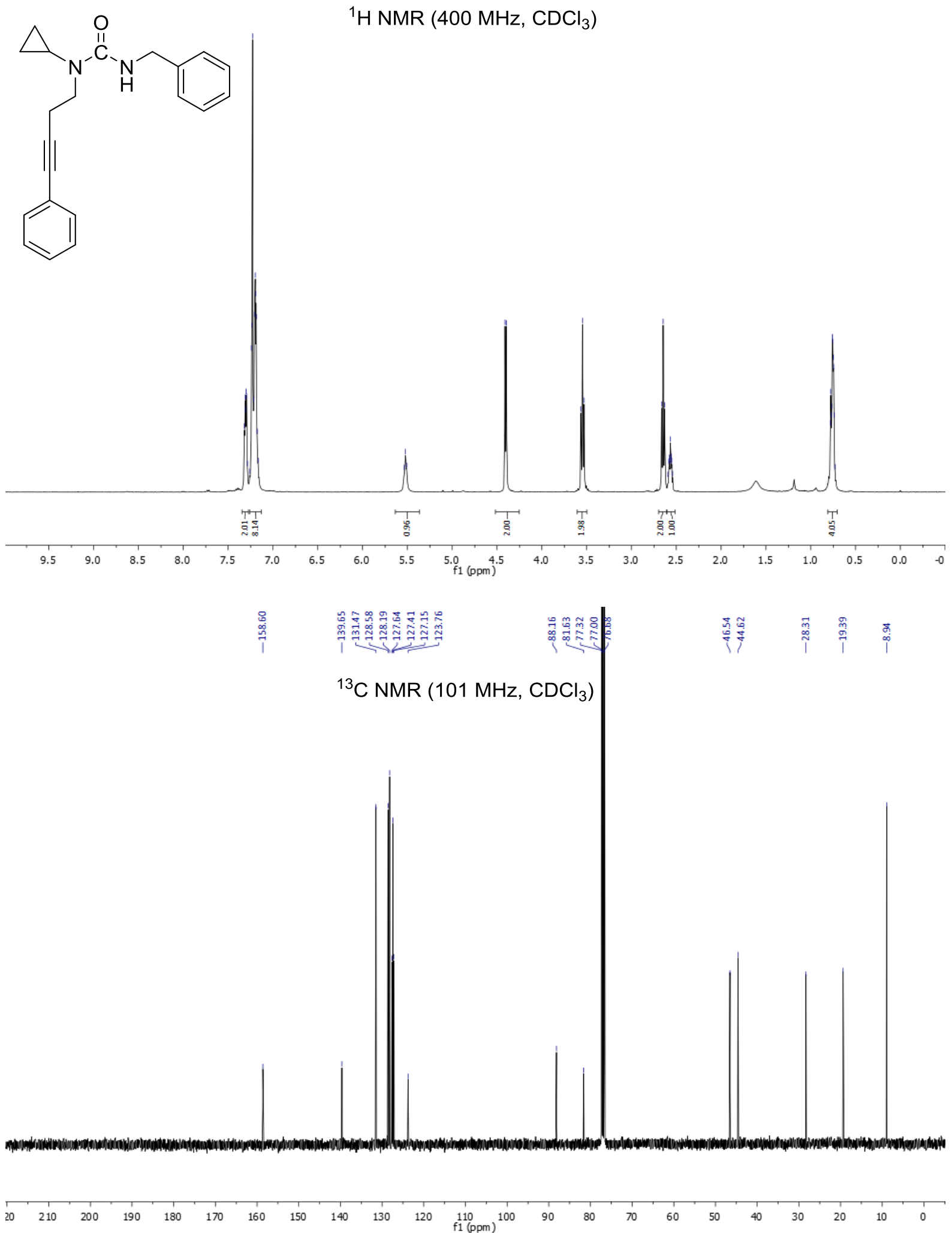
(E)-2-Benzyl-6-benzylidenehexahydro-1H-pyrrolo[1,2-c][1,3]diazepine-1,3(2H)-dione 6p

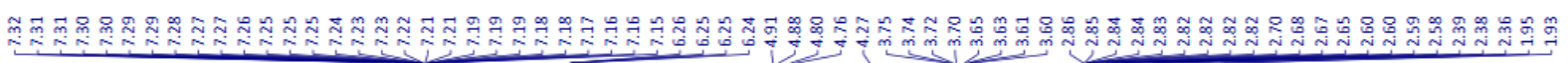

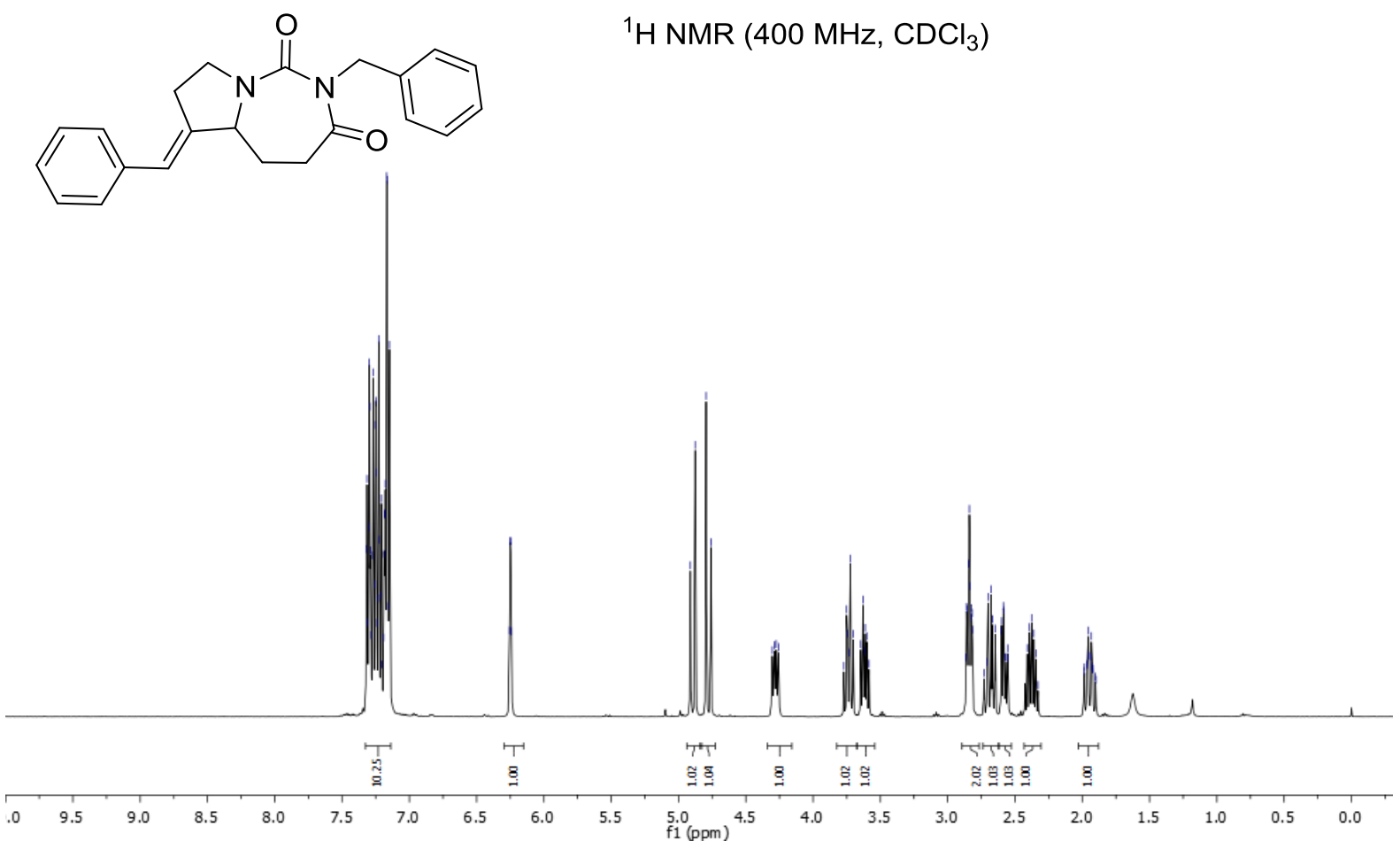

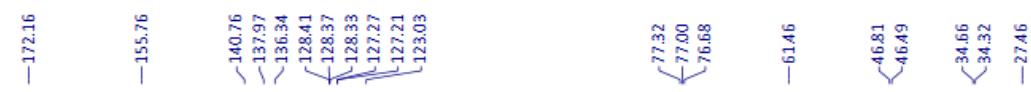

${ }^{13} \mathrm{C}$ NMR $\left(101 \mathrm{MHz}, \mathrm{CDCl}_{3}\right)$

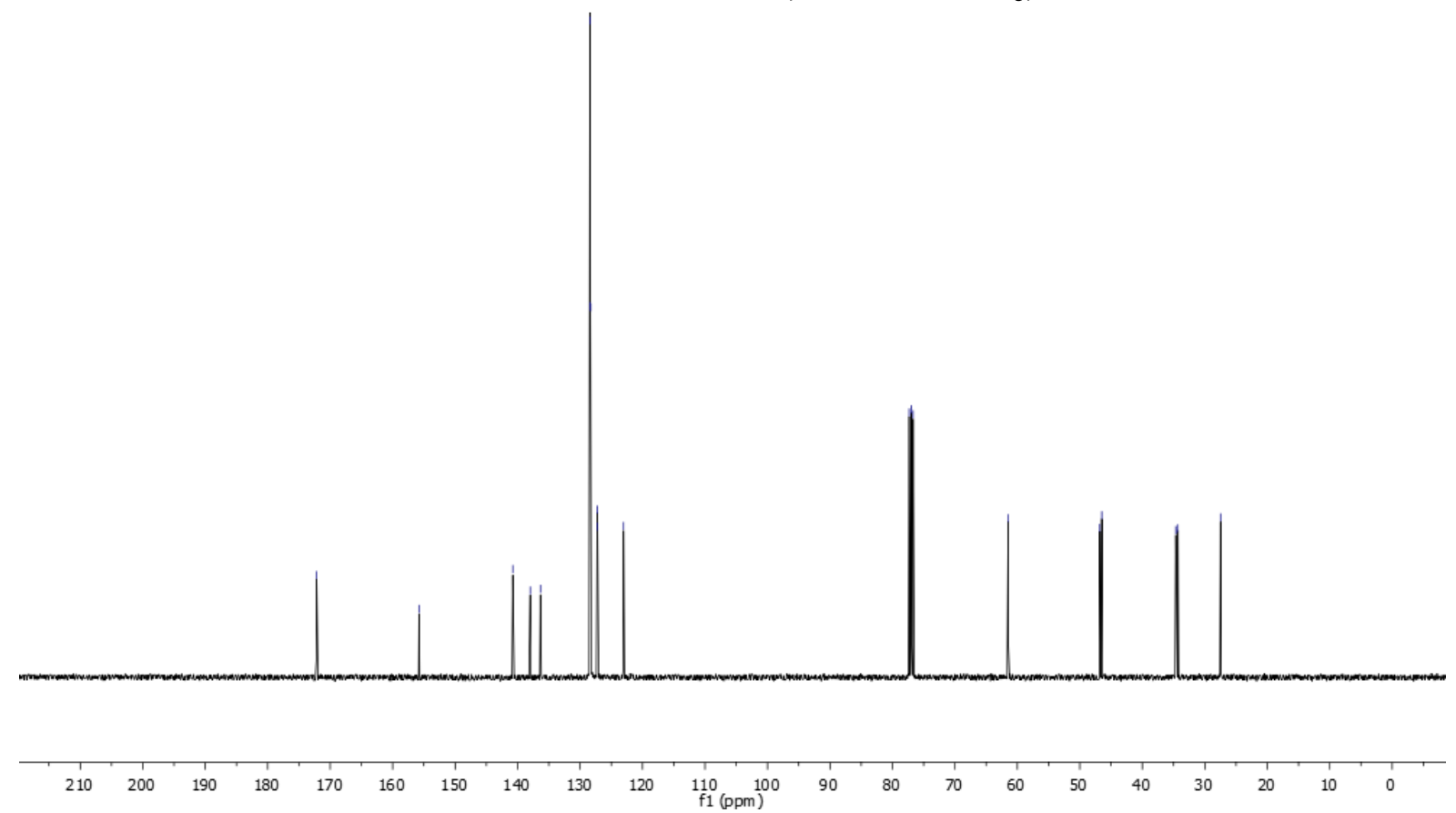


(E)- $N$-Cyclopropyl- $N$-(hexa-3,5-dien-1-yl)-1H-pyrrole-3-carboxamide 5q

总<smiles>C=C/C=C\CCN(C(=O)c1cc[nH]c1)C1CC1</smiles>

${ }^{1} \mathrm{H}$ NMR $\left(400 \mathrm{MHz}, \mathrm{CDCl}_{3}\right)$

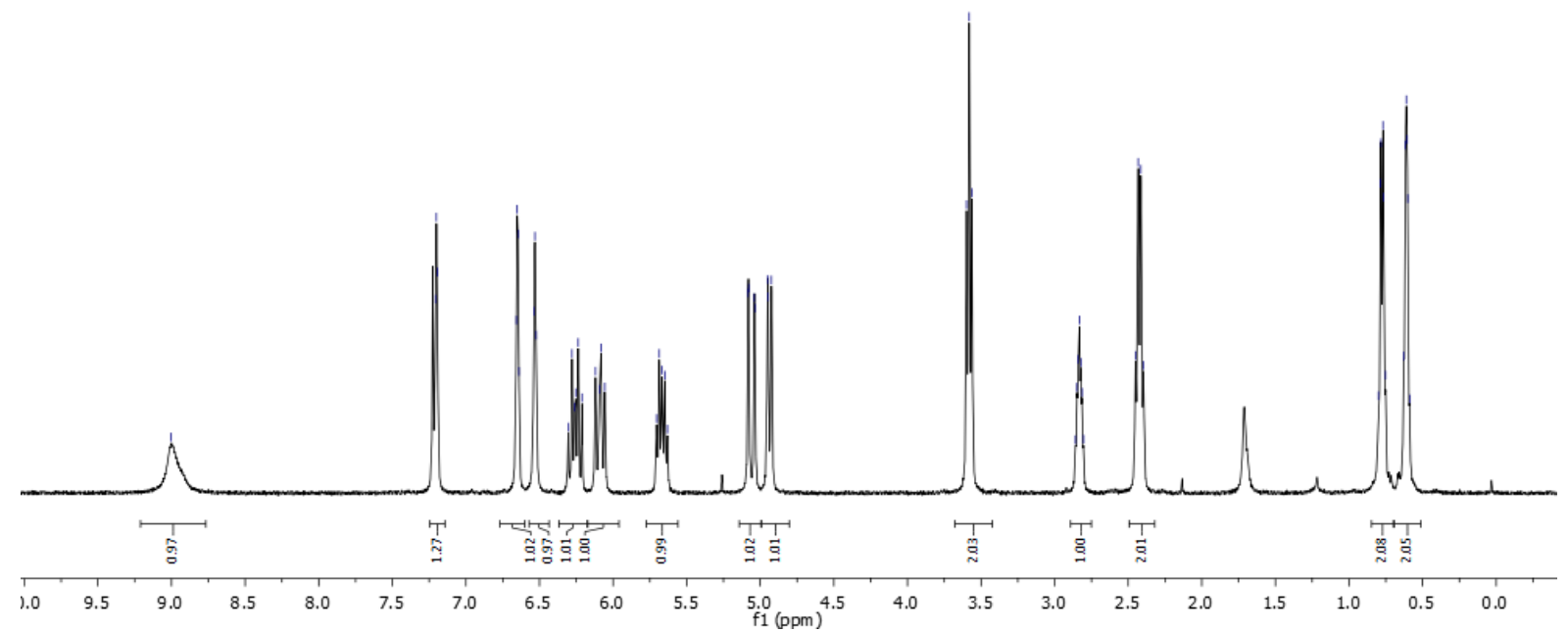

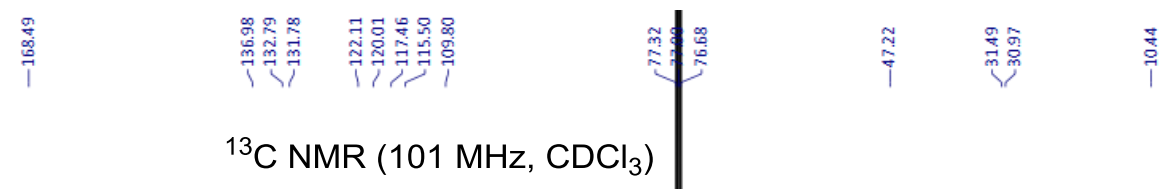

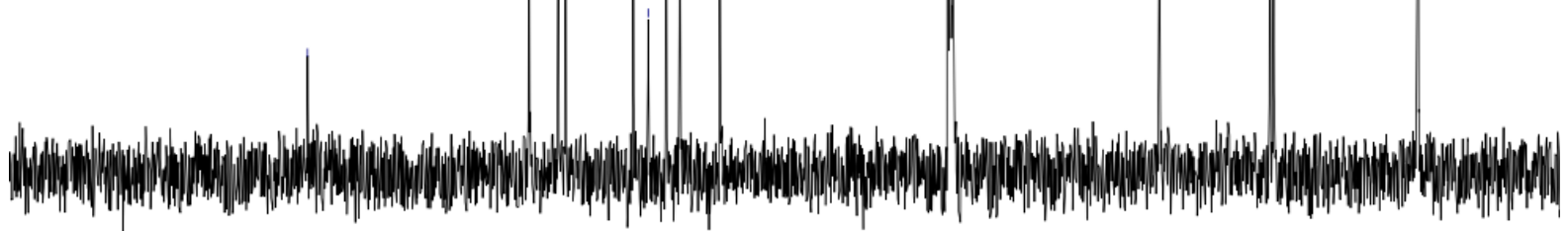

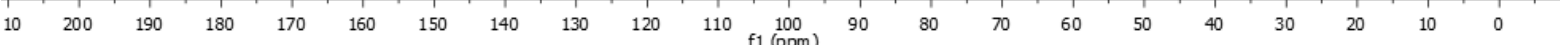


$\left(6 a S^{*}, 7 S^{*}\right)-7-M e t h y l-5,6,6 a, 7,10,11-h e x a h y d r o-3 H$-azepino[1,2-a]pyrrolo[2,3-f]azocine-

\section{4,13-dione 6q}

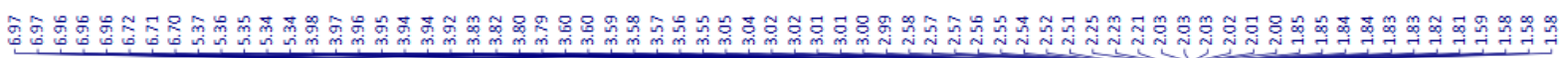
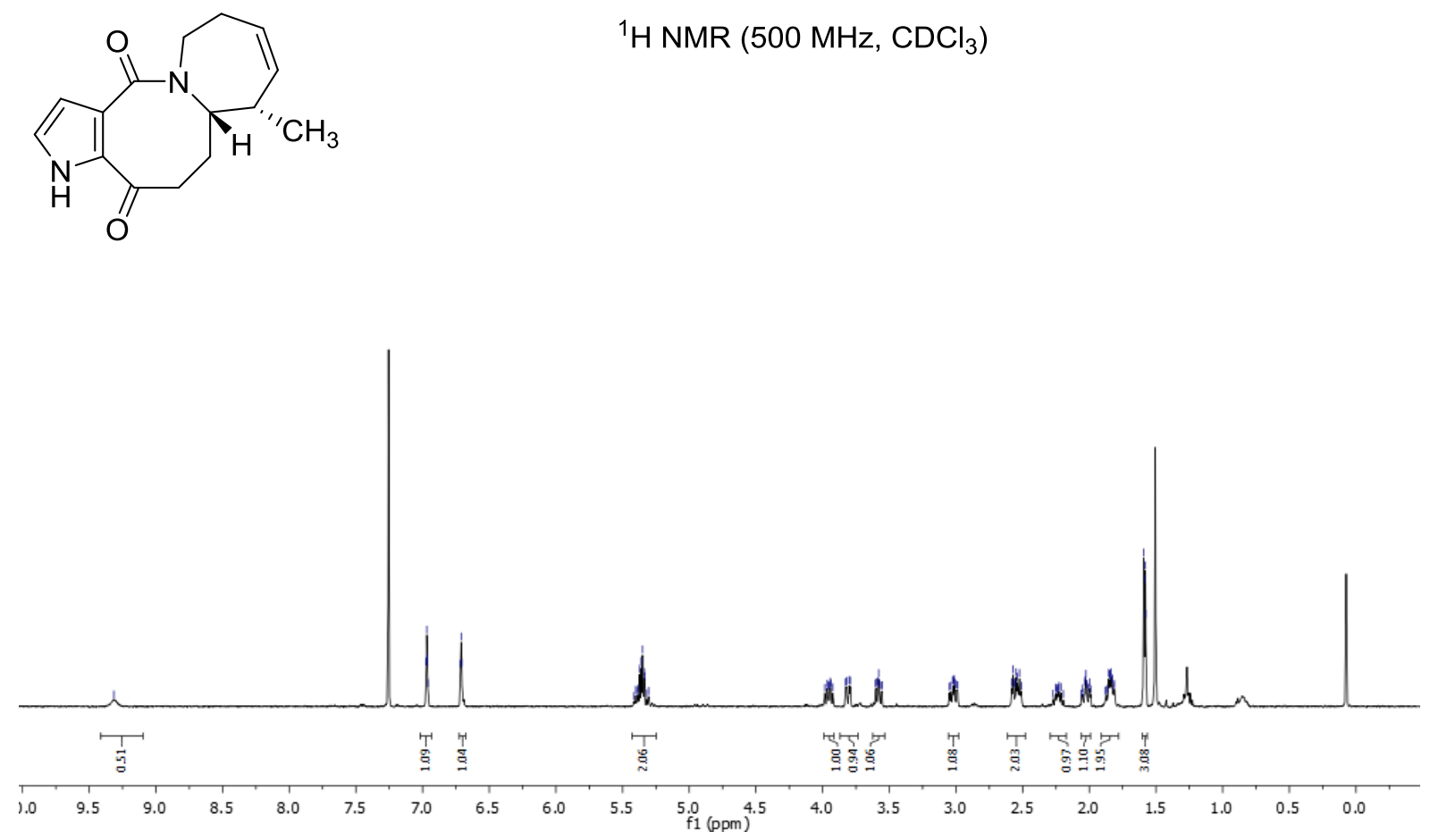

兽 兽

${ }^{13} \mathrm{C}$ NMR $\left(101 \mathrm{MHz}, \mathrm{CDCl}_{3}\right)$

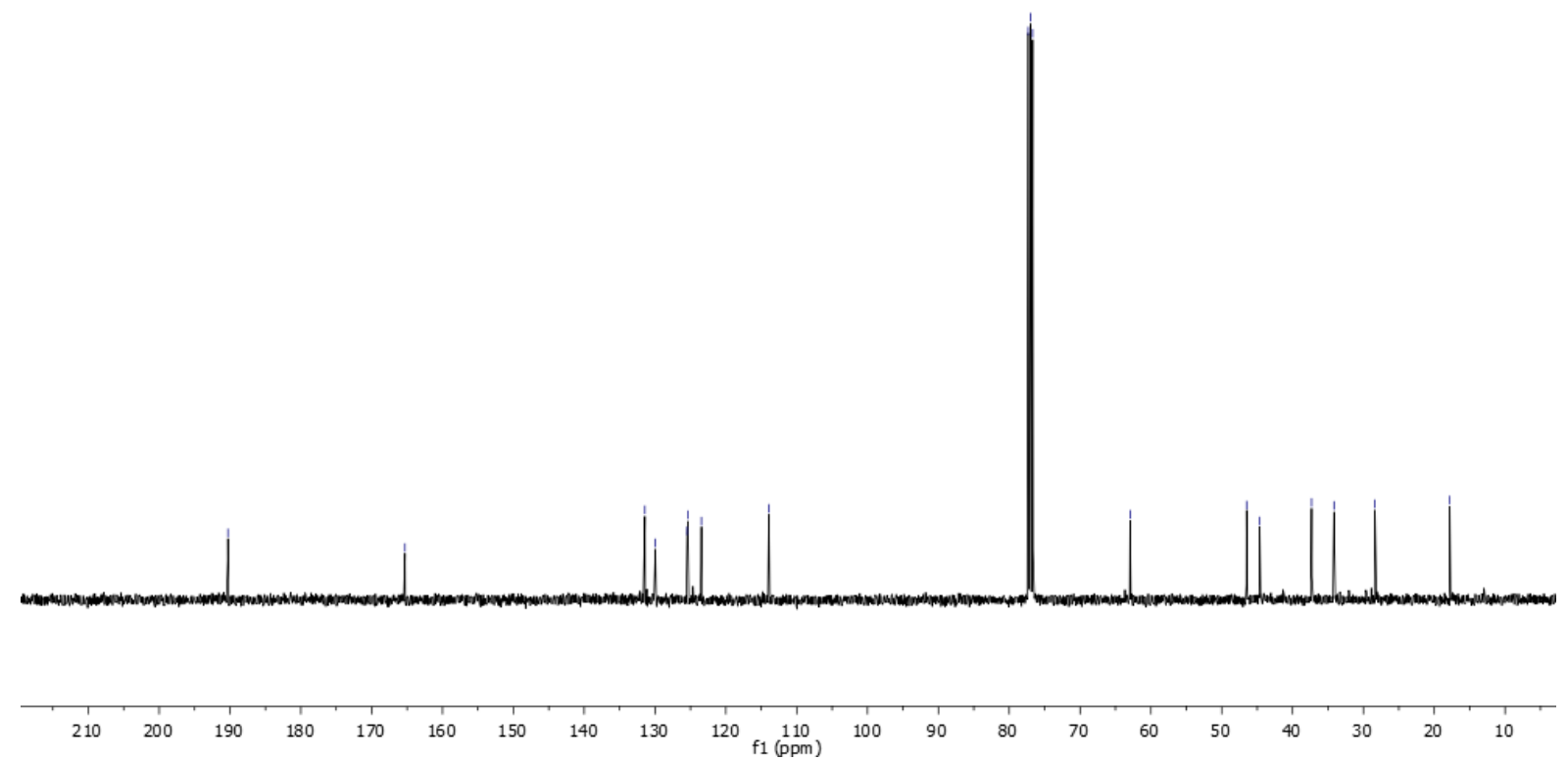


$N$-Benzyl- $N$-ethyl-1H-indole-1-carboxamide 7

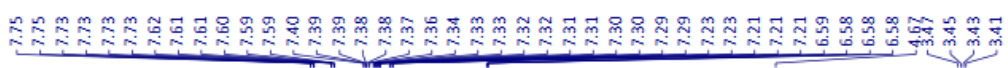<smiles>CCN(Cc1ccccc1)C(=O)n1ccc2ccccc21</smiles>

${ }^{1} \mathrm{H}$ NMR $\left(400 \mathrm{MHz}, \mathrm{CDCl}_{3}\right)$

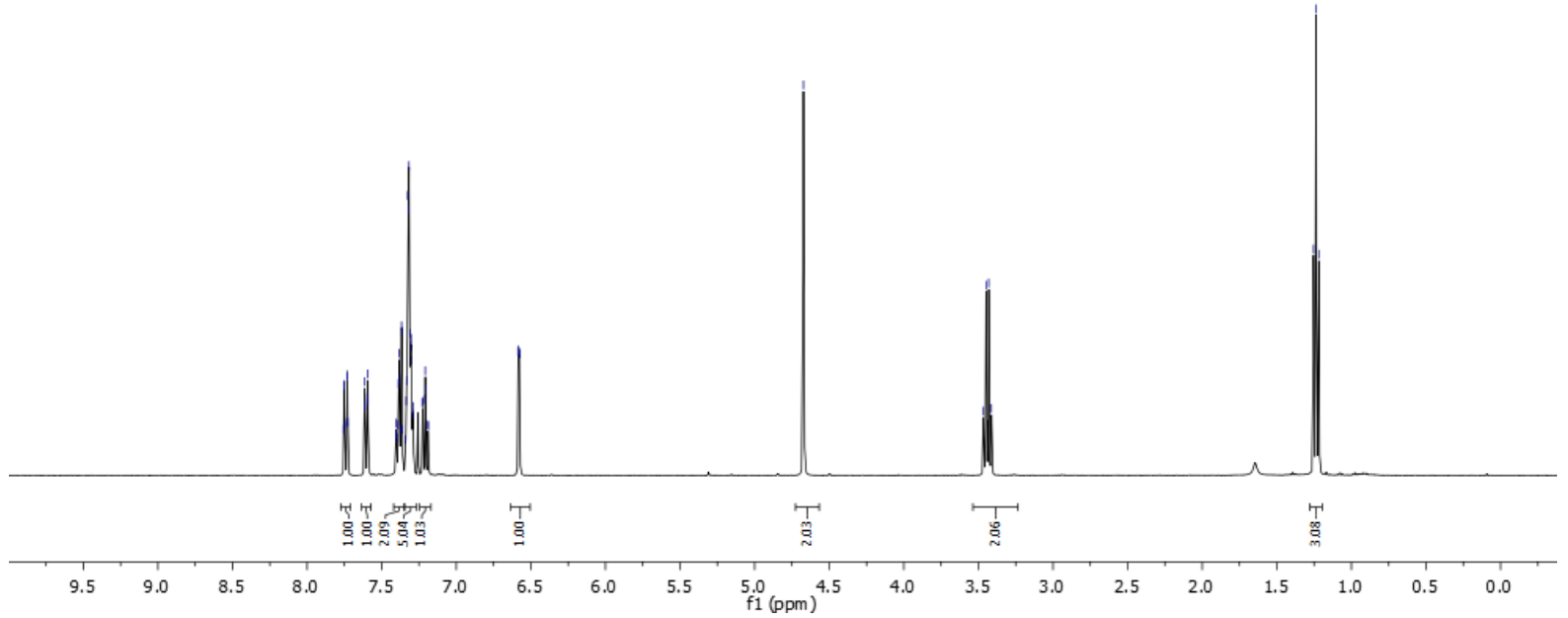

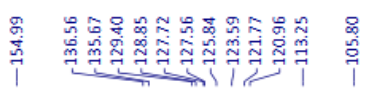

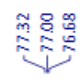

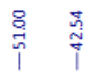

$\underset{\sim}{\stackrel{乛}{i}}$

${ }^{13} \mathrm{C}$ NMR $\left(101 \mathrm{MHz}, \mathrm{CDCl}_{3}\right)$

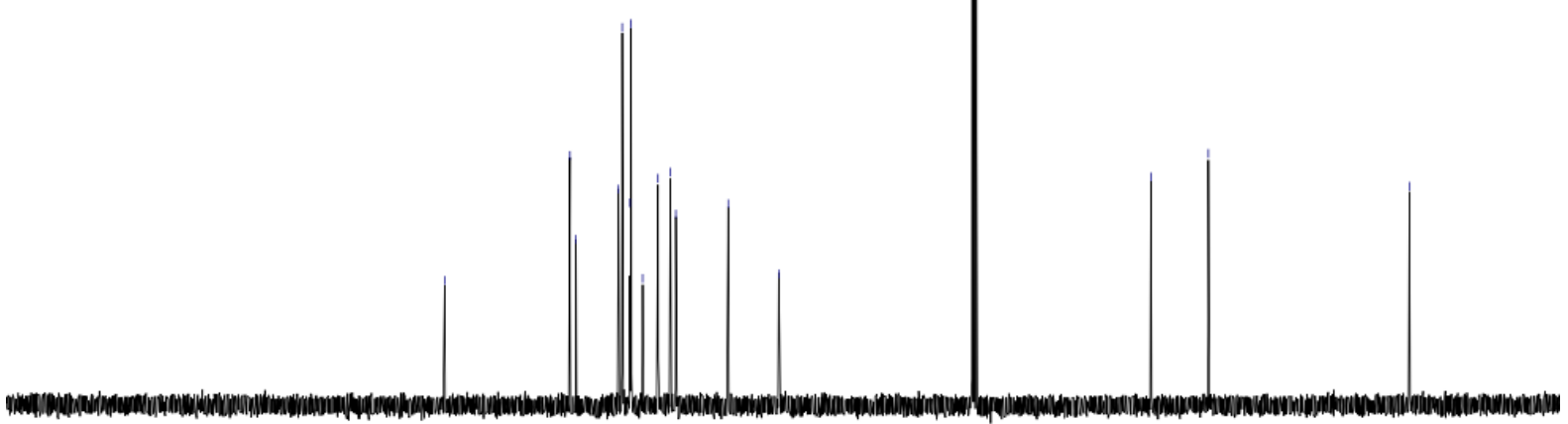

$\begin{array}{lllllllllll}210 & 200 & 190 & 180 & 170 & 160 & 150 & 140 & 130 & 120 & 110 \\ \mathrm{f} 1(\mathrm{ppm}) & 100\end{array}$ 
Selected Reaction Optimization Results
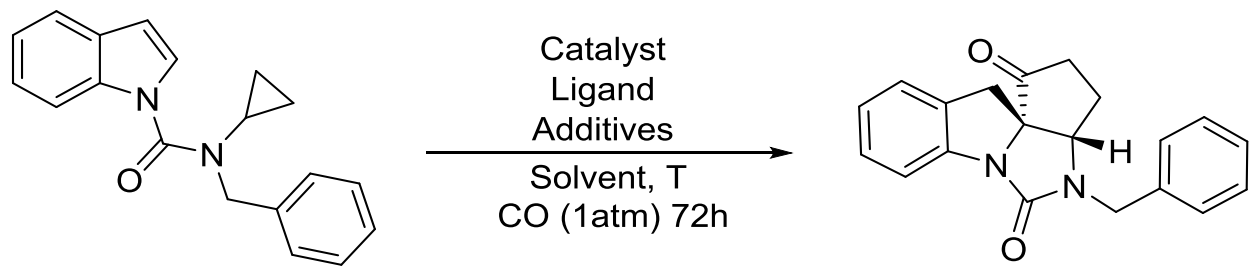

\begin{tabular}{|c|c|c|c|c|c|c|}
\hline Catalyst (\%) & Ligand $(\%)$ & Additive (\%) & Solvent (M) & $\begin{array}{c}\mathbf{T} \\
\left({ }^{\circ} \mathbf{C}\right)\end{array}$ & $\begin{array}{l}\text { S.M } \\
(\%)\end{array}$ & $\begin{array}{c}\text { Product } \\
(\%)\end{array}$ \\
\hline $\mathrm{Rh}(\operatorname{cod})_{2} \mathrm{OTf}(7.5)$ & $\mathrm{P}-\left(3,5-\left(\mathrm{CF}_{3}\right)_{2} \mathrm{C}_{6} \mathrm{H}_{3}\right)_{3}(15)$ & None & $\operatorname{DCB}(0.2)$ & 140 & $>90$ & trace \\
\hline$[\mathrm{Rh}(\operatorname{cod}) \mathrm{Cl}]_{2}(3.75)$ & P- $\left(3,5-\left(\mathrm{CF}_{3}\right)_{2} \mathrm{C}_{6} \mathrm{H}_{3}\right)_{3}(15)$ & None & $\operatorname{DCB}(0.2)$ & 140 & $>95$ & 0 \\
\hline $\mathrm{Rh}(\operatorname{cod})_{2} \mathrm{OTf}(7.5)$ & P- $\left(3,5-\left(\mathrm{CF}_{3}\right)_{2} \mathrm{C}_{6} \mathrm{H}_{3}\right)_{3}(15)$ & None & $\mathrm{PhCN}(0.2)$ & 140 & $>95$ & 0 \\
\hline $\mathrm{Rh}(\operatorname{cod})_{2} \mathrm{OTf}(7.5)$ & P- $\left(3,5-\left(\mathrm{CF}_{3}\right)_{2} \mathrm{C}_{6} \mathrm{H}_{3}\right)_{3}(15)$ & $\mathrm{C}_{6} \mathrm{H}_{5} \mathrm{OCH}_{2} \mathrm{COOH}(30)$ & $\operatorname{DCB}(0.2)$ & 140 & 80 & 10 \\
\hline $\mathrm{Rh}(\operatorname{cod})_{2} \mathrm{OTf}(7.5)$ & P- $\left(4-(\mathrm{F}) \mathrm{C}_{6} \mathrm{H}_{4}\right)_{3}(15)$ & $\mathrm{C}_{6} \mathrm{H}_{5} \mathrm{OCH}_{2} \mathrm{COOH}(30)$ & $\mathrm{DCB}(0.2)$ & 140 & 77 & 11 \\
\hline $\mathrm{Rh}(\operatorname{cod})_{2} \mathrm{OTf}(7.5)$ & $\mathrm{P}-\left(\mathrm{C}_{6} \mathrm{~F}_{5}\right)_{3}(15)$ & $\mathrm{C}_{6} \mathrm{H}_{5} \mathrm{OCH}_{2} \mathrm{COOH}(30)$ & $\mathrm{DCB}(0.2)$ & 140 & 0 & 46 \\
\hline $\mathrm{Rh}(\operatorname{cod})_{2} \mathrm{OTf}(7.5)$ & $\mathrm{PPh}_{3}(15)$ & $\mathrm{C}_{6} \mathrm{H}_{5} \mathrm{OCH}_{2} \mathrm{COOH}(30)$ & $\operatorname{DCB}(0.2)$ & 140 & $>90$ & trace \\
\hline $\mathrm{Rh}(\operatorname{cod})_{2} \mathrm{OTf}(7.5)$ & $\mathrm{P}-\left(4-(\mathrm{OMe}) \mathrm{C}_{6} \mathrm{H}_{4}\right)_{3}(15)$ & $\mathrm{C}_{6} \mathrm{H}_{5} \mathrm{OCH}_{2} \mathrm{COOH}(30)$ & $\operatorname{DCB}(0.2)$ & 140 & $>95$ & 0 \\
\hline $\mathrm{Rh}(\operatorname{cod})_{2} \mathrm{OTf}(7.5)$ & Binap (7.5) & $\mathrm{C}_{6} \mathrm{H}_{5} \mathrm{OCH}_{2} \mathrm{COOH}(30)$ & $\mathrm{DCB}(0.2)$ & 140 & $>95$ & 0 \\
\hline $\mathrm{Rh}(\operatorname{cod})_{2} \mathrm{OTf}(7.5)$ & $\mathrm{P}-\left(\mathrm{C}_{6} \mathrm{~F}_{5}\right)_{3}(15)$ & $\mathrm{C}_{6} \mathrm{H}_{5} \mathrm{OCH}_{2} \mathrm{COOH}(30)$ & $\operatorname{DCB}(0.2)$ & 130 & 25 & 38 \\
\hline $\mathrm{Rh}(\operatorname{cod})_{2} \mathrm{OTf}(7.5)$ & $\mathrm{P}-\left(\mathrm{C}_{6} \mathrm{~F}_{5}\right)_{3}(15)$ & $\mathrm{C}_{6} \mathrm{H}_{5} \mathrm{OCH}_{2} \mathrm{COOH}(15)$ & $\mathrm{DCB}(0.2)$ & 130 & 8 & 53 \\
\hline $\mathrm{Rh}(\operatorname{cod})_{2} \mathrm{OTf}(7.5)$ & $\mathrm{P}-\left(\mathrm{C}_{6} \mathrm{~F}_{5}\right)_{3}(15)$ & $\mathrm{C}_{6} \mathrm{H}_{5} \mathrm{OCH}_{2} \mathrm{COOH}(50)$ & $\mathrm{DCB}(0.2)$ & 130 & 8 & 47 \\
\hline $\mathrm{Rh}(\operatorname{cod})_{2} \mathrm{OTf}(7.5)$ & $\mathrm{P}-\left(\mathrm{C}_{6} \mathrm{~F}_{5}\right)_{3}(15)$ & TsOH (15) & $\mathrm{DCB}(0.2)$ & 130 & 25 & 12 \\
\hline $\mathrm{Rh}(\operatorname{cod})_{2} \mathrm{OTf}(7.5)$ & $\mathrm{P}-\left(\mathrm{C}_{6} \mathrm{~F}_{5}\right)_{3}(15)$ & $\left(\mathrm{C}_{6} \mathrm{H}_{5} \mathrm{O}\right)_{2} \mathrm{POOH}(15)$ & $\operatorname{DCB}(0.2)$ & 130 & 37 & 12 \\
\hline $\mathrm{Rh}(\operatorname{cod})_{2} \mathrm{OTf}(7.5)$ & $\mathrm{P}-\left(\mathrm{C}_{6} \mathrm{~F}_{5}\right)_{3}(15)$ & $\mathrm{CH}_{3}\left(\mathrm{CH}_{2}\right)_{4} \mathrm{COOH}(15)$ & $\operatorname{DCB}(0.2)$ & 130 & 21 & 34 \\
\hline $\mathrm{Rh}(\operatorname{cod})_{2} \mathrm{OTf}(7.5)$ & $\mathrm{P}-\left(\mathrm{C}_{6} \mathrm{~F}_{5}\right)_{3}(15)$ & Fumic acid (10) & $\mathrm{DCB}(0.2)$ & 130 & 15 & 45 \\
\hline $\mathrm{Rh}(\operatorname{cod})_{2} \mathrm{OTf}(7.5)$ & $\mathrm{P}-\left(\mathrm{C}_{6} \mathrm{~F}_{5}\right)_{3}(15)$ & $\mathrm{o}-\mathrm{NO}_{2} \mathrm{C}_{6} \mathrm{H}_{5} \mathrm{COOH}(15)$ & $\operatorname{DCB}(0.2)$ & 130 & 19 & 37 \\
\hline $\mathrm{Rh}(\operatorname{cod})_{2} \mathrm{OTf}(7.5)$ & $\mathrm{P}-\left(\mathrm{C}_{6} \mathrm{~F}_{5}\right)_{3}(15)$ & $\mathrm{C}_{6} \mathrm{H}_{5} \mathrm{COOH}(15)$ & $\operatorname{DCB}(0.2)$ & 130 & 9 & 43 \\
\hline $\mathrm{Rh}(\operatorname{cod})_{2} \mathrm{OTf}(7.5)$ & $\mathrm{P}-\left(\mathrm{C}_{6} \mathrm{~F}_{5}\right)_{3}(15)$ & $\begin{array}{c}2,3,5-\left(\mathrm{CH}_{3}\right)_{3} \mathrm{C}_{6} \mathrm{H}_{2} \mathrm{COOH} \\
(15)\end{array}$ & $\operatorname{DCB}(0.2)$ & 130 & 12 & 40 \\
\hline $\mathrm{Rh}(\operatorname{cod})_{2} \mathrm{OTf}(7.5)$ & $\mathrm{P}-\left(\mathrm{C}_{6} \mathrm{~F}_{5}\right)_{3}(15)$ & 4- $\mathrm{NMe}_{2} \mathrm{C}_{6} \mathrm{H}_{4} \mathrm{COOH}$ (15) & $\mathrm{DCB}(0.2)$ & 130 & 5 & 55 \\
\hline $\mathrm{Rh}(\operatorname{cod})_{2} \mathrm{OTf}(7.5)$ & None & 4- $\mathrm{NMe}_{2} \mathrm{C}_{6} \mathrm{H}_{4} \mathrm{COOH}$ (15) & $\mathrm{DCB}(0.2)$ & 130 & 0 & 71 \\
\hline $\mathrm{Rh}(\operatorname{cod})_{2} \mathrm{OTf}(7.5)$ & None & 4- $\mathrm{NMe}_{2} \mathrm{C}_{6} \mathrm{H}_{4} \mathrm{COOH}(30)$ & $\operatorname{DCB}(0.2)$ & 130 & 6 & 68 \\
\hline $\mathrm{Rh}(\operatorname{cod})_{2} \mathrm{OTf}(7.5)$ & None & 4- $\mathrm{NMe}_{2} \mathrm{C}_{6} \mathrm{H}_{4} \mathrm{COOH}(100)$ & $\mathrm{DCB}(0.2)$ & 130 & 33 & 41 \\
\hline $\mathrm{Rh}(\operatorname{cod})_{2} \mathrm{OTf}(7.5)$ & None & 4-NMe $2 \mathrm{C}_{6} \mathrm{H}_{4} \mathrm{COOH}(30)$ & $\mathrm{DCB}(0.1)$ & 130 & 0 & 73 \\
\hline $\mathrm{Rh}(\operatorname{cod})_{2} \mathrm{OTf}(7.5)$ & None & $\begin{array}{c}4-\mathrm{NMe}_{2} \mathrm{C}_{6} \mathrm{H}_{4} \mathrm{COOH} \\
(30)+\mathrm{Na}_{2} \mathrm{SO}_{4}(100) \\
\end{array}$ & $\operatorname{DCB}(0.1)$ & 130 & 0 & 81 \\
\hline $\operatorname{Rh}(\operatorname{cod})_{2} \operatorname{BARf}(7.5)$ & None & $\begin{array}{c}4-\mathrm{NMe}_{2} \mathrm{C}_{6} \mathrm{H}_{4} \mathrm{COOH} \\
(30)+\mathrm{Na}_{2} \mathrm{SO}_{4}(100)\end{array}$ & $\operatorname{DCB}(0.1)$ & 130 & 47 & 27 \\
\hline $\mathrm{Rh}(\operatorname{cod})_{2} \mathrm{BF}_{4}(7.5)$ & None & $\begin{array}{c}4-\mathrm{NMe}_{2} \mathrm{C}_{6} \mathrm{H}_{4} \mathrm{COOH} \\
(30)+\mathrm{Na}_{2} \mathrm{SO}_{4}(100)\end{array}$ & $\operatorname{DCB}(0.1)$ & 130 & 77 & 16 \\
\hline $\mathrm{Rh}(\operatorname{cod})_{2} \mathrm{OTf}(7.5)$ & P-(4-(F) $\left.\mathrm{C}_{6} \mathrm{H}_{4}\right)_{3}(15)$ & $\begin{array}{c}4-\mathrm{NMe}_{2} \mathrm{C}_{6} \mathrm{H}_{4} \mathrm{COOH} \\
(30)+\mathrm{Na}_{2} \mathrm{SO}_{4}(100)\end{array}$ & $\operatorname{DCB}(0.1)$ & 130 & 6 & 40 \\
\hline
\end{tabular}




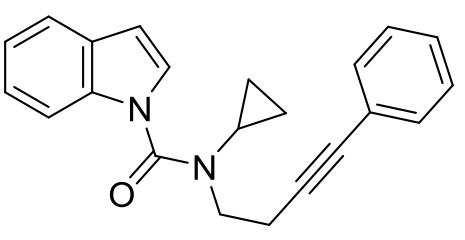

$\mathbf{X X}$

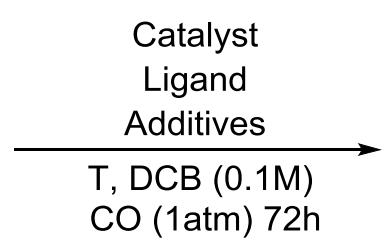

CO (1atm) $72 \mathrm{~h}$

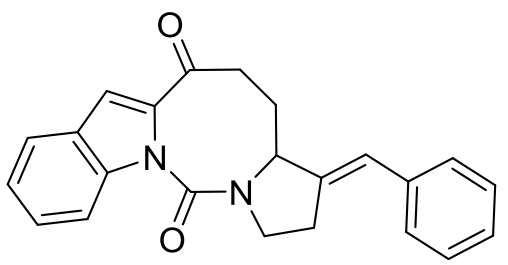

$\mathbf{X X}$

\begin{tabular}{|c|c|c|c|c|c|}
\hline Catalyst (\%) & Ligand (\%) & Additive (\%) & $\mathbf{T}\left({ }^{\circ} \mathbf{C}\right)$ & $\begin{array}{l}\text { S.M } \\
(\%) \\
\end{array}$ & $\begin{array}{c}\text { Product } \\
(\%)\end{array}$ \\
\hline $\operatorname{Rh}(\operatorname{cod})_{2} \mathrm{OTf}(7.5)$ & None & $\begin{array}{c}4-\mathrm{NMe}_{2} \mathrm{C}_{6} \mathrm{H}_{4} \mathrm{COOH} \\
(30)+\mathrm{Na}_{2} \mathrm{SO}_{4}(100) \\
\end{array}$ & 130 & Trace & 0 \\
\hline $\operatorname{Rh}(\operatorname{cod})_{2} \mathrm{OTf}(7.5)$ & $\mathrm{P}\left(4-(\mathrm{F}) \mathrm{C}_{6} \mathrm{H}_{4}\right)_{3}(15)$ & $\begin{array}{c}4-\mathrm{NMe}_{2} \mathrm{C}_{6} \mathrm{H}_{4} \mathrm{COOH} \\
(30)+\mathrm{Na}_{2} \mathrm{SO}_{4}(100)\end{array}$ & 130 & Trace & 26 \\
\hline $\mathrm{Rh}(\operatorname{cod})_{2} \mathrm{OTf}(7.5)$ & $\mathrm{P}\left(\mathrm{C}_{6} \mathrm{H}_{5}\right)_{3}(15)$ & $\begin{array}{c}4-\mathrm{NMe}_{2} \mathrm{C}_{6} \mathrm{H}_{4} \mathrm{COOH} \\
(30)+\mathrm{Na}_{2} \mathrm{SO}_{4}(100)\end{array}$ & 130 & Trace & 30 \\
\hline $\operatorname{Rh}(\operatorname{cod})_{2} \mathrm{OTf}(7.5)$ & $\mathrm{P}\left(4-(\mathrm{OMe}) \mathrm{C}_{6} \mathrm{H}_{4}\right)_{3}(15)$ & $\begin{array}{c}4-\mathrm{NMe}_{2} \mathrm{C}_{6} \mathrm{H}_{4} \mathrm{COOH} \\
(30)+\mathrm{Na}_{2} \mathrm{SO}_{4}(100)\end{array}$ & 130 & Trace & 25 \\
\hline $\operatorname{Rh}(\operatorname{cod})_{2} \mathrm{OTf}(7.5)$ & $\mathrm{P}\left(3,5-\left(\mathrm{CH}_{3}\right)_{2} \mathrm{C}_{6} \mathrm{H}_{3}\right)_{3}(15)$ & $\begin{array}{c}4-\mathrm{NMe}_{2} \mathrm{C}_{6} \mathrm{H}_{4} \mathrm{COOH} \\
(30)+\mathrm{Na}_{2} \mathrm{SO}_{4}(100) \\
\end{array}$ & 130 & 19 & 40 \\
\hline $\operatorname{Rh}(\operatorname{cod})_{2} \mathrm{OTf}(7.5)$ & $\mathrm{PCH}_{3}\left(\mathrm{C}_{6} \mathrm{H}_{5}\right)_{2}(15)$ & $\begin{array}{c}4-\mathrm{NMe}_{2} \mathrm{C}_{6} \mathrm{H}_{4} \mathrm{COOH} \\
(30)+\mathrm{Na}_{2} \mathrm{SO}_{4}(100) \\
\end{array}$ & 130 & 14 & 26 \\
\hline $\mathrm{Rh}(\operatorname{cod})_{2} \mathrm{OTf}(7.5)$ & $\mathrm{P}\left(\mathrm{C}_{6} \mathrm{H}_{13}\right)_{3}(15)$ & $\begin{array}{c}4-\mathrm{NMe}_{2} \mathrm{C}_{6} \mathrm{H}_{4} \mathrm{COOH} \\
(30)+\mathrm{Na}_{2} \mathrm{SO}_{4}(100) \\
\end{array}$ & 130 & 50 & Trace \\
\hline $\mathrm{Rh}(\operatorname{cod})_{2} \mathrm{OTf}(7.5)$ & $\mathrm{P}\left(3,5-\left(\mathrm{CH}_{3}\right)_{2} \mathrm{C}_{6} \mathrm{H}_{3}\right)_{3}(15)$ & $\begin{array}{c}4-\mathrm{NMe}_{2} \mathrm{C}_{6} \mathrm{H}_{4} \mathrm{COOH} \\
(30)+\mathrm{Na}_{2} \mathrm{SO}_{4}(100)\end{array}$ & 120 & 11 & 48 \\
\hline $\mathrm{Rh}(\operatorname{cod})_{2} \mathrm{OTf}(7.5)$ & $\mathrm{P}\left(3,5-\left(\mathrm{CH}_{3}\right)_{2} \mathrm{C}_{6} \mathrm{H}_{3}\right)_{3}(15)$ & $\begin{array}{c}4-\mathrm{NMe}_{2} \mathrm{C}_{6} \mathrm{H}_{4} \mathrm{COOH} \\
(15)+\mathrm{Na}_{2} \mathrm{SO}_{4}(100)\end{array}$ & 120 & 7 & 55 \\
\hline
\end{tabular}

\section{References}

1. Yoakim, C.; Ogilvie, W. W.; Cameron, D. R.; Chabot, C.; Guse, I.; Haché, B.; Naud, J.; O'Meara, J. A.; Plante, R.; Déziel, R. $\beta$-Lactam derivatives as inhibitors of human cytomegalovirus protease. J. Med. Chem. 1998, 41, 2882-2891.

2. McCreanor, N. G.; Stanton, S.; Bower J. F. Capture-collapse heterocyclization: 1,3diazepanes by $\mathrm{C}-\mathrm{N}$ reductive elimination from rhodacyclopentanones. J. Am. Chem. Soc. 2016, 138, 11465-11468.

3. Shaw, M. H.; McCreanor, N. G.; Whittingham, W. G.; Bower, J. F. Reversible C-C bond activation enables stereocontrol in Rh-catalyzed carbonylative cycloadditions of aminocyclopropanes. J. Am. Chem. Soc. 2015, 137, 463-468.

4. Wu, X. W.; Ji, H. T. Ruthenium(II)-catalyzed regio- and stereoselective $\mathrm{C}-\mathrm{H}$ allylation of indoles with allyl alcohols. Org. Lett. 2018, 20, 2224-2227. 
5. Schaus, S. E.; Brandes, B. D.; Larrow, J. F.; Tokunaga, M.; Hansen, K. B.; Gould, A. E.; Furrow, M. E.; Jacobsen, E. N. Highly selective hydrolytic kinetic resolution of terminal epoxides catalyzed by chiral (salen)Co ${ }^{\mathrm{III}}$ complexes. Practical synthesis of enantioenriched terminal epoxides and 1,2-diols. J. Am. Chem. Soc. 2007, 124, 1307-1315.

6. Ji, Y.-Y.; Lin, S.-D.; Wang, Y.-J.; Su, M.-B.; Zhang, W.; Gunosewoyo, H.; Yang, F.; Li, J.; Tang, J.; Zhou, Y.-B.; Yu, L.-F. Tying up tranylcypromine: novel selective histone lysine specific demethylase 1 (LSD1) inhibitors. Eur. J. Med. Chem. 2017, 141, 101-112.

7. Su, X.; Sun, Y. H.; Yao, J. N.; Chen, H.; Chen, C. Acid-promoted bicyclization of arylacetylenes to benzobicyclo[3.2.1] octanes through cationic rearrangements. Chem. Commun. 2016, 52, 4537-4540.

8. Motika, S. E.; Wang, Q. Y.; Akhmedov, N. G.; Wojtas, L.; Shi, X. D. Regioselective amine-borane cyclization: towards the synthesis of 1,2-BN-3-cyclohexene by copper-assisted triazole/gold catalysis. Angew. Chem. Int. Ed. 2016, 55, 11582-11586.

9. Shaw, M. H.; Melikhova, E. Y.; Kloer, D. P.; Whittingham, W. G.; Bower, J. F. Directing group enhanced carbonylative ring expansions of aminosubstituted cyclopropanes: rhodiumcatalyzed multicomponent synthesis of $\mathrm{N}$-heterobicyclic enones. J. Am. Chem. Soc. 2013, $135,4992-4995$.

10. Boger, D. L.; Patel, M. Total synthesis of prodigiosin, prodigiosene, and desmethoxyprodigiosin: Diels-Alder reactions of heterocyclic azadienes and development of an effective palladium(II)-promoted 2,2'-bipyrrole coupling procedure. J. Org. Chem. 1988, $53,1405-1415$.

11. Eggers, M. E.; Jog, P. V.; Bates, D. K. Intramolecular sulfoxide electrophilic sulfenylation in 2- and 3-indoleanilides. Tetrahedron 2007, 63, 12185-12194.

12. Polic, V.; Cheong, K. J.; Hammerer, F.; Auclair, K. Regioselective epoxidations by cytochrome P450 3A4 using a theobromine chemical auxiliary to predictably produce Nprotected $\beta$ - or $\gamma$-amino epoxides. Adv. Synth. Catal. 2017, 359, 3983-3989.

13. Hiroto, K.; Takahiro, I.; Risa, K.; Shiho, M.; Yu, W.; Makoto, I.; Takeshi, N.; Mitsuyo, H.; Tetsuto, T. A. Method to prepare optically active acyclic $\alpha$-benzyl ketones by thermodynamically controlled deracemization. Eur. J. Org. Chem. 2013, 36, 8208-8213.

14. Brand, J. P.; Chevalley, C.; Scopelliti, R.; Waser, J. Ethynyl benziodoxolones for the direct alkynylation of heterocycles: structural requirement, improved procedure for pyrroles, and insights into the mechanism. Chem. Eur. J. 2012, 18, 5655-5666. 
15. Zhou, X. K.; Luo, Y. X.; Kong, L. H.; Xu, Y. W.; Zheng, G. F.; Lan, Y.; Li, X. W. $\mathrm{Cp}^{*} \mathrm{Co}^{\mathrm{III}}$-catalyzed branch-selective hydroarylation of alkynes via $\mathrm{C}-\mathrm{H}$ activation: efficient access to $\alpha^{-}$gem-vinylindoles. ACS Catal. 2017, 7, 7296-7304.

16. Wang, G.-W.; Bower, J. F. Modular access to azepines by directed carbonylative C-C bond activation of aminocyclopropanes. J. Am. Chem. Soc. 2018, 140, 8, 2743-2747.

17. Cotton, F. A., Wilkinson, G., Murillo, C. A. \& Bochmann, M. Advanced Inorganic Chemistry, 6th ed. (Wiley, New York, 1999).

18. Becke, A. D. Density-functional thermochemistry. III. The role of exact exchange. J. Chem. Phys. 1993, 98, 5648-52. 